FINAL REPORT

U.S. Department of Energy

\title{
Isolation of Metals from Liquid Wastes: Reactive Scavenging in Turbulent Thermal Reactors
}

\author{
Principal Investigator: J ost O.L. Wendt \\ Department of Chemical and Environmental Engineering \\ The University of A rizona, Tucson Az. \\ Collaborators: \\ Alan R. Kerstein \\ Sandia Laboratories, Livermore, Ca. \\ A lexander Scheeline \\ Department of Chemistry \\ University of Illinois at Urbana-Champaign \\ Arne J. Pearlstein \\ Department of Mechanical and Industrial Engineering \\ University of Illinois at Urbana-Champaign \\ William P. Linak \\ A ir Pollution Technology Branch \\ National Risk Management Research Laboratory \\ US Environmental Protection Agency
}

Project Number: 60326

Grant Number: DE-FG 07-97E R14831

Grant Project Officers:

SC Project Officer: Roland Hirsch

EM Project Officer: Chet Miller

Project Duration: September 15, 1997 to September 30, 2001 


\section{Table of Contents}

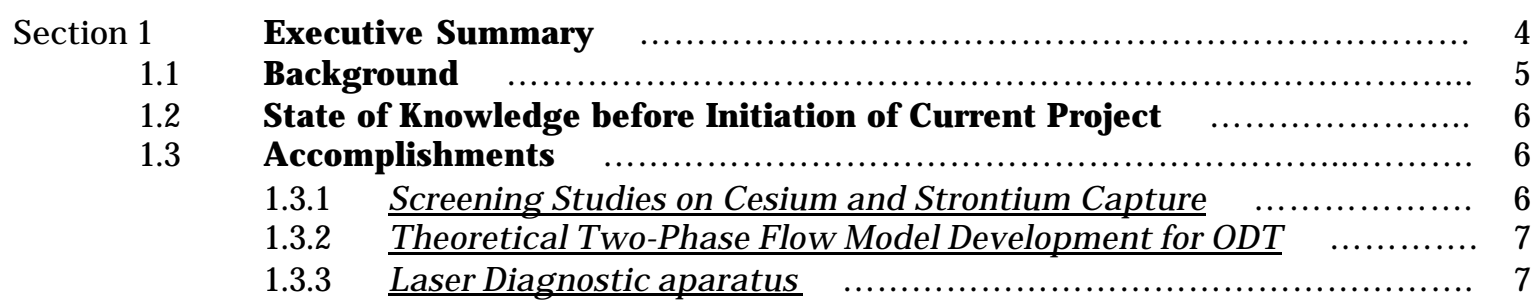

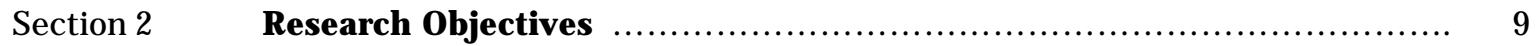

2.1. A Generic Technology for treatment of DOE

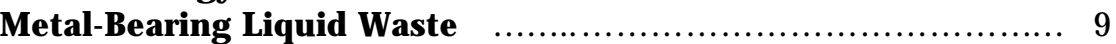

2.2. Community Acceptability of High-Temperature Waste Processing $\quad \ldots \ldots \quad 10$

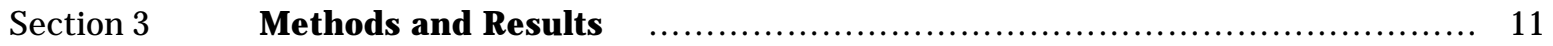

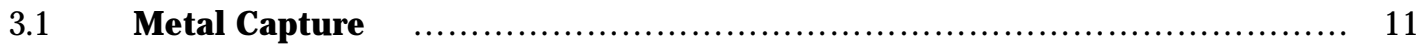

3.1.1 Experimental Methods: Screening Studies on Cesium

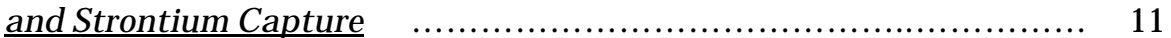

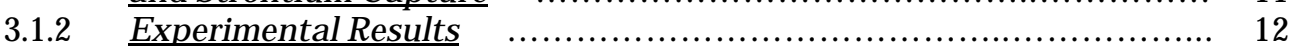

3.2 MathematicalModeling of Individual Droplet Trajectories $\ldots \ldots \ldots \ldots \ldots \ldots . \quad 13$

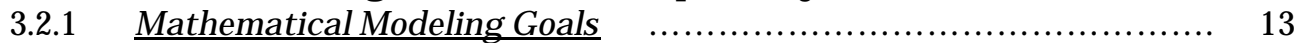

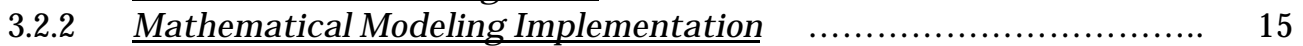

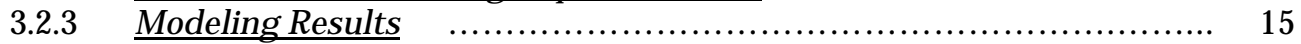

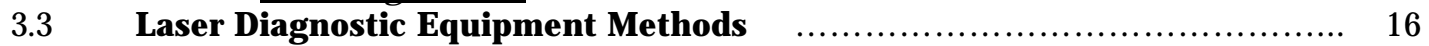

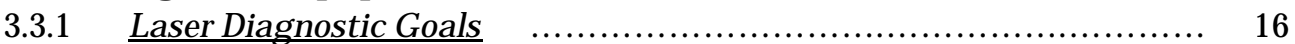

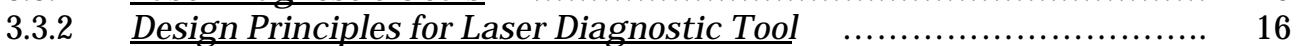

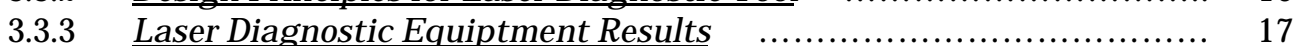

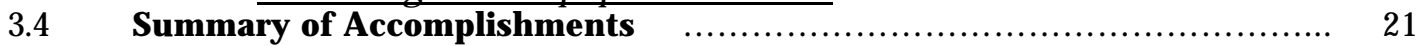

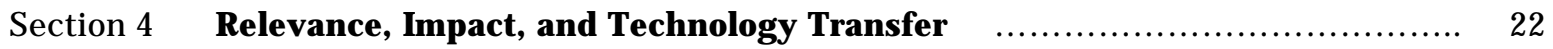

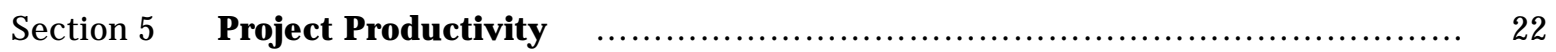

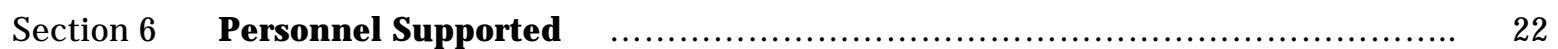

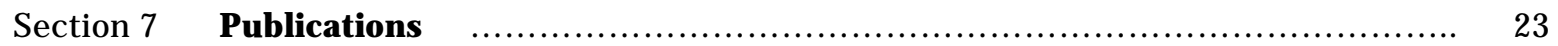

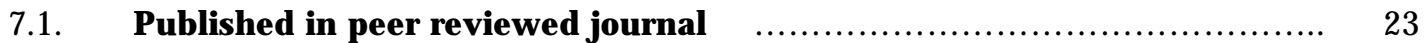

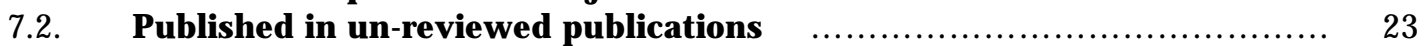

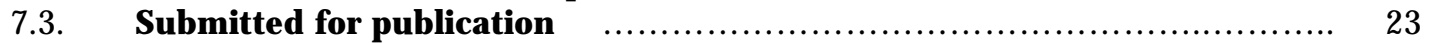

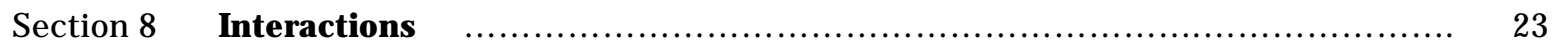

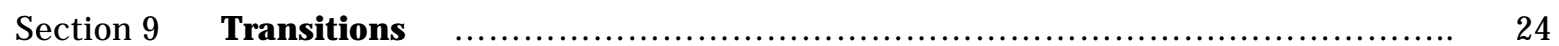

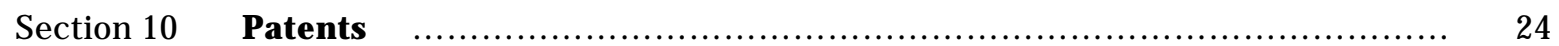

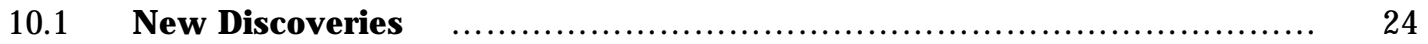

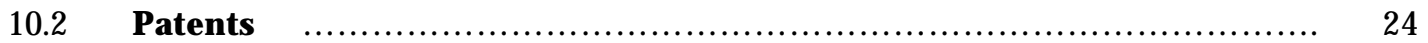

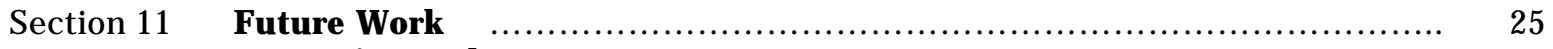

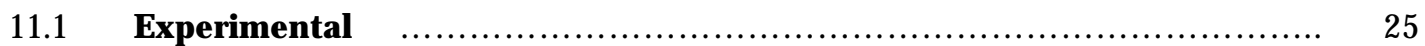

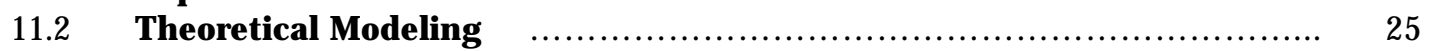

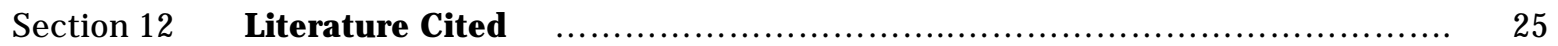


Appendix A Manuscript of "High Temperature Reactive Capture of Cesium and Strontium by Sorbents" J. M. Amos, Department of Chemical and Environmental Engineering, University of Arizona, 1999

Appendix B Manuscript of "Trajectories of Evaporating Droplets in a Turbulent Combustor Using the One Dimensional Turbulence Model" J .R.Schmidt, The University of Arizona, 2000

Appendix C "Trajectories of Evaporating Droplets in a Turbulent Combustor Using the One Dimensional Turbulence Model” J.R Schmidt, J.O.L.Wendt, and A.R.Kerstein, unpublished manuscript 


\section{Section 1 \\ Executive Summary}

This report describes results from a multi-institutional research project involving the University of Arizona (UA), the University of Illinois at Urbana Champaign (UIUC), the U.S. Environmental Protection Agency, National Risk Management Research Laboratory (EPA,NRM RL) and Sandia National Laboratories (Sandia). The objective was to provide the underlying scientific infrastructure behind the use of turbulent thermal reactors for the scavenging of cesium and strontium wastes. Thermal reactors allow these metals to be vaporized, and subsequently to react on kaolinite based substrates to form unleachable metal containing alumino-silicates. To this end, the project consisted of the following components. Chemistry of interaction was to be investigated at the UA by personal from both UA and UIUC. Theoretical models describing the motions of drops and particles in turbulent flow -fields were to be developed at UA working with Sandia and at UIUC. It was hoped that progress in both areas would allow:

1) modeling of the contacting of every last piece of metal waste with the kaolinite sorbent and;

2) prediction of the chemistry of interaction.

Finally, EPA, NRML was to conduct tests in a turbulent flow thermal reactor to confirm the findings of the more fundamental investigations. The goal was to develop a modeling tool that it can be used to help guarantee safe and effective operation of turbulent flow reactors (Wendt, 1992) that use kaolinite pow ders to scavenge and isolate radioactive cesium and strontium.

The overall project demonstrated that toxic metals (cesium Cs and strontium $\mathrm{Sr}$ ) in aqueous and organic wastes can be isolated from the environment through reaction with kaolinite based sorbent substrates in high temperature reactor environments. In addition, a state-of-the-art laser diagnostic tool to measure droplet characteristic in practical 'dirty' laboratory environments was developed, and was featured on the cover of a recent edition of the scientific journal "Applied Spectroscopy" [Cabalo et al., 2002]. Furthermore, great strides have been made in developing a theoretical model that has the potential to allow prediction of the position and life history of every 
particle of waste in a high temperature, turbulent flow field, a very challenging problem involving as it does, the fundamentals of two phase turbulence and of particle drag physics.

\subsection{Background}

Metal-bearing solutions and slurries can pose environmental hazards. Among the most acute of these hazards is the accumulation of radioactive wastes, held in underground steel tanks at locations such as Hanford, WA and the Savannah River Site at Aiken, SC [Terrell, 1994; Zorpette, 1996; Stew art, 1997]. Vitrification of radioactive wastes, either as glass logs [Tollison \& Finger 1997] or cement [Gougar et al. 1999] is the approach most actively being follow ed to sequester radionuclides over the millennia required for decay to negligible activity levels. An important step in preparation of vitrified material is removal of water and organic material, resulting in volume reduction. A mong the ways to perform solvent removal, that simultaneously destroys organic matter, is thermal treatment. This process simply consists of spraying the waste into a high temperature ( $T>1800 \mathrm{~K}$ ) reactor and adding pow dered sorbent material. Water is vaporized, organics are oxidized to water and carbon dioxide, and metallic species oxidized. Scavenging of metal ions by kaolinite [Davis et al. 1999; Davis et al. 1998; Davis and Wendt 2000A; Davis and Wendt 2000B] prior to cooling and oxide formation is a promising technology for high efficiency collection of gas-phase radionuclides. Under optimized conditions, Destruction/Recovery Efficiencies (DRE's) of up to $99.99 \%$ of metal ions in an entering waste stream can be scavenged.

It has been observed that in the incineration of liquid hazardous wastes there exist "rogue" droplets ( $>300$ ? $\mathrm{m}$ diameter) which penetrate past the flame zone and burn as isolated droplets in the postflame gasses [Mulholland et al. 1991]. Detailed knowledge of the droplet burnout points are essential to keeping the destruction removal efficiency in excess of the $99.99 \%$ required by law. These drops avoid hot zones in the reactor, resulting in either incomplete vaporization or vaporization in a zone with insufficient temperature to vaporize the metal contents.

The processes considered all require the metal in question to be volatilized in a hot chamber and then to be reactively scavenged by inexpensive sorbents, which are introduced downstream. The metal is isolated within the molten like particles, which are subsequently collected. In order for this 
process to be rendered effective and safe two issues must be resolved. First, the optimum application and choice of sorbents must be determined; second, there must be a method to guarantee that not one particle of radioactive waste (or, for that matter, any undesirable byproduct) can escape the system. The research performed in this project was designed to create the underlying knowledge that allows these issues to be addressed in practice so that the process can be realized in the field.

\subsection{State of Knowledge before Initiation of Current Project}

Prior to the current research grant, the nature of suitable sorbents for DOE relevant metals had yet to be identified, although, previous w ork on sodium capture by kaolinite suggested that sorbent to be a possible candidate for cesium sorption. No sorbent had been identified for strontium, another metal of interest to DOE. It was required to show that high temperature scavenging reactions betw een cesium and strontium metal vapors and dispersed kaolinite pow ders, were sufficiently rapid to allow isolation of both metals. Prediction of droplet trajectories and of contacting between metal vapor and dispersed kaolinite is also required to make this process viable. Available experimental results [Mulholland et al, 1991] showed that turbulence exerted a profound effect on the trajectories and final burnout points of identically injected droplets. A newly developed model framew ork, the One Dimensional Turbulence (ODT) model created by Kerstein, (1999) appeared to show promise of being able to predict this phenomenon, without requiring the unreasonable (in practice) computational effort of Direct Numerical Simulation (DNS) for the high level of turbulence which the waste would be exposed to during incineration. We believed this model could be extended to predict the individual trajectories of individual droplets and, for that matter, of fluid particles in hot turbulent flow-field. The development of a state-of-the-art Laser diagnostic apparatus was necessary to measure drop size as a function of position, drop and gas-phase temperature, and to observe speciation in the reactor including free atoms, small molecules, and soot. This would create the science supporting a future methodology that would be able to guarantee safe operation of the proposed process for metal scavenging in turbulent thermal reactors.

\subsection{Accomplishments}

1.3.1 Screening Studies on Cesium and Strontium Capture. Screening studies suggested that thermal treatment might be effective in capturing both cesium and strontium on dispersed kaolinite 
pow ders, at high temperatures. Strontium required addition of chlorine to enable metal vaporization at moderately high temperatures, and it was show $n$ that even under those conditions, significant capture was possible. These results are described in more detail in the Master's Thesis of J onathon A mos [A mos, 1999], attached in its entirety as A ppendix A. These results consist of:

a) theoretical equilibrium calculations;

b) experimental studies on cesium and strontium partitioning in the presence of kaolinite sorbent in a $17 \mathrm{~kW}$ laminar flow reactor; and

c) inferences on pertinent mechanisms based on particle morphologies.

Fundamental kinetic rates and mechanisms of the sorption process were beyond the scope of these screening studies., but w ould properly be the subject of future work.

1.3.2 Theoretical Two-Phase Flow Model Development for ODT. Results of this portion of the project are found in the University of Arizona Master's Thesis of J ohn R. Schmidt [Schmidt, 2000], which is attached as Appendix B. The major accomplishments of this research are the development of a tw ophase flow model, which is consistent with the phenomenology of ODT for predicting the particle trajectories of individual droplets and the implementation of many of these drops with burning to simulate individual evaporating droplet streams injected into a turbulent combustor. The spread in trajectory endpoints of these droplet streams was investigated numerically. Results are in good agreement with the measurements and consisted of:

a) Theoretical development of a two-phase flow model which is consistent with the phenomenology of ODT

b) Implementation of a droplet burning model into ODT.

c) A parametric study of how burnout points are effected by initial droplet size, initial droplet velocity, interdroplet spacing, and droplet injection angle.

1.3.3 Laser Diagnostic apparatus. It was desired to measure droplet trajectories in turbulent thermal reactors. To this end a laser diagnostic apparatus was designed, built, and tested. The major accomplishments of this portion of the work was to build a laser imaging instrument for emission, fluorescence, and scattering diagnostics of a $17 \mathrm{~kW}$ gas/air thermal reactor which consisted of: 
a) Design of a laser apparatus using Z-pair parabolic (reflective) mirrors.

b) Building of this apparatus in a dirty lab environment.

c) Demonstration of the laser tool's imaging capabilities

Examples of data show non-axisymmetric flow in a nominally laminar flow system, drop shapes in situ, and Mie scattering from soot. Details of the state-of-the-art laser apparatus, which was also featured on the cover of the volume of the journal, are published in Cabalo et al. [2002], 


\section{Section 2 \\ Research Objectives}

\subsection{A Generic Technology for treatment of DOE Metal-Bearing Liquid Waste}

The DOE metal-bearing liquid waste inventory is large and diverse, both $w$ ith respect to the metals (heavy metals, transuranics, radionuclides) themselves, and the nature of the other species (annions, organics, etc.) present. Separation and concentration of metals is of interest from the standpoint of reducing the volume of waste that will require special treatment or isolation, as well as, potentially, from the standpoint of returning some materials to commerce by recycling.

The variety of metal-bearing liquid waste in the DOE complex is so great that it is unlikely that any one process (or class of processes) will be suitable for all material. How ever, processes capable of dealing with a wide variety of wastes will have major advantages in terms of process development, capital, and operating costs, as well as in environmental and safety permitting. Moreover, to the extent that a process operates w ell with a variety of metal-bearing liquid feedwastes, its performance is likely to be relatively robust with respect to the inevitable composition variations in each waste feed.

One such class of processes involves high-temperature treatment of atomized liquid waste to promote reactive capture of volatile metallic species on collectable particulate substrates injected downstream of a flame zone. Compared to low-temperature processes that remove metals from the original liquid phase by extraction, precipitation, ion exchange, etc., some of the attractive features of high-temperature reactive scavenging are:

- The organic constituents of some metal-bearing liquid wastes (in particular, some low-level mixed wastes) must be treated thermally in order to meet the requirements of the Resource Conservation and Recovery Act (RCRA) and Toxic Substances Control Act (TSCA), and the law s of various states.

- No species need be added to an already complex liquid system. This is especially important in light of the fact that DOE has already experienced problems with organic complexants added to precipitate radionuclides. For example, the Defense Nuclear Facilities Safety Board has expressed, in a formal Recommendation to the Secretary of Energy, its concern about the evolution of benzene vapor in concentrations greater then the low er flammability limit from tanks to which sodium tetraphenylborate has been added to precipitate ${ }^{137} \mathrm{Cs}$ in the "In-Tank Precipitation" (ITP) process at the Savannah River Site. Other species added to the waste in the ITP process are sodium titanate (to adsorb ${ }^{90} \mathrm{Sr}$ and $\mathrm{Pu}$ ), and oxalic acid. A voiding addition of organics to radioactive waste has the additional advantage that is likely to significantly reduce the rate of radiolytic and radiolytically- induced hydrogen generation (c.f. Meisel et al., [1993], in which it is show $n$ that removal of oranics reduces the rate of hydrogen generation in simulated waste from Hanford tank $241-5 Y-101$ by over $70 \%)$.

- Organic species already present are destroyed with very high efficiency. This attribute is especially attractive with respect to high-level tank waste at the Hanford Site, in which large amounts of citrate, glyoxylate, EDTA (ethylenediaminetetraacetic acid), and HEDTA [N-(2hydroxyethyl)-ethylenediaminetriacetic acid] were added to precipitate radionuclides. These organic species are important in the thermal and radiolytic generation of methane, hydrogen, and nitrous oxide, flammable mixtures of which are episodically vented from 25 tanks on Hanford's Fammable Gas Watch List [Hopkins, 1994].

- The same basic approach can be used to treat a broad range of liquid wastes, in each case concentrating the metals (regardless of liquid-phase oxidation state or association with chelators or absorbents) using a collectable sorbent, and destroying any organic species present. In common with the Army's approach (see section 2.2) to the thermal destruction of a 
range of chemical warfare agents (GB, VX, and two blister agents), this may drastically simplify process and plant design and facility permitting, and reduce capital costs, by avoiding development of a separate "w et" process for each type of liquid w aste source.

- The expected robustness of the process with respect to gross feedwaste composition suggests a relatively high degree of tolerance with respect to inevitable variations in the composition of a given metal-bearing feedwaste.

For these reasons, high-temperature reactive scavenging is a potentially attractive approach to the removal of metals from liquid waste in the DOE complex.

\subsection{Community Acceptability of High-Temperature Waste Processing}

One of the issues affecting the viability of large-scale nuclear waste remediation processes is community (i.e. political) acceptability. If a process is not and cannot be made acceptable to a community, it will not operate there. In light of the financial and potential environmental costs of moving large quantities of liquid waste from sites at which a particular treatment process is unacceptable, it is fair to conclude that waste from such a site will not be destroyed by that process anyw here. If a process is unacceptable at all sites where treatable waste is stored, then the process is unusable. In this section, we discuss issues relevant to acceptability, with special emphasis on high temperature processing of extremely hazardous materials.

High-temperature treatment of non-nuclear hazardous waste has been plagued by problems of community acceptability for some time. In some cases, permitting of waste incinerators has been delayed so long that the would-be operators have abandoned the application process. As discussed below, there are, however, cases in which high-temperature treatment facilities have been successfully build and operated. The similarities of those cases to DOE's problems are important.

The primary public concern with regard to thermal treatment is emissions. Under proper conditions, the problem is both technically and politically solvable. This has been proven by the Army's Office of the Program Manager for Chemical Demilitarization (formally the Army Chemical Materiel Destruction Agency) which is successfully using incineration to destroy large volumes of liquid chemical warfare agents (nerve and blister agents). The technology underwent a four-stage Operational Verification Test at J ohnston A toll during 1990-1993. Since then, it has been successfully demonstrated on a production scale at J ohnston A toll Chemical A gent Disposal Facility (CADF) in the Pacific (over 1 million $\mathrm{kg}$ of agent destroyed), and since A ugust 1996 at the Tooele CADF in Utah (over $380,000 \mathrm{~kg}$ of agent destroyed). These facilities have been designed, built, and permitted to meet all applicable civilian regulations (including RCRA, TSCA, the Clean A ir Act, the clean Water Act, and the Occupational Safety and Health Act). In February 1997, a \$586 million systems contract (for design, construction, operation, and closure) was awarded to Raytheon Demilitarization Company for a thermal treatment plant at Umatilla, Oregon.

With passage of the National Defense Authorization Act for Fiscal Year 1992 (Public Law 102190), Congress prohibited the transport of stockpiled chemical warfare agents. After that date, the major factor in gaining community acceptance of incinerators in Utah, Oregon, Alabama, and A rkansas has been the realization that the alternatives are a) thermal destruction; b) further research on alternative technologies, followed by process development and testing, during which time munitions, canisters, and other containers would continue to degrade; or c) indefinite continued storage, again during this time munitions, canisters, and other containers would continue to degrade. The relative attractiveness of proven technology, coupled $w$ ith diligence in operations and a concerted A rmy effort at community involvement and outreach, has led to the present state of affairs.

This project demonstrated how CS and St could be captured by kaolinite. It also took large strides in developing a novel tw o-phase flow model able to predict individual droplet trajectories and environments in combustor turbulent flow fields. This was not merely a random process, since we 
wished to capture the physics of turbulence and dominant turbulent mixing and eddy characteristics without DNS (direct numerical simulation). Kerstein's [1999] One Dimensional Turbulence (ODT) Model appeared to posses the correct attributes to do this. Like DNS, the ODT method requires multiple realizations. How ever, unlike DNS, ODT for droplet trajectories in a practical configuration, is currently "doable" for the turbulent intensities seen in turbulent combustors. We needed to produce a laser diagnostic apparatus to both give in-situ measurements of droplets in an actual combustor and capable of aiding the kinetic studies with in-situ gas phase species measurements. This requires the ability to generate drops, both as sprays and as monodisperse streams, to measure drop size as a function of position, to measure drop and gas-phase temperature, and to observe speciation in the reactor including free atoms, small molecules, and soot.

\subsection{Metal Capture}

\section{Section 3 Methods and Results}

3.1.1 Experimental methods: Screening Studies on Cesium and Strontium Capture. Screening studies focused on capture of cesium and strontium (two important metallic wastes of interest to DOE ) and consisted of:

a) theoretical equilibrium calculations;

b) experimental studies on cesium and strontium partitioning in the presence of kaolinite sorbent in a $17 \mathrm{~kW}$ laminar flow reactor; and

c) inferences on pertinent mechanisms based on particle morphologies.

Fundamental kinetic rates and mechanisms of the sorption process were beyond the scope of these screening studies.

Thermodynamic Predictions. Equilibrium calculations were performed to predict the metal dew points and speciation. Calculations were performed with the NASA Chemical Equilibrium and Applications (CEA) code. Calculations were performed to match experimental conditions $\{100 \mathrm{ppm}$ metal, Stoichiometric Ratio $(S R)=1.2,1000 \mathrm{ppm}$ of dopant gas $\}$. Input thermodynamic data for cesium and strontium compounds were added to the database [Barin, 1994]. Twenty-three strontium and seventeen cesium compounds were considered [A mos, 1999 and A ppendix A]. Cesium by itself has a dew point of $675 \mathrm{~K}$, which categorizes it on the low end of semi-volatile. The presence of chlorine in the system raises the dewpoint to $900 \mathrm{~K}$. This is slightly unusual, in that the presence of chlorine tends to lower the dewpoint of most metals. When sulfur is added to the system, it raises the dew point even higher, to $1300 \mathrm{~K}$. On its ow n, strontium has a dew point of $2000 \mathrm{~K}$. This classifies strontium as a refractory metal. In the presence of chlorine, strontium vaporizes $w$ ith a dew point of $1400 \mathrm{~K}$. This indicates that the presence of chlorine may be necessary in order to reactively capture strontium.

Experimental Studies. Experiments were performed in the University of Arizona 17kW laminar, downflow furnace, operated a $S R=1.2$, with time-temperature profiles similar to those of practical combustors. Metals were injected into a natural gas flame as aqueous solutions of metal salts (cesium acetate and strontium nitrate). Flow rates were set to provide a metal concentration of $100 \mathrm{ppm}$ in the furnace. The sorbent pow der (kaolinite, lime) was injected below flame using a water-cooled probe. The sorbent $w$ as fed at a rate of $2 \mathrm{~g} / \mathrm{min}$. The furnace temperature at the sorbent injection point was $1400 \mathrm{~K}$. Dopant gases $\left(\mathrm{Cl}_{2}, \mathrm{SO}_{2}\right)$ were mixed with the combustion air prior to entering the furnace. Samples were withdraw $n$ from the furnace with a water-cooled isokinetic-sampling probe. The samples were withdraw $n$ after a two second residence time in the furnace, and at a temperature of $1120 \mathrm{~K}$. The sampling rate was 1.3 liters per minute, and samples were collected for ten minutes. The sample stream was diluted with nitrogen at a ratio of 18:1 to quench reactions. Gas samples were passed through a Berner Low-Pressure Impactor (LPI) to segregate particles by size [Hillamo and Kauppinen, 1991], and particles were collected on greased polycarbonate substrates. The substrates were then digested in a strong acid solution prior to analysis. Strontium, aluminum, and calcium were analyzed by flame atomic adsorption spectroscopy, and cesium was analyzed by flame atomic emission spectroscopy. Reactive capture on sorbents was determined by sampling above the 
predicted metal dew point and by using the aerosol size fractionation methods described by Davis et al. [1998]. Strontium, aluminum, and calcium were analyzed by flame atomic adsorption spectroscopy, and cesium was analyzed by flame atomic emission spectroscopy.

3.1.2 Screening Experimental Results. Key results of the cesium tests are shown in Figures 1 and 2, which show the response of the cesium Particle Size Distribution (PSD) when kaolinite sorbent are added to the system. Without sorbent, the cesium alone forms a peak near 0.06 microns, indicating that the cesium is completely vaporized, and then nucleates to form a fume upon cooling in the probe [Davis et al, 1998]. The aerosol size fractionation method identifies the area under the curve, at approximately $1 \mu \mathrm{m}$, as the fraction of cesium that is captured, and the area to the left of this, in the nano-particle size range, as that fraction of the cesium that remains uncaptured [Davis and Wendt, $2000 \mathrm{~A}, \mathrm{~B}]$. In Figure 1, when kaolinite is added to the system, the cesium peak shifts to near 1 micron because it has reacted with the substrate particles. Figure 2 shows that the presence of chlorine diminishes the Cs capture, probably because of the formation of the cesium chloride in the gas phase, although this must be confirmed through in-situ measurements. When lime is added to the system, which is indicated by the calcium peak, there is no shift in the cesium PSD, meaning the cesium does not react with the lime. Data were also obtained with sulfur present, the main effect of $w$ hich is to raise the metal dew point and diminish the time available for reaction.

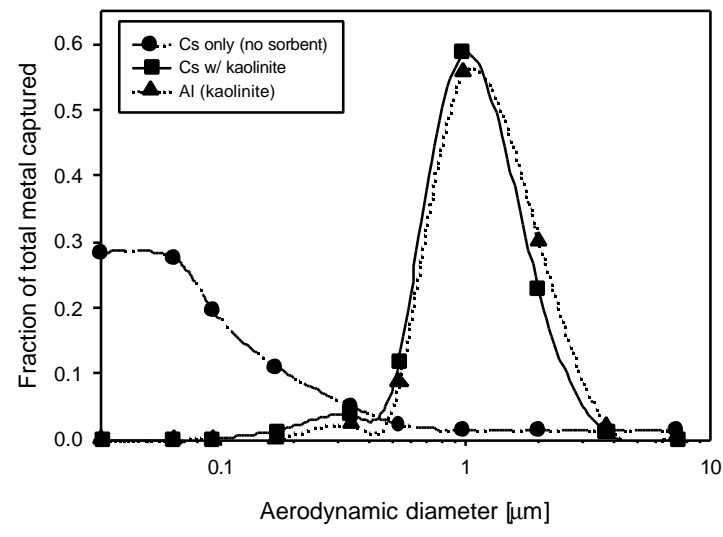

Figure 1. Cesium capture by kaolinite. No chlorine.

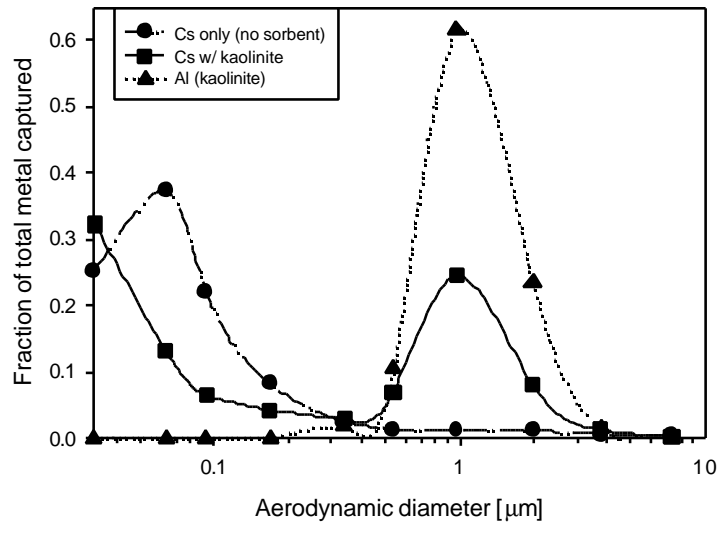

Figure 2 Diminished cesium capture by kaolinite in presence of chlorine.

The key strontium results are show $\mathrm{n}$ in Figure 3. Without chlorine present, the strontium does not volatilize and therefore cannot be captured by a sorbent. It forms large residual particles of unreacted strontium, as show $n$ on the LHS of Figure 3. The presence of chlorine serves to volatilize strontium, as show $\mathrm{n}$ on the RHS of Figure 3, and this does allow capture, if not complete capture, as demonstrated by aerosol size fractionation and the narrow psd on the RHS of Figure 3. Lime as a sorbent showed no capture either with or without chlorine. Sulfur complicated the metal/sorbent interactions.

Figure 4 shows the morphological changes that occur upon reaction of cesium and strontium with kaolinite. In all cases where reaction occurs, a glass melt is formed (center and right), while very little (probably due to impurities) was formed when the kaolinite passed through the same temperature zone alone (left). These experimental screening results are very significant. First, they show that cesium and strontium can be scavenged by an inexpensive sorbent, kaolinite. Second, they show that chlorine is required to devolatilize strontium, but this can also diminish the reaction of cesium. Clearly, additional fundamental data on the pertinent kinetic mechanisms is required in order 
to optimize the use of high temperature kaolinite to reactively scavenge both cesium and strontium in mixed wastes.
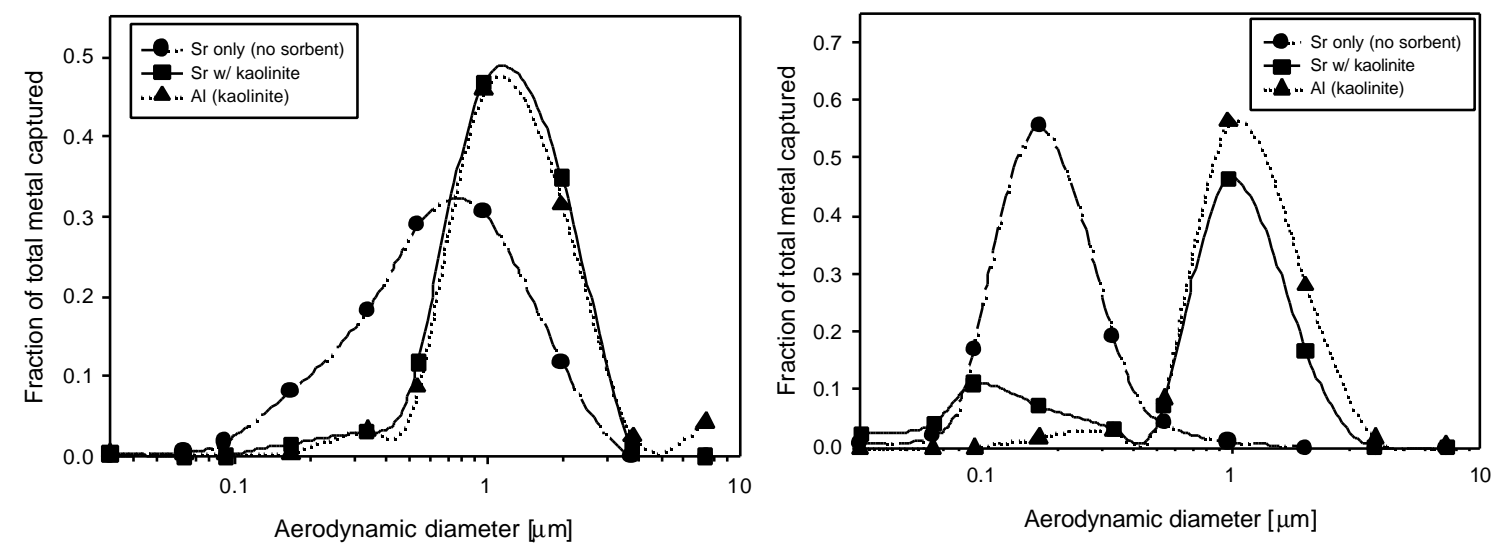

Figure 3. Strontium capture by kaolinite does not occur w ithout chlorine (LHS) but does occur with chlorine (RHS).
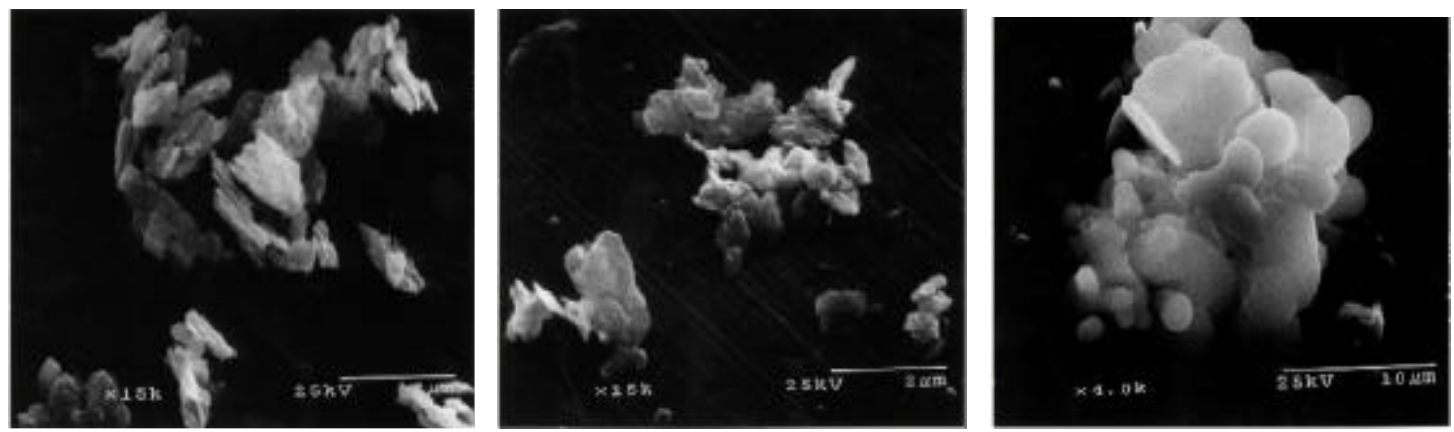

Figure 4. M orphology of unreacted kaolinite (left); product of scavenged cesium, no chlorine (center); product scavenged strontium with chlorine present (right).

\subsection{Mathematical Modeling of Individual Droplet Trajectories.}

3.2.1 Modeling Goals. We wished to determine whether it was possible to predict individual droplet trajectories and environments in combustor turbulent flow fields. This was not merely only a random process, since we wished to capture the physics of turbulence and dominant turbulent mixing and eddy characteristics without DNS (direct numerical simulation). Kerstein's [1999] One Dimensional Turbulence (ODT) Model appeared to posses the correct attributes to do this. Like DNS, the ODT method requires multiple realizations. How ever, unlike DNS, ODT for droplet trajectories in a practical configuration, is currently "doable."

The Problem. The following issues can be raised:

- Experimental data show wide range of droplet endpoints for identical initial conditions [Mulholland et al, 1991]

- We wished to predict individual, as distinct from average, droplet burnout points. 
- We adapted Kerstein's [1999] One Dimensional Turbulence (ODT) model for droplet trajectories.

- We developed new two-phase flow model to be incorporated within ODT, consistent with phenomenology and assumptions in ODT.

There were three phases:

1.

To confirm that ODT can adequately reproduce the axial evolution of average temperature and $z$ velocity as measured by Mulholland. Calibration with experimental data occurred only through initial conditions. The model requires measured mean $v_{z}$ and mean $T$ at $z=0$, and then only one free parameter to be chosen to allow reasonable agreement betw een predicted and measured mean gas axial velocities and temperatures downstream. It is worth noting that, although a triplet map assumes a specific form of stretching and distortion due to turbulence, ODT is implemented such that turbulent energies and momenta are conserved. Triplet maps are modeled to occur instantaneously; yet there is a "turn over" time associated with them that is used to compute the transverse velocity component. ODT contains enough of the physics to replicate the dow nstream laminarization of the turbulent flow as measured also by Mulholland et al [1991].

2. To develop a droplet trajectory model which is consistent with the linearity of ODT. This consumed a major portion of the theoretical modeling effort of the current project, and required implementation of several innovative approaches. It is important to note that $\mathbf{N O}$ additional adjustable parameter was required.

3. To implement burning droplets into the ODT domain and track many realizations of the drop (of order 100) w hile keeping track of the burnout points.

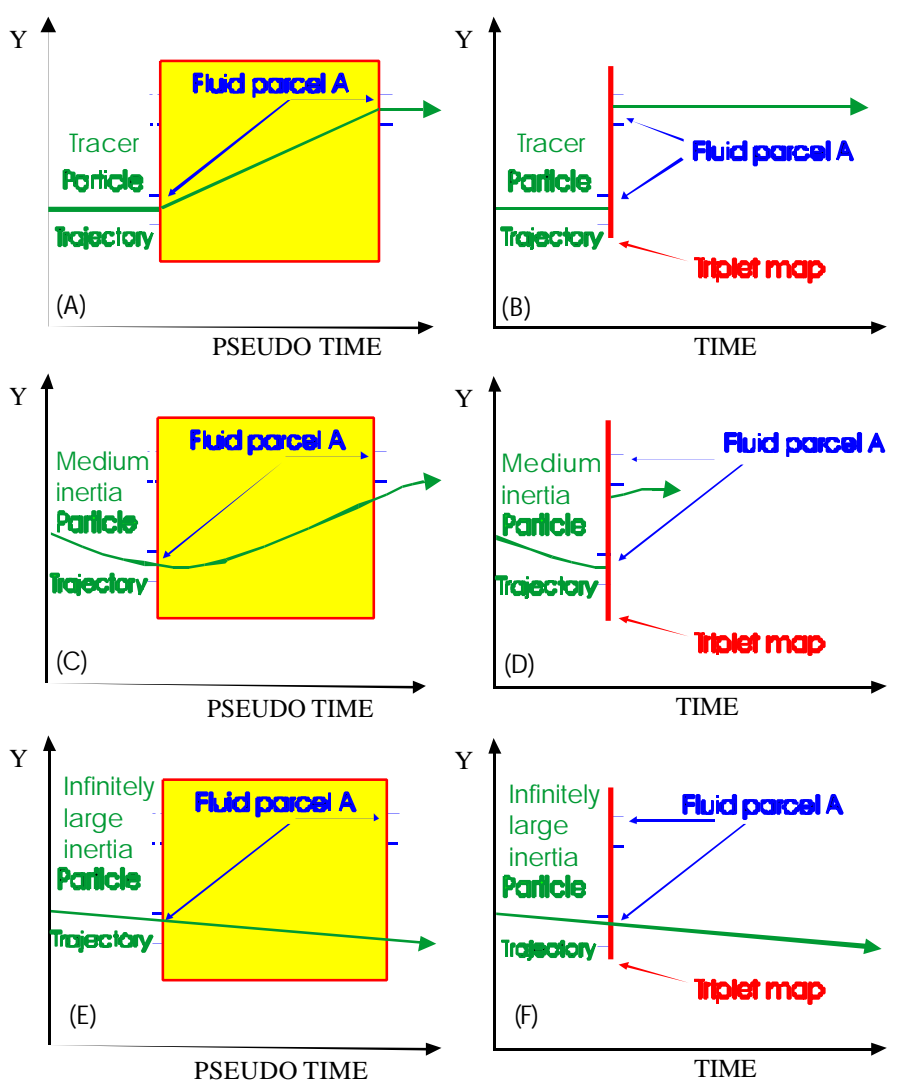

Figure 5. Implementation of two phase flow into ODT. Upper, middle and low er panels show interactions betw een triplet map and low, medium and high inertia droplets respectively. 
3.2.2 Implementation of Droplet Trajectory Model in ODT. Figure 5 depicts the major model development in this research. It schematically shows how droplet drag is implemented in the model. A particle traversing the ODT distance-time domain now can interact with areas (boxes, LHS of Figure 5) rather then lines (w hich are infinitesimally thin, RHS of Figure 5). Low inertia particles (top) follow the gas; medium inertia particles (center panel) do not, and are affected by the drag law, while high inertia droplets show no interaction with local turbulence (as postulated through the local triplet map). A nother issue $w$ as to determine $w$ hen and $w$ here a droplet actually did enter a triplet map. This was complex and is described by Schmidt [2000] and Schmidt, Wendt and Kerstein [2001A, B].

Conservation equations for the heat and mass transfer with burning were solved to predict the burning rates of the drop. The following approximations are used: spherical symmetry, infinitely fast chemical reaction at the flame sheet, pseudo steady state, adiabatic flame temperature. The authors direct you to Schmidt [2000] or A ppendix B for further details on the burning model.

3.2.3 Results for ODT Model. Results are shown on Figure 6. For an ensemble size of 350, experimental results depict the predicted axial spread for the four variations of initial stream conditions, namely diameter, velocity, droplet spacing, and injection angle. A nalysis show s excellent agreement of the predicted spread $w$ ith the measured spread for the variation in initial diameter. The agreement with the variation in initial velocity is also good with both the simulated and experimental data showing a dow nw ard trend as the initial velocity increases. The model show s a marked change in behavior of the spread as the initial spacing/diameter $\left(s / D_{0}\right)$ changes. A bove a value of $s / D_{0}$ of 10 , the particles behave independently. There is very good agreement in the second grouping (the last three cases) of the spread as compared to the measured data. The first grouping is not a good match.
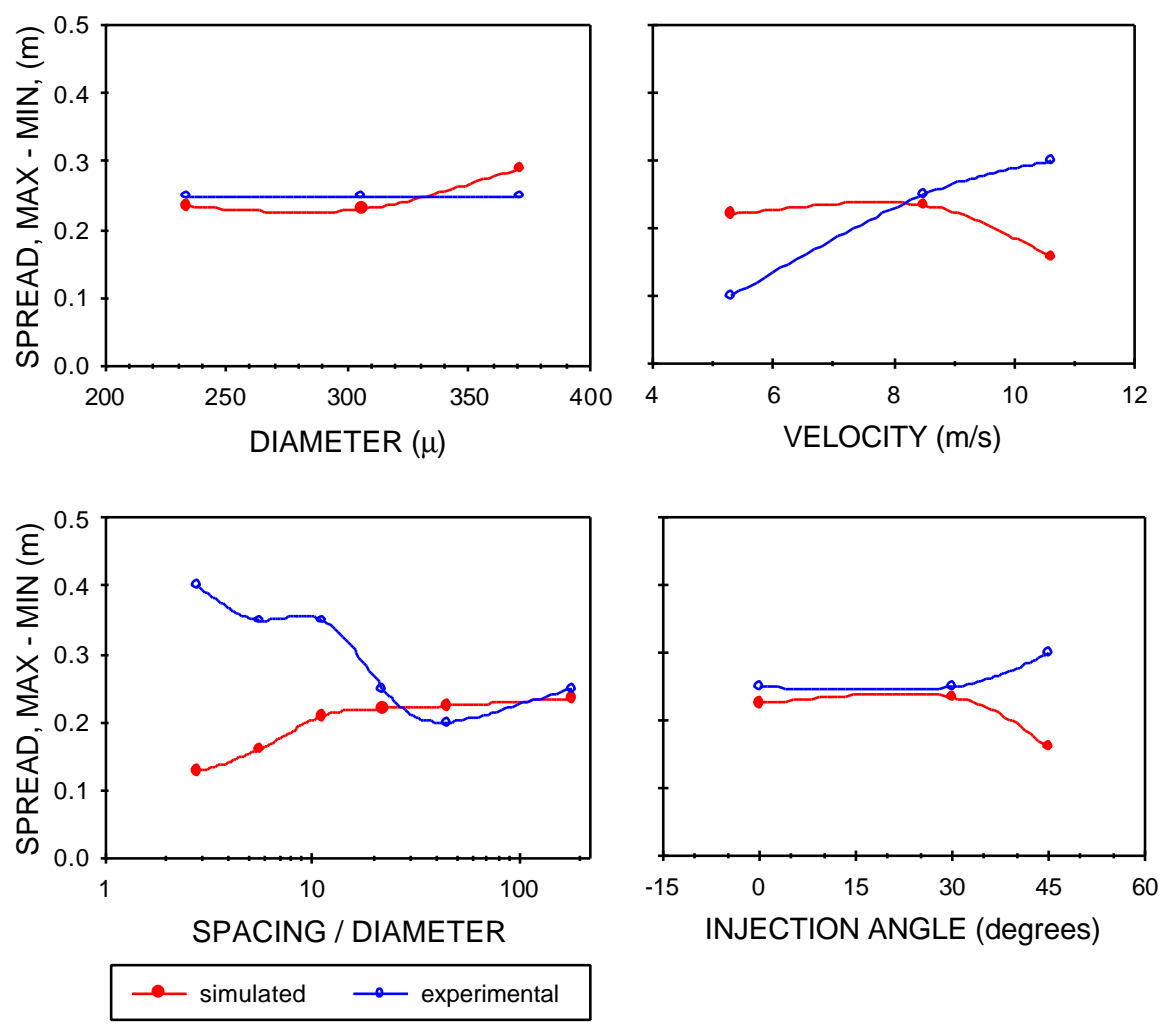

Figure 6: Results: comparison betw een the experimentally measured spread in burn out end points and the spread predicted by the cimulation This simulated data romes from an encemhle size nf 350 
The lack of $s / D_{0}$ parameterization in the ODT droplet submodel is probably the cause of this discrepancy. The agreement for the zero and thirty-degree injection angles is good. The trend of the simulated data is significantly different from the measured data. This is probably due to the artifact created in ODT by the assumption that eddies are instantaneous in time. As the injection angle increases the $y$-component of the particle velocity increases and the error due to this artifact becomes more pronounced.

\subsection{Laser Diagnostic Equipment Methods}

3.3.1 Laser Diagnostic Goals. We needed to produce a laser diagnostic apparatus to test the rogue drop hypothesis. This required the ability to generate drops, both as sprays and as monodisperse streams, to measure drop size as a function of position, to measure drop and gas-phase temperature, and to observe speciation in the reactor including free atoms, small molecules, and soot. With the exception that a lack of a tunable laser prevented observation of free atoms, the apparatus reported here is capable of all these measurements. Drop generation is by use of an electronically-driven oscillating piezoelectric bimorph [Mulholland et al. 1991; Clampitt \& Hieftje, 1972]. Temperature measurement within drops is based on exciplex fluorescence [Melton, 1983 and 1993; Murray \& Melton, 1985; Gossage \& Melton, 1987; Nickle \& M elton, 1989; Bai \& M elton, 1997; Lu \& M elton, 2000]. While we had hoped to measure soot temperature via laser-induced incandescence (LII) [Melton, 1984; Wainner, 1999; Witze et al., 2001], the dispersion of the available spectrograph is more appropriate for atomic or molecular line/band emission rather than the LII continuum.

3.3.2 Design Principles for Laser Diagnostic Tool. To have freedom from chromatic aberration and because of the success of all-reflective spatial and temporal resolution spectrometric systems in recent years, only reflective focusing elements were employed. The only transmissive components are $w$ indow $s$ in the sides of the reactor and one beam splitter.

The excitation laser beam was focused to a sheet illuminating a plane passing through the axis of the cylindrical reactor (Figure 7). A cylindrical mirror, dielectric-coated for maximum reflectivity at $308 \mathrm{~nm}$, converted the rectangular $(24 \mathrm{~mm}$ by $6 \mathrm{~mm}$ ) beam from the excimer excitation laser to a $50 \mathrm{~mm}$ high, $1.6 \mathrm{~mm}$ thick illumination sheet. The central plane of this sheet is the object plane for the observation optics.

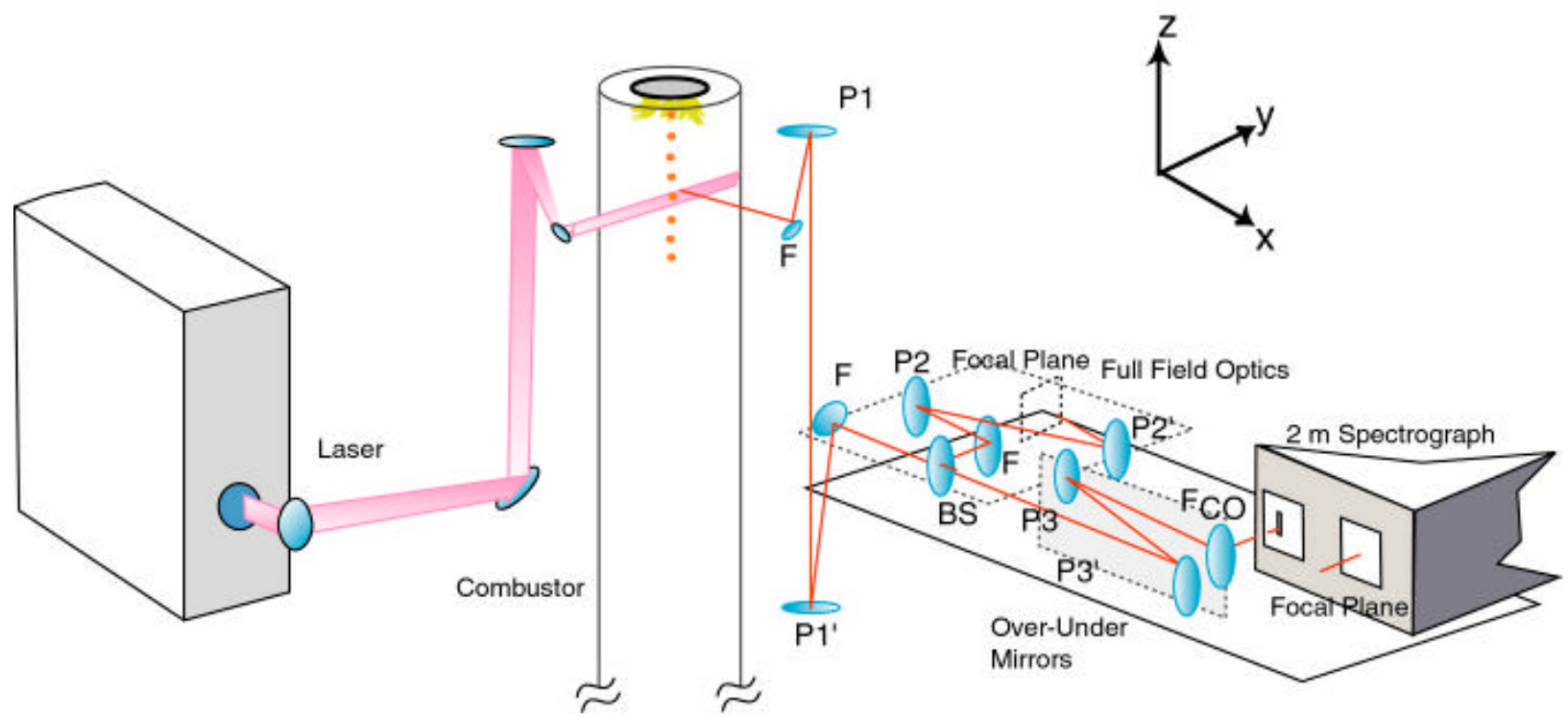

Figure 7. Optical Layout. Two planar folding mirrors direct the $308 \mathrm{~nm}, 25 \mathrm{~ns} 400 \mathrm{~mJ}$ beam to a cylindrical mirror that focuses the beam through a quartz window into the center of the reactor. The observation optics are orthogonal to the illumination optics, directing the fluoresced/emitted/scattered light to either a non-dispersed focal plane or to a $2 \mathrm{~m}$ spectrograph. 
Stigmatic imaging was based on the work of Goldstein and Walters [1976] as later implemented by Klueppel et al. [1978], Hsu et al. [1989], Scheeline et al. [1984], and Scheeline et al. [1991]. In this arrangement, images are relayed from point to point using parabolic mirrors, illuminated off-axis. Mirrors of nearly identical focal length are used in Z-pairs, so that each pair has no third-order coma and a controlled amount of astigmatism. Parabolic figure guarantees freedom from first order spherical aberration. In contrast to most previous implementations of this design, closed-form approximations in the placement of optics have been supplemented by ray traces using Zemax (Focus Softw are, Tucson, AZ).

For some experiments, either wavelength-integrated measurements or measurements limited only by bandpass filters are desirable. For other measurements, sub-nanometer resolution as most conveniently provided by a scanning spectrograph is desirable. The optical system was designed with two focal planes (Figure 7), one of which used a $2 \mathrm{~m}$ Czerny-Turner spectrograph for dispersion (M inuteman Laboratories, A cton, MA), the other of which simply focused an image onto an intensified CCD camera. Because of the $10.92 \mathrm{~mm}$ astigmatism of the spectrograph, different astigmatism compensation optics were designed for the two focal planes. The initial image transfer was from a single Z-pair of mirrors; just beyond the astigmatic focal region for this pair was located a UV beam splitter.

In contrast to typical spectroscopic work, the optical system resided in a lab that can most succinctly be described as "dirty." Coal dust from experiments and infiltrating sand particles from the A rizona desert are frequent specimens in the reactor bay. UV laser optics are highly susceptible to damage from surface dust, yet dust was a concomitant of nearly all experiments. We thus covered the optical system with Lucite (which also served to enhance eye safety, as polymethylmethacrylate absorbs $308 \mathrm{~nm}$ laser light), and pumped dry HEPA-filtered air into the enclosure. Despite months of experiments on coal dust combustion taking place on both the reactor in which we observed drop dynamics and in an adjacent reactor, which together left a thick coating of dust on the outside of the spectrograph and Lucite box housings, the optical path remained free of detectable dust and the mirrors never suffered damage.

The only excitation yet employed on this apparatus is the $308 \mathrm{~nm} \mathrm{XeCl}$ laser band (Lambda Physik COM Pex 201, Ft. Lauderdale, FL). Ultraviolet wavelengths were chosen so that signals w ould be well away from the blackbody continuum emitted by the reactor walls. Pulsed illumination assisted in temporal selection against continuum while assisting in obtaining strong signals from single drops. Signal-to-noise ratio calculations suggested that illumination at over $10 \mathrm{~mJ}$ per pulse w ould be required to obtain adequate LIF, LII, and Mie scattering signals.

3.3.3 Laser Diagnostic Equiptment Results. To date, work has focused on the use of filtered full-field images to characterize drop trajectories and sooting behavior. Spectrographic/high resolution data have not been useful for single laser firings, as no atomic or diatomic molecular species present in the reactor absorb and efficiently fluoresce at $308 \mathrm{~nm}$. Both the $\mathrm{Al} \mathrm{I}$ and $\mathrm{OH}^{\bullet} X^{2} \Pi_{i} \rightarrow A^{2} \Sigma^{+}$band overlaps with excimer emission, however, several conditions (see Cabalo et al., 2002) precluded observation.

We thus confine discussion to observations of drops, gas flows, and soot. Drops of either water or diesel fuel were used. Water had an acitone (5\%) tracer added. No tracer was required with diesel drops because of naturally occurring aromatic hydrocarbons that are present in diesel fuel and are strongly pumped at $308 \mathrm{~nm}$, fluorescing strongly as show $\mathrm{n}$ in Figure 8.

The drop sizing capability of the apparatus was investigated by comparing the size measured by the apparatus with known drop sizes. Results are shown in Table I. In all cases, optically determined sizes are less than those expected, typically by approximately 1 pixel or $5 \%$. Considering that some evaporative loss is expected and that optical magnification is calculated to be 1.003 , this is acceptable. In addition to drop sizing, drop imaging was quite effective in determining drop shapes. Smaller drops $(\mathrm{d}<500 ? \mathrm{~m})$ tended to be spherical, and larger drops tended to be slightly flattened. Drop shape and size are extremely important for determining drop drag coefficients that help predict 


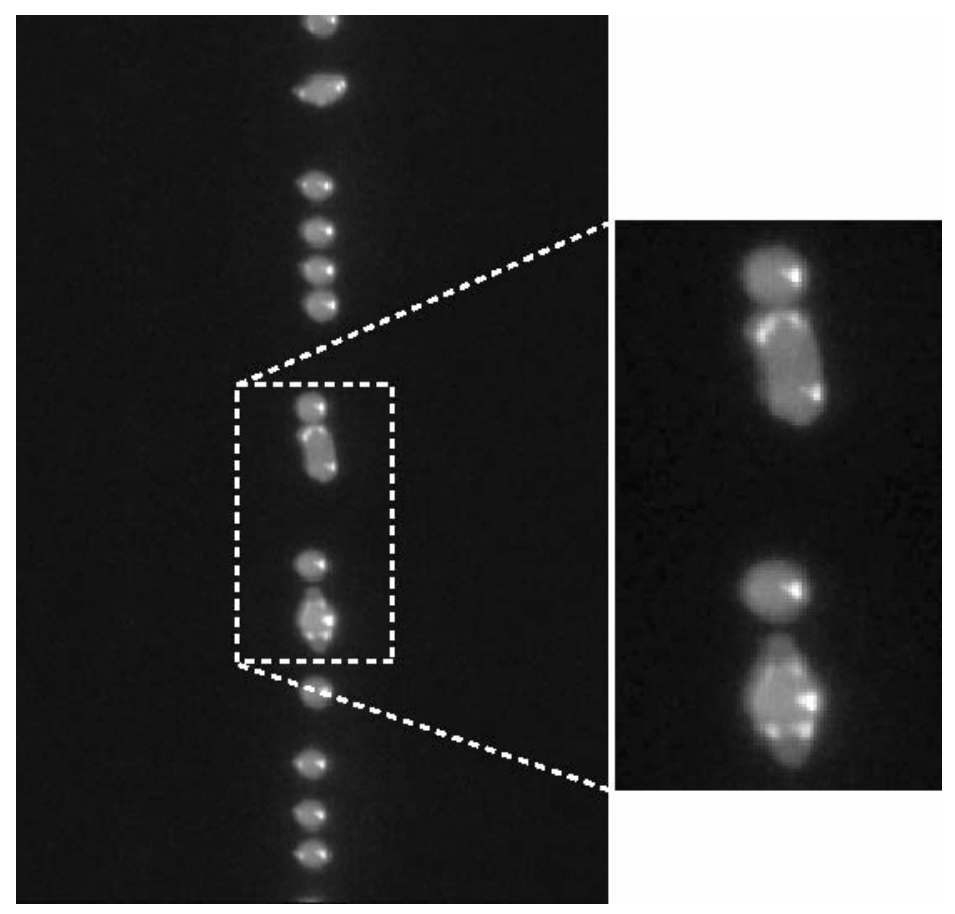

Figure 8 Drop stream showing agglomeration of adjacent drops.

the penetration of drops into the combustor. The drop size and shape also affect the transport of vapor material aw ay from the drop.

At sufficiently high spray rates, drop collisions and coagulation can be seen (Figure 8). Drop grow th as well as vaporization can thus be observed. The fate of diesel drop groups was observed at three points positioned along the centerline of the furnace $12.1 \mathrm{~cm}, 42.6 \mathrm{~cm}$, and $73.0 \mathrm{~cm}$ dow nstream from the burner. At low gas flow rates, drop groups tended to remain intact even dow $n$ to the third port, with no coagulation evident. However, as the gas flow was increased, the drop stream was broken up, the drops were observed to form clump like formations, and coagulation was observed at the second and third ports. Except at the highest flow rates, the drop groups were not broken up at the first port. We attribute the breakup of drop formations to turbulence. Except at the low est flow rates, drops were rarely observed at the third port, and these drops no longer remained in linear formation.

A nother issue of importance for thermal destruction of liquid waste is the gas phase transport of material originating from the liquid drops and possible uptake by sorbent particles. This was investigated by observing the transport of PAH from diesel drops, as shown in Figure 9. With the Schott BG-25 and WG-320 filters, the camera was optimized for imaging light in the range of $350 \mathrm{~nm}$ to $450 \mathrm{~nm}$, the emission wavelength range for PAH. The most intense PAH signal arises from the liquid phase, where the strong attenuation of laser light through the thickness of the drops appears as the signal rapidly decays from the right to the left in Figure $9 \mathrm{~A}$. Fluorescence from the gas phase PAH in a gas phase concentration map clearly show s the transport of vapor aw ay from the drop array.

Figure 10 shows the radial cross sections of PAH fluorescence from the middle of the drop stream to the edge of the observable volume. The low er curve is the signal through the saddle point between drops, and the upper curve is a cross section through the middle of a drop. The signal through the saddle point decreases in an exponential fashion until it reaches a cusp, which incidentally is where this curve intersects the cross section through the drop. The position of this intersection point does not change with respect to drop injection velocity. This can be explained by the fact that the decay in PAH signal is a combination of diffusive and convective transport aw ay from 

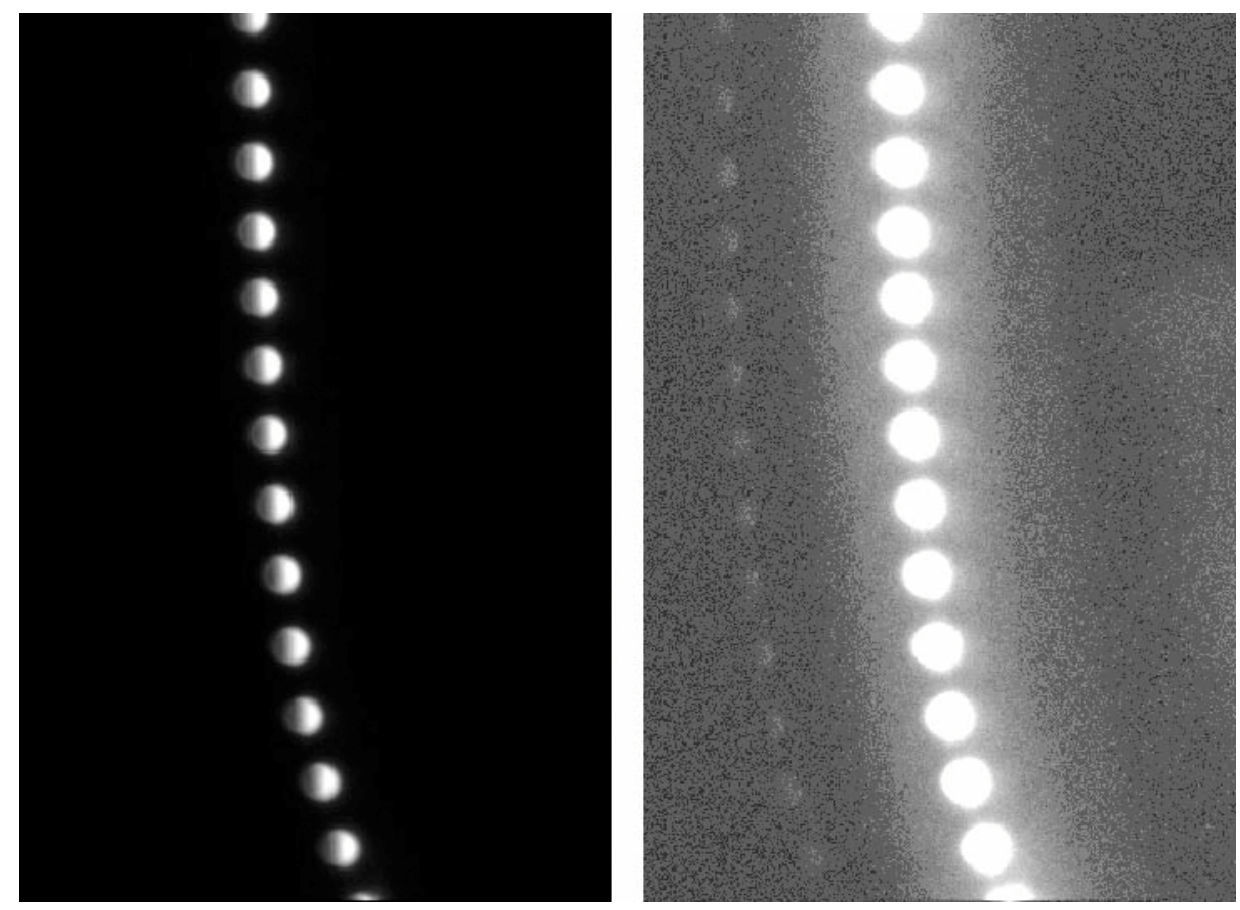

Figure 9 PAH fluorescence both in drops and in the atmosphere surrounding drop trajectories.

the drop stream and the thermal degradation of the PAH. Material vaporizing from the drop stream should experience rapid heating from the diesel wet bulb temperature to $\sim 1700 \mathrm{~K}$. Thus, thermal degradation should be a dominant factor in the decay of PA H signal.

In the case of combustion, soot formation is an important process. Thus, it is important that this system also have the capability of detecting small particles. Soot $w$ as imaged, as show $n$ in Figure 11. Though the CCD camera does not have the resolution to image individual particles less than 25 $? \mathrm{~m}$ in size, the scattered light intensity was much more intense than the fluorescence signals. The apparatus is capable of detecting zones containing sufficient concentrations of particles. Soot appeared in clouds surrounding the drop streams. Only for lean conditions did soot appear to be forming in the wake of individual drops. Otherwise, the soot appeared to be formed from the diesel vapor from previous drops. Some of the images show ed clumping of the soot. Soot was also helpful in visualizing the motion of the gas surrounding the drop stream. At the second and third port, the soot also revealed turbulent eddies, as well as how diesel drops also interacted with the surrounding gas. It is clear from Figure 11 that even at port 2 the drops pulled soot material into their wakes when they were still traveling faster than the surrounding gas.

Table I. Observed vs. computed drop diameter.

\begin{tabular}{|l|l|}
\hline M easured diameter (w eighed drop stream) & A pparent diameter \\
\hline $405 \mu \mathrm{m}$ & $375 \mu \mathrm{m}$ \\
\hline $511 \mu \mathrm{m}$ & $500 \mu \mathrm{m}$ \\
\hline $606 \mu \mathrm{m}$ & $575 \mu \mathrm{m}$ \\
\hline
\end{tabular}



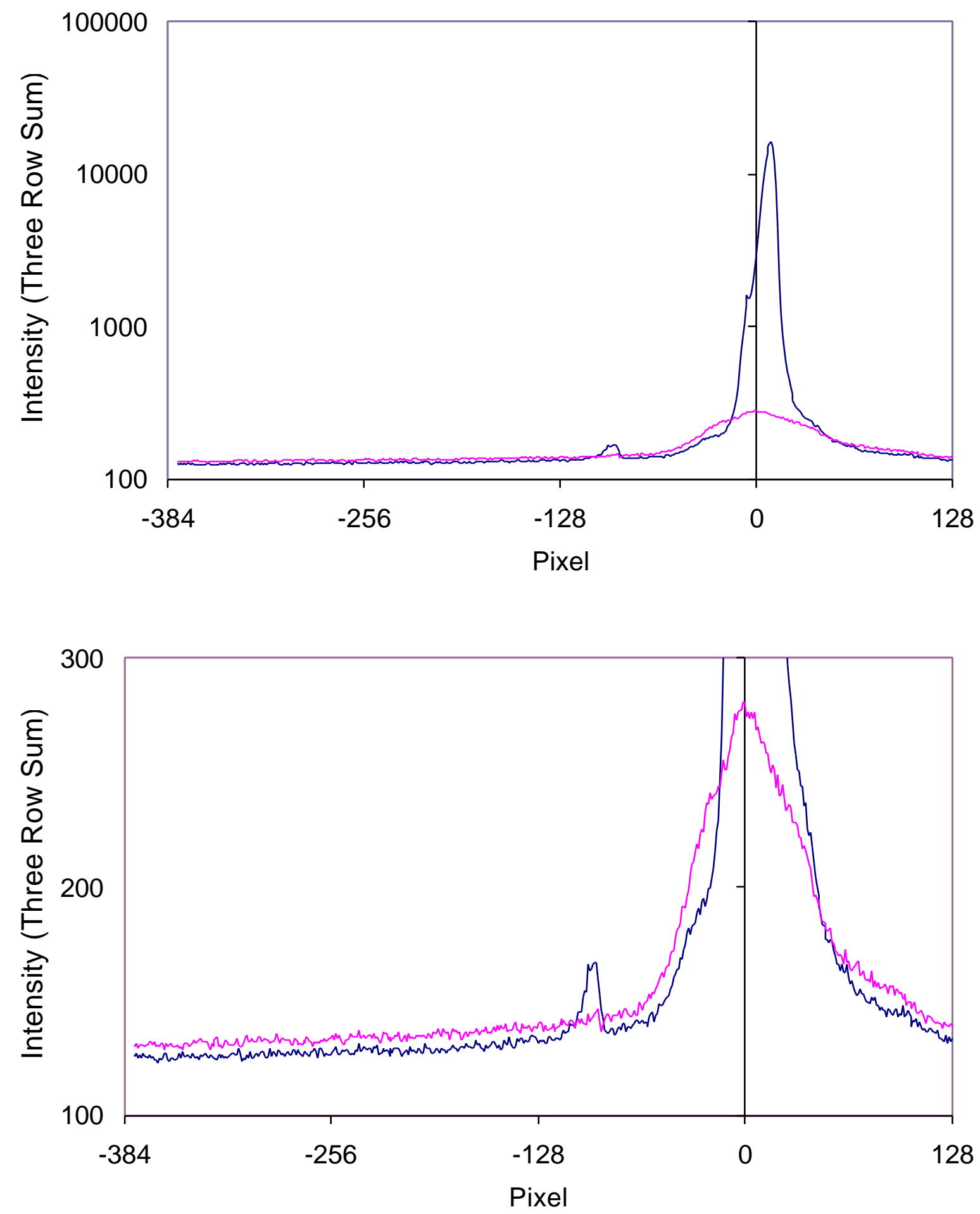

Figure 10 Cross sections of the PAH fluorescence in Figure 9. The ordinate axis position marks the midpoint of the drop array. 


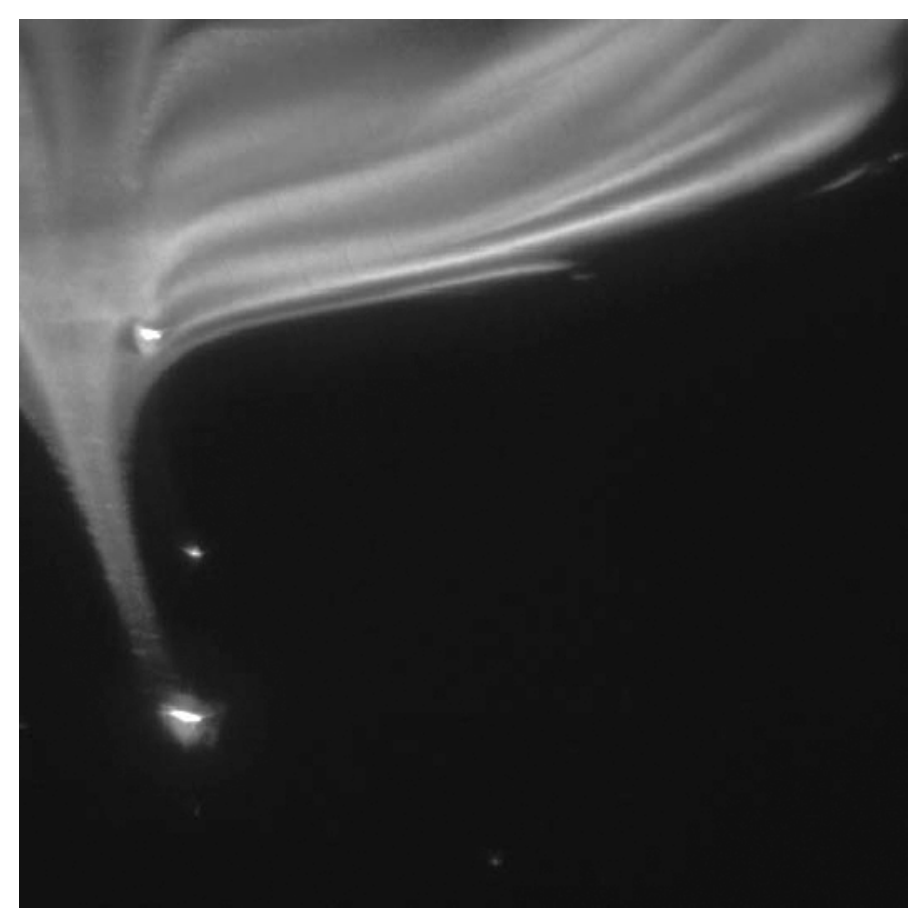

Figure 11 Image of scattered laser light from diesel drops passing through clouds of soot recorded at the second port. The soot material entrained in the drop wake indicates the high drop velocity with respect to the surrounding gases. Note that the drop acts as a lens and focuses laser light to a bright spot.

\subsection{Summary of Accomplishments of Current Grant.}

Research results from the current grant are encouraging. The experimental screening studies show that kaolinite will reactively capture cesium and strontium, to form glassy melts. The conditions under which this capture are complex, and for mixtures of cesium and strontium, require optimum levels of chlorine. The mechanism and kinetics of the process have not been delineated. To accomplish this will require in-situ measurements of metal species within the laminar flow combustor.

The One Dimensional Turbulence Model of Kerstein promises to make it possible one day to track every particle of waste, and every droplet injected into a hot turbulent flow reactor. A turbulent flow reactor would be the practical realization of this process. Public acceptance and process safety of this process would be greatly enhanced if the trajectories and instantaneous locations of all waste and sorbent particles were to be predicted, and the local chemistry know $\mathrm{n}$.

A new, high fidelity imaging apparatus has been constructed for the observation of drop dynamics and vapor transport properties in multi-kilow att thermal reactors. The apparatus is capable of optically determining the size and shape of drops for the determination of drag coefficients. Important events such as drop coagulation can be observed. In addition, suspended and vapor phase material, such as soot and PAH vapor surrounding the drops that pertain to gas phase transport, have been imaged. Soot images showed the interaction between the drops and the surrounding combustion gases as the drops ploughed through. The instrument was also show $n$ to be capable of recording emission spectra within the combustor. 


\section{Section 4 \\ Relevance, Impact, and Technology Transfer}

The relevance of this project lies in its relationship to providing solid scientific underpinnings required to make thermal treatment of radioactive metal wastes a viable and publicly acceptable technology. Two impediments to this technology must be addressed:

1. Lack of knowledge whether the chemistry of interaction between metal vapors and sorbent substrates is appropriate to make the process practical

2. Inability to quantitatively predict the physical processes of interactions between droplets, turbulent flows, metal vapors and dispersed particles, so that there is complete confidence that all the metal vapor is contacted by sorbent within the proper temperature regime.

This project was designed to surmount these two impediments, and to provide predictive tools that allow safety guarantees for thermal treatment processes.

The short term impact of this project is curtailed because, even though great progress has been made, further work was still required to meet these very ambitious goals, and thermal treatment of radioactive wastes remains an anathema both to the public and to practitioners in the field. The long term impact of this work may be substantial, because this work led to a basis for productive future research that may w ell make thermal treatment more acceptable. It also has led to advances in understanding the fundamentals of turbulent tw o phase flow .

Technology transfer to the scientific community is detailed below in Section 7. There has not been significant technology transfer to the field at this time, because application of the project results required further work.

\section{Section 5 Project Productivity}

This project was extremely ambitious and therefore fell somew hat short of meeting all its stated goals. Both the turbulence modeling and the laser diagnostics w ere at the cutting edge of their respective fields, and, not unexpectedly, very difficult problems in each area emerged, and were solved, but at the cost of time and money. They are described in detail in the appendices attached.

The successes of the project can be listed as follow s:

1. Thermal scavenging of both cesium and strontium $w$ as show $n$ to be a viable option for isolating those metals from the environment.

2. A new approach to model two phase turbulent flow was developed, and show $n$ to be the only method currently available to predict individual droplet trajectories that have been measured in combusting turbulent flow fields.

3. A diagnostic tool was developed that allowed droplet positions to be observed nonintrusively. This work was featured on the cover of the October 2002 volume of the scientific journal "Applied Spectroscopy". With additional work, this tool could be improved to allow simultaneous measurement of droplet positions and species concentrations in a turbulent flow thermal reactor.

Details of all of the above are in the Appendices.

\section{Section 6 Personnel Supported}

Personnel who were supported with this research effort at the University of A rizona were:

- Dr. Jost O.L. Wendt, Professor and Head, Department of Chemical and Environmental Engineering, University of A rizona

- J ohn R. Schmidt, Graduate Student, U of A 
- J onathon M. A mos, Graduate Student, U of A

- Dr. Sheldon B. Davis, Post-Doc, U of A

- Dr. J erry Cabalo, Post-Doc (w ork completed at UA, funded through UIUC)

\section{Section 7 \\ Publications}

\subsection{Published in peer-reviewed J ournal.}

"Spectrometric System for Characterizing Drop and Powder Trajectories and Chemistry in Reactive Flow s," by J erry C. Cabalo, J ohn Schmidt, J ost O. L. Wendt, and A lexander Scheeline

Published in A pplied Spectroscopy, J anuary, 2002

\subsection{Published in unreviewed publications.}

Preliminary results for this project were presented at the following symposia and published in the corresponding unreview ed proceeding.

- The First J oint Meeting of the US Sections of the Combustion Institute, George Washington University, Washington, DC March 1999 [A mos \& Wendt, 1999].

- The Sixth International Congress on Toxic Combustion By-Products, Invited lecture, University of Karlsruhe, Germany, J une 1999.[Wendt, 1999].

- The $4^{\text {th }}$ International Syposium \& Exhibition on Gas Cleaning at High Temperatures, University of Karlsruhe (TH),September 1999. [Davis et al. 1999].

- American Flame Research Council Semi-Annual Meeting, Tucson, Arizona, 1999. Papers distributed at the meeting. [A mos \& Wendt, 1999B].

- 2000 Spring Meeting Western States Section The combustion Institute, March 2000. [Schmidt et al., $2000 \mathrm{~A}]$.

- 2000 A merican Flame Research Committee International Symposium, September 2000. [Schmidt et al., $2000 \mathrm{~B}$.

- The Second J oint M eeting of the US Section of the Combustion Institute, March 2001. [Schmidt et al., 2001].

\subsection{Submitted/in preparation for publication.}

A $n$ article dealing with the numerical modeling portion of the completed project was submitted to the peer-reviewed journal. It is entitled, "Trajectories of Evaporating Droplets in a Turbulent Combustor Using the One Dimensional Turbulence Model". The manuscript submitted for publication is reproduced in its entirety in A ppendix C.

A second article dealing with the experimental portion of the completed project is in preparation. It will be submitted to the peer-review ed journal Environmental Science and Technology [A mos et al., in preparation].

\section{Section 8}

\section{Interactions}

This project resulted in a close working relationship between UA and Sandia, to the extent that Dr. Alan Kerstein acted as a co-advisor to Schmidt's MS Thesis and Ph.D. Dissertation, and participated in all the exams. This collaboration is enduring.

This project also required close collaboration between UIUC where the laser diagnostic tool was constructed and UA where the tool was made to work and put to use. This collaboration will also continue. 
Presentations of the preliminary results from this research w ere given at:

- ESMP w orkshop Chicago, J uly, 1998

- The First J oint Meeting of the US Section of the Combustion Institute, March 1999

- The Sixth International Congress on Toxic Combustion By-Products, Germany, J une 1999.

- The $4^{\text {th }}$ International Syposium \& Exhibition on Gas Cleaning at High Temperatures, (TH), September 1999.

- ESMP w orkshop, A tlanta, A pril 2000

- Western States Section The combustion Institute, March 2000.

- A merican Flame Research Committee International Symposium, September 2000.

- The Second J oint M eeting of the US Section of the Combustion Institute, M arch 2001.

\section{Section 9 Transitions}

The results form this w ork need further refinement (see future w ork Section 11 ) in order to undergo the transition to a hazardous waste incineration technology.

\section{Section 10 Patents}

\subsection{New Discoveries}

Cesium alone vaporizes readily in the flame, and subsequently nucleates in the sampling probe to form sub-micron particles. Cesium is almost completely captured in the presence of kaolinite. It is not captured by lime. Chlorine raises the dew point of cesium. This is unusual, in that chlorine tends to low er the dew point of most metals. The presence of chlorine does appear to have some effect on the reactive capture of cesium by kaolinite. It does not appear to affect the capture of cesium by lime. The presence of sulfur also raises the dew point of cesium. How ever, this appears to have little effect on the capture by kaolinite. It does enhance the capture of cesium by lime. This is probably due to the speciation of the cesium compounds.

Strontium alone does not vaporize in the flame, and is only minimally captured on kaolinite and lime, probably by physical coagulation processes and not reactive scavenging. The presence of chlorine enhances the vaporization of strontium and subsequent capture by kaolinite. In this sense, strontium behaves similarly to nickel. How ever, this vaporization does not promote capture on lime. In contrast to the equilibrium predictions, sulfur enhances the volatilization of strontium. How ever, it does not aid in capture.

A novel two-phase flow model was added to the ODT turbulence model utilizing one-way coupling between gas flow and particle trajectories. Correlation between the spread in the burnout points and initial droplet size, droplet velocity, interdroplet spacing, and droplet injection angle are examined. The numerical investigation also includes a droplet heating/burning model. Results are in good agreement with the measurements of Mulholland et al. [1991].

An instrument for emission, fluorescence, and scattering diagnostics of a $17 \mathrm{~kW}$ gas/air thermal reactor was successfully designed buildt and tested. Z-pair parabolic mirrors provide diffractionlimited imaging in field center, with worst-case blur over a $12.8 \mathrm{~mm}$ field of view having a radius of $0.044 \mathrm{~mm}$ RMS. Either full-field filtered or transverse line dispersed view ing can occur simultaneously. Dispersion is achieved using a $2 \mathrm{~m}$ spectrograph f-matched to the feed optics. Examples of data show non-axisymmetric flow in a nominally laminar flow system, drop shapes in situ, and Mie scattering from soot.

\subsection{Patents}

None 


\section{Section 11 \\ Future Work}

\subsection{Experimental}

Given the continuing funding, the UA could conduct laminar flow reactor studies in the $17 \mathrm{~kW}$ downflow reactor to determine the kinetic parameters that control the scavenging of cesium and strontium vapors by kaolinite. In situ diagnostics would be developed by UIUC. Obtaining a tunable dye laser in the future would do this. The only components that would have to be changed to accommodate a tunable operation are the mirrors that relay and focus the laser beam to the mid-plane of the reactor. With a tunable laser radiation source, the transport properties of various materials such as heavy metals and their oxides whose properties are not completely known can be directly measured under combustion conditions. The objective would be to elucidate the mechanism of interaction between cesium or strontium and kaolinite both in the absence and in the presence of chlorine. In-situ measurements would consist of elemental composition of particles, using LIBS, and also gas phase metal speciation, using PLIF.

\subsection{Theoretical Modeling}

Theoretical modeling will focus of further validation of the two phase ODT version. We have already show $\mathrm{n}$ that we can simulate accurately the spread in droplet trajectories that $\mathrm{w}$ as measured by Mulholland et al. (1991). However, this was a rather unique experimental configuration and additional validation is essential, since the model does contain many assumptions. There is a paucity of experimental data analogous to Mulholland's, and so we propose to compare particle flow results from ODT with similar results from DNS (Direct Numerical Simulations). Then we shall incorporate the improved drag coefficients of Pearlstein into the model, and use them to determine maximum penetrations of large deformable droplets in a thermal reactor. Finally we may develop an ODT version that accounts for two separate streams of particles (droplets and sorbent particles) introduced at different locations, and determine the interactions between the two. As before, this work will be jointly supervised by Dr Alan Kerstein, who will co-advise Ph.D. students with Professor Wendt. These Ph.D. candidates will also spend time at Sandia, to learn from Dr. Kerstein. Finally, this model will be compared to experimental data to be obtained through yet another collaboration with $\mathrm{Dr}$. William P. Linak of US EPA in North Carolina.

\section{Section 12 Literature Cited}

A mos, J , M., "High Temperature Reactive Capture of Cesium and Strontium by Sorbents", M.S. Thesis, University of Arizona, Department of Chemical and Environmental Engineering, 1999.

A mos J .M., Davis, S.B., and Wendt, J.O.L. "Evaluation of Sorbent to Reduce Radionuclide Emissions from Mixed Waste Incineration", In preparation, to be submitted to Environmental Science and Technology.

A mos, J .M. and Wendt J .O.L., "High Temperature Scavenging of Cesium and Strontium by Sorbents", Presented at Joint Meeting of the US Sections of the Combustion Institute, George Washington University, Washington, DC March 1999 A.

A mos, J onathan and Wendt J .O.L. "High-Temperature Capture of Cesium and Strontium by Sorbents", A merican Flame Research Council Semi-A nnual Meeting, Tucson, A rizona, 1999 B.

Bai, F. and Melton, L. A "High-temperature, Oxygen-resistant Molecular Fluorescence Thermometers," A ppl. Spectrosc. 51, 1276-1280 (1997).

Barin, I., Thermochemical Data of Pure Substances. Weinheim, FRG, VCH (1995). 
Cabalo, J.C., Schmidt, J.R., Wendt, J.O.L., Scheeline, A., "Spectrometric System for Characterizing Drop and Powder Trajectories and Chemistry in Reactive Flows," Applied Spectroscopy 56, 10, pp $1345-1353,2002$.

Clampitt, N.C., and Hieftje, G. M., "Mechanism of Desolvation of Sample Droplets in Flame Spectrometry," A nal. Chem. 44, 1211-1219 (1972).

Davis, S.B., Amos, J.M., Gale, T.K., Wendt, J.O.L., Biermann, J J .B. and Voogt, N., "Use of Sorbents from Paper recycling Wastes for the High Temperature Capture of Multiple Metals in the Presence of Chlorine" pp 784-796, Proceedings of $4^{\text {th }}$ International Syposium \& Exhibition on Gas Cleaning at High Temperatures, , University of Karlsruhe (TH),Sep 22-24, 1999.

Davis, S. B., Gale, T.K., Wendt, J.O.L. and Linak, W.P., "Multi-Component Coagulation and Condensation of Toxic Metals in Combustors" "Proceedings of the Combustion Institute, Vol 27, pp1785-1791, The Combustion Institute, Pittsburgh, 1998.

Davis, S. B., Gale, T.K. and Wendt, J.O.L. "Competition for Sodium and Toxic Metals Capture on Sorbents," Aerosol Sci. Technol. 32, 142-151 (2000).

Davis, S. B., Wendt, J.O.L. and Linak, W.P. "Interactions between Lead Vapor and Sorbents at High Temperatures," Chem. Phys. Processes Combust. 157-159 (1999).

Davis, S.B. and Wendt, J.O.L. "Mechanism and Kinetics of Lead Capture by Kaolinite in a Downflow Combustor", in press, Proceedings of the Combustion Institute, $\mathrm{Vol} 28(2000 \mathrm{~A})$.

Davis, S.B. and Wendt, J O.L. "Quantitative A nalysis of High Temperature Toxic Metal Sorption Rates using A erosol Fractionation", A erosol Sci. and Technol.,33:536-543 (2000 B).

Goldstein, S. A., and Walters, J .P. "A Review of Considerations for High-fidelity Imaging of Laboratory Spectroscopic Sources. Part I," Spectrochim. Acta, 31B, 201-220 (1976).

Gossage, H. E., and Melton, L.A. "Fluorescence Thermometers Using Intermolecular Exciplexes." A ppl. Opt. 26, 2256-2259 (1987).

Gougar, M. L. D., Scheetz, B.E. and Siemer, D.D. "A Novel Waste Form for Disposal of Spent-nuclearfuel Reprocessing Waste: a Vitrifiable Cement," Nucl Technol. 125, 93-103 (1999).

Hillamo, R. and Kauppinen, E. "On the Performance of the Berner Low Pressure Impactor. "Aerosol Science and Technology 14: 33-47(1991).

Hopkins, J.D. (1994) "Criteria For Flammable Gas Watch List Tanks," WHC-EP-0702, Rev. 0, Westinghouse Hanford Company.

Hsu, W. H., Sainz, M.A. and Coleman, D.M., "An A berration-Corrected Time- and Spatially-Resolved Spectrometer for Studies of Transient Discharges," Spectrochim. Acta, 44B, 109-121 (1989).

Kerstein, Alan R., "One-dimensional turbulence: model formulation and application to homogeneous turbulence, shear flow s, and buoyant stratified flow s." J . Fluid M ech. v392,. pp. 277-334 (1999).

Klueppel, R. J., Coleman, D.M. Eaton, W.S., Goldstein, S.A., Sacks, R.D., and Walters, J.P., "A Spectrometer for Time-gated, Spatially-resolved Study of Repetitive Electrical Discharge," Spectrochim. Acta, 33B, 1-30 (1978). 
Lu, Q. -Z., and Melton, L.A., "Measurement of Transient Temperature Field within a Falling Droplet," AIAA J . 38, 95-101 (2000).

Meisel, D., J onah, C.D., Kapoor, S., Matheson, M.S., and Sauer, M.C., "Radiolytic and Radiolytically Induced Generation of Gates from Synthetic Wastes," A NL-93/43, A rgonne National Laboratory (1993).

Melton, L. A., "Spectrally Separated Fluorescence Emissions for Diesel Fuel Droplets and Vapor," A ppl. Opt. 22, 2224-2226 (1983).

Melton, L. A ., "Soot Diagnostics Based on Laser Heating,"A ppl. Opt. 23, 2201-2208 (1984).

Melton, L. A. "Exciplex-based Vapor/Liquid Visualization Systems Appropriate for Automotive Gasolines," A ppl. Spectrosc. 47, 782-786 (1993).

Mulholland, J.A., Srivasta, R.K., Wendt, J.O.L., Agrawal, S.R., and Lanier, W.S., "Trajectory and Incineration of Rogue Droplets in a Turbulent Diffusion Flame." Combust. Flame, v86, pp. 297-310 (1991).

Murray, A. M., and Melton, L.A., "Fluorescence Methods for Determination of Temperature in Fuel Sprays," A ppl. Opt. 24, 2783-2787 (1985).

Nickle, S. K., and M elton, L.A., "Fluorescence Lifetime and Quenching Rates for $\mathrm{N}, \mathrm{N}, \mathrm{N}$ ',N '-tetramethylp-phenylenediamine in the Vapor Phase,"A ppl. Spectrosc. 43, 1406-1409 (1989).

Scheeline, A., Bye, C.A., Miller, D.L., Rynders, S.W., and Owen, R.C., "Design and Characterization of an Echelle Spectrometer for Fundamental and Applied Emission Spectrochemical Analysis," Appl. Spectrosc. 45, 334-341 (1991).

Scheeline, A., Kamla, G.J., and Zoellner, M.J., "Electron Concentration Measurement in the High Voltage Spark Using Stark Broadening of H? Emission, "'Spectrochim. A cta, 39B, 677-691 (1984).

Schmidt, J.R., Trajectories of Evaporating Droplets in a Turbulent Combustor Using the One Dimensional Trubulence M odel, M asters Thesis, University of A rizona, 2000.

Schmidt, J.R., Wendt, J.O.L., and Kerstein, A.R., "Trajectories of Evaporating Droplets in Highly Turbulent Flow s using the One Dimensional Turbulence Model", 2000 Spring Meeting Western States Section The combustion Institute, March $14 \& 14,2000$ A.

Schmidt, J.R., Wendt, J O.L., and Kerstein, A.R., "Capturing the Spatial Variation of Burning Droplets in Highly Turbulent Flows using the One Dimensional Turbulence Model", 2000 American Flame Research Committee International Symposium, September 18-21, 2000 B.

Schmidt, J .R., Wendt, J O.L., and Kerstein, A.R., "Capturing the Spatial Variation of Burning Droplets in Highly Turbulent Flows using the One Dimensional Turbulence Model", The Proceeding of The Second J oint M eeting of the US Section of the Combustion Institute, March 25-28, 2001.

Schmidt, J.R., Wendt, J .O.L., and Kerstein, A.R., "Trajectories of Evaporating Droplets in a Turbulent Combustor Using the One Dimensional Turbulence Model", submitted for publication.

Stew art, T. L., "Overview of U. S. Radioactive Tank Problem, "Book of Abstracts, $214^{\text {th }}$ ACS National Meeting, Las Vegas, NV I\&EC-055 (1997).

Terrell, C.W., "High Level Waste Management, Processing, and Vitrification at the Savannah River Site," Nucl. Mater. Manage., 23, 233-237 (1994). 
Tollison, S.A. and Finger, S.M., "Glass - the Ultimate Waste Form," Radiat. Prot. Manage. 14, 45-49 (1997).

Wainner, R. T., An A nalytical and Quantitative Analysis of the Laser-Induced Incandescence of Soot, Ph.D. Thesis, Georgia Institute of Technology (1999).

Wendt, J .O.L., Invited lecture "The Fate of Toxic Metals in High Temperature Combustion Systems", Invited Topical Review, The Sixth International Congress on Toxic Combustion By-Products, J une 2730, 1999, University of Karlsruhe, Germany.

Witze, P. O., Hochgreb, S., Kayes, D., Michelsen, H.A., and Shaddix, C.R., "Time-resolved Laserinduced Incandescence and Laser Elastic-scattering Measurements in a Propane Diffusion Flame," Appl. Opt. 40, 2443-2452 (2001).

Zorpette, G., "Confronting the Nuclear Legacy - Part II. Hanford's N uclear Wasteland," Sci. A m. 274(5), 88-97 (1996).

\section{Section 13}

Feedback

There is no feedback at this time. 


\title{
Appendix A
}

\section{Manuscript of \\ “High Temperature Reactive Capture of Cesium and Strontium by Sorbents"}

\author{
J. M. Amos \\ Department of Chemical and Environmental Engineering \\ The University of Arizona \\ 1999 \\ (Self paginated)
}


High Temperature Reactive Capture of Cesium and Strontium by Sorbents

$$
\text { by }
$$

Jonathan Michael Amos

\author{
A Thesis Submitted to the Faculty of the \\ DEPARTMENT OF CHEMICAL AND ENVIRONMENTAL ENGINEERING \\ In Partial Fulfillment of the Requirements \\ For the Degree of \\ MASTER OF SCIENCE \\ WITH A MAJOR IN CHEMICAL ENGINEERING \\ In the Graduate College \\ THE UNIVERSITY OF ARIZONA
}

1999 


\section{STATEMENT BY AUTHOR}

This thesis has been submitted in partial fulfillment of requirements for an advanced degree at The University of Arizona and is deposited in the University Library to be made available to borrowers under rules of the Library.

Brief quotations from this thesis are allowable without special permission, provided that accurate acknowledgment of source is made. Requests for permission for extended quotation from or reproduction of this manuscript in whole or in part may be granted by the head of the major department or the Dean of the Graduate College when in his or her judgement the proposed use of the material is in the interests of scholarship. In all other instances, however, permission must be obtained from the author.

SIGNED:

\section{APPROVAL BY THESIS DIRECTOR}

This thesis has been approved on the date shown below:

Jost O. L. Wendt

Date

Professor of Chemical Engineering 


\section{ACKNOWLEDGEMENTS}

I would like to start by thanking my advisor, Dr. Jost O. L. Wendt. Without the vision and direction of the good doctor, this project would have gone nowhere.

Remember, I was in it for the beer.

Lorenzo Lujan is the man! His skill in fabricating the various and sundry widgets that were needed in the lab was unsurpassed. Even more important, however, was his patience and effort in instructing me in the use of the various shop machines. His patience was especially appreciated when I would break his tools.

I would like to thank the rest of the combustion group for their assistance and for the stimulating exchange of information and ideas they engendered. This motley bunch includes Sheldon Davis, Tom Gale, Greg Ogden, Wayne Seams, John Schmidt, and Art Fernandez.

Thanks are also due to Gary Chandler, for instruction and guidance with the SEM portions of this work.

I would also like to thank the horde of undergraduates who helped with the preparation and analysis grunt work: Cathie Timper, Chris Hammond, John Logan, Danny Sanchez, Daryl Nauman, Jam Sorooshian, Ellen Kitchen, Steve Erickson, Steve Finley, and Blake Norstead.

Nina Welch, Jo Leeming, Pat Elias, Arla Allen, and Rose Myers never receive enough recognition for the administrative support they provide to the department. I would like to thank them for assisting my endeavors here. 


\section{DEDICATION}

This thesis is dedicated to my parents,

Candyce and Michael,

for all their love and support over the years. 


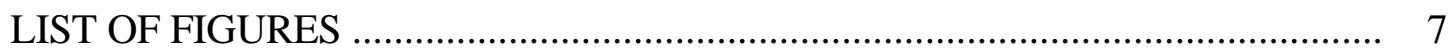

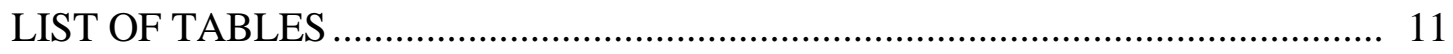

ABSTRACT

1. INTRODUCTION AND BACKGROUND ………………........................... 13

1.1 Cesium and Strontium as Hazardous Waste …………………………............. 13

1.2 Metals in Combustion.............................................................................. 15

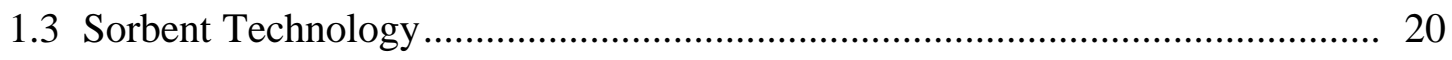

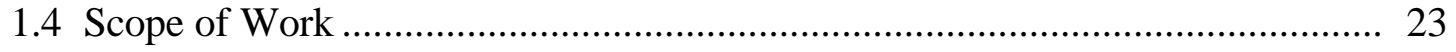

2. THERMODYNAMIC CALCULATIONS..................................................... 24

3. EXPERIMENTAL APPROACH.............................................................. 39

3.1 Furnace Description.......................................................................... 39

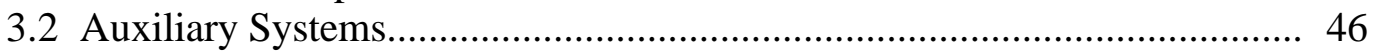

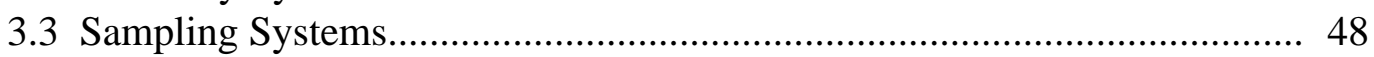

3.4 AA/AE Analysis ............................................................................... 53

3.5 SEM/EDS Procedures........................................................................ 54

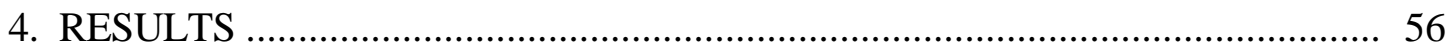

4.1 Introductory Comments ......................................................................... 56

4.2 Cesium Results ................................................................................ 57

4.3 Strontium Results.................................................................................... 72

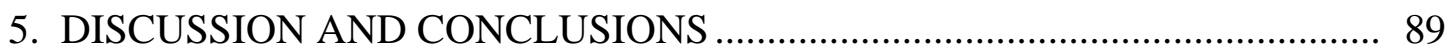

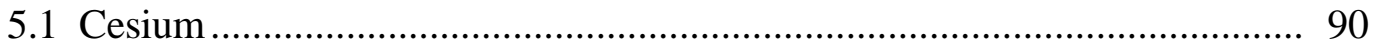

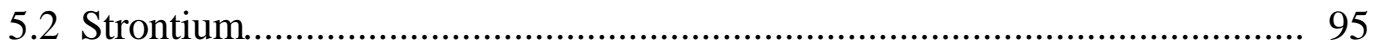

5.3 Areas for Future Work ……………………………………………….... 98

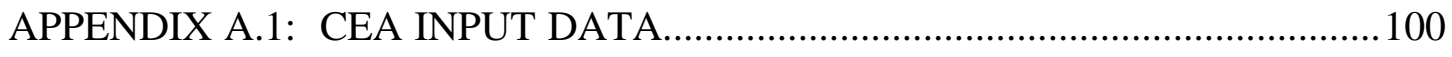

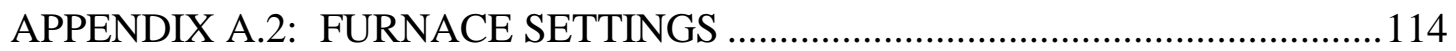

APPENDIX A.3: EXPERIMENTAL CONDITIONS .............................................115 


\section{TABLE OF CONTENTS - Continued}

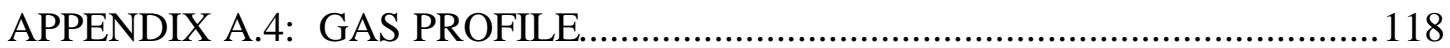

APPENDIX A.5: RAW EXPERIMENTAL DATA........................................... 119

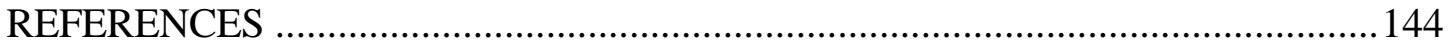




\section{LIST OF FIGURES}

FIGURE 1.1, Potential Metal Interactions in Combustion Systems........................ 16

FIGURE 2.1, Cesium dewpoint calculations ................................................28

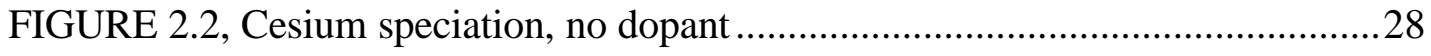

FIGURE 2.3, Cesium speciation, in presence of chlorine ................................... 30

FIGURE 2.4, Cesium speciation, in presence of sulfur ...................................... 30

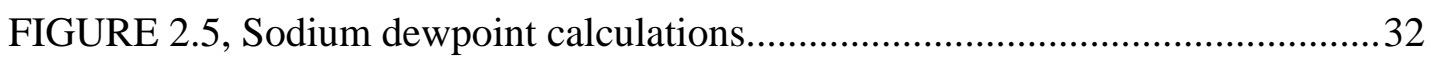

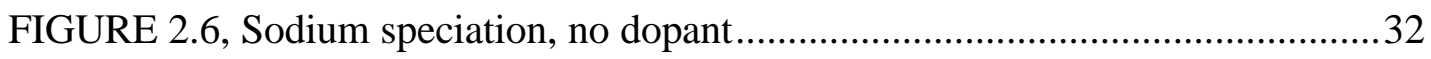

FIGURE 2.7, Sodium speciation, in presence of chlorine ....................................33

FIGURE 2.8, Sodium speciation, in presence of sulfur......................................33

FIGURE 2.9, Strontium dewpoint calculations ............................................... 35

FIGURE 2.10, Strontium speciation, no dopant .............................................. 35

FIGURE 2.11, Strontium speciation, in presence of chlorine ….............................36

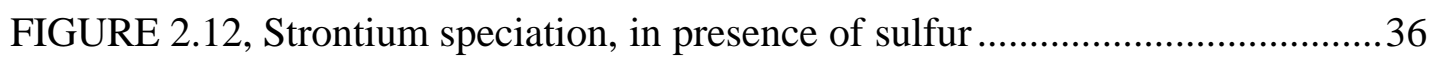

FIGURE 3.1, Laboratory downflow combustor and support apparatus ..................40

FIGURE 3.2, Schematic of furnace materials ............................................... 42

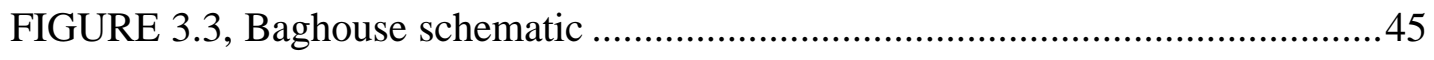

FIGURE 3.4, Average temperature profile at $18 \mathrm{~kW}$ load ..................................49

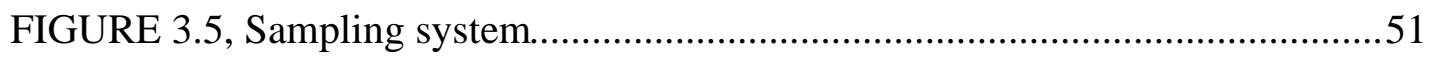

FIGURE 3.6, Berner low pressure cascade impactor ..........................................52

FIGURE 4.1, Cesium and kaolinite, no dopant ...............................................58 


\section{LIST OF FIGURES - Continued}

FIGURE 4.2, SEM micrograph of unreacted kaolinite.....................................58

FIGURE 4.3, SEM micrograph of cesium and kaolinite particles .........................59

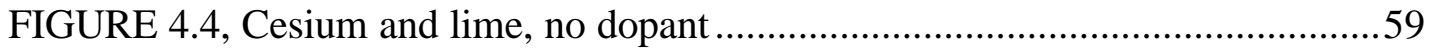

FIGURE 4.5, Cesium and mixed sorbent, no dopant ....................................... 61

FIGURE 4.6, Cesium and CDEM, no dopant................................................... 61

FIGURE 4.7, Cesium and kaolinite, with chlorine ...........................................63

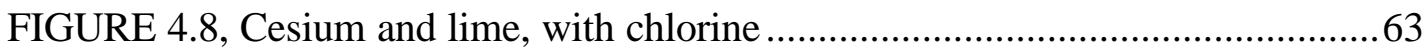

FIGURE 4.9, Cesium and mixed sorbent, with chlorine ...................................65

FIGURE 4.10, Cesium and CDEM, with chlorine ........................................6 65

FIGURE 4.11, Time evolution of cesium/sulfur particles..................................66

FIGURE 4.12, SEM micrograph of cesium particles with sulfur..........................66

FIGURE 4.13, SEM micrograph of cesium particles with sulfur..........................68

FIGURE 4.14, Cesium and kaolinite, with sulfur..............................................6

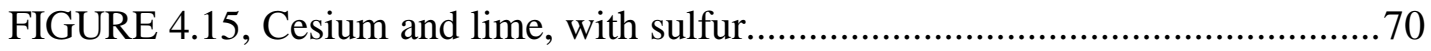

FIGURE 4.16, SEM micrograph of unreacted lime......................................... 70

FIGURE 4.17, SEM micrograph of cesium and lime particles, with sulfur .............71

FIGURE 4.18, Cesium and mixed sorbent, with sulfur..................................... 71

FIGURE 4.19, Cesium and CDEM, with sulfur .................................................. 73

FIGURE 4.20, Strontium and kaolinite, no dopant ............................................ 73

FIGURE 4.21, SEM micrograph of residual strontium particles........................... 74 


\section{LIST OF FIGURES - Continued}

FIGURE 4.22, SEM micrograph of strontium and kaolinite particles ....................74

FIGURE 4.23, Strontium and lime, no dopant ............................................ 76

FIGURE 4.24, SEM micrograph of strontium and lime particles ..........................76

FIGURE 4.25, Strontium and mixed sorbent, no dopant...................................... 77

FIGURE 4.26, Strontium and CDEM, no dopant............................................. 77

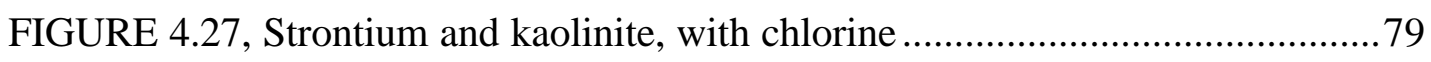

FIGURE 4.28, SEM micrograph of strontium and kaolinite, with chlorine .............79

FIGURE 4.29, SEM micrograph of strontium and kaolinite, with chlorine ..............80

FIGURE 4.30, Strontium and lime, with chlorine ........................................ 80

FIGURE 4.31, Strontium and mixed sorbent, with chlorine ............................... 82

FIGURE 4.32, Strontium and CDEM, with chlorine .......................................... 82

FIGURE 4.33, Time evolution of strontium/sulfur particles ...............................83

FIGURE 4.34, SEM micrograph of strontium particles, with sulfur.......................83

FIGURE 4.35, Strontium and kaolinite, with sulfur......................................... 85

FIGURE 4.36, SEM micrograph of strontium and kaolinite, with sulfur.................85

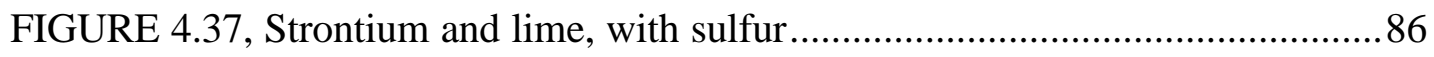

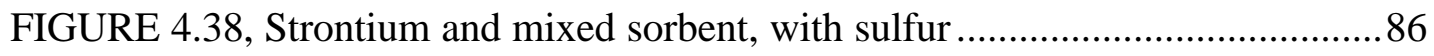

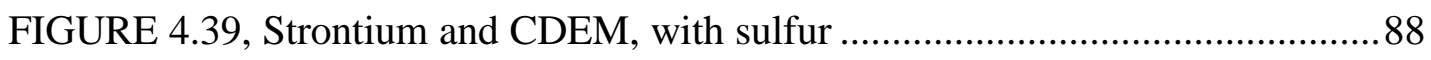

FIGURE 5.1, Weight Percent Oxide vesus Diameter for Cesium on Kaolinite........91

FIGURE 5.2, Weight Percent Oxide vesus Diameter for Cesium on Lime ..............91 


\section{LIST OF FIGURES - Continued}

FIGURE 5.3, Weight Percent Oxide vesus Diameter

for Cesium on Mixed Sorbent ..................................................... 93

FIGURE 5.4, Weight Percent Oxide vesus Diameter for Cesium on CDEM .......... 93

FIGURE 5.5, Weight Percent Oxide vesus Diameter

for Strontium Capture Cases.................................................... 96

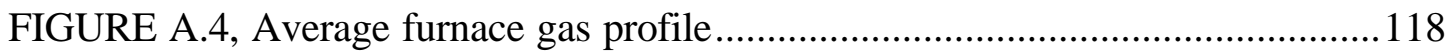




\section{LIST OF TABLES}

TABLE 1.1, Physical Properties of Cesium and Strontium................................... 18

TABLE 2.1, Compounds considered in equilibrium calculations ..........................26

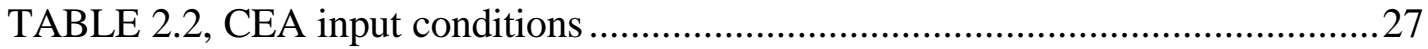

TABLE 3.1, Sorbent Characteristics ............................................................. 47

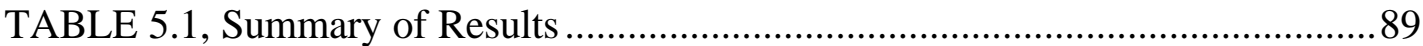




\begin{abstract}
High-temperature reactive scavenging by sorbents is one possible treatment strategy for metal-bearing liquid wastes. The current work focuses on cesium and strontium, two metals that are present in the DOE waste inventory.

To explore the behavior of the metals and the expected speciation, equilibrium calculations were performed prior to the experimental work. Experiments were performed in an $18 \mathrm{~kW}$ laminar downflow furnace. Aqueous solutions of the metals were atomized and injected into the flame. Powdered sorbents were injected into the postflame region of the furnace. The sorbents investigated were kaolinite, lime, a 50/50 mix of lime and kaolinite, and the CDEM product. Particles were sampled from the furnace isokinetically and passed through a Berner low-pressure impactor. The samples were then analyzed with flame atomic absorption spectroscopy and scanning electron microscopy. The effects of adding chlorine and sulfur to the waste stream were also explored.
\end{abstract}




\section{$\underline{\text { Chapter } 1}$}

$\underline{\text { Introduction }}$

\subsection{Cesium and Strontium as Hazardous Waste}

A significant portion of the U.S. Department of Energy's (DOE) hazardous waste inventory is composed of metal-bearing liquid wastes (Serne et al. 1996). These wastes are quite varied with respect to the hazardous constituents (metals, organics, anions, etc.). Release of these wastes into the public domain, through air, water, or ground releases, would have a large detrimental impact on public health. As such, it is very costly to properly store and maintain the facilities for these wastes. A reduction in the volume of these wastes is highly desirable.

Two of the metals present in these wastes are cesium and strontium (Serne et al. 1996). Cesium-137 and strontium-90 are both radioactive wastes that are produced from fission reactions, with half-lives of 30 years and 28 years, respectively. They are often found in reactor wastes, as well as fallout from nuclear accidents. The relatively long half-lives (with respect to human lifetimes) mean these wastes can pose a significant threat to human health over a long period. Any treatment method that is used with these species must be able to immobilize the metals from all possible pathways (air, surface water, ground water, etc.).

There are several different treatment methods available for reducing and destroying these wastes. These include incineration, precipitation reactions, membrane

processes, biological processes, and ion-exchange processes. One of the more promising 
methods is incineration. There are several advantages to this method of treatment. First, the organic portions of the wastes are destroyed with a very high efficiency. For some components of the organic waste, thermal destruction is required by current environmental legislation anyway. Second, no chemical species need to be added to the waste system. The DOE has experienced problems with some precipitation-type treatment methods producing additional hazardous components (benzene and hydrogen) after the addition of the complexing agents. A third advantage to this process is that the same basic approach can be used to treat a variety of wastes, with widely varying compositions. This will help keep costs low, and will simplify the design of any treatment facilities. Fourth, this treatment process is robust, being able to accommodate a large degree of variation in the feedwaste and operating parameters. Finally, incineration has existed for a long time, so the process and its limitations are much better understood than other treatment methods

There are some disadvantages to this treatment method. Metals cannot be destroyed by incineration. Either the metals remain with the non-combustible portion of the waste or they vaporize, depending on the volatility of the metals. Any gaseous metal constituents either condense onto larger particles in the waste stream which are removed by the air pollution control equipment, or nucleate and form sub-micron aerosols of their own. These particles can often penetrate standard air pollution control equipment. These sub-micron particles are enriched in the toxic species, and are easily inhaled into the lungs. They can also deposit into surface reservoirs or food crops. For these reasons, 
incineration is often contested by the public as a viable treatment option (Costner and Thornton 1990).

Sorbent material can be injected into incineration systems to deal with the presence of metals. The metals react with the sorbent particles to form a metal/sorbent complex of some variety. The sorbent materials are inexpensive, readily available materials, such as kaolinite or lime, that have the potential to react with metals. The relatively large sorbent particles are easily removed from the exhaust stream with standard air pollution control equipment. The desire is to form a water insoluble product to prevent the metal from leaching into drinking water supplies after final disposal.

\subsection{Metals in Combustion}

Metals can occur in many different fuels and wastes, including coal, fuel oil, municipal wastes, hazardous wastes, etc. Extensive work has been performed to determine the behavior and fate of metals in combustion systems (Furr et al. 1977; Sill 1988; Fernandez et al. 1992; Ho et al. 1993; Linak and Wendt 1993; Linak et al. 1994; Rizeq et al. 1994; Bool et al. 1995; Gallagher et al. 1996; Karimanal and Hall 1996; Linak et al. 1996; Buckley et al. 1998; Davis et al. 1998; Linak and Wendt 1998; Yang et al. 1998). The review by Linak and Wendt provides a thorough overview of the issues encountered with metals in combustion systems (1993). Figure 1.1, which is adapted from their Figure 4, provides a summary of the possible mechanisms that may govern in a particular system. Based on the volatility of the metal, it will end up in either the supermicron fraction of the ash, which is easily collected and disposed of, or in the 


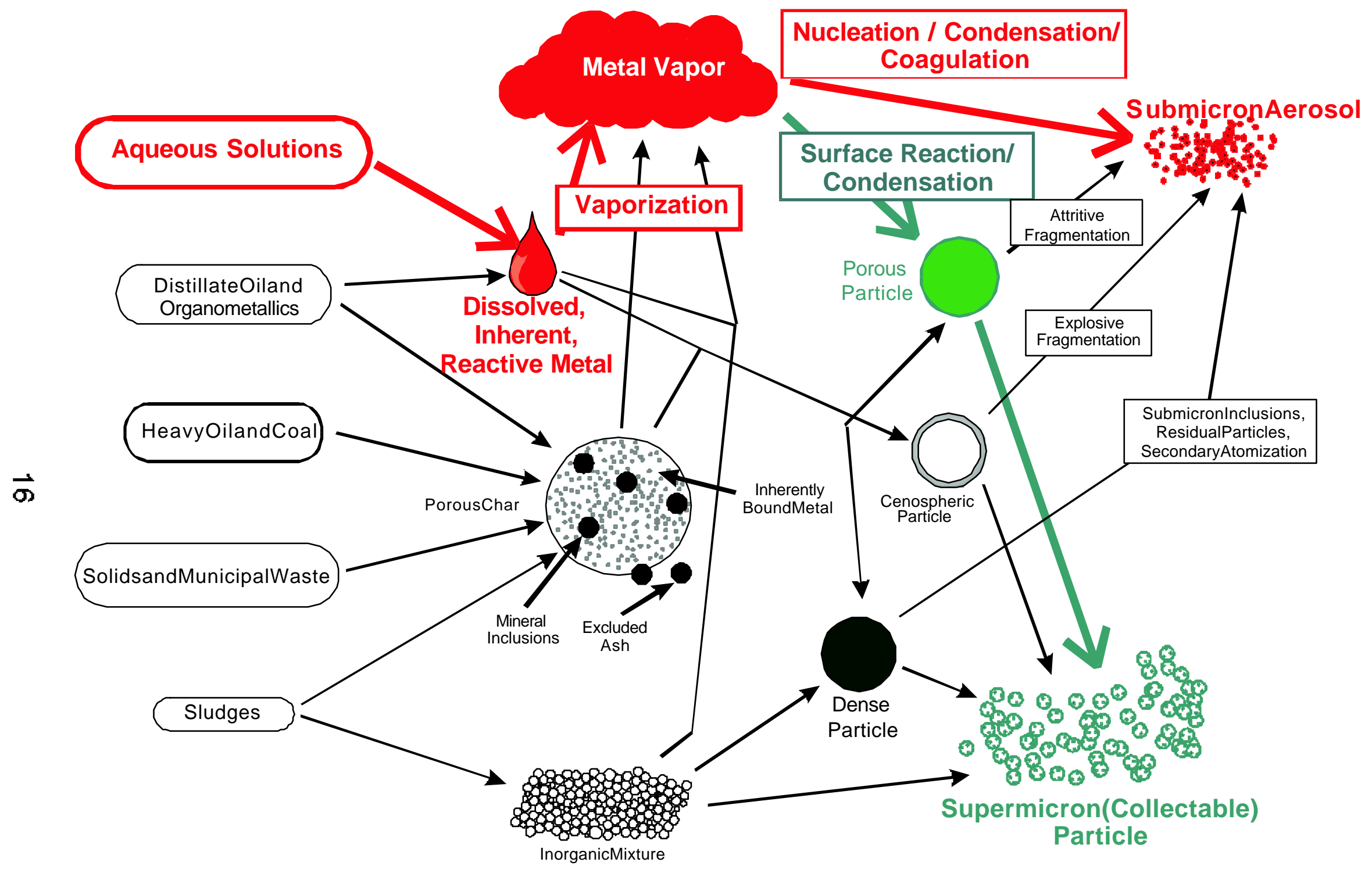

Figure 1.1 - Potential Metal Interactions in Combustion Systems 
submicron aerosols, which are difficult to collect and can easily come in contact with the general population. The authors also discuss in some detail the aerosol mechanisms of coagulation, condensation, and nucleation, which determine the final form of the metals in the system. Finally, they discuss the use of sorbents to capture the metals and remove them from the combustion wastes. This topic will be discussed more in the next section.

The volatility of the metals plays an important role in determining the final form of the metal during combustion. Rizeq et. al. (1994) classified several metals into three categories: volatile, semi-volatile, and refractory. Each category exhibits certain characteristics when exposed to combustion environments. Volatile metals, like mercury and selenium, form vapors readily, and may remain in the gaseous state even when passed through the pollution control equipment. Semi-volatile metals partition in the furnace, with a portion of the metals vaporizing in the flame of the incinerator and then condensing further down the furnace to form a sub-micron fume. This fume may pass through air pollution control equipment, releasing an easily respirable aerosol that is concentrated in the metal. Refractory metals have low vapor pressures, even in the high temperature zone of the furnace. They tend not to vaporize and are generally found in the larger sized ash.

There has been little work with cesium or strontium in combustion systems. There has been no work with sorbent interactions of strontium and only some thermogravimetric studies of cesium and sorbents. Due to this dearth of prior experimental data, it would be useful to examine other metals that may have similar 
characteristics to cesium and strontium. Here, strontium will be compared to nickel, and sodium will be considered an analogue to cesium.

Strontium is considered a refractory metal. It has a dewpoint near $2000 \mathrm{~K}$ at a metal vapor concentration of $100 \mathrm{ppm}$. The relevance of the dewpoint on metals behavior will be discussed further in the next section. Some useful physical data for both strontium and cesium are shown in Table 1.1.

\begin{tabular}{|l|c|c|}
\hline Physical Property & Strontium & Cesium \\
\hline Abbreviation & $\mathrm{Sr}$ & $\mathrm{Cs}$ \\
\hline Atomic Number & 38 & 55 \\
\hline Atomic Weight & 87.6 & 132.9 \\
\hline Melting Point, ${ }^{\circ} \mathrm{C}$ at $1 \mathrm{~atm}$ & 777 & 28.44 \\
\hline Boiling Point, ${ }^{\circ} \mathrm{C}$ at $1 \mathrm{~atm}$ & 1382 & 671 \\
\hline Vapor Pressure, atm at $300 \mathrm{~K}$ & $4.99 \mathrm{E}-23$ & $2.4 \mathrm{E}-9$ \\
\hline Vapor Pressure, atm at $1500 \mathrm{~K}$ & 0.529 & 40.89 \\
\hline Density, g/cm & 2.64 & 1.93 \\
\hline $\mathrm{C}_{\mathrm{p}}, \mathrm{J} / \mathrm{mol} \mathrm{K}$ & 26.4 & 32.2 \\
\hline$\Delta_{\mathrm{f}} \mathrm{H}^{\circ}, \mathrm{kJ} / \mathrm{mol}(\mathrm{gas})$ & 164.4 & 76.5 \\
\hline$\Delta_{\mathrm{f}} \mathrm{G}^{\circ}, \mathrm{kJ} / \mathrm{mol}(\mathrm{gas})$ & 130.9 & 49.6 \\
\hline $\mathrm{S}^{\circ}, \mathrm{J} / \mathrm{mol} \mathrm{K}$ (gas) & 164.6 & 175.6 \\
\hline
\end{tabular}

\section{Table 1.1 - Physical Properties of Cesium and Strontium (Data from (Lide 1995))}

There has been some work that has studied the behavior of nickel, another refractory metal (Linak et al. 1994; Linak and Wendt 1998). In this work, the metal was injected into a furnace, and the resulting aerosol was sampled and analyzed. It was found that the nickel did not vaporize, and the nickel particle size distribution formed a peak around one micron. When chlorine was added to the system, the nickel did vaporize and formed smaller particles when cooled. The results of these previous works may be used to help explain the behavior of strontium. 
Cesium is considered a low-end semi-volatile metal, with a dewpoint near $700 \mathrm{~K}$ at $100 \mathrm{ppm}$ of metal vapor. Cesium is in the same column of the periodic chart as sodium, so the chemical behavior of cesium should be similar to that of sodium. The chemical similarities between cesium and sodium will be explored further in Chapter 2 . There has been some work on the fate of sodium from coal combustion (Gallagher et al. 1996). The ash aerosol from coal combustion was sampled and analyzed. The sodium was found to be rather volatile, with the majority of the sodium appearing on the submicron plates of the impactor. It was also found that the sodium tended to associate with the silica in the ash.

There has been some work to determine the partitioning behavior of cesium and strontium at high temperatures. There have been studies on species present in coal ash (Furr et al. 1977), but these have focused on the presence of a large number of elements, and have not focused on cesium and strontium in particular. Sill (1988) performed volatility experiments on cesium and strontium from synthetic basalt. The volatility of cesium increased linearly as the temperature increased, with some volatility evident even at low temperatures $\left(400^{\circ} \mathrm{C}\right)$. The strontium did not volatilize at any temperature studied.

Yang et. al. (1998) studied the behavior of several different metals. They performed both thermogravimetric studies and small-scale combustor tests. They used cesium chloride for their thermogravimetric tests, and found that the cesium did eventually vaporize $\left(750^{\circ} \mathrm{C}\right)$. This temperature is low for a combustion system. Their small-scale furnace results show that the majority of the strontium ends up in the bottom 
ash of the incinerator. A larger portion of the cesium ended up downstream of the incinerator. However, their experiments were performed at temperatures that are low $\left(850{ }^{\circ} \mathrm{C}\right)$ compared with practical incinerators $\left(1200{ }^{\circ} \mathrm{C}\right)$.

Buckley et. al. (1998) studied the fate of cesium and strontium in the combustion of a biomass fuel. Their experiments were performed on a $100 \mathrm{~kW}$ down flow combustor. Samples were extracted isokinetically and passed through a multi-orifice impactor (MOUDI). Filters were weighed to determine mass collected, and beta activity was measured using an automated alpha/beta counter. The radionuclides tended to concentrate in the sub-micron range ( $\sim 0.4$ microns) when the furnace was operated at higher temperatures $\left(1200{ }^{\circ} \mathrm{C}\right)$. At lower temperatures $\left(800-1000^{\circ} \mathrm{C}\right)$, the radioactivity was spread over a large particle size range, with some concentration in the larger particle sizes. Their analysis techniques did not allow them to separate the cesium and strontium components of the beta radiation contribution. Therefore, the exact distributions of the individual components cannot be determined.

\subsection{Sorbent Technology}

One of the major obstacles towards public acceptance of incineration technologies is the presence of metals in the incinerated wastes, and the potential release of the metals into the environment. Metal capture by sorbents is a promising method of metal control. A lot of work has been done in this area (Ho et al. ; Lee and Johnson 1980; Shadman and Punjak 1988; Punjak et al. 1989; Rizeq and Shadman 1989; Uberoi et al. 1990; Uberoi and Shadman 1990; Uberoi and Shadman 1991; Crosley and Kedl 1992; Scotto 1992; 
Scotto et al. 1992; Scotto et al. 1994; Jozewicz and Gullett 1995; Linak et al. 1995; Wu et al. 1995; Ghosh-Dastidar et al. 1996; Mwabe and Wendt 1996; Venkatesh et al. 1996; Aganihotri et al. 1998; Biswas and Wu 1998; Davis 1999). These studies include both thermogravimetric work and pilot-scale incinerator experiments.

Biswas and $\mathrm{Wu}$ (1998) wrote a review of sorbent control technology. The majority of the paper discusses general metals interactions in combustion environments. A small section does cover the use of sorbents introduced to the post-flame region of furnaces to control the metals. The majority of the sorbent discussion relates to sorbents formed in the furnace by gas-phase precursors, not the introduction of solid sorbents, which is explored in this work.

There has been a relatively larger body of work that has focused on thermogravimetric experiments to determine metals capture by sorbent (Lee and Johnson 1980; Punjak et al. 1989; Uberoi et al. 1990; Uberoi and Shadman 1990; Uberoi and Shadman 1991; Crosley and Kedl 1992; Wu et al. 1995; Ghosh-Dastidar et al. 1996; Aganihotri et al. 1998). Often, these tests are run at temperatures that are lower than typical combustion environments. The contact time between the metal vapors and the sorbents is also rather long, ranging from two to forty hours, compared to a few seconds in an incineration environment. Since these tests are often easier to set up and run than tests on laboratory-scale incinerators, they serve well as screening tests for further research. They are also useful for identifying possible reaction products that may form. Crosley and Kedl (1992) performed thermogravimetric tests on cesium and silica. They heated a sample of $\mathrm{CsCl}$ and passed the cesium vapor over a bed of silica. The 
silica was weighed before and after the experiments were performed, and weight gain by the sorbent was taken as a sign of reaction. They found that cesium does sorb on to silica. They used inductively coupled plasma atomic adsorption spectroscopy to verify the presence of cesium in the sorbent. However, they did not use any other technique to try and determine the final form of the cesium compounds. The authors did propose a method for making educated guesses about particular metal and sorbent combinations, based on finding a stable compound on the phase diagrams of the metal/sorbent systems. This method assumes the final compound is in an equilibrium state, however, and which may not be the case if a glass-like compound is formed. Therefore, this method can serve as a starting point for further investigation, but should not be relied upon for definitive conclusions.

There have also been several researchers who have studied sorbent capture technology in laboratory scale and semi-industrial scale incinerators (Scotto 1992; Scotto et al. 1992; Scotto et al. 1994; Linak et al. 1995; Mwabe and Wendt 1996; Venkatesh et al. 1996; Davis 1999 - not yet in print). Linak, et. al. investigated the capture of nickel onto kaolinite (1995). They found that the nickel would only capture when in the presence of chlorine. Without chlorine present, the nickel did not vaporize and capture. Mwabe and Wendt (1996) performed experiments to determine the capture of sodium onto kaolinite. Sodium was injected as an aqueous solution into a $17 \mathrm{~kW}$ laminar down flow reactor. The sorbent was injected as a disperse powder. Samples were collected isokinetically and passed through an Anderson cascade impactor. They found that sodium does capture onto kaolinite, and they determined a global rate constant for this 
reaction. They also explored the effect of chlorine and sulfur on the capture of the sodium. Both dopants acted to decrease the amount of capture in the system. Chlorine was found to change the speciation of the reacting metal, effectively reducing the amount of metal available for capture. Sulfur was found to increase the dewpoint, which reduces the time the sodium has to react.

\subsection{Scope of This Work}

The purpose of the present work reported here is to explore the behavior of cesium and strontium in combustion systems. Experiments will be performed to determine the metal distribution when the cesium and strontium are injected as salts in aqueous solutions. Various sorbents will be added to attempt to capture the metals and force the metals into a larger particle size distribution. These sorbents are kaolinite, lime, a kaolinite/lime mix, and CDEM sorbent. CDEM sorbent is a paper recycling waste, and its characteristics will be discussed further in the experimental section. In addition, the effects of chlorine and sulfur on the experimental systems will be explored. These two elements are often found in waste streams, and they could have a significant effect on the behavior of the metals in these systems. These are first level screening tests to determine the reactions involved and to determine which combinations of metals, sorbents, and dopants warrant further study. No attempt has been made to optimize the operating conditions to maximize metal capture. Optimization of the process is a subject for future work. 


\section{Chapter 2}

\section{Thermodynamic Calculations}

Prior to beginning experimental work, having some idea of the expected system behavior is useful. In order to accomplish this, thermodynamic calculations were performed. Other researchers have used this technique, both to predict the behavior of chemical species in their experimental systems and to develop a better understanding of the chemistry involved (Linak and Wendt 1993; Frandsen et al. 1994; Durlak et al. 1997; Davis et al. 1998; Linak and Wendt 1998). Buckley et. al. (1998) have performed equilibrium calculations for cesium and strontium species. Their results will be discussed later in this section.

This equilibrium approach does have several limitations that must be kept in mind. First, these calculations model the equilibrium condition of the system. It does not take into account the kinetics of the reactions involved. If the residence time in the real system is not long enough, the calculations will not match the experimental data. Second, this approach assumes that the reactor is thoroughly mixed. In real systems, turbulence and flow patterns may prevent this condition from existing. Finally, all the important chemical species must be included in the input data, or the final results will be misleading.

It is believed that the metal/ sorbent interactions that occur are between a gas phase metal and a solid sorbent (Scotto et al. 1992; Mwabe and Wendt 1996; Davis 1999). As such, it is useful to know when the metal exists in the furnace as a vapor. The dewpoint of the metals, or the point at which the first traces of metal begin to condense, 
represents an ending point for the capture reaction. Below the dewpoint, condensation begins to occur, potentially competing with the capture reaction. Once the temperature drops below the bubble point of the metal, the metal is no longer in a form that will react with the sorbent. The dewpoint of the metals is one of the essential pieces of information that can be obtained from the thermodynamic calculations.

Metals may be considered to fall into three basic categories, based on their volatility (Rizeq et al. 1994). Refractory metals have high dewpoints, and will not exist as vapor in practical combustors under normal circumstances. The dewpoints of refractory metals are usually around $2000 \mathrm{~K}$ or higher at concentrations of approximately $100 \mathrm{ppm}$ of metal vapor in the gaseous mixture. This category includes nickel, chromium, barium, and beryllium. Semi-volatile metals are those metals that will normally vaporize in the flame zone of a furnace, but return to a solid form at the cooler end of the combustor. This category includes lead, cadmium, arsenic, and antimony. The dewpoints of these metals, at a concentration of $100 \mathrm{ppm}$ of metal vapor, range between $1800 \mathrm{~K}$ and $1000 \mathrm{~K}$. Volatile metals are those metals that exist as gases, even in the relatively cool exhaust sections of the combustor. This category includes mercury and selenium, and exhibit dewpoints of less than $1000 \mathrm{~K}$ at $100 \mathrm{ppm}$ of metal.

The computer code used to perform the current calculations was the NASA Chemical Equilibrium and Applications (CEA) code (Gordon and McBride 1994). The numerical algorithm used by CEA is based on the minimization of Gibbs free energy. This allows the use of temperature and pressure as the primary thermodynamic variables, and it permits the chemical species to be treated independently, without specifying the 
chemical reactions beforehand. Some important assumptions are made by CEA. The code considers all gaseous species ideal, and the gaseous mixture is considered ideal as well. All condensed species are treated as pure. It is also important to note that the results obtained from these calculations are the results as they would occur if given infinite time to react. Kinetic and transport limitations may be present in the actual furnace that would prevent the system from achieving thermodynamic equilibrium.

\begin{tabular}{|c|c|}
\hline Cesium compounds considered & Strontium compounds considered \\
\hline $\mathrm{Cs}, \mathrm{CsCl}, \mathrm{CsO}, \mathrm{CsOH}, \mathrm{Cs}_{2}, \mathrm{Cs}_{2} \mathrm{Cl}_{2}, \mathrm{Cs}_{2} \mathrm{O}$, & $\mathrm{Sr}, \mathrm{SrCl}, \mathrm{SrCl}, \mathrm{SrO}, \mathrm{SrOH}, \mathrm{Sr}(\mathrm{OH})_{2}, \mathrm{SrS}$, \\
\hline $\mathrm{Cs}_{2}(\mathrm{OH})_{2}, \mathrm{Cs}_{2} \mathrm{SO}_{4}, \mathrm{Cs}(\mathrm{cr}), \mathrm{Cs}(\mathrm{L}), \mathrm{CsCl}(\mathrm{s})$, & $\mathrm{Sr}(\mathrm{s}), \mathrm{Sr}(\mathrm{L}), \mathrm{SrCl}_{2}(\mathrm{~s}), \mathrm{SrCl}_{2}(\mathrm{~L}), \mathrm{SrO}(\mathrm{s})$, \\
\hline $\mathrm{CsCl}(\mathrm{L}), \mathrm{CsOH}(\mathrm{s}), \mathrm{CsOH}(\mathrm{L}), \mathrm{Cs}_{2} \mathrm{SO}_{4}(\mathrm{~s})$, & $\mathrm{SrO}(\mathrm{L}), \mathrm{Sr}(\mathrm{OH})_{2}(\mathrm{~s}), \mathrm{Sr}(\mathrm{OH})_{2}(\mathrm{~L}), \mathrm{SrS}(\mathrm{s})$, \\
\hline \multirow[t]{2}{*}{$\mathrm{Cs}_{2} \mathrm{SO}_{4}(\mathrm{~L})$} & $\mathrm{SrO}_{2}(\mathrm{~s}), \mathrm{Sr}_{3} \mathrm{~N}_{2}(\mathrm{~s}), \mathrm{SrC}_{2}(\mathrm{~s}), \mathrm{SrH}_{2}(\mathrm{~s}), \mathrm{SrSO}_{4}$, \\
\hline & $\mathrm{SrCO}_{3}(\mathrm{~s}), \mathrm{SrSO}_{4}(\mathrm{~s})$ \\
\hline
\end{tabular}

Table 2.1 - Compounds considered in equilibrium calculations

Thermodynamic data must be supplied to CEA in order to perform the calculations. These data are the specific heat, the enthalpy, and the entropy of each species, as functions of temperature. These data are supplied to the program as a set of least-squares coefficients. These coefficients are placed in a seven-term polynomial for specific heat, with corresponding equations for enthalpy and entropy. The quality of the calculations performed by CEA is a result of the input data supplied. For example, if an important chemical specie is excluded from the input data, CEA will give erroneous results. In addition to the standard database, seventeen cesium compounds and twentythree strontium compounds were added to the input data (Barin 1995). These compounds 
are shown in Table 2.1. The actual input data for the cesium, strontium, and sodium species considered here, are included in Appendix A.1.

The equilibrium calculations were set up to match experimental feed conditions. Methane and air were used as the fuel and oxidizer, with a stoichiometric ratio of 1.2. The pressure was set to one atmosphere, and the temperature was varied. The metal concentration in each case was set to $100 \mathrm{ppm}$. The remainder of the calculation input conditions are shown in Table 2.2.

\begin{tabular}{|l|l|l|}
\hline \multicolumn{1}{|c|}{ Metal } & \multicolumn{1}{c|}{ Dopant } & \multicolumn{1}{c|}{ Figure } \\
\hline $\mathrm{Cs}$ & None & 2.2 \\
\hline $\mathrm{Cs}$ & $1000 \mathrm{ppm} \mathrm{Cl}$ & 2.3 \\
\hline $\mathrm{Cs}$ & $1000 \mathrm{ppm} \mathrm{S}$ & 2.4 \\
\hline $\mathrm{Na}$ & None & 2.6 \\
\hline $\mathrm{Na}$ & $1000 \mathrm{ppm} \mathrm{Cl}$ & 2.7 \\
\hline $\mathrm{Na}$ & $1000 \mathrm{ppm} \mathrm{S}$ & 2.8 \\
\hline $\mathrm{Sr}$ & None & 2.10 \\
\hline $\mathrm{Sr}$ & $1000 \mathrm{ppm} \mathrm{Cl}$ & 2.11 \\
\hline $\mathrm{Sr}$ & $1000 \mathrm{ppm} \mathrm{S}$ & 2.12 \\
\hline
\end{tabular}

\section{Table 2.2 - CEA input conditions}

The cesium results are shown in Figure 2.1 through 2.4. Figure 2.1 shows an overview of the dewpoint of the cesium species. Cesium alone has a relatively low dewpoint, approximately $675 \mathrm{~K}$, which classifies it as a low-end semi-volatile metal. The addition of chlorine tends to raise the dewpoint to around $900 \mathrm{~K}$. This is slightly unusual, as the addition of chlorine tends to decrease the dewpoint of most metals (Scotto et al. 1992; Mwabe and Wendt 1996). This is important for two reasons. First, in the case of sodium, it is believed that the capture reaction that occurs is between $\mathrm{NaOH}$ and the 


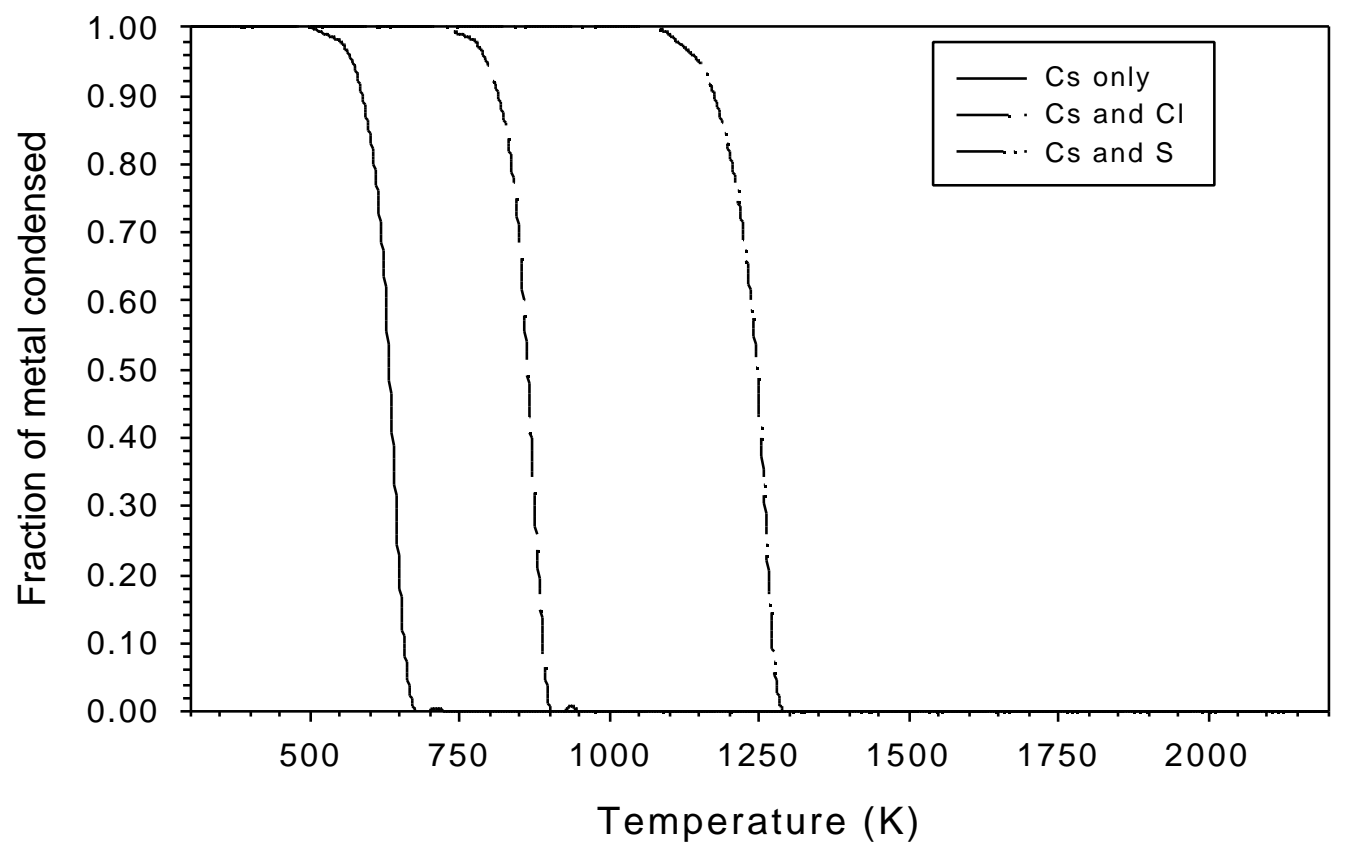

Figure 2.1 - Cesium dewpoint calculations

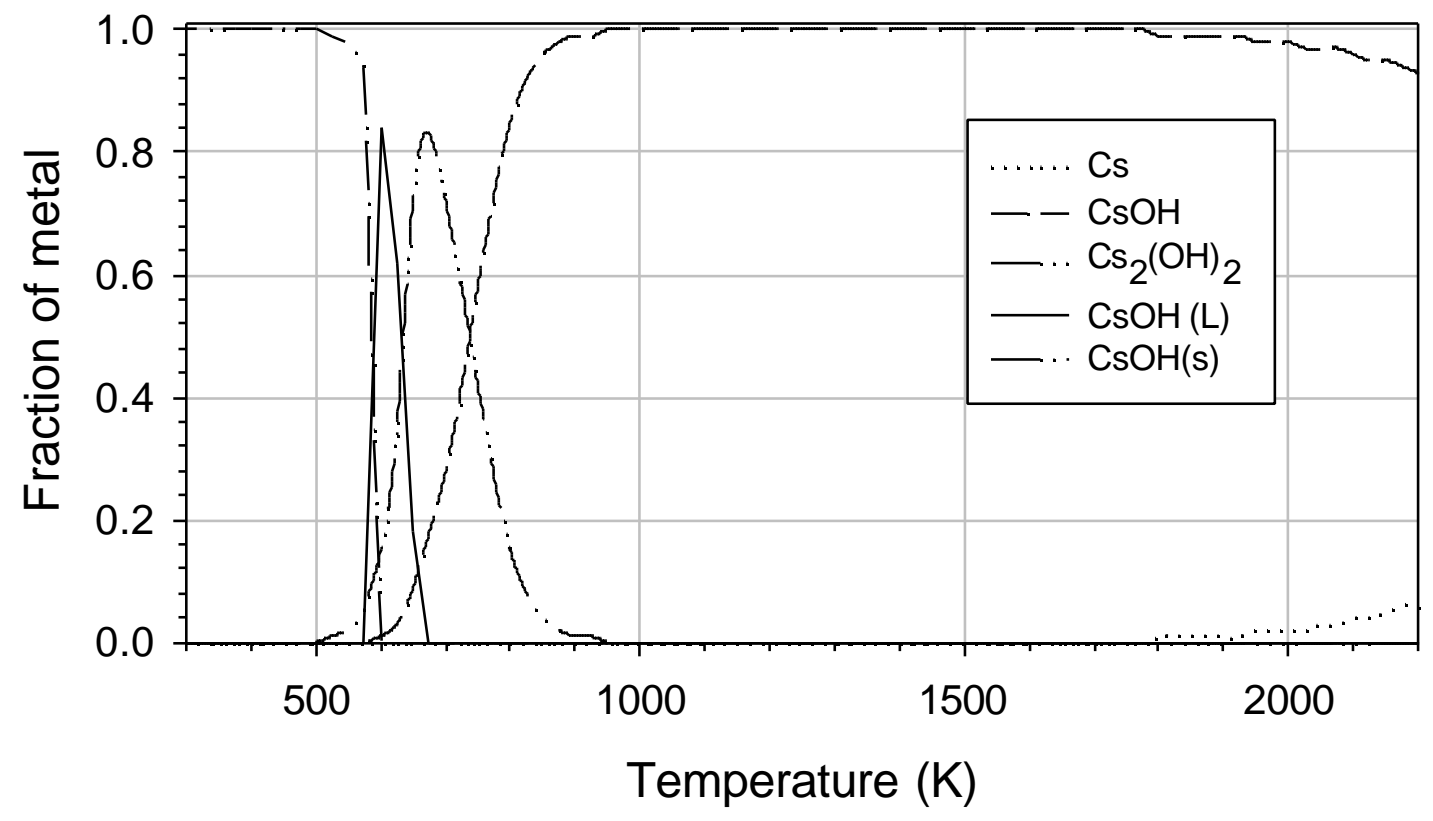

Figure 2.2 - Cesium speciation, no dopant 
kaolinite particle. Since sodium and cesium are in the same period of the periodic table, it might be expected that the two metals will show similar chemical behavior. Comparison calculations for sodium will be presented later. The presence of chlorine reduces the $\mathrm{NaOH}$ concentration in the furnace, decreasing the overall rate of the capture reaction. Second, since the dewpoint of the sodium is reduced, the time for reaction to occur has increased, which helps offset the lower $\mathrm{NaOH}$ concentration. In the case of cesium, however, the dewpoint increases, reducing the time to react as well as the $\mathrm{CsOH}$ concentration in the furnace. The combination of these two factors could have a large impact on the capture of cesium. The addition of sulfur raises the dewpoint of cesium even further, to around $1300 \mathrm{~K}$. This increase in dewpoint can significantly reduce the time the metal and sorbent have to react.

Figures 2.2, 2.3, and 2.4 show the speciation of cesium for each of the three cases examined. With no dopant present, the cesium exists in the gas phase as $\mathrm{CsOH}$. As the temperature decreases, the $\mathrm{CsOH}$ condenses to first liquid, then solid form. When chlorine is present in the system, the cesium exists primarily as $\mathrm{CsCl}$ in the gas phase. The condensed phase is predicted to be $\mathrm{CsCl}$, as well. When sulfur is added to the system, the cesium is present as $\mathrm{CsOH}$ at higher temperatures. As the temperature drops, the equilibrium shifts to $\mathrm{Cs}_{2} \mathrm{SO}_{4}$ as the gaseous specie. The $\mathrm{Cs}_{2} \mathrm{SO}_{4}$ then condenses and forms the prevalent specie in the solid phase. This indicates that cesium in the presence of sulfur may be captured at high temperatures, but the favorable capture conditions do not last for long. 


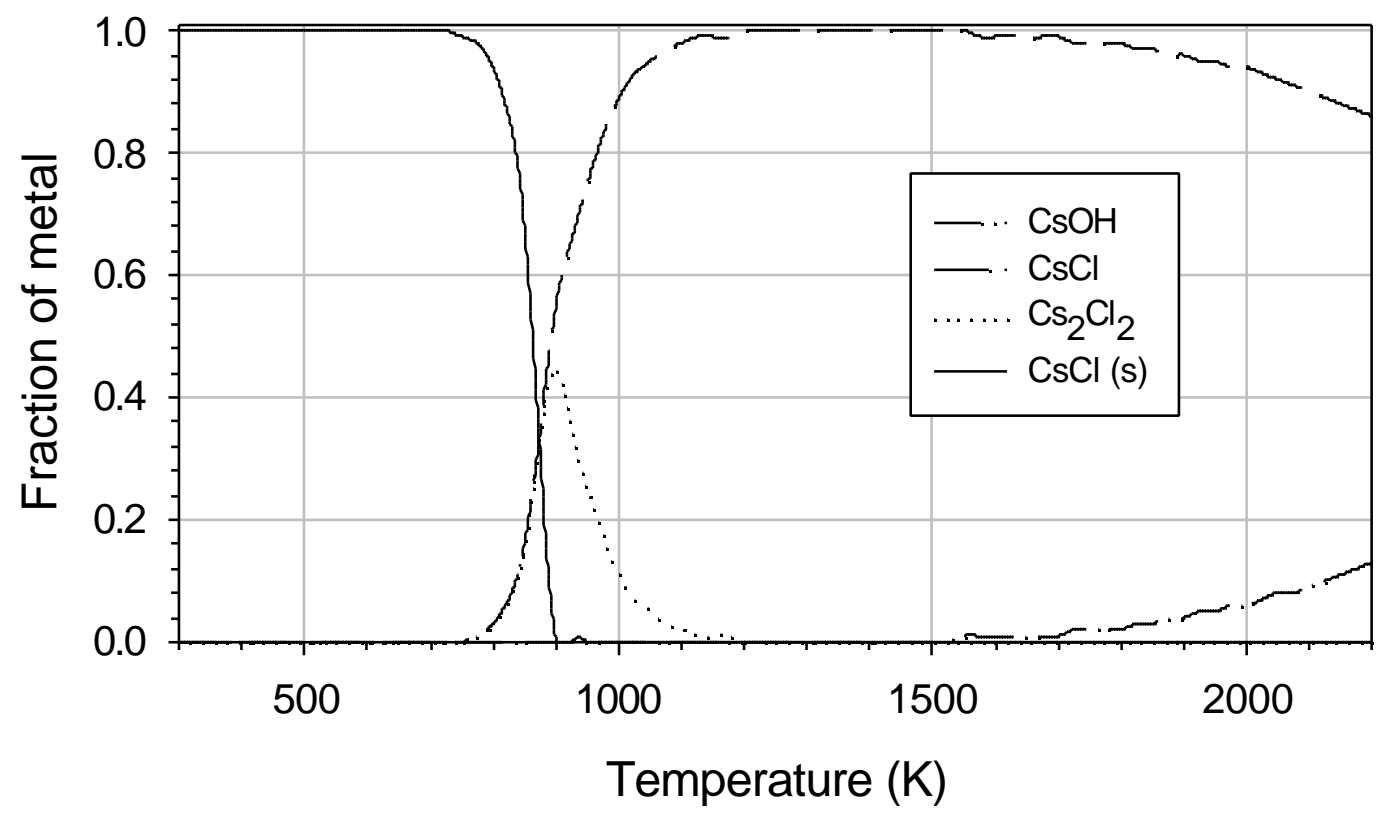

Figure 2.3 - Cesium speciation, in presence of chlorine

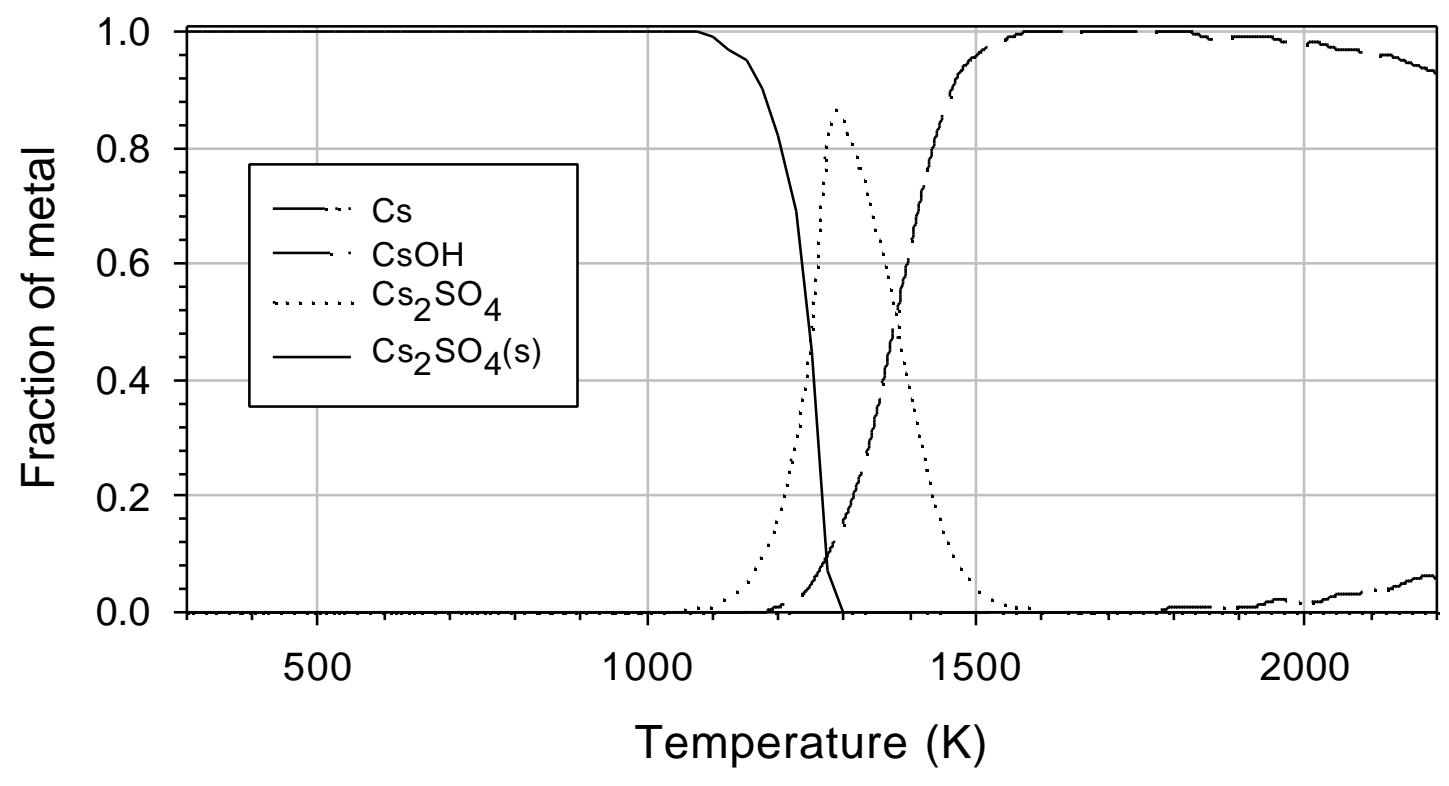

Figure 2.4 - Cesium speciation, in presence of sulfur 
For comparison, sodium calculations were performed to match the cesium conditions. These results are shown in Figures 2.5 through 2.8. Figure 2.5 illustrates the dewpoint behavior of sodium. With no dopant gases present, sodium has a dewpoint near $1200 \mathrm{~K}$. This is higher than the dewpoint of cesium. When chlorine is added to the system, the sodium dewpoint drops, which is similar to the behavior of most metals, to around $1000 \mathrm{~K}$. This number is within $100 \mathrm{~K}$ of the value for cesium. With sulfur present, the dewpoint of sodium jumps to near $1500 \mathrm{~K}$, which is about $200 \mathrm{~K}$ higher than in the case of cesium.

The sodium speciation plots are shown in Figures 2.6, 2.7, and 2.8. Without chlorine or sulfur present, sodium exists primarily as $\mathrm{NaOH}$ in the gas phase. When the sodium condenses, it forms liquid $\mathrm{Na}_{2} \mathrm{CO}_{3}$, which then quickly solidifies. This varies from the cesium results. The condensed cesium tends to form the solid hydroxide, and not the carbonate. However, it should be noted that $\mathrm{Cs}_{2} \mathrm{CO}_{3}$ is not included in the input data for cesium. The addition of this specie could change the results of the equilibrium calculation. When chlorine is added to the system, the majority of the sodium exists as $\mathrm{NaCl}$ in the gas phase. As the temperature drops, the sodium shifts briefly towards $\mathrm{Na}_{2} \mathrm{Cl}_{2}$ before it condenses into solid $\mathrm{NaCl}$. This behavior mirrors the cesium results almost exactly. Therefore, we can expect cesium to behave similarly to sodium when chlorine is present. When sulfur is added to the system, the sodium exists chiefly as $\mathrm{NaOH}$ in the gas phase. The equilibrium shifts towards $\mathrm{Na}_{2} \mathrm{SO}_{4}$ as the temperature drops. When the temperature drops further, the sodium condenses to liquid $\mathrm{Na}_{2} \mathrm{SO}_{4}$ and finally solidifies. This is also similar to the behavior exhibited by cesium, except the cesium 


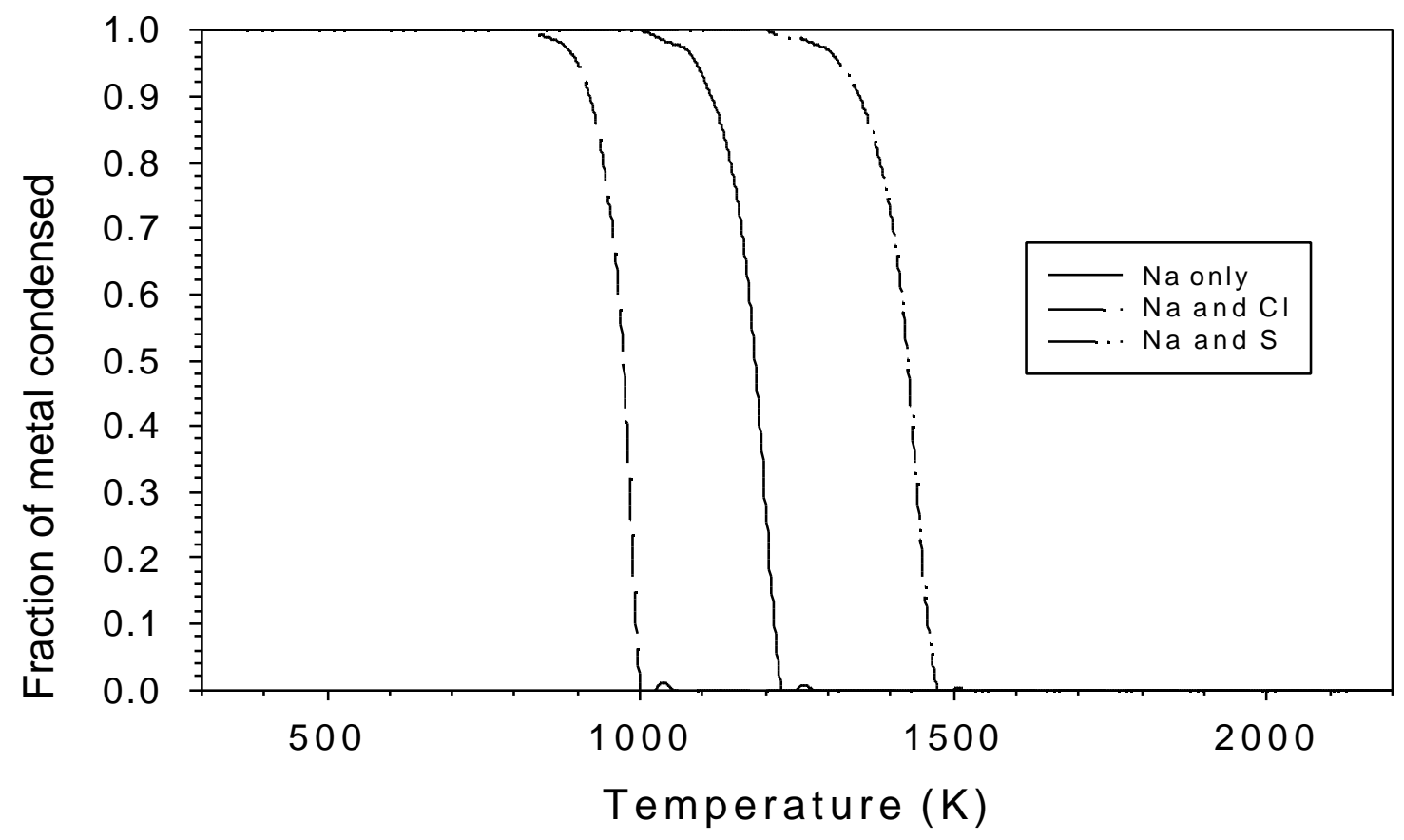

Figure 2.5 - Sodium dewpoint calculations

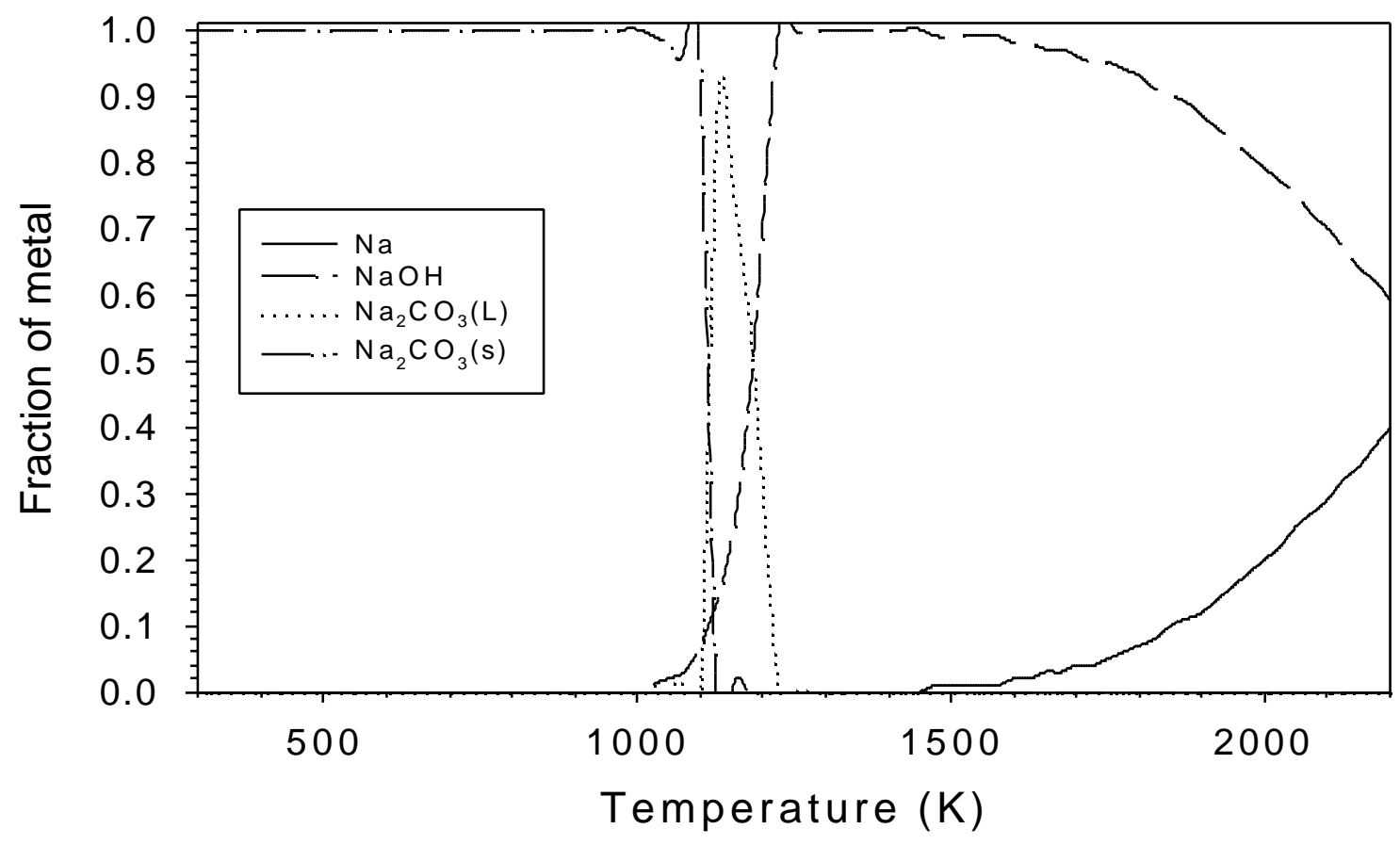

Figure 2.6 - Sodium speciation, no dopant 


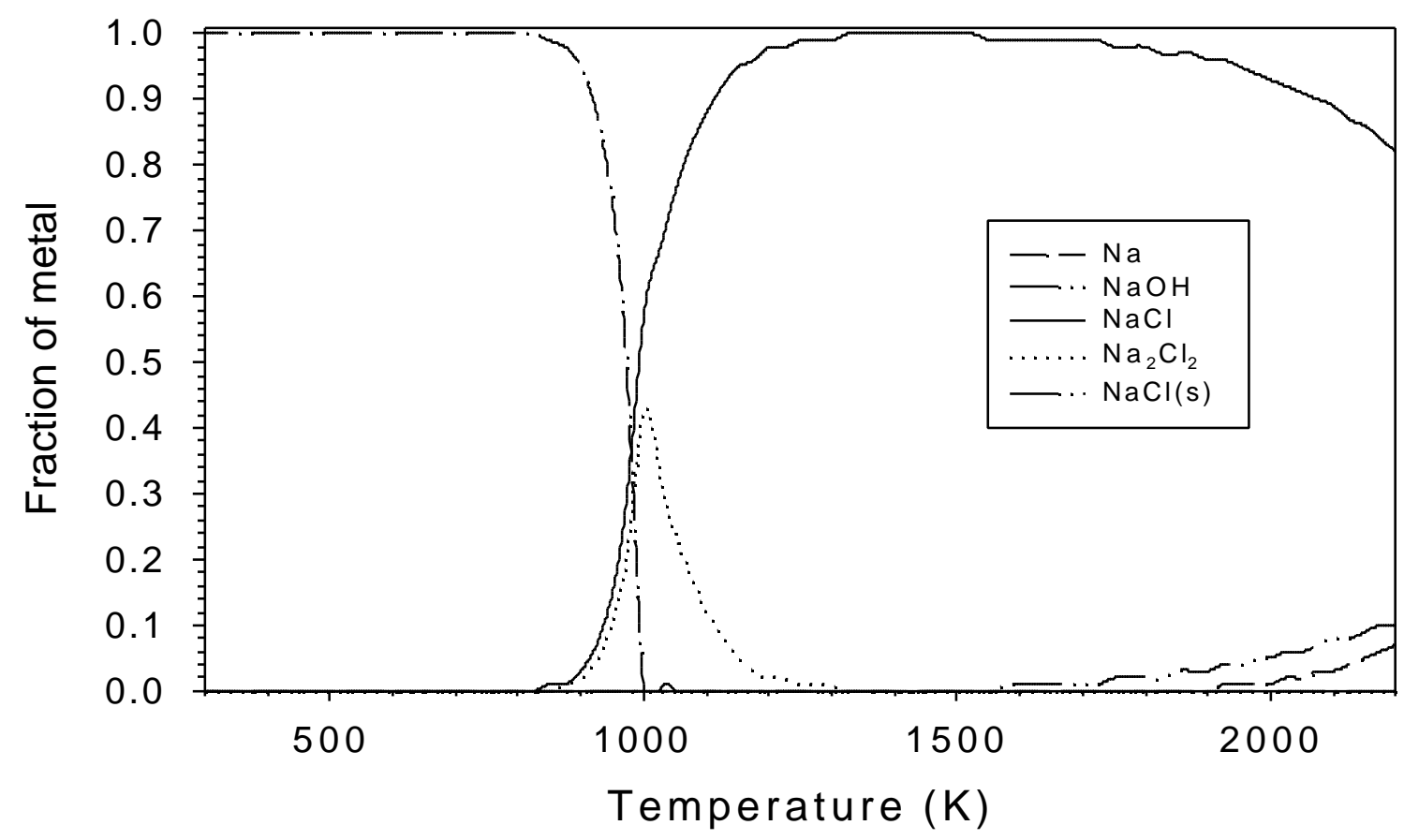

Figure 2.7 - Sodium speciation, in presence of chlorine

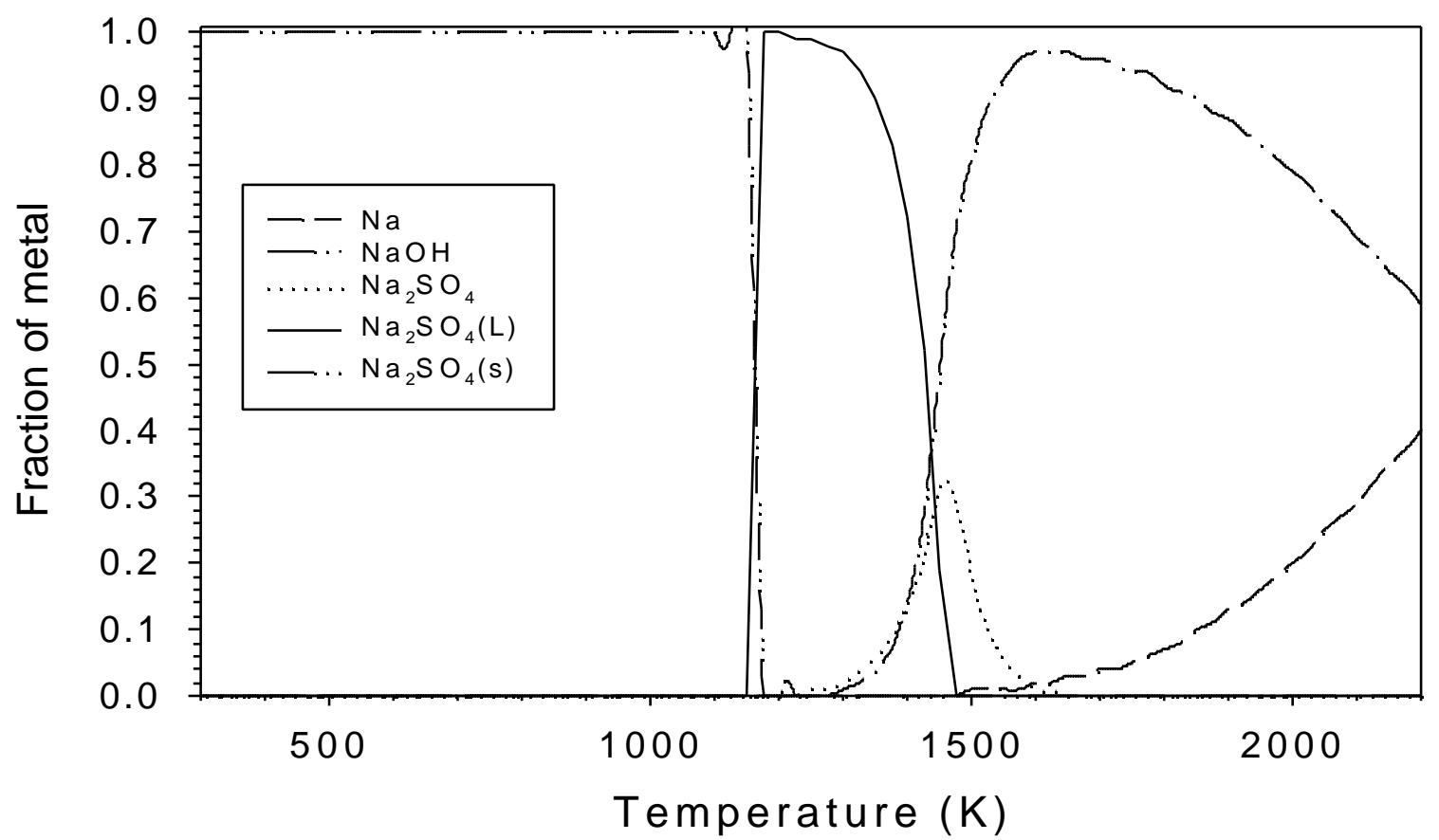

Figure 2.8 - Sodium speciation, in presence of sulfur 
condenses directly to the solid phase, and does not form a liquid. These results suggest that cesium may indeed behave similarly to sodium when reacted with kaolinite sorbent.

The strontium calculations are shown in Figures 2.9 through 2.12. Figure 2.9 is an overview of the dewpoint calculations. With no dopant gas present, strontium has a high dewpoint at $2000 \mathrm{~K}$. This classifies strontium as a refractory metal. Temperatures this high are seldom seen in practical combustors. This suggests that the water in the droplets of the injected strontium solution will evaporate, leaving a residual strontium particle behind. Since it is believed that the capture reactions occur between a gaseous metal and a solid sorbent, no reaction will occur if the strontium does not vaporize. When chlorine is added to the system, the dewpoint is reduced to around $1400 \mathrm{~K}$. This suggests that strontium will be vaporized and may be captured by sorbents when chlorine is present in the system. This behavior is similar to that seen in nickel, another refractory metal (Linak and Wendt 1998). There appears to be little change in the dewpoint when sulfur is added to the system, which again would suggest that no capture will occur.

The speciation plots for strontium are shown in Figures 2.10, 2.11, and 2.12. $\mathrm{Sr}(\mathrm{OH})_{2}$ is the primary gaseous specie present when no dopant is added to the system. The strontium then condenses as $\mathrm{SrO}$. As the temperature continues to drop, the equilibrium shifts to condensed $\mathrm{SrCO}_{3}$. When chlorine is present, the primary gaseous strontium specie is $\mathrm{SrCl}$. This then condenses to a liquid, and as the temperature continues to drop, solid $\mathrm{SrCl}$ is formed. With sulfur present in the system, the strontium exists as $\mathrm{Sr}(\mathrm{OH})_{2}$ in the gas phase, which then condenses to form solid SrO. This is the 


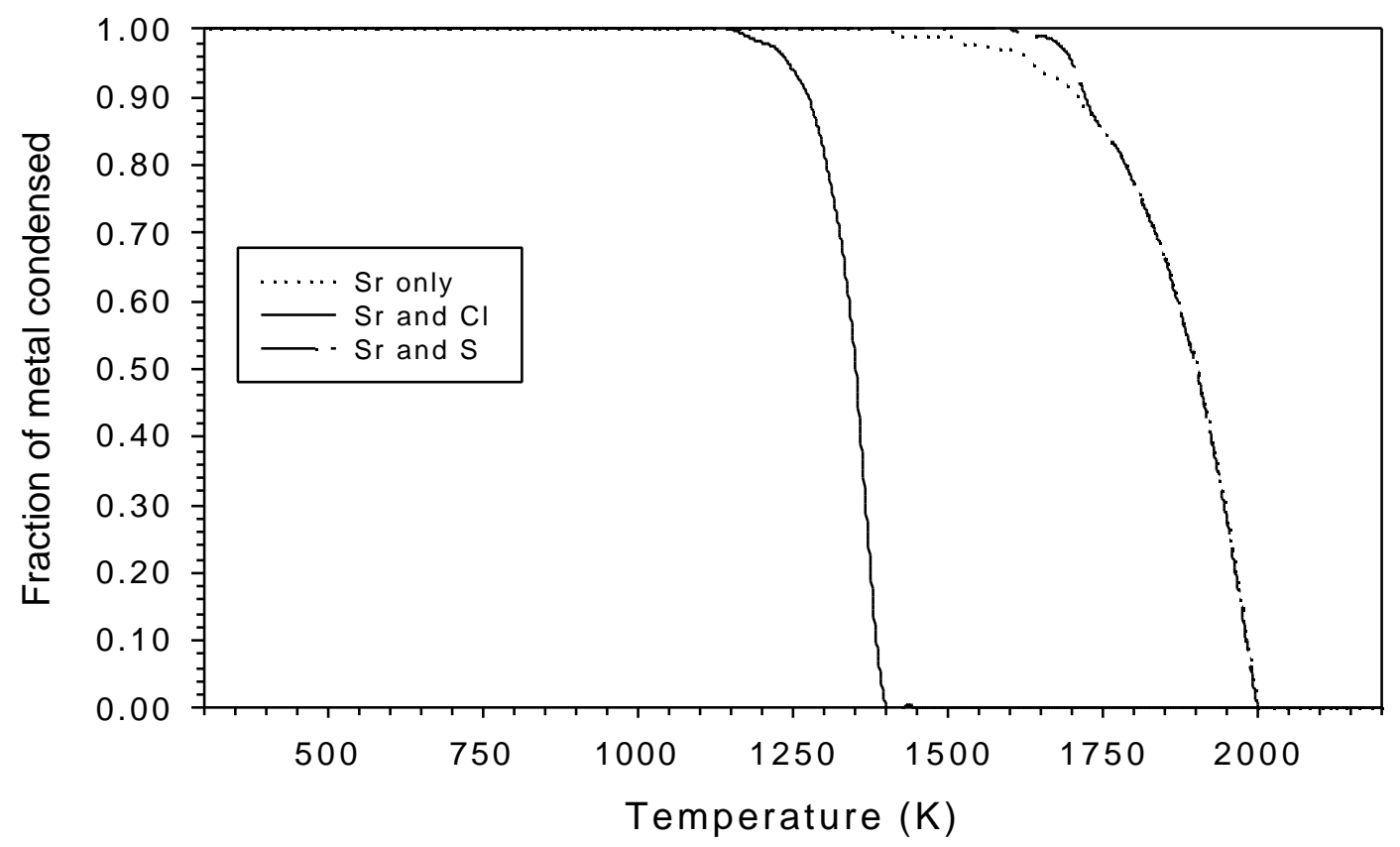

Figure 2.9 - Strontium dewpoint calculations

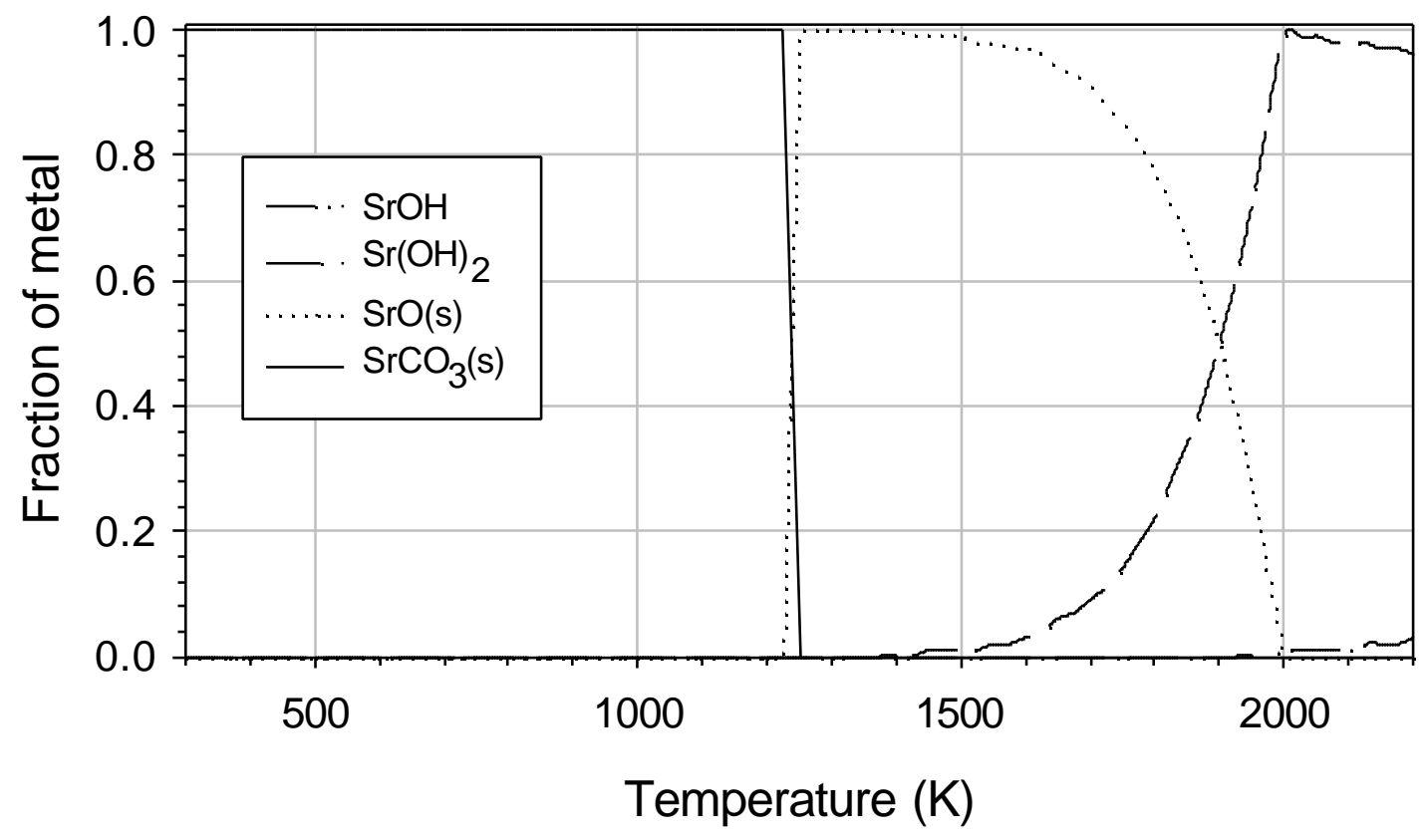

Figure 2.10 - Strontium speciation, no dopant 


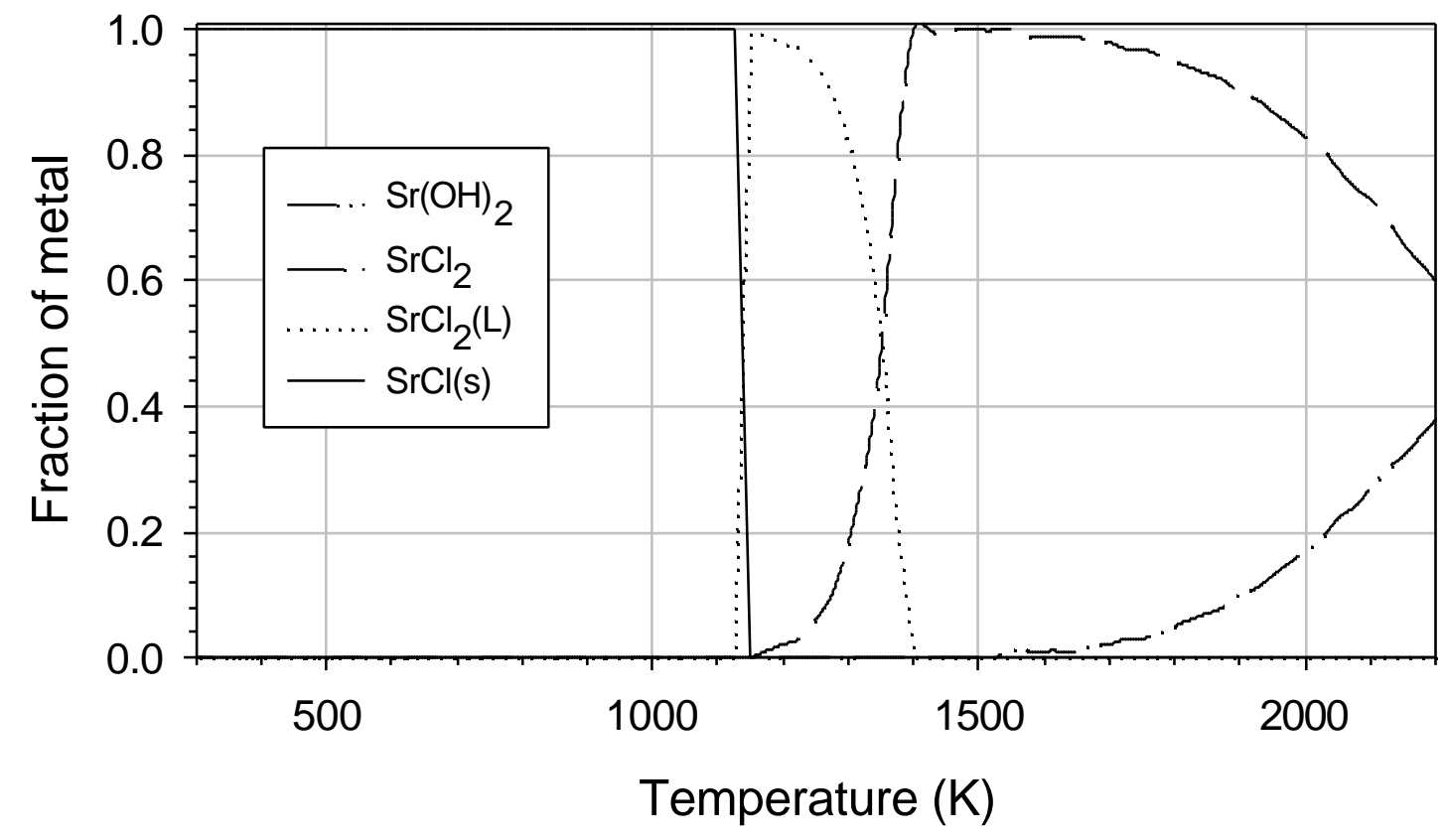

Figure 2.11 - Strontium speciation, in presence of chlorine

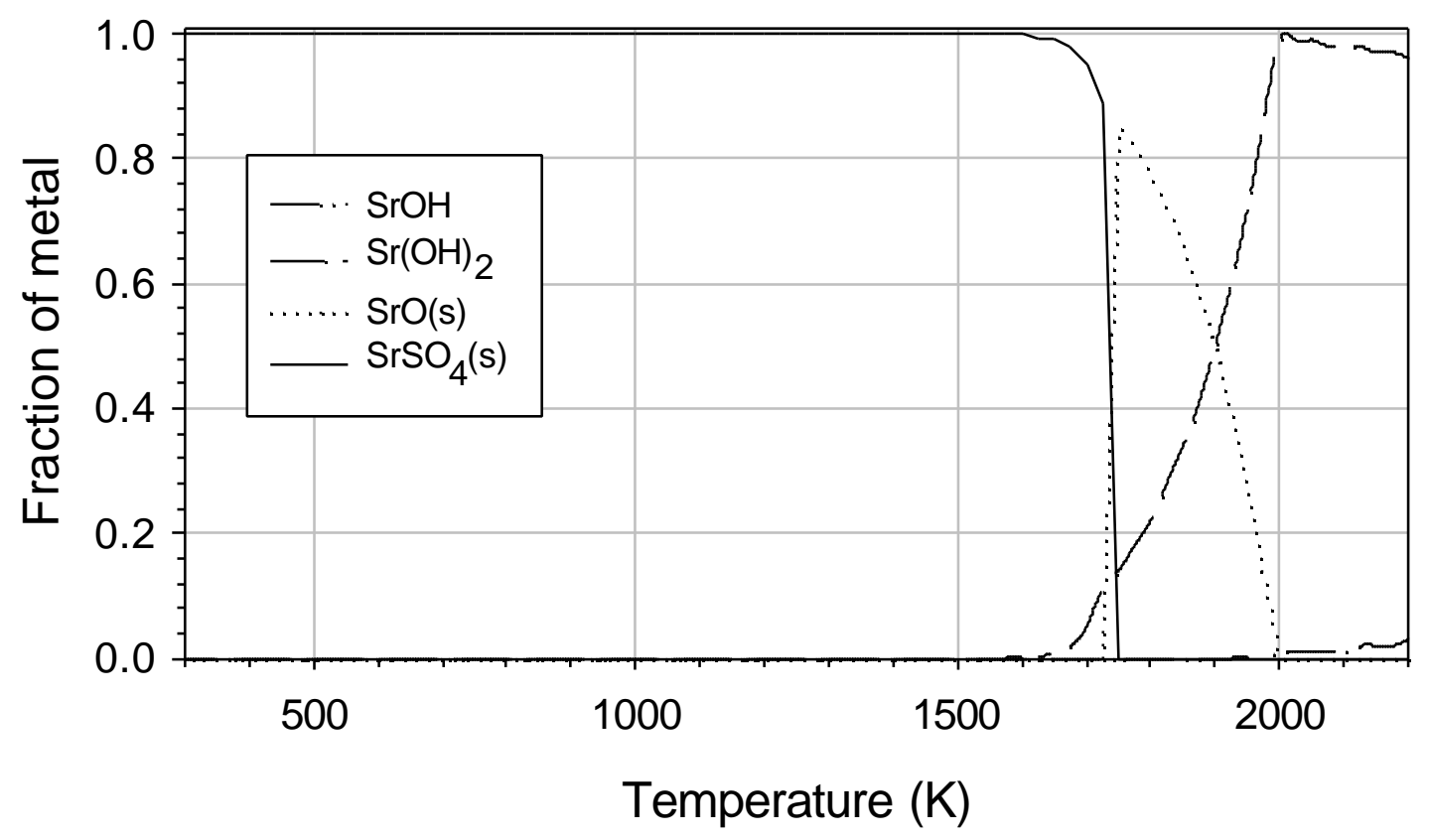

Figure 2.12 - Strontium speciation, in presence of sulfur 
same behavior as when no dopant gas is present in the system. However, as the temperature decreases, the equilibrium shifts so that $\mathrm{SrSO}_{4}$ is the prevalent solid specie.

These results provide some insight into the behavior that can be expected from the experimental research. Cesium should be present in the furnace in a gaseous form over the entire length of the furnace. Only when sulfur is added to the system will conditions be such that solid cesium will exist in the furnace. In addition, the chemical speciation of the cesium should be similar to that of sodium, and hence its capture characteristics should be similar as well. The strontium, on the other hand, will probably never vaporize in the furnace, except when chlorine is added. It is believed that in order for metal to react with sorbents, the metal must be present in the gas phase. If the metal never vaporizes in the furnace, it will not react with the sorbents. This suggests that in order to obtain reactive capture of strontium, chlorine must be present in the system.

Buckley, et. al. (1998) performed similar equilibrium calculations for cesium and strontium. There are some substantial differences between their results and the present work. Their calculations were performed using the Stanjan equilibrium code, and their input data was taken from the JANAF tables. The list of cesium and strontium species corresponds well, except their work lacks several of the sulfate species. Also, they were attempting to model a more complex situation, a biomass fuel with trace amounts of cesium and strontium present. As such, the presence of other chemical species, such as sodium, probably affected the behavior of the various species. These are effects that would not necessarily be seen in the simple situations modeled here. The results they obtained for strontium closely match those calculated in this work. The cesium results, 
however, show some differences. They predict that the cesium primarily exists as $\mathrm{CsOH}$ in the gaseous phase, and then condenses as liquid $\mathrm{CsCl}$. The temperature at which this occurs is significantly higher than the temperature predicted in this work. It is likely that these differences are due to two different factors: the presence of other chemical species in the system and the difference in the concentrations of the metals between the two studies. Sodium, potassium, and possibly magnesium are species that would compete, chemically, with cesium and strontium to bind with chlorine, sulfur, and other elements. This would lead to differences in the dewpoints and speciation of the various chemical species. Also, the dewpoint of the metal is dependent on the concentration of the metal present. 


\section{Chapter 3}

\section{Experimental Approach}

\subsection{Furnace Description}

Experiments were performed on the University of Arizona $18 \mathrm{~kW}$ laminar, downflow, swirl-flame combustor. This furnace was designed to have a time/temperature profile similar to those found in practical boilers and incinerators. This furnace was originally described by Scotto (1992). However, due to a catastrophic failure in the furnace wall, major portions of the furnace had to be rebuilt. The terminal crack was discovered in early July of 1998, and the rebuild occurred over the next four months. The furnace was operational again in November of 1998.

A diagram of the furnace and its support apparatus is shown in Figure 3.1. A variable swirl burner is mounted to the top of the furnace. The furnace is approximately seven meters tall and has an inner diameter of 0.15 meters. Seventeen sampling ports are spaced down the front of the furnace at approximately 30 centimeters intervals. The ports have a five-centimeter inner diameter and are capped by two-inch ball valves. A removable ash trap is attached to the bottom. A baghouse removes the particulate matter from the exhaust stream. The exhaust then passes through a condenser and a mist eliminator to remove any water from the gas and to protect the exhaust fan.

The upper section of the furnace is the portion that needed to be rebuilt. This encompasses the top three meters of the furnace structure, reaching from the burner to below the upper-level floor. A schematic of the new furnace construction, as well as the 
Liquid:

\begin{tabular}{l|l}
$\mathrm{H}_{2} \mathrm{O} / \mathrm{M}$ etal Salt \\
Natural & -Atomizing Air \\
Gas & CombustionAir/Dopant Gas
\end{tabular}

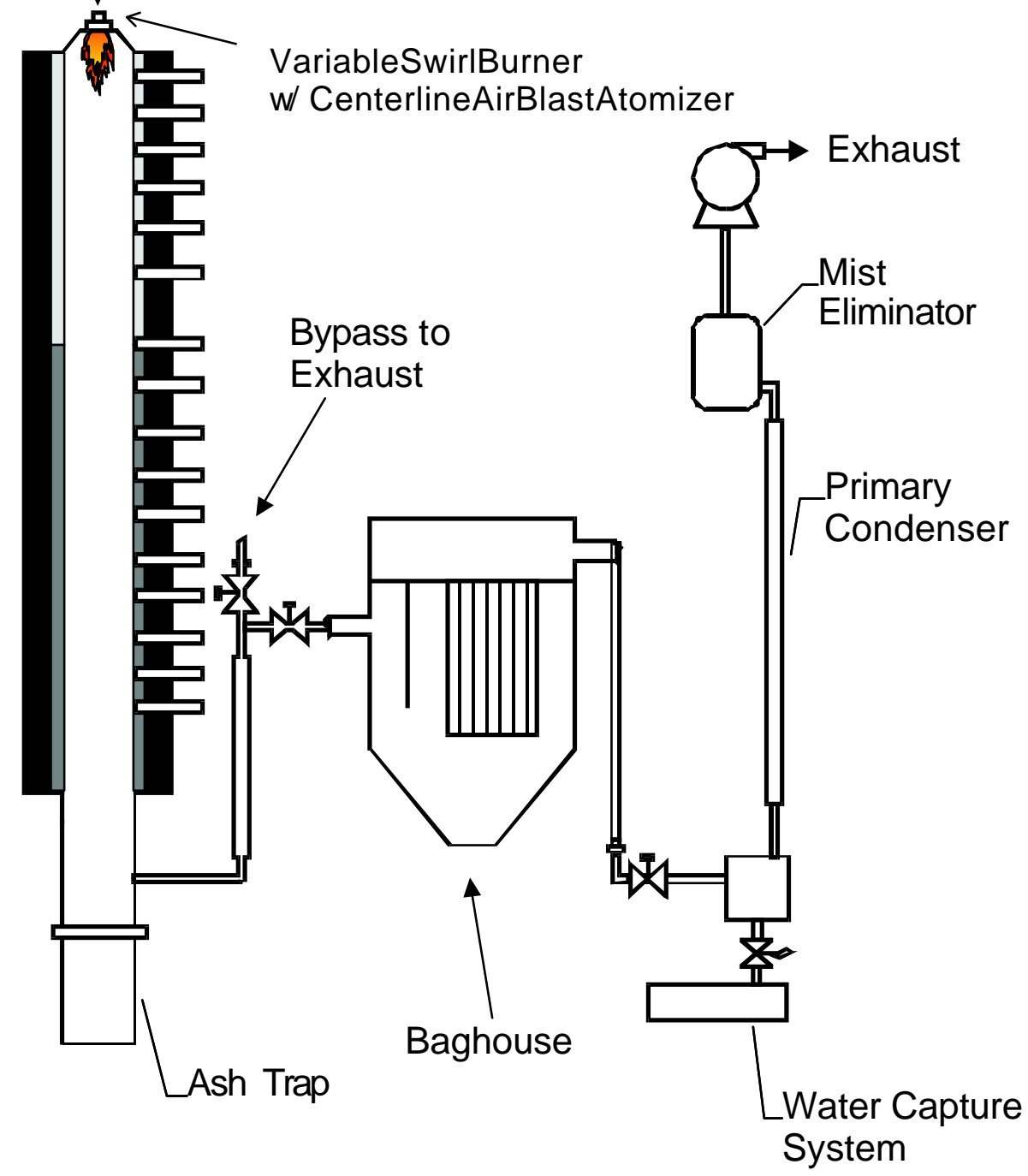

Figure 3.1 - Laboratory downflow combustor and support a pparatus 
materials used, is shown in Figure 3.2. The main portion of the upper furnace is cast of Thermal Ceramics HPM-95, a high-density slag resistant castable refractory. The upper two feet of this block has an inner diameter of six inches and an outer diameter of fourteen inches. The bottom foot of the block has an outer diameter of eighteen inches, which allows the block to rest on the stainless steel support structure. Three ports are cast into this section. The upper cone section of the furnace is cast of Thermal Ceramics 2800-LI insulating refractory cement. An insulating cement was used for this section in order to protect the various pieces of support equipment (burner, atomizer, UV flame detector, etc.) that are attached to the top of the furnace. The two refractory cement sections are joined together with a thin layer of Zircar type SALI moldable alumina insulation.

The upper furnace structure rests on a frame made from 2" $\mathrm{x} 4$ " hollow rectangular stainless steel beam, with a 1/4" wall thickness. The stainless steel beams are attached to the existing furnace frame and support the upper four feet of the new furnace. Placed on top of the stainless steel beams are lengths of 2" x 2" Zircar RS-100 coil post. This is a high-density fiber reinforced alumina composite. This material has a high compressive strength and is able to withstand the temperatures produced by the furnace. This layer helps protect the stainless steel supports from the high temperatures seen at the outer surface of the upper furnace section. Layers of Fiberfrax felt are placed on top of the RS-100 post to help protect the post surfaces from abrasion and to level the top section of the furnace. 


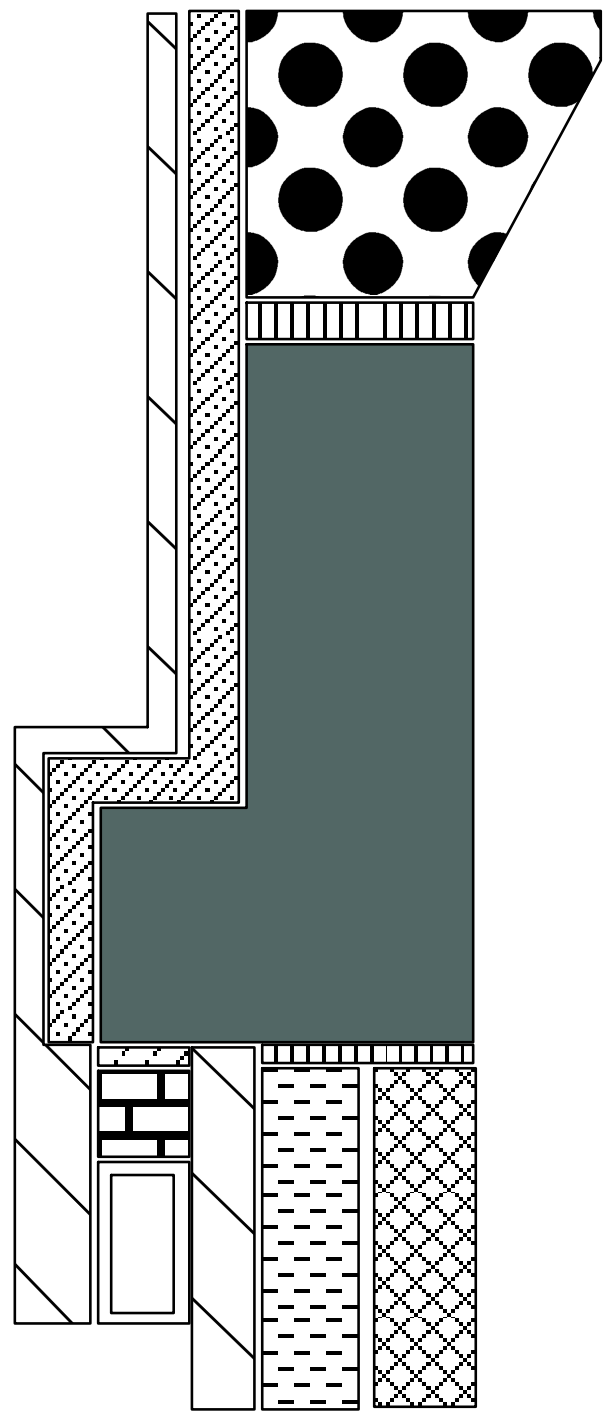

$12800-4$

IIII SAU moldable

HPM-95

II RS-100 post

2 Fiberfraxfelt

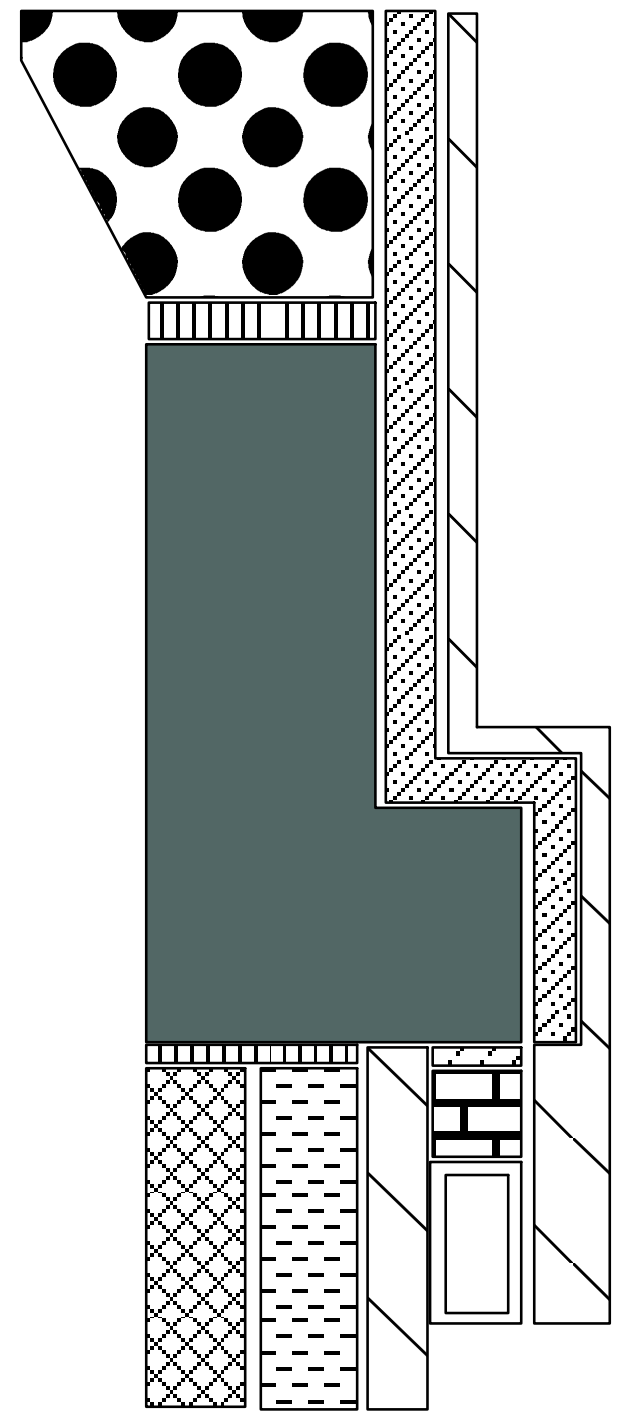

18 TypeALC-AA alumina-silica

Type ASHalumina-silica

$\triangle$ Kaowool insulation

Cerachem insulation

$\square \quad$ Stainless steel beam

Figure 3.2 - Schematic of furnace materials 
The first furnace section above the floor and the first section below the floor were also rebuilt. This section is constructed from Zircar alumina insulating cylinders. The inner cylinders are made of Zircar type ALC "AA" high-purity alumina bond in order to withstand the inner furnace temperatures. These cylinders have an inner diameter of six inches and an outer diameter of eight inches. The outer cylinders, which have an inner diameter of eight inches and an outer diameter of ten inches, are made of Zircar type ASH alumina-silica insulation. This material is significantly less expensive than the type ALC "AA" insulation, but cannot withstand the temperatures on the inner wall of the furnace. All the cylinders are in one-foot sections and are joined together with the type SALI moldable alumina, with the cylinder seams offset to prevent leaks. The alumina cylinders are sealed to the HPM-95 refractory block by a layer of type SALI moldable.

The port cylinders are constructed of the type SALI moldable alumina that has been coated with Zircar alumina rigidizer/hardener, which provides additional rigidity to the material. The port cylinders are sealed into the furnace body with additional SALI moldable.

The upper four feet of the furnace is insulated with a two-inch thick layer of Thermal Ceramics Cerachem blanket. This is a high temperature insulation composed of alumina-silica-zirconia fiber. Around this layer is a two-inch thick layer of Thermal Ceramics Kaowool insulation. This is a lower cost insulation, but cannot withstand the temperatures generated at the outside of the HPM-95 block. The lower sections of the furnace are insulated with a four-inch layer of the Kaowool insulation. The insulation is held in place by steel wire and wire mesh. 
The baghouse has also been rebuilt. A schematic of the new baghouse is shown in Figure 3.3. This baghouse is constructed of carbon steel, rather than stainless steel, which is what the old baghouse was made of. The carbon steel was less expensive and easier to work with than stainless steel. The exhaust enters the baghouse and flows around a baffle. This attempts to distribute the airflow over the entire surface area of the bags. The exhaust then passes through woven Teflon fiber bags with a surface air velocity of nine feet per minute. The bags are manufactured by GoreTex and can withstand temperatures up to 250 degrees $C$. The particulate matter is retained on the bags, and the gas passes out through the exhaust.

In order to remove the particles from the bags, the baghouse is equipped with an air pulse line. This provides a pressurized pulse of air that flows counter to the exhaust gases. This air pulse cleans the bags and knocks the particulate material into the base of the baghouse. Each pulse has a pressure of 30 psi and lasts for 0.1 seconds. An access port allows for periodic emptying of the baghouse. A magnehelic gauge monitors the pressure drop across the bags, which provides an indication of plugging in the bags. When the pressured drop across the bags has increased by $0.5 " \mathrm{H}_{2} 0$, the baghouse is pulsed. The baghouse is also equipped with electric heaters, which prevent water from condensing in the baghouse during furnace warm-up. If water were to condense on the bags, it would mix with the sorbent particles collected by the cartridges, forming a cement-like mixture that would foul the bags. Two K-type thermocouples are used to monitor the inlet and outlet temperatures in the baghouse. 


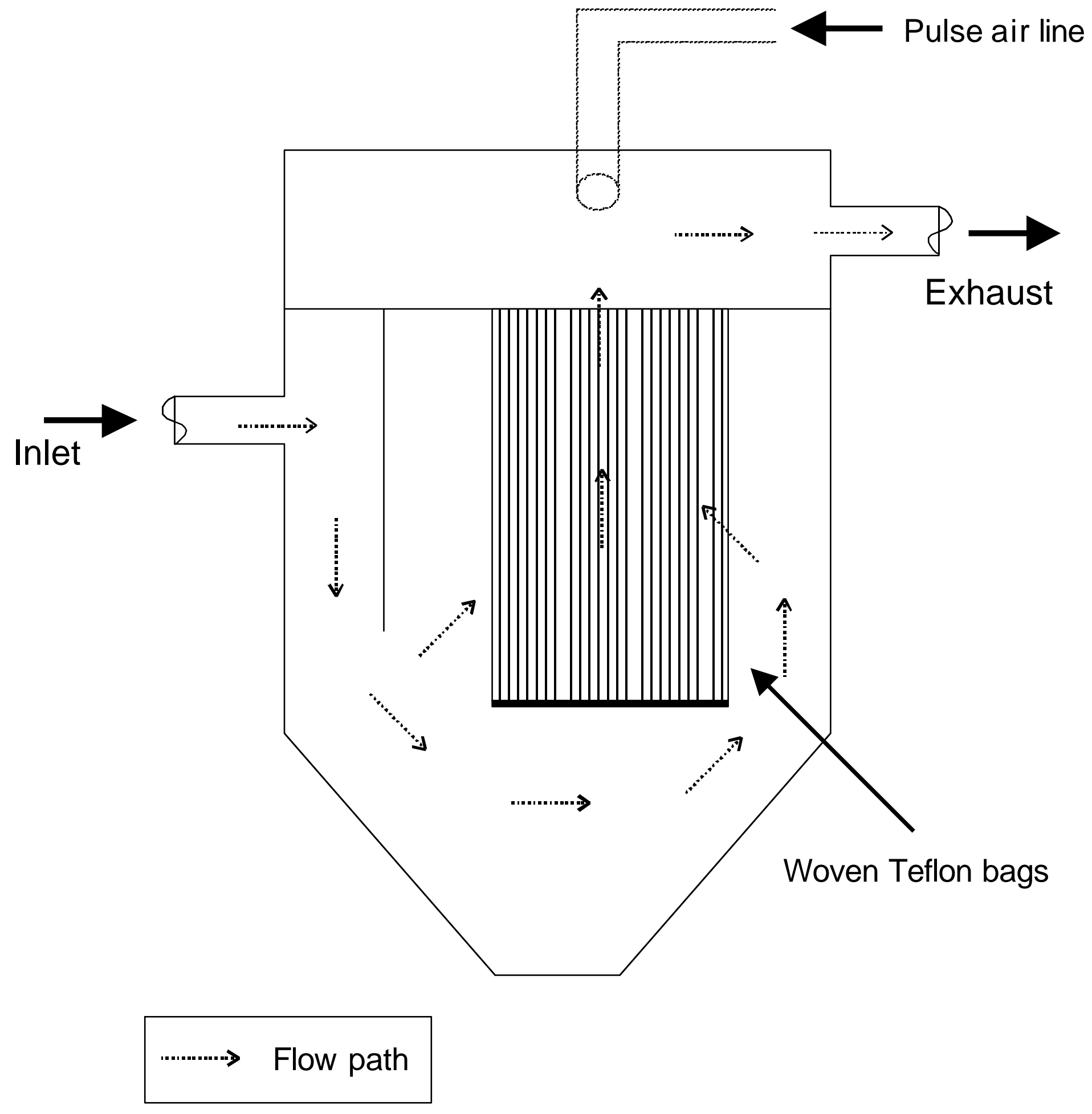

Figure 3.3 - Baghouse schematic 


\subsection{Auxiliary Systems}

Metals were injected into the furnace as aqueous solutions. All metals used in these experiments were non-radioactive isotopes. This fact should not significantly affect the results of these experiments, as the chemical kinetics and transport properties of the metals are not a function of the radioactivity of the species. Cesium solutions were prepared from cesium acetate salt, $\mathrm{CsCH}_{3} \mathrm{CO}_{2}$, and strontium solutions were prepared with strontium nitrate, $\mathrm{Sr}\left(\mathrm{NO}_{3}\right)_{2}$, both of which were obtained from Aldrich Chemical Company. These salts were chosen because they are sufficiently soluble in water, yet are free of chemical species that may interfere with the reactions of interest $(\mathrm{Cl}, \mathrm{S}$, etc.). The salts were added to distilled water and mixed thoroughly. The solution was then injected into the furnace using a Fluid Metering, Inc. FM-100 positive-displacement pump with a QVC pump head. The solution traveled to a plain-jet air-blast atomizer located at the furnace through the center of the burner, with the tip extending $1 / 4$ " from the methane nozzle.

Several sorbents were used for these experiments. The physical properties of the sorbents are listed in Table 3.1. The first was a kaolinite powder. The primary kaolinite used was called Burgess No. 40, and is referred to as kaolinite throughout the rest of this report. For some experiments, other kaolinite powders were used. The Burgess No. 80 and the 6104-B have different characteristics than the kaolinite. The third sorbent used was a pulverized hydrated lime. The next sorbent used is called CDEM. Four Dutch paper manufacturers founded CDEM Holland B. V. in order to address environmental concerns of the paper recycling industries. A de-inking residue is passed through a 
fluidized bed combustor. This process destroys the organic portion of the residue, drives off the water, and leaves the mineral fraction in a cement-like form. For further information on the CDEM sorbent, see Biermann et. al. (1997). Experiments involving the "mixed" sorbent used a 50/50 mechanical mixture of the kaolinite and lime described above. This mixture simulated the CDEM sorbent, and provided some insight into the role of the individual constituents of the CDEM. For further information on the physical characteristics of the sorbent, such as particle and pore size distributions, see Davis (1999).

\begin{tabular}{|l|l|l|c|}
\hline Sorbent & Supplier & Composition & $\mathbf{d}_{\text {ave }}(\mu \mathbf{m})$ \\
\hline Kaolinite (B-40) & Burgess Pigment Company & $\mathrm{Al}_{2} \mathrm{O}_{3} \bullet 2 \mathrm{SiO}_{2} \bullet 2 \mathrm{H}_{2} \mathrm{O}$ & 1.4 \\
\hline B-80 & Burgess Pigment Company & $\mathrm{Al}_{2} \mathrm{O}_{3} \bullet 2 \mathrm{SiO}_{2} \bullet 2 \mathrm{H}_{2} \mathrm{O}$ & 0.6 \\
\hline 6104-B & Burgess Pigment Company & $\mathrm{Al}_{2} \mathrm{O}_{3} \bullet 2 \mathrm{SiO}_{2} \bullet 2 \mathrm{H}_{2} \mathrm{O}$ & 27 \\
\hline Hydrated Lime & Global Stone, Inc. & $\mathrm{Ca}(\mathrm{OH})_{2}$ & 6 \\
\hline CDEM & CDEM Holland $\mathrm{B} . \mathrm{V}$. & $\begin{array}{l}\mathrm{CaCO} 33 \% \\
\mathrm{CaO} \quad 21 \% \\
\mathrm{SiO}\end{array}$ & $\begin{array}{l}\mathrm{Al}_{2} \mathrm{O}_{3} \quad 15 \% \\
\text { Other } 1 \%\end{array}$ \\
\hline Mixed Sorbent & As above & $\begin{array}{l}50 \% \text { kaolinite } \\
50 \% \text { lime }\end{array}$ & As above \\
\hline
\end{tabular}

Table 3.1 - Sorbent characteristics

The sorbents were pneumatically injected into the furnace by a water-cooled probe. A K-Tron twin-screw feeder was calibrated, and the rotation rate was set to deliver a set sorbent flow rate to the probe. Due to the different handling characteristics 
of each sorbent, a calibration curve was produced for each. The feeder dropped the sorbent into a pneumatic transfer device, where a high velocity air stream intercepted the sorbent and carried it through the probe.

Dopant gases, $\mathrm{Cb}_{2}$ and $\mathrm{SO}_{2}$, were used to explore the effects of sulfur and chlorine on the metal/sorbent systems. $\mathrm{Cl}_{2}$ and $\mathrm{SO}_{2}$ were metered out through a Matheson 602 rotameter. The gaseous dopants were then mixed with the combustion air prior to entering the furnace.

The typical furnace settings, as well as a listing of the experimental conditions for each experimental run, are listed in Appendix A.2 and A.3, respectively.

\subsection{Sampling Systems}

Two systems were used to characterize the furnace. A probe composed of an Rtype bare wire thermocouple encased in a ceramic sheath measured the temperature along the centerline of the furnace. These measurements were not corrected for radiation. An average temperature profile is shown in Figure 3.4. The error bars denote the $99 \%$ confidence interval. Also shown are the sorbent injection and sampling points that were used for the majority of the experiments. The furnace was allowed to achieve thermal equilibrium prior to each set of experimental runs. Equilibrium was achieved when the temperature of the furnace varied by no more than two degrees in an hour.

The second system used to classify the furnace was the gas sampling system. A water-cooled stainless steel probe withdrew the gas samples from the furnace ports. The gas stream then passed through a refrigerated knock-out pot and a coalescing filter to 


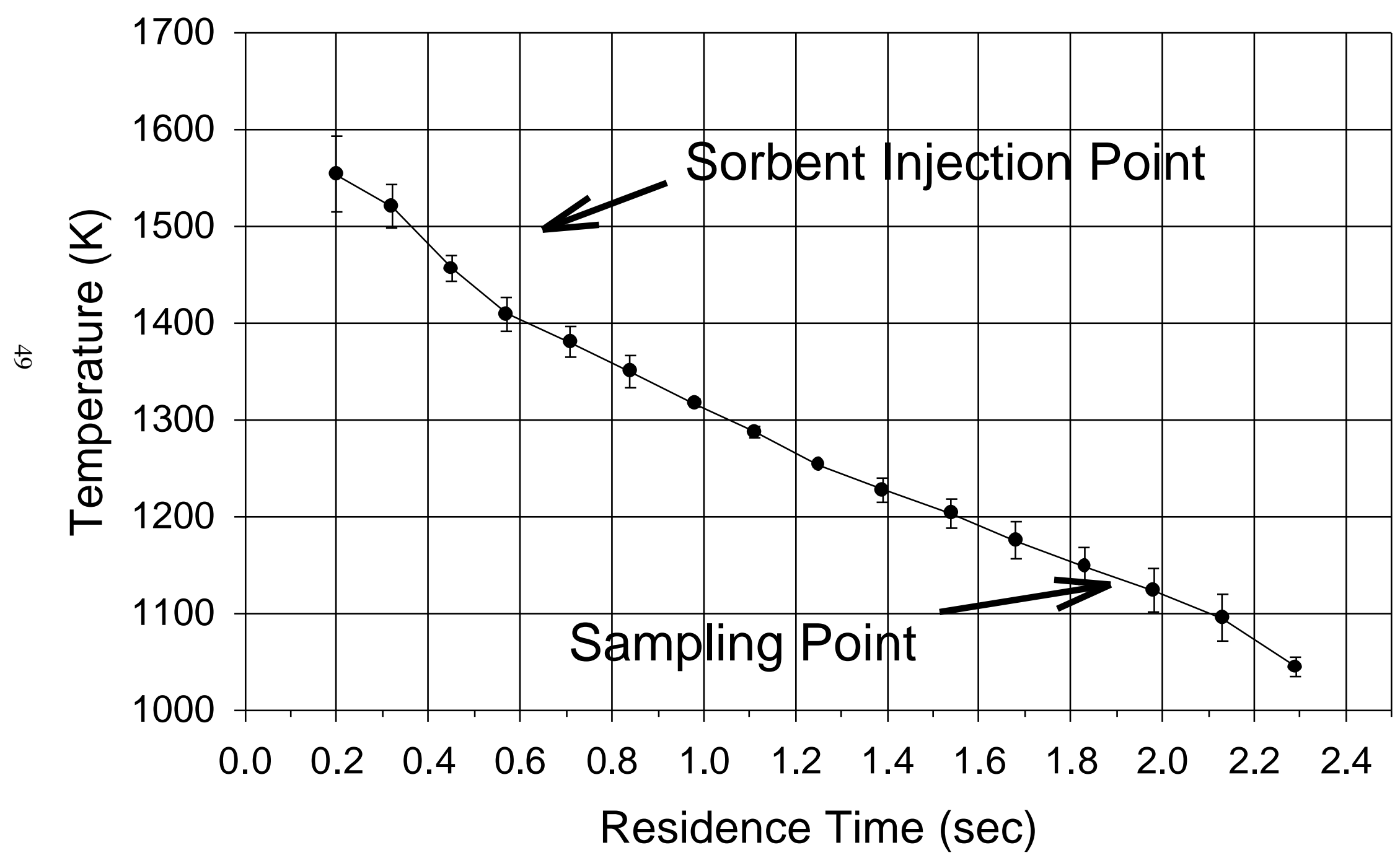

Figure 3.4 - Average temperature profile at $18 \mathrm{~kW}$ load 
remove the water from the sample. The gas sample then entered a bank of continuous emissions monitors (CEMs) that were used to determine the gas concentrations. The CEMs were calibrated with certified calibration gases of known concentrations. $\mathrm{CO}$ and $\mathrm{CO}_{2}$ were measured with a California Analytical ZRH infrared gas analyzer. A Rosemont Analytical Model 755D paramagnetic oxygen analyzer measured the $\mathrm{O}_{2}$ in the sample. A typical gas profile can be found in Appendix A.4.

A schematic of the particulate sampling system is shown in Figure 3.5. Samples were withdrawn from the appropriate sampling port using a water-cooled sampling probe and a large vacuum pump. Nitrogen was injected into the probe at a ratio of about 20:1 to dilute and cool the sample. This method provides a rapid cooling rate that should freeze any chemical reactions at the point of sampling and prevent water from condensing in the sampling system. A mass flow controller maintained the nitrogen flow rate. The sampling flow rate was determined by calculating the isokinetic sampling rate based on the current furnace conditions. The sampling rate was monitored by a mass flow meter.

After being removed from the furnace, the sample passed through a Berner lowpressure impactor (LPI) (Hillamo and Kauppinen 1991). A diagram of the impactor showing the general arrangement and the cut diameter of each stage is shown in Figure 3.6. This cascade impactor was designed so that several of the stages operate at pressures below atmospheric, allowing the segregation of particles down to 0.032 microns. There were ten stages in the set-up used, with the Plate 11 data disregarded due to poor collection ability. A Nucleopore polycarbonate filter, obtained from Osmonics, was 


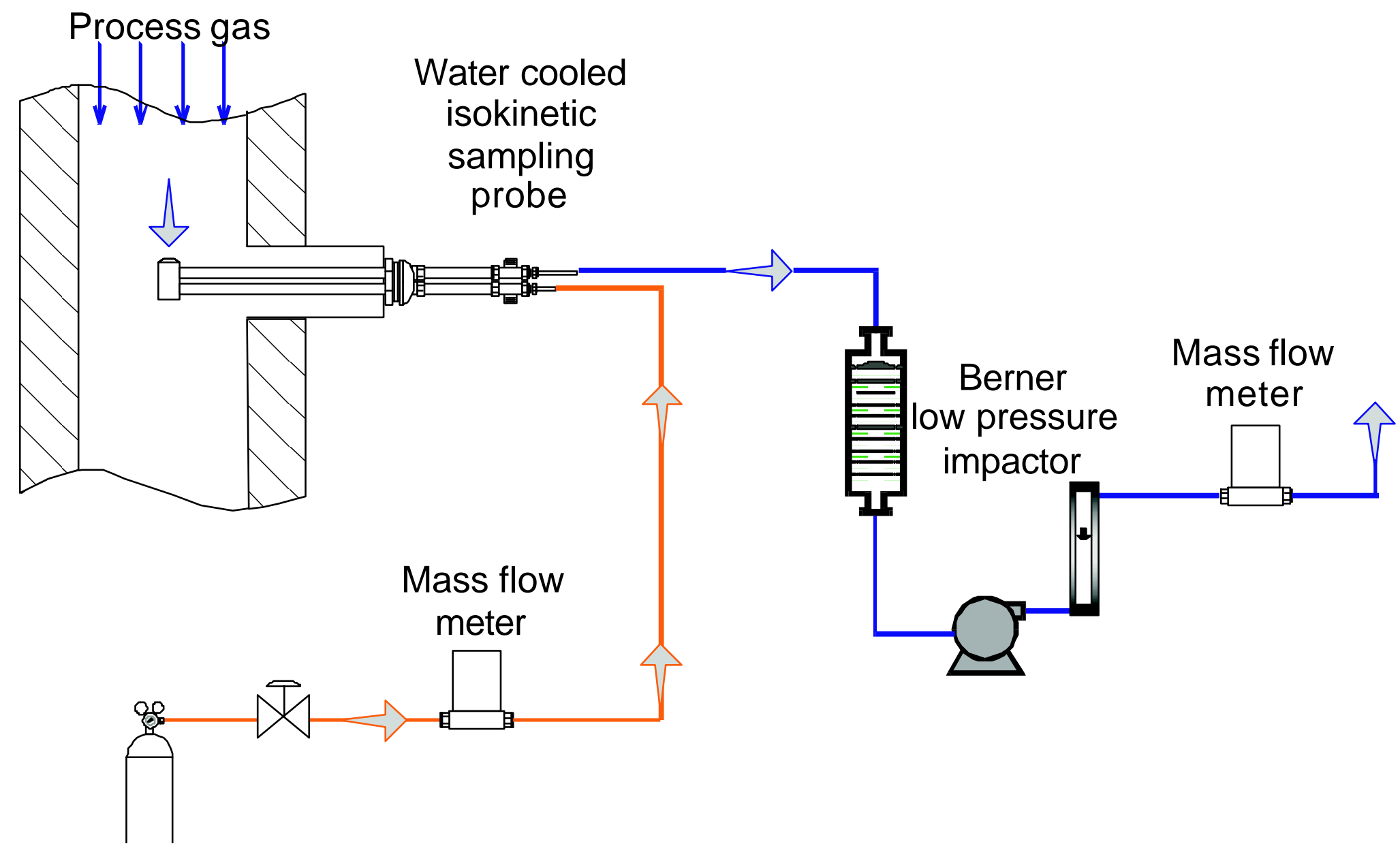

Nitrogen quench gas

Figure 3.5 - Sampling system 
Aerodynamic $50 \%$ Cut-Off

Diameter $[\mu \mathrm{m}]$

Plate $11 \quad 15.7$

Plate $10 \quad 7.33$

Plate $9 \quad 3.77$

Plate $8 \quad 1.98$

Plate $7 \quad 0.973$

Plate $6 \quad 0.535$

Plate $5 \quad 0.337$

Plate $4 \quad 0.168$

Plate $3 \quad 0.0926$

Plate $2 \quad 0.0636$

Plate $1 \quad 0.0324$

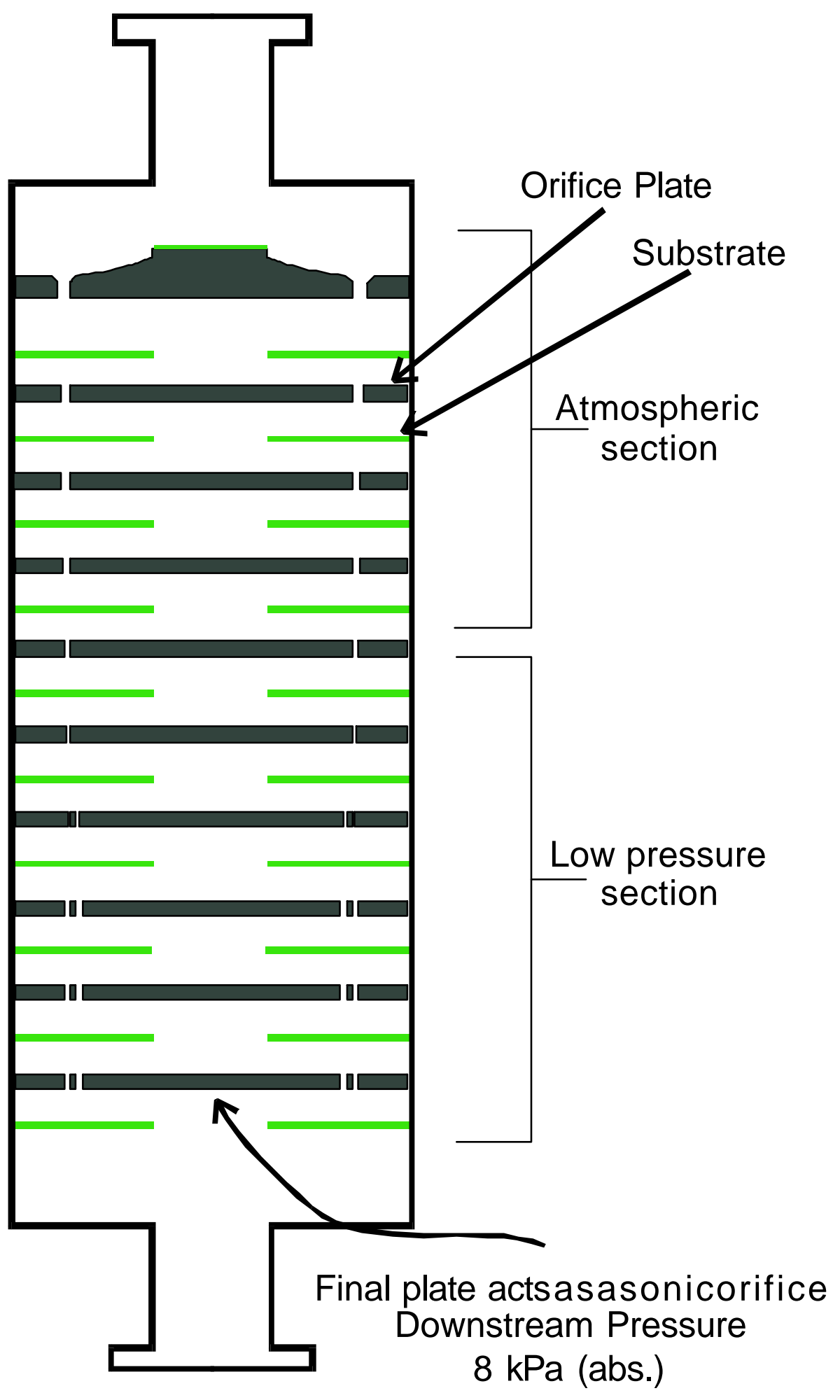

Figure 3.6 - Berner low pressure cascade impactor 
placed on each impaction stage. Each filter was lightly airbrushed with a mixture of Apiezon-L vacuum grease and toluene, to prevent particle bounce-off. The substrates were then vacuum desiccated for 24 hours prior to use, in order to remove the solvent.

A full discussion of the particle sampling method can be found elsewhere (Davis et al. 1998).

\subsection{AA/AE Analysis}

Once the samples were collected, the substrates were removed from the LPI and placed in polyethylene vials. A $15 \mathrm{~mL}$ aliquot of digestion acid was then added to each vial. The digestion acid was composed of a 5:3:1:1 mixture of ultrapure water, hydrofluoric acid, nitric acid, and hydrochloric acid. The samples were placed in a ultrasonic cleaner for ten minutes, and the samples were allowed to sit for a period of 24 hours. After the 24 hour waiting period, a $15 \mathrm{~mL}$ aliquot of a boric acid solution was added. The boric acid binds with the excess fluorine in the samples, helping to protect the components of the atomic adsorption system. The composition of the boric acid solution was dependent upon the contents of the samples. The boric acid solution added to the samples containing strontium was composed of $56.6 \mathrm{~g}$ of boric acid crystals, one liter of ultrapure water, and $0.78 \mathrm{~g}$ of $\mathrm{CsNO}_{3}$, added to reduce ionization interferences. The boric acid solution added to those samples containing cesium was the same, except the $\mathrm{CsNO}_{3}$ was replaced with $0.6 \mathrm{~g}$ of $\mathrm{KCl}$. The $\mathrm{KCl}$ was substituted for the $\mathrm{CsNO}_{3}$ into those runs that contain cesium because the amount of cesium added in the digestion step 
cannot be measured precisely enough to correct for. The samples were again sonicated for ten minutes, after which the samples were ready for analysis.

The majority of the elements of interest were analyzed for using flame atomic absorption spectroscopy (AAS). This was performed on a Perkin-Elmer Model 2380 Atomic Absorption Spectrophotometer. Hollow cathode lamps were used for the light sources. The spectral lines examined were: $309.3 \mathrm{~nm}$ for aluminum, $422.7 \mathrm{~nm}$ for calcium, $251.6 \mathrm{~nm}$ for silica, and $460.7 \mathrm{~nm}$ for strontium. Cesium was analyzed using flame atomic emission spectroscopy (AES). The same instrument was used as before, except no lamp was used. An optical filter was installed on the spectrometer prior to use. The spectral line of $852.1 \mathrm{~nm}$ was examined. For all analysis, standards were prepared from purchased standard metal solutions. To prevent matrix errors, the preparation solutions were matched to the dissolving acid used on the samples. More information on the theory of atomic absorption spectroscopy, atomic emission spectroscopy, and the specific settings used for the Perkin-Elmer machine can be found elsewhere (Siemer 1986) (1982).

\subsection{SEM/EDS Procedures}

Samples generated for scanning electron microscopy and energy dispersive spectroscopy (SEM/EDS) analysis were collected using the same sampling equipment. Instead of the polycarbonate substrates used for AAS, ungreased aluminum foil substrates were used. Samples were collected for one minute. After collection, a small piece of the substrate was cut and mounted on an aluminum pin mount using a carbon-based glue tab. 
The samples were then coated with an approximately 200-angstrom layer of gold/palladium using an Anatech Ltd. Hummer VI sputter coater. The samples were stored in a dessicator when not in use.

The microscope used was a Hitachi Model S-2460N scanning electron microscope equipped with a Noran Instruments SiLi x-ray detector. The SEM was operated such that high-magnification images of the samples were obtained. For more information, consult the appropriate equipment manuals (Hitachi 1992), (1995). No attempt was made to perform quantitative EDS on the particles. 


\section{Chapter 4}

\section{Results}

\subsection{Introductory Comments}

The results are presented in the following series of graphs. The raw data from which these figures are constructed are presented in Appendix A.5. Each of the curves presented is plotted based on the average of two separate experimental runs. The variation between each of the experimental runs was no greater than $15 \%$. For the sake of clarity, error bars were not included in the figures.

Each of the results figures is constructed in a similar manner. The $\mathrm{x}$-axis presents the aerodynamic diameter of the particles in microns. Since this characteristic spans four orders of magnitude, this axis is presented in a logarithmic scale. The aerodynamic diameter assumes a particle density of one, so the actual size of particles on a plate may vary based on the density. The y-axis is the fraction of total metal captured. Thus, each

point represents the mass of metal on a stage, divided by the sum of the masses found on all stages. This graphic presentation gives an indication of the distribution of the metals of interest over the range of the impactor. Shifts in the shape and position of these curves indicate the activity of the metals present.

In each of these figures, the particle size distribution (PSD) of the metal of interest is plotted without the presence of sorbent. This gives a baseline indication to compare with, in order to determine the effect of sorbent on the system. The second curve on the graphs is the metal in the presence of the particular sorbent. The sorbent 
size distribution is shown on these graphs by plotting the major element of each of the sorbents: aluminum for kaolinite and calcium for lime. For the mixed sorbent and the CDEM sorbent, both aluminum and calcium curves are shown, as the chemical behavior of the two constituents can be significantly different.

\subsection{Cesium Results}

The results of the cesium experiments are shown in Figures 4.1 through 4.19. The first of these figures shows the behavior of cesium with the sorbents but with no dopant gases added.

Figure 4.1 shows the behavior of cesium with kaolinite. The cesium alone exhibits a curve with a peak near 0.05 microns. This indicates that the cesium metal vaporizes in the flame. When the gaseous cesium is withdrawn from the furnace, it quickly nucleates in the probe to form a fume of fine metal particles. These results coincide well with the behavior predicted by the thermodynamic calculations. When kaolinite is injected into the furnace, the cesium peak shifts to near one micron. This peak almost perfectly overlaps the aluminum peak from the sorbent. This shift and overlap of the cesium peak indicates that the cesium is reacting with the kaolinite in the furnace, forming larger particles.

Figures 4.2 and 4.3 are SEM micrographs of kaolinite and cesium with kaolinite. Unless noted otherwise, all SEM images are taken from samples on Plate 6 of the impactor. The morphology of kaolinite is seen in Figure 4.2. This kaolinite was injected in the furnace and collected with the particulate sampling system, but no metals were 


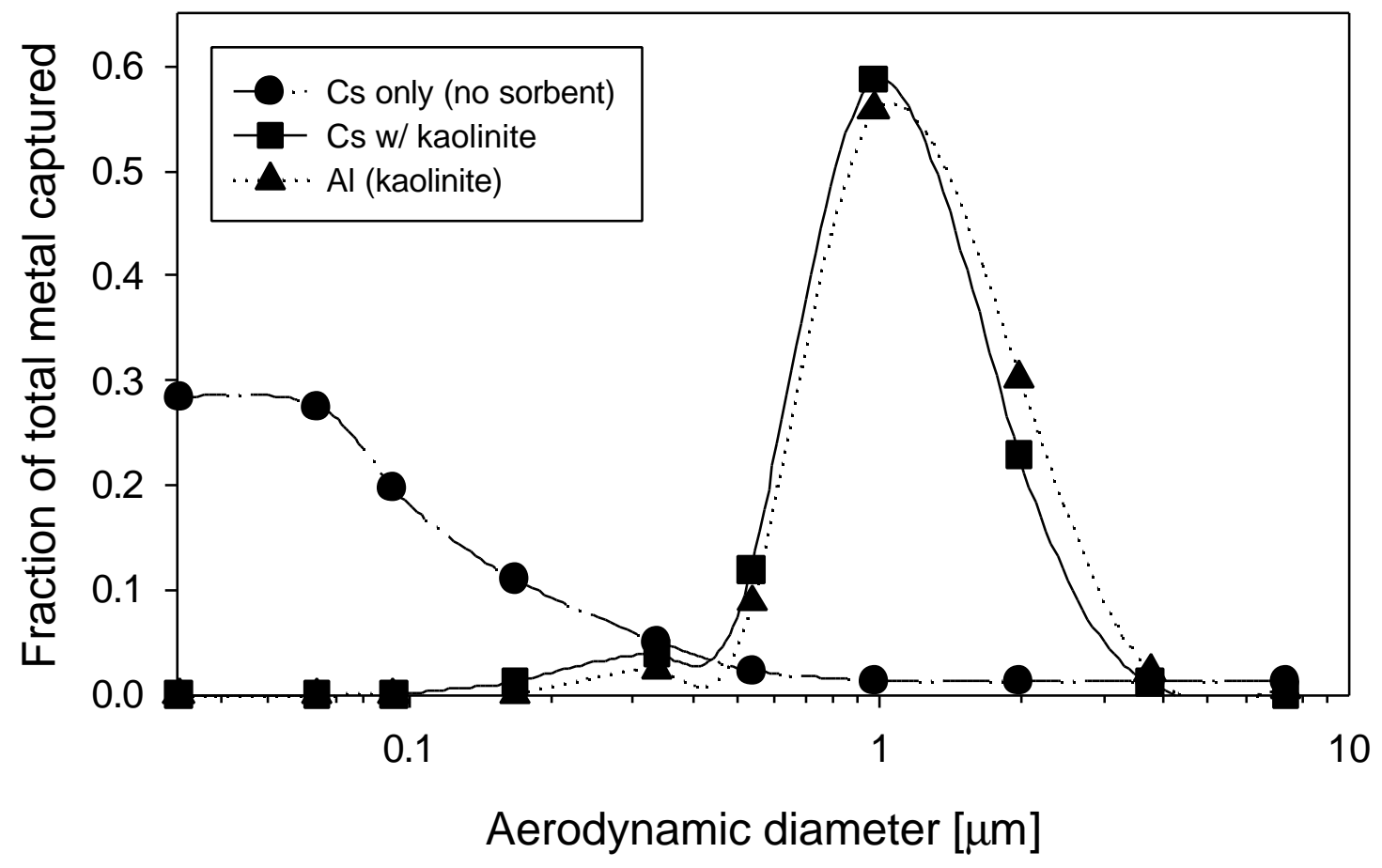

Figure 4.1 - Cesium and kaolinite, no dopant

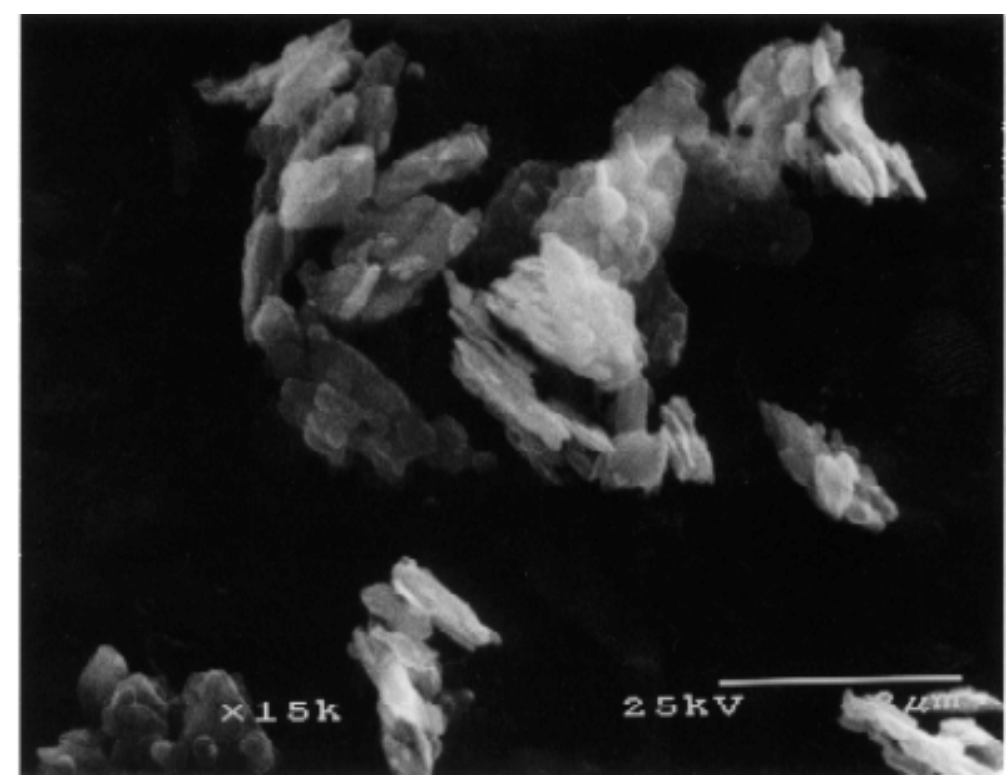

Figure 4.2 - SEM micrograph of unreacted kaolinite 


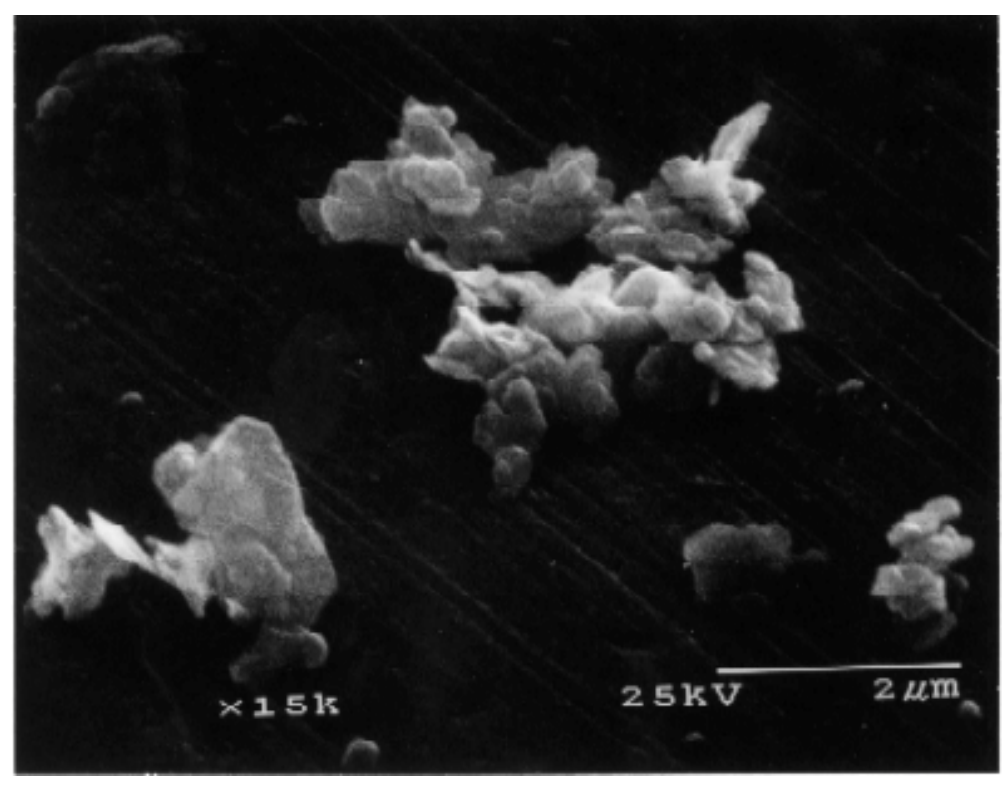

Figure 4.3 - SEM micrograph of cesium and kaolinite particles

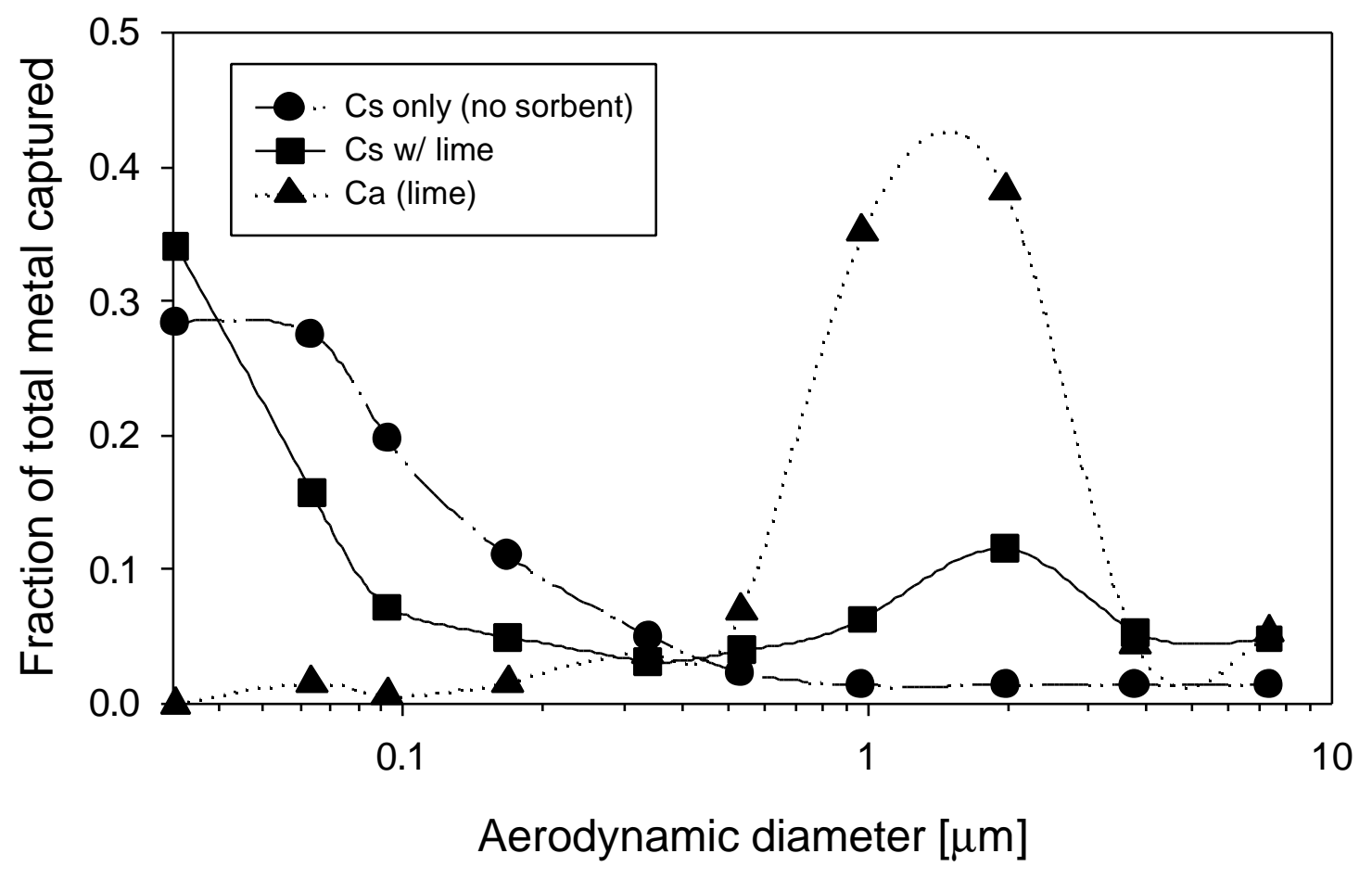

Figure 4.4 - Cesium and lime, no dopant 
added with the sorbent. The kaolinite particles show a jagged and angular morphology. A sample from the cesium and kaolinite system is shown in Figure 4.3. The PSD analysis indicated that the cesium reacts with the kaolinite, so some evidence of this reaction might be expected in the SEM image. The particles again have a jagged and angular appearance. Very little difference in the morphology can be seen between the two samples.

The results of cesium with lime are shown in Figure 4.4. Here, the cesium exhibits a large peak near the small end of the impactor, and a short peak at around two microns. This suggests that there may be some reaction of the cesium with the lime. However, the rate of the reaction is probably very slow, preventing a significant fraction of the cesium from reacting. The majority of the metal ends up as a fume in the probe, similar to what is seen when there is no sorbent present.

The results of experiments in the presence of the mixed sorbent are shown in Figure 4.5. The cesium shows a peak near one micron, similar to that seen in the presence of kaolinite. This implies that the cesium is reacting with the kaolinite in the mixture, and not with the lime. Since the degree of reaction with kaolinite was much greater than with lime, this result is not surprising.

Figure 4.6 shows the results of injecting cesium and CDEM together in the furnace. The cesium forms a double peak, with a large peak near 0.07 microns, and a shorter peak near 1.5 micron. This result is similar to that seen in the lime experiments. This could indicate the cesium is reacting only with the calcium products in the sorbent 


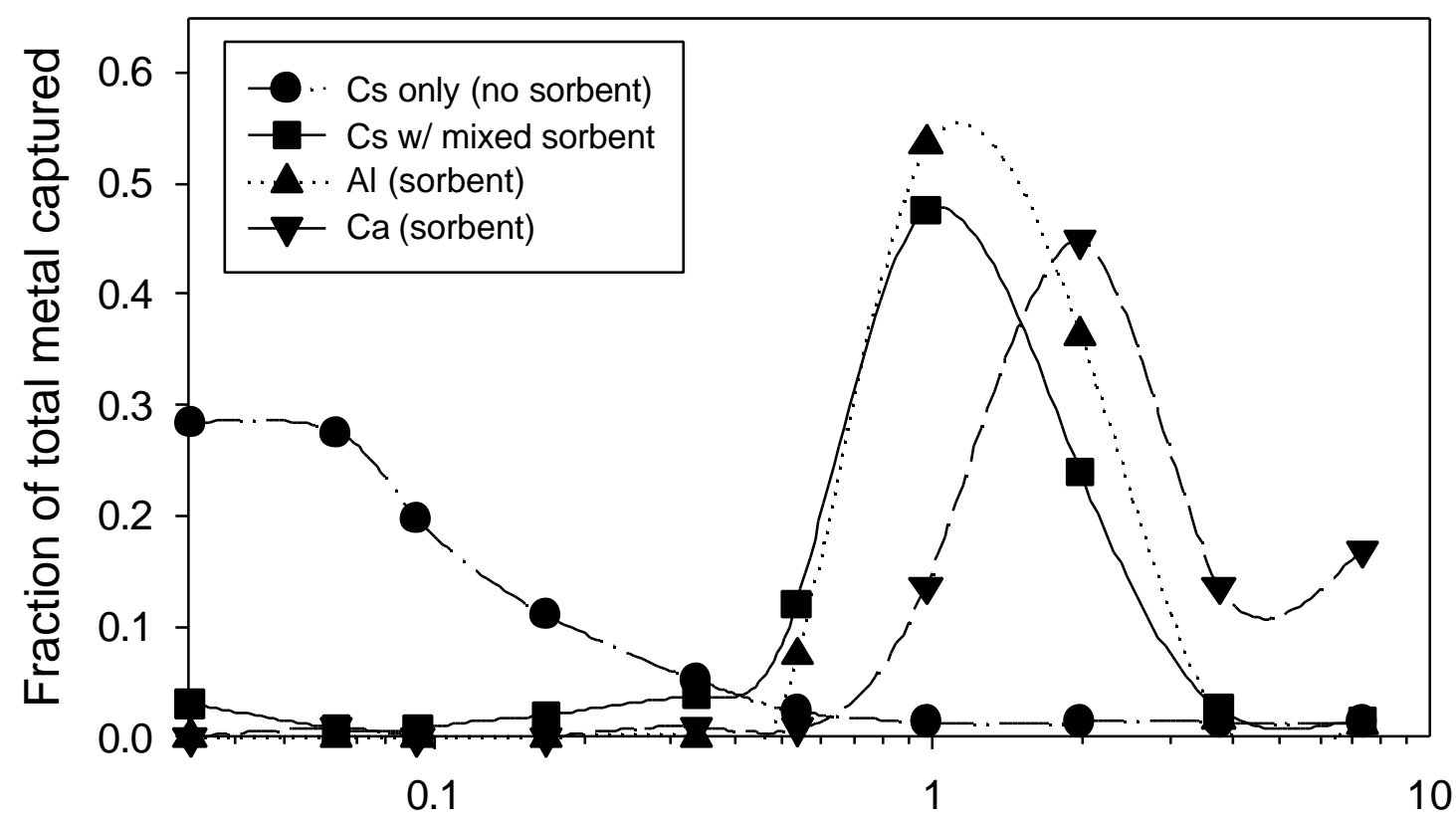

Aerodynamic diameter [ $\mu \mathrm{m}]$

Figure 4.5 - Cesium and mixed sorbent, no dopant

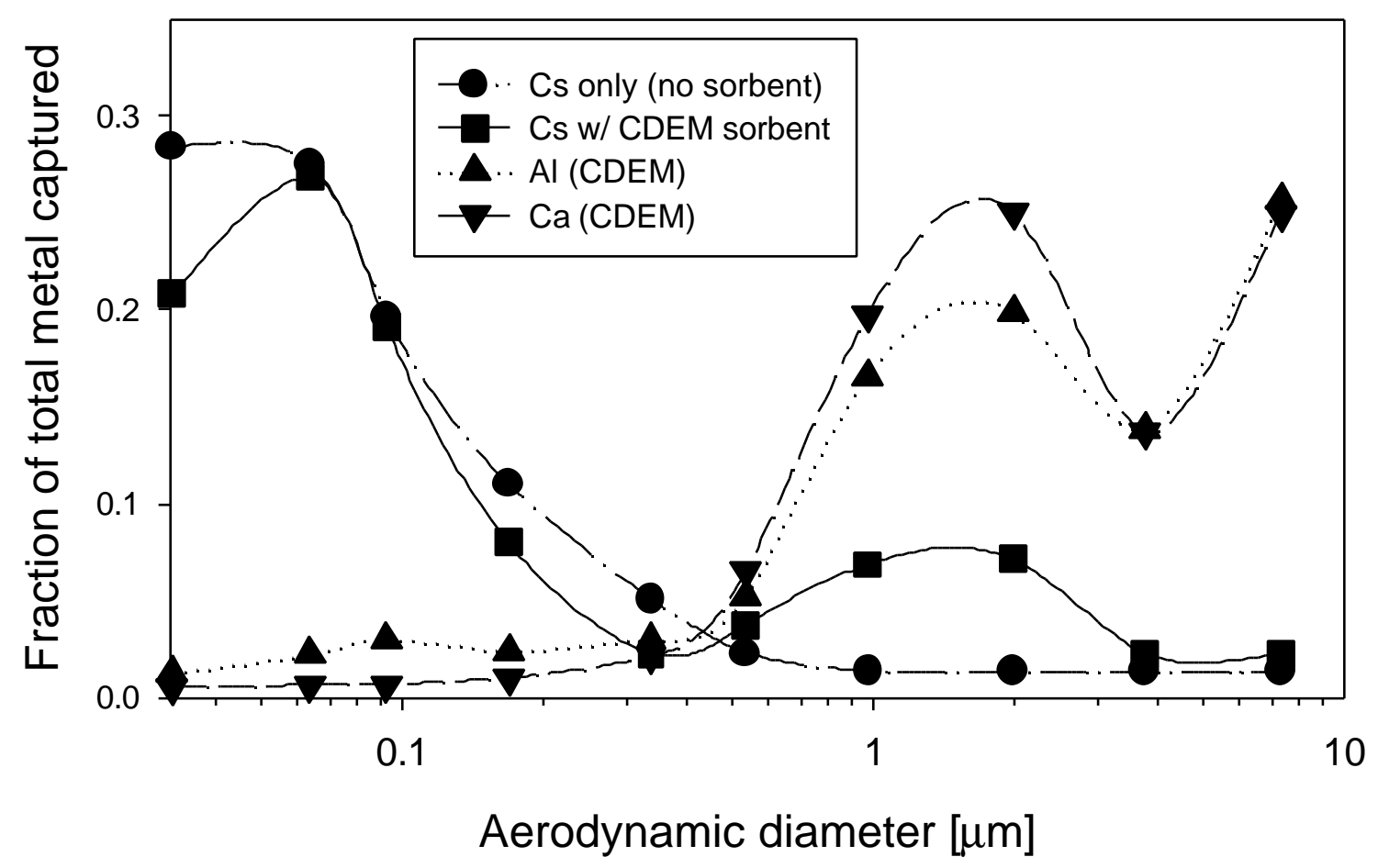

Figure 4.6 - Cesium and CDEM, no dopant 
or that the cesium is blocked in some manner from reacting with the alumino-silicate portion of the sorbent.

The next set of figures display the results of the experiments with cesium in the presence of chlorine gas. Figure 4.7 displays the results of cesium with kaolinite in the presence of chlorine. Cesium and chlorine alone, with no sorbent present, exhibits a peak near 0.09 microns. This peak is slightly different from that seen when there was no chlorine in the system. The cesium peak with no chlorine was butted right up to the small end of the impactor, indicating that the fume that formed was composed of relatively undeveloped particles. With the chlorine present, the peak was fully within the impactor, suggesting a slightly more mature aerosol. This is consistent with the thermodynamic calculations, which indicated that the dewpoint of the cesium would rise.

When the kaolinite was added to the cesium/chlorine system, a double peak formed. The first peak trails off the small end of the impactor, and the second peak forms around one micron. This indicates that the cesium is still reacting with the kaolinite, but to a lesser extent. The remaining cesium forms a sub-micron fume. This could be explained by the gaseous cesium species present. It is suspected that $\mathrm{CsOH}$ is the product that reacts with the kaolinite. When an excess amount of chlorine is present, a large fraction of the cesium is present in the form of $\mathrm{CsCl}$, which does not react with the sorbent. Equilibrium is established between the gaseous $\mathrm{CsOH}$ and $\mathrm{CsCl}$. As the $\mathrm{CsOH}$ reacts with the sorbent, some of the $\mathrm{CsCl}$ forms $\mathrm{CsOH}$ to replace that lost to the sorbent. So, the cesium is still reacting with the kaolinite, but at a much slower rate. 


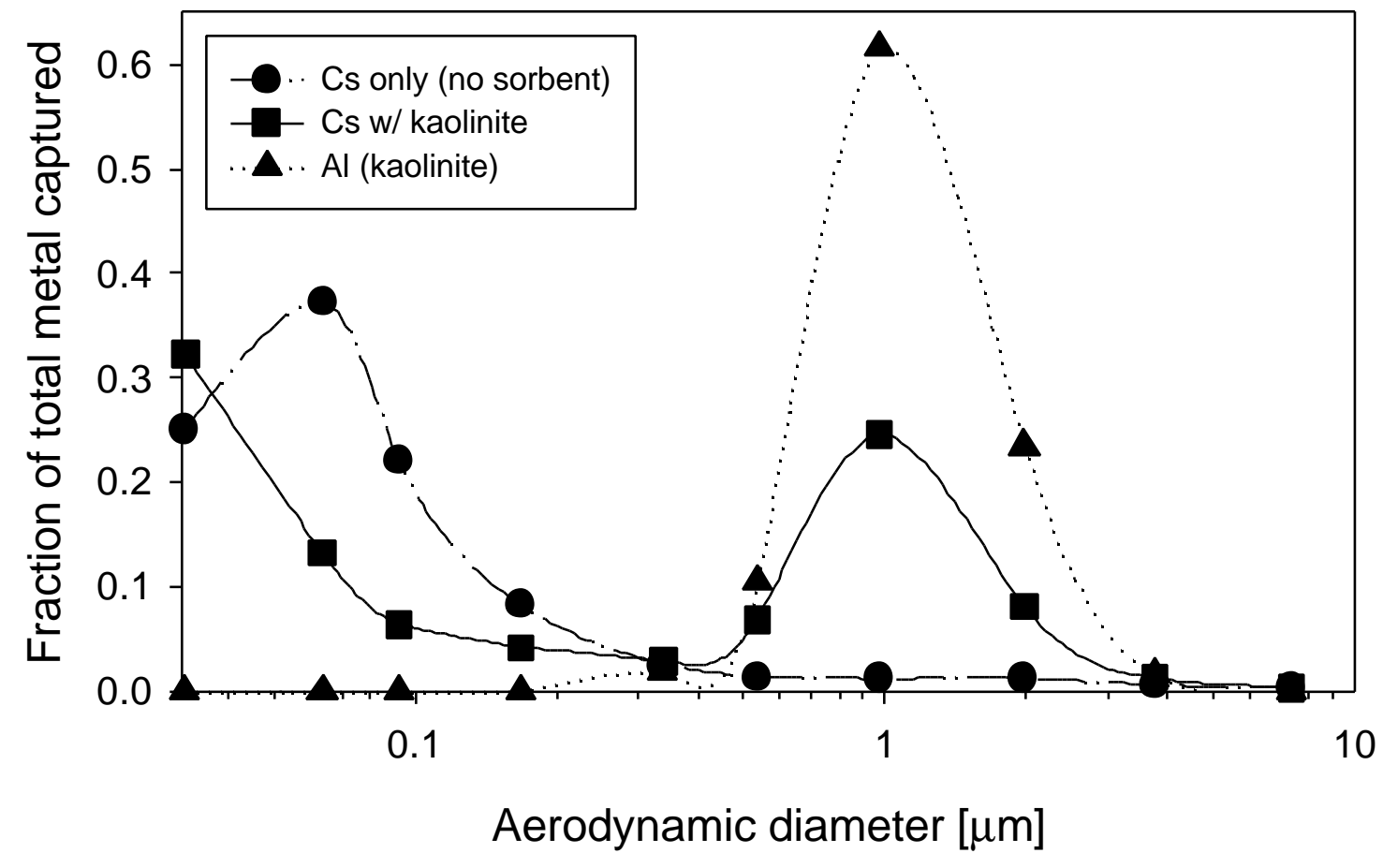

Figure 4.7 - Cesium and kaolinite, with chlorine

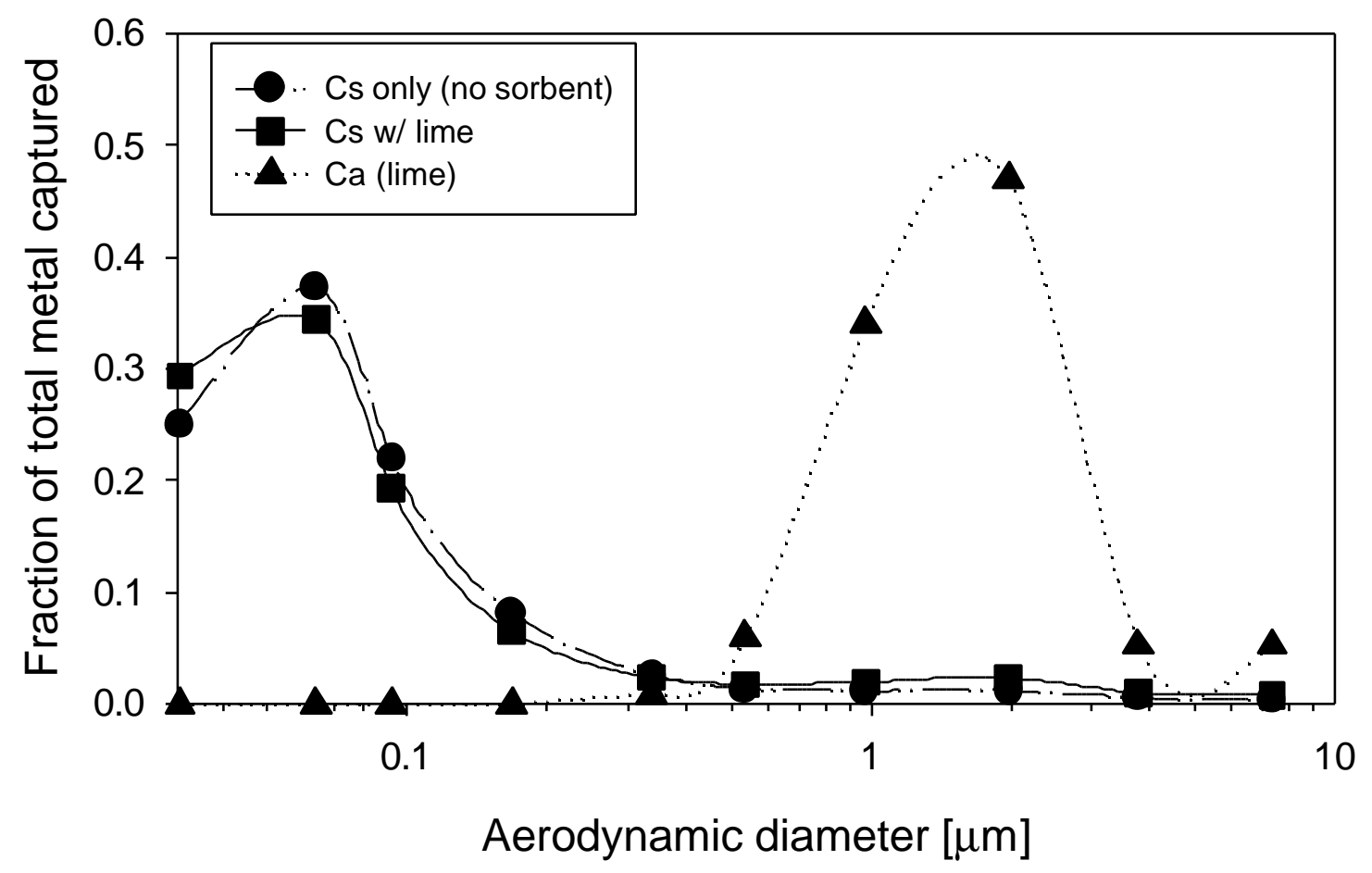

Figure 4.8 - Cesium and lime, with chlorine 
The results of the cesium/chlorine system experiments with injected lime are shown in Figure 4.8. The cesium in this case forms only a single peak near 0.08 microns. This peak exactly matches the peak formed with no sorbent present. This means that the cesium does not react with lime when chlorine is present in the system.

Figure 4.9 shows the cesium/chlorine/mixed sorbent experiment results. The cesium again forms a double peak. The first peak trails out the small end of the impactor, suggesting the formation of a fume, and the second peak is around one micron. The second peak forms near the aluminum peak from the kaolinite. These results indicate that the cesium is reacting with the kaolinite in the sorbent and not with the lime. This is consistent based on the results from the previous two figures.

The results in Figure 4.10 are from the cesium/chlorine/CDEM test system. The cesium in this case forms a single peak near 0.08 microns, matching the peak that forms without sorbent present. This indicates that the cesium is not reacting with the sorbent. In addition, there was a change in the appearance of the sorbent after being exposed to the chlorine. The sorbent started as a beige/white powder, prior to injection in the furnace. After being exposed to the chlorine, the sorbent appeared more yellowish, with some evidence of crystallization. Thus, the chlorine appears to react with the sorbent, changing its chemical characteristics.

The results from the cesium and sulfur tests are depicted in the next group of figures. Shown in Figure 4.11 are the data that demonstrate the effect of sulfur on cesium particulate formation. The one curve shows the results when cesium in the presence of sulfur is sampled from the furnace at Port 14, which is where all the previous samples 


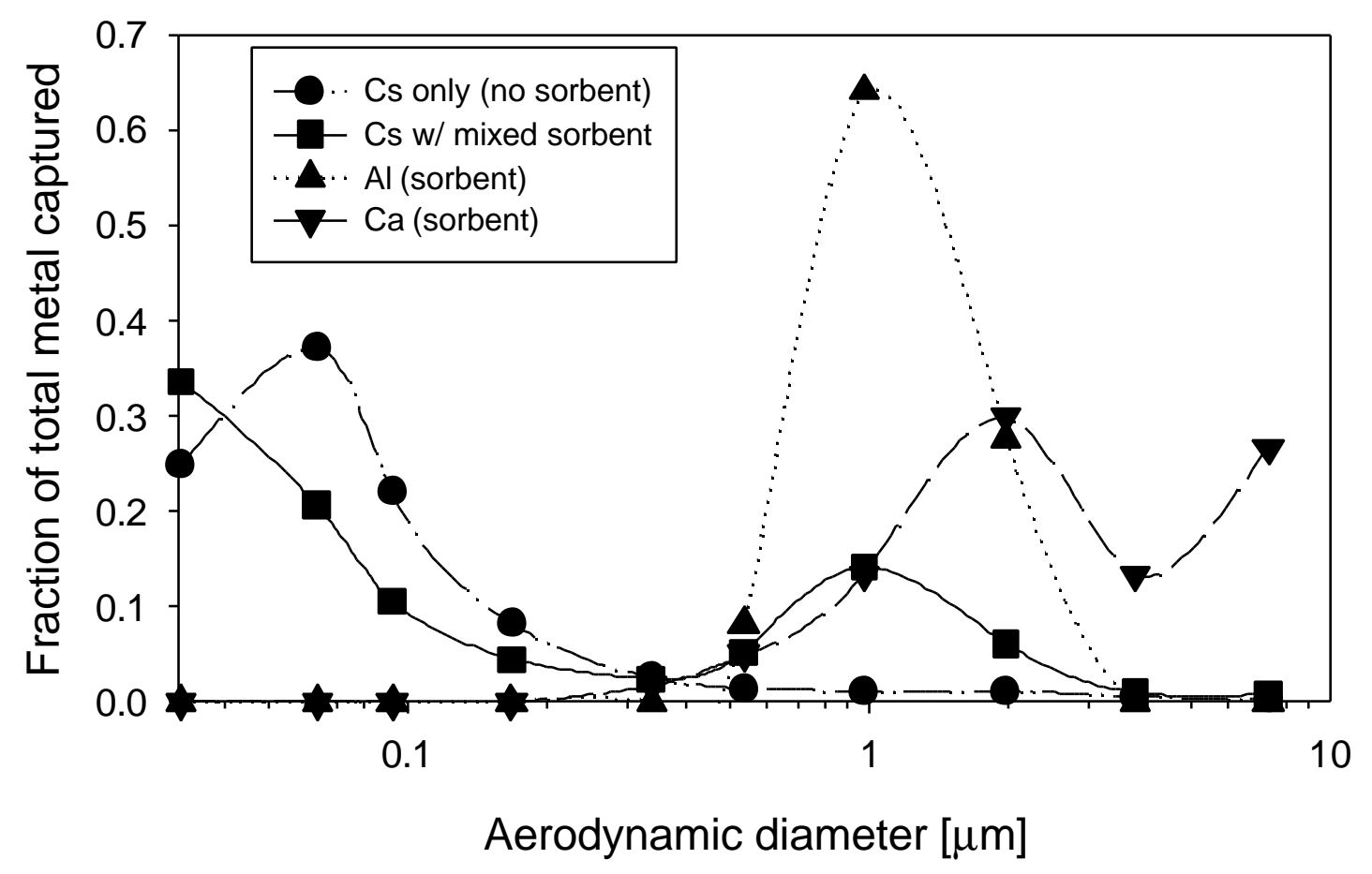

Figure 4.9 - Cesium and mixed sorbent, with chlorine

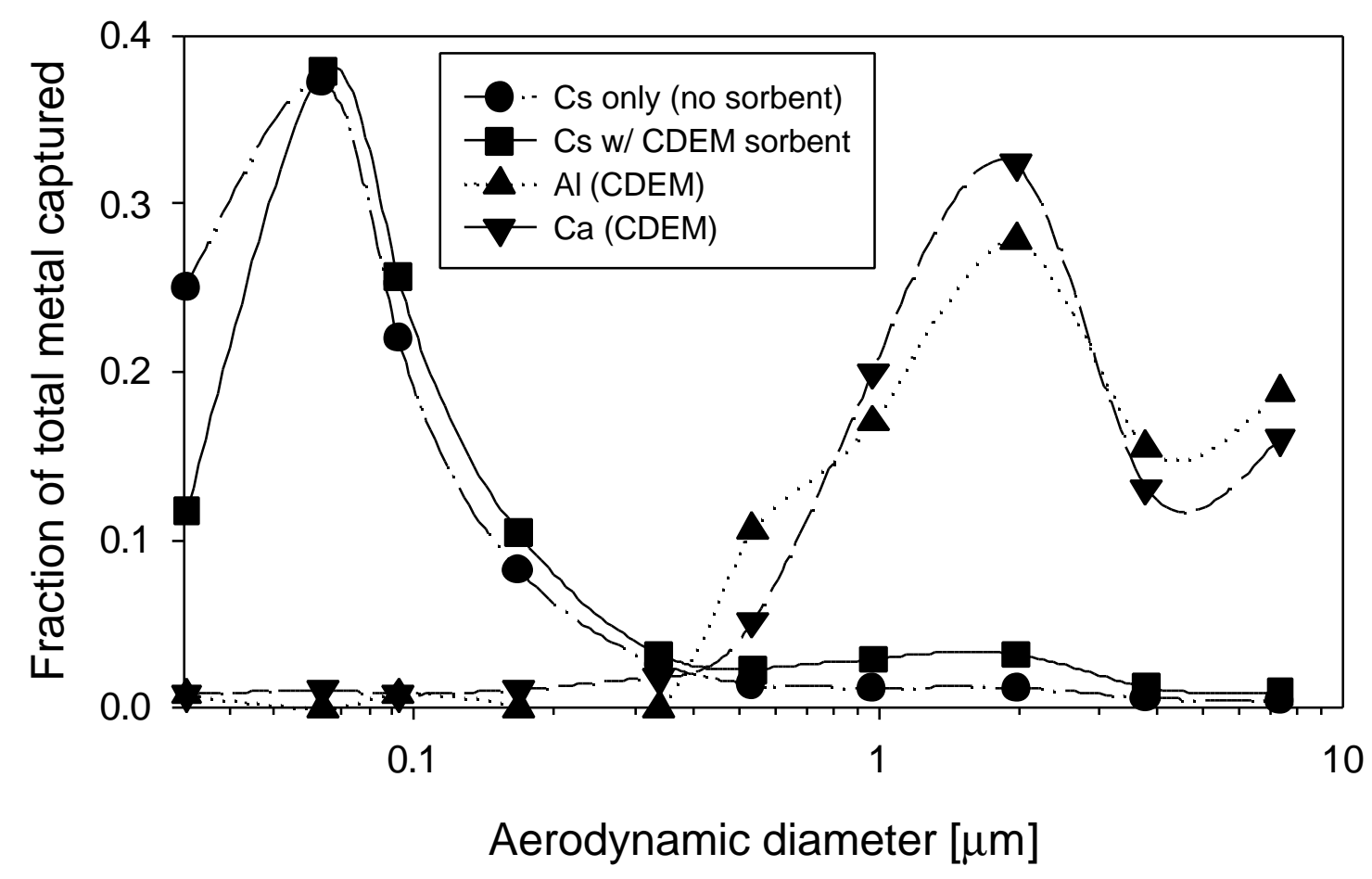

Figure 4.10 - Cesium and CDEM, with chlorine 


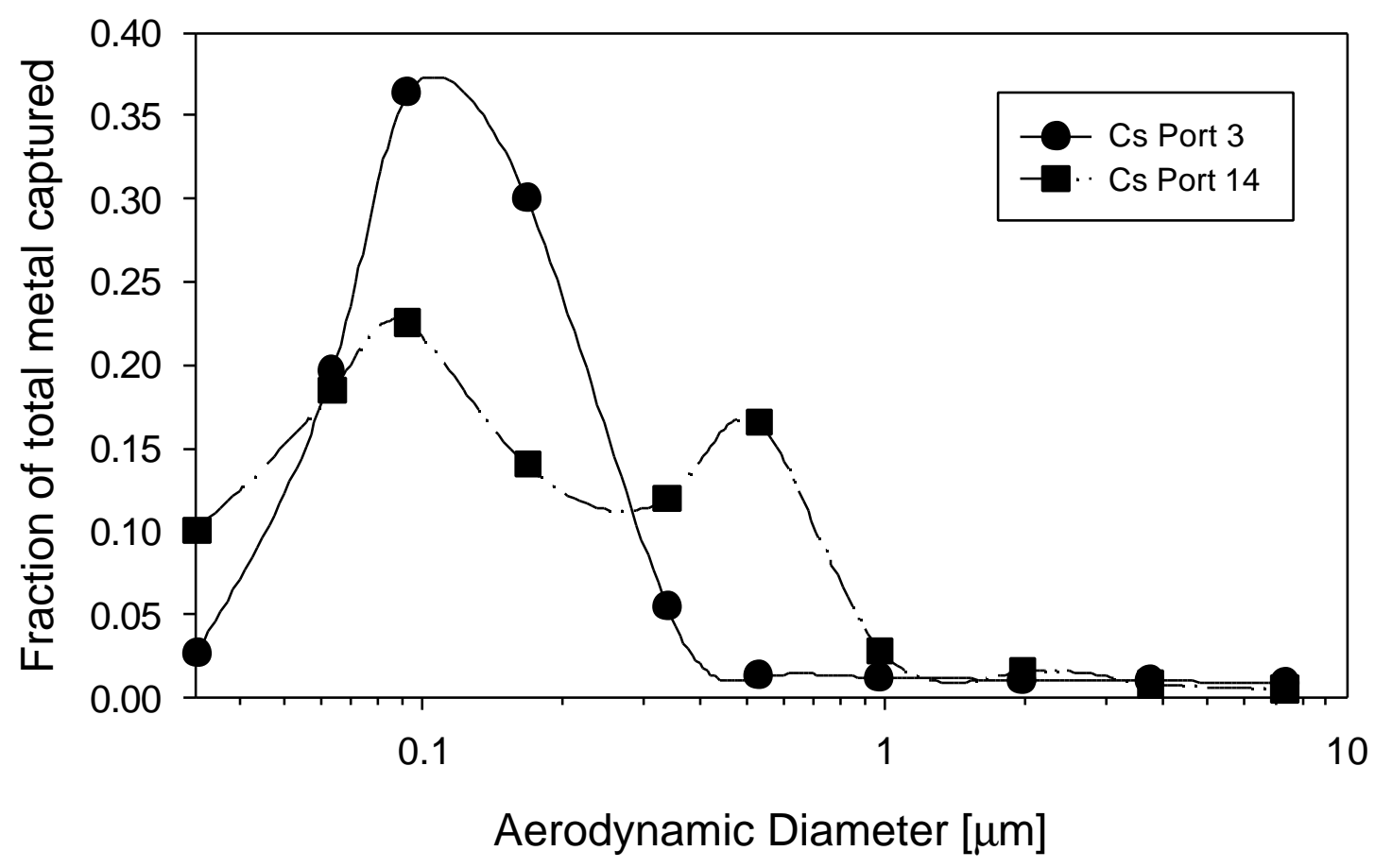

Figure 4.11 - Time evolution of cesium/sulfur particles

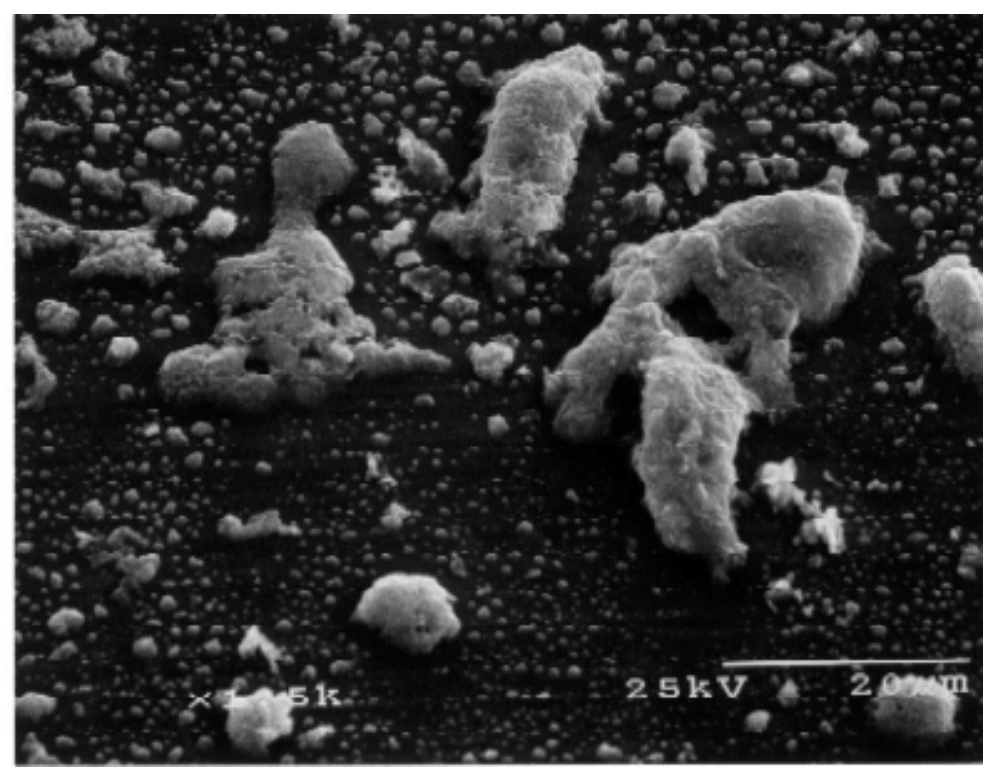

Figure 4.12 - SEM micrograph of cesium particles with sulfur 
have been collected. This point in the furnace has a temperature of about $1125 \mathrm{~K}$ and a residence time of about two seconds. The cesium shows a double peaked curve, with the peaks forming at 0.1 and 0.5 microns. To better understand the nature of these results, a second sample was taken at exactly the same conditions, except the aerosol was sampled at Port 3. This port has a temperature of $1450 \mathrm{~K}$ and a residence time of about 0.4 seconds. This sample exhibits a curve with a single peak at 0.1 microns. This suggests that when the cesium enters the furnace, it is completely vaporized. As the cesium passes down the length of the furnace, the temperature drops and the cesium starts to nucleate and form particles. As time progresses, additional cesium condenses onto the existing cesium particles. Therefore, the first peak seen at Port 14 is due to nucleation, and the second peak is due to condensation.

SEM images of the cesium/sulfur tests are shown in Figures 4.12 and 4.13. Figure 4.12 is an image taken at a relatively low magnification. Very large ( 20 micron) particle clusters are evident. This size of particle, had it existed in the sample stream, would have been collected on a higher plate, most likely Plate 10. These particles appear to be agglomerations of smaller particles that formed due to impaction in the impactor. Figure 4.13 is a close-up of some of the smaller particles on the plate. These particles also appear to be built-up and show some agglomeration. These particles represent the second peak of the PSD curve. This morphologic evidence supports the theory that these particles are formed by nucleation of the gas phase cesium, followed by condensation.

The results when kaolinite is added to the system are shown in Figure 4.14. The cesium shows a strong peak near one micron. This peak follows the aluminum peak from 


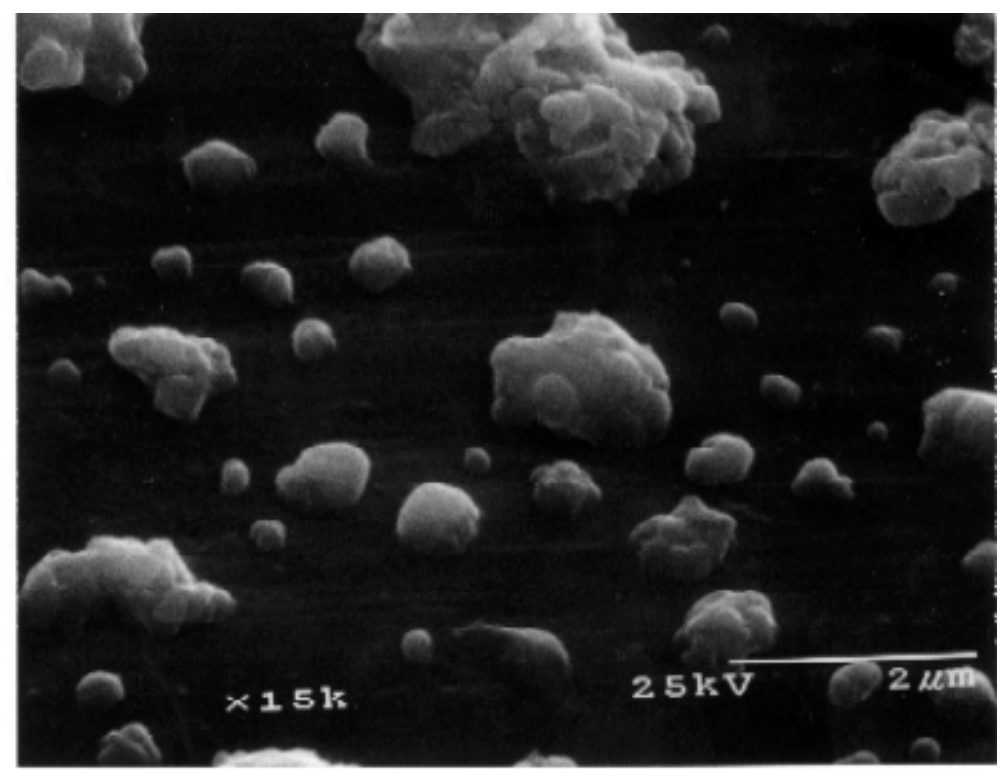

Figure 4.13 - SEM micrograph of cesium particles with sulfur

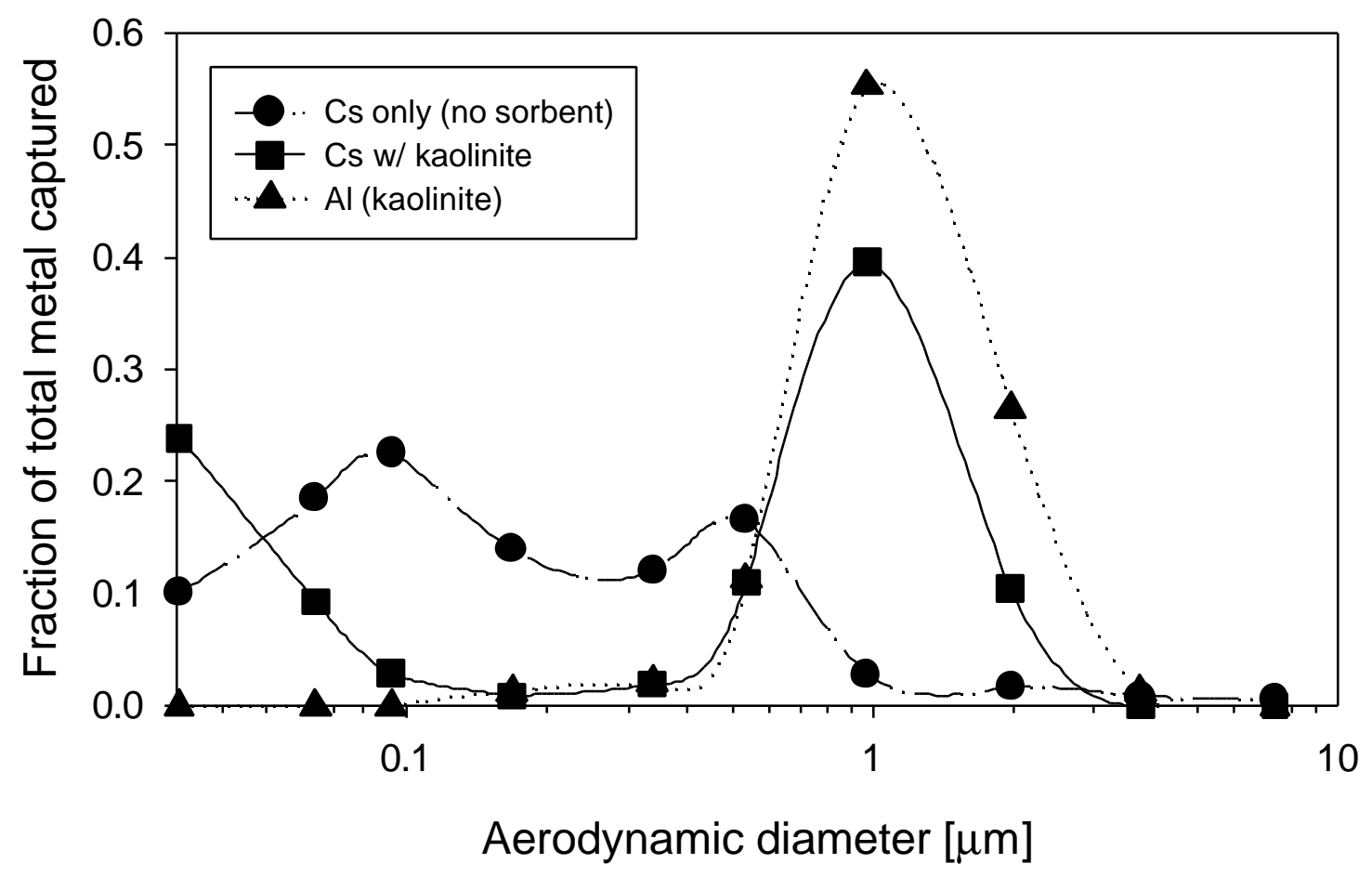

Figure 4.14 - Cesium and kaolinite, with sulfur 
the sorbent. This suggests that the cesium is reactively captured. There is also a trailing peak at the low end of the impactor, indicating that not all of the cesium has reacted. This coincides with the thermodynamic predictions. The presence of sulfur increased the dewpoint of the cesium. This allowed less time in the reactor for the cesium to react with the sorbent, hence reducing the total capture of the cesium.

The results of the cesium/sulfur/lime experiments are shown in Figure 4.15. A double peak is formed, with peaks at the trailing end and at two microns. This indicates that the cesium is reacting with the lime, but at a finite rate, leaving a portion of the metal unreacted. Thus, it appears that the presence of sulfur enhances the ability of lime to capture cesium.

Figures 4.16 and 4.17 are micrographs that show the cesium/sulfur/lime samples. Figure 4.16 is a sample of lime, with no metal or dopant present. The particles in this picture have a chunky appearance, with some degree of angularity. The edges are a little more rounded than those seen in the kaolinite. Figure 4.17 shows the cesium/sulfur/lime sample, which was taken from Plate 7 of the impactor. There are two different particle morphologies present in this sample. Particles in the upper left and lower left corners have an angular shape. These are lime particles that show little evidence of melting or reaction. The particles in the lower center of the picture have a rounded appearance. These appear to be cesium particles that have agglomerated onto the sorbent particles, but that have not reacted with the lime.

In Figure 4.18 are the results of cesium and sulfur with the mixed sorbent. Again, the double peak is formed, with one peak trailing out the bottom of the impactor and the 


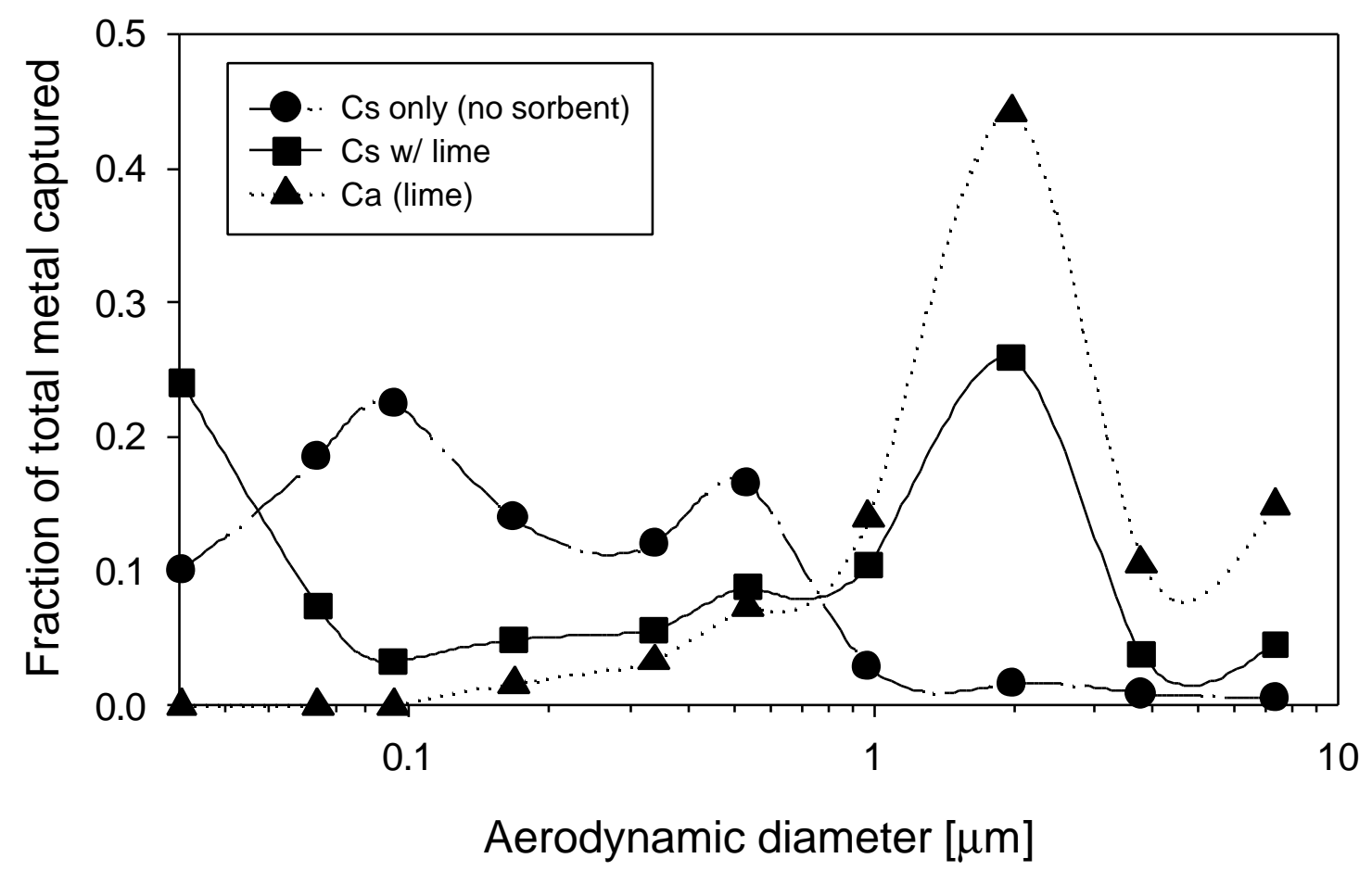

Figure 4.15 - Cesium and lime, with sulfur

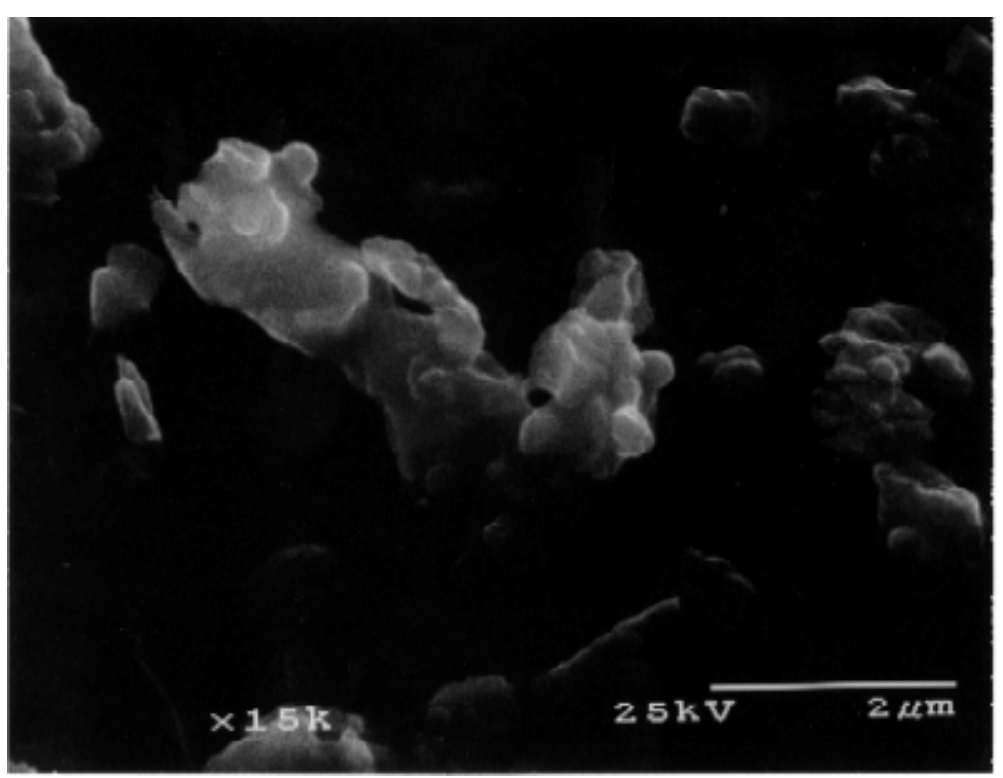

Figure 4.16 - SEM micrograph of unreacted lime 


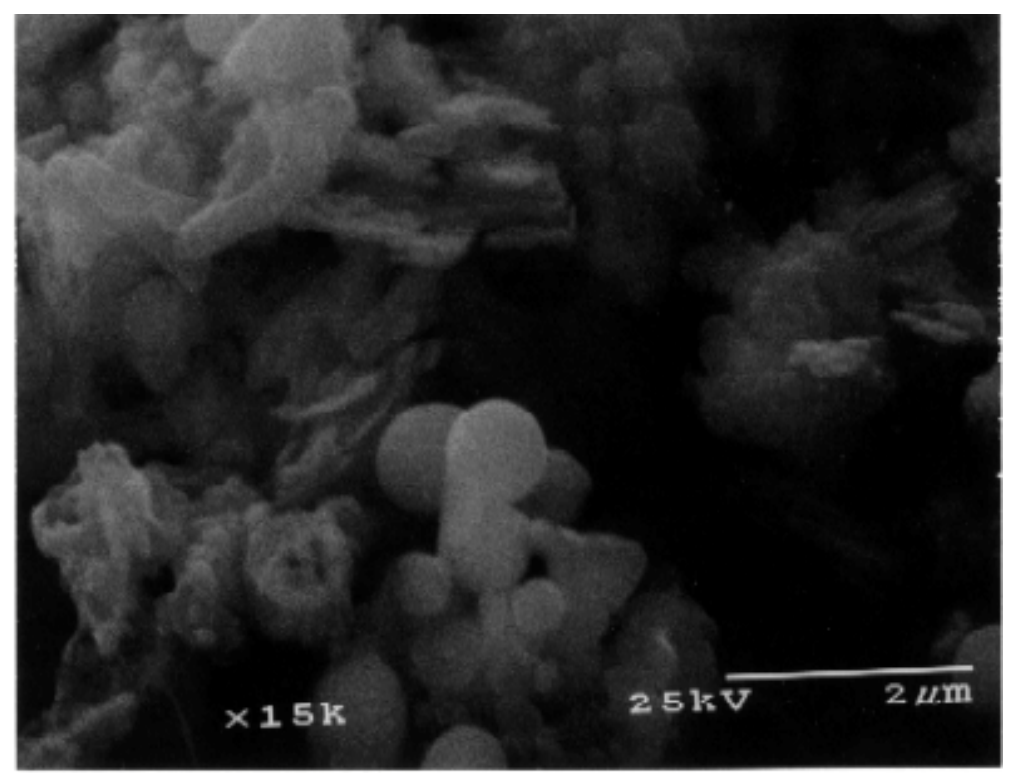

Figure 4.17 - SEM micrograph of cesium and lime particles, with sulfur

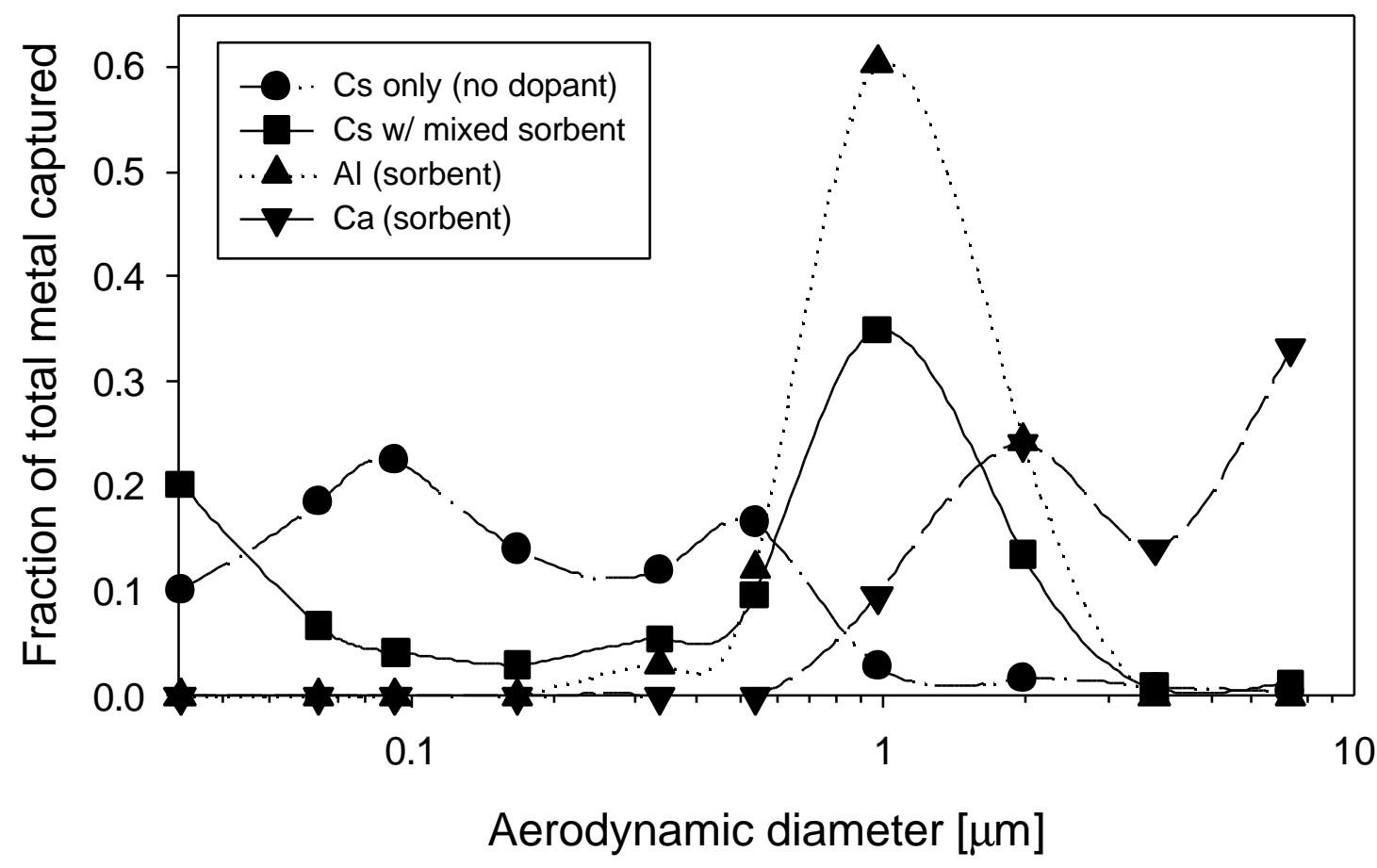

Figure 4.18 - Cesium and mixed sorbent, with sulfur 
other forming at one micron. This shows that the cesium is preferentially reacting with the kaolinite in the system, but is still being captured.

Figure 4.19 shows the results from the cesium/sulfur/CDEM tests. There is a large peak formed near two microns, indicating that the CDEM sorbent is fairly effective in capturing cesium in the presence of sulfur. There is also a small peak at the trailing end, showing that the reaction is occurring at some finite rate.

\subsection{Strontium Results}

The results of the strontium tests are shown in Figures 4.20 through 4.39. The first of these figures represent the results of the tests with strontium only, without the addition of dopants.

Figure 4.20 displays the results of the strontium and kaolinite system, with no dopant gas. Strontium on its own forms a broad peak at 0.8 microns, with a long tail extending to the smaller particle size. This most likely represents residual strontium from the aqueous solution. The water from the droplets evaporates, leaving the strontium behind. When kaolinite is added to the system, the peak shifts to a slightly larger (one micron) particle diameter and the long trailing tail disappears. This might represent capture, but the other evidence suggests that the strontium does not vaporize, preventing it from reacting. The strontium is probably collected on the kaolinite particles by some physical mechanism, such as coagulation or impaction.

The particles shown in Figure 4.21 are strontium particles collected without sorbent present. These particles show a very rounded morphology. This shape is 


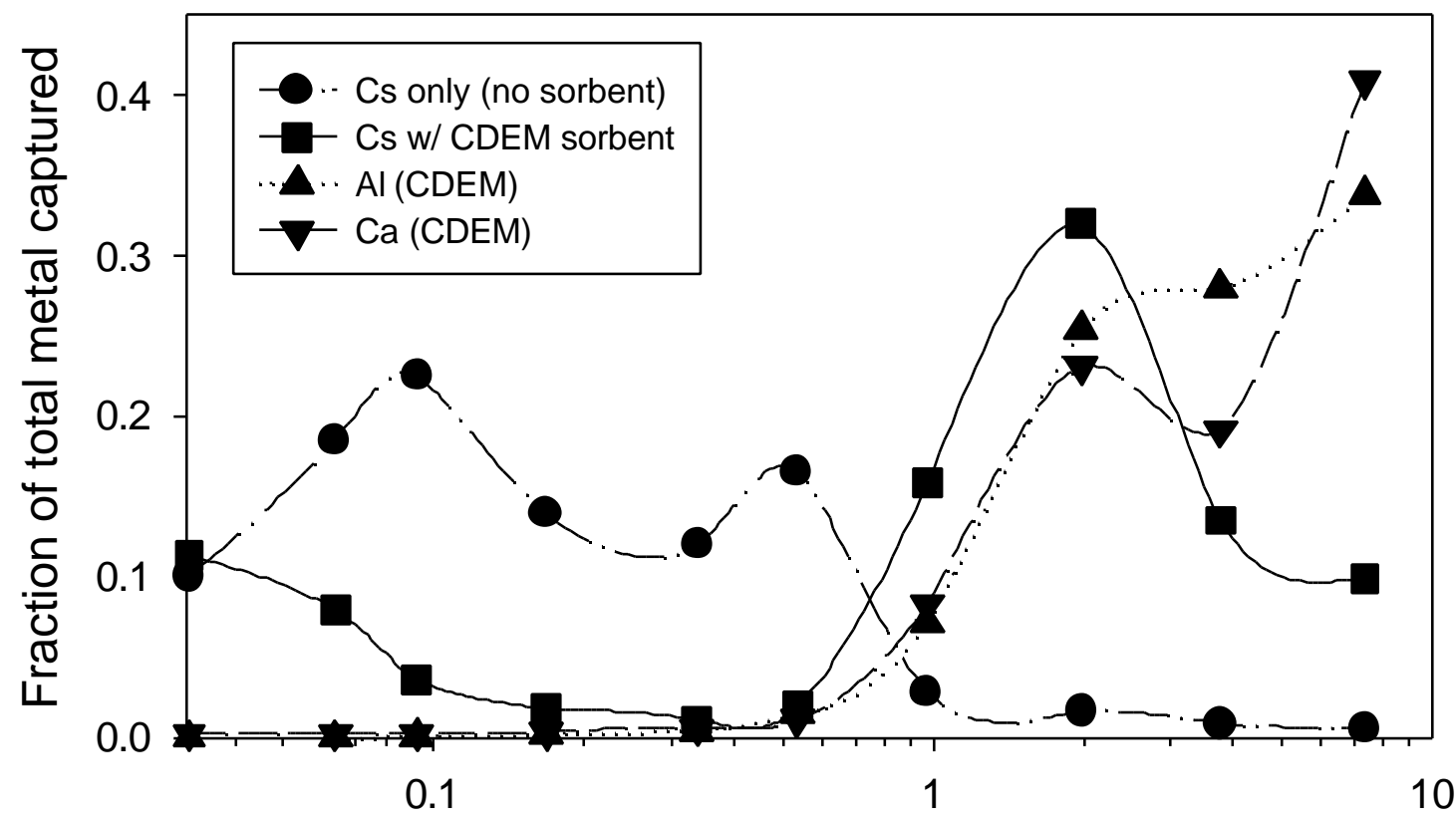

Aerodynamic diameter $[\mu \mathrm{m}]$

Figure 4.19 - Cesium and CDEM, with sulfur

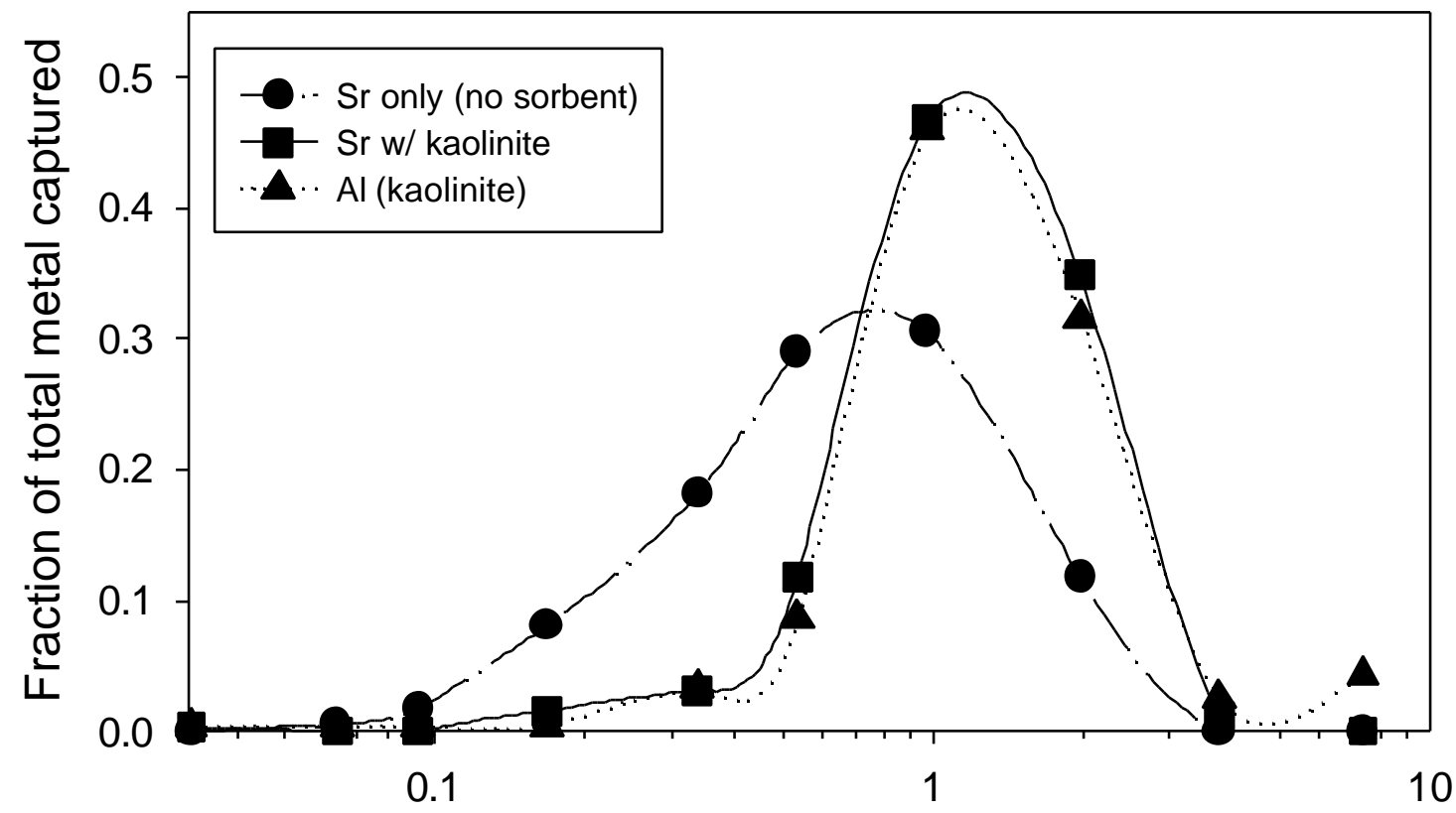

Aerodynamic diameter $[\mu \mathrm{m}]$

Figure 4.20 - Strontium and kaolinite, no dopant 


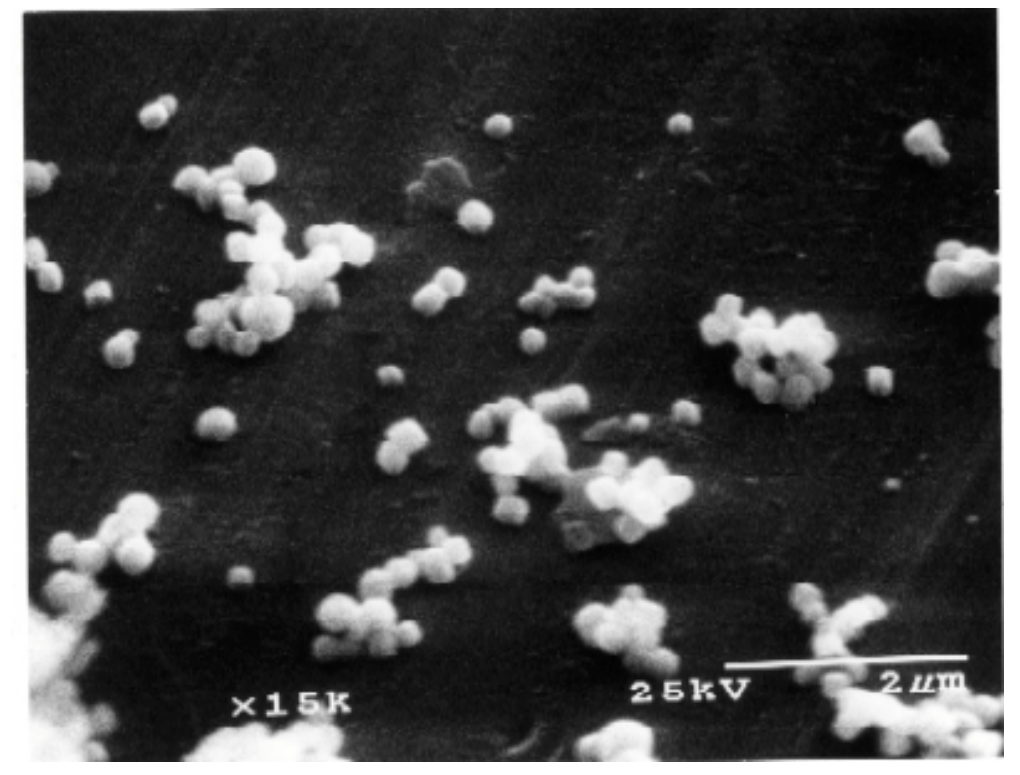

Figure 4.21 - SEM micrograph of residual strontium particles

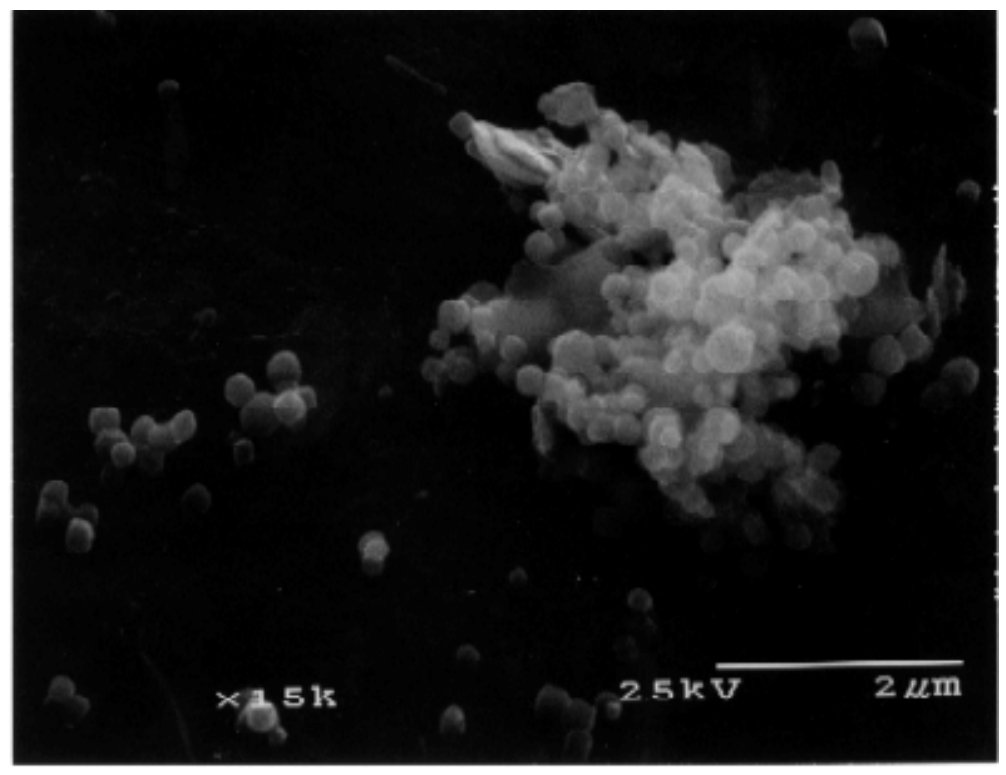

Figure 4.22 - SEM micrograph of strontium and kaolinite particles 
consistent with the idea that the water from the spray droplets evaporates, leaving the strontium behind. The results when kaolinite is added to the system are shown in Figure 4.22. The residual strontium particles are quite evident. In addition, obscured by the strontium particles clinging to their surface, are kaolinite particles that show the jagged, angular appearance of unreacted kaolinite. Thus, it appears that no reaction occurs between the strontium and kaolinite.

The results of the strontium and lime experiments are shown in Figure 4.23. The strontium forms a peak at around one micron, with some evidence of a tail in the smaller size range. Again, the strontium appears to be collected by some physical mechanism. This is supported by the fact that the strontium peak does not exactly follow the lime peak. Figure 4.24 is a micrograph of this system. As in the case of strontium and kaolinite, the residual strontium particles are seen clinging to the sorbent particles, which show no evidence of chemical reaction.

The strontium and mixed sorbent results are depicted in Figure 4.25. The strontium again forms a peak at around one micron, with the smaller sized tail significantly reduced. Again, this appears to be a physical mechanism shifting the PSD. This is supported by the fact that the strontium does not exactly match the distribution of either of the components of the sorbent.

Shown in Figure 4.26 are the results of the strontium and CDEM tests. The peak in this case appears similar to that in the other sorbent cases. However, there is a small tail that appears on the larger end of the distribution. Because the size of the CDEM 


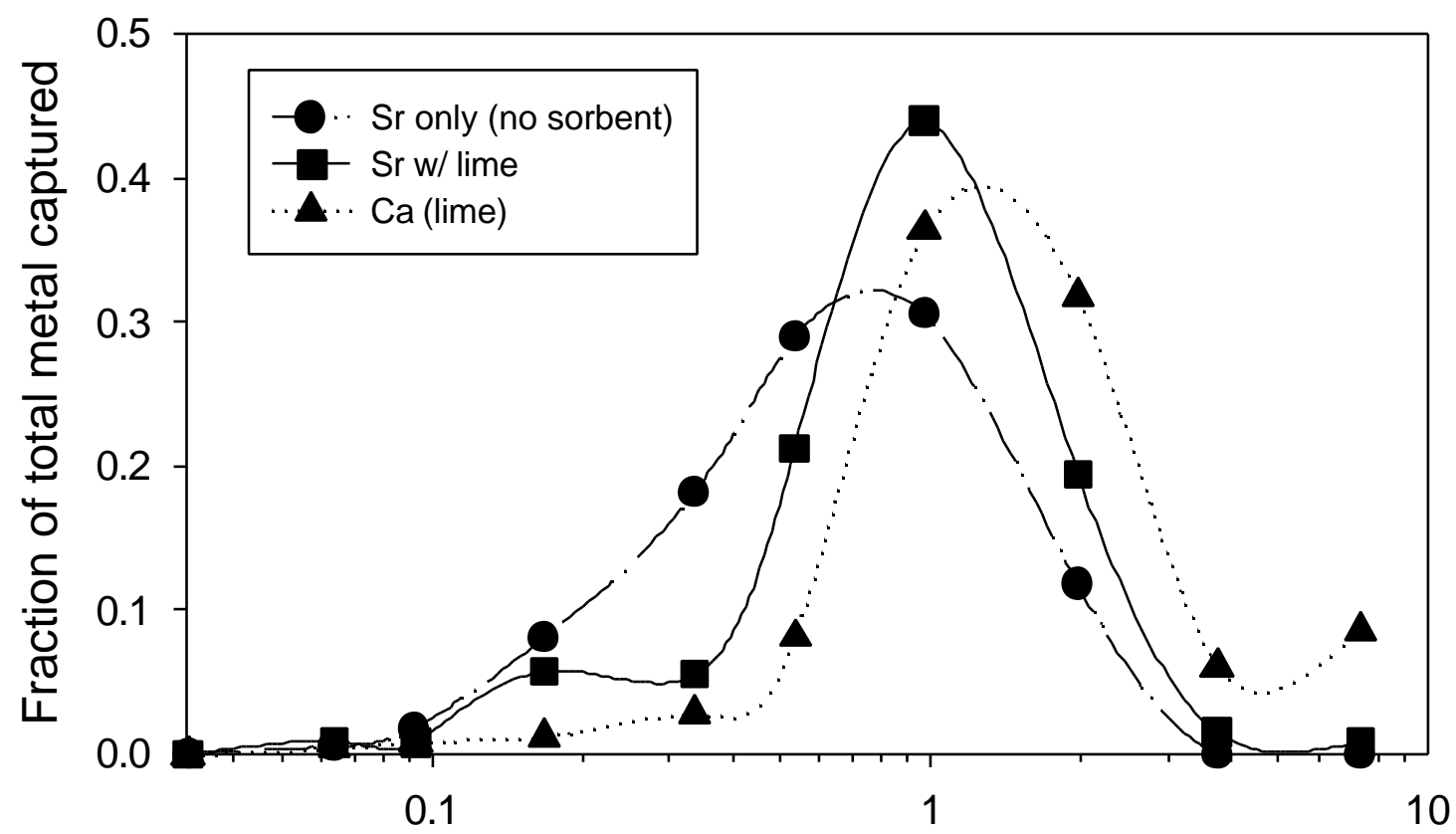

Aerodynamic diameter $[\mu \mathrm{m}]$

Figure 4.23 - Strontium and lime, no dopant

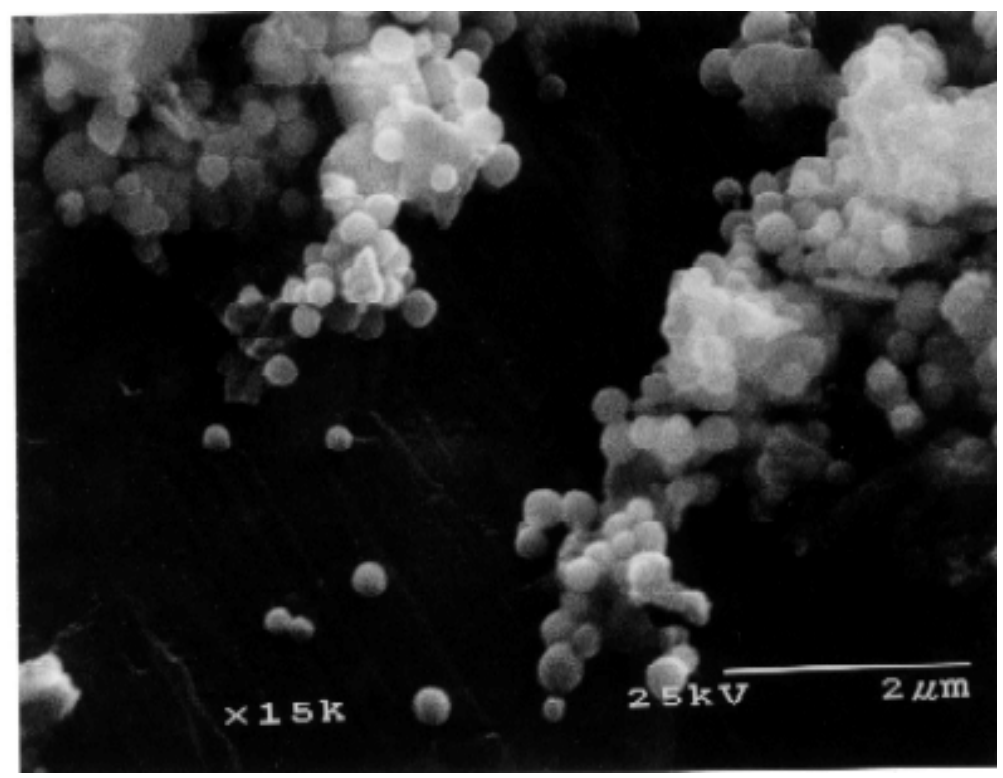

Figure 4.24 - SEM micrograph of strontium and lime particles 


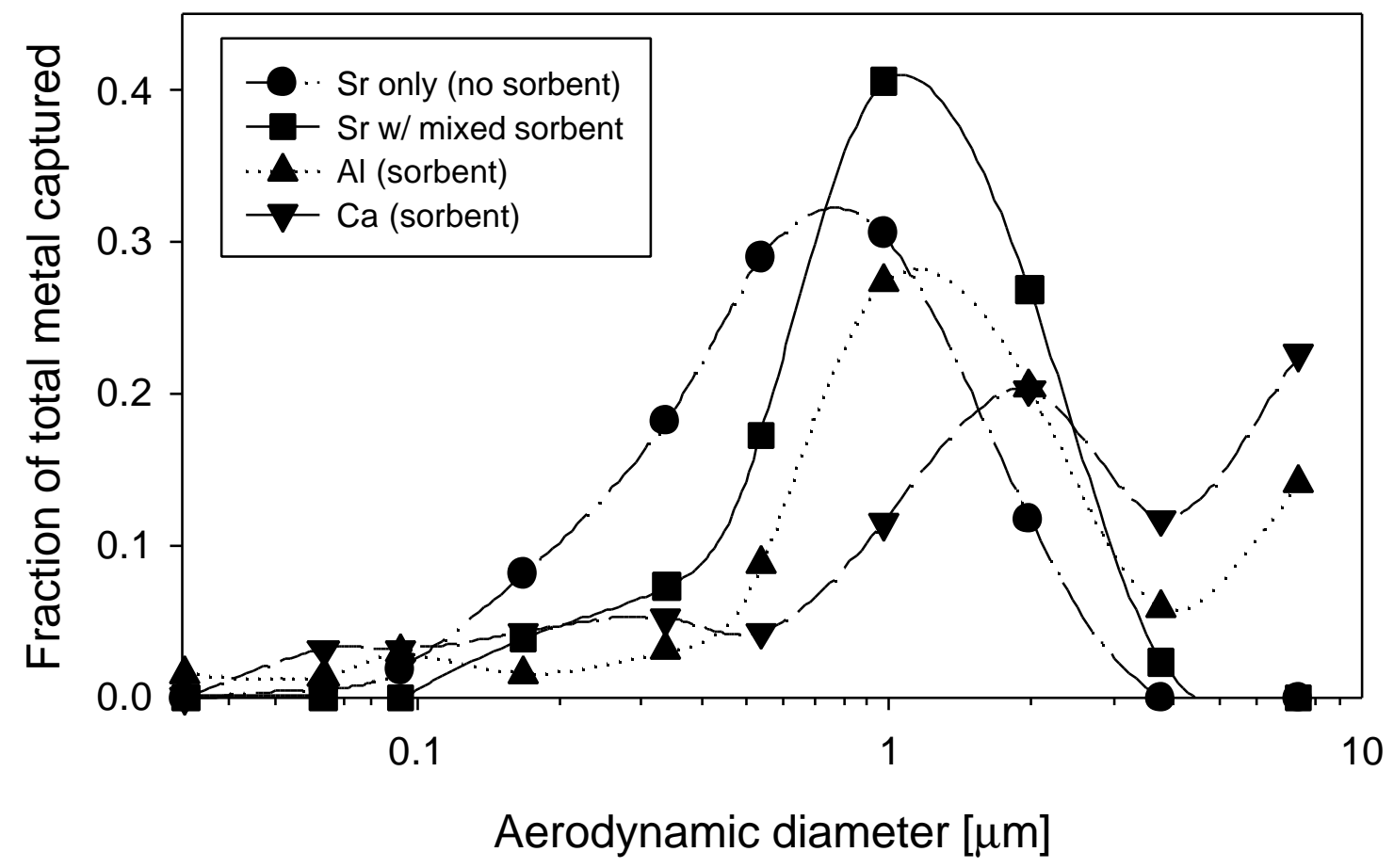

Figure 4.25 - Strontium and mixed sorbent, no dopant

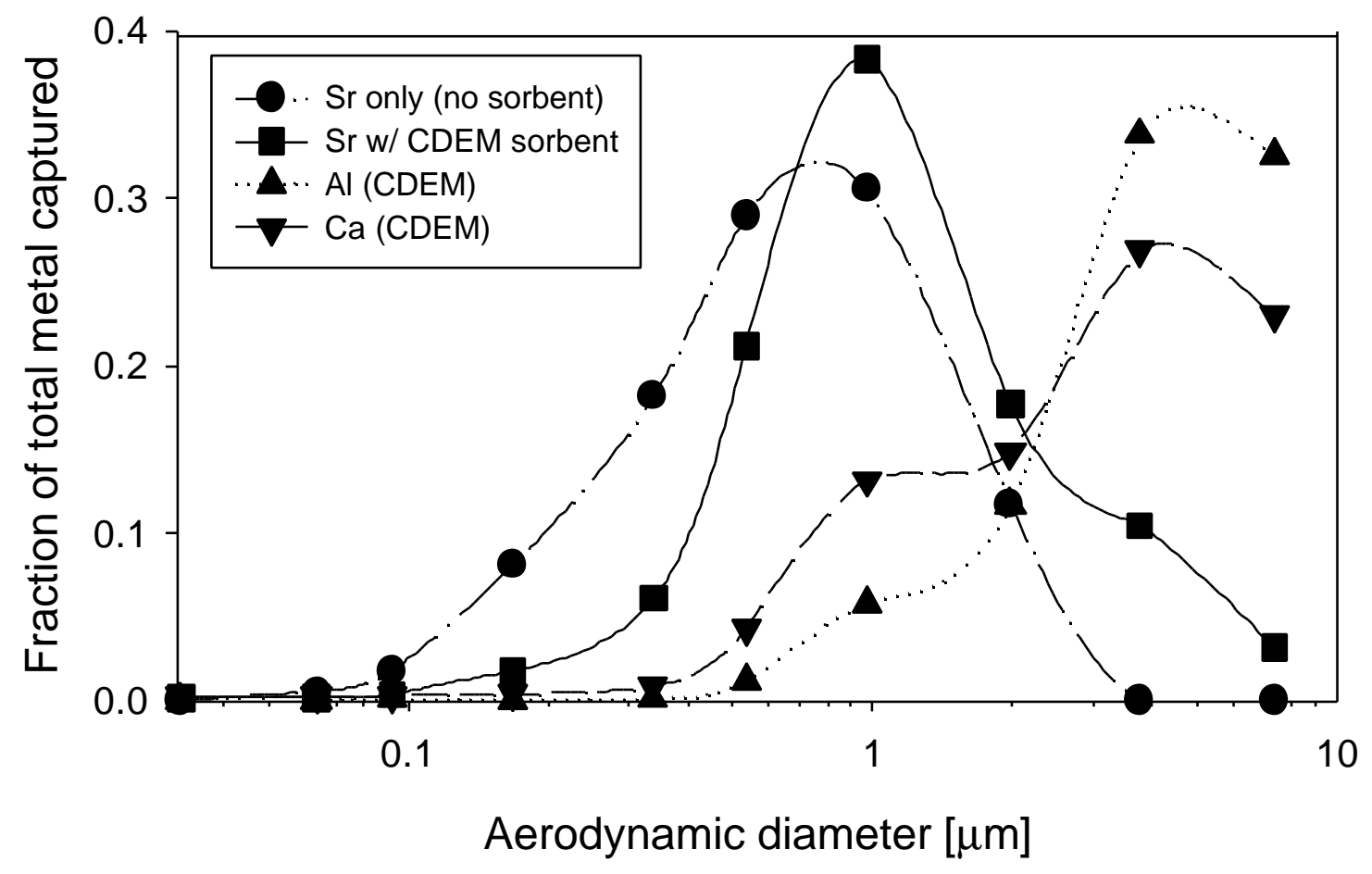

Figure 4.26 - Strontium and CDEM, no dopant 
sorbent is larger, this tail is not unexpected. This is also consistent with the physical mechanism explanation.

The results for the strontium and kaolinite tests with chlorine present in the system are shown in Figure 4.27. The strontium with no sorbent present forms a curve with the peak around 0.2 microns. This indicates that the sorbent is actually vaporizing in the furnace. This was predicted by the thermodynamic calculations. When kaolinite is added to the system, the strontium peak shifts to around one micron. This peak closely matches the aluminum peak from the kaolinite. This shows that reactive capture is occurring with chlorine present.

SEM images of this situation are seen in Figures 4.28 and 4.29. Figure 4.28 is an image of the particles at a relatively low magnification. The metal and sorbent mix appears to be a fairly large ( 15 microns) agglomeration, with significant melting of the sorbent particles. This agglomeration must have occurred on the impactor plate, as a particle of that size would have been separated from the impactor earlier had it formed in the furnace or probe. Figure 4.29 is an image taken at higher magnification. This image also shows significant melting.

The strontium/lime/chlorine results are shown in Figure 4.30. The strontium peak remains near 0.2 microns and closely follows the strontium peak when no sorbent is present. This demonstrates that no reaction occurs between the strontium and lime, even in the presence of chlorine.

Figure 4.31 shows the results of the strontium and mixed sorbent results, in the presence of chlorine. The strontium displays a double peaked curve, with peaks at 0.2 


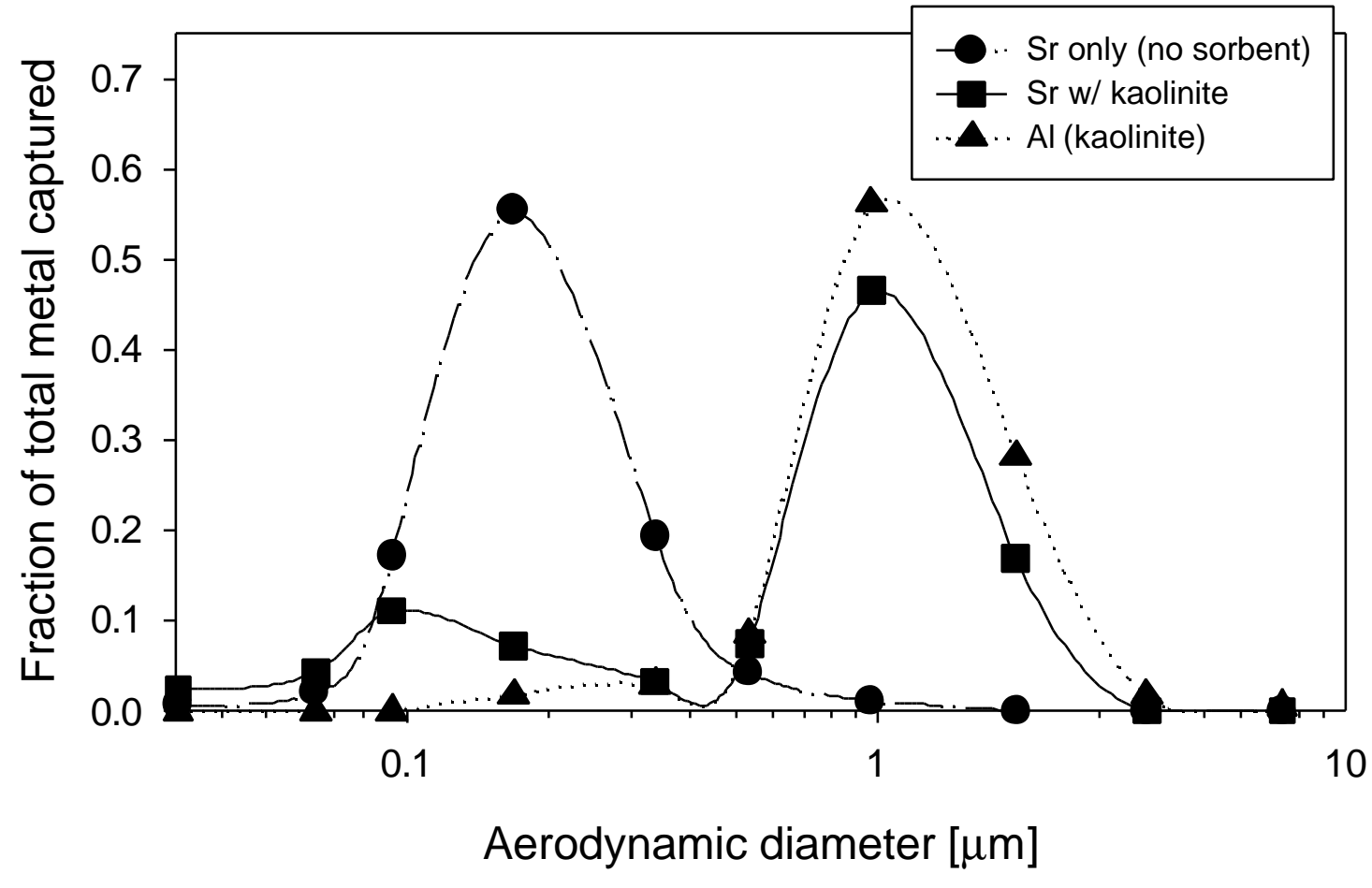

Figure 4.27 - Strontium and kaolinite, with chlorine

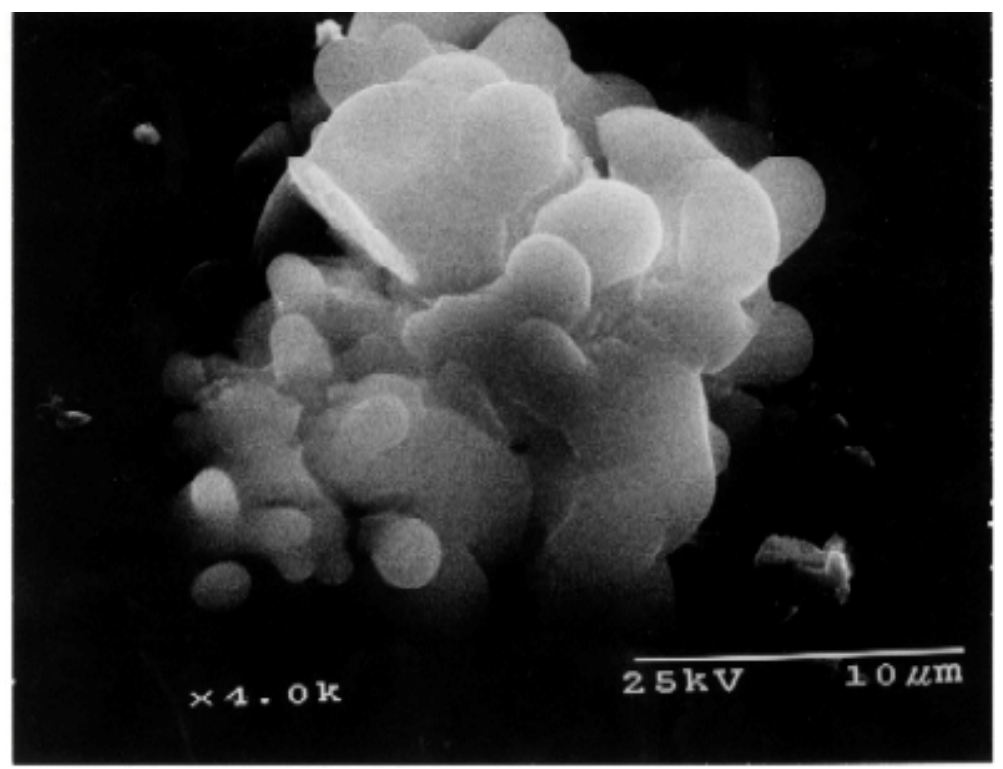

Figure 4.28 - SEM micrograph of strontium and kaolinite, with chlorine 


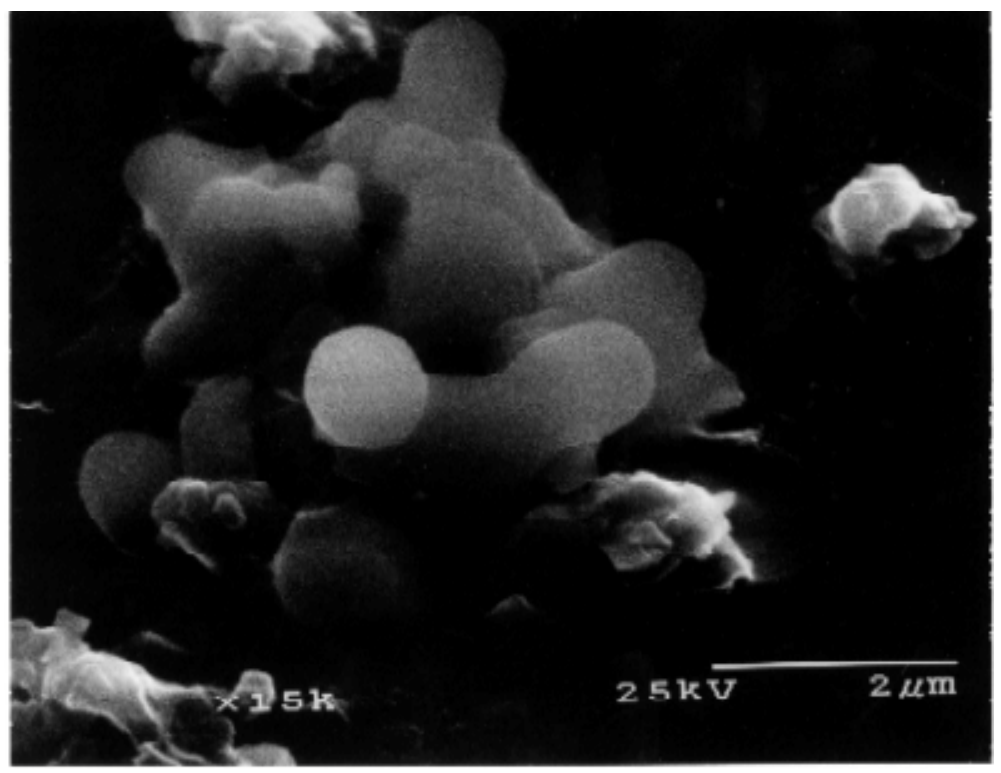

Figure 4.29 - SEM micrograph of strontium and kaolinite, with chlorine

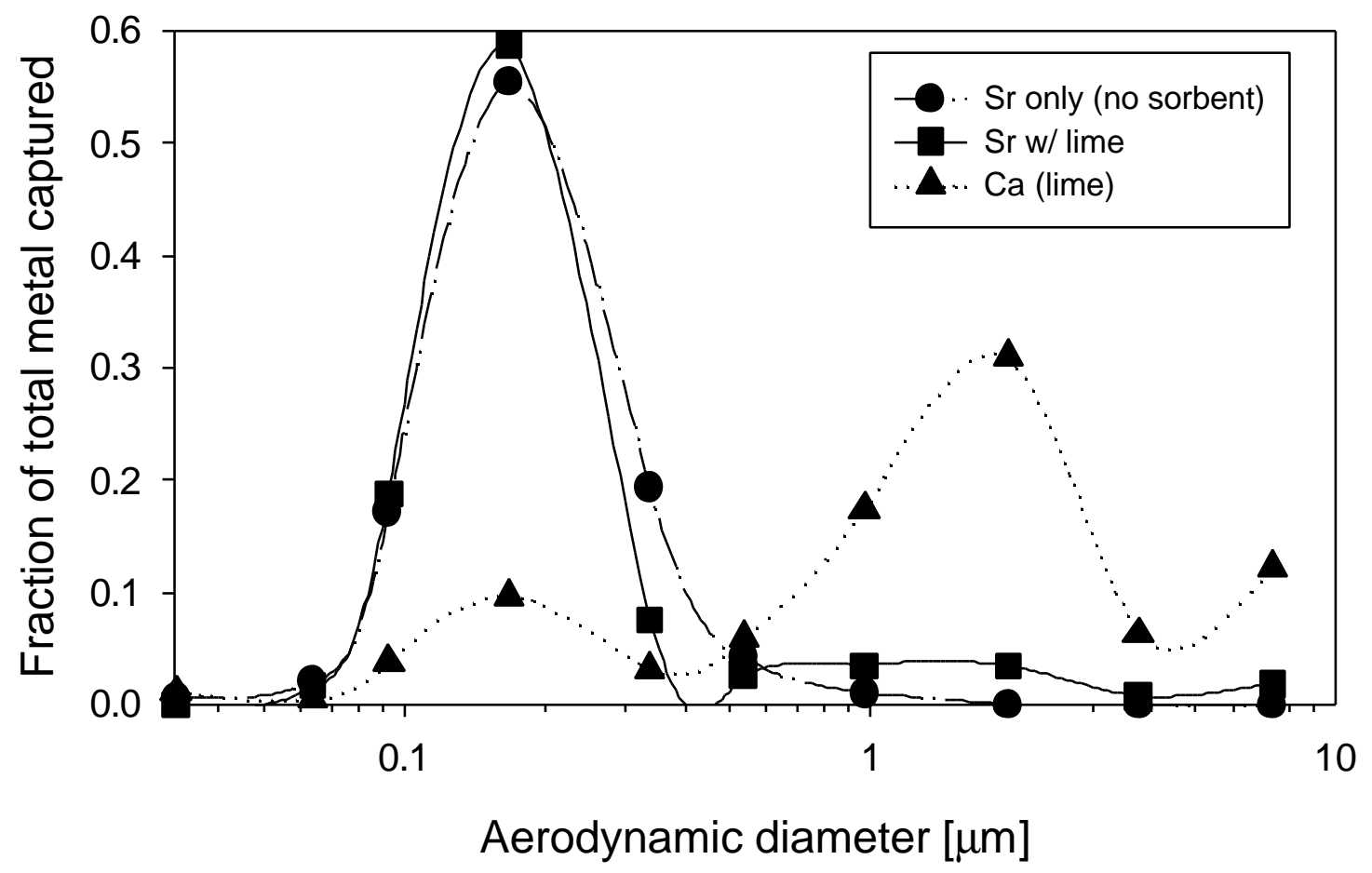

Figure 4.30 - Strontium and lime, with chlorine 
microns and one micron. This change in the strontium peak represents partial reaction with the sorbent. The strontium is reacting with the kaolinite portion of the sorbent, and not reacting with the lime. Higher sorbent feed rates would probably lead to increased capture, but only on the kaolinite fraction.

The CDEM and strontium results with chlorine present are shown in Figure 4.32. The strontium peak is formed near 0.2 microns, with a little bit of tail on the larger size end. This indicates that the strontium may be reacting to a small extent with the sorbent, but the majority of the metal is unreacted. This may be related to the changes in the sorbent due to the presence of chlorine. Some of the strontium may be showing reaction with the alumino-silicate portion of the sorbent even after it has been affected by the chlorine, which would explain the tail of the curve.

Figure 4.33 presents the results of strontium when exposed to sulfur. The strontium, when sampled at Port 14, displays a double peaked curve, with the peaks forming at 0.2 microns and 0.7 microns. To further explore this situation, a sample was taken from Port 3. This curve shows a large peak at around one micron, and a small peak near 0.1 microns. This would suggest that the strontium enters the furnace as residual strontium from the aqueous spray. As the strontium comes in contact with the sulfur in the combustion air, the residual strontium particles are etched or decomposed in some way by the sulfur. Smaller strontium particles result. The residence time in the furnace is not sufficient to allow a complete decomposition of the particles, hence the double peaked curve seen at the base of the furnace. 


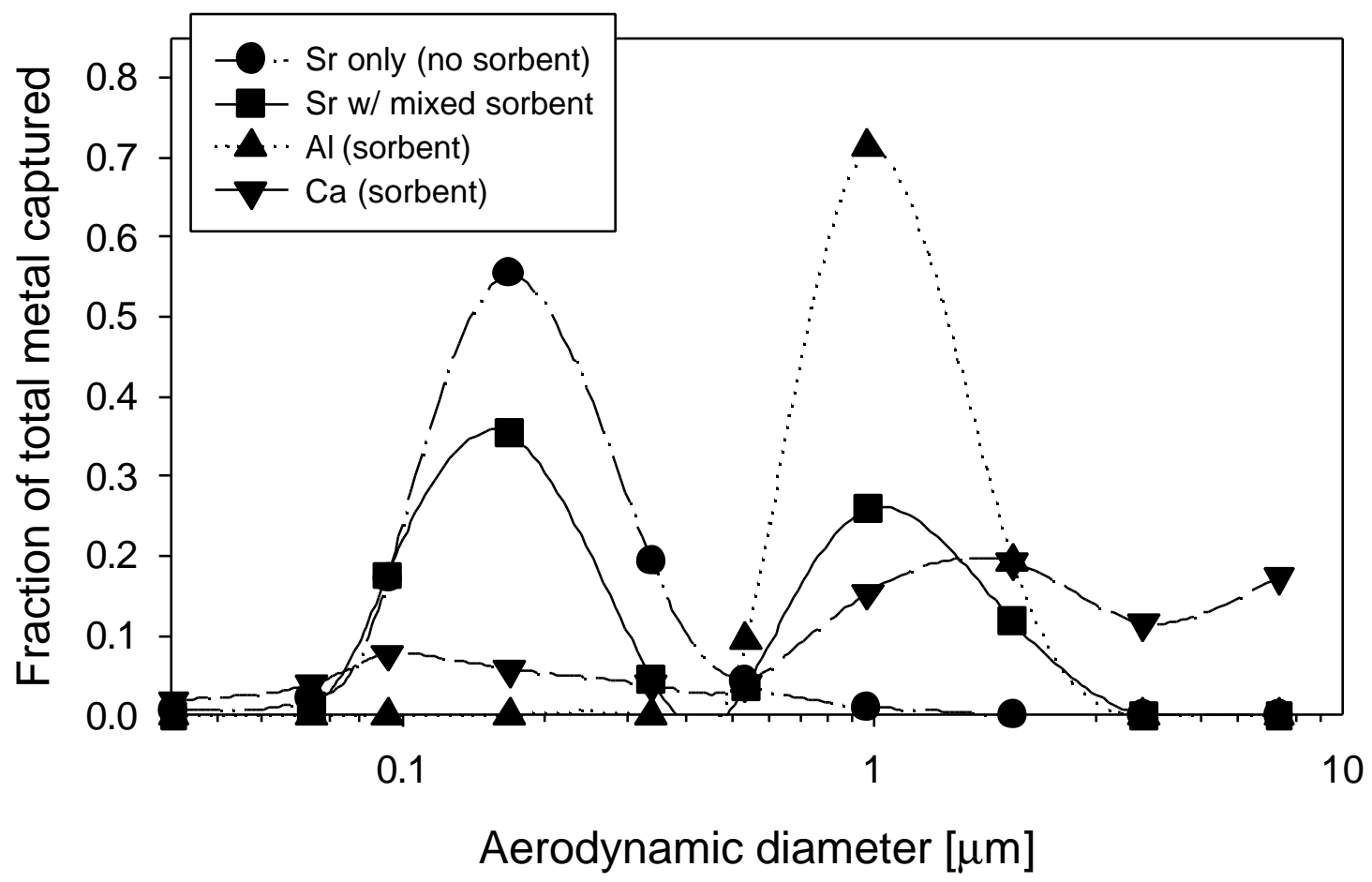

Figure 4.31 - Strontium and mixed sorbent, with chlorine

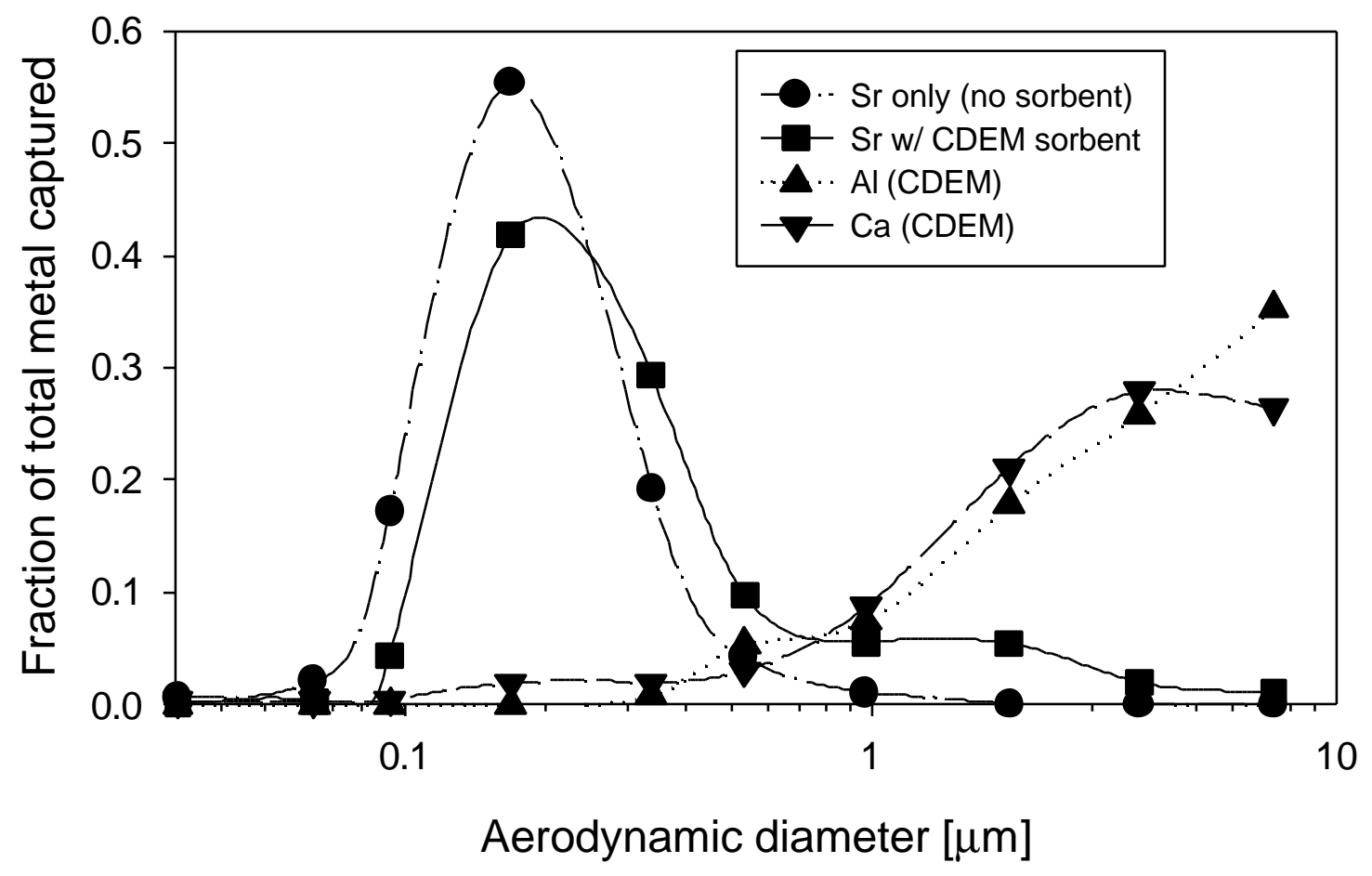

Figure 4.32 - Strontium and CDEM, with chlorine 


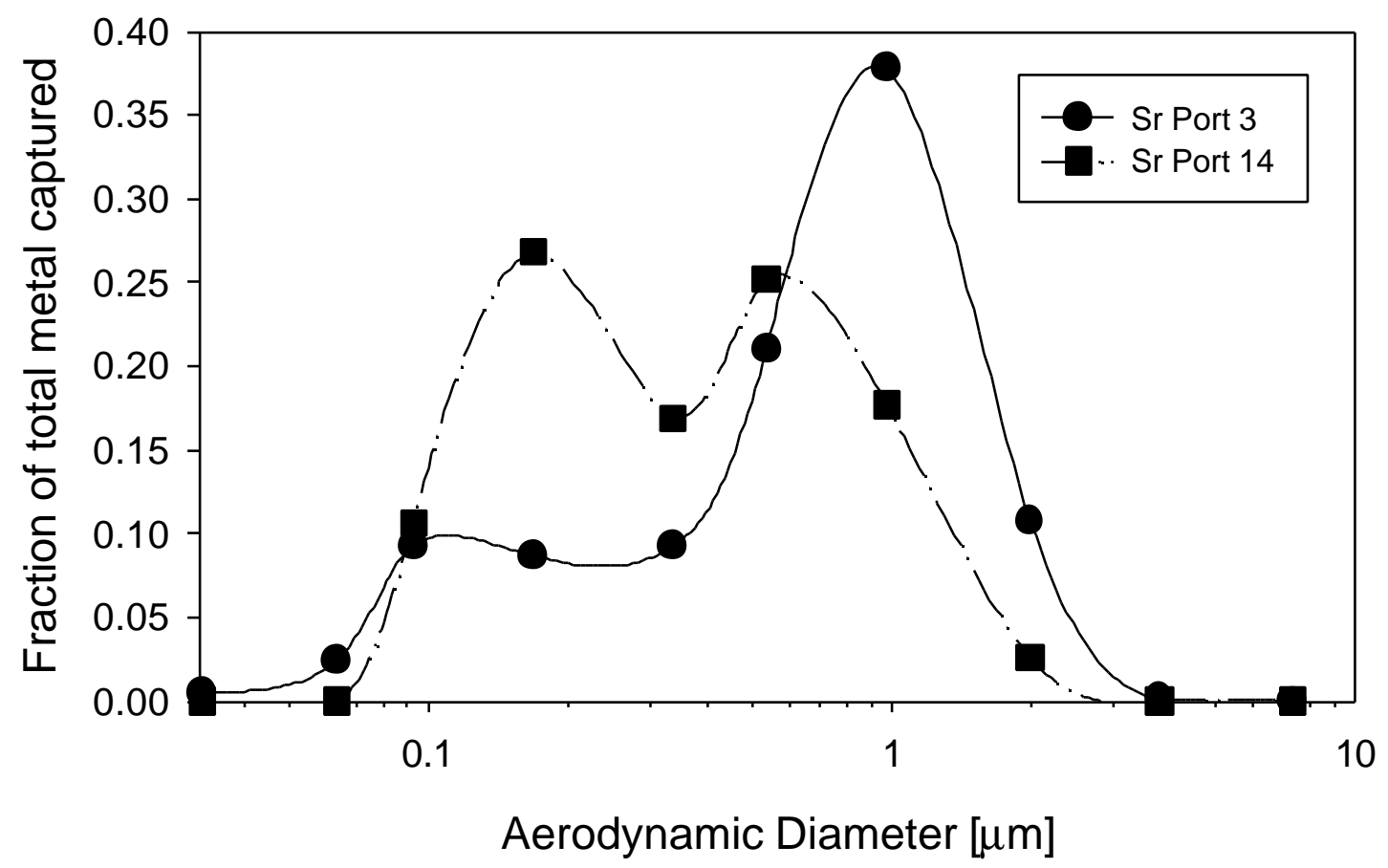

Figure 4.33 - Time evolution of strontium/sulfur particles

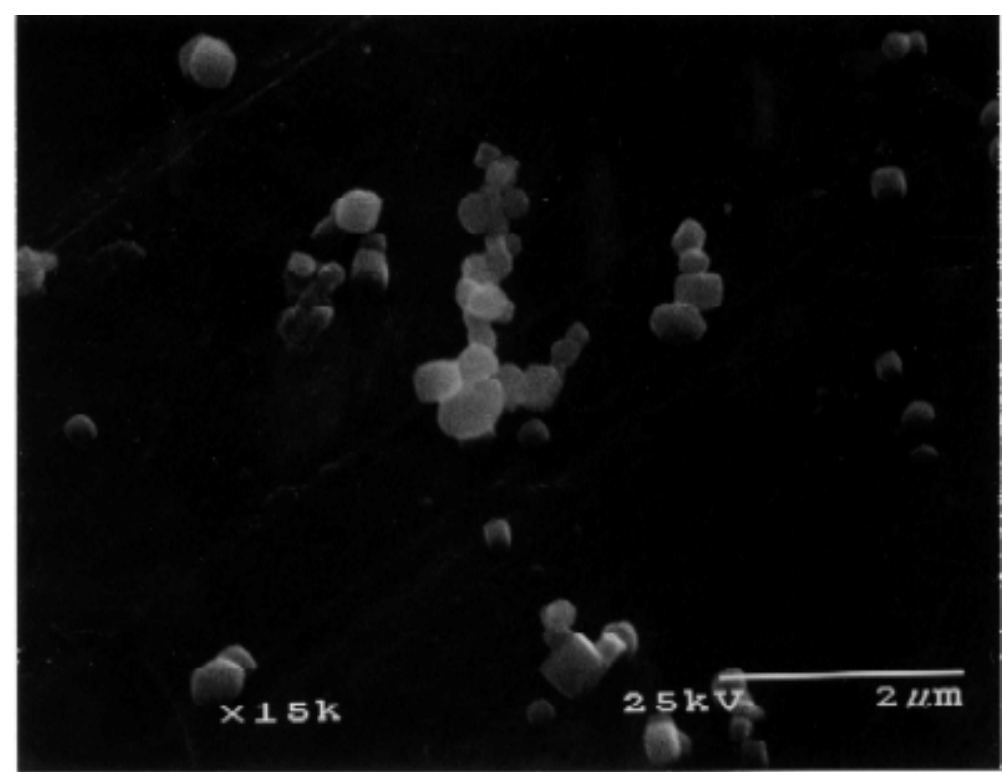

Figure 4.34 - SEM micrograph of strontium particles, with sulfur 
A SEM image of the strontium/sulfur experiments is shown in Figure 4.34. The particles are similar in appearance to the strontium only particles seen in Figure 4.21. However, the particles in this case do show some signs of angularity. Some of the particles almost appear to be cubical, as if some degree of crystallization of the metal is occurring.

When kaolinite is added to the system, the curve in Figure 4.35 is the result. The strontium still shows the double peaked curve, but the peaks are slightly shifted. The peak on the smaller end of the curve is only slightly larger than 0.2 microns, and the peak on the upper end is shifted closer to one micron. This is most likely due to some physical interaction, similar to that exhibited when sulfur was not present in the system. Figure 4.36 is a micrograph of this condition. The smaller strontium particles are seen clinging to the large angular sorbent particles. This also supports the theory that the metal is not reacting with the sorbent, and is only being gathered by some physical mechanism.

The results of the strontium/lime/sulfur system, shown in Figure 4.37, are similar to those of the strontium/kaolinite sulfur system. The peak at the larger end of the size distribution is shifted to a slightly larger peak, near two microns. The other peak has not shifted in this case. Once again, this shift is probably due to some physical mechanism, and is not the result of reactive capture of the metal.

The results of the strontium and mixed sorbent tests with sulfur are shown in Figure 4.38. The strontium again shows the double peaked curve that is shifted slightly by the presence of the sorbent. The upper peak is formed near one micron, and the lower peak has not moved. This shift matches the shift seen by the kaolinite and the lime 


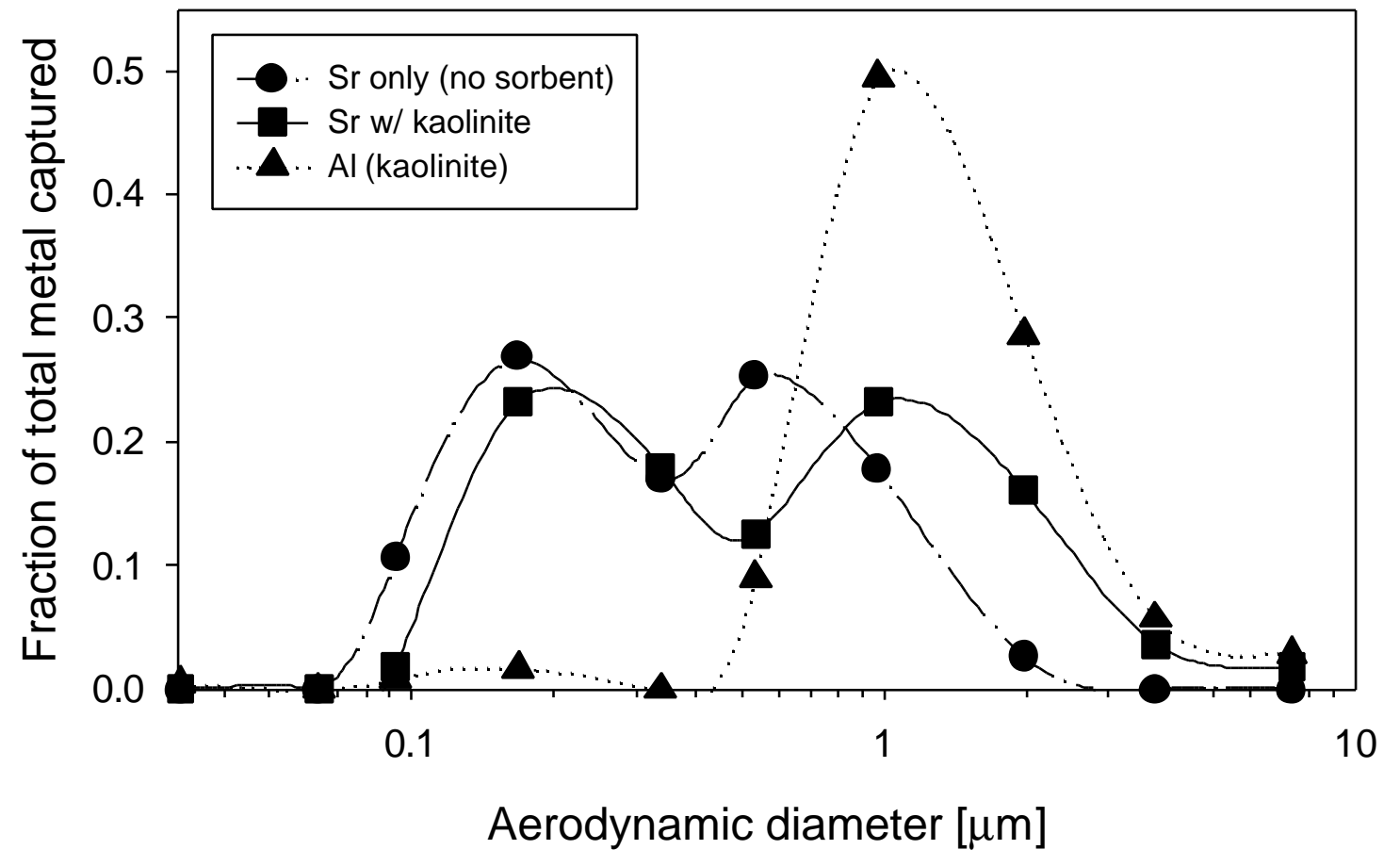

Figure 4.35 - Strontium and kaolinite, with sulfur

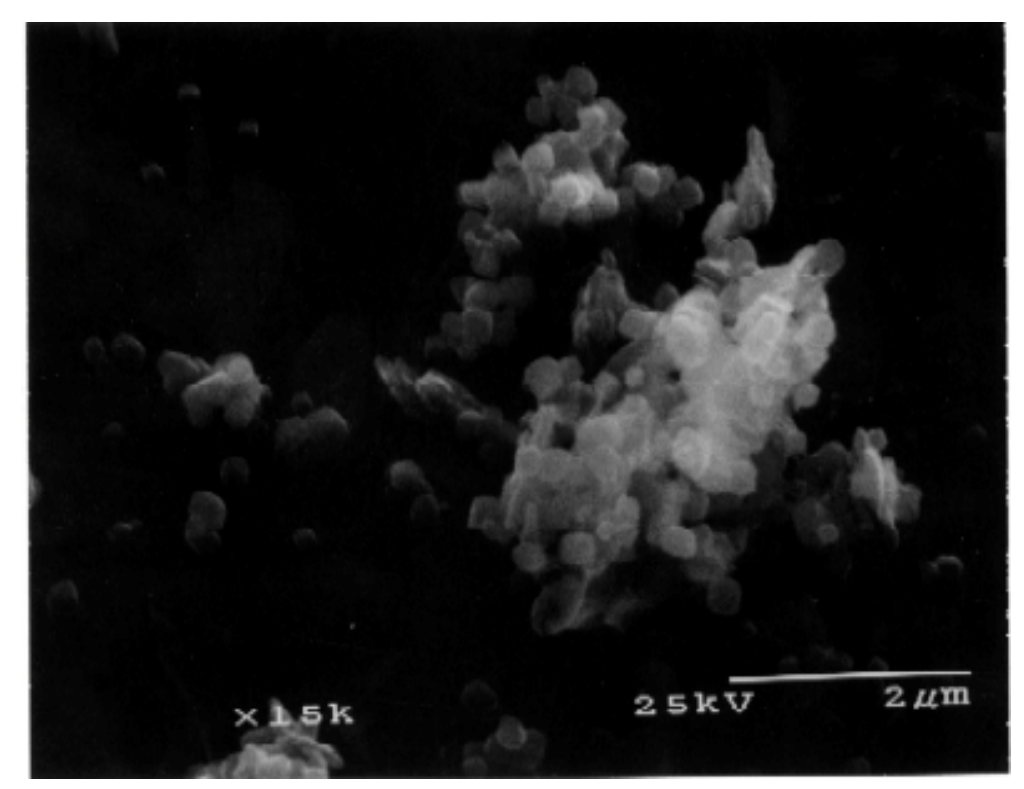

Figure 4.36 - SEM micrograph of strontium and kaolinite, with sulfur 


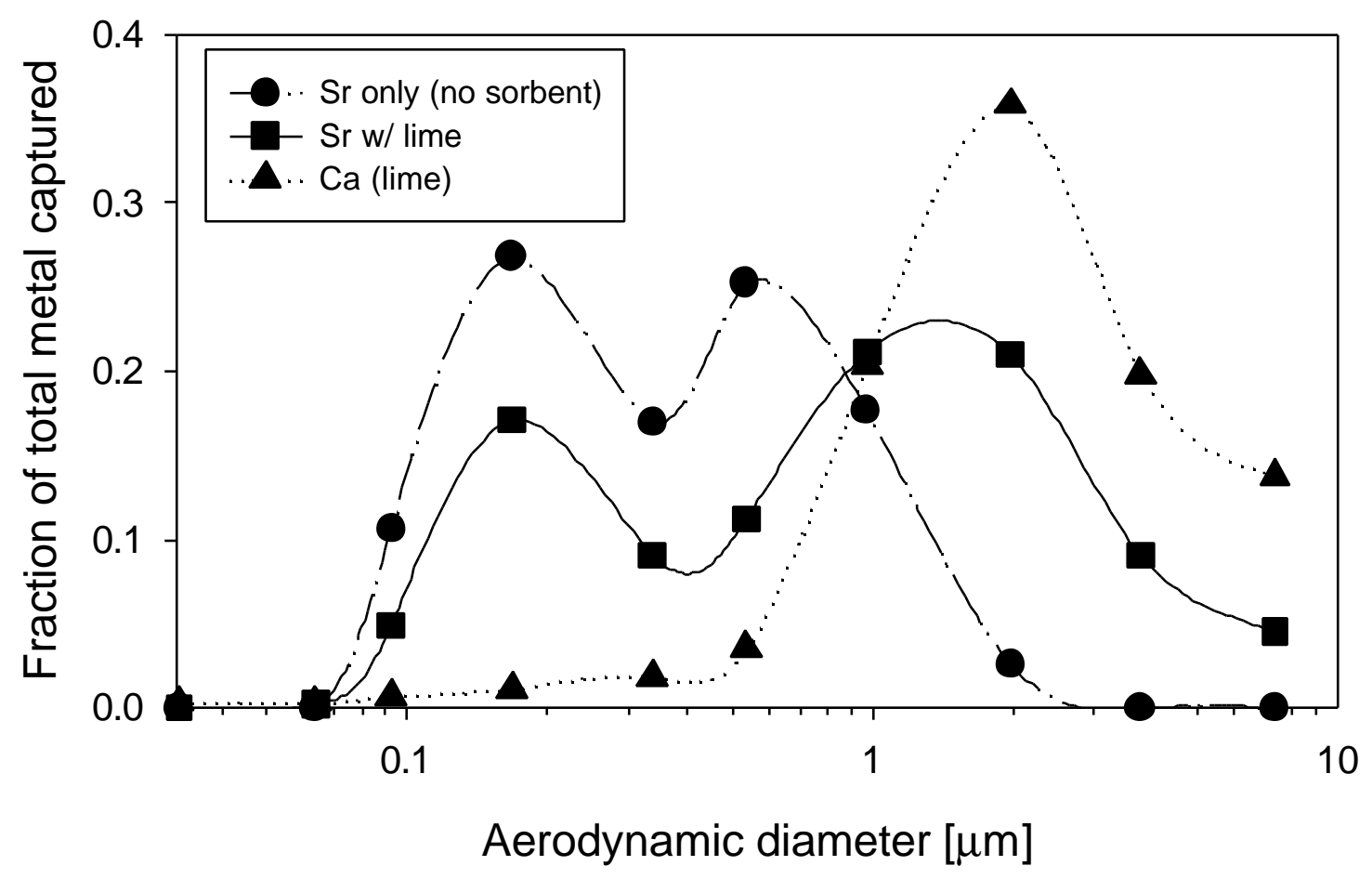

Figure 4.37 - Strontium and lime, with sulfur

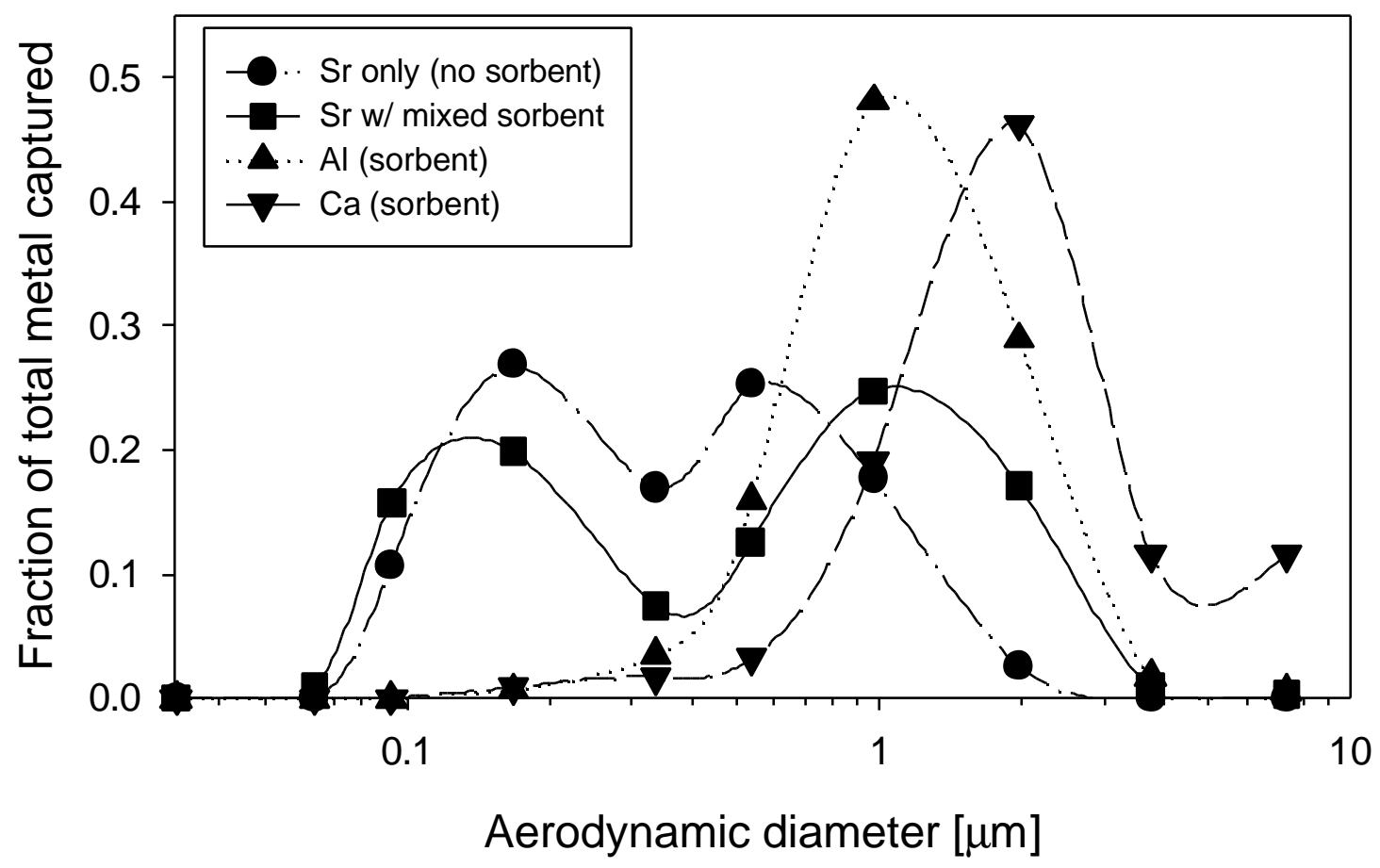

Figure 4.38 - Strontium and mixed sorbent, with sulfur 
separately. The effect of the two sorbents on the strontium is not additive, which lends additional support to the idea that the shift in the curve is due to some physical mechanism, and not due to some form of chemical reaction.

Figure 4.39 shows the results of the strontium and sulfur system when CDEM is the added sorbent. There is a double peak formed, but it is different in shape than those seen previously. The larger of the two peaks is centered around one micron, and has more of a tail towards the larger particle sizes. The second peak is substantially smaller, and is centered near 0.1 microns. It is possible that the smaller strontium particles are reacting with the CDEM sorbent, which would explain the diminished lower peak. The larger strontium particles are probably only being shifted by the same physical mechanisms seen previously. 


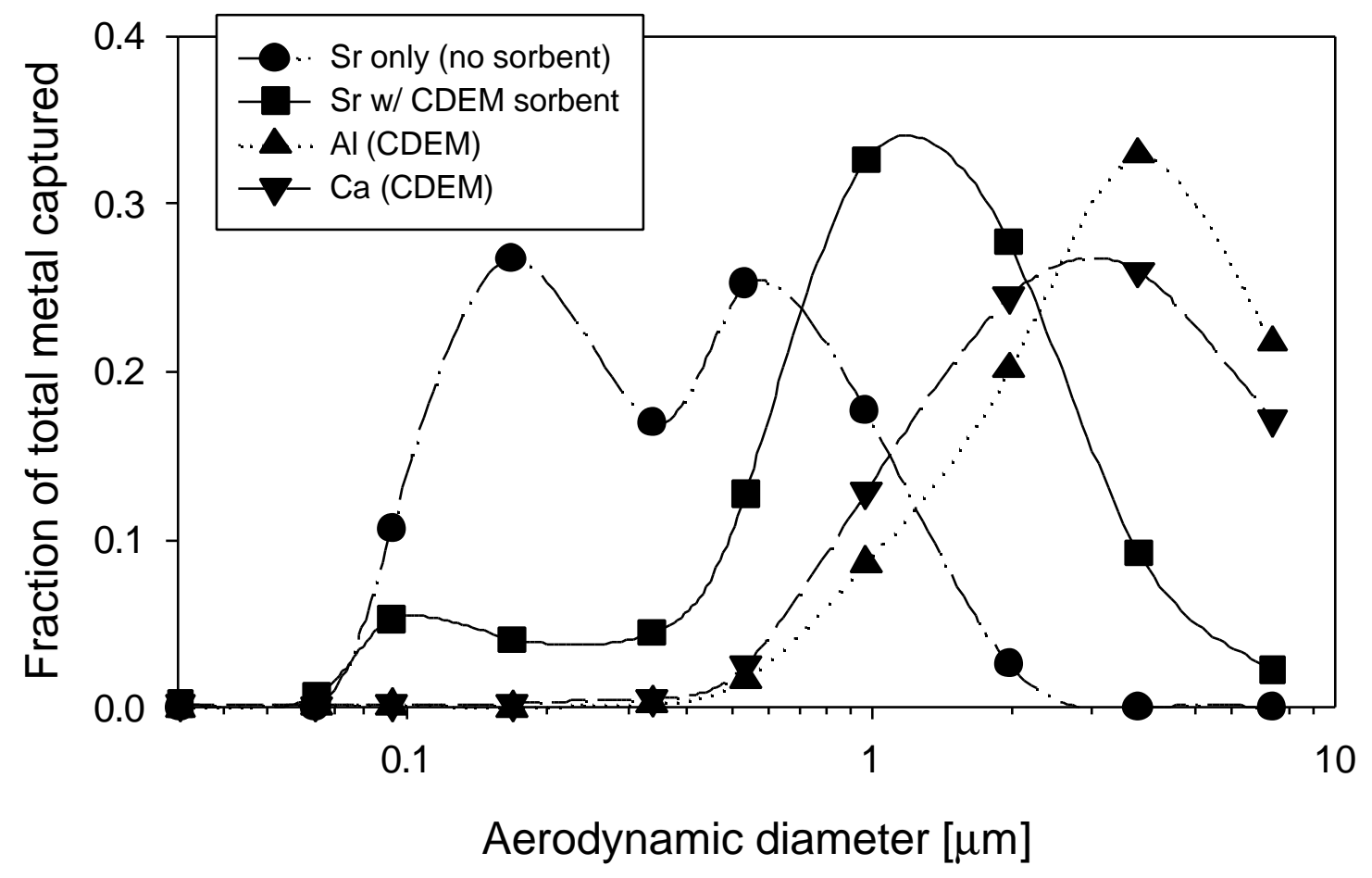

Figure 4.39 - Strontium and CDEM, with sulfur 


\section{$\underline{\text { Chapter } 5}$}

\section{$\underline{\text { Conclusions }}$}

This work has explored the reactions of two metal species, cesium and strontium, with several different sorbents in a combustion system. The effects produced by adding other chemical species, chlorine and sulfur, were also explored. Several conclusions can be drawn from this work. A summary of the results is presented in Table 5.1.

\begin{tabular}{|c|c|c|c|}
\hline Metal & Dopant & Sorbent & Interaction \\
\hline Cesium & None & Kaolinite & Capture \\
\hline Cesium & None & Lime & Slight Capture \\
\hline Cesium & None & Mixed & Capture \\
\hline Cesium & None & CDEM & Slight Capture \\
\hline Cesium & Chlorine & Kaolinite & Some Capture \\
\hline Cesium & Chlorine & Lime & None \\
\hline Cesium & Chlorine & Mixed & Slight Capture \\
\hline Cesium & Chlorine & CDEM & None \\
\hline Cesium & Sulfur & Kaolinite & Capture \\
\hline Cesium & Sulfur & Lime & Capture \\
\hline Cesium & Sulfur & Mixed & Capture \\
\hline Cesium & Sulfur & CDEM & Capture \\
\hline Strontium & None & Kaolinite & Coagulation \\
\hline Strontium & None & Lime & Coagulation \\
\hline Strontium & None & Mixed & Coagulation \\
\hline Strontium & None & CDEM & Coagulation \\
\hline Strontium & Chlorine & Kaolinite & Capture \\
\hline Strontium & Chlorine & Lime & None \\
\hline Strontium & Chlorine & Mixed & Some Capture \\
\hline Strontium & Chlorine & CDEM & None \\
\hline Strontium & Sulfur & Kaolinite & Coagulation \\
\hline Strontium & Sulfur & Lime & Coagulation \\
\hline Strontium & Sulfur & Mixed & Coagulation \\
\hline Strontium & Sulfur & CDEM & Possible Capture \\
\hline
\end{tabular}

Table 5.1 - Summary of Results 


\subsection{Cesium}

In order to gain a fuller understanding of the possible metal transformation mechanisms that may be occurring, the data were analyzed according to Figures 5.1 through 5.4. For those cases where capture appeared to occur, the weight percent metal oxide on a plate was plotted against the aerodynamic diameter of the particle. Only those plates where sorbent was found were included on the plots. If the data shows a $1 / d_{p}$ dependence, as indicated by a line with a slope of -1 on the logarithmic plots, the capture mechanism is surface reaction or pore diffusion controlled in the continuum regime $\left(d_{p}>0.5 \mu \mathrm{m}\right)$. If the line on the logarithmic plots has a slope of -2 , due to a $1 / d_{p}{ }^{2}$ dependence, then the mechanism is film diffusion controlled, probably an indication of rapid condensation. A thorough explanation of this methodology can be found elsewhere (Linak and Wendt 1993).

The data for cesium on kaolinite are shown in Figure 5.1 It appeared that kaolinite would capture cesium in all instances. The capture appeared to be reduced with the addition of chlorine or sulfur. The data in Figure 5.1 indicate there is a weak (less than $1 / d_{p}$ ) diameter dependence for the cesium/kaolinite and the cesium/kaolinite/chlorine cases. This suggests that the mechanism is not surface reaction or pore diffusion controlled. The data for the cesium/kaolinite/sulfur case might indicate a $1 / d_{p}$ dependence, although the data are too sparse to draw a definitive conclusion.

Figure 5.2 illustrates similar data for the cesium and lime cases. Lime appeared to capture cesium with no dopant and with sulfur present, but not with chlorine added. The cesium/lime case shows no diameter dependence. The cesium/lime/sulfur case does 


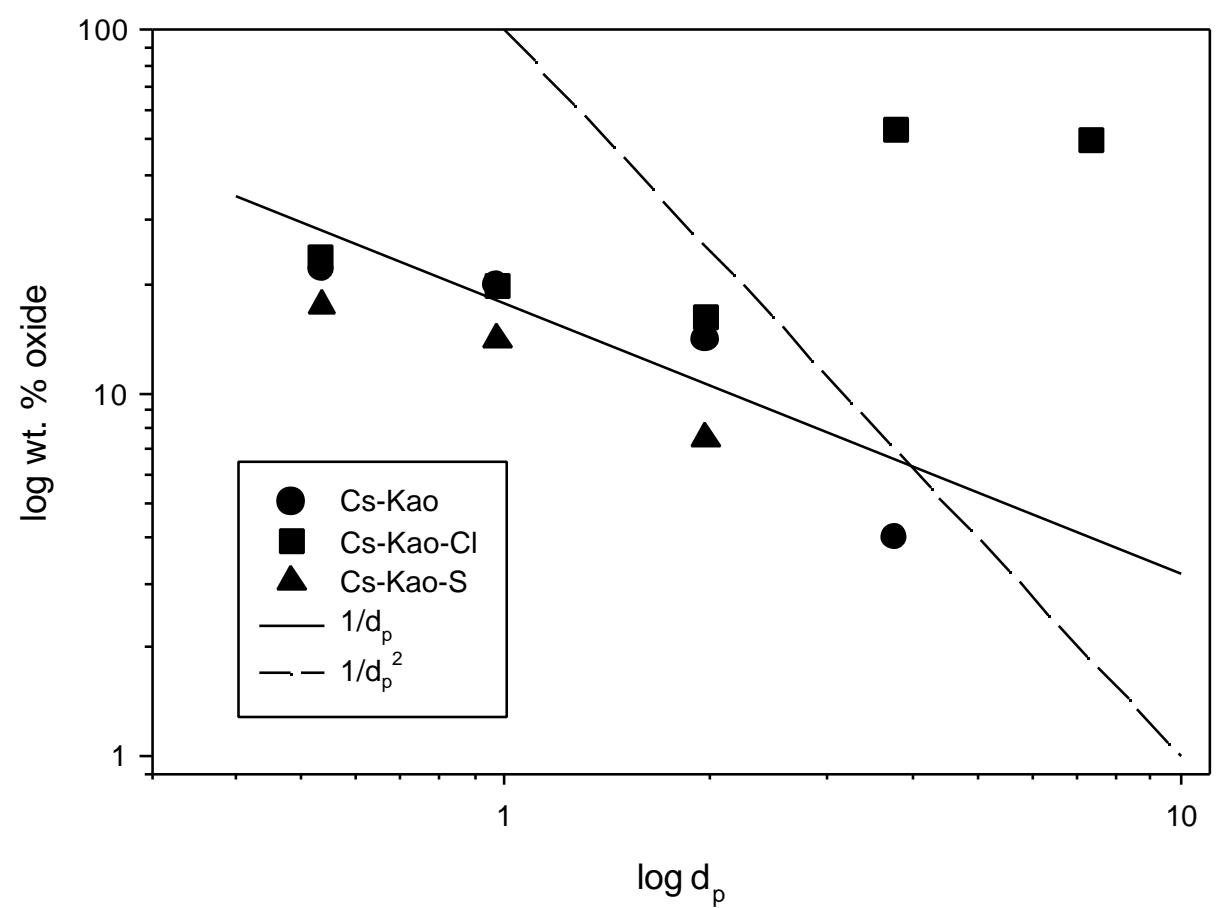

Figure 5.1 - Weight Percent Oxide versus Diameter for Cesium on Kaolinite

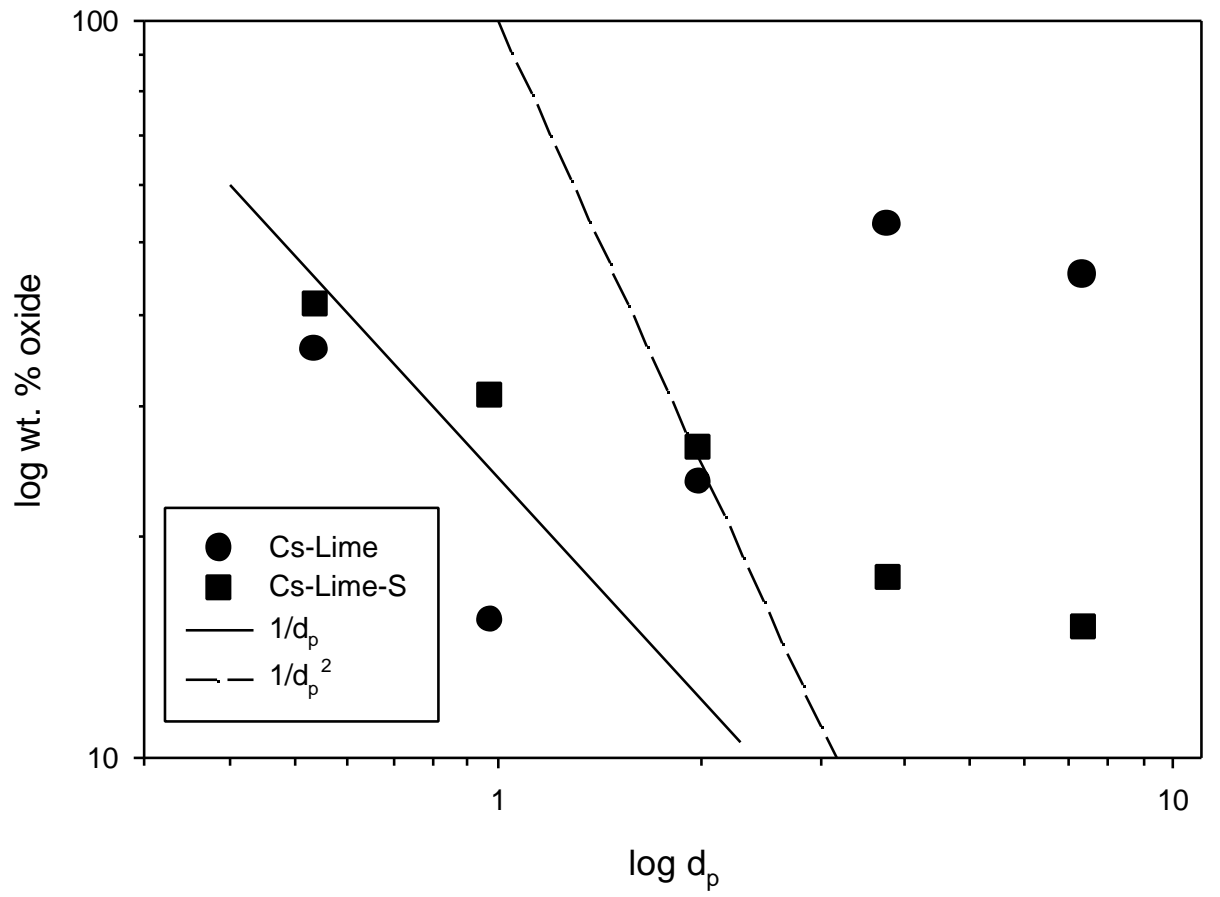

Figure 5.2 - Weight Percent Oxide versus Diameter for Cesium on Lime 
appear to have some diameter dependence. However, the slope of the line indicates a dependence on $d_{p}$ less than $1 / d_{p}$, the ramifications of which are not clear.

The data for the cesium and mixed sorbent is shown in Figure 5.3. Capture appeared to occur under all three dopant conditions. The cesium/mixed/sulfur case does not appear to have a clear diameter dependence. The cesium/mixed and cesium/mixed/chlorine cases appear to have a diameter dependence that is very close to $1 / d_{p}$. This indicates that the mechanism is surface reaction or pore diffusion controlled.

For CDEM and cesium, capture only appeared to occur with the cesium/CDEM and cesium/CDEM/sulfur cases. These data are plotted in Figure 5.4. In both cases, there appears to be a $1 / d_{p}$ dependence of the data. This dependence is very good for the cesium/CDEM/sulfur case. Again, this suggests that the mechanism is controlled by surface reaction or pore diffusion.

Thermodynamic calculations were performed prior to beginning experimental work. This was done in order to gain some understanding of the potential behavior of the metals in the combustion system. Cesium was predicted to behave as a semi-volatile metal. The dewpoint of the cesium with no dopants present was predicted to be fairly low. The addition of chlorine to the system was expected to push the dewpoint of cesium to a higher temperature, and the addition of sulfur was predicted to push the dewpoint even higher. The experimental evidence suggests that the thermodynamic calculations were fairly accurate. Cesium alone exhibited a fairly low dewpoint, forming a submicron fume in the probe when extracted from the bottom of the furnace. Chlorine did appear to increase the dewpoint of the metal. The addition of sulfur appeared to push the 


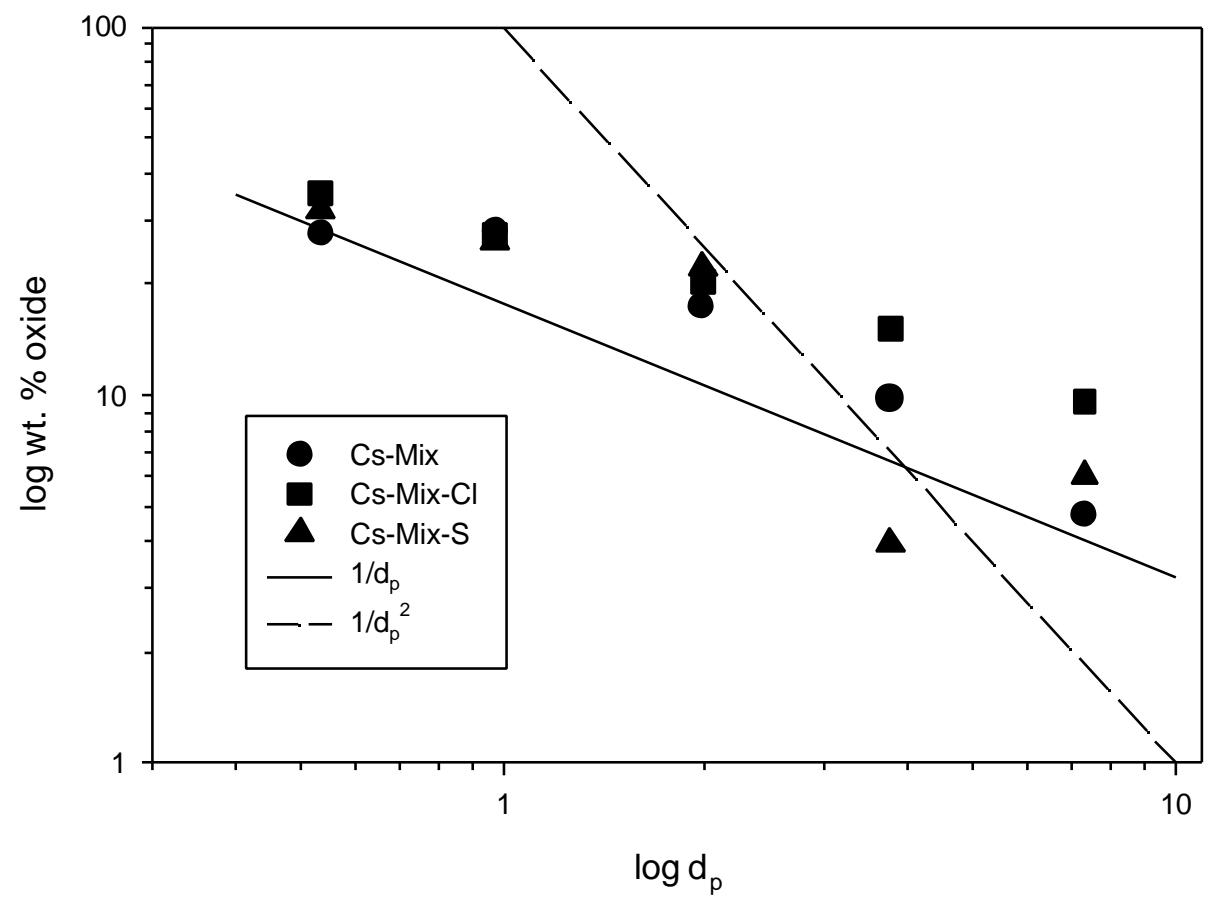

Figure 5.3 - Weight Percent Oxide versus Diameter for Cesium on Mixed Sorbent

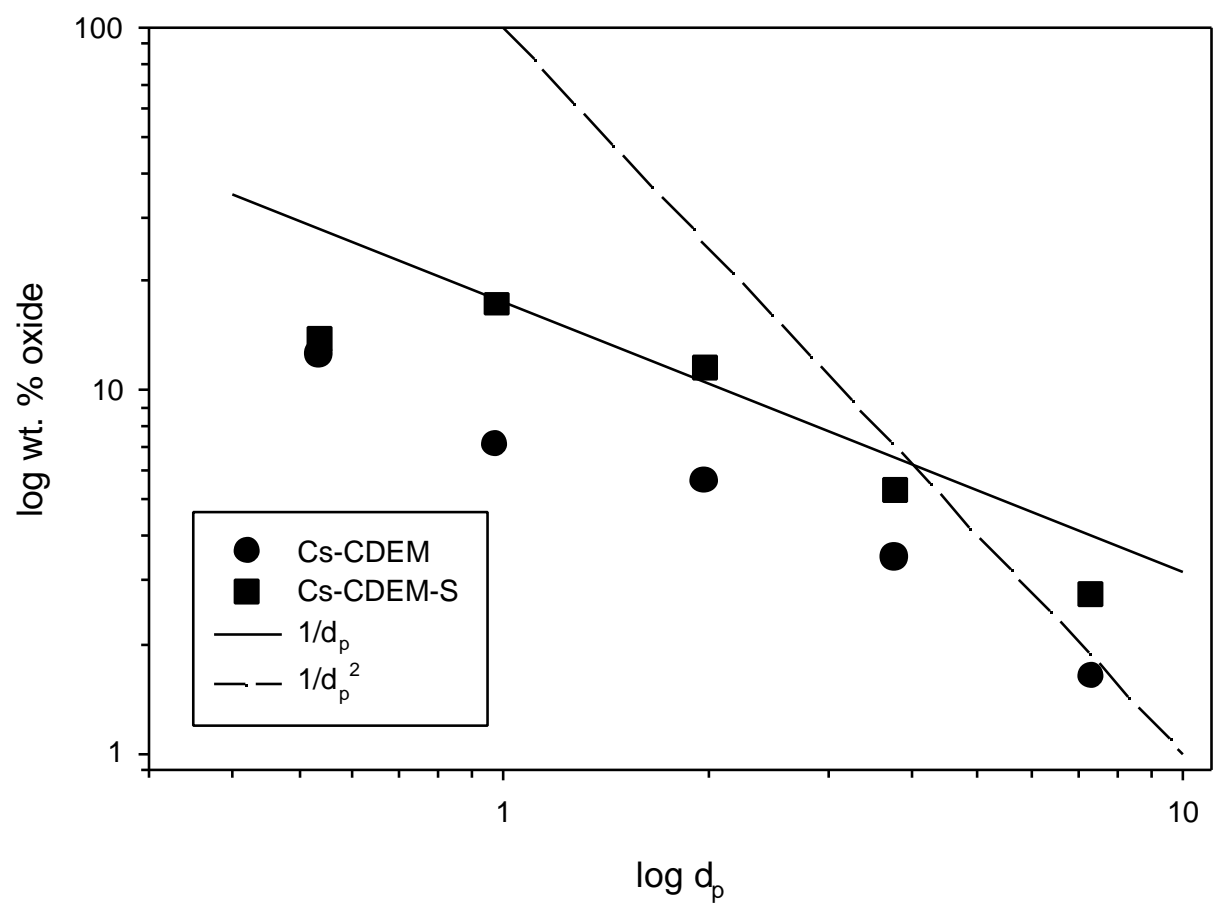

Figure 5.4 - Weight Percent Oxide versus Diameter for Cesium on CDEM 
dewpoint even higher, which can be inferred from the change in the curves in Figure 4.11. Overall, the thermodynamic predictions for cesium appeared to match the experimental data fairly well.

Earlier in this work, sodium was introduced as an analogue to cesium. Little work had been done with cesium up to this point. Sodium is in the same period of the periodic table as cesium, and there has been some work that has examined the capture of sodium by sorbents (Mwabe and Wendt 1996). Therefore, in order to gain some idea of the possible behavior of cesium, the existing sodium results were examined.

The thermodynamic calculations showed some difference between the two metals. Sodium has a higher dewpoint when no dopant gases are present. There is some difference in the speciation of the two metals, particularly in the solid phase, which may account for the difference in the dewpoint temperatures.. With the dopants added, the cesium and sodium behavior was very similar, both in terms of speciation and dewpoint temperatures. The thermodynamic database for sodium is slightly larger, so some of the variation may be due to deficiencies in the cesium data.

The experimental results also appear to match fairly well. Cesium, like sodium appears to be reactively captured by kaolinite. When chlorine is added to the furnace, the capture of both metals is reduced. For sodium, this is due to the reduction in gas phase $\mathrm{NaOH}$ due to the presence of the chlorine. This is slightly offset by the decreased dewpoint temperature. For cesium, not only is the gas phase $\mathrm{CsOH}$ reduced, but the dewpoint is also increased, which reduces the time for capture. With the addition of 
sulfur, capture is reduced for sodium due to the increased dewpoint of the metal. This also appears to be the case with cesium.

It appears that kaolinite is the best choice of sorbent to capture cesium. It has the highest capture capacity over all conditions explored. The lime only captured cesium when sulfur was present, and then only moderately. The CDEM sorbent worked well when sulfur was added, but performed poorly in the other conditions.

\subsection{Strontium}

The thermodynamic calculations that were performed for strontium appeared to match the experimental results fairly well. When no dopant was present, the strontium did not vaporize in the furnace, forming residual particles, as was predicted. When chlorine was added, the strontium did vaporize and was able to react with kaolinite, which was suggested by the thermodynamic results. However, when sulfur was added as the dopant, the results did not match as closely. The thermodynamic calculations predicted that sulfur would have no effect on the strontium dewpoint, and it would form the residual particles as were seen in the no dopant case. The experimental results suggest that there is some vaporization of the strontium occurring, as seen in Figure 4.33. This discrepancy is most likely due to an important missing specie in the thermodynamic database.

There were only a few cases where capture of strontium appeared to occur. These cases are illustrated in Figure 5.5. Strontium did appear to be captured by kaolinite in the presence of chlorine. The capture that occurred in the strontium/mixed/chlorine case is 


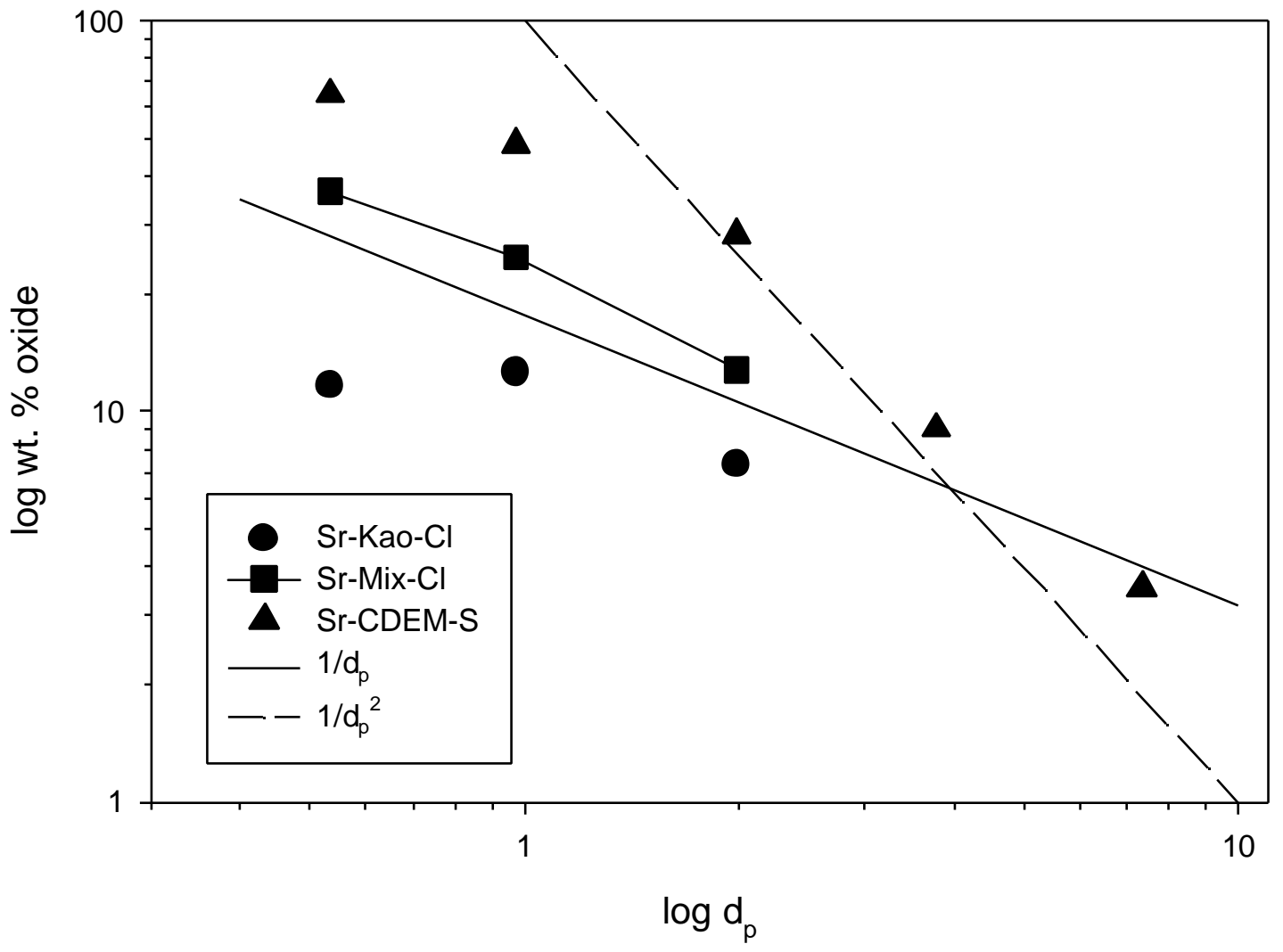

Figure 5.5 - Weight Percent Oxide versus Diameter for Strontium Capture Cases 
most likely due to the kaolinite, with no contribution from the lime. There may be some capture in the strontium/CDEM/sulfur case, so its data were included here. There may be a strong contribution from a physical mechanism in this case, however. The strontium/kaolinite/chlorine and strontium/CDEM/sulfur cases do not appear to have any diameter dependence. The strontium/mixed/chlorine case may have a $1 / d_{p}$ dependence. This result may be skewed, however, due to the presence of the non-reactive lime.

In several cases with the strontium, it appears that coagulation may play a role in the final metal distribution. These include the cases where no dopant was used, and where the dopant was sulfur. The thermodynamic predictions and the SEM images support this conclusion. In order to better understand the mechanisms that are important, further aerosol modeling and experimental work may need to be performed.

Strontium and nickel were compared earlier to gain some idea of the behavior of strontium in the combustion system. Very little work had been done on strontium, but there had been some work using nickel (Linak et al. 1994; Linak et al. 1995; Linak and Wendt 1998).

The thermodynamic calculations performed for strontium for this work will be compared to the nickel calculations in Linak and Wendt (1998). There is fairly good agreement between the two metals. Both have a high dewpoint with no dopant gases present. When chlorine is introduced to the system, the dewpoints of both nickel and strontium drop considerably. This reduction in dewpoint may enable the capture of these metals in a real system. When sulfur is added to the system, there is no change in the dewpoint from the initial values. However, experimental evidence suggests that there is 
some volatilization of strontium in the presence of sulfur that was not identified in the thermodynamic calculations. This is probably due to the lack of an important specie in the thermodynamic data base. For nickel, no experiments were performed with the addition of sulfur. Therefore, it is possible that nickel may exhibit the same behavior as strontium.

Kaolinite may be the best choice of sorbent for strontium capture. This reaction only occurs when chlorine is present in the system. Lime remains non-reactive in all cases, and the mixed sorbent only exhibits capture when chlorine is present. There may be some reaction with the CDEM sorbent, but only when sulfur is present, and only to a small extent.

\subsection{Areas for Future Work}

Based on this work, several areas for future exploration suggest themselves. First, now that particular capture situations have been identified, further experiments can be performed to determine the kinetics of these reactions. In particular, the cesium reactions with kaolinite, both with and without dopants, and the strontium/chlorine/kaolinite reactions should be examined further. Second, the partitioning of the metals should be determined based on the form the metal takes in the feed. The final partitioning could depend upon whether the metals start as aqueous solutions, chelated complexes, or organo-metallic species bound in the fuel. Third, leaching tests can be performed with identified capture situations to determine whether the metals are bound in an insoluble form in the sorbent. This could have an impact on the acceptance of this methodology as 
a form of treatment technology. Fourth, aerosol modeling, using an algorithm like MAEROS, should be done in order to more fully understand the behavior of strontium with the sorbents. In particular, the issue of whether coagulation is a realistic possibility to explain the shift in the strontium curves. Finally, it appears that the dopant gases may react with the CDEM sorbent. Chlorine appears to destroy the capture capability of the sorbent, while sulfur may enhance this ability in some situations. These relationships warrant further exploration. 


\section{Appendix A.1 : CEA INPUT DATA}

\section{Cesium Species}

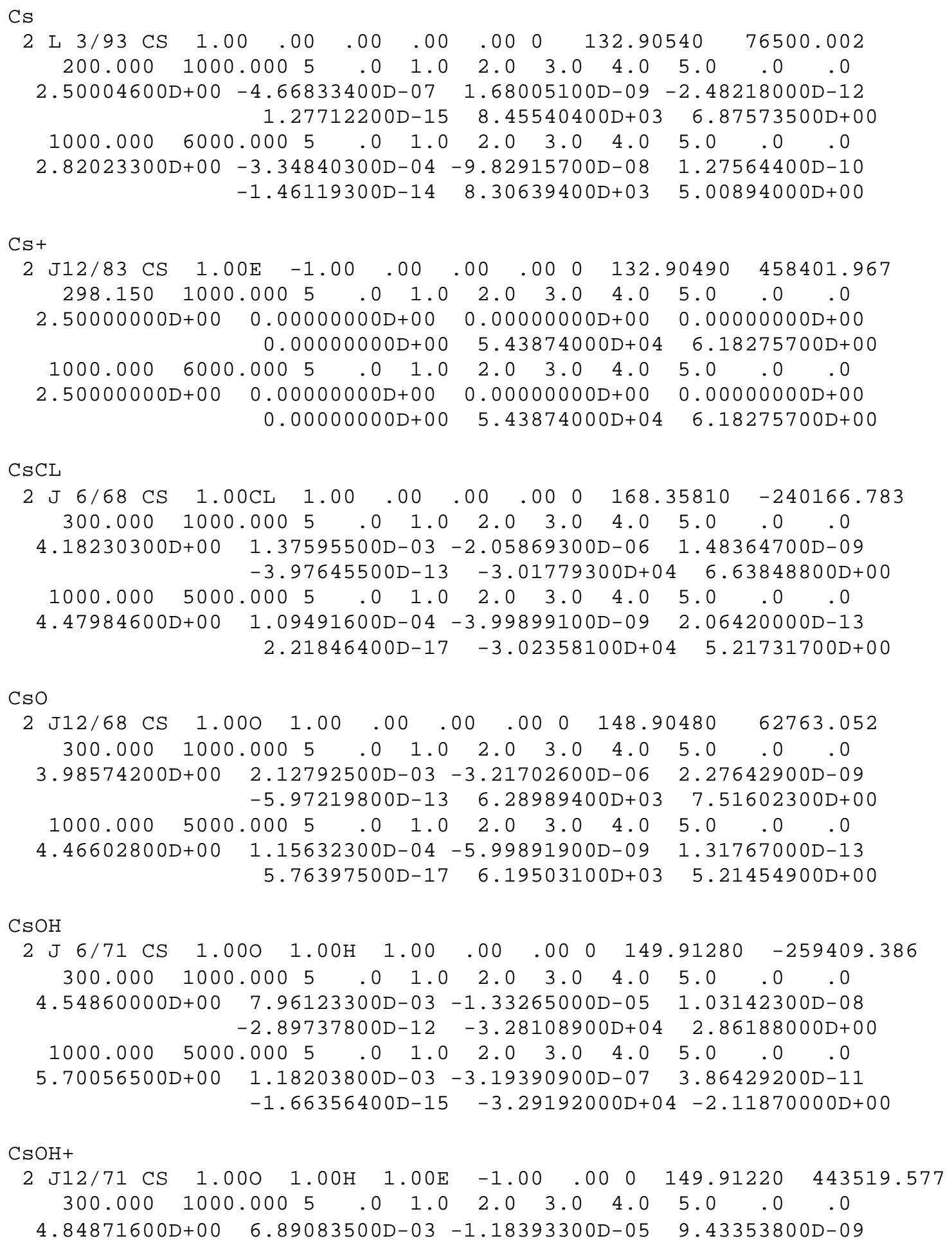




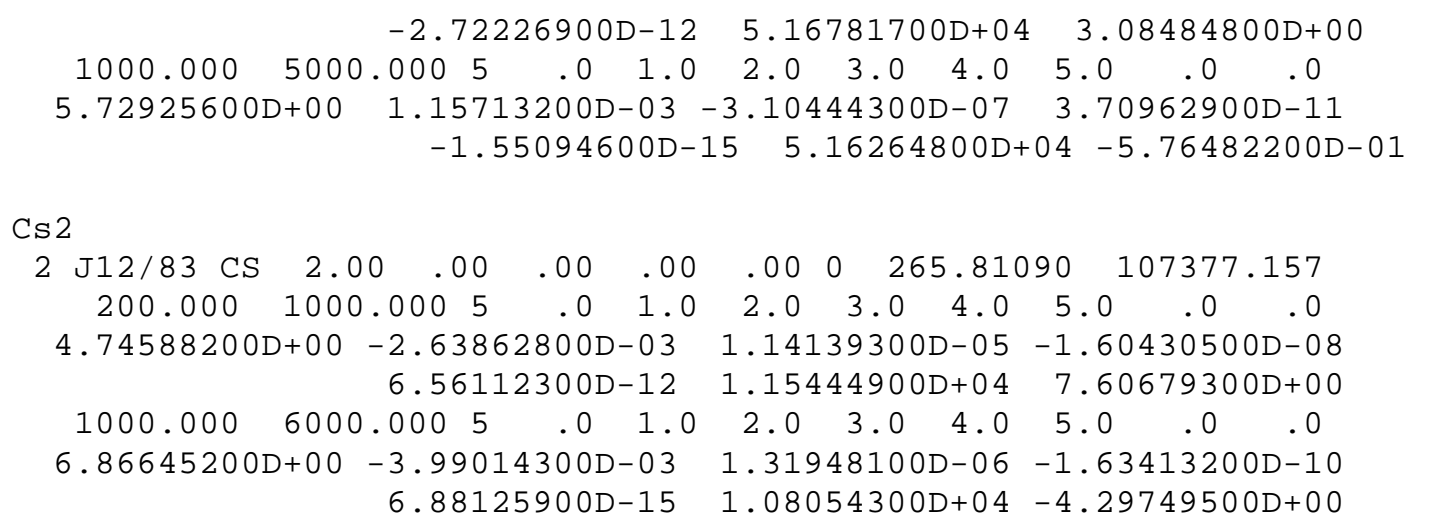

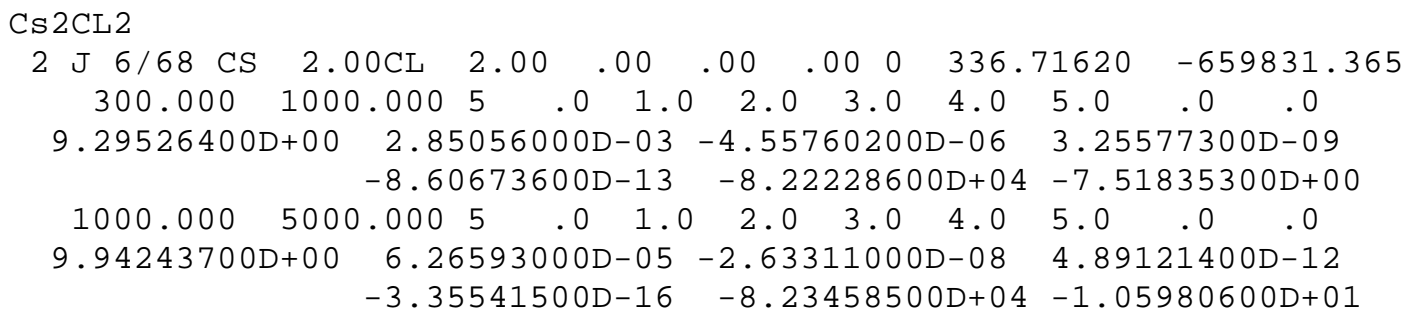




\section{$8.33415900 \mathrm{D}-10-9.80844400 \mathrm{D}+02-8.10866800 \mathrm{D}+00$}

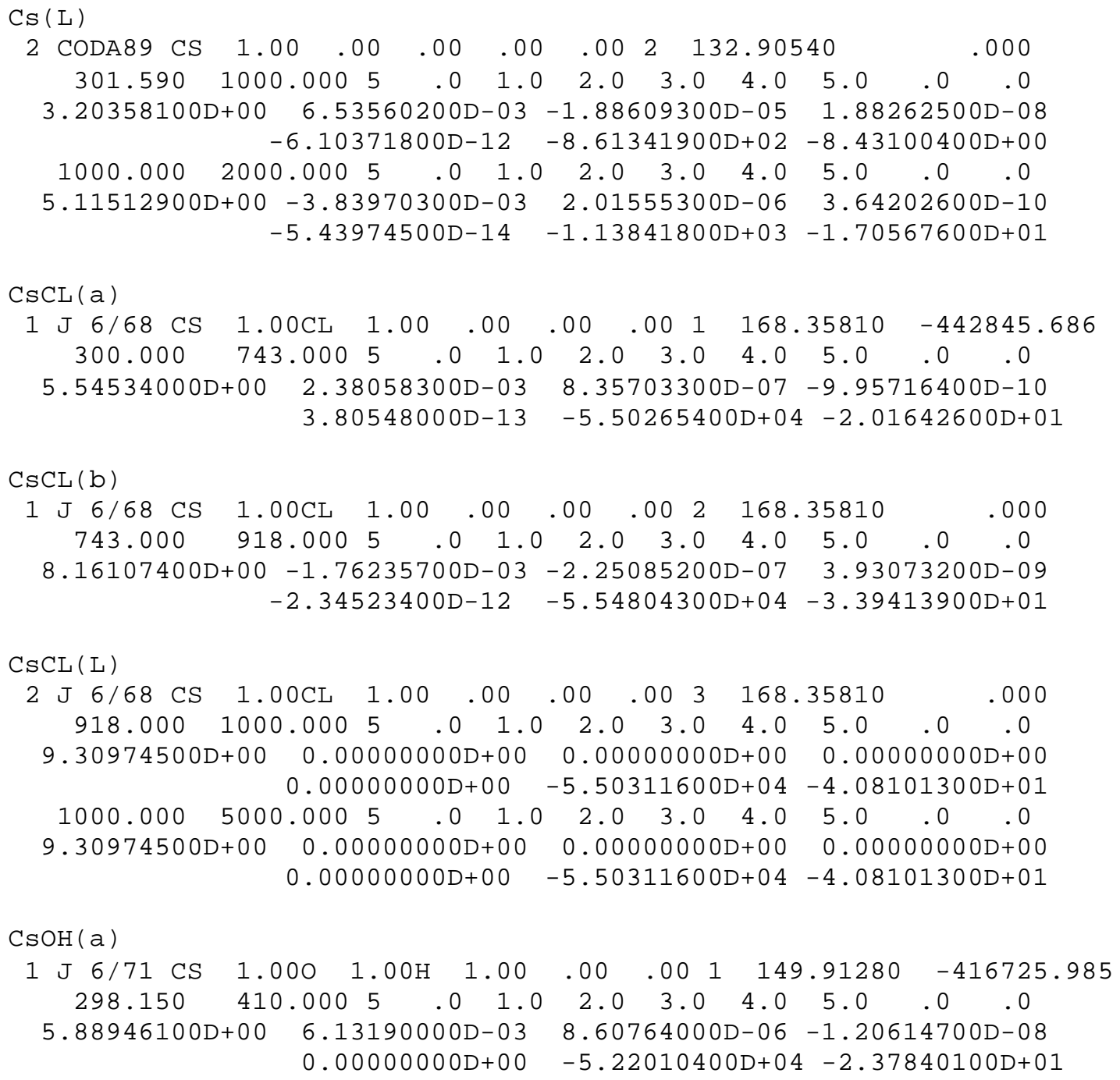




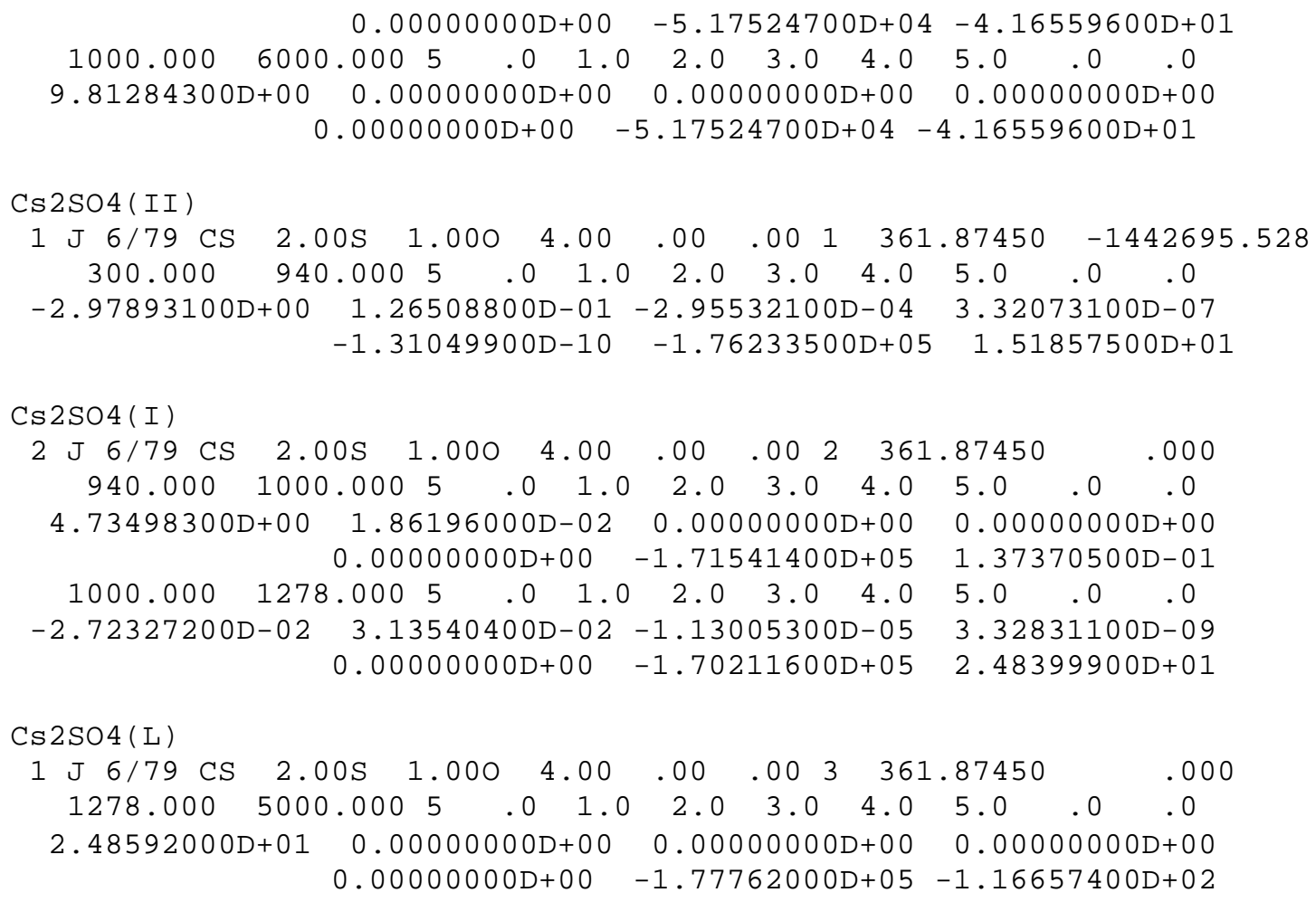

\section{Sodium Species}

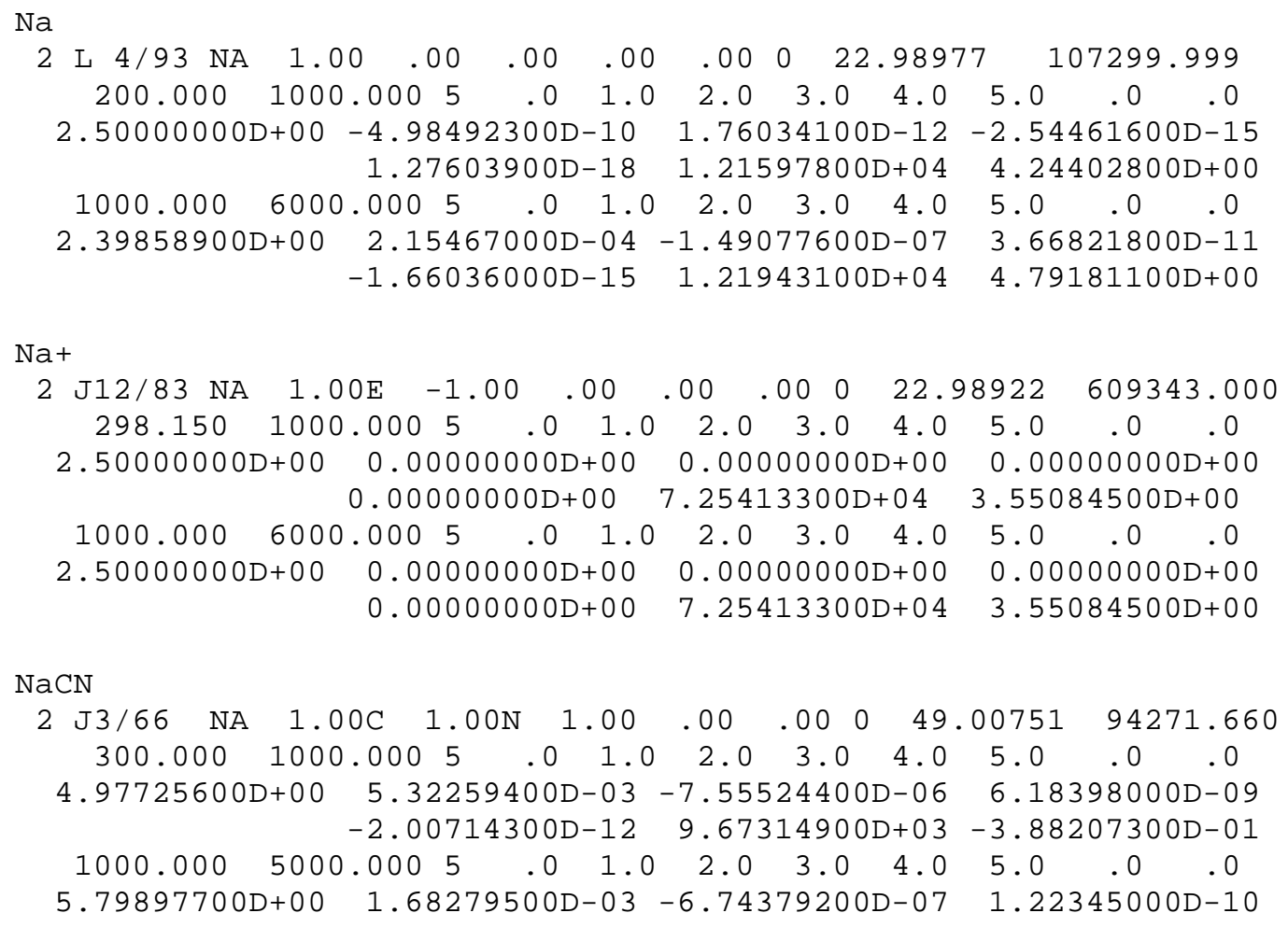




$$
-8.29660900 \mathrm{D}-15 \quad 9.49334500 \mathrm{D}+03-4.34426000 \mathrm{D}+00
$$

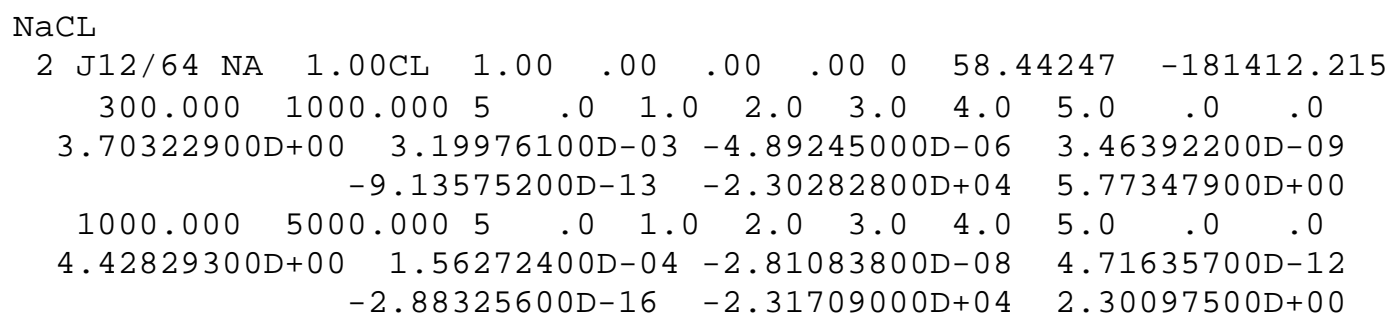




$$
-2.67804600 D-14-1.29673600 D+05-4.76569200 D+01
$$

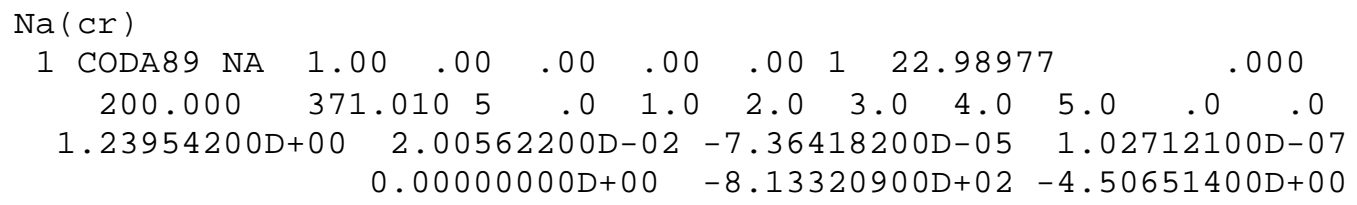

$\mathrm{NaOH}(\mathrm{L})$ 


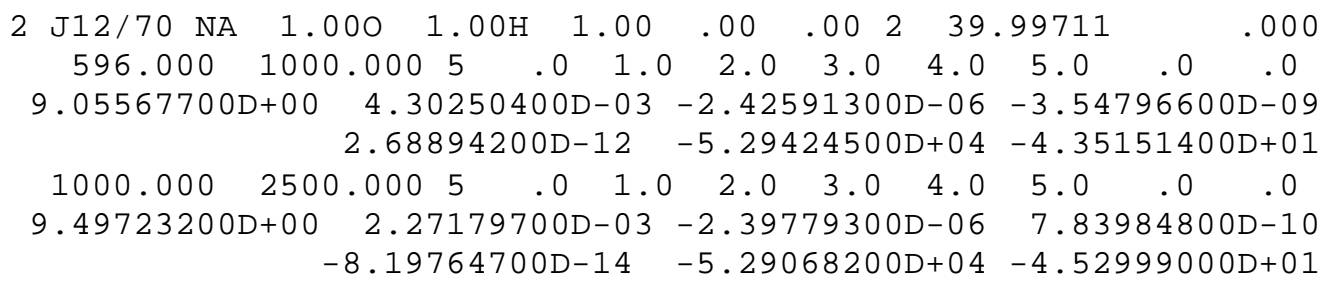

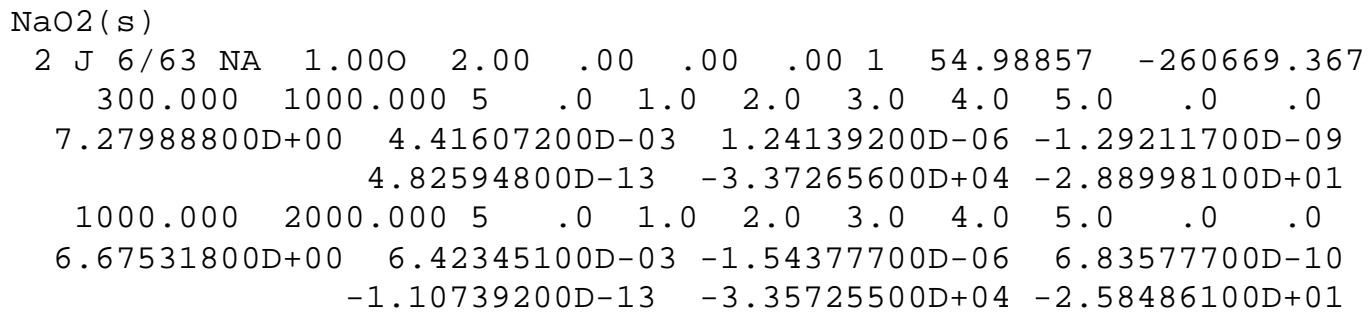

$\mathrm{Na} 2 \mathrm{O}(\mathrm{L})$ 


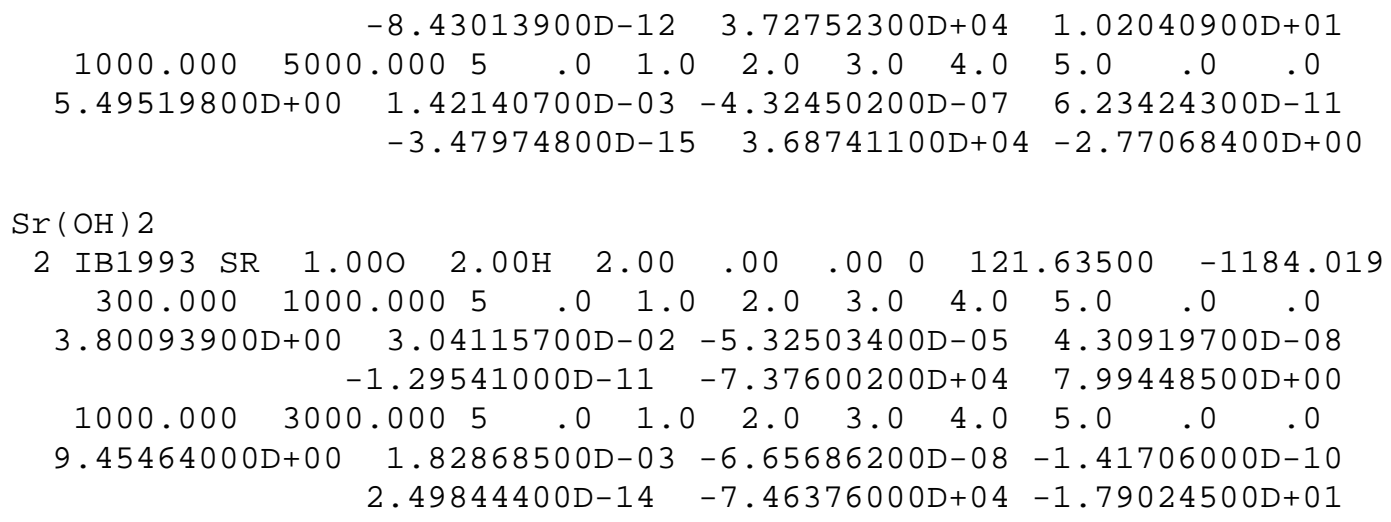




$$
0.00000000 D+00-1.89885000 D+05-1.10075200 D+02
$$

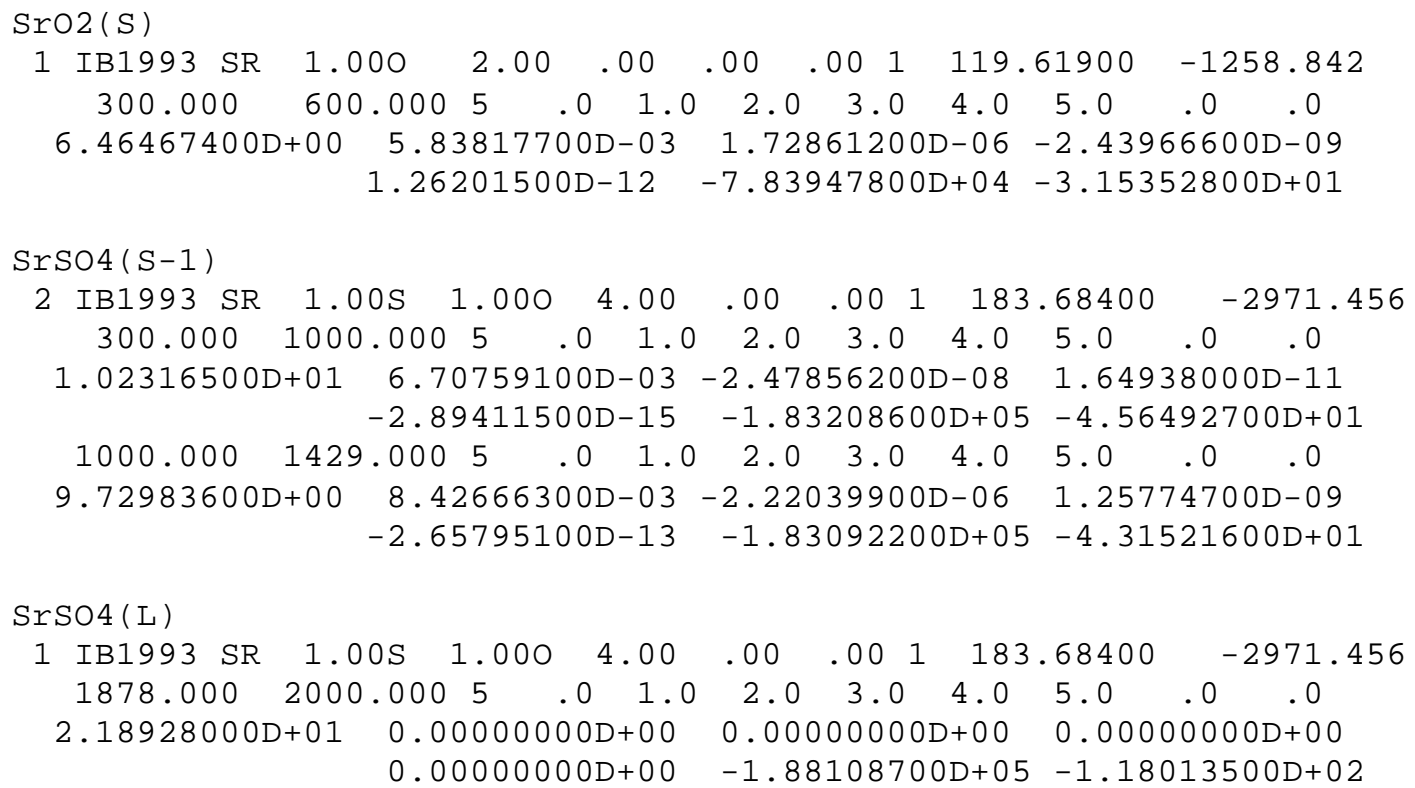

$\operatorname{SrCO} 3(\mathrm{~S}-\mathrm{B})$ 


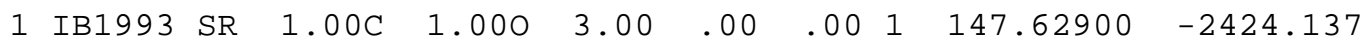

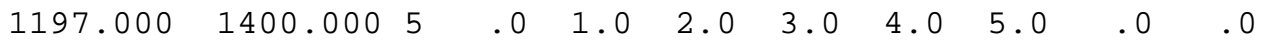
$\begin{array}{lllll}1.71117300 D+01 & 0.00000000 D+00 & 0.00000000 D+00 & 0.00000000 D+00\end{array}$ $0.00000000 D+00-1.52694300 D+05-8.96734500 D+01$

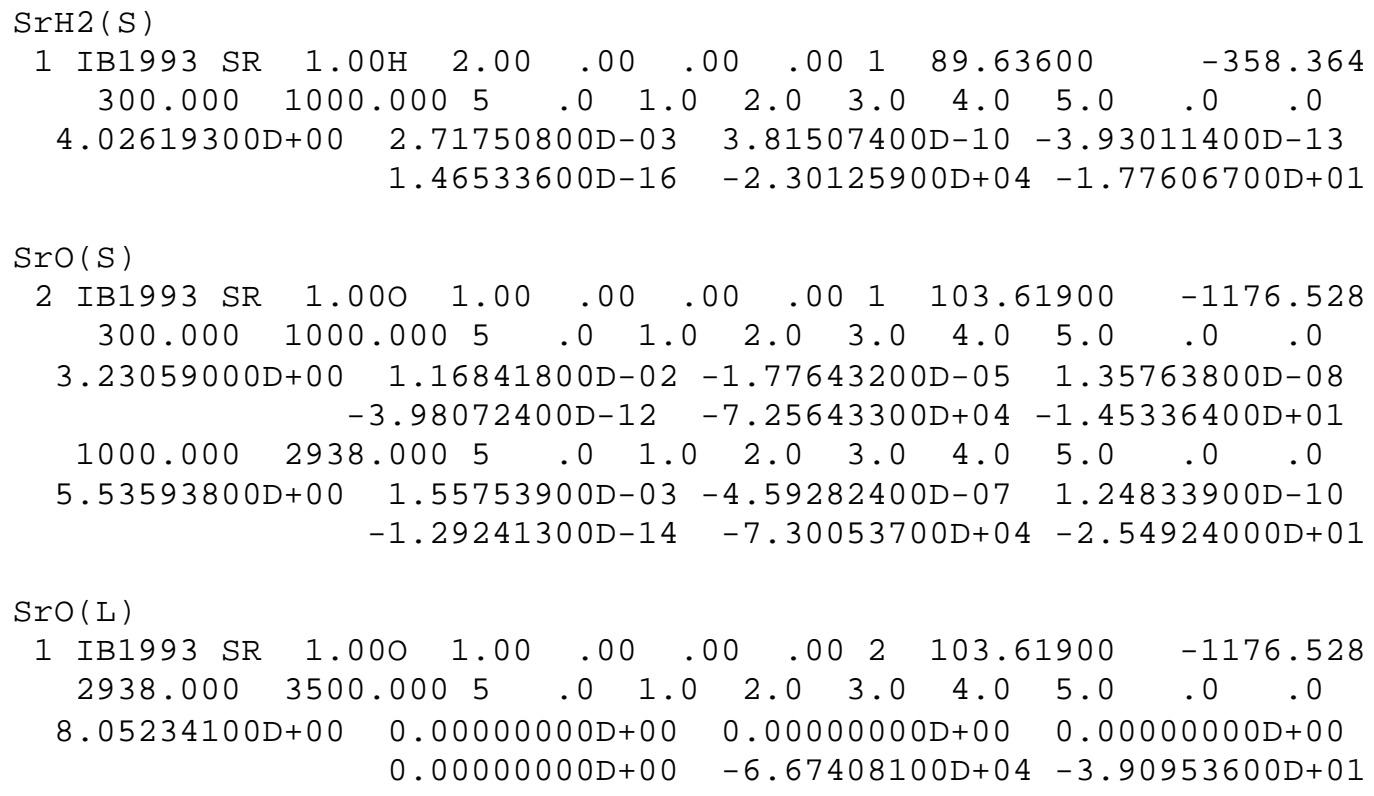


APPENDIX A.2 : FURNACE SETTINGS

\begin{tabular}{|c|c|}
\hline $\begin{array}{l}\text { Furnace Parameters } \\
\text { Stoichiometric Ratio } \\
\text { Air Temperature } \\
\text { Primary Air Pressure } \\
\text { Methane Rotameter } \\
\text { UV Sweep Air } \\
\text { Draft Fan Frequency } \\
\text { Secondary Air Pressure } \\
\text { Lower Air Monometer }\end{array}$ & $\begin{array}{l}1.2 \\
78 \text { deg F } \\
50 \text { psig } \\
18 \text { units } \\
110 \text { units } \\
45 \mathrm{~Hz} \\
20 \text { psig } \\
1.85 \text { in } \mathrm{H} 20\end{array}$ \\
\hline $\begin{array}{c}\text { Metal Injection Parameters } \\
\text { Atomizer Pressure } \\
\text { Atomizer Flow } \\
\text { Solution CsAct } \\
\text { SrNit } \\
\text { Injection Rate } \\
\end{array}$ & $\begin{array}{c}50 \mathrm{psig} \\
95 \mathrm{units} \\
15.5 \mathrm{~g} / \mathrm{L} \\
17.1 \mathrm{~g} / \mathrm{L} \\
20 \mathrm{~mL} / \mathrm{min}\end{array}$ \\
\hline $\begin{array}{l}\text { Sorbent Parameters } \\
\text { Transport Air Pressure } \\
\text { Transport Air Flow } \\
\text { Injection Rate } \\
\text { Feeder Rotation Reading } \\
\text { Kaolinite } \\
\text { Lime } \\
\text { Mixture } \\
\text { CDEM } \\
\text { B-80 } \\
6104-B \\
\end{array}$ & $\begin{array}{c}50 \mathrm{psig} \\
125 \text { units GB } \\
2 \mathrm{~g} / \mathrm{min} \\
1150 \mathrm{rpm} \\
775 \mathrm{rpm} \\
1000 \mathrm{rpm} \\
610 \mathrm{rpm} \\
1800 \mathrm{rpm} \\
750 \mathrm{rpm} \\
\end{array}$ \\
\hline $\begin{array}{r}\text { Dopant Parameters } \\
\text { Flowrate } \mathrm{SO} 2 \\
\mathrm{Cl} 2 \\
\end{array}$ & $\begin{array}{l}420 \mathrm{~mL} / \mathrm{min} \\
210 \mathrm{~mL} / \mathrm{min}\end{array}$ \\
\hline $\begin{array}{l}\text { Sampling Port } \\
\text { Target Sampling Rate } \\
\text { LPI Backside Pressure } \\
\text { LPI Flowrate } \\
\text { Sampling Time } \\
\text { Impactor }\end{array}$ & $\begin{array}{c}14 \\
1.25 \mathrm{Lpm} \\
27 \mathrm{in} \mathrm{Hg} \\
22 \mathrm{Lpm} \\
10 \mathrm{~min} \\
\text { EPA-LPI, UA-LPI, PSI-LPI }\end{array}$ \\
\hline
\end{tabular}




\section{APPENDIX A.3 : EXPERIMENTAL CONDITIONS}

\begin{tabular}{|c|c|c|c|c|c|c|c|c|c|c|}
\hline Run \# & $\begin{array}{c}\text { Fluid [ ppm } \\
\text { Cs }\end{array}$ & $\begin{array}{c}\text { gas] } \\
\mathrm{Sr}\end{array}$ & Sorbent & $\begin{array}{c}\text { Sorbent } \\
\text { Flowrate } \\
\text { [g/min] }\end{array}$ & Dopant & $\begin{array}{r}\text { Time } \\
\text { [min] }\end{array}$ & Substrate & $\begin{array}{c}\text { Sorbent } \\
\text { Injection } \\
\text { Port }\end{array}$ & $\begin{array}{c}\text { Sample } \\
\text { Port }\end{array}$ & $\begin{array}{c}\text { Date } \\
\text { Completed }\end{array}$ \\
\hline $\mathrm{C} 2-1$ & 100 & - & - & - & - & 10 & PC & - & 14 & $01 / 05 / 1999$ \\
\hline C2-2 & 100 & - & - & - & - & 10 & $P C$ & - & 14 & 01/05/1999 \\
\hline C2-3 & 100 & - & - & - & $\mathrm{Cl}-1000$ ppm & 10 & PC & - & 14 & 01/05/1999 \\
\hline C2-4 & 100 & - & - & - & $\mathrm{Cl}-1000$ ppm & 10 & PC & - & 14 & 01/05/1999 \\
\hline C2-5 & 100 & - & - & - & S - 1000 ppm & 10 & PC & - & 14 & 01/05/1999 \\
\hline C2-6 & 100 & - & - & - & S - 1000 ppm & 10 & PC & - & 14 & 01/06/1999 \\
\hline $\mathrm{C} 2-7$ & 100 & - & $\mathrm{KAO}$ & 2 & - & 10 & $P C$ & 4 & 14 & 01/05/1999 \\
\hline C2-8 & 100 & - & $\mathrm{KAO}$ & 2 & - & 10 & PC & 4 & 14 & 01/05/1999 \\
\hline C2-9 & 100 & - & $\mathrm{KAO}$ & 2 & $\mathrm{Cl}-1000$ ppm & 10 & $P C$ & 4 & 14 & 01/05/1999 \\
\hline C2-10 & 100 & - & $\mathrm{KAO}$ & 2 & $\mathrm{Cl}-1000$ ppm & 10 & PC & 4 & 14 & 01/05/1999 \\
\hline C2-11 & 100 & - & $\mathrm{KAO}$ & 2 & S - 1000 ppm & 10 & $\mathrm{PC}$ & 4 & 14 & 01/05/1999 \\
\hline C2-12 & 100 & - & $\mathrm{KAO}$ & 2 & S - 1000 ppm & 10 & PC & 4 & 14 & 01/05/1999 \\
\hline C2-13 & 100 & - & Lime & 2 & - & 10 & PC & 4 & 14 & 01/06/1999 \\
\hline C2-14 & 100 & - & Lime & 2 & - & 10 & PC & 4 & 14 & 01/06/1999 \\
\hline C2-15 & 100 & - & Lime & 2 & $\mathrm{Cl}-1000$ ppm & 10 & PC & 4 & 14 & 01/06/1999 \\
\hline C2-16 & 100 & - & Lime & 2 & $\mathrm{Cl}-1000$ ppm & 10 & $P C$ & 4 & 14 & 01/06/1999 \\
\hline $\mathrm{C} 2-17$ & 100 & - & Lime & 2 & S - 1000 ppm & 10 & PC & 4 & 14 & 01/06/1999 \\
\hline C2-18 & 100 & - & Lime & 2 & S - 1000 ppm & 10 & PC & 4 & 14 & 01/06/1999 \\
\hline C2-19 & 100 & - & CDEM & 2 & - & 10 & $P C$ & 4 & 14 & 02/03/1999 \\
\hline C2-20 & 100 & - & CDEM & 2 & - & 10 & PC & 4 & 14 & 02/03/1999 \\
\hline C2-21 & 100 & - & CDEM & 2 & $\mathrm{Cl}-1000$ ppm & 10 & PC & 4 & 14 & 02/03/1999 \\
\hline C2-22 & 100 & - & CDEM & 2 & $\mathrm{Cl}-1000$ ppm & 10 & PC & 4 & 14 & 02/03/1999 \\
\hline C2-23 & 100 & - & CDEM & 2 & S - 1000 ppm & 10 & $P C$ & 4 & 14 & - \\
\hline C2-24 & 100 & - & CDEM & 2 & S - 1000 ppm & 10 & PC & 4 & 14 & - \\
\hline C2-25 & 100 & - & Mixture & 2 & - & 10 & PC & 4 & 14 & 01/07/1999 \\
\hline C2-26 & 100 & - & Mixture & 2 & - & 10 & PC & 4 & 14 & 01/07/1999 \\
\hline C2-27 & 100 & - & Mixture & 2 & $\mathrm{Cl}-1000$ ppm & 10 & PC & 4 & 14 & 01/07/1999 \\
\hline C2-28 & 100 & - & Mixture & 2 & $\mathrm{Cl}-1000$ ppm & 10 & PC & 4 & 14 & 01/07/1999 \\
\hline C2-29 & 100 & - & Mixture & 2 & S - 1000 ppm & 10 & PC & 4 & 14 & 01/07/1999 \\
\hline $\mathrm{C} 2-30$ & 100 & - & Mixture & 2 & S - 1000 ppm & 10 & $\mathrm{PC}$ & 4 & 14 & 01/07/1999 \\
\hline
\end{tabular}


APPENDIX A.3 : EXPERIMENTAL CONDITIONS - Continued

\begin{tabular}{|c|c|c|c|c|c|c|c|c|c|c|}
\hline \multicolumn{3}{|c|}{ Fluid [ppm in gas] } & \multirow[t]{2}{*}{ Sorbent } & \multirow{2}{*}{$\begin{array}{c}\text { Sorbent } \\
\text { Flowrate } \\
\text { [g/min] }\end{array}$} & \multirow[t]{2}{*}{ Dopant } & \multirow{2}{*}{$\begin{array}{r}\text { Time } \\
\text { [min] }\end{array}$} & \multirow[t]{2}{*}{ Substrate } & \multirow{2}{*}{$\begin{array}{c}\text { Sorbent } \\
\text { Injection } \\
\text { Port }\end{array}$} & \multirow{2}{*}{$\begin{array}{c}\text { Sample } \\
\text { Port }\end{array}$} & \multirow{2}{*}{$\begin{array}{c}\text { Date } \\
\text { Completed }\end{array}$} \\
\hline Run \# & Cs & $\mathrm{Sr}$ & & & & & & & & \\
\hline S2-1 & - & 100 & - & - & - & 10 & $\mathrm{PC}$ & - & 14 & $12 / 31 / 1998$ \\
\hline S2-2 & - & 100 & - & - & - & 10 & PC & - & 14 & 12/31/1998 \\
\hline S2-3 & - & 100 & - & - & $\mathrm{Cl}-1000$ ppm & 10 & $\mathrm{PC}$ & - & 14 & $12 / 31 / 1998$ \\
\hline S2-4 & - & 100 & - & - & $\mathrm{Cl}-1000$ ppm & 10 & $\mathrm{PC}$ & - & 14 & $12 / 31 / 1998$ \\
\hline S2-5 & - & 100 & - & - & S - 1000 ppm & 10 & PC & - & 14 & 12/31/1998 \\
\hline S2-6 & - & 100 & - & - & S - 1000 ppm & 10 & PC & - & 14 & 12/31/1998 \\
\hline S2-7 & - & 100 & $\mathrm{KAO}$ & 2 & - & 10 & PC & 4 & 14 & 01/02/1999 \\
\hline S2-8 & - & 100 & $\mathrm{KAO}$ & 2 & - & 10 & PC & 4 & 14 & 01/02/1999 \\
\hline S2-9 & - & 100 & $\mathrm{KAO}$ & 2 & $\mathrm{Cl}-1000$ ppm & 10 & PC & 4 & 14 & 01/02/1999 \\
\hline S2-10 & - & 100 & $\mathrm{KAO}$ & 2 & $\mathrm{Cl}-1000$ ppm & 10 & $\mathrm{PC}$ & 4 & 14 & 01/02/1999 \\
\hline $\mathrm{S} 2-11$ & - & 100 & $\mathrm{KAO}$ & 2 & S - 1000 ppm & 10 & PC & 4 & 14 & 01/02/1999 \\
\hline S2-12 & - & 100 & $\mathrm{KAO}$ & 2 & S - 1000 ppm & 10 & PC & 4 & 14 & 01/02/1999 \\
\hline $\mathrm{S} 2-13$ & - & 100 & Lime & 2 & - & 10 & PC & 4 & 14 & 01/06/1999 \\
\hline $\mathrm{S} 2-14$ & - & 100 & Lime & 2 & - & 10 & PC & 4 & 14 & 01/06/1999 \\
\hline $\mathrm{S} 2-15$ & - & 100 & Lime & 2 & $\mathrm{Cl}-1000$ ppm & 10 & PC & 4 & 14 & 01/06/1999 \\
\hline S2-16 & - & 100 & Lime & 2 & $\mathrm{Cl}-1000$ ppm & 10 & PC & 4 & 14 & 01/06/1999 \\
\hline S2-17 & - & 100 & Lime & 2 & S - 1000 ppm & 10 & $P C$ & 4 & 14 & 01/06/1999 \\
\hline S2-18 & - & 100 & Lime & 2 & S - 1000 ppm & 10 & PC & 4 & 14 & 01/06/1999 \\
\hline S2-19 & - & 100 & CDEM & 2 & - & 10 & PC & 4 & 14 & 02/03/1999 \\
\hline S2-20 & - & 100 & CDEM & 2 & - & 10 & $P C$ & 4 & 14 & 02/03/1999 \\
\hline S2-21 & - & 100 & CDEM & 2 & $\mathrm{Cl}-1000$ ppm & 10 & PC & 4 & 14 & 02/03/1999 \\
\hline S2-22 & - & 100 & CDEM & 2 & $\mathrm{Cl}-1000$ ppm & 10 & PC & 4 & 14 & 02/03/1999 \\
\hline S2-23 & - & 100 & CDEM & 2 & S - 1000 ppm & 10 & PC & 4 & 14 & - \\
\hline S2-24 & - & 100 & CDEM & 2 & S - 1000 ppm & 10 & PC & 4 & 14 & - \\
\hline S2-25 & - & 100 & Mixture & 2 & - & 10 & PC & 4 & 14 & 01/06/1999 \\
\hline S2-26 & - & 100 & Mixture & 2 & - & 10 & $P C$ & 4 & 14 & 01/06/1999 \\
\hline S2-27 & - & 100 & Mixture & 2 & $\mathrm{Cl}-1000$ ppm & 10 & PC & 4 & 14 & 01/06/1999 \\
\hline S2-28 & - & 100 & Mixture & 2 & $\mathrm{Cl}-1000$ ppm & 10 & $P C$ & 4 & 14 & 01/07/1999 \\
\hline S2-29 & - & 100 & Mixture & 2 & S - 1000 ppm & 10 & PC & 4 & 14 & 01/07/1999 \\
\hline $\mathrm{S} 2-30$ & - & 100 & Mixture & 2 & S - 1000 ppm & 10 & $\mathrm{PC}$ & 4 & 14 & 01/07/1999 \\
\hline
\end{tabular}


APPENDIX A.3 : EXPERIMENTAL CONDITIONS - Continued

\begin{tabular}{|c|c|c|c|c|c|c|c|c|c|c|}
\hline \multicolumn{3}{|c|}{ Fluid [ ppm in gas] } & \multirow[t]{2}{*}{ Sorbent } & \multirow{2}{*}{$\begin{array}{c}\text { Sorbent } \\
\text { Flowrate } \\
\text { [g/min] }\end{array}$} & \multirow[t]{2}{*}{ Dopant } & \multirow{2}{*}{$\begin{array}{l}\text { Time } \\
\text { [min] }\end{array}$} & \multirow[t]{2}{*}{ Substrate } & \multirow{2}{*}{$\begin{array}{c}\text { Sorbent } \\
\text { Injection } \\
\text { Port }\end{array}$} & \multirow{2}{*}{$\begin{array}{c}\text { Sample } \\
\text { Port }\end{array}$} & \multirow{2}{*}{$\begin{array}{c}\text { Date } \\
\text { Completed }\end{array}$} \\
\hline Run \# & Cs & $\mathrm{Sr}$ & & & & & & & & \\
\hline C2-23 & 100 & - & CDEM & 2 & S - 1000 ppm & 10 & $P C$ & 4 & 14 & $05 / 10 / 1999$ \\
\hline C2-24 & 100 & - & CDEM & 2 & S - 1000 ppm & 10 & PC & 4 & 14 & 05/10/1999 \\
\hline C3-1 & 100 & - & - & - & S - 1000 ppm & 10 & PC & - & 3 & 05/10/1999 \\
\hline C3-2 & 100 & - & - & - & S - 1000 ppm & 10 & PC & - & 3 & 05/10/1999 \\
\hline S2-19 & - & 100 & CDEM & 2 & - & 10 & PC & 4 & 14 & 05/10/1999 \\
\hline S2-20 & - & 100 & CDEM & 2 & - & 10 & $\mathrm{PC}$ & 4 & 14 & 05/10/1999 \\
\hline S2-23 & - & 100 & CDEM & 2 & S - 1000 ppm & 10 & $P C$ & 4 & 14 & 05/10/1999 \\
\hline S2-24 & - & 100 & CDEM & 2 & S - 1000 ppm & 10 & PC & 4 & 14 & 05/10/1999 \\
\hline S3-3 & - & 100 & - & - & S - 1000 ppm & 10 & PC & - & 3 & 05/10/1999 \\
\hline S3-4 & - & 100 & - & - & S - 1000 ppm & 10 & PC & - & 3 & 05/10/1999 \\
\hline S3-5 & - & 100 & B-80 & 2 & $\mathrm{Cl}-1000$ ppm & 10 & PC & 4 & 14 & 05/10/1999 \\
\hline S3-6 & - & 100 & B-80 & 2 & $\mathrm{Cl}-1000$ ppm & 10 & PC & 4 & 14 & 05/10/1999 \\
\hline C3-3 & 100 & - & B-80 & 2 & - & 10 & PC & 4 & 14 & 05/10/1999 \\
\hline C3-4 & 100 & - & B-80 & 2 & - & 10 & $P C$ & 4 & 14 & 05/10/1999 \\
\hline C3-5 & 100 & - & 1040 & 2 & - & 10 & PC & 4 & 14 & 05/12/1999 \\
\hline C3-6 & 100 & - & 1040 & 2 & - & 10 & $\mathrm{PC}$ & 4 & 14 & 05/12/1999 \\
\hline S3-7 & - & 100 & 1040 & 2 & $\mathrm{Cl}-1000$ ppm & 10 & $P C$ & 4 & 14 & 05/12/1999 \\
\hline S3-8 & - & 100 & 1040 & 2 & $\mathrm{Cl}-1000$ ppm & 10 & PC & 4 & 14 & 05/12/1999 \\
\hline S3-1 & - & 100 & Kao & 2 & S - 1000 ppm & 10 & PC & 4 & 14 & 05/12/1999 \\
\hline S3-2 & - & 100 & $\mathrm{Kao}$ & 2 & S - 1000 ppm & 10 & $\mathrm{PC}$ & 4 & 14 & 05/12/1999 \\
\hline
\end{tabular}


Appedix A.4 - GAS PROFILE

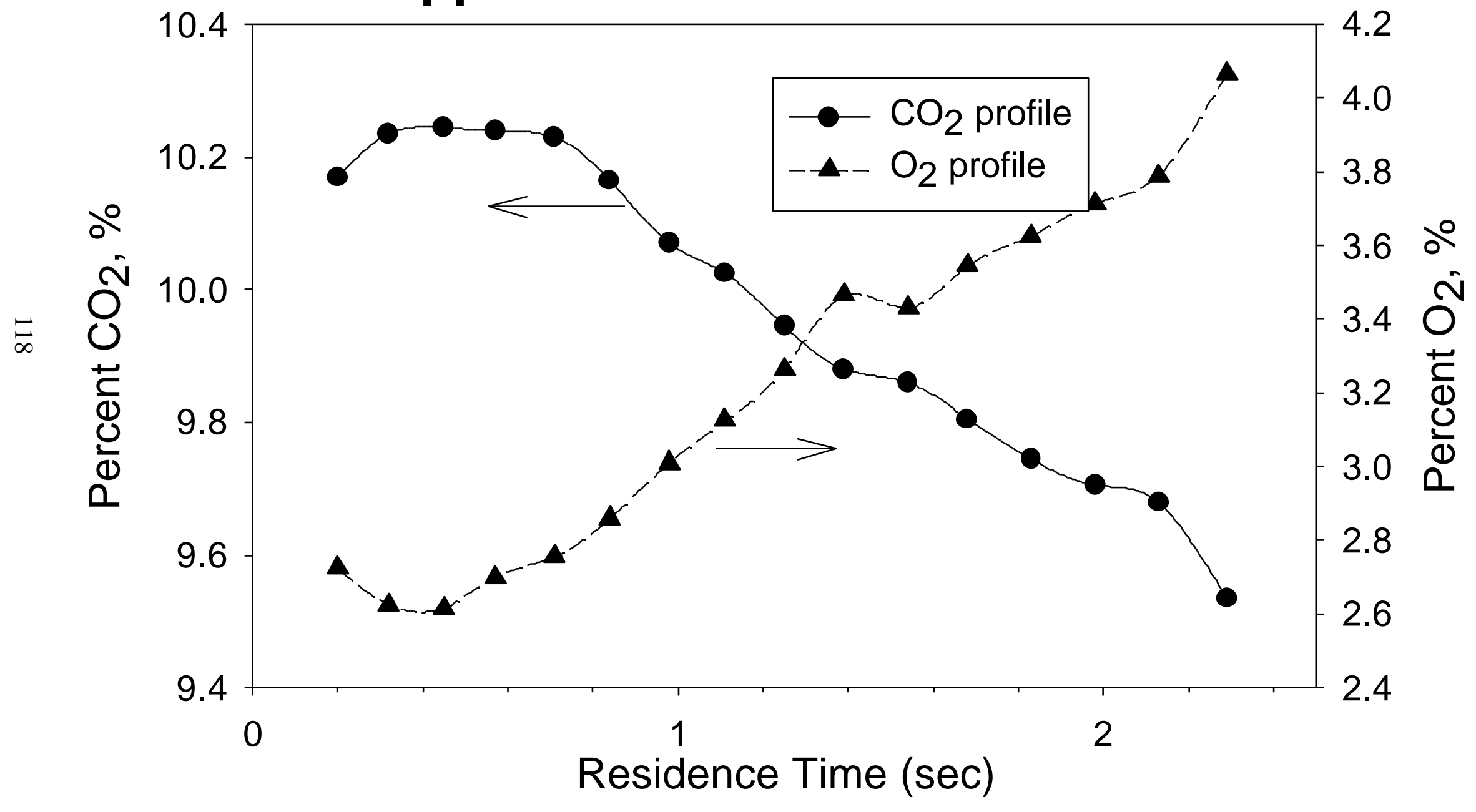

Figure A.4 - Average furnace gas profile 


\section{APPENDIX A.5 : RAW EXPERIMENTAL DATA}

\begin{tabular}{|c|c|c|c|c|c|c|c|c|}
\hline \multirow{4}{*}{$\begin{array}{l}\text { Run } \\
\text { Date } \\
\text { Initials }\end{array}$} & \multicolumn{2}{|c|}{$\mathrm{C} 2-1$} & \multicolumn{3}{|c|}{ Figures where data appears: } & \multicolumn{3}{|c|}{$4.1,4.4,4.5,4.6$} \\
\hline & \multirow{2}{*}{\multicolumn{2}{|c|}{$\begin{array}{c}\text { Cs } \\
\text { \#\#\#\#\#\#\# } \\
\text { JMA }\end{array}$}} & \multirow{2}{*}{\multicolumn{2}{|c|}{$\mathrm{Si}$}} & \multirow{2}{*}{\multicolumn{2}{|c|}{$\mathrm{Al}$}} & \multirow{2}{*}{\multicolumn{2}{|c|}{$\mathrm{Ca}$}} \\
\hline & & & & & & & & \\
\hline & \begin{tabular}{|l|} 
conc. \\
\end{tabular} & s.d. & conc. & s.d. & conc. & s.d. & conc. & s.d. \\
\hline Plate 1 & 2.9 & 0.14 & & & & & & \\
\hline Plate 2 & 3 & 0.16 & & & & & & \\
\hline Plate 3 & 2 & 0.1 & & & & & & \\
\hline Plate 4 & 1.3 & 0.07 & & & & & & \\
\hline Plate 5 & 0.7 & 0.14 & & & & & & \\
\hline Plate 6 & 0.3 & 0.09 & & & & & & \\
\hline Plate 7 & 0.1 & 0.08 & & & & & & \\
\hline Plate 8 & 0.1 & 0.05 & & & & & & \\
\hline Plate 9 & 0.1 & 0.04 & & & & & & \\
\hline Plate 10 & 0.1 & 0.06 & & & & & & \\
\hline Plate 11 & 0 & 0.07 & & & & & & \\
\hline
\end{tabular}

Run

C2-2

Figures where data appears: $4.1,4.4,4.5,4.6$

Date

Initials

Plate 1

Plate 2

Plate 3

Plate 4

Plate 5

Plate 6

Plate 7

Plate 8

Plate 9

Plate 10

Plate 11

\begin{tabular}{|c|c|c|c|c|c|c|c|}
\hline $\begin{array}{c} \\
\# \# \# \# \# \# \\
\text { JMA } \\
\end{array}$ & & \multicolumn{2}{|c|}{$\mathrm{Si}$} & \multicolumn{2}{|c|}{$\overline{\mathrm{Al}}$} & \multicolumn{2}{|c|}{$\mathrm{Ca}$} \\
\hline conc. & s.d. & conc. & s.d. & conc. & s.d. & conc. & s.d. \\
\hline 3.3 & 0.11 & & & & & & \\
\hline 3 & 0.12 & & & & & & \\
\hline 2.3 & 0.12 & & & & & & \\
\hline 1.1 & 0.12 & & & & & & \\
\hline 0.4 & 0.09 & & & & & & \\
\hline 0.2 & 0.05 & & & & & & \\
\hline 0.2 & 0.07 & & & & & & \\
\hline 0.2 & 0.06 & & & & & & \\
\hline 0.2 & 0.05 & & & & & & \\
\hline 0.2 & 0.04 & & & & & & \\
\hline 0.1 & 0.04 & & & & & & \\
\hline
\end{tabular}

Run

C2-3

Figures where data appears: $4.7,4.8,4.9,4.10$

Date

Initials

Plate 1

Plate 2

Plate 3

Plate 4

Plate 5

Plate 6

Plate 7

Plate 8

\begin{tabular}{|c|c|c|c|c|c|c|c|}
\hline $\begin{array}{c}\text { \#\#\#\#\#\# } \\
\text { JMA }\end{array}$ & & \multicolumn{2}{|c|}{$\mathrm{Si}$} & \multicolumn{2}{|c|}{$\mathrm{Al}$} & \multicolumn{2}{|c|}{$\mathrm{Ca}$} \\
\hline conc. & s.d. & conc. & s.d. & conc. & s.d. & conc & s.d. \\
\hline 7.7 & 0.14 & & & & & & \\
\hline 9.3 & 0.15 & & & & & & \\
\hline 4.9 & 0.17 & & & & & & \\
\hline 2 & 0.09 & & & & & & \\
\hline 0.6 & 0.06 & & & & & & \\
\hline 0.3 & 0.08 & & & & & & \\
\hline 0.3 & 0.07 & & & & & & \\
\hline 0.3 & 0.08 & & & & & & \\
\hline 0.2 & 0.08 & & & & & & \\
\hline
\end{tabular}

Plate 9 


\begin{tabular}{|c|c|c|c|c|c|c|c|c|}
\hline $\begin{array}{l}\text { Plate } 10 \\
\text { Plate } 11\end{array}$ & $\begin{array}{l}0.1 \\
0.1 \\
\end{array}$ & $\begin{array}{l}0.08 \\
0.05 \\
\end{array}$ & & & & & & \\
\hline Run & $\mathrm{C} 2-4$ & & Figures & re da & pears: & $7,4.8$ &, 4.10 & \\
\hline $\begin{array}{l}\text { Date } \\
\text { Initials }\end{array}$ & $\begin{array}{c}\text { C } \\
\text { \#\#\#\#\#\# } \\
\text { JMA }\end{array}$ & & & & & & & \\
\hline & conc. & s.d. & conc & s.d. & conc. & s.d. & conc. & s.d. \\
\hline Plate 1 & 5.1 & 0.06 & & & & & & \\
\hline Plate 2 & 9.7 & 0.18 & & & & & & \\
\hline Plate 3 & 6.3 & 0.07 & & & & & & \\
\hline Plate 4 & 2.2 & 0.05 & & & & & & \\
\hline Plate 5 & 0.7 & 0.11 & & & & & & \\
\hline Plate 6 & 0.4 & 0.03 & & & & & & \\
\hline Plate 7 & 0.3 & 0.05 & & & & & & \\
\hline Plate 8 & 0.3 & 0.07 & & & & & & \\
\hline Plate 9 & 0.1 & 0.03 & & & & & & \\
\hline Plate 10 & 0.1 & 0.06 & & & & & & \\
\hline Plate 11 & 0 & 0.06 & & & & & & \\
\hline
\end{tabular}

\begin{tabular}{|c|c|c|c|c|c|c|c|c|}
\hline \multirow{3}{*}{$\begin{array}{l}\text { Date } \\
\text { Initials }\end{array}$} & \multicolumn{2}{|l|}{ C2-5 } & \multicolumn{3}{|c|}{ Figures where data appears: } & \multicolumn{3}{|c|}{$4.11,4.14,4.15,4.18,4.19$} \\
\hline & $\begin{array}{c}\text { \#\#\#\#\#\# } \\
\text { JMA }\end{array}$ & & & & & & & \\
\hline & conc. & s.d. & conc. & s.d. & conc. & s.d. & conc. & s.d. \\
\hline Plate 1 & 1.6 & 0.09 & & & & & & \\
\hline Plate 2 & 3 & 0.09 & & & & & & \\
\hline Plate 3 & 4.2 & 0.05 & & & & & & \\
\hline Plate 4 & 2.6 & 0.12 & & & & & & \\
\hline Plate 5 & 2.1 & 0.06 & & & & & & \\
\hline Plate 6 & 2.6 & 0.1 & & & & & & \\
\hline Plate 7 & 0.2 & 0.03 & & & & & & \\
\hline Plate 8 & 0.3 & 0.07 & & & & & & \\
\hline Plate 9 & 0.2 & 0.06 & & & & & & \\
\hline Plate 10 & 0.1 & 0.06 & & & & & & \\
\hline Plate 11 & 0.1 & 0.06 & & & & & & \\
\hline
\end{tabular}

\begin{tabular}{|c|c|c|c|c|c|c|c|c|}
\hline \multirow{3}{*}{$\begin{array}{l}\text { Date } \\
\text { Initials }\end{array}$} & \multicolumn{2}{|l|}{$\mathrm{C} 2-6$} & \multicolumn{3}{|c|}{ Figures where data appears: } & \multicolumn{3}{|c|}{$4.11,4.14,4.15,4.18,4.19$} \\
\hline & $\begin{array}{c}\text { C } \\
\text { \#\#\#\#\#\#\# } \\
\text { JMA }\end{array}$ & & & & & & & \\
\hline & conc. & s.d. & conc. & s.d. & conc. & s.d. & conc. & s.d. \\
\hline Plate 1 & 2.1 & 0.07 & & & & & & \\
\hline Plate 2 & 3.8 & 0.09 & & & & & & \\
\hline Plate 3 & 4 & 0.09 & & & & & & \\
\hline Plate 4 & 2.5 & 0.05 & & & & & & \\
\hline Plate 5 & 2.3 & 0.08 & & & & & & \\
\hline Plate 6 & 3.5 & 0.11 & & & & & & \\
\hline Plate 7 & 0.9 & 0.09 & & & & & & \\
\hline
\end{tabular}




\begin{tabular}{|c|c|c|c|c|c|c|c|c|}
\hline $\begin{array}{l}\text { Plate } 8 \\
\text { Plate } 9 \\
\text { Plate } 10 \\
\text { Plate } 11\end{array}$ & $\begin{array}{c}0.3 \\
0.1 \\
0.1 \\
0\end{array}$ & $\begin{array}{l}0.13 \\
0.05 \\
0.04 \\
0.03\end{array}$ & & & & & & \\
\hline Run & $\mathrm{C} 2-7$ & & Figures & re dat & appears: & & & \\
\hline $\begin{array}{l}\text { Date } \\
\text { Initials }\end{array}$ & $\begin{array}{c} \\
\# \# \# \# \# \# \\
\text { JMA }\end{array}$ & & $\begin{array}{c}\text { \#\#\#\#\# } \\
\text { DS }\end{array}$ & & $\begin{array}{c}\text { \#\#\#\#\#\# } \\
\text { JMA }\end{array}$ & & & \\
\hline & conc. & s.d. & conc. & s.d. & conc. & s.d. & conc. & s.d. \\
\hline Plate 1 & 0 & 0.04 & 0.3 & 0.59 & 0 & 0.06 & & \\
\hline Plate 2 & 0 & 0.05 & 0.4 & 0.86 & 0 & 0.04 & & \\
\hline Plate 3 & 0 & 0.05 & 0 & 0.75 & 0 & 0.05 & & \\
\hline Plate 4 & 0.1 & 0.03 & 0.7 & 0.87 & 0 & 0.07 & & \\
\hline Plate 5 & 0.3 & 0.07 & 1 & 0.75 & 0.2 & 0.07 & & \\
\hline Plate 6 & 1 & 0.07 & 1.7 & 0.62 & 0.9 & 0.06 & & \\
\hline Plate 7 & 4.7 & 0.04 & 6.5 & 0.75 & 4.8 & 0.07 & & \\
\hline Plate 8 & 1.9 & 0.07 & 4.5 & 0.5 & 2.9 & 0.12 & & \\
\hline Plate 9 & 0.1 & 0.05 & 1.1 & 0.59 & 0.4 & 0.06 & & \\
\hline Plate 10 & 0 & 0.03 & 1.6 & 0.47 & 0.1 & 0.08 & & \\
\hline Plate 11 & 0 & 0.07 & 0.9 & 0.55 & 0 & 0.05 & & \\
\hline
\end{tabular}

\begin{tabular}{|c|c|c|c|c|c|c|c|c|}
\hline \multirow{3}{*}{$\begin{array}{l}\text { Run } \\
\text { Date } \\
\text { Initials }\end{array}$} & \multicolumn{2}{|l|}{ C2-8 } & \multicolumn{3}{|c|}{ Figures where data appears: } & \multicolumn{2}{|c|}{4.1} & \\
\hline & $\begin{array}{c}\text { \#\#\#\#\#\# } \\
\text { JMA }\end{array}$ & & $\begin{array}{c}\text { \#\#\#\#\# } \\
\text { DS }\end{array}$ & & $\begin{array}{c}\# \# \# \# \# \\
\text { JMA }\end{array}$ & & & \\
\hline & conc. & s.d. & conc. & s.d. & conc. & s.d. & conc. & s.d. \\
\hline Plate 1 & 0 & 0.03 & 0.6 & 0.34 & 0 & 0.05 & & \\
\hline Plate 2 & 0 & 0.06 & 0.7 & 0.65 & 0 & 0.08 & & \\
\hline Plate 3 & 0 & 0.03 & 0.6 & 0.66 & 0 & 0.05 & & \\
\hline Plate 4 & 0.1 & 0.07 & 1.2 & 0.27 & 0 & 0.09 & & \\
\hline Plate 5 & 0.4 & 0.05 & 0 & 0.64 & 0.2 & 0.06 & & \\
\hline Plate 6 & 1.1 & 0.04 & 0.9 & 0.7 & 0.6 & 0.09 & & \\
\hline Plate 7 & 5.6 & 0.09 & 5.8 & 0.57 & 4.5 & 0.09 & & \\
\hline Plate 8 & 2.1 & 0.09 & 3.4 & 0.48 & 2.2 & 0.07 & & \\
\hline Plate 9 & 0.1 & 0.04 & 1 & 0.52 & 0 & 0.07 & & \\
\hline Plate 10 & 0 & 0.04 & 0.9 & 0.33 & 0 & 0.04 & & \\
\hline Plate 11 & 0 & 0.07 & 1.3 & 0.49 & 0 & 0.05 & & \\
\hline
\end{tabular}

\begin{tabular}{|c|c|c|c|c|c|c|c|c|}
\hline \multirow{2}{*}{$\begin{array}{l}\text { Run } \\
\text { Date } \\
\text { Initials }\end{array}$} & \multicolumn{2}{|l|}{ C2-9 } & \multicolumn{3}{|c|}{ Figures where data appears: } & \multicolumn{2}{|c|}{4.7} & \\
\hline & $\begin{array}{c} \\
\text { \#\#\#\#\#\# } \\
\text { JMA }\end{array}$ & & $\begin{array}{c}\text { \#\#\#\#\#\# } \\
\text { DS }\end{array}$ & & $\begin{array}{c}\# \# \# \# \# \\
\text { JMA }\end{array}$ & & & \\
\hline & conc. & s.d. & conc. & s.d. & conc. & s.d. & conc. & s.d. \\
\hline Plate 1 & 5.1 & 0.07 & 0.5 & 0.63 & 0 & 0.05 & & \\
\hline Plate 2 & 2.2 & 0.07 & 0 & 0.65 & 0 & 0.06 & & \\
\hline Plate 3 & 1.3 & 0.07 & 0 & 0.54 & 0 & 0.05 & & \\
\hline Plate 4 & 0.8 & 0.06 & 0.5 & 0.78 & 0 & 0.06 & & \\
\hline Plate 5 & 0.5 & 0.08 & 0.8 & 0.42 & 0 & 0.07 & & \\
\hline
\end{tabular}




\begin{tabular}{|c|c|c|c|c|c|c|c|c|}
\hline Plate 6 & 1 & 0.05 & 0.9 & 0.44 & 0.5 & 0.1 & & \\
\hline Plate 7 & 3.6 & 0.11 & 3 & 0.87 & 3.2 & 0.08 & & \\
\hline Plate 8 & 1.2 & 0.04 & 1.5 & 0.64 & 1.2 & 0.06 & & \\
\hline Plate 9 & 0.2 & 0.06 & 0 & 0.82 & 0.1 & 0.08 & & \\
\hline Plate 10 & 0 & 0.04 & 0 & 0.7 & 0 & 0.04 & & \\
\hline Plate 11 & 0 & 0.09 & 0 & 0.68 & 0.1 & 0.06 & & \\
\hline \multirow{3}{*}{$\begin{array}{l}\text { Run } \\
\text { Date } \\
\text { Initials }\end{array}$} & $\mathrm{C} 2-10$ & & \multicolumn{6}{|c|}{ Figures where data appears: } \\
\hline & $\begin{array}{c}\text { \#\#\#\#\#\#\# } \\
\text { JMA }\end{array}$ & & $\begin{array}{c}\text { \#\#\#\#\#\# } \\
\text { DS }\end{array}$ & & $\begin{array}{c}\text { \#\#\#\#\#\# } \\
\text { JMA }\end{array}$ & & & \\
\hline & conc. & s.d. & conc. & s.d. & conc. & s.d. & conc. & s.d. \\
\hline Plate 1 & 4.9 & 0.06 & 0.6 & 0.74 & 0 & 0.04 & & \\
\hline Plate 2 & 1.9 & 0.07 & 0.4 & 0.59 & 0 & 0.04 & & \\
\hline Plate 3 & 0.7 & 0.07 & 0.7 & 0.76 & 0 & 0.05 & & \\
\hline Plate 4 & 0.5 & 0.05 & 1 & 0.95 & 0 & 0.07 & & \\
\hline Plate 5 & 0.4 & 0.04 & 0.8 & 0.56 & 0.3 & 0.05 & & \\
\hline Plate 6 & 1.1 & 0.06 & 1.3 & 0.79 & 0.9 & 0.06 & & \\
\hline Plate 7 & 4 & 0.11 & 6 & 0.83 & 4.9 & 0.09 & & \\
\hline Plate 8 & 1.3 & 0.07 & 2.4 & 0.65 & 1.9 & 0.09 & & \\
\hline Plate 9 & 0.2 & 0.03 & 0 & 0.59 & 0.1 & 0.06 & & \\
\hline Plate 10 & 0.1 & 0.05 & 0 & 0.61 & 0 & 0.05 & & \\
\hline Plate 11 & 0 & 0.05 & 0 & 0.46 & 0 & 0.03 & & \\
\hline
\end{tabular}

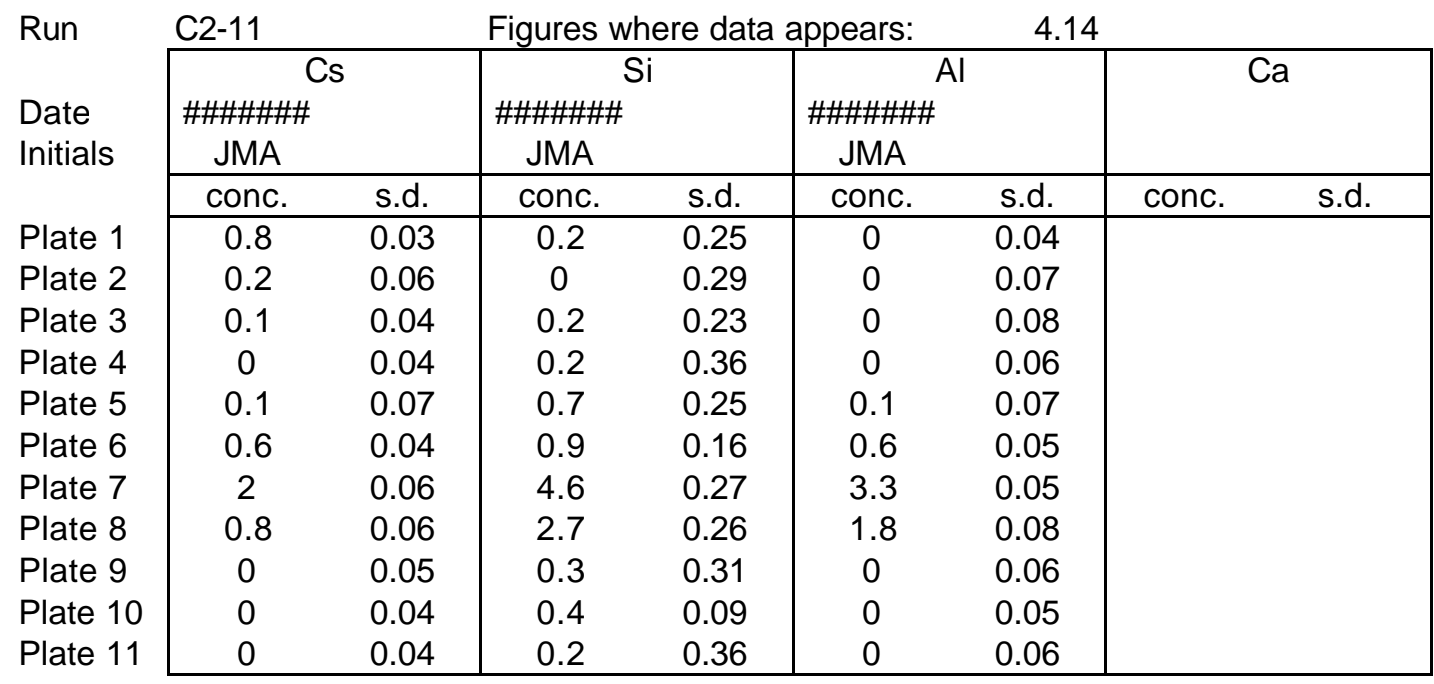

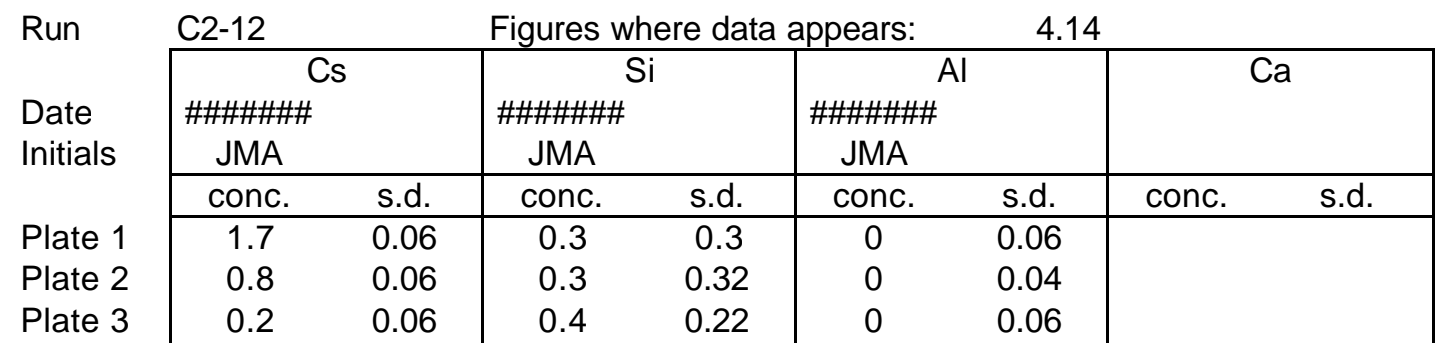




\begin{tabular}{l|cc|cc|cc|c|} 
Plate 4 & 0.1 & 0.03 & 0.3 & 0.33 & 0.1 & 0.06 & \\
Plate 5 & 0.1 & 0.03 & 0.5 & 0.27 & 0.1 & 0.05 & \\
Plate 6 & 0.5 & 0.04 & 0.7 & 0.21 & 0.5 & 0.05 & \\
Plate 7 & 2 & 0.07 & 3 & 0.24 & 2.2 & 0.07 & \\
Plate 8 & 0.2 & 0.04 & 0.6 & 0.29 & 0.9 & 0.04 & \\
Plate 9 & 0 & 0.03 & 0 & 0.19 & 0.1 & 0.1 & \\
Plate 10 & 0 & 0.04 & 0 & 0.31 & 0 & 0.04 & \\
Plate 11 & 0 & 0.03 & 0 & 0.33 & 0.2 & 0.05 & \\
\cline { 2 - 7 }
\end{tabular}




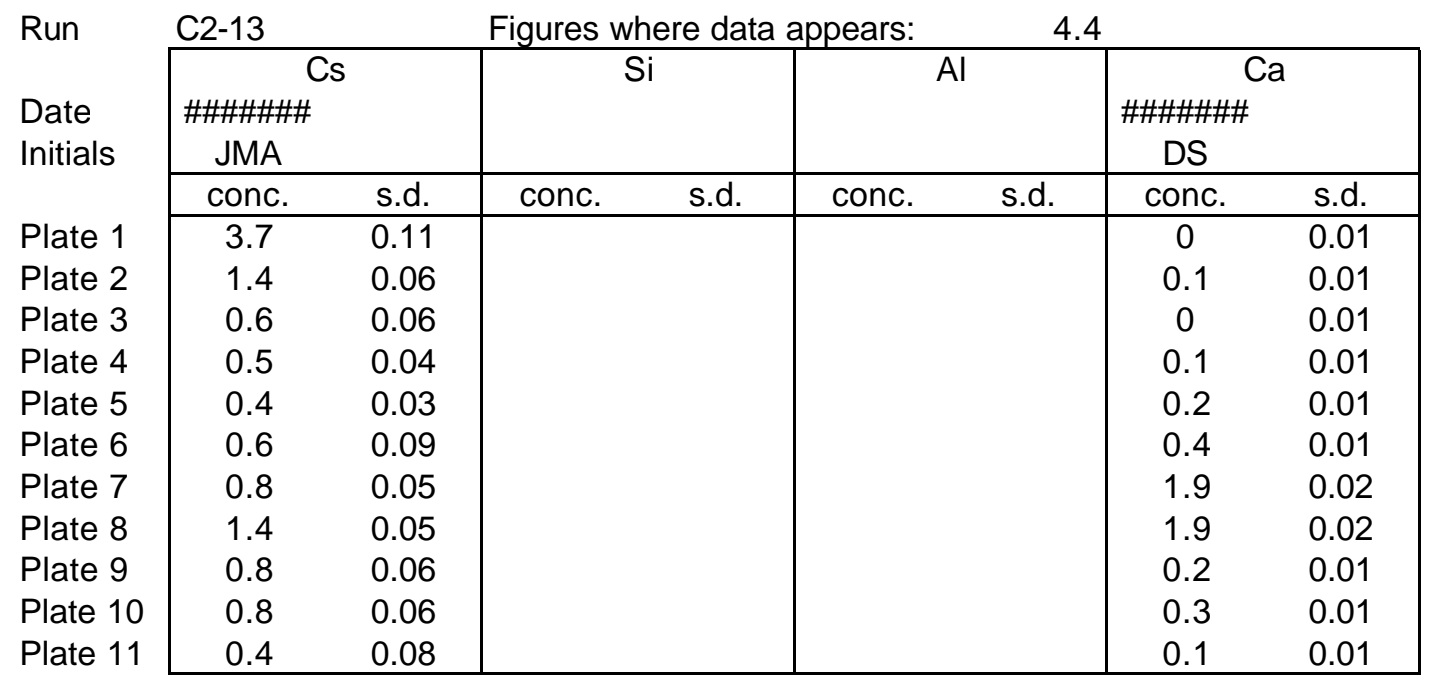

\begin{tabular}{|c|c|c|c|c|c|c|c|c|}
\hline \multirow{3}{*}{$\begin{array}{l}\text { Run } \\
\text { Date } \\
\text { Initials }\end{array}$} & \multicolumn{2}{|l|}{ C2-14 } & \multicolumn{3}{|c|}{ Figures where data appears: } & \multicolumn{2}{|c|}{4.4} & \\
\hline & $\begin{array}{c}\text { \#\#\#\#\#\# } \\
\text { JMA } \\
\end{array}$ & & & & & & $\begin{array}{c}\text { \#\#\#\#\#\# } \\
\text { DS }\end{array}$ & \\
\hline & conc. & s.d. & conc. & s.d. & conc. & s.d. & conc. & s.d. \\
\hline Plate 1 & 3.9 & 0.1 & & & & & 0 & 0.01 \\
\hline Plate 2 & 2.1 & 0.06 & & & & & 0.1 & 0.01 \\
\hline Plate 3 & 1 & 0.05 & & & & & 0.1 & 0.01 \\
\hline Plate 4 & 0.6 & 0.07 & & & & & 0.1 & 0.01 \\
\hline Plate 5 & 0.3 & 0.06 & & & & & 0.3 & 0.01 \\
\hline Plate 6 & 0.3 & 0.05 & & & & & 0.5 & 0.01 \\
\hline Plate 7 & 0.6 & 0.03 & & & & & 2.7 & 0.02 \\
\hline Plate 8 & 1.2 & 0.09 & & & & & 3.2 & 0.04 \\
\hline Plate 9 & 0.4 & 0.09 & & & & & 0.4 & 0.01 \\
\hline Plate 10 & 0.3 & 0.04 & & & & & 0.4 & 0.01 \\
\hline Plate 11 & 0.2 & 0.07 & & & & & 0.2 & 0.01 \\
\hline
\end{tabular}

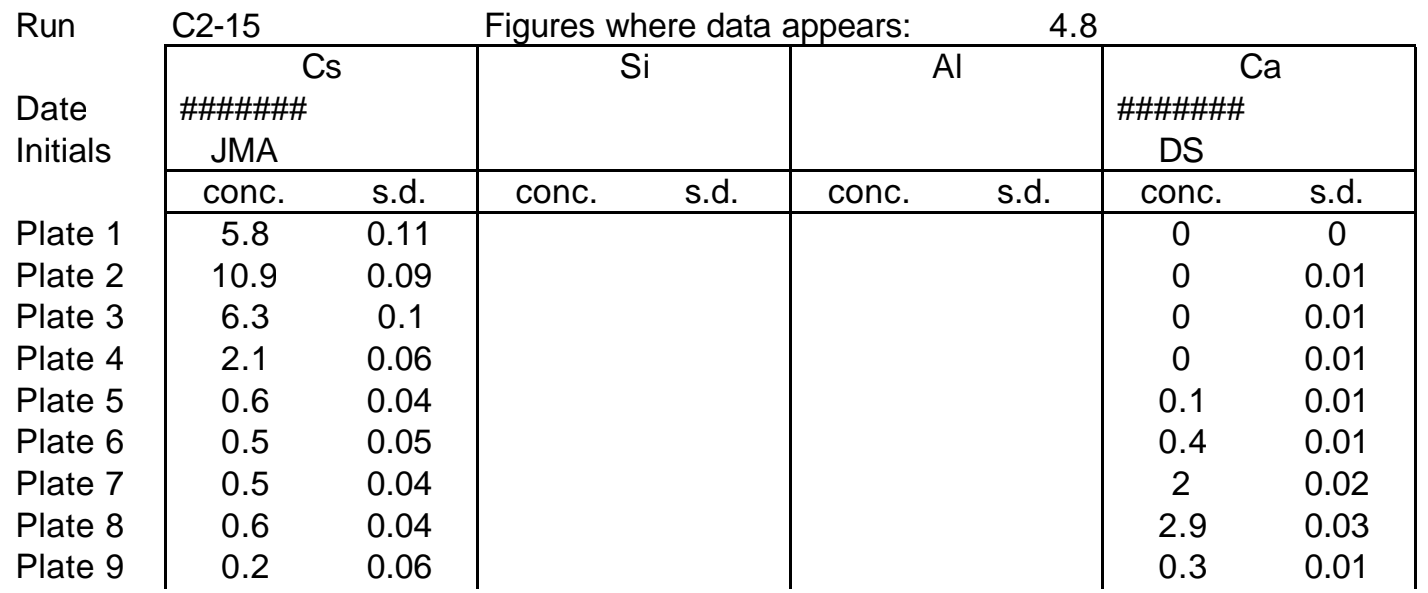




\begin{tabular}{|c|c|c|c|c|c|c|c|c|}
\hline \multirow{3}{*}{$\begin{array}{l}\text { Plate } 10 \\
\text { Plate } 11 \\
\text { Run }\end{array}$} & 0.1 & 0.04 & & & & & 0.3 & 0.01 \\
\hline & 0 & 0.06 & & & & & 0.1 & 0.01 \\
\hline & C2-16 & & \multicolumn{4}{|c|}{ Figures where data appears: } & \\
\hline $\begin{array}{l}\text { Date } \\
\text { Initials }\end{array}$ & $\begin{array}{c}\text { \#\#\#\#\#\#\# } \\
\text { JMA }\end{array}$ & & \multicolumn{2}{|c|}{$\mathrm{Si}$} & \multicolumn{2}{|c|}{$\mathrm{Al}$} & \multicolumn{2}{|c|}{$\begin{array}{c}\text { Ca } \\
\text { \#\#\#\#\#\# } \\
\text { DS } \\
\end{array}$} \\
\hline & conc. & s.d. & conc. & s.d. & conc. & s.d. & conc. & s.d. \\
\hline Plate 1 & 9 & 0.17 & & & & & 0 & 0.01 \\
\hline Plate 2 & 7 & 0.11 & & & & & 0 & 0.01 \\
\hline Plate 3 & 3.8 & 0.08 & & & & & 0 & 0.01 \\
\hline Plate 4 & 1.3 & 0.08 & & & & & 0 & 0.01 \\
\hline Plate 5 & 0.6 & 0.04 & & & & & 0 & 0.01 \\
\hline Plate 6 & 0.4 & 0.05 & & & & & 0.3 & 0.01 \\
\hline Plate 7 & 0.5 & 0.04 & & & & & 1.9 & 0.02 \\
\hline Plate 8 & 0.6 & 0.05 & & & & & 2.5 & 0.02 \\
\hline Plate 9 & 0.3 & 0.06 & & & & & 0.3 & 0.01 \\
\hline Plate 10 & 0.3 & 0.04 & & & & & 0.3 & 0.01 \\
\hline Plate 11 & 0.1 & 0.04 & & & & & 0.1 & 0.01 \\
\hline
\end{tabular}

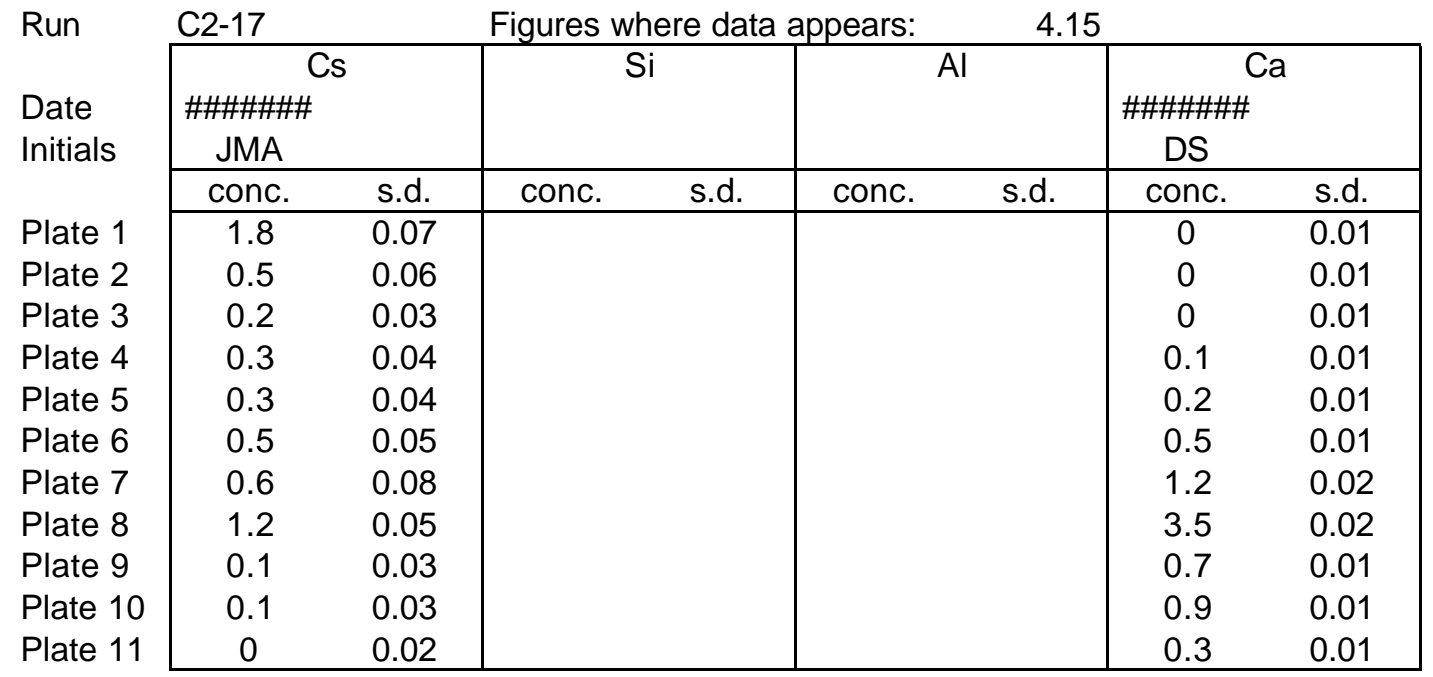

\begin{tabular}{|c|c|c|c|c|c|c|c|c|}
\hline \multirow{3}{*}{$\begin{array}{l}\text { Run } \\
\text { Date } \\
\text { Initials }\end{array}$} & \multicolumn{2}{|l|}{$\mathrm{C} 2-18$} & \multicolumn{3}{|c|}{ Figures where data appears: } & \multicolumn{2}{|c|}{4.15} & \\
\hline & $\begin{array}{c}\text { \#\#\#\#\#\# } \\
\text { JMA }\end{array}$ & & & & & & $\begin{array}{c}\text { \#\#\#\#\#\# } \\
\text { DS }\end{array}$ & \\
\hline & conc. & s.d. & conc & s.d. & conc. & s.d. & conc. & s.d. \\
\hline Plate 1 & 1.1 & 0.05 & & & & & 0 & 0.01 \\
\hline Plate 2 & 0.4 & 0.03 & & & & & 0 & 0.01 \\
\hline Plate 3 & 0.2 & 0.02 & & & & & 0 & 0.01 \\
\hline Plate 4 & 0.3 & 0.04 & & & & & 0.1 & 0.01 \\
\hline Plate 5 & 0.4 & 0.03 & & & & & 0.2 & 0.01 \\
\hline Plate 6 & 0.6 & 0.03 & & & & & 0.4 & 0.01 \\
\hline Plate 7 & 0.7 & 0.03 & & & & & 0.6 & 0.01 \\
\hline
\end{tabular}




\begin{tabular}{|c|c|c|c|c|c|c|c|c|}
\hline $\begin{array}{l}\text { Plate } 8 \\
\text { Plate } 9 \\
\text { Plate } 10 \\
\text { Plate } 11\end{array}$ & $\begin{array}{l}2.1 \\
0.4 \\
0.5 \\
0.2 \\
\end{array}$ & $\begin{array}{l}0.05 \\
0.07 \\
0.01 \\
0.03 \\
\end{array}$ & & & & & $\begin{array}{l}2.1 \\
0.6 \\
0.9 \\
0.2 \\
\end{array}$ & $\begin{array}{l}0.02 \\
0.01 \\
0.01 \\
0.01 \\
\end{array}$ \\
\hline Run & C2-19 & & \multicolumn{4}{|c|}{ Figures where data appears: } & & \\
\hline $\begin{array}{l}\text { Date } \\
\text { Initials }\end{array}$ & $\begin{array}{c}\text { C } \\
\text { \#\#\#\#\#\# } \\
\text { JS }\end{array}$ & & $\begin{array}{c}\text { \#\#\#\#\#\# } \\
\text { JS }\end{array}$ & & $\begin{array}{l} \\
\text { \#\#\#\#\#\# } \\
\text { SF } \\
\end{array}$ & & $\begin{array}{c}\text { \#\#\#\#\#\#\# } \\
\text { JS } \\
\end{array}$ & \\
\hline & conc. & s.d. & conc. & s.d. & conc. & s.d. & conc. & s.d. \\
\hline Plate 1 & 3.8 & 0.06 & 0 & 0.31 & 0.1 & 0.18 & 0.1 & 0.01 \\
\hline Plate 2 & 5.2 & 0.08 & 0 & 0.45 & 0.4 & 0.14 & 0.2 & 0.01 \\
\hline Plate 3 & 3.1 & 0.06 & 0 & 0.32 & 0.4 & 0.32 & 0.2 & 0.01 \\
\hline Plate 4 & 1.4 & 0.04 & 0.3 & 0.3 & 0.3 & 0.25 & 0.3 & 0.02 \\
\hline Plate 5 & 0.4 & 0.02 & 0 & 0.68 & 0.4 & 0.26 & 0.7 & 0.01 \\
\hline Plate 6 & 0.6 & 0.02 & 0 & 0.41 & 0.3 & 0.25 & 1.9 & 0.03 \\
\hline Plate 7 & 1.2 & 0.03 & 1.2 & 0.33 & 1.3 & 0.28 & 6.3 & 0.08 \\
\hline Plate 8 & 1.1 & 0.04 & 1.9 & 0.49 & 1.5 & 0.2 & 7.9 & 0.14 \\
\hline Plate 9 & 0.4 & 0.03 & 1.7 & 0.38 & 1.2 & 0.22 & 5.1 & 0.08 \\
\hline Plate 10 & 0.4 & 0.02 & 4.1 & 0.43 & 2.4 & 0.24 & 8.8 & 0.2 \\
\hline Plate 11 & 0.1 & 0.02 & 0.2 & 0.39 & 0.6 & 0.16 & 1.8 & 0.05 \\
\hline
\end{tabular}

\begin{tabular}{|c|c|c|c|c|c|c|c|c|}
\hline \multirow{3}{*}{$\begin{array}{l}\text { Run } \\
\text { Date } \\
\text { Initials }\end{array}$} & \multicolumn{2}{|l|}{ C2-20 } & \multicolumn{3}{|c|}{ Figures where data appears: } & \multicolumn{2}{|c|}{4.6} & \\
\hline & $\begin{array}{c}\text { \#\#\#\#\#\# } \\
\text { JS }\end{array}$ & & $\begin{array}{c}\text { \#\#\#\#\#\# } \\
\text { JS }\end{array}$ & & $\begin{array}{c}\text { \#\#\#\#\# } \\
\text { SF }\end{array}$ & & $\begin{array}{c}\text { \#\#\#\#\#\# } \\
\text { JS }\end{array}$ & \\
\hline & conc. & s.d. & conc. & s.d. & conc. & s.d. & conc. & s.d. \\
\hline Plate 1 & 3.5 & 0.07 & 0 & 0.38 & 0.1 & 0.28 & 0.2 & 0.01 \\
\hline Plate 2 & 4.2 & 0.09 & 0.1 & 0.33 & 0 & 0.21 & 0.2 & 0.01 \\
\hline Plate 3 & 3.6 & 0.05 & 0.3 & 0.33 & 0.1 & 0.23 & 0.2 & 0.02 \\
\hline Plate 4 & 1.4 & 0.04 & 0.4 & 0.41 & 0.1 & 0.19 & 0.3 & 0.01 \\
\hline Plate 5 & 0.4 & 0.02 & 0.4 & 0.39 & 0.1 & 0.14 & 0.6 & 0.02 \\
\hline Plate 6 & 0.7 & 0.02 & 0 & 0.6 & 0.5 & 0.15 & 2 & 0.02 \\
\hline Plate 7 & 1.2 & 0.02 & 0.6 & 0.51 & 1.3 & 0.18 & 5.6 & 0.08 \\
\hline Plate 8 & 1.4 & 0.03 & 1.5 & 0.68 & 1.6 & 0.23 & 7.2 & 0.14 \\
\hline Plate 9 & 0.4 & 0.02 & 0.2 & 0.49 & 1 & 0.35 & 3.3 & 0.07 \\
\hline Plate 10 & 0.4 & 0.02 & 2.3 & 0.42 & 1.7 & 0.16 & 6.4 & 0.13 \\
\hline Plate 11 & 0.1 & 0.02 & 0 & 0.39 & 0.5 & 0.18 & 1.3 & 0.03 \\
\hline
\end{tabular}

\begin{tabular}{|c|c|c|c|c|c|c|c|c|}
\hline \multirow{3}{*}{$\begin{array}{l}\text { Run } \\
\text { Date } \\
\text { Initials }\end{array}$} & \multicolumn{2}{|l|}{ C2-21 } & \multicolumn{3}{|c|}{ Figures where data appears: } & \multicolumn{2}{|c|}{4.10} & \\
\hline & $\begin{array}{c} \\
\# \# \# \# \# \# \\
\text { JS }\end{array}$ & & $\begin{array}{c}\text { \#\#\#\#\# } \\
\text { JS }\end{array}$ & & $\begin{array}{c}\text { \#\#\#\#\# } \\
\text { SF }\end{array}$ & & $\begin{array}{c} \\
\text { \#\#\#\#\#\#\# } \\
\text { JS }\end{array}$ & \\
\hline & conc. & s.d. & conc. & s.d. & conc. & s.d. & conc. & s.d. \\
\hline Plate 1 & 4.5 & 0.08 & 0 & 0.32 & 0.1 & 0.13 & 0.1 & 0.01 \\
\hline Plate 2 & 12.6 & 0.11 & 0.1 & 0.4 & 0 & 0.12 & 0.2 & 0.02 \\
\hline Plate 3 & 7.5 & 0.1 & 0 & 0.44 & 0.1 & 0.3 & 0.2 & 0.01 \\
\hline Plate 4 & 2.7 & 0.06 & 0.3 & 0.34 & 0 & 0.24 & 0.2 & 0.02 \\
\hline Plate 5 & 1 & 0.02 & 0 & 0.27 & 0 & 0.17 & 0.4 & 0.02 \\
\hline
\end{tabular}




\begin{tabular}{|c|c|c|c|c|c|c|c|c|}
\hline Plate 6 & 0.7 & 0.03 & 0.4 & 0.55 & 0.8 & 0.25 & 1 & 0.02 \\
\hline Plate 7 & 1 & 0.05 & 0.7 & 0.47 & 1.2 & 0.24 & 4.4 & 0.09 \\
\hline Plate 8 & 1 & 0.02 & 1.3 & 0.4 & 1.6 & 0.23 & 6.4 & 0.06 \\
\hline Plate 9 & 0.4 & 0.04 & 0.7 & 0.59 & 1 & 0.19 & 2.8 & 0.04 \\
\hline Plate 10 & 0.3 & 0.04 & 1.1 & 0.55 & 1 & 0.22 & 2.9 & 0.04 \\
\hline Plate 11 & 0.2 & 0.02 & 0.4 & 0.31 & 0.7 & 0.26 & 1.5 & 0.03 \\
\hline Run & C2-22 & & \multicolumn{6}{|c|}{ Figures where data appears: $\quad 4.10$} \\
\hline $\begin{array}{l}\text { Date } \\
\text { Initials }\end{array}$ & $\begin{array}{c}\text { \#\#\#\#\#\# } \\
\text { JS } \\
\end{array}$ & & 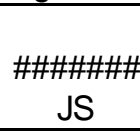 & & $\begin{array}{c} \\
\text { \#\#\#\#\#\#\# } \\
\text { SF } \\
\end{array}$ & & $\begin{array}{c}\text { \#\#\#\#\#\#\# } \\
\text { JS } \\
\end{array}$ & \\
\hline & conc. & s.d. & conc. & s.d. & conc. & s.d. & conc. & s.d. \\
\hline Plate 1 & 3.2 & 0.06 & 0 & 0.38 & 0 & 0.25 & 0.2 & 0.01 \\
\hline Plate 2 & 12.5 & 0.07 & 0 & 0.33 & 0 & 0.24 & 0.2 & 0.01 \\
\hline Plate 3 & 9.6 & 0.1 & 0.2 & 0.64 & 0 & 0.18 & 0.1 & 0.01 \\
\hline Plate 4 & 4.3 & 0.1 & 0.1 & 0.57 & 0 & 0.19 & 0.2 & 0.02 \\
\hline Plate 5 & 1.1 & 0.04 & 0.1 & 0.62 & 0 & 0.16 & 0.3 & 0.01 \\
\hline Plate 6 & 0.8 & 0.08 & 0.7 & 0.36 & 0.4 & 0.2 & 1 & 0.03 \\
\hline Plate 7 & 0.9 & 0.03 & 0.6 & 0.34 & 0.7 & 0.26 & 3.4 & 0.07 \\
\hline Plate 8 & 1.1 & 0.04 & 1.3 & 0.63 & 1.4 & 0.14 & 6.2 & 0.11 \\
\hline Plate 9 & 0.4 & 0.02 & 0.4 & 0.62 & 0.7 & 0.16 & 2.3 & 0.05 \\
\hline Plate 10 & 0.3 & 0.02 & 1.6 & 0.49 & 1 & 0.14 & 3.3 & 0.05 \\
\hline Plate 11 & 0.2 & 0.03 & 0.5 & 0.46 & 0.3 & 0.23 & 1.6 & 0.03 \\
\hline
\end{tabular}

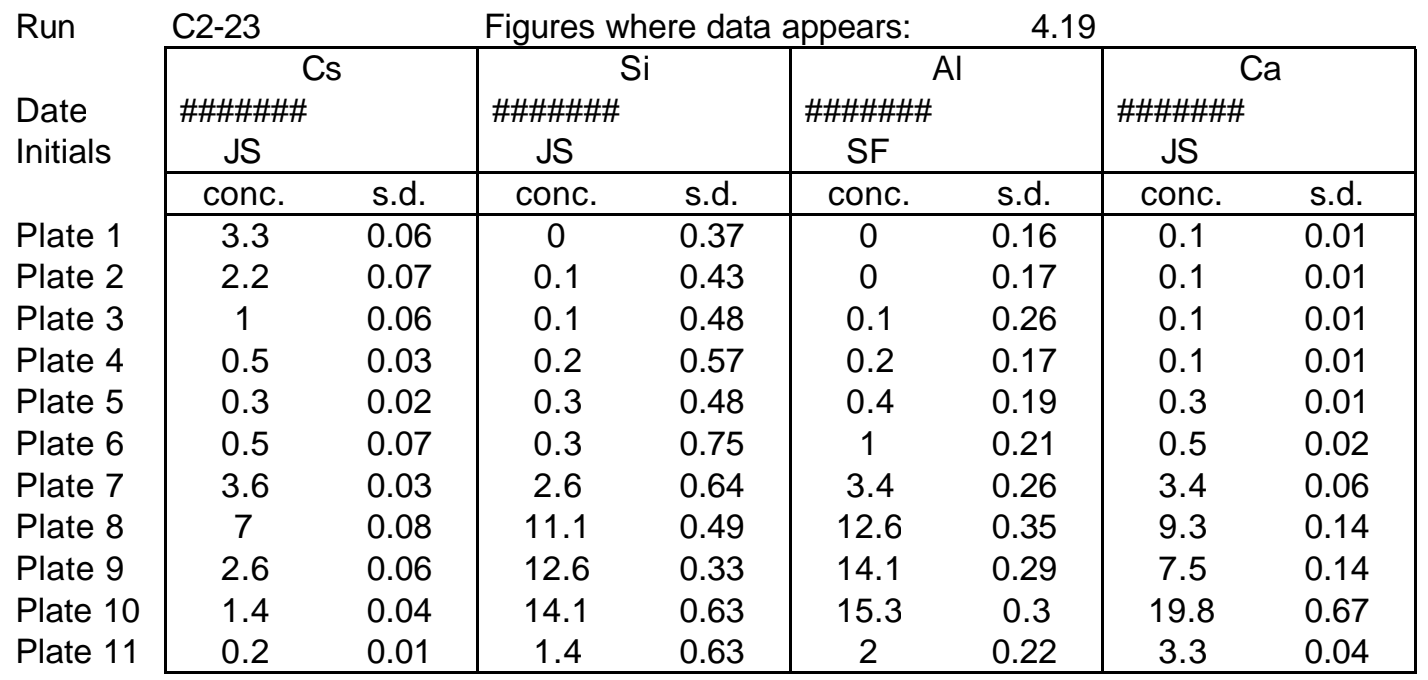

\begin{tabular}{|c|c|c|c|c|c|c|c|c|}
\hline Run & C2-24 & & Figures & re dat & appears: & 4. & & \\
\hline $\begin{array}{l}\text { Date } \\
\text { Initials }\end{array}$ & 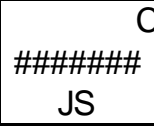 & & $\begin{array}{c}\text { \#\#\#\#\# } \\
\text { JS }\end{array}$ & & $\begin{array}{c}\text { \#\#\#\#\#\# } \\
\text { SF }\end{array}$ & & $\begin{array}{c}\text { C\#\#\#\#\# } \\
\text { JS } \\
\end{array}$ & \\
\hline & conc. & s.d. & conc. & s.d. & conc. & s.d. & conc. & s.d. \\
\hline Plate 1 & 2.9 & 0.05 & 0 & 0.41 & 0 & 0.12 & 0.2 & 0.01 \\
\hline Plate 2 & 2.2 & 0.04 & 0 & 0.46 & 0 & 0.1 & 0.2 & 0.01 \\
\hline Plate 3 & 1 & 0.04 & 0.1 & 0.4 & 0 & 0.23 & 0.2 & 0.02 \\
\hline
\end{tabular}




\begin{tabular}{l|cc|cc|cc|cc|} 
Plate 4 & 0.5 & 0.02 & 0 & 0.35 & 0 & 0.26 & 0.3 & 0.02 \\
Plate 5 & 0.3 & 0.02 & 0.2 & 0.49 & 0 & 0.19 & 0.3 & 0.01 \\
Plate 6 & 0.6 & 0.02 & 0.7 & 0.42 & 0.4 & 0.29 & 0.7 & 0.03 \\
Plate 7 & 5.6 & 0.09 & 4 & 0.3 & 3.6 & 0.18 & 5.6 & 0.07 \\
Plate 8 & 11.7 & 0.11 & 11.8 & 0.62 & 12.1 & 0.36 & 15.7 & 0.25 \\
Plate 9 & 5.5 & 0.12 & 13.1 & 0.43 & 13.1 & 0.25 & 13.3 & 0.14 \\
Plate 10 & 4.8 & 0.08 & 18.3 & 0.51 & 17.6 & 0.26 & 23.1 & 0.26 \\
Plate 11 & 0.3 & 0.03 & 1.4 & 0.45 & 1.3 & 0.21 & 2.3 & 0.04 \\
\hline
\end{tabular}




\begin{tabular}{|c|c|c|c|c|c|c|c|c|}
\hline \multirow{3}{*}{$\begin{array}{l}\text { Run } \\
\text { Date } \\
\text { Initials }\end{array}$} & \multicolumn{2}{|l|}{$\mathrm{C} 2-25$} & \multicolumn{3}{|c|}{ Figures where data appears: } & \multicolumn{2}{|c|}{4.5} & \\
\hline & $\begin{array}{c}\text { } \\
\text { \#\#\#\#\#\# } \\
\text { JMA }\end{array}$ & & $\begin{array}{c}\text { \#\#\#\#\# } \\
\text { BN }\end{array}$ & & $\begin{array}{c}\text { \#\#\#\#\#\# } \\
\text { JMA }\end{array}$ & & $\begin{array}{c}\text { \#\#\#\#\# } \\
\text { DS }\end{array}$ & \\
\hline & conc. & s.d. & conc. & s.d. & conc. & s.d. & conc. & s.d. \\
\hline Plate 1 & 0.5 & 0.03 & 0.5 & 0.87 & 0 & 0.02 & 0 & 0.02 \\
\hline Plate 2 & 0.1 & 0.03 & 0.5 & 0.85 & 0 & 0.04 & 0 & 0.01 \\
\hline Plate 3 & 0.1 & 0.02 & 2.4 & 0.62 & 0 & 0.06 & 0 & 0.01 \\
\hline Plate 4 & 0.3 & 0.04 & 2.7 & 0.78 & 0 & 0.09 & 0 & 0.01 \\
\hline Plate 5 & 0.5 & 0.03 & 3.2 & 0.67 & 0 & 0.07 & 0 & 0.01 \\
\hline Plate 6 & 1.5 & 0.07 & 1.9 & 0.75 & 0.3 & 0.06 & 0 & 0.01 \\
\hline Plate 7 & 5.8 & 0.07 & 5.1 & 1.01 & 2.5 & 0.03 & 0.5 & 0.01 \\
\hline Plate 8 & 2.9 & 0.12 & 4 & 0.73 & 1.6 & 0.05 & 1.7 & 0.02 \\
\hline Plate 9 & 0.3 & 0.02 & 0.8 & 0.79 & 0 & 0.06 & 0.5 & 0.01 \\
\hline Plate 10 & 0.2 & 0.02 & 2 & 0.83 & 0.1 & 0.06 & 0.7 & 0.01 \\
\hline Plate 11 & 0.3 & 0.03 & 3.5 & 0.72 & 0 & 0.06 & 0.7 & 0.01 \\
\hline
\end{tabular}

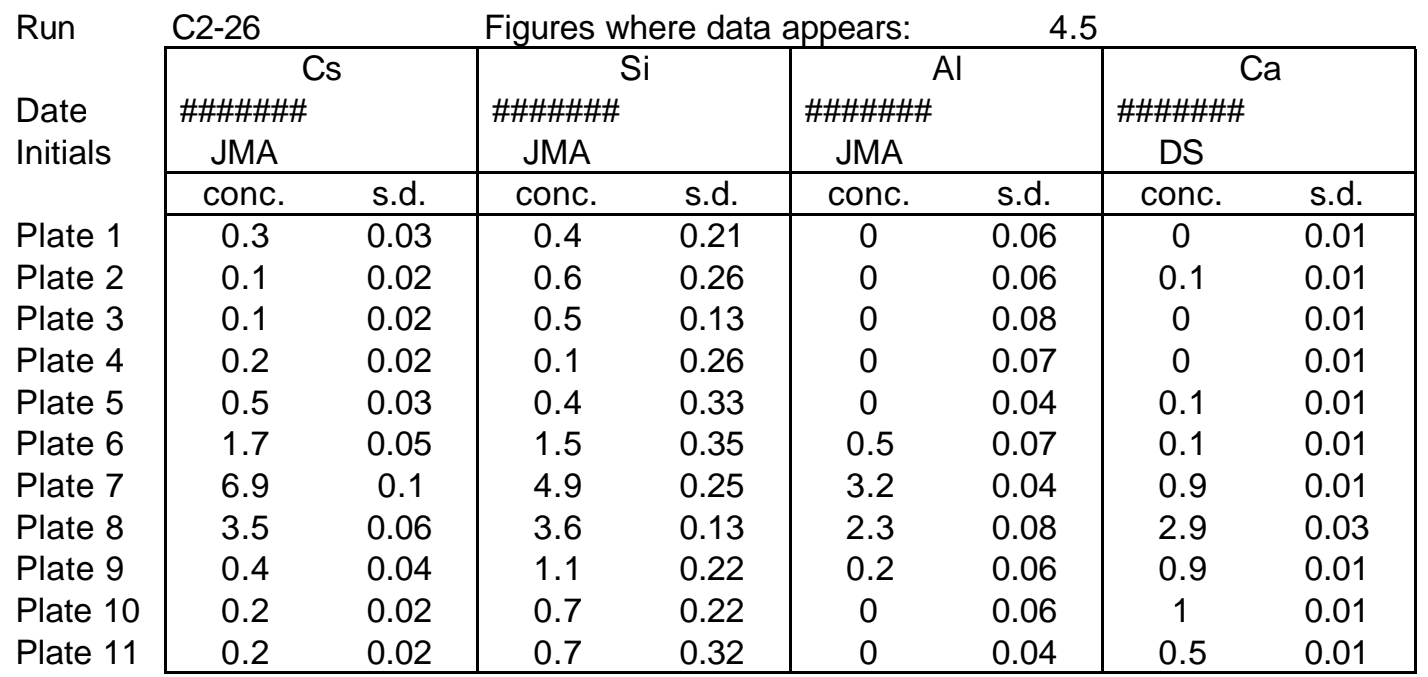

\begin{tabular}{|c|c|c|c|c|c|c|c|c|}
\hline \multirow{3}{*}{$\begin{array}{l}\text { Run } \\
\text { Date } \\
\text { Initials }\end{array}$} & \multicolumn{2}{|l|}{ C2-27 } & \multicolumn{3}{|c|}{ Figures where data appears: } & \multicolumn{2}{|c|}{4.9} & \\
\hline & $\begin{array}{c}\text { \#\#\#\#\# } \\
\text { JMA }\end{array}$ & & $\begin{array}{r}\text { \#\#\#\#\# } \\
\text { JMA }\end{array}$ & & $\begin{array}{c}\text { \#\#\#\#\# } \\
\text { JMA }\end{array}$ & & $\begin{array}{c}\text { \#\#\#\#\# } \\
\text { DS }\end{array}$ & \\
\hline & conc. & s.d. & conc. & s.d. & conc. & s.d. & conc. & s.d. \\
\hline Plate 1 & 6.5 & 0.14 & 0 & 0.32 & 0 & 0.04 & 0 & 0.01 \\
\hline Plate 2 & 4.1 & 0.08 & 0.1 & 0.26 & 0 & 0.07 & 0 & 0.01 \\
\hline Plate 3 & 2 & 0.07 & 0.2 & 0.18 & 0 & 0.09 & 0 & 0.01 \\
\hline Plate 4 & 0.7 & 0.03 & 0 & 0.29 & 0 & 0.05 & 0 & 0.01 \\
\hline Plate 5 & 0.5 & 0.06 & 0.2 & 0.15 & 0 & 0.07 & 0.1 & 0.01 \\
\hline Plate 6 & 1 & 0.04 & 0.7 & 0.3 & 0.1 & 0.07 & 0.1 & 0.01 \\
\hline Plate 7 & 3 & 0.09 & 2.5 & 0.21 & 1.4 & 0.06 & 0.4 & 0.01 \\
\hline Plate 8 & 1.6 & 0.05 & 1.8 & 0.28 & 0.7 & 0.04 & 1 & 0.02 \\
\hline Plate 9 & 0.3 & 0.08 & 0.5 & 0.21 & 0 & 0.05 & 0.4 & 0.01 \\
\hline
\end{tabular}




\begin{tabular}{|c|c|c|c|c|c|c|c|c|}
\hline Plate 10 & 0.2 & 0.02 & 0.7 & 0.27 & 0 & 0.04 & 0.8 & 0.01 \\
\hline Plate 11 & 0.1 & 0.02 & 0.4 & 0.19 & 0 & 0.05 & 0.2 & 0.01 \\
\hline Run & C2-28 & & \multicolumn{6}{|c|}{ Figures where data appears: $\quad 4.9$} \\
\hline $\begin{array}{l}\text { Date } \\
\text { Initials }\end{array}$ & $\begin{array}{c}\text { C } \\
\text { \#\#\#\#\#\# } \\
\text { JMA }\end{array}$ & & $\begin{array}{c}\text { \#\#\#\#\#\# } \\
\text { JMA }\end{array}$ & & $\begin{array}{c}\text { \#\#\#\#\#\# } \\
\text { JMA }\end{array}$ & & $\begin{array}{c}\text { \#\#\#\#\# } \\
\text { DS } \\
\end{array}$ & \\
\hline & conc. & s.d. & conc. & s.d. & conc. & s.d. & conc. & s.d. \\
\hline Plate 1 & 5.7 & 0.11 & 0.4 & 0.24 & 0 & 0.06 & 0 & 0.01 \\
\hline Plate 2 & 3.4 & 0.1 & 0.1 & 0.36 & 0 & 0.06 & 0 & 0.01 \\
\hline Plate 3 & 1.8 & 0.05 & 0.1 & 0.26 & 0 & 0.06 & 0 & 0.01 \\
\hline Plate 4 & 0.9 & 0.03 & 0.2 & 0.29 & 0 & 0.05 & 0 & 0.01 \\
\hline Plate 5 & 0.4 & 0.02 & 0.4 & 0.27 & 0 & 0.04 & 0 & 0.01 \\
\hline Plate 6 & 0.9 & 0.05 & 0.6 & 0.3 & 0.2 & 0.06 & 0.1 & 0.01 \\
\hline Plate 7 & 2.2 & 0.07 & 1.7 & 0.29 & 1.1 & 0.07 & 0.2 & 0.01 \\
\hline Plate 8 & 0.7 & 0.04 & 0.6 & 0.14 & 0.4 & 0.07 & 0.4 & 0.01 \\
\hline Plate 9 & 0.1 & 0.03 & 0.1 & 0.35 & 0 & 0.06 & 0.2 & 0.01 \\
\hline Plate 10 & 0.1 & 0.02 & 0 & 0.33 & 0 & 0.06 & 0.4 & 0.01 \\
\hline Plate 11 & 0.1 & 0.02 & 0 & 0.31 & 0 & 0.04 & 0.2 & 0.01 \\
\hline
\end{tabular}

\begin{tabular}{|c|c|c|c|c|c|c|c|c|}
\hline \multirow{3}{*}{$\begin{array}{l}\text { Run } \\
\text { Date } \\
\text { Initials }\end{array}$} & \multicolumn{2}{|l|}{ C2-29 } & \multicolumn{3}{|c|}{ Figures where data appears: } & \multicolumn{2}{|c|}{4.18} & \\
\hline & $\begin{array}{c}\text { \#\#\#\#\#\# } \\
\text { JMA }\end{array}$ & & \multicolumn{2}{|c|}{\begin{tabular}{l}
\multicolumn{1}{c}{ Si } \\
$\# \# \# \# \# \#$ \\
JMA
\end{tabular}} & \multicolumn{2}{|c|}{$\begin{array}{l}\text { Al } \\
\text { \#\#\#\#\#\#\# } \\
\text { JMA }\end{array}$} & \multicolumn{2}{|c|}{$\begin{array}{l}\text { Ca } \\
\text { \#\#\#\#\#\#\# } \\
\text { DS }\end{array}$} \\
\hline & conc. & s.d. & conc. & s.d. & conc & s.d. & conc. & s.d. \\
\hline Plate 1 & 0.8 & 0.05 & 0 & 0.25 & 0 & 0.06 & 0 & 0.01 \\
\hline Plate 2 & 0.2 & 0.02 & 0 & 0.3 & 0 & 0.06 & 0 & 0.01 \\
\hline Plate 3 & 0.2 & 0.03 & 0 & 0.27 & 0 & 0.08 & 0 & 0.01 \\
\hline Plate 4 & 0.1 & 0.02 & 0 & 0.19 & 0 & 0.04 & 0 & 0.01 \\
\hline Plate 5 & 0.3 & 0.02 & 0 & 0.32 & 0 & 0.06 & 0 & 0 \\
\hline Plate 6 & 0.3 & 0.03 & 0.1 & 0.29 & 0.2 & 0.06 & 0 & 0.01 \\
\hline Plate 7 & 1.3 & 0.07 & 1.1 & 0.32 & 1.1 & 0.05 & 0.1 & 0.02 \\
\hline Plate 8 & 0.6 & 0.03 & 0.4 & 0.31 & 0.4 & 0.03 & 0.3 & 0.01 \\
\hline Plate 9 & 0 & 0.02 & 0 & 0.29 & 0 & 0.05 & 0.1 & 0.01 \\
\hline Plate 10 & 0.1 & 0.02 & 0.1 & 0.2 & 0 & 0.06 & 0.3 & 0.01 \\
\hline Plate 11 & 0 & 0.01 & 0.1 & 0.29 & 0 & 0.08 & 0.2 & 0.01 \\
\hline
\end{tabular}

\begin{tabular}{|c|c|c|c|c|c|c|c|c|}
\hline \multirow{3}{*}{$\begin{array}{l}\text { Run } \\
\text { Date } \\
\text { Initials }\end{array}$} & \multicolumn{2}{|l|}{$\mathrm{C} 2-30$} & \multicolumn{6}{|c|}{ Figures where data appears: } \\
\hline & $\begin{array}{l} \\
\text { \#\#\#\#\#\# } \\
\text { JMA }\end{array}$ & & $\begin{array}{c}\text { \#\#\#\#\#\# } \\
\text { JMA }\end{array}$ & & $\begin{array}{c}\text { \#\#\#\#\# } \\
\text { JMA }\end{array}$ & & $\begin{array}{c}\# \# \# \# \#+ \\
\text { DS }\end{array}$ & \\
\hline & conc. & s.d. & conc. & s.d. & conc. & s.d. & conc. & s.d. \\
\hline Plate 1 & 1.2 & 0.05 & 0 & 0.23 & 0 & 0.07 & 0 & 0.01 \\
\hline Plate 2 & 0.5 & 0.02 & 0 & 0.27 & 0 & 0.08 & 0 & 0.01 \\
\hline Plate 3 & 0.2 & 0.02 & 0.1 & 0.26 & 0 & 0.04 & 0 & 0.01 \\
\hline Plate 4 & 0.2 & 0.04 & 0.2 & 0.24 & 0 & 0.06 & 0 & 0.01 \\
\hline Plate 5 & 0.2 & 0.03 & 0.1 & 0.27 & 0.1 & 0.06 & 0 & 0.01 \\
\hline Plate 6 & 0.7 & 0.03 & 0.7 & 0.38 & 0.2 & 0.05 & 0 & 0.01 \\
\hline Plate 7 & 2.2 & 0.05 & 1.7 & 0.24 & 0.9 & 0.06 & 0.1 & 0.01 \\
\hline
\end{tabular}




\begin{tabular}{|c|c|c|c|c|c|c|c|c|}
\hline Plate 8 & 0.7 & 0.03 & 0.7 & 0.15 & 0.4 & 0.04 & 0.2 & 0.01 \\
\hline Plate 9 & 0.1 & 0.01 & 0.4 & 0.31 & 0 & 0.07 & 0.2 & 0.01 \\
\hline Plate 10 & 0 & 0.03 & 0.2 & 0.27 & 0 & 0.05 & 0.4 & 0.01 \\
\hline Plate 11 & 0 & 0.03 & 0.4 & 0.2 & 0 & 0.06 & 0.2 & 0.01 \\
\hline Run & C3-1 & & \multicolumn{6}{|c|}{ Figures where data appears: } \\
\hline $\begin{array}{l}\text { Date } \\
\text { Initials }\end{array}$ & \begin{tabular}{|c} 
Cs \\
$\begin{array}{l}\text { \#\#\#\#\#\# } \\
\text { JS }\end{array}$ \\
\end{tabular} & & & & & & & \\
\hline & conc. & s.d. & conc. & s.d. & conc. & s.d. & conc. & s.d. \\
\hline Plate 1 & 1.2 & 0.03 & & & & & & \\
\hline Plate 2 & 9.1 & 0.07 & & & & & & \\
\hline Plate 3 & 18 & 0.14 & & & & & & \\
\hline Plate 4 & 18.7 & 0.19 & & & & & & \\
\hline Plate 5 & 3.5 & 0.08 & & & & & & \\
\hline Plate 6 & 0.8 & 0.03 & & & & & & \\
\hline Plate 7 & 0.7 & 0.04 & & & & & & \\
\hline Plate 8 & 0.7 & 0.03 & & & & & & \\
\hline Plate 9 & 0.8 & 0.03 & & & & & & \\
\hline Plate 10 & 0.6 & 0.03 & & & & & & \\
\hline Plate 11 & 0.2 & 0.02 & & & & & & \\
\hline
\end{tabular}

\begin{tabular}{|c|c|c|c|c|c|c|c|c|}
\hline \multirow{3}{*}{$\begin{array}{l}\text { Run } \\
\text { Date } \\
\text { Initials }\end{array}$} & \multicolumn{2}{|l|}{ C3-2 } & \multicolumn{4}{|c|}{ Figures where data appears: } & \multicolumn{2}{|c|}{4.11} \\
\hline & $\begin{array}{c} \\
\text { \#\#\#\#\#\#\# } \\
\text { JS } \\
\end{array}$ & & & & & & & \\
\hline & conc. & s.d. & conc. & s.d. & conc. & s.d. & conc. & s.d. \\
\hline Plate 1 & 1.6 & 0.04 & & & & & & \\
\hline Plate 2 & 11.1 & 0.19 & & & & & & \\
\hline Plate 3 & 19.5 & 0.24 & & & & & & \\
\hline Plate 4 & 12.6 & 0.14 & & & & & & \\
\hline Plate 5 & 2.2 & 0.03 & & & & & & \\
\hline Plate 6 & 0.6 & 0.02 & & & & & & \\
\hline Plate 7 & 0.5 & 0.02 & & & & & & \\
\hline Plate 8 & 0.4 & 0.03 & & & & & & \\
\hline Plate 9 & 0.3 & 0.02 & & & & & & \\
\hline Plate 10 & 0.3 & 0.02 & & & & & & \\
\hline Plate 11 & 0.1 & 0.01 & & & & & & \\
\hline
\end{tabular}

\begin{tabular}{|c|c|c|c|c|c|c|c|c|}
\hline \multirow{3}{*}{$\begin{array}{l}\text { Run } \\
\text { Date } \\
\text { Initials }\end{array}$} & \multicolumn{2}{|l|}{ C3-3 } & \multicolumn{6}{|c|}{ Figures where data appears: } \\
\hline & $\begin{array}{c}\text { \#\#\#\#\#\# } \\
\text { JS }\end{array}$ & & $\begin{array}{c}\text { \#\#\#\#\#\# } \\
\text { JS }\end{array}$ & & $\begin{array}{c}\text { \#\#\#\#\# } \\
\text { SF }\end{array}$ & & & \\
\hline & conc. & s.d. & conc. & s.d. & conc. & s.d. & conc. & s.d. \\
\hline Plate 1 & 0.4 & 0.02 & 0 & 0.35 & 0 & 0.19 & & \\
\hline Plate 2 & 0.2 & 0.01 & 0 & 0.48 & 0 & 0.18 & & \\
\hline Plate 3 & 0.2 & 0.02 & 0.1 & 0.46 & 0.1 & 0.18 & & \\
\hline Plate 4 & 0.2 & 0.01 & 0.2 & 0.44 & 0.3 & 0.2 & & \\
\hline Plate 5 & 0.7 & 0.03 & 1 & 0.4 & 1 & 0.16 & & \\
\hline
\end{tabular}




\begin{tabular}{|c|c|c|c|c|c|c|c|c|}
\hline Plate 6 & 5.2 & 0.06 & 6.5 & 0.55 & 6.9 & 0.35 & & \\
\hline Plate 7 & 11.9 & 0.17 & 14 & 0.36 & 16.5 & 0.38 & & \\
\hline Plate 8 & 4.4 & 0.06 & 7 & 0.46 & 7.9 & 0.21 & & \\
\hline Plate 9 & 0.7 & 0.02 & 1 & 0.54 & 2.1 & 0.24 & & \\
\hline Plate 10 & 0.2 & 0.02 & 0.2 & 0.43 & 1.3 & 0.25 & & \\
\hline Plate 11 & 0.1 & 0.01 & 0 & 0.59 & 0.8 & 0.24 & & \\
\hline Run & C3-4 & & \multicolumn{6}{|c|}{ Figures where data appears: } \\
\hline $\begin{array}{l}\text { Date } \\
\text { Initials }\end{array}$ & $\begin{array}{c}\text { \#\#\#\#\#\# } \\
\text { JS } \\
\end{array}$ & & $\begin{array}{c} \\
\# \# \# \# \# \# \\
\text { JS } \\
\end{array}$ & & $\begin{array}{c} \\
\text { \#\#\#\#\#\# } \\
\text { SF } \\
\end{array}$ & & & \\
\hline & conc. & s.d. & conc. & s.d. & conc. & s.d. & conc. & s.d. \\
\hline Plate 1 & 0.3 & 0.02 & 0.1 & 0.52 & 0.2 & 0.24 & & \\
\hline Plate 2 & 0.1 & 0.02 & 0 & 0.52 & 0.1 & 0.22 & & \\
\hline Plate 3 & 0.1 & 0.01 & 0.1 & 0.51 & 0 & 0.21 & & \\
\hline Plate 4 & 0.2 & 0.02 & 0 & 0.5 & 0.2 & 0.22 & & \\
\hline Plate 5 & 0.9 & 0.03 & 0.5 & 0.58 & 1.1 & 0.23 & & \\
\hline Plate 6 & 4.4 & 0.05 & 6.8 & 0.51 & 6.5 & 0.19 & & \\
\hline Plate 7 & 8.8 & 0.07 & 10.9 & 0.37 & 10.8 & 0.16 & & \\
\hline Plate 8 & 4.2 & 0.08 & 7.4 & 0.37 & 7.1 & 0.25 & & \\
\hline Plate 9 & 0.8 & 0.04 & 2 & 0.41 & 1.8 & 0.27 & & \\
\hline Plate 10 & 0.2 & 0.02 & 1.5 & 0.5 & 1.1 & 0.19 & & \\
\hline Plate 11 & 0.1 & 0.01 & 0.3 & 0.42 & 0.2 & 0.16 & & \\
\hline
\end{tabular}

Run $\quad$ C3-5 $\quad$ Figures where data appears:

\begin{tabular}{|c|c|c|c|c|c|c|c|c|}
\hline \multirow[t]{2}{*}{$\begin{array}{l}\text { Date } \\
\text { Initials }\end{array}$} & \multicolumn{2}{|c|}{$\begin{array}{l}\text { Cs } \\
\text { \#\#\#\#\#\#\# } \\
\text { JS }\end{array}$} & \multicolumn{2}{|c|}{$\begin{array}{l}\text { Si } \\
\text { \#\#\#\#\#\#\# } \\
\text { JS }\end{array}$} & \multicolumn{2}{|c|}{$\begin{array}{l}\text { Al } \\
\text { \#\#\#\#\#\#\# } \\
\text { SF }\end{array}$} & \multicolumn{2}{|c|}{$\mathrm{Ca}$} \\
\hline & conc. & s.d. & conc. & s.d. & conc. & s.d. & conc. & s.d. \\
\hline Plate 1 & 0.3 & 0.02 & 0 & 0.38 & 0 & 0.2 & & \\
\hline Plate 2 & 0.7 & 0.03 & 0 & 0.48 & 0 & 0.12 & & \\
\hline Plate 3 & 0.7 & 0.02 & 0 & 0.47 & 0.1 & 0.29 & & \\
\hline Plate 4 & 0.5 & 0.03 & 0 & 0.58 & 0.1 & 0.13 & & \\
\hline Plate 5 & 0.7 & 0.02 & 0.3 & 0.37 & 0.7 & 0.2 & & \\
\hline Plate 6 & 3 & 0.03 & 3.9 & 0.27 & 3.3 & 0.18 & & \\
\hline Plate 7 & 9.5 & 0.14 & 12 & 0.68 & 12 & 0.26 & & \\
\hline Plate 8 & 8.9 & 0.1 & 16 & 0.51 & 17.2 & 0.39 & & \\
\hline Plate 9 & 6.1 & 0.1 & 15 & 0.37 & 15.8 & 0.35 & & \\
\hline Plate 10 & 5.3 & 0.07 & 18.7 & 0.5 & 20.3 & 0.36 & & \\
\hline Plate 11 & 0.4 & 0.02 & 2.6 & 0.53 & 2 & 0.27 & & \\
\hline
\end{tabular}

\begin{tabular}{|c|c|c|c|c|c|c|c|c|}
\hline \multirow{3}{*}{$\begin{array}{l}\text { Run } \\
\text { Date } \\
\text { Initials }\end{array}$} & \multicolumn{2}{|l|}{ C3-6 } & \multicolumn{6}{|c|}{ Figures where data appears: } \\
\hline & \begin{tabular}{|c} 
\#\#\#\#\#\# \\
JS
\end{tabular} & & $\begin{array}{c}\text { \#\#\#\#\# } \\
\text { JS }\end{array}$ & & $\begin{array}{c}\text { \#\#\#\#\#\# } \\
\text { SF }\end{array}$ & & & \\
\hline & conc. & s.d. & conc. & s.d. & conc. & s.d. & conc. & s.d. \\
\hline Plate 1 & 3.3 & 0.06 & 0.3 & 0.27 & 0 & 0.25 & & \\
\hline Plate 2 & 2.1 & 0.06 & 0.2 & 0.54 & 0 & 0.21 & & \\
\hline Plate 3 & 0.8 & 0.02 & 0.1 & 0.35 & 0 & 0.17 & & \\
\hline
\end{tabular}




\begin{tabular}{l|cc|cc|cc|c|} 
Plate 4 & 0.6 & 0.02 & 0 & 0.41 & 0 & 0.1 & \\
Plate 5 & 0.8 & 0.02 & 1.1 & 0.37 & 0.3 & 0.19 & \\
Plate 6 & 2.8 & 0.04 & 3.1 & 0.38 & 2.2 & 0.22 & \\
Plate 7 & 7.3 & 0.11 & 7.8 & 0.57 & 6.3 & 0.25 & \\
Plate 8 & 7.3 & 0.06 & 11 & 0.68 & 10.1 & 0.29 & \\
Plate 9 & 3.8 & 0.06 & 9.9 & 0.39 & 9.2 & 0.24 & \\
Plate 10 & 3.3 & 0.05 & 15 & 0.52 & 15.4 & 0.29 & \\
Plate 11 & 0.3 & 0.02 & 2.2 & 0.54 & 1.1 & 0.26 & \\
\cline { 2 - 8 }
\end{tabular}




\begin{tabular}{|c|c|c|c|c|c|c|c|c|}
\hline \multirow{3}{*}{$\begin{array}{l}\text { Date } \\
\text { Initials }\end{array}$} & \multicolumn{2}{|l|}{ S2-1 } & \multicolumn{3}{|c|}{ Figures where data appears: } & \multicolumn{3}{|c|}{$.20,4.23,4.25,4.26$} \\
\hline & $\begin{array}{r}\mathrm{s} \\
\# \# \# \# \# \# \\
\text { JMA }\end{array}$ & & & & & & & \\
\hline & conc. & s.d. & conc & s.d. & conc. & s.d. & conc. & s.d. \\
\hline Plate 1 & 0 & 0 & & & & & & \\
\hline Plate 2 & 0 & 0.01 & & & & & & \\
\hline Plate 3 & 0.2 & 0.02 & & & & & & \\
\hline Plate 4 & 0.9 & 0.04 & & & & & & \\
\hline Plate 5 & 2.2 & 0.05 & & & & & & \\
\hline Plate 6 & 3.8 & 0.07 & & & & & & \\
\hline Plate 7 & 4.5 & 0.1 & & & & & & \\
\hline Plate 8 & 1.6 & 0.06 & & & & & & \\
\hline Plate 9 & 0 & 0.02 & & & & & & \\
\hline Plate 10 & 0 & 0.01 & & & & & & \\
\hline Plate 11 & 0 & 0.04 & & & & & & \\
\hline
\end{tabular}

Run S2-2 Figures where data appears: $\quad 4.20,4.23,4.25,4.26$

Date Initials

Plate 1

Plate 2

Plate 3

Plate 4

Plate 5

Plate 6

Plate 7

Plate 8

Plate 9

Plate 10

Plate 11

\begin{tabular}{|c|c|c|c|c|c|c|c|}
\hline 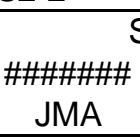 & & \multicolumn{2}{|c|}{$\mathrm{Si}$} & \multicolumn{2}{|c|}{$\mathrm{Al}$} & \multicolumn{2}{|c|}{$\mathrm{Ca}$} \\
\hline conc. & s.d. & conc. & s.d. & conc. & s.d. & conc. & s.d. \\
\hline 0 & 0.01 & & & & & & \\
\hline 0.1 & 0.01 & & & & & & \\
\hline 0.2 & 0.01 & & & & & & \\
\hline 0.9 & 0.02 & & & & & & \\
\hline 1.9 & 0.11 & & & & & & \\
\hline 2.8 & 0.06 & & & & & & \\
\hline 2.6 & 0.07 & & & & & & \\
\hline 1.1 & 0.02 & & & & & & \\
\hline 0 & 0.01 & & & & & & \\
\hline 0 & 0.01 & & & & & & \\
\hline 0 & 0.01 & & & & & & \\
\hline
\end{tabular}

Run

S2-3

Figures where data appears: $4.27,4.30,4.31,4.32$

Date

Initials

Plate 1

Plate 2

Plate 3

Plate 4

Plate 5

Plate 6

Plate 7

Plate 8

Plate 9

Plate 10

\begin{tabular}{|c|c|c|c|c|c|c|c|}
\hline $\begin{array}{l}\text { S } \\
\text { \#\#\#\#\#\#\# } \\
\text { JMA }\end{array}$ & & \multicolumn{2}{|c|}{$\mathrm{Si}$} & \multicolumn{2}{|c|}{$\mathrm{Al}$} & \multicolumn{2}{|c|}{$\mathrm{Ca}$} \\
\hline conc. & s.d. & conc. & s.d. & conc. & s.d. & conc. & s.d. \\
\hline 0.1 & 0.01 & & & & & & \\
\hline 0.2 & 0.02 & & & & & & \\
\hline 2.4 & 0.06 & & & & & & \\
\hline 9.7 & 0.2 & & & & & & \\
\hline 3.7 & 0.09 & & & & & & \\
\hline 0.9 & 0.04 & & & & & & \\
\hline 0.2 & 0.01 & & & & & & \\
\hline 0 & 0.02 & & & & & & \\
\hline 0 & 0.02 & & & & & & \\
\hline 0 & 0.01 & & & & & & \\
\hline
\end{tabular}




\begin{tabular}{|c|c|c|c|c|c|c|c|c|}
\hline Plate 11 & 0 & 0.01 & & & & & & \\
\hline Run & S2-4 & & Figures & re da & opears: & 27,4 & $4.31,4$ & \\
\hline $\begin{array}{l}\text { Date } \\
\text { Initials }\end{array}$ & 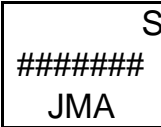 & & & & & & & \\
\hline & conc. & s.d. & conc. & s.d. & conc. & s.d. & conc. & s.d. \\
\hline Plate 1 & 0.1 & 0.01 & & & & & & \\
\hline Plate 2 & 0.4 & 0.03 & & & & & & \\
\hline Plate 3 & 2.5 & 0.05 & & & & & & \\
\hline Plate 4 & 6.7 & 0.14 & & & & & & \\
\hline Plate 5 & 2.1 & 0.07 & & & & & & \\
\hline Plate 6 & 0.4 & 0.03 & & & & & & \\
\hline Plate 7 & 0.1 & 0.03 & & & & & & \\
\hline Plate 8 & 0 & 0.02 & & & & & & \\
\hline Plate 9 & 0 & 0.01 & & & & & & \\
\hline Plate 10 & 0 & 0.01 & & & & & & \\
\hline Plate 11 & 0 & 0.02 & & & & & & \\
\hline
\end{tabular}

Run S2-5

Figures where data appears: $\quad 4.33,4.35,4.37,4.38,4.39$

Date

Initials

Plate 1

Plate 2

Plate 3

Plate 4

Plate 5

Plate 6

Plate 7

Plate 8

Plate 9

Plate 10

Plate 11

\begin{tabular}{|c|c|c|c|c|c|c|c|}
\hline $\begin{array}{c} \\
\text { \#\#\#\#\#\# } \\
\text { JMA }\end{array}$ & & & & & & & \\
\hline conc. & s.d. & conc. & s.d. & conc. & s.d. & conc. & s.d. \\
\hline 0 & 0.01 & & & & & & \\
\hline 0 & 0.04 & & & & & & \\
\hline 0.7 & 0.02 & & & & & & \\
\hline 1.7 & 0.06 & & & & & & \\
\hline 1.1 & 0.02 & & & & & & \\
\hline 1.5 & 0.03 & & & & & & \\
\hline 1.2 & 0.04 & & & & & & \\
\hline 0.2 & 0.02 & & & & & & \\
\hline 0 & 0.01 & & & & & & \\
\hline 0 & 0.01 & & & & & & \\
\hline 0 & 0.02 & & & & & & \\
\hline
\end{tabular}

Run

Date

Initials

Plate 1

Plate 2

Plate 3

Plate 4

Plate 5

Plate 6

Plate 7

Plate 8

S2-6

Figures where data appears: $4.33,4.35,4.37,4.38,4.39$

Plate 9

\begin{tabular}{|c|c|c|c|c|c|c|c|}
\hline \multicolumn{2}{|c|}{$\begin{array}{l}\text { Sr } \\
\text { \#\#\#\#\#\#\# } \\
\text { JMA }\end{array}$} & \multicolumn{2}{|c|}{$\mathrm{Si}$} & \multicolumn{2}{|c|}{$\overline{\mathrm{Al}}$} & \multicolumn{2}{|c|}{$\mathrm{Ca}$} \\
\hline conc. & s.d. & conc. & s.d. & conc. & s.d. & conc. & s.d. \\
\hline 0 & 0.01 & & & & & & \\
\hline 0 & 0.01 & & & & & & \\
\hline 0.5 & 0.02 & & & & & & \\
\hline 1.3 & 0.04 & & & & & & \\
\hline 0.8 & 0.02 & & & & & & \\
\hline 1.3 & 0.04 & & & & & & \\
\hline 0.8 & 0.04 & & & & & & \\
\hline 0.1 & 0.02 & & & & & & \\
\hline 0 & 0.01 & & & & & & \\
\hline
\end{tabular}




\begin{tabular}{|c|c|c|c|c|c|c|c|c|}
\hline $\begin{array}{l}\text { Plate } 10 \\
\text { Plate } 11\end{array}$ & $\begin{array}{l}0 \\
0\end{array}$ & $\begin{array}{l}0.01 \\
0.01\end{array}$ & & & & & & \\
\hline Run & S2-7 & & Figures & re da & appears: & 4. & & \\
\hline $\begin{array}{l}\text { Date } \\
\text { Initials }\end{array}$ & $\begin{array}{c} \\
\text { \#\#\#\#\#\#\# } \\
\text { BN }\end{array}$ & & 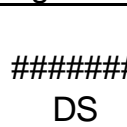 & & $\begin{array}{c}\text { \#\#\#\#\#\# } \\
\text { JS }\end{array}$ & & & \\
\hline & conc. & s.d. & conc. & s.d. & conc. & s.d. & conc. & s.d. \\
\hline Plate 1 & 0.1 & 0.05 & 0.5 & 0.4 & 0.1 & 0.08 & & \\
\hline Plate 2 & 0 & 0.07 & 2.4 & 0.39 & 0 & 0.1 & & \\
\hline Plate 3 & 0 & 0.04 & 2.4 & 0.67 & 0 & 0.08 & & \\
\hline Plate 4 & 0.3 & 0.05 & 2 & 0.5 & 0 & 0.09 & & \\
\hline Plate 5 & 0.8 & 0.04 & 2.6 & 0.51 & 0.3 & 0.06 & & \\
\hline Plate 6 & 2 & 0.08 & 0 & 0.52 & 0.9 & 0.11 & & \\
\hline Plate 7 & 7.1 & 0.12 & 5.5 & 0.51 & 5.1 & 0.1 & & \\
\hline Plate 8 & 4.6 & 0.08 & 4.1 & 0.61 & 3.3 & 0.11 & & \\
\hline Plate 9 & 0.2 & 0.04 & 1 & 0.39 & 0.5 & 0.08 & & \\
\hline Plate 10 & 0 & 0.04 & 1.9 & 0.47 & 1 & 0.09 & & \\
\hline Plate 11 & 0.1 & 0.06 & 1.2 & 0.38 & 0.5 & 0.09 & & \\
\hline
\end{tabular}

\begin{tabular}{|c|c|c|c|c|c|c|c|c|}
\hline \multirow{3}{*}{$\begin{array}{l}\text { Run } \\
\text { Date } \\
\text { Initials }\end{array}$} & \multicolumn{2}{|l|}{ S2-8 } & \multicolumn{3}{|c|}{ Fiqures where data appears: } & \multicolumn{2}{|c|}{4.20} & \\
\hline & $\begin{array}{c}\text { \#\#\#\#\#+ } \\
\text { BN }\end{array}$ & & $\begin{array}{c}\text { \#\#\#\#\#\# } \\
\text { DS }\end{array}$ & & $\begin{array}{c}\text { \#\#\#\#\#\# } \\
\text { JS }\end{array}$ & & & \\
\hline & conc. & s.d. & conc. & s.d. & conc. & s.d. & conc. & s.d. \\
\hline Plate 1 & 0 & 0.05 & 0.7 & 0.45 & 0 & 0.07 & & \\
\hline Plate 2 & 0 & 0.05 & 1.5 & 0.35 & 0.1 & 0.06 & & \\
\hline Plate 3 & 0 & 0.04 & 2.3 & 0.48 & 0.1 & 0.09 & & \\
\hline Plate 4 & 0.1 & 0.04 & 2.2 & 0.71 & 0.1 & 0.07 & & \\
\hline Plate 5 & 0.1 & 0.05 & 3 & 0.46 & 0.5 & 0.12 & & \\
\hline Plate 6 & 1 & 0.04 & 1.6 & 0.63 & 1.2 & 0.08 & & \\
\hline Plate 7 & 4.5 & 0.06 & 6.7 & 0.3 & 6.1 & 0.12 & & \\
\hline Plate 8 & 3.8 & 0.05 & 6.1 & 0.46 & 4.4 & 0.06 & & \\
\hline Plate 9 & 0.1 & 0.05 & 2.6 & 0.36 & 0.1 & 0.11 & & \\
\hline Plate 10 & 0 & 0.06 & 2.5 & 0.49 & 0 & 0.1 & & \\
\hline Plate 11 & 0 & 0.05 & 2.6 & 0.39 & 0 & 0.06 & & \\
\hline
\end{tabular}

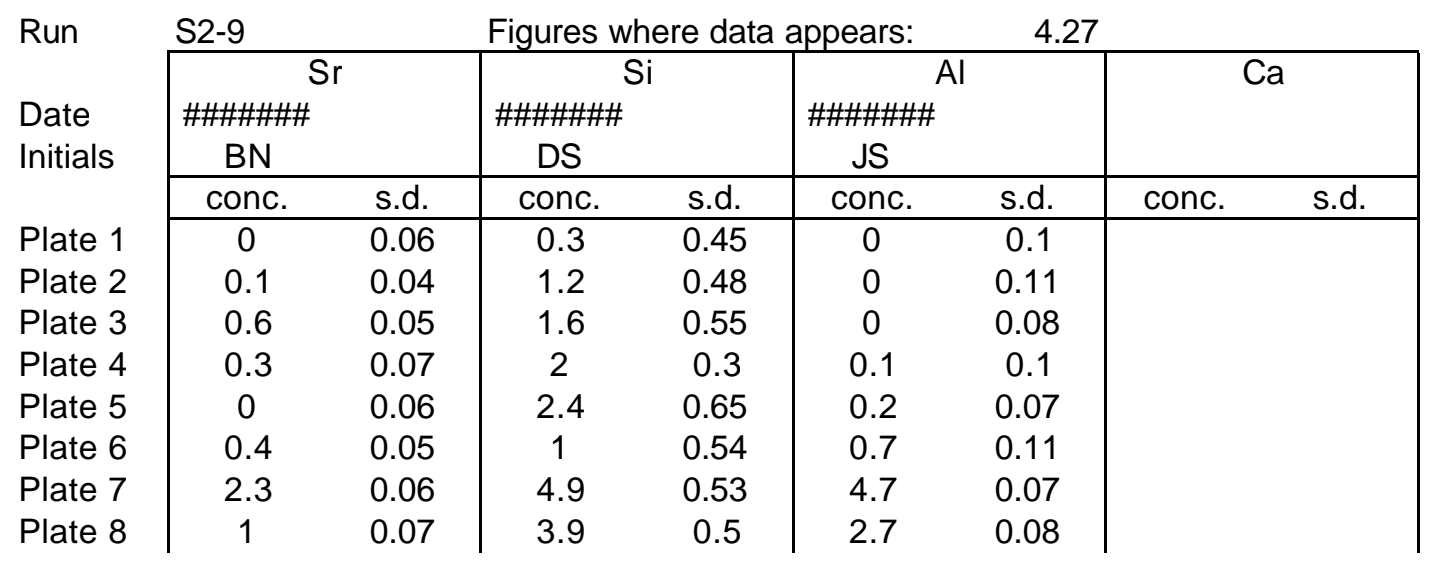




\begin{tabular}{|c|c|c|c|c|c|c|c|c|}
\hline Plate 9 & 0 & 0.03 & 1.3 & 0.3 & 0.2 & 0.07 & & \\
\hline Plate 10 & 0 & 0.06 & 1.9 & 0.44 & 0.1 & 0.11 & & \\
\hline Plate 11 & 0.1 & 0.08 & 0.8 & 0.5 & 0.1 & 0.1 & & \\
\hline Run & S2-10 & & Figures & re dat & ppears: & 4.2 & & \\
\hline $\begin{array}{l}\text { Date } \\
\text { Initials }\end{array}$ & $\begin{array}{c}\mathrm{s} \\
\text { \#\#\#\#\#\# } \\
\text { BN }\end{array}$ & & $\begin{array}{c}\text { \#\#\#\#\# } \\
\text { DS }\end{array}$ & & $\begin{array}{c}\text { \#\#\#\#\#\#\# } \\
\text { JS }\end{array}$ & & & \\
\hline & conc. & s.d. & conc. & s.d. & conc. & s.d. & conc. & s.d. \\
\hline Plate 1 & 0.3 & 0.06 & 8.3 & 0.43 & 0 & 0.1 & & \\
\hline Plate 2 & 0.4 & 0.06 & 0.5 & 0.61 & 0 & 0.1 & & \\
\hline Plate 3 & 0.6 & 0.05 & 1.3 & 0.35 & 0 & 0.07 & & \\
\hline Plate 4 & 0.5 & 0.03 & 1.6 & 0.34 & 0.2 & 0.07 & & \\
\hline Plate 5 & 0.4 & 0.06 & 1.6 & 0.45 & 0.3 & 0.09 & & \\
\hline Plate 6 & 0.4 & 0.1 & 1 & 0.39 & 0.8 & 0.09 & & \\
\hline Plate 7 & 2.8 & 0.04 & 5.8 & 0.51 & 5.3 & 0.13 & & \\
\hline Plate 8 & 0.8 & 0.05 & 4.2 & 0.97 & 2.3 & 0.1 & & \\
\hline Plate 9 & 0 & 0.04 & 1.1 & 0.42 & 0.1 & 0.1 & & \\
\hline Plate 10 & 0 & 0.07 & 1.3 & 0.32 & 0 & 0.09 & & \\
\hline Plate 11 & 0 & 0.05 & 1.6 & 0.58 & 0 & 0.08 & & \\
\hline
\end{tabular}

\begin{tabular}{|c|c|c|c|c|c|c|c|c|}
\hline \multirow{3}{*}{$\begin{array}{l}\text { Run } \\
\text { Date } \\
\text { Initials }\end{array}$} & S2-11 & & \multicolumn{3}{|c|}{ Figures where data appears: } & \multicolumn{2}{|c|}{4.35} & \\
\hline & \multicolumn{2}{|c|}{ 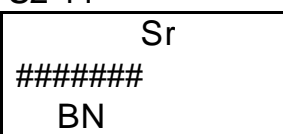 } & \multicolumn{2}{|c|}{\begin{tabular}{l}
\multicolumn{1}{c}{ Si } \\
\#\#\#\#\#\#\# \\
DS
\end{tabular}} & \multicolumn{2}{|c|}{$\begin{array}{l}\text { Al } \\
\text { \#\#\#\#\#\#\# } \\
\text { JS }\end{array}$} & \multicolumn{2}{|c|}{$\mathrm{Ca}$} \\
\hline & conc. & s.d. & conc. & s.d. & conc. & s.d. & conc. & s.d. \\
\hline Plate 1 & 0 & 0.03 & 0.2 & 0.57 & 0 & 0.08 & & \\
\hline Plate 2 & 0 & 0.07 & 0.8 & 0.37 & 0.1 & 0.08 & & \\
\hline Plate 3 & 0.9 & 0.05 & 1 & 0.29 & 0 & 0.08 & & \\
\hline Plate 4 & 3.3 & 0.09 & 1.2 & 0.3 & 0 & 0.08 & & \\
\hline Plate 5 & 0.4 & 0.06 & 1.6 & 0.5 & 0.2 & 0.05 & & \\
\hline Plate 6 & 0.2 & 0.12 & 1.6 & 0.54 & 1.3 & 0.07 & & \\
\hline Plate 7 & 0.2 & 0.06 & 5.9 & 0.44 & 5.2 & 0.08 & & \\
\hline Plate 8 & 0.2 & 0.06 & 3.3 & 0.54 & 2.4 & 0.1 & & \\
\hline Plate 9 & 0.2 & 0.05 & 0.8 & 0.39 & 0.3 & 0.05 & & \\
\hline Plate 10 & 0.2 & 0.06 & 1 & 0.32 & 0.2 & 0.1 & & \\
\hline Plate 11 & 0 & 0.1 & 1 & 0.62 & 0.3 & 0.11 & & \\
\hline
\end{tabular}

\begin{tabular}{|c|c|c|c|c|c|c|c|c|}
\hline \multirow{3}{*}{$\begin{array}{l}\text { Run } \\
\text { Date } \\
\text { Initials }\end{array}$} & \multirow{2}{*}{\multicolumn{2}{|c|}{$\begin{array}{l}\text { S2-12 } \\
\begin{array}{c}\text { Sr } \\
\# \# \# \# \# \# \\
\text { BN }\end{array}\end{array}$}} & \multicolumn{6}{|c|}{ Figures where data appears: } \\
\hline & & & $\begin{array}{c}\text { \#\#\#\#\# } \\
\text { DS }\end{array}$ & & $\begin{array}{c}\text { \#\#\#\#\# } \\
\text { JS }\end{array}$ & & & \\
\hline & conc. & s.d. & conc. & s.d. & conc. & s.d. & conc. & s.d. \\
\hline Plate 1 & 0 & 0.1 & 0 & 0.52 & 0 & 0.06 & & \\
\hline Plate 2 & 0 & 0.06 & 0.5 & 0.61 & 0.1 & 0.08 & & \\
\hline Plate 3 & 0 & 0.05 & 0.8 & 0.47 & 0.1 & 0.1 & & \\
\hline Plate 4 & 0.2 & 0.06 & 1.3 & 0.34 & 0.1 & 0.07 & & \\
\hline Plate 5 & 0.6 & 0.05 & 1.7 & 0.44 & 0.3 & 0.1 & & \\
\hline Plate 6 & 0.9 & 0.08 & 0.8 & 0.58 & 1.2 & 0.12 & & \\
\hline Plate 7 & 2.2 & 0.04 & 4.7 & 0.59 & 4.5 & 0.14 & & \\
\hline
\end{tabular}




\begin{tabular}{l|cc|cc|cc|c|} 
Plate 8 & 0.9 & 0.09 & 2.9 & 0.46 & 1.7 & 0.09 & \\
Plate 9 & 0 & 0.08 & 1.8 & 0.4 & 0.1 & 0.11 & \\
Plate 10 & 0 & 0.07 & 1.9 & 0.41 & 0.1 & 0.09 & \\
Plate 11 & 0 & 0.07 & 1.2 & 0.49 & 0.1 & 0.1 & \\
\cline { 2 - 7 } & & & & &
\end{tabular}




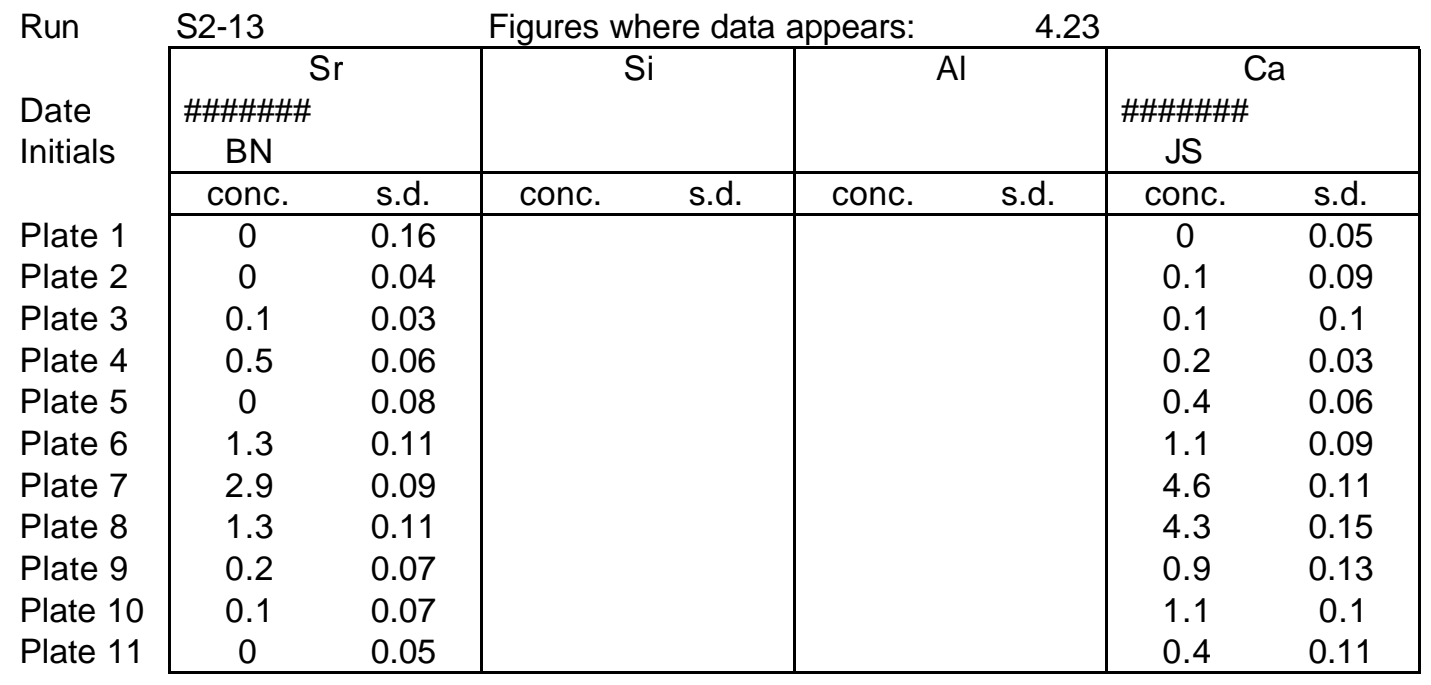

Run

S2-14

Figures where data appears:

Date

Initials

Plate 1

Plate 2

Plate 3

Plate 4

Plate 5

Plate 6

Plate 7

Plate 8

Plate 9

Plate 10

Plate 11

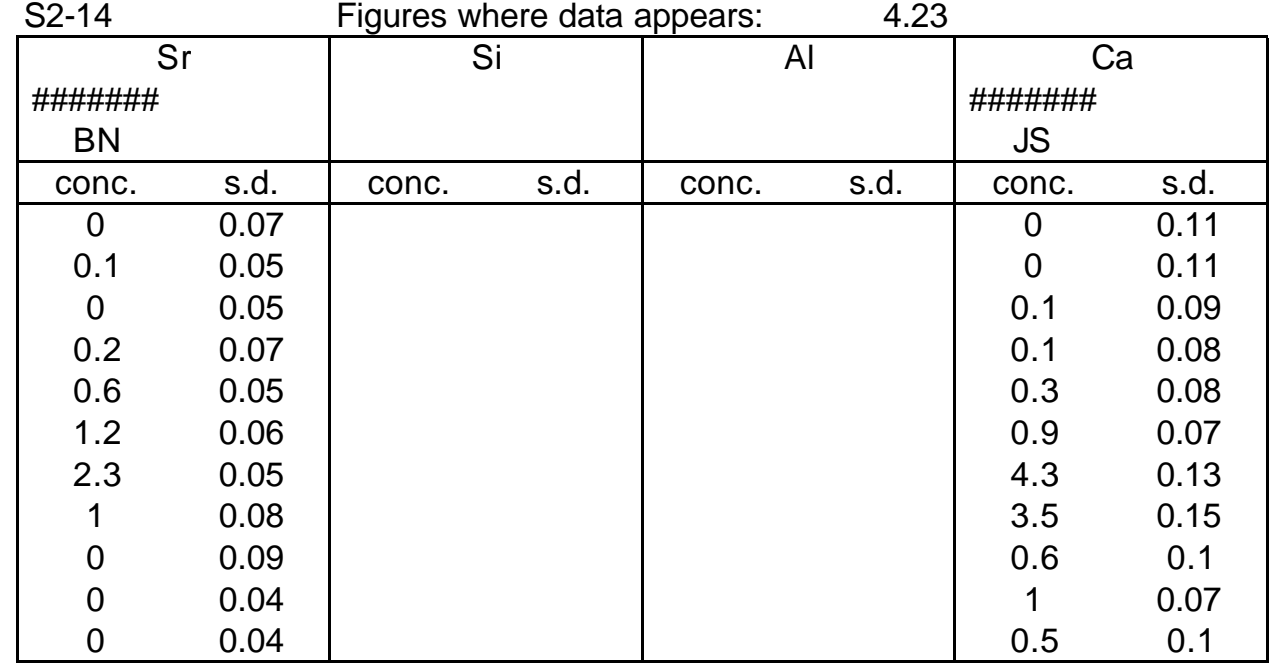

Run

S2-15

Figures where data appears:

Date
Initials

Plate 1

Plate 2

Plate 3

Plate 4

Plate 5

Plate 6

Plate 7

Plate 8

Plate 9

\begin{tabular}{|c|c|c|c|c|c|c|c|}
\hline \multicolumn{2}{|c|}{$\begin{array}{c}\mathrm{Sr} \\
\text { \#\#\#\#\#\#\# } \\
\text { BN }\end{array}$} & \multicolumn{2}{|c|}{$\mathrm{Si}$} & \multicolumn{2}{|c|}{$\mathrm{Al}$} & \multicolumn{2}{|c|}{$\begin{array}{c}\text { Ca } \\
\text { \#\#\#\#\#\# } \\
\text { JS } \\
\end{array}$} \\
\hline conc. & s.d. & conc. & s.d & conc. & s.d. & conc. & s.d. \\
\hline 0 & 0.06 & & & & & 0.1 & 0.07 \\
\hline 0.1 & 0.07 & & & & & 0.1 & 0.1 \\
\hline 0.9 & 0.07 & & & & & 0.4 & 0.06 \\
\hline 3.3 & 0.06 & & & & & 1.1 & 0.08 \\
\hline 0.3 & 0.07 & & & & & 0.4 & 0.15 \\
\hline 0.2 & 0.05 & & & & & 0.6 & 0.17 \\
\hline 0.2 & 0.06 & & & & & 1.8 & 0.1 \\
\hline 0.2 & 0.08 & & & & & 3.3 & 0.12 \\
\hline 0 & 0.07 & & & & & 0.8 & 0.05 \\
\hline 0.2 & 0.05 & & & & & 1.5 & 0.08 \\
\hline
\end{tabular}

\begin{tabular}{l|ll} 
Plate 10 & 0.2 & 0.05
\end{tabular} 


\begin{tabular}{|c|c|c|c|c|c|c|c|c|}
\hline Plate 11 & 0 & 0.05 & & & & & 0.5 & 0.09 \\
\hline Run & S2-16 & & Figures & re da & ppears: & & & \\
\hline $\begin{array}{l}\text { Date } \\
\text { Initials }\end{array}$ & $\begin{array}{c}\mathrm{s} \\
\# \# \# \# \# \# \\
\text { BN }\end{array}$ & & & & & & $\begin{array}{c}\text { \#\#\#\#\#\# } \\
\text { JS }\end{array}$ & \\
\hline & conc. & s.d. & conc. & s.d. & conc. & s.d. & conc. & s.d. \\
\hline Plate 1 & 0 & 0.06 & & & & & 0.1 & 0.06 \\
\hline Plate 2 & 0.1 & 0.06 & & & & & 0 & 0.1 \\
\hline Plate 3 & 1.3 & 0.07 & & & & & 0.3 & 0.08 \\
\hline Plate 4 & 3.5 & 0.04 & & & & & 0.7 & 0.06 \\
\hline Plate 5 & 0.6 & 0.05 & & & & & 0.2 & 0.14 \\
\hline Plate 6 & 0.1 & 0.04 & & & & & 0.5 & 0.09 \\
\hline Plate 7 & 0.2 & 0.05 & & & & & 1.4 & 0.1 \\
\hline Plate 8 & 0.2 & 0.07 & & & & & 2.4 & 0.17 \\
\hline Plate 9 & 0.1 & 0.06 & & & & & 0.4 & 0.07 \\
\hline Plate 10 & 0 & 0.08 & & & & & 0.8 & 0.13 \\
\hline Plate 11 & 0.1 & 0.07 & & & & & 1 & 0.14 \\
\hline
\end{tabular}

Run S2-17 Figures where data appears: 4.37

Date Initials

Plate 1

Plate 2

Plate 3

Plate 4

Plate 5

Plate 6

Plate 7

Plate 8

Plate 9

Plate 10

Plate 11

\begin{tabular}{|c|c|c|c|c|c|c|c|}
\hline \multicolumn{2}{|c|}{$\begin{array}{l}\text { Sr } \\
\text { \#\#\#\#\#\#\# } \\
\text { JS }\end{array}$} & \multicolumn{2}{|c|}{$\mathrm{Si}$} & \multicolumn{2}{|c|}{$\overline{A l}$} & \multicolumn{2}{|c|}{$\begin{array}{l}\mathrm{Ca} \\
\text { \#\#\#\#\#\# } \\
\text { JS }\end{array}$} \\
\hline conc. & s.d. & conc. & s.d. & conc & s.d. & conc. & s.d. \\
\hline 0 & 0.05 & & & & & 0.1 & 0.11 \\
\hline 0.1 & 0.07 & & & & & 0.1 & 0.12 \\
\hline 0.8 & 0.09 & & & & & 0.2 & 0.12 \\
\hline 2.8 & 0.05 & & & & & 0.5 & 0.15 \\
\hline 1.5 & 0.04 & & & & & 1 & 0.1 \\
\hline 1.8 & 0.08 & & & & & 2.5 & 0.15 \\
\hline 4.1 & 0.04 & & & & & 15.8 & 0.37 \\
\hline 5.1 & 0.07 & & & & & 24.3 & 0.24 \\
\hline 3.2 & 0.06 & & & & & 25.6 & 0.31 \\
\hline 1.5 & 0.08 & & & & & 13.9 & 0.35 \\
\hline 0.3 & 0.06 & & & & & 1.2 & 0.12 \\
\hline
\end{tabular}

Run

S2-18

Figures where data appears:

Date
Initials

Plate 1

Plate 2

Plate 3

Plate 4

Plate 5

Plate 6

Plate 7

Plate 8

\begin{tabular}{|c|c|c|c|c|c|c|c|}
\hline $\begin{array}{c}\text { \#\#\#\#\#\# } \\
\text { JS }\end{array}$ & & & & & & $\begin{array}{c}\text { \#\#\#\#\#\# } \\
\text { JS }\end{array}$ & \\
\hline conc. & s.d. & conc. & s.d. & conc. & s.d. & conc. & s.d. \\
\hline 0 & 0.08 & & & & & 0.1 & 0.11 \\
\hline 0 & 0.05 & & & & & 0.1 & 0.07 \\
\hline 0.6 & 0.06 & & & & & 0.2 & 0.08 \\
\hline 2.1 & 0.07 & & & & & 0.3 & 0.08 \\
\hline 1.1 & 0.07 & & & & & 0.5 & 0.09 \\
\hline 1.4 & 0.06 & & & & & 0.8 & 0.15 \\
\hline 2.3 & 0.06 & & & & & 4.4 & 0.13 \\
\hline 1.8 & 0.05 & & & & & 8.5 & 0.12 \\
\hline 0.3 & 0.06 & & & & & 1.9 & 0.1 \\
\hline
\end{tabular}




\begin{tabular}{|c|c|c|c|c|c|c|c|c|}
\hline Plate 10 & 0.2 & 0.06 & & & & & 2.2 & 0.15 \\
\hline Plate 11 & 0.2 & 0.05 & & & & & 0.7 & 0.1 \\
\hline Run & S2-19 & & Figures & re dat & appears: & 4. & & \\
\hline $\begin{array}{l}\text { Date } \\
\text { Initials }\end{array}$ & $\begin{array}{c}\text { \#\#\#\#\# } \\
\text { JS } \\
\end{array}$ & & $\begin{array}{c}\text { \#\#\#\#\# } \\
\text { JS } \\
\end{array}$ & & $\begin{array}{c}\text { \#\#\#\#\#\# } \\
\text { SF }\end{array}$ & & $\begin{array}{c}\text { \#\#\#\#\# } \\
\text { JS } \\
\end{array}$ & \\
\hline & conc. & s.d. & conc. & s.d. & conc. & s.d. & conc. & s.d. \\
\hline Plate 1 & 0.1 & 0.01 & 0 & 0.31 & 0 & 0.35 & 0.3 & 0.01 \\
\hline Plate 2 & 0.1 & 0.01 & 0 & 0.62 & 0 & 0.24 & 0.3 & 0.01 \\
\hline Plate 3 & 0.4 & 0.02 & 0.1 & 0.57 & 0.1 & 0.41 & 0.4 & 0.02 \\
\hline Plate 4 & 1.7 & 0.04 & 0.2 & 0.48 & 0 & 0.28 & 0.5 & 0.01 \\
\hline Plate 5 & 5.9 & 0.13 & 0.8 & 0.38 & 0.1 & 0.26 & 1.3 & 0.02 \\
\hline Plate 6 & 21.2 & 0.37 & 0.8 & 0.48 & 0.6 & 0.32 & 6.7 & 0.07 \\
\hline Plate 7 & 45.6 & 0.71 & 5.8 & 0.32 & 3.6 & 0.23 & 23.4 & 0.24 \\
\hline Plate 8 & 27.7 & 1.06 & 12.1 & 0.55 & 8.1 & 0.37 & 30.2 & 0.39 \\
\hline Plate 9 & 10.7 & 0.28 & 24.9 & 0.68 & 16.8 & 0.33 & 43.7 & 0.61 \\
\hline Plate 10 & 3.8 & 0.11 & 29.4 & 0.87 & 18.5 & 0.47 & 42 & 0.7 \\
\hline Plate 11 & 0.5 & 0.06 & 8.6 & 0.48 & 5.7 & 0.39 & 20.3 & 0.17 \\
\hline
\end{tabular}

\begin{tabular}{|c|c|c|c|c|c|c|c|c|}
\hline \multirow{3}{*}{$\begin{array}{l}\text { Run } \\
\text { Date } \\
\text { Initials }\end{array}$} & \multicolumn{2}{|l|}{ S2-20 } & \multicolumn{3}{|c|}{ Fiqures where data appears: } & \multicolumn{2}{|c|}{4.26} & \\
\hline & $\begin{array}{c}\text { \#\#\#\#\#\#\# } \\
\text { JS }\end{array}$ & & $\begin{array}{c}\text { \#\#\#\#\#\# } \\
\text { JS }\end{array}$ & & $\begin{array}{c}\text { \#\#\#\#\# } \\
\text { SF } \\
\end{array}$ & & $\begin{array}{c}\text { \#\#\#\#\#\# } \\
\text { JS }\end{array}$ & \\
\hline & conc. & s.d. & conc. & s.d. & conc. & s.d. & conc. & s.d. \\
\hline Plate 1 & 0.2 & 0.02 & 0.1 & 0.42 & 0 & 0.25 & 0.5 & 0.02 \\
\hline Plate 2 & 0.2 & 0.01 & 0.1 & 0.41 & 0 & 0.28 & 0.4 & 0.01 \\
\hline Plate 3 & 0.3 & 0.03 & 0.1 & 0.42 & 0 & 0.31 & 0.5 & 0.02 \\
\hline Plate 4 & 1.7 & 0.04 & 0.1 & 0.28 & 0 & 0.29 & 0.6 & 0.01 \\
\hline Plate 5 & 5.9 & 0.21 & 0 & 0.31 & 0 & 0.25 & 1.4 & 0.02 \\
\hline Plate 6 & 19.9 & 0.45 & 0.9 & 0.5 & 0.5 & 0.22 & 6.7 & 0.07 \\
\hline Plate 7 & 31 & 0.8 & 2.9 & 0.54 & 2.1 & 0.27 & 18.2 & 0.21 \\
\hline Plate 8 & 9.7 & 0.37 & 4.5 & 0.52 & 3.5 & 0.3 & 17.2 & 0.36 \\
\hline Plate 9 & 9.7 & 0.26 & 22.7 & 0.64 & 15.5 & 0.26 & 40.7 & 0.55 \\
\hline Plate 10 & 2.6 & 0.1 & 19.6 & 0.89 & 13.1 & 0.41 & 31 & 0.2 \\
\hline Plate 11 & 0.6 & 0.02 & 11.1 & 0.3 & 8 & 0.4 & 28.1 & 0.43 \\
\hline
\end{tabular}

\begin{tabular}{|c|c|c|c|c|c|c|c|c|}
\hline \multirow{2}{*}{$\begin{array}{l}\text { Run } \\
\text { Date } \\
\text { Initials }\end{array}$} & \multicolumn{2}{|l|}{ S2-21 } & \multicolumn{6}{|c|}{ Figures where data appears: } \\
\hline & $\begin{array}{c}\text { \#\#\#\#\# } \\
\text { JS }\end{array}$ & & $\begin{array}{c}\text { \#\#\#\#\# } \\
\text { JS }\end{array}$ & & $\begin{array}{c}\# \# \# \# \# \\
\text { SF }\end{array}$ & & $\begin{array}{l} \\
\text { \#\#\#\#\#\#\# } \\
\text { JS }\end{array}$ & \\
\hline & conc. & s.d. & conc. & s.d. & conc. & s.d. & conc. & s.d. \\
\hline Plate 1 & 0 & 0.01 & 0 & 0.31 & 0 & 0.26 & 0.1 & 0.01 \\
\hline Plate 2 & 0 & 0.01 & 0.2 & 0.41 & 0 & 0.16 & 0.1 & 0.01 \\
\hline Plate 3 & 1 & 0.03 & 0.3 & 0.57 & 0 & 0.23 & 0.2 & 0.01 \\
\hline Plate 4 & 9 & 0.26 & 0 & 0.51 & 0 & 0.36 & 0.7 & 0.02 \\
\hline Plate 5 & 7.7 & 0.82 & 0 & 0.62 & 0 & 0.26 & 0.8 & 0.02 \\
\hline Plate 6 & 2.1 & 0.06 & 0 & 0.41 & 0.3 & 0.41 & 1 & 0.03 \\
\hline Plate 7 & 1.6 & 0.06 & 0 & 0.31 & 0.5 & 0.18 & 3.3 & 0.04 \\
\hline Plate 8 & 1.6 & 0.05 & 0.9 & 0.35 & 1 & 0.24 & 8 & 0.13 \\
\hline
\end{tabular}




\begin{tabular}{|c|c|c|c|c|c|c|c|c|}
\hline Plate 9 & 0.5 & 0.02 & 0.9 & 0.55 & 1.4 & 0.38 & 10.1 & 0.13 \\
\hline Plate 10 & 0.2 & 0.02 & 1.8 & 0.46 & 1.5 & 0.17 & 8.2 & 0.13 \\
\hline Plate 11 & 0.1 & 0.01 & 0.2 & 0.45 & 0.2 & 0.28 & 1.9 & 0.02 \\
\hline Run & S2-22 & & \multicolumn{6}{|c|}{ Figures where data appears: 4.32} \\
\hline $\begin{array}{l}\text { Date } \\
\text { Initials }\end{array}$ & $\begin{array}{c}\text { \#\#\#\#\# } \\
\text { JS } \\
\end{array}$ & & \begin{tabular}{|c} 
\#\#\#\#\#\# \\
JS
\end{tabular} & & $\begin{array}{l} \\
\text { \#\#\#\#\#\# } \\
\text { SF } \\
\end{array}$ & & $\begin{array}{c}\text { \#\#\#\#\#\# } \\
\text { JS } \\
\end{array}$ & \\
\hline & conc. & s.d. & conc. & s.d. & conc. & s.d. & conc. & s.d. \\
\hline Plate 1 & 0 & 0.01 & 0.6 & 0.48 & 0 & 0.25 & 0.1 & 0.01 \\
\hline Plate 2 & 0.1 & 0.01 & 0.5 & 0.3 & 0 & 0.33 & 0.1 & 0.02 \\
\hline Plate 3 & 1 & 0.04 & 0.1 & 0.35 & 0 & 0.25 & 0.1 & 0.02 \\
\hline Plate 4 & 10.3 & 0.3 & 0.4 & 0.23 & 0 & 0.19 & 0.7 & 0.02 \\
\hline Plate 5 & 5.9 & 0.2 & 0.7 & 0.4 & 0.1 & 0.4 & 0.6 & 0.02 \\
\hline Plate 6 & 2.4 & 0.07 & 0.9 & 0.4 & 0.3 & 0.29 & 1.2 & 0.02 \\
\hline Plate 7 & 1 & 0.02 & 1.2 & 0.33 & 0.3 & 0.29 & 3 & 0.03 \\
\hline Plate 8 & 0.9 & 0.04 & 2 & 0.48 & 1 & 0.32 & 7.3 & 0.09 \\
\hline Plate 9 & 0.4 & 0.02 & 2.6 & 0.52 & 1.5 & 0.33 & 10.2 & 0.62 \\
\hline Plate 10 & 0.3 & 0.02 & 6 & 0.43 & 2.6 & 0.26 & 11.2 & 0.21 \\
\hline Plate 11 & 0.1 & 0.02 & 1.2 & 0.35 & 0.7 & 0.28 & 4.1 & 0.07 \\
\hline
\end{tabular}

\begin{tabular}{|c|c|c|c|c|c|c|c|c|}
\hline \multirow{3}{*}{$\begin{array}{l}\text { Run } \\
\text { Date } \\
\text { Initials }\end{array}$} & \multicolumn{2}{|l|}{ S2-23 } & \multicolumn{3}{|c|}{ Figures where data appears: } & \multicolumn{2}{|c|}{4.39} & \\
\hline & $\begin{array}{c}\text { \#\#\#\#\# } \\
\text { JS }\end{array}$ & & $\begin{array}{c}\text { \#\#\#\#\# } \\
\text { JS }\end{array}$ & & $\begin{array}{c}\text { \#\#\#\#\# } \\
\text { SF }\end{array}$ & & $\begin{array}{c}\text { \#\#\#\#\# } \\
\text { JS }\end{array}$ & \\
\hline & conc. & s.d. & conc. & s.d. & conc. & s.d. & conc. & s.d. \\
\hline Plate 1 & 0.3 & 0.01 & 0.8 & 0.46 & 0 & 0.31 & 0.2 & 0.02 \\
\hline Plate 2 & 0.9 & 0.02 & 0.5 & 0.34 & 0.1 & 0.31 & 0.1 & 0.01 \\
\hline Plate 3 & 6.4 & 0.17 & 1 & 0.55 & 0.1 & 0.23 & 0.2 & 0.01 \\
\hline Plate 4 & 5 & 0.16 & 0.8 & 0.58 & 0 & 0.28 & 0.2 & 0.02 \\
\hline Plate 5 & 5.4 & 0.2 & 0.9 & 0.38 & 0 & 0.26 & 0.5 & 0.01 \\
\hline Plate 6 & 15.6 & 0.58 & 1.2 & 0.34 & 0.5 & 0.36 & 2.6 & 0.05 \\
\hline Plate 7 & 37.2 & 0.92 & 4.5 & 0.53 & 3 & 0.24 & 13.2 & 0.03 \\
\hline Plate 8 & 30.1 & 0.99 & 9 & 0.48 & 6.8 & 0.21 & 22 & 0.26 \\
\hline Plate 9 & 9.3 & 0.27 & 15.2 & 0.98 & 11.6 & 0.26 & 24 & 0.34 \\
\hline Plate 10 & 2.8 & 0.1 & 12.7 & 0.7 & 9.3 & 0.32 & 20.6 & 0.32 \\
\hline Plate 11 & 1 & 0.02 & 7.4 & 0.52 & 5.3 & 0.33 & 17.6 & 0.25 \\
\hline
\end{tabular}

\begin{tabular}{|c|c|c|c|c|c|c|c|c|}
\hline \multirow{3}{*}{$\begin{array}{l}\text { Run } \\
\text { Date } \\
\text { Initials }\end{array}$} & \multicolumn{2}{|c|}{ S2-24 } & \multicolumn{6}{|c|}{ Figures where data appears: } \\
\hline & \multicolumn{2}{|c|}{$\begin{array}{l}\mathrm{Sr} \\
\text { \#\#\#\#\#\# } \\
\text { JS }\end{array}$} & \multicolumn{2}{|c|}{\begin{tabular}{l}
\multicolumn{1}{c}{$\mathrm{Si}$} \\
\#\#\#\#\#\#\# \\
JS
\end{tabular}} & \multicolumn{2}{|c|}{$\begin{array}{l}\text { Al } \\
\text { \#\#\#\#\#\#\# } \\
\text { SF }\end{array}$} & \multicolumn{2}{|c|}{ 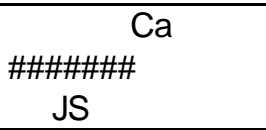 } \\
\hline & conc. & s.d. & conc. & s.d. & conc. & s.d. & conc. & s.d. \\
\hline Plate 1 & 0.2 & 0.02 & 0.1 & 0.48 & 0 & 0.37 & 0.2 & 0.02 \\
\hline Plate 2 & 0.7 & 0.03 & 0 & 0.26 & 0 & 0.27 & 0.2 & 0.02 \\
\hline Plate 3 & 5.8 & 0.23 & 0.2 & 0.46 & 0 & 0.37 & 0.2 & 0.01 \\
\hline Plate 4 & 4.4 & 0.14 & 0 & 0.38 & 0 & 0.39 & 0.3 & 0.01 \\
\hline Plate 5 & 5 & 0.23 & 0.4 & 0.53 & 0.2 & 0.32 & 0.7 & 0.02 \\
\hline Plate 6 & 14 & 0.45 & 0.7 & 0.6 & 0.9 & 0.41 & 3.3 & 0.03 \\
\hline Plate 7 & 38.9 & 0.93 & 4.8 & 0.26 & 4.2 & 0.29 & 17.2 & 0.22 \\
\hline
\end{tabular}




\begin{tabular}{l|cc|cc|cc|cc|} 
Plate 8 & 34.7 & 0.91 & 12.4 & 0.48 & 10.1 & 0.25 & 37 & 0.48 \\
Plate 9 & 12.2 & 0.35 & 21.1 & 0.39 & 15.9 & 0.38 & 38.5 & 0.43 \\
Plate 10 & 2.4 & 0.08 & 11.4 & 0.45 & 8.4 & 0.22 & 19.1 & 0.21 \\
Plate 11 & 0.6 & 0.02 & 9.8 & 0.62 & 6.6 & 0.39 & 19.3 & 0.41 \\
\cline { 2 - 8 }
\end{tabular}




\begin{tabular}{|c|c|c|c|c|c|c|c|c|}
\hline \multirow{2}{*}{$\begin{array}{l}\text { Run } \\
\text { Date } \\
\text { Initials }\end{array}$} & \multicolumn{2}{|l|}{ S2-25 } & \multicolumn{6}{|c|}{ Figures where data appears: } \\
\hline & \multicolumn{2}{|c|}{ 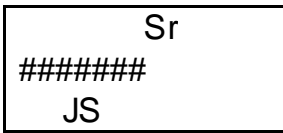 } & \multicolumn{2}{|c|}{$\begin{array}{l}\mathrm{Si} \\
\# \# \# \# \# \# \\
\text { BN } \\
\end{array}$} & \multicolumn{2}{|c|}{\begin{tabular}{|l} 
Al \\
$\# \# \# \# \# \#$ \\
JS \\
\end{tabular}} & \multicolumn{2}{|c|}{$\begin{array}{l}\text { Ca } \\
\text { \#\#\#\#\#\# } \\
\text { JS } \\
\end{array}$} \\
\hline & conc. & s.d. & conc. & s.d. & conc. & s.d. & conc. & s.d. \\
\hline Plate 1 & 0 & 0.07 & 0.2 & 0.43 & 0.1 & 0.07 & 0 & 0.2 \\
\hline Plate 2 & 0 & 0.06 & 0.3 & 0.24 & 0 & 0.11 & 0.4 & 0.06 \\
\hline Plate 3 & 0 & 0.06 & 1 & 0.39 & 0.1 & 0.08 & 0.4 & 0.05 \\
\hline Plate 4 & 0.2 & 0.04 & 2.1 & 0.35 & 0.1 & 0.07 & 0.5 & 0.11 \\
\hline Plate 5 & 0.3 & 0.06 & 3.7 & 0.42 & 0.2 & 0.08 & 0.7 & 0.07 \\
\hline Plate 6 & 0.7 & 0.05 & 0.9 & 0.39 & 0.3 & 0.09 & 0.5 & 0.07 \\
\hline Plate 7 & 1.6 & 0.07 & 2 & 0.52 & 0.8 & 0.06 & 1.2 & 0.08 \\
\hline Plate 8 & 1.2 & 0.08 & 1.1 & 0.6 & 0.7 & 0.07 & 2.4 & 0.09 \\
\hline Plate 9 & 0.2 & 0.05 & 1.3 & 0.4 & 0.2 & 0.1 & 1.4 & 0.09 \\
\hline Plate 10 & 0 & 0.04 & 1.7 & 0.6 & 0.3 & 0.09 & 1.8 & 0.1 \\
\hline Plate 11 & 0.1 & 0.03 & 2.1 & 0.73 & 0.4 & 0.11 & 1.3 & 0.12 \\
\hline
\end{tabular}

\begin{tabular}{|c|c|c|c|c|c|c|c|c|}
\hline \multirow{3}{*}{$\begin{array}{l}\text { Run } \\
\text { Date } \\
\text { Initials }\end{array}$} & \multicolumn{2}{|l|}{ S2-26 } & \multicolumn{3}{|c|}{ Figures where data appears: } & \multicolumn{2}{|c|}{4.25} & \\
\hline & \multicolumn{2}{|c|}{$\begin{aligned} \mathrm{Sr} \\
\text { \#\#\#\#\#\#\# } \\
\text { JMA }\end{aligned}$} & \multicolumn{2}{|c|}{$\begin{array}{l}\text { Si } \\
\text { \#\#\#\#\#\# } \\
\text { BN } \\
\end{array}$} & \multicolumn{2}{|c|}{$\begin{array}{l}\text { Al } \\
\text { \#\#\#\#\#\#\# } \\
\text { JS }\end{array}$} & \multicolumn{2}{|c|}{$\begin{array}{l}\text { Ca } \\
\text { \#\#\#\#\#\#\# } \\
\text { JS }\end{array}$} \\
\hline & conc. & s.d. & conc. & s.d. & conc. & s.d. & conc. & s.d. \\
\hline Plate 1 & 0 & 0.01 & 0 & 0.61 & 0 & 0.09 & 0 & 0.09 \\
\hline Plate 2 & 0 & 0.03 & 0.7 & 0.42 & 0.1 & 0.08 & 0.2 & 0.08 \\
\hline Plate 3 & 0 & 0.02 & 0.7 & 0.44 & 0.1 & 0.1 & 0.2 & 0.04 \\
\hline Plate 4 & 0.2 & 0.01 & 1 & 0.58 & 0 & 0.04 & 0.3 & 0.1 \\
\hline Plate 5 & 0.5 & 0.03 & 1.4 & 0.52 & 0 & 0.1 & 0.3 & 0.1 \\
\hline Plate 6 & 1.2 & 0.04 & 0.5 & 0.55 & 0.3 & 0.1 & 0.3 & 0.07 \\
\hline Plate 7 & 2.9 & 0.07 & 2.2 & 0.48 & 1.1 & 0.06 & 0.9 & 0.1 \\
\hline Plate 8 & 1.7 & 0.07 & 2 & 0.38 & 0.7 & 0.11 & 1.4 & 0.09 \\
\hline Plate 9 & 0 & 0.03 & 1.2 & 0.48 & 0.2 & 0.04 & 0.8 & 0.1 \\
\hline Plate 10 & 0 & 0.01 & 2.6 & 0.45 & 0.7 & 0.01 & 2.2 & 0.13 \\
\hline Plate 11 & 0.1 & 0.01 & 1.8 & 0.36 & 0.5 & 0.07 & 1.2 & 0.13 \\
\hline
\end{tabular}

\begin{tabular}{|c|c|c|c|c|c|c|c|c|}
\hline \multirow{3}{*}{$\begin{array}{l}\text { Run } \\
\text { Date } \\
\text { Initials }\end{array}$} & \multicolumn{2}{|l|}{ S2-27 } & \multicolumn{3}{|c|}{ Figures where data appears: } & \multicolumn{2}{|c|}{4.31} & \\
\hline & $\begin{array}{c}\text { \#\#\#\#\# } \\
\text { JMA }\end{array}$ & & $\begin{array}{c}\text { \#\#\#\#\# } \\
\text { BN }\end{array}$ & & $\begin{array}{c}\text { \#\#\#\#\# } \\
\text { JS }\end{array}$ & & $\begin{array}{c}\text { \#\#\#\#\#\#\# } \\
\text { JS }\end{array}$ & \\
\hline & conc. & s.d. & conc. & s.d. & conc. & s.d. & conc. & s.d. \\
\hline Plate 1 & 0 & 0.03 & 0 & 0.42 & 0 & 0.11 & 0.1 & 0.08 \\
\hline Plate 2 & 0 & 0.01 & 0.2 & 0.23 & 0 & 0.13 & 0.1 & 0.09 \\
\hline Plate 3 & 0.7 & 0.04 & 0.7 & 0.49 & 0 & 0.08 & 0.3 & 0.08 \\
\hline Plate 4 & 1.5 & 0.03 & 0.6 & 0.32 & 0 & 0.06 & 0.1 & 0.08 \\
\hline Plate 5 & 0.1 & 0.01 & 1 & 0.37 & 0 & 0.07 & 0 & 0.06 \\
\hline Plate 6 & 0.1 & 0.01 & 0 & 0.4 & 0.1 & 0.09 & 0.2 & 0.11 \\
\hline Plate 7 & 1.1 & 0.03 & 0.6 & 0.5 & 0.8 & 0.13 & 0.4 & 0.05 \\
\hline Plate 8 & 0.5 & 0.02 & 1.2 & 0.48 & 0 & 0.1 & 0.4 & 0.09 \\
\hline Plate 9 & 0 & 0.02 & 0.3 & 0.46 & 0 & 0.12 & 0.3 & 0.09 \\
\hline Plate 10 & 0 & 0.01 & 0.8 & 0.31 & 0 & 0.09 & 0.5 & 0.07 \\
\hline
\end{tabular}




\begin{tabular}{|c|c|c|c|c|c|c|c|c|}
\hline \multirow{2}{*}{$\begin{array}{l}\text { Plate } 11 \\
\text { Run }\end{array}$} & 0 & 0.01 & 1.1 & 0.4 & 0 & 0.1 & 0.2 & 0.11 \\
\hline & S2-28 & & \multicolumn{4}{|c|}{ Figures where data appears: } & \multicolumn{2}{|c|}{4.31} \\
\hline \multirow[t]{2}{*}{$\begin{array}{l}\text { Date } \\
\text { Initials }\end{array}$} & 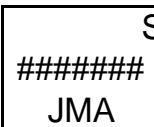 & & $\begin{array}{c}\text { \#\#\#\#\#\# } \\
\text { BN }\end{array}$ & & $\begin{array}{c}\text { \#\#\#\#\#\# } \\
\text { JS }\end{array}$ & & $\begin{array}{c}\text { \#\#\#\#\#\# } \\
\text { JS }\end{array}$ & \\
\hline & conc. & s.d. & conc. & s.d. & conc. & s.d. & conc. & s.d. \\
\hline Plate 1 & 0 & 0.02 & 0 & 0.43 & 0 & $0.0 s$ & 0 & 0.1 \\
\hline Plate 2 & 0.1 & 0.01 & 0 & 0.47 & 0 & 0.11 & 0.1 & 0.08 \\
\hline Plate 3 & 0.8 & 0.01 & 0 & 0.32 & 0 & 0.08 & 0.1 & 0.11 \\
\hline Plate 4 & 1.5 & 0.05 & 0.7 & 0.41 & 0 & 0.07 & 0.2 & 0.04 \\
\hline Plate 5 & 0.3 & 0.01 & 1.4 & 0.61 & 0 & 0.05 & 0.2 & 0.09 \\
\hline Plate 6 & 0.2 & 0.02 & 0 & 0.51 & 0.1 & 0.08 & 0 & 0.11 \\
\hline Plate 7 & 1.1 & 0.05 & 1.1 & 0.5 & 0.7 & 0.08 & 0.4 & 0.1 \\
\hline Plate 8 & 0.5 & 0.02 & 1.4 & 0.53 & 0.5 & 0.08 & 0.6 & 0.06 \\
\hline Plate 9 & 0 & 0.01 & 1.3 & 0.46 & 0 & 0.08 & 0.3 & 0.07 \\
\hline Plate 10 & 0 & 0.01 & 1.9 & 0.31 & 0 & 0.11 & 0.4 & 0.15 \\
\hline Plate 11 & 0 & 0.02 & 1.9 & 0.23 & 0 & 0.1 & 0.3 & 0.06 \\
\hline
\end{tabular}

\begin{tabular}{|c|c|c|c|c|c|c|c|c|}
\hline \multirow{3}{*}{$\begin{array}{l}\text { Run } \\
\text { Date } \\
\text { Initials }\end{array}$} & \multicolumn{2}{|l|}{ S2-29 } & \multicolumn{3}{|c|}{ Figures where data appears: } & \multicolumn{2}{|c|}{4.38} & \\
\hline & $\begin{array}{c}\text { \#\#\#\#\# } \\
\text { JMA }\end{array}$ & & $\begin{array}{c} \\
\# \# \# \# \# \\
\text { BN }\end{array}$ & & $\begin{array}{c} \\
\text { \#\#\#\#\#\# } \\
\text { JS }\end{array}$ & & $\begin{array}{c}\text { \#\#\#\#\#\# } \\
\text { JS }\end{array}$ & \\
\hline & conc. & s.d. & conc. & s.d. & conc. & s.d. & conc. & s.d. \\
\hline Plate 1 & 0 & 0.01 & 0.1 & 0.3 & 0 & 0.1 & 0 & 0.08 \\
\hline Plate 2 & 0.2 & 0.02 & 0 & 0.34 & 0 & 0.08 & 0 & 0.08 \\
\hline Plate 3 & 2.8 & 0.05 & 0.2 & 0.39 & 0 & 0.06 & 0 & 0.06 \\
\hline Plate 4 & 2.8 & 0.08 & 0.2 & 0.42 & 0.1 & 0.09 & 0.1 & 0.12 \\
\hline Plate 5 & 1.1 & 0.05 & 0.3 & 0.22 & 0.3 & 0.08 & 0.1 & 0.09 \\
\hline Plate 6 & 1.9 & 0.04 & 0.9 & 0.36 & 1.3 & 0.09 & 0.2 & 0.11 \\
\hline Plate 7 & 3.8 & 0.07 & 4 & 0.42 & 4.1 & 0.12 & 1.2 & 0.09 \\
\hline Plate 8 & 2.4 & 0.06 & 1.9 & 0.51 & 2.2 & 0.17 & 2.6 & 0.17 \\
\hline Plate 9 & 0.1 & 0.02 & 0 & 0.3 & 0 & 0.09 & 0.6 & 0.11 \\
\hline Plate 10 & 0.1 & 0.01 & 0 & 0.21 & 0 & 0.07 & 0.8 & 0.12 \\
\hline Plate 11 & 0.1 & 0.02 & 0 & 0.47 & 0 & 0.1 & 0.4 & 0.11 \\
\hline
\end{tabular}

Run

S2-30 Figures where data appears:

Date

Initials

Plate 1

Plate 2

Plate 3

Plate 4

Plate 5

Plate 6

Plate 7

Plate 8

\begin{tabular}{|c|c|c|c|c|c|c|c|}
\hline S2-30 & & \multicolumn{3}{|c|}{ Figures where data appears: } & \multicolumn{2}{|c|}{4.38} & \\
\hline $\begin{array}{c}\mathrm{s} \\
\text { \#\#\#\#\#\#\# } \\
\text { JMA }\end{array}$ & & $\begin{array}{c}\text { \#\#\#\#\# } \\
\text { BN }\end{array}$ & & $\begin{array}{c}\text { \#\#\#\#\# } \\
\text { JS }\end{array}$ & & $\begin{array}{c}\text { \#\#\#\#\#\# } \\
\text { JS }\end{array}$ & \\
\hline conc. & s.d. & conc. & s.d. & conc. & s.d. & conc. & s.d. \\
\hline 0 & 0.02 & 0 & 0.32 & 0 & 0.09 & 0 & 0.1 \\
\hline 0.1 & 0.01 & 0 & 0.44 & 0 & 0.14 & 0 & 0.07 \\
\hline 2.4 & 0.08 & 0 & 0.34 & 0 & 0.05 & 0 & 0.09 \\
\hline 3.9 & 0.16 & 0 & 0.38 & 0 & 0.1 & 0 & 0.12 \\
\hline 1.4 & 0.06 & 0.2 & 0.59 & 0.3 & 0.08 & 0.1 & 0.11 \\
\hline 2.3 & 0.07 & 1 & 0.5 & 1.4 & 0.11 & 0.2 & 0.07 \\
\hline 4.5 & 0.07 & 3.4 & 0.35 & 4 & 0.11 & 1.1 & 0.1 \\
\hline 3.4 & 0.08 & 2.3 & 0.4 & 2.7 & 0.1 & 3 & 0.13 \\
\hline 0.2 & 0.02 & 0.6 & 0.53 & 0.3 & 0.1 & 0.8 & 0.1 \\
\hline
\end{tabular}




\begin{tabular}{|c|c|c|c|c|c|c|c|c|}
\hline \multirow{3}{*}{$\begin{array}{l}\text { Plate } 10 \\
\text { Plate } 11 \\
\text { Run }\end{array}$} & 0 & 0.01 & 0 & 0.25 & 0.1 & 0.09 & 0.6 & 0.1 \\
\hline & 0 & 0.01 & 0 & 0.31 & 0.1 & 0.07 & 0.3 & 0.1 \\
\hline & \multicolumn{2}{|l|}{ S3-1 } & \multicolumn{4}{|c|}{ Figures where data appears: } & \multicolumn{2}{|c|}{4.35} \\
\hline \multirow[t]{2}{*}{$\begin{array}{l}\text { Date } \\
\text { Initials }\end{array}$} & $\begin{array}{c}\text { \#\#\#\#\#\# } \\
\text { JS }\end{array}$ & & \multicolumn{2}{|c|}{$\mathrm{Si}$} & $\begin{array}{c}\text { \#\#\#\#\#\# } \\
\text { SF }\end{array}$ & & \multicolumn{2}{|c|}{$\mathrm{Ca}$} \\
\hline & conc. & s.d. & conc. & s.d. & conc. & s.d. & conc & s.d. \\
\hline Plate 1 & 0.5 & 0.02 & 0 & 0.53 & 0.4 & 0.27 & & \\
\hline Plate 2 & 2.4 & 0.07 & 0.1 & 0.64 & 0.2 & 0.21 & & \\
\hline Plate 3 & 2.7 & 0.06 & 0 & 0.56 & 0 & 0.2 & & \\
\hline Plate 4 & 1.6 & 0.03 & 0.3 & 0.64 & 0.4 & 0.17 & & \\
\hline Plate 5 & 2.1 & 0.05 & 0.1 & 0.68 & 0.4 & 0.23 & & \\
\hline Plate 6 & 6.7 & 0.18 & 1.2 & 0.41 & 1.8 & 0.26 & & \\
\hline Plate 7 & 8.7 & 0.18 & 8.3 & 0.82 & 8.6 & 0.39 & & \\
\hline Plate 8 & 2.6 & 0.07 & 4.6 & 0.7 & 4.5 & 0.22 & & \\
\hline Plate 9 & 0.5 & 0.02 & 0.4 & 0.58 & 1.5 & 0.3 & & \\
\hline Plate 10 & 0.3 & 0.01 & 1.2 & 0.51 & 1.3 & 0.25 & & \\
\hline Plate 11 & 0 & 0.06 & 0 & 0.45 & 0.5 & 0.35 & & \\
\hline
\end{tabular}

\begin{tabular}{|c|c|c|c|c|c|c|c|c|}
\hline \multirow{3}{*}{$\begin{array}{l}\text { Run } \\
\text { Date } \\
\text { Initials }\end{array}$} & \multicolumn{2}{|l|}{ S3-2 } & \multicolumn{3}{|c|}{ Fiqures where data appears: } & 4.35 & \\
\hline & $\begin{array}{c}\text { \#\#\#\#\#\# } \\
\text { JS }\end{array}$ & & $\begin{array}{c}\text { \#\#\#\#\#\# } \\
\text { JS }\end{array}$ & & $\begin{array}{c}\text { \#\#\#\#\#\# } \\
\text { SF }\end{array}$ & & & \\
\hline & conc. & s.d. & conc. & s.d. & conc. & s.d. & conc. & s.d. \\
\hline Plate 1 & 0.5 & 0.01 & 0 & 0.38 & 0.2 & 0.27 & & \\
\hline Plate 2 & 2.6 & 0.1 & 0 & 0.45 & 0.1 & 0.26 & & \\
\hline Plate 3 & 3.1 & 0.12 & 0 & 0.48 & 0.2 & 0.27 & & \\
\hline Plate 4 & 1.7 & 0.06 & 0.3 & 0.59 & 0.3 & 0.41 & & \\
\hline Plate 5 & 2.2 & 0.06 & 0.4 & 0.49 & 0.3 & 0.32 & & \\
\hline Plate 6 & 4.3 & 0.2 & 2 & 0.47 & 1.9 & 0.26 & & \\
\hline Plate 7 & 11 & 0.27 & 15.7 & 0.74 & 13.7 & 0.44 & & \\
\hline Plate 8 & 3.3 & 0.11 & 9.1 & 0.68 & 7.6 & 0.31 & & \\
\hline Plate 9 & 0.6 & 0.02 & 2.9 & 0.8 & 2.4 & 0.27 & & \\
\hline Plate 10 & 0.5 & 0.02 & 2.4 & 0.67 & 2 & 0.26 & & \\
\hline Plate 11 & 0.1 & 0.01 & 0.5 & 0.69 & 0.5 & 0.22 & & \\
\hline
\end{tabular}

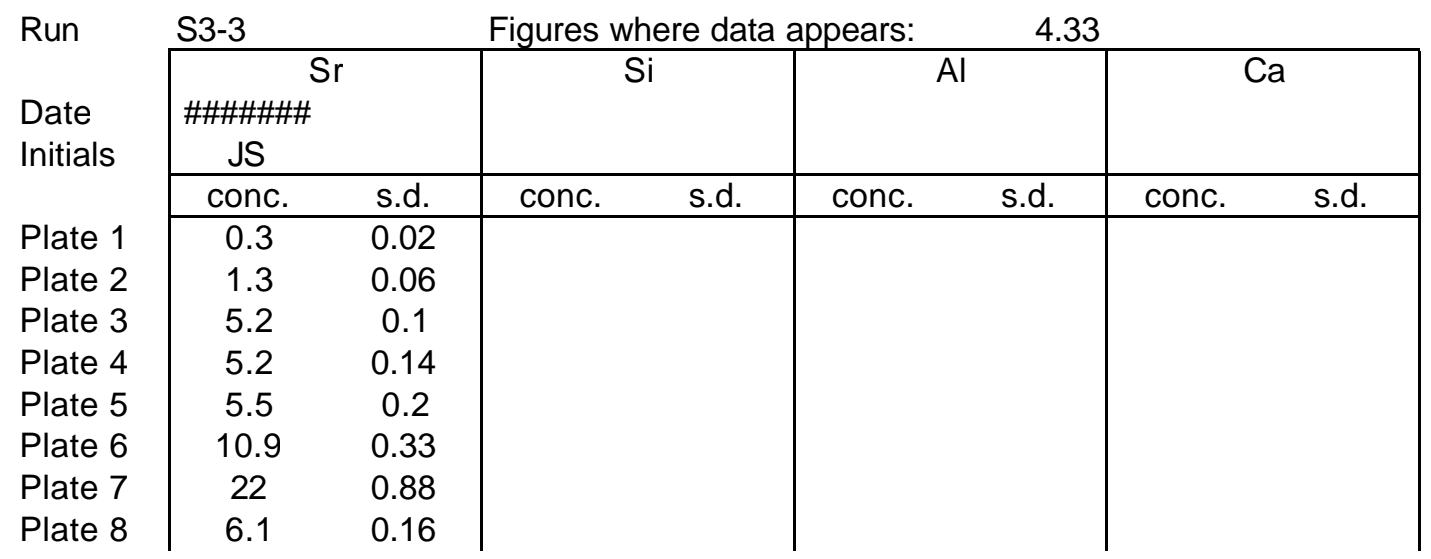




\begin{tabular}{|c|c|c|c|c|c|c|c|c|}
\hline $\begin{array}{l}\text { Plate } 9 \\
\text { Plate } 10 \\
\text { Plate } 11\end{array}$ & $\begin{array}{c}0.1 \\
0 \\
0\end{array}$ & $\begin{array}{l}0.02 \\
0.01 \\
0.02\end{array}$ & & & & & & \\
\hline Run & S3-4 & & Figures & re da & pears: & & & \\
\hline $\begin{array}{l}\text { Date } \\
\text { Initials }\end{array}$ & \begin{tabular}{|c} 
S \\
\#\#\#\#\#\# \\
JS
\end{tabular} & & & & & & & \\
\hline & conc. & s.d. & conc. & s.d. & conc. & s.d. & conc. & s.d. \\
\hline Plate 1 & 0.3 & 0.02 & & & & & & \\
\hline Plate 2 & 1.5 & 0.04 & & & & & & \\
\hline Plate 3 & 5.6 & 0.21 & & & & & & \\
\hline Plate 4 & 4.8 & 0.15 & & & & & & \\
\hline Plate 5 & 5.3 & 0.22 & & & & & & \\
\hline Plate 6 & 13.5 & 0.58 & & & & & & \\
\hline Plate 7 & 21.9 & 0.5 & & & & & & \\
\hline Plate 8 & 6.4 & 0.22 & & & & & & \\
\hline Plate 9 & 0.1 & 0.02 & & & & & & \\
\hline Plate 10 & 0 & 0.02 & & & & & & \\
\hline Plate 11 & 0 & 0.01 & & & & & & \\
\hline
\end{tabular}

\begin{tabular}{|c|c|c|c|c|c|c|c|c|}
\hline \multirow{3}{*}{$\begin{array}{l}\text { Run } \\
\text { Date } \\
\text { Initials }\end{array}$} & \multirow{2}{*}{\multicolumn{2}{|c|}{ 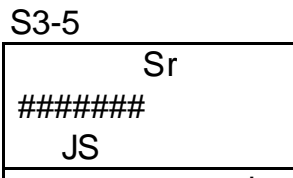 }} & \multicolumn{6}{|c|}{ Figures where data appears: } \\
\hline & & & \multicolumn{2}{|c|}{ 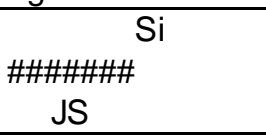 } & \multicolumn{2}{|c|}{ 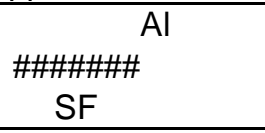 } & \multicolumn{2}{|c|}{$\mathrm{Ca}$} \\
\hline & conc. & s.d. & conc. & s.d. & conc. & s.d. & conc. & s.d. \\
\hline Plate 1 & 0.1 & 0.01 & 0.4 & 0.77 & 0.5 & 0.24 & & \\
\hline Plate 2 & 0.1 & 0.01 & 0.6 & 0.34 & 0.5 & 0.19 & & \\
\hline Plate 3 & 0.2 & 0.02 & 0.1 & 0.68 & 0.5 & 0.37 & & \\
\hline Plate 4 & 0.5 & 0.02 & 0.2 & 0.5 & 0.6 & 0.17 & & \\
\hline Plate 5 & 0.9 & 0.03 & 0.7 & 0.51 & 0.6 & 0.19 & & \\
\hline Plate 6 & 2.6 & 0.09 & 7.5 & 0.6 & 5.8 & 0.33 & & \\
\hline Plate 7 & 6.1 & 0.18 & 16.9 & 0.96 & 14.3 & 0.39 & & \\
\hline Plate 8 & 5 & 0.2 & 16 & 0.6 & 13.6 & 0.28 & & \\
\hline Plate 9 & 0.3 & 0.02 & 2.4 & 0.46 & 1.3 & 0.28 & & \\
\hline Plate 10 & 0.1 & 0.02 & 1 & 0.48 & 0.4 & 0.24 & & \\
\hline Plate 11 & 0.1 & 0.02 & 0.3 & 0.55 & 0.2 & 0.32 & & \\
\hline
\end{tabular}

Run S3-6 Figures where data appears:

\begin{tabular}{|c|c|c|c|c|c|c|c|c|}
\hline \multirow[t]{2}{*}{$\begin{array}{l}\text { Date } \\
\text { Initials }\end{array}$} & \multicolumn{2}{|c|}{$\begin{array}{c}\text { Sr } \\
\text { \#\#\#\#\#\#\# } \\
\text { JS }\end{array}$} & \multicolumn{2}{|c|}{$\begin{array}{l}\text { Si } \\
\text { \#\#\#\#\#\#\# } \\
\text { JS }\end{array}$} & \multicolumn{2}{|c|}{$\begin{array}{l}\text { Al } \\
\text { \#\#\#\#\#\#\# } \\
\text { SF }\end{array}$} & \multicolumn{2}{|c|}{$\mathrm{Ca}$} \\
\hline & conc. & s.d. & conc. & s.d. & conc. & s.d. & conc. & s.d. \\
\hline Plate 1 & 0 & 0.02 & 0 & 0.56 & 0.1 & 0.46 & & \\
\hline Plate 2 & 0.1 & 0.01 & 0 & 0.58 & 0.1 & 0.25 & & \\
\hline Plate 3 & 0.2 & 0.01 & 0 & 0.67 & 0 & 0.31 & & \\
\hline Plate 4 & 0.4 & 0.02 & 0.3 & 0.6 & 0.3 & 0.25 & & \\
\hline Plate 5 & 0.6 & 0.02 & 0.5 & 0.56 & 0.8 & 0.26 & & \\
\hline Plate 6 & 2.4 & 0.04 & 7.2 & 0.54 & 5.8 & 0.42 & & \\
\hline Plate 7 & 8.5 & 0.28 & 18 & 0.66 & 16.2 & 0.31 & & \\
\hline
\end{tabular}




\begin{tabular}{l|ll|ll|ll|l|} 
Plate 8 & 6.2 & 0.16 & 17.7 & 0.4 & 15.8 & 0.35 & \\
Plate 9 & 0.8 & 0.05 & 2.8 & 0.45 & 2.6 & 0.36 & \\
Plate 10 & 0.2 & 0.02 & 1.4 & 0.57 & 0.9 & 0.31 & \\
Plate 11 & 1.8 & 0.08 & 4.3 & 0.66 & 3.4 & 0.27 & \\
\hline
\end{tabular}




\begin{tabular}{|c|c|c|c|c|c|c|c|c|}
\hline \multirow{3}{*}{$\begin{array}{l}\text { Run } \\
\text { Date } \\
\text { Initials }\end{array}$} & \multirow{2}{*}{\multicolumn{2}{|c|}{$\begin{array}{l}\text { S3-7 } \\
\begin{array}{|c}\text { Sr } \\
\text { \#\#\#\#\#\#\# } \\
\text { JS }\end{array} \\
\end{array}$}} & \multicolumn{6}{|c|}{ Figures where data appears: } \\
\hline & & & \multicolumn{2}{|c|}{$\begin{array}{l}\text { Si } \\
\text { \#\#\#\#\#\#\# } \\
\text { JS } \\
\end{array}$} & \multicolumn{2}{|c|}{$\begin{array}{l}\mathrm{Al} \\
\# \# \# \# \# \# \\
\text { SF }\end{array}$} & \multicolumn{2}{|c|}{$\mathrm{Ca}$} \\
\hline & conc. & s.d. & conc. & s.d. & conc. & s.d. & conc. & s.d. \\
\hline Plate 1 & 0.1 & 0.01 & 0 & 0.44 & 0 & 0.34 & & \\
\hline Plate 2 & 0.5 & 0.01 & 0.9 & 0.28 & 0 & 0.28 & & \\
\hline Plate 3 & 1.6 & 0.07 & 0.2 & 0.6 & 0 & 0.24 & & \\
\hline Plate 4 & 3.5 & 0.11 & 0 & 0.52 & 0 & 0.27 & & \\
\hline Plate 5 & 4.7 & 0.13 & 0.6 & 0.52 & 0.1 & 0.25 & & \\
\hline Plate 6 & 5.7 & 0.15 & 2 & 0.67 & 1.5 & 0.33 & & \\
\hline Plate 7 & 6.1 & 0.2 & 11.8 & 0.71 & 10.3 & 0.39 & & \\
\hline Plate 8 & 5.1 & 0.17 & 12.4 & 0.53 & 11.2 & 0.32 & & \\
\hline Plate 9 & 0.9 & 0.03 & 5.3 & 0.6 & 4.8 & 0.2 & & \\
\hline Plate 10 & 0.7 & 0.03 & 11.1 & 0.51 & 9.7 & 0.42 & & \\
\hline Plate 11 & 0.2 & 0.01 & 4.5 & 0.74 & 4.1 & 0.35 & & \\
\hline
\end{tabular}

Run

S3-8

Figures where data appears:

Date

Initials

Plate 1

Plate 2

Plate 3

Plate 4

Plate 5

Plate 6

Plate 7

Plate 8

Plate 9

Plate 10

Plate 11

\begin{tabular}{|c|c|c|c|c|c|c|c|}
\hline \multicolumn{2}{|c|}{$\begin{array}{l}\text { Sr } \\
\text { \#\#\#\#\#\#\# } \\
\text { JS }\end{array}$} & \multicolumn{2}{|c|}{$\begin{array}{l}\text { Si } \\
\text { \#\#\#\#\#\#\# } \\
\text { JS }\end{array}$} & \multicolumn{2}{|c|}{$\begin{array}{l}\text { Al } \\
\text { \#\#\#\#\#\#\# } \\
\text { SF }\end{array}$} & \multicolumn{2}{|c|}{$\mathrm{Ca}$} \\
\hline conc. & s.d. & conc. & s.d. & conc. & s.d. & conc. & s.d. \\
\hline 0.1 & 0.01 & 0 & 0.74 & 0.1 & 0.32 & & \\
\hline 0.4 & 0.02 & 0.1 & 0.51 & 0 & 0.28 & & \\
\hline 2.3 & 0.11 & 0 & 0.39 & 0 & 0.31 & & \\
\hline 4.4 & 0.1 & 0 & 0.56 & 0 & 0.24 & & \\
\hline 4.8 & 0.1 & 0 & 0.53 & 0.1 & 0.3 & & \\
\hline 8.5 & 0.31 & 1.6 & 0.55 & 1.7 & 0.32 & & \\
\hline 7.1 & 0.27 & 11.4 & 0.69 & 10.7 & 0.33 & & \\
\hline 5.6 & 0.19 & 12.7 & 0.57 & 11 & 0.29 & & \\
\hline 0.7 & 0.04 & 2.7 & 0.6 & 3 & 0.24 & & \\
\hline 0.6 & 0.03 & 7.7 & 0.51 & 6.9 & 0.16 & & \\
\hline 0.2 & 0.02 & 5.6 & 0.7 & 5.5 & 0.22 & & \\
\hline
\end{tabular}




\section{REFERENCES}

Aganihotri, R., et al. (1998). "Selenium Removal Using Ca-Based Sorbents: Reaction Kinetics.” Environmental Science and Technology 32: 1841-1846.

Barin, I. (1995). Thermochemical Data of Pure Substances. Weinheim, FRG, VCH.

Biermann, J. J. P., et al. (1997). Thermal Conversion of Deinking Residue to Produce Valuable Cement Products. 18th VDI German/Dutch Engineering meeting.

Biswas, P. and C. Y. Wu (1998). "Control of Toxic Metal Emissions from Combustors Using Sorbents: A Review." Journal of the Air and Waste Management Association 48(February): 113-127.

Bool, L. E., et al. (1995). "The Partitioning of Iron during the Combustion of Pulverized Coal." Combustion and Flame 100: 262-270.

Buckley, S. G., et al. (1998). Fate of Sr and Cs in Biomass Combustion. 1998 Spring Technical Meeting of the Western States Section of the Combustion Institute, U.C. Berkeley, Berkeley, CA.

Costner, P. and J. Thornton (1990). Playing with Fire: Hazardous Waste Incineration. $\underline{A}$ Greenpeace Report, Washington D.C., Greenpeace U.S.A.

Crosley, S. M. and R. J. Kedl (1992). Laboratory Measurements of Metal Adsorption from Simulated Incinerator Flue Gases: Sorbent Selection for Cesium Capture. 1992 Incineration Conference, Albuquerque, NM.

Davis, S. B. (1999 - not yet in print). Interactions Between Semi-Volatile Toxic Metals and Sorbents in Combustors. Chemical and Environmental Engineering, Tucson, AZ, University of Arizona.

Davis, S. B., et al. (1998). Multi-Component Coagulation and Condensation of Toxic Metals in Combustors. 27th Symposium (International) on Combustion, University of Colorado at Boulder.

Durlak, S. K., et al. (1997). "Equilibrium Analysis of the Affect of Temperature, Moisture, and Sodium Content on Heavy Metal Emissions from Municipal Solid Waste Incinerators." Journal of Hazardous Materials 56: 1-20.

Fernandez, M. A., et al. (1992). "Behavior of Heavy Metals in the Combustion Gases of Urban Waste Incinerators." Environmental Science and Technology 26: 10401047. 
Frandsen, F., et al. (1994). "Trace Elements From Combustion and Gasification of Coal An Equilibrium Approach." Progress in Energy and Combustion Science 20: 115138.

Furr, A. K., et al. (1977). "National Survey of Elements and Radioactivity in Fly Ashes: Absorption of Elements by Cabbage Grown in Fly Ash-Soil Mixtures." Environmental Science and Technology 11(13): 1194-1201.

Gallagher, N. B., et al. (1996). Sodium Partitioning in a Pulverized Coal Combustion Environment. Twenty-Sixth Symposium (International) on Combustion.

Ghosh-Dastidar, A., et al. (1996). "Selenium Capture Using Sorbent Powders: Mechanism of Sorption by Hydrated Lime." Environmental Science and Technology 30: 447-452.

Gordon, S. and B. J. McBride (1994). Computer Program for Calculation of Complex Chemical Equilibrium Compositions and Applications, Cleveland, Ohio, National Aeronautics and Space Administration.

Hillamo, R. and E. Kauppinen (1991). "On the Performance of the Berner Low Pressure Impactor." Aerosol Science and Technology 14: 33-47.

Hitachi (1992). Instrument Manual for Model S-2460N, Hitachi.

Ho, T. C., et al. (1993). "Metal Volatilization and Separation During Incineration." Waste Management 13: 455-466.

Ho, T. C., et al. "Characteristics of Metal Capture during Fluidized Bed Incineration." AIChE Symposium Series 87(281): 118-126.

Noran Instruments. (1995). Voyager Handbook, Noran Instruments.

Jozewicz, W. and B. K. Gullett (1995). "Reaction Mechanisms of Dry Ca-Based Sorbents with Gaseous HCl.” Industrial and Engineering Chemistry Research 34: 607-612.

Karimanal, K. V. and M. J. Hall (1996). "Effect of Temperature and Flow on the Volatilization of Elemental Lead and Cadmium." Hazardous Waste and Hazardous Materials 13(1): 63-71.

Lee, S. H. D. and I. Johnson (1980). "Removal of Gaseous Alkali Metal Compounds from Hot Flue Gas by Particulate Sorbents." Journal of Engineering for Power 102(April): 397-402.

Lide, D. R. (1995). CRC Handbook of Chemistry and Physics. Boca Raton, FL, CRC Press. 
Linak, W. P., et al. (1996). "Formation and Destruction of Hexavalent Chromium in a Laboratory Swirl Flame Incinerator.” Combustion Science and Technology 116117: 479-498.

Linak, W. P., et al. (1994). "Metal Aerosol Formation in a Laboratory Swirl Flame Incinerator." Combustion Science and Technology 101: 7-27.

Linak, W. P., et al. (1995). "Sorbent Capture of Nickel, Lead, and Cadmium in a Laboratory Swirl Flame Incinerator.” Combustion and Flame 100: 241-250.

Linak, W. P. and J. O. L. Wendt (1993). "Toxic Metal Emissions From Incineration: Mechanisms and Control." Progress in Energy and Combustion Science 19: 145185.

Linak, W. P. and J. O. L. Wendt (1998). "Partitioning of the Refractory Metals, Nickel and Chromium, in Combustion Systems." Combustion Science and Technology: 29.

Mwabe, P. O. and J. O. L. Wendt (1996). Mechanisms Governing Trace Sodium Capture by Kaolinite in a Downflow Combustor. Twenty-Sixth Symposium (International) on Combustion, Naples, Italy.

Perkin-Elmer (1982). User's Manual for the Model 2380 Atomic Absorption Spectrophotometer, Perkin-Elmer.

Punjak, W. A., et al. (1989). "High-Temperature Adsorption of Alkali Vapors on Solid Sorbents.” AIChE Journal 35(7, July): 1186-1194.

Rizeq, R. G., et al. (1994). "Predictions of metals emissions and partitioning in coal-fired combustion systems.” Fuel Processing Technology 39: 219-236.

Rizeq, R. G. and F. Shadman (1989). "Alkali-Induced Agglomeration of Solid Particles in Coal Combustors and Gasifiers." Chemical Engineering Communication 81: 83-96.

Scotto, M. V. (1992). Mechanisms Governing the Abatement of Metal Emissions From Waste Incineration. Chemical Engineering, Tucson, University of Arizona: 360.

Scotto, M. V., et al. (1992). Hazardous Waste Incineration: The In-situ Capture of Lead by Sorbents in a Laboratory Down-Flow Combustor. Twenty-Fourth Symposium (International) on Combustion.

Scotto, M. V., et al. (1994). "Metal Capture by sorbents in combustion processes." Fuel Processing Technology 39: 357-372. 
Serne, R. J., et al. (1996). Characterization of Radionuclide-Chelating Agent Complexes Found in Low-Level Radioactive Decontamination Waste, Richland, WA, Pacific Northwest National Laboratory: 147.

Shadman, F. and W. A. Punjak (1988). "Thermochemistry of Alkali Interactions with Refractory Adsorbents.” Thermochimica Acta: 141-152.

Siemer, D. D. (1986). Atomic Absorption Spectroscopy. Materials Characterization. R. E. Whan, ASM International. 10: 43-59.

Sill, C. W. (1988). "Volatility of Cesium and Strontium from a Synthetic Basalt." Nuclear and Chemical Waste Management 8: 97-105.

Uberoi, M., et al. (1990). "The Kinetics and Mechanism of Alkali Removal from Flue Gases by Solid Sorbents." Progress in Energy and Combustion Science 16: 205211.

Uberoi, M. and F. Shadman (1990). "Sorbents for Removal of Lead Compounds from Hot Flue Gases.” AIChE Journal 36(2): 307-309.

Uberoi, M. and F. Shadman (1991). "High-Temperature Removal of Cadmium Compounds Using Solid Sorbents.” Environmental Science and Technology 25(7): 1285-1289.

Venkatesh, S., et al. (1996). "Evaluation of Mineral-Based Additives as Sorbents for Hazardous Trace Metal Capture and Immobilization in Incineration Processes." Hazardous Waste and Hazardous Materials 13(1): 73-94.

Wu, B., et al. (1995). "Multi-Functional Sorbents for the Removal of Sulfur and Metallic Contaminants from High-Temperature Gases." Environmental Science and Technology 29: 1660-1665.

Yang, H. C., et al. (1998). "Behavior of Hazardous and Radioactive Metals in a Laboratory Furnace and a Demonstration-Scale Incinerator." Environmental Engineering Science 15(4): 299-311. 


\section{Appendix B}

Manuscript of

“Trajectories of Evaporating Droplets in a Turbulent Combustor Using the One Dimensional Turbulence Model"

$$
\text { J. R. Schmidt }
$$

Department of Chemical and Environmental Engineering

The University of Arizona

2000

(Self paginated) 
TRAJECTORIES OF EVAPORATING DROPLETS IN A TURBULENT COMBUSTOR USING THE ONE DIMENSIONAL TURBULENCE MODEL

by

John Richard Schmidt

\author{
A Thesis Submitted to the Faculty of the \\ DEPARTMENT OF CHEMICAL AND ENVIRONMENTAL ENGINEERING \\ In Partial Fulfillment of the Requirements \\ For the Degree of \\ MASTER OF SCIENCE \\ WITH A MAJOR IN CHEMICAL ENGINEERING \\ In the Graduate College \\ THE UNIVERSITY OF ARIZONA
}

2000 


\section{STATEMENT BY THE AUTHOR}

This thesis has been submitted in partial fulfillment of requirements for an advanced degree at the University of Arizona and is deposited in the University Library to be made available to borrowers under rules of the Library.

Brief quotations from this thesis are allowable without special permission, provided that accurate acknowledgment of source is made. Requests for permission for extended quotation from or reproduction of this manuscript in whole or in part may be granted by the head of the major department or the Dean of the Graduate College when in his or her judgement the proposed use of the material is in the interest of scholarship. In all other instances, however, permission must be obtained from the author.

\section{SIGNED:}

APPROVAL BY THESIS DIRECTOR

This thesis has been approved on the date shown below:

Jost Otto Lutz Wendt, Ph D

Date

Professor and Head

Department of Chemical and

Environmental Engineering 


\section{ACKNOWLEDGEMENTS}

It is with much gratitude that I acknowledge professor Jost Wendt for his support, guidance and help. Most of all for allowing me the opportunity to return to my studies after a long absence in industry. Returning to the rigors and discipline of the academic life was not easy, but Dr. Wendt remained patient and understanding.

Also, the completion of this work would have never come about in a timely manner, if it were not for the advice and guidance of Dr. Alan Kerstein of Sandia National Laboratories. His keen interest in this project and the sharing of his technical brilliance were of great help.

Funding from the Department of Energy under project number DEFG0797ER14831 was greatly appreciated.

Dr. Tom Dreeben was kind enough to give me a highly developed ODT proper FORTRAN code which was the starting point for the two-phase flow modeling I did. I also thank him for his patience as I learned his code. Many people offered up valuable advice and help at various stages of my research. While I cannot possibly thank everyone by name, I would like to name Marvin Landis, Dr. Sheldon Davis, Scot Wunsch, Vebjorn Nilsen, Tereck Echekki, Art Fernandez, John Hewson, Tom Gale, Greg Ogden and Wayne Seams.

I thank my mother, Aida Villagran Schmidt, for her love faith and encouragement were essential in bringing this work to completion. The kindness, love and encouragement of my brother (Henry) and sisters (Frances, Aida, Anne, and Maria [niece]) were of great comfort in times of distress.

I thank my many friends, both recent and lifelong for their help and encouragement.

Most importantly I thank God, the source of life, for the plethora of gifts which he has bestowed on me. Both the natural gifts which allowed me to do the engineering portion of this work and the everyday gifts (e.g. food, clothing, ...). His church has also brought me much support, encouragement, and consolation over the many years of my studies. Also the patronage of Mary has been instrumental in my studies, for which I am very grateful. 
"Burn from my brain and from my breast

Sloth, and the cowardice that clings, And stiffness and the soul's arrest:

And feed my brain with better things."

- Gilbert Keith Chesterton 


\section{TABLE OF CONTENTS}

Page

List of Figures 8

List of Tables

Abstract $\quad$................................................................. 13

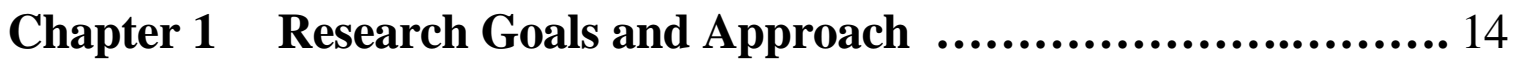

Chapter 2 One Dimensional Turbulence Model (ODT) ............. 25

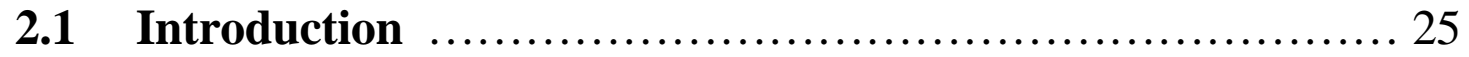

2.2 Definition of the triplet map ............................. 26

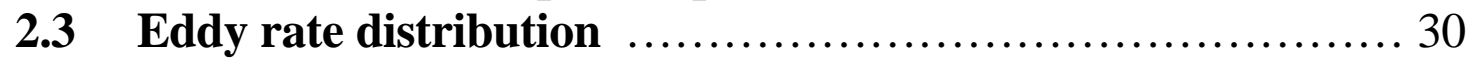

2.4 Dynamic feedback ........................................ 32

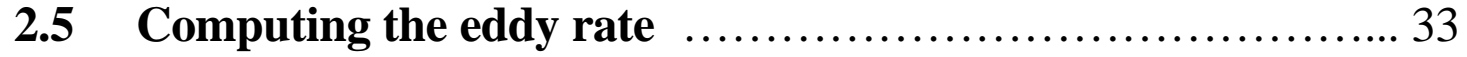

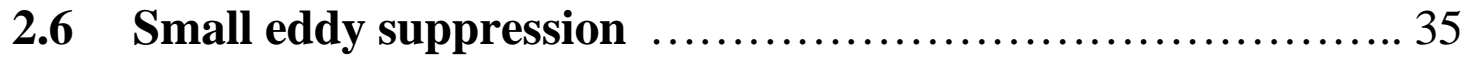

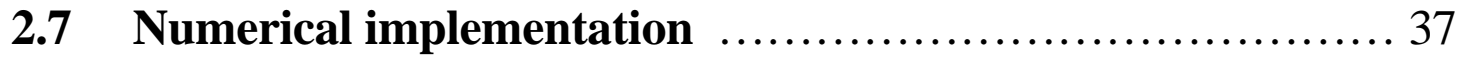

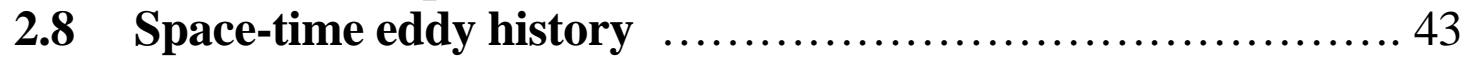

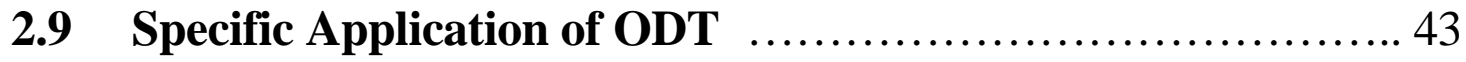

2.10 Conclusion ............................................ 46

Chapter 3 Two-Phase Flow Addition to the ODT Model ........... 52

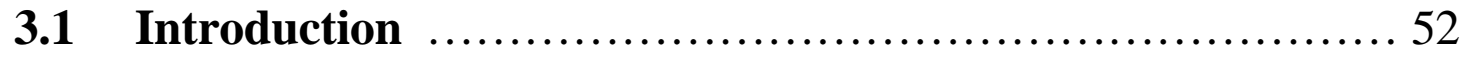

3.2 Y component of the droplet-drag law ...................... 57

3.3 X component of the droplet-drag law .......................5 59

3.4 Particle-eddy interactions in ODT .........................6 61

3.5 Particle-eddy interaction model ..........................66 66

3.6 Effect of pseudotime integration in the real time domain ....6 67

3.7 Alternative representations of particle-eddy interactions .... 75

3.8 Eddy lifetime ............................................. 79

3.9 Duration of the particle-eddy interaction ................... 81

3.9.1 Particle-eddy interactions in the $y$ direction ............ 81

3.9.2 Particle-eddy interactions in the $x$ direction $\ldots \ldots \ldots \ldots . . .82$ 


\section{TABLE OF CONTENTS -- continued}

3.9.3 Spatial $(x$ and $y)$ particle-eddy interaction box $\ldots \ldots \ldots . \ldots 88$

3.10 Conclusion and summary ............................... 96

3.11 Two-phase Flow Model Extension - type-C interactions ... 99

3.12 Numerical implementation ............................. 105

Chapter 4 Droplet Burning Model .................................. 119

4.1 Introduction ................................................ 119

4.2 Droplet heating model used by Mulholland et al. ........... 122

4.3 Introduction of the burning rate parameter ................ 127

4.4 Review of droplet-drag law used in Mulholland et al. ........ 128

4.5 Modification of trajectory model for this application ........ 130

4.6 Conclusion ............................................. 134

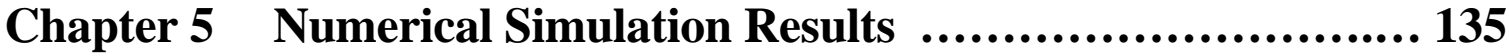

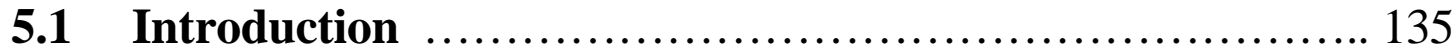

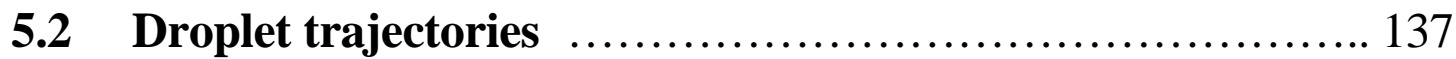

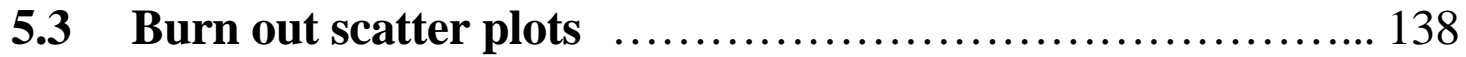

5.4 Summary of burn out points by method varied ............ 144

Chapter 6 Effects of the Two-phase Flow Parameter $\beta \quad \ldots . . . . . . . .173$

6.1 Droplet model free parameter $\beta$......................... 173

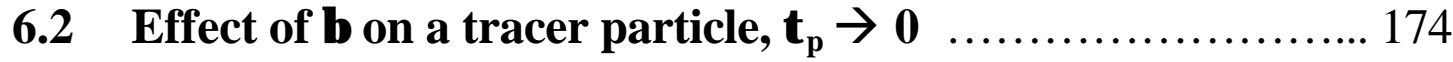

6.3 Effect on a finite inertia particle exiting the

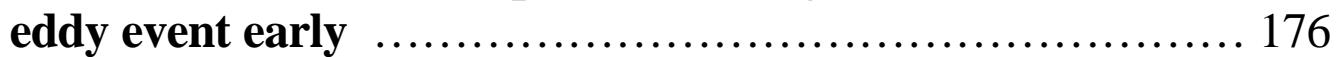

6.4 Particles with infinitely large inertia ..................... 177

6.5 Numerical results for 12 cases studied .................... 178

6.6 Conclusions ................................................ 181

Chapter 7 Conclusions and Future Work ......................... 202

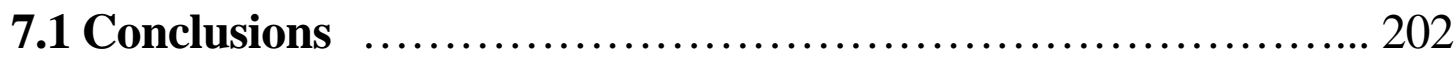

7.2 Future Work ......................................... 203 


\section{TABLE OF CONTENTS -- continued}

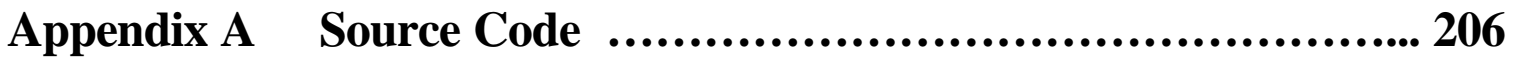

A.1 Source code for ODT and droplet submodel ................ 207

A.2 Source code for the initial conditions (Mulholland et al. "like" model) ................................................ 278

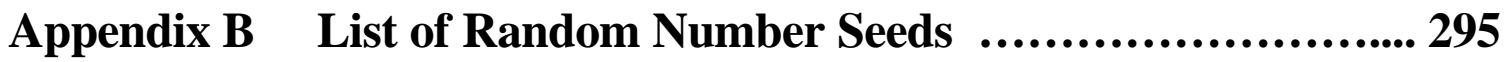

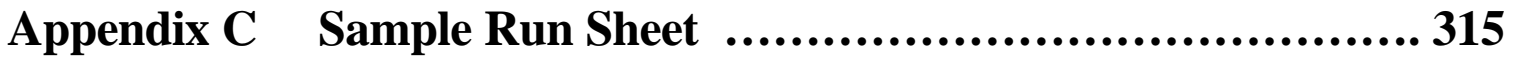

C.1 Sample input sheet for 12 cases $(\max 15)$

two-phase flow sub model .............................. 316

C.2 Sample input sheet for ODT program proper .............. 321

C.3 Sample long output sheet for one case, one realization.

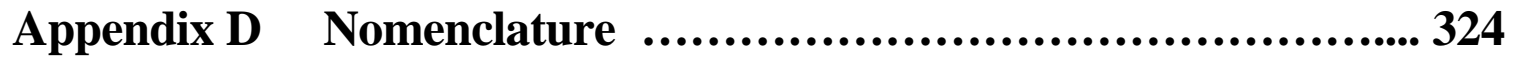

References $\quad$.............................................................. 327 


\section{List of Figures}

Figure 1.1: Drawing of the $100 \mathrm{~kW}$ furnace. ......................... 19

Figure 1.2: Measured trajectories for the nominal stream condition (Case 1) in a Type $\mathrm{C}$ turbulent flame. ............................ 20

Figure 2.1: A sample velocity profile undergoing a triplet map "eddy". .. 27

Figure 2.2: Effect of the triplet map on an initially linear velocity profile $u(y, t)$.

Figure 2.3: Illustration of a pointer, "clock" choosing the next logical process code to execute. ....................................... 38

Figure 2.4: General logic flow for an ODT sub program with the droplet submodel added.

Figure 2.5: Pictorial representing the triplet maps in space-time coordinates. 45

Figure 2.6: Measured mean temperature and axial velocity profiles. ..... 47

Figure 2.7: Selected mean velocity profiles. ......................... 48

Figure 2.8: Selected mean temperature profiles. ....................... 49

Figure 2.9: Mean and instantaneous velocity profiles. ................... 50

Figure 2.10: Mean and instantaneous temperature profiles. ............. 51

Figure 3.1: Tracer particle paths .................................. 54

Figure 3.2: Particles with an initial velocity $\ldots \ldots \ldots \ldots \ldots \ldots \ldots \ldots \ldots . \ldots 6 . \ldots \ldots$

Figure 3.3: The procedure for implementing particle-eddy interactions which avoids double counting. ................................. 70

Figure 3.4: Particle-eddy interactions cause the path to deviate and may exit the interaction box by one of the horizontal surfaces before the eddy lifetime has expired.

Figure 3.5: The trajectories of particles with varying inertia (low to high) are shown in pseudo time during particle-eddy interactions $(\mathrm{A}),(\mathrm{C})$, and $(\mathrm{E})$.

Figure 3.6: Diagram A shows the trajectory of a tracer particle for a particle-eddy interaction using the simple model.

Figure 3.7: Particle paths can exit the interaction box and reenter the box due to particle eddy interactions.

Figure 3.8: Effect of non-zero $x$ component eddy velocity on the particle-eddy interaction time.

Figure 3.9: Time series qualitatively demonstrating the various affects of eddy velocities on particle trajectories. 


\section{List of Figures -- continued}

Figure 3.10: Particle trajectories in the moving reference frame of the eddy. ..................................................... 95

Figure 3.11: Multiple type-C interactions may occur at the same time. . 102 Figure 3.12: Possible type-I and type-C interactions for two different initial velocities. ................................................. 103

Figure 3.13: Actual $\Delta t_{\text {drop }}$ is only a fraction of the maximum $\Delta t_{\text {drop }}$ due to the implementation strategy of the two-phase flow submodel. ...... 109

Figure 5.1: Trajectories from Case 1 chosen to exemplify the extremes of spread in the $x$ and $y$ directions of the burn out points. ............ 150

Figure 5.2: Burn out points for variation in initial diameter, D. ........ 152

Figure 5.3: Burn out points for variation in initial diameter, D. ........ 153

Figure 5.4: Burn out points for variation in initial diameter, D. ........ 154

Figure 5.5: Burn out points for variation in initial velocity, V. ......... 155

Figure 5.6: Burn out points for variation in initial velocity, V. ......... 156

Figure 5.7: Burn out points for variation in initial velocity, V. ......... 157

Figure 5.8: Burn out points for variation in initial droplet spacing, s/D. 158

Figure 5.9: Burn out points for variation in initial droplet spacing, s/D. 159

Figure 5.10: Burn out points for variation in initial droplet spacing, s/D. 160

Figure 5.11: Burn out points for variation in initial injection angle, $\theta$. .. 161

Figure 5.12: Burn out points for variation in initial injection angle, $\theta$. .. 162

Figure 5.13: Burn out points for variation in initial injection angle, $\theta$. .. 163

Figure 5.14: Comparison of experimental measurements and simulated burning droplets.

Figure 5.15: Comparison of experimental measurements and simulated burning droplets.

Figure 5.16: Comparison of experimental measurements and simulated burning droplets. 166

Figure 5.17: Comparison between the experimentally measured spread in burn out end points and the spread predicted by the simulation. . . 167

Figure 5.18: Comparison between the experimentally measured spread in burn out end points and the spread predicted by the simulation. ... 168

Figure 5.19: Comparison between the experimentally measured spread in burn out end points and the spread predicted by the simulation. . . 169

Figure 5.20: Variation in the Y component of the droplet position as a function of the input conditions. 


\section{List of Figures -- continued}

Figure 5.21: Variation in the $Y$ component of the droplet position as a function of the input conditions.

Figure 5.22: Variation in the $Y$ component of the droplet position as a function of the input conditions.

Figure 6.1: Effects of the free parameter $\beta$ on the eddy interaction box (A) and on the particle velocity (B) at the moment of the eddy interaction.

Figure 6.2: Effects of $\beta$ on one real particle with an initial y velocity. ... 176

Figure 6.3: Variation of case 1 as a function of $\beta$. .................. 182

Figure 6.4: Variation of case 2 as a function of $\beta$. ................... 183

Figure 6.5: Variation of case 3 as a function of $\beta . \ldots \ldots \ldots \ldots \ldots \ldots . \ldots 184$

Figure 6.6: Variation of case 4 as a function of $\beta$. ................... 185

Figure 6.7: Variation of case 5 as a function of $\beta$. ................... 186

Figure 6.8: Variation of case 6 as a function of $\beta . \ldots \ldots \ldots \ldots \ldots \ldots \ldots . \ldots \ldots 7$

Figure 6.9: Variation of case 7 as a function of $\beta$. .................. 188

Figure 6.10: Variation of case 8 as a function of $\beta$. .................. 189

Figure 6.11: Variation of case 9 as a function of $\beta$. ................. 190

Figure 6.12: Variation of case 10 as a function of $\beta$. .................. 191

Figure 6.13: Variation of case 11 as a function of $\beta$. .................. 192

Figure 6.14: Variation of case 12 as a function of $\beta$. .................. 193

Figure 6.15: Variation in the $Y$ direction as a function of

Diameter and $\beta$.

Figure 6.16: Variation in the $\mathrm{Y}$ direction as a function of velocity and $\beta$.

Figure 6.17: Variation in the $Y$ direction as a function of initial interdroplet spacing and $\beta$.

Figure 6.18: Variation in the $Y$ direction as a function of initial

.....injection angle and $\beta$.

Figure 6.19: Variation in the axial direction as a function of initial diameter and $\beta$.

Figure 6.20: Variation in the axial direction as a function of initial velocity and $\beta$.

Figure 6.21: Variation in the axial direction as a function of initial droplet spacing and $\beta$. 


\section{List of Figures -- continued}

Figure 6.22: Variation in the axial direction as a function of

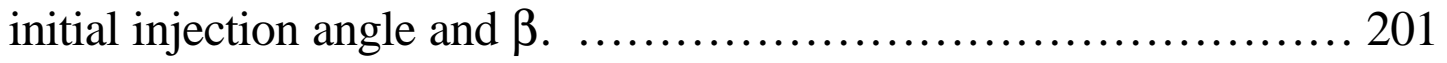




\section{List of Tables}

Table 5.1 Droplet Stream Test Conditions $\ldots \ldots \ldots \ldots \ldots \ldots \ldots \ldots \ldots \ldots$ 


\begin{abstract}
In the incineration of liquid hazardous wastes there exist "rogue" droplets (>300 $\mu \mathrm{m}$ diameter) which penetrate past the flame zone and burn as isolated droplets in the postflame gasses. Detailed knowledge of the droplet burnout points are essential to keeping the destruction removal efficiency in excess of the $99.99 \%$ required. The spread in trajectory endpoints of individual evaporating droplet streams injected into a turbulent combustor was investigated numerically. Results are in good agreement with the measurements. Correlation between the spread in the burnout points and initial droplet size, initial droplet velocity, interdroplet spacing, and droplet injection angle were investigated. The numerical investigation utilizes the novel One Dimensional Turbulence (ODT) $\{$ Kerstein (1999) $\}$ for the time developing fluid velocity and temperature fields with a new two phase flow model for predicting particle trajectories. The droplet heating/burning model used by Mulholland et al. (1991) is modified for application to this thesis.
\end{abstract}




\section{Chapter 1}

\section{Research Goals and Approach}

Two-phase flows are designated as flows in which there are two distinct phases [Sirignano (1999)]. Gas-liquid and solid-gas are the two most common. Examples include bubbles rising in a liquid; liquid being sprayed into a gas phase, such as a fuel into a combustor; and a dusty gas, solid particles dispersed in a gas phase. Most two-phase flow applications involve one of the phases occupying a relatively small volume fraction. The phase with the largest volume fraction is referred to as the continuous phase. The other phase is referred to as the discrete phase. The example of bubbly flow given above would have the gas phase as the discrete phase, and the liquid phase as the continuous phase. However, the example of fuel being sprayed into a combustor would be arranged in the opposite order. The liquid phase is the discrete phase and the gas phase is the continuous phase.

The modeling of two-phase flow systems accurately is of economic importance because of the multitude of industrial manufacturing world-wide

which uses this technology (Sirignano, 1999; Stock, 1996). Just a few examples are, spray drying, spray coating, pulverized coal combustion, and even such obscure applications as the entrainment of prescription drugs as an 
aerosol so that patients may ingest the drugs through their lungs rather then through the stomach. Using this method enables the drug to get into the blood stream much more quickly and lessens the possibility of destructive reactions with the gastric fluids.

Another area of two-phase flow modeling which is of major importance is in the area of combustion. Here the challenge of turbulence modeling is added to the already difficult modeling of two phase flows.

The motivation of the present work lies in the existence of a large volume of liquid hazardous waste in virtually perpetual storage by the Department of Energy (DOE). Some of the storage containers housing these hazardous wastes have leaked and contaminated the surrounding soil. This has motivated the DOE to look at other ways to handle, perhaps dispose of these liquid hazardous wastes. A good method of disposing of these wastes is thermal reaction or incineration. Specifically the incineration of metal containing liquid hazardous wastes provided the funding for this project. The United States law in many cases (such as for principal organic hazardous constituents) requires a $99.99 \%$ destruction of the organic hazardous material (Wendt, 1994). In a few cases (e.g. polychlorobiphenyls) a destruction level of $99.9999 \%$ is required by law. 
Even though this destruction level must only be demonstrated during test burns, this places a tremendous burden on the proper design and implementation of these incineration systems to assure the safety of those working in the incineration plant and those people living in the area. Typically the incineration system consists of an ignited gas fired combustor into which the liquid hazardous waste is sprayed through a relatively large bore spray nozzle. Since these are wastes, there are large variations in the composition and viscosity of these wastes. It is this wide range of viscosity that requires the use of a large bore spray nozzle so the spray nozzle does not clog up and cause a furnace shutdown and cleaning out of the nozzle by a HazMat team (Kramlich, et al., 1984).

Studies of the incineration systems showed the existence of large "rogue" droplets which penetrated the flame zone and burn as individual droplets (Wendt, 1994). The existence of rogue droplets, which are approximately $10 \mathrm{X}$ the size of the mean droplet, causes a legitimate concern for the safety of those who may come in contact with the exhaust fumes of the furnace, if the droplets traverse the hot zone of the furnace with out being combusted. One rogue droplet (10X the mean radius) which is only $80 \%$ converted out of two million mean size droplets is enough to fail the $99.99 \%$ conversion 
requirement. The systematic study and modeling of rogue droplets is essential to the safe design of these systems. Motivated by this, Mulholland et al. (1991) performed a number of systematic experiments and some numerical modeling with the intention of improving the state-of-the-art design technology.

Mulholland et al. took great pains in the experimental arrangement to be able to reproduce droplet streams of nearly identical initial diameter, velocity, inter-droplet spacing, and injection angle into a $100 \mathrm{~kW}$ (lab scale) turbulent combustor. A fuel oil was used as the hazardous waste surrogate. The droplets themselves were designed to be representative of the rogue droplets with an initial diameter greater than $300 \mu$. The stream was injected through an opening $\sim 14 \mathrm{~cm}$ above the centerline. The centerline of the reactor is also the position of the gas burner. The burner was set to produce a type $\mathrm{C}$ flame. A type $\mathrm{C}$ flame uses a high combustion air swirl (swirl number $=1.3$ in this case) therefore the flame is stabilized close to the burner. The injected droplet stream proceeded in a straight line trajectory until it reached the high shear layer of the combustion gas flow, at which point the droplet stream broke into what appeared to be random individual drops. The droplets passed through the flame zone and subsequently ignited 
and burned out. Mulholland et al. performed careful position measurements of the droplet ignition and burnout. They found a surprising large spread (maximum axial distance traveled before complete burning minus the minimum axial distance traveled before complete burning) in the burnout points for all cases. A schematic of the experimental apparatus used by Mulholland et al. is reproduced in Figure 1.1. It shows a drawing of the 100 $\mathrm{kW}$ combustor and relevant equipment. Figure 1.2 shows the observed range of trajectory endpoints (location at which burn out was completed) for one specific test case (the nominal case). The authors were surprised because of the droplet streams themselves were highly reproducible and in the absence of turbulence one would expect the spread in the burnout to be small. Hence, the large spread was properly attributed to the highly turbulent nature of the fluid flow in the lab scale kiln. Attempts by the authors to model the mean trajectory of the droplets were of limited success They could not hope to predict the spread in the endpoints because of the failure of their model to capture properly the turbulent flow in the reactor.

What is needed in order to render incineration completely safe, is a tool that can predict the time/temperature/environment history of every single fragment of waste that is introduced into the reactor. In turbulent flows, 


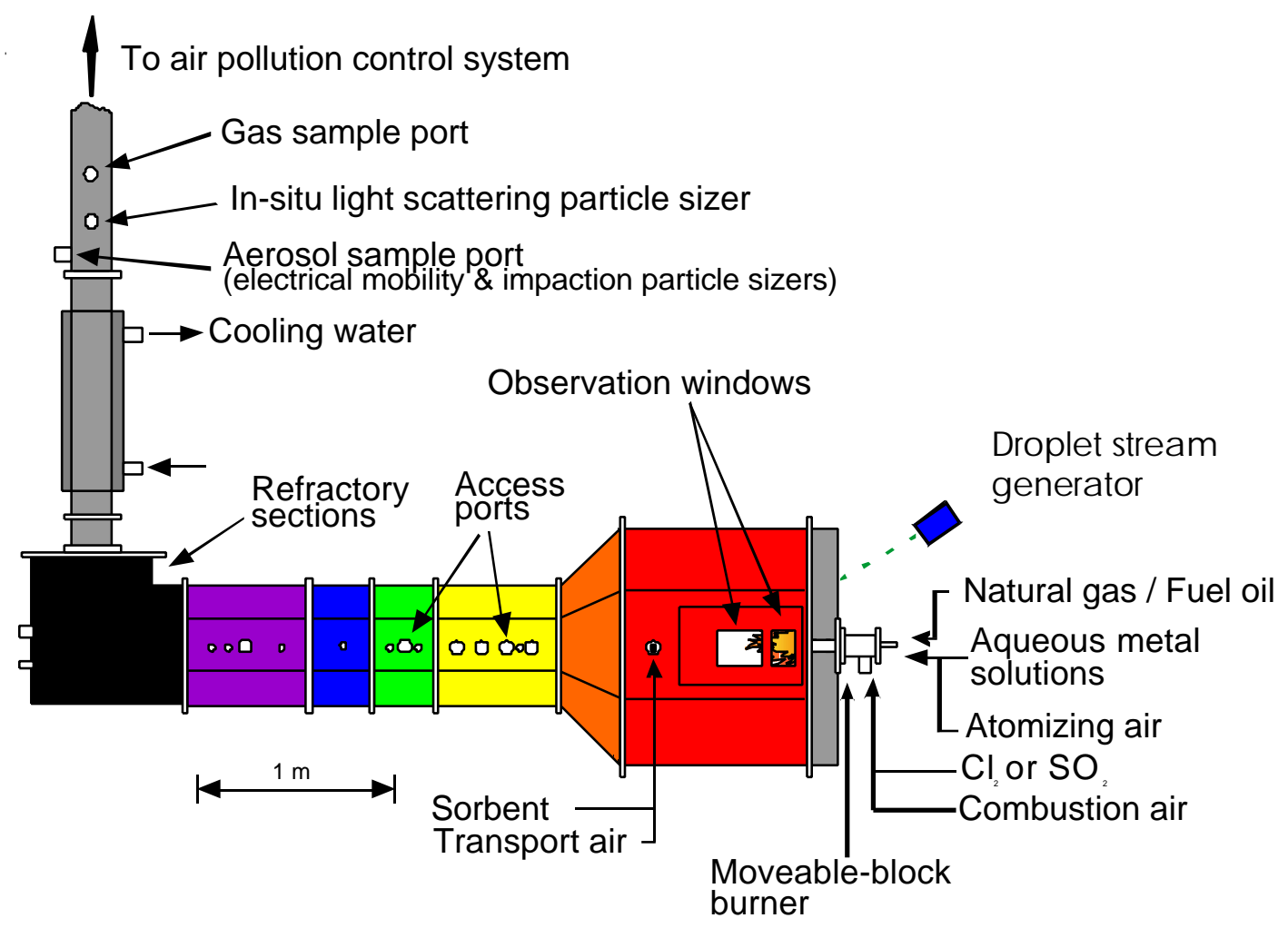

Figure 1.1: Drawing of the $100 \mathrm{~kW}$ furnace. Modular furnace for turbulent diffusion flame study, with monodisperse droplet stream generator.

different fragments of waste that are introduced in an identical manner have different time/temperature/environment histories. See Figure 1.2 These are not completely random. A description of the mean or median time/ temperature/environment history is not adequate. Rather it is required to find a modeling method which captures the relevant physics of the turbulent fluid flow and the two-phase flow, in as simple a formulation as possible. Also a model for predicting the droplet heating and evaporation is necessary 

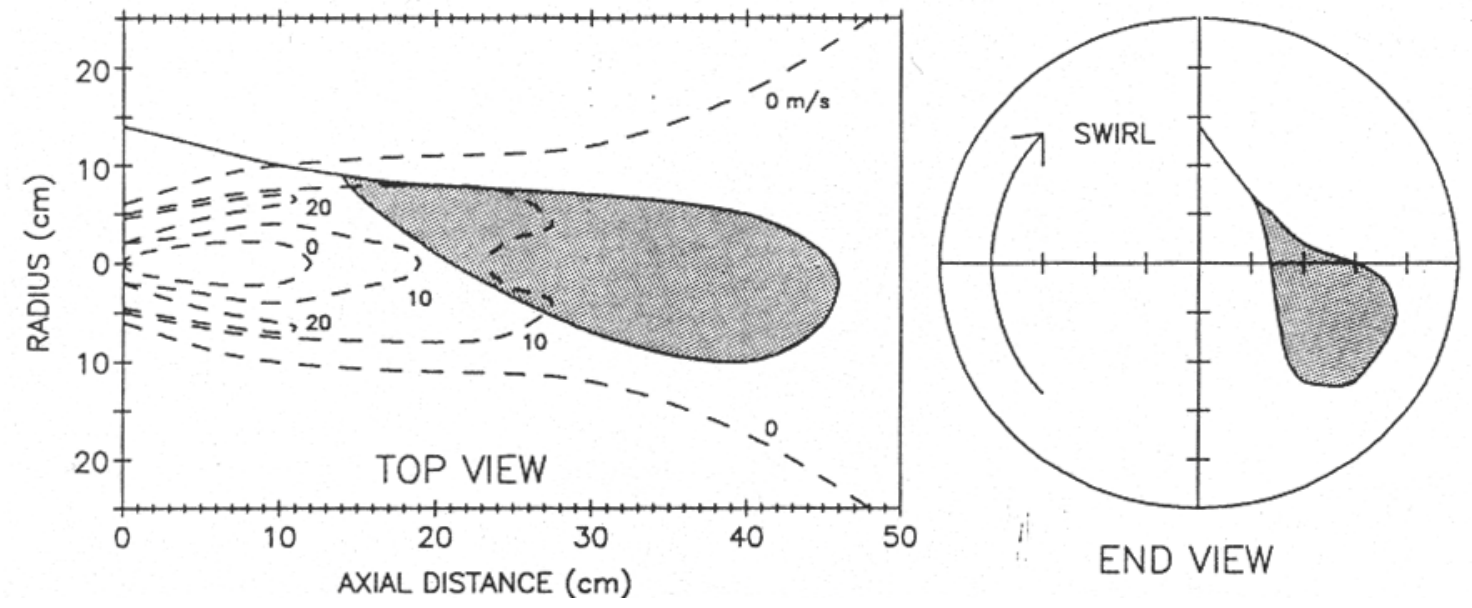

Figure 1.2: Measured trajectories for the nominal stream condition (Case 1) in a Type $C$ turbulent flame. The shaded area represents the range of trajectory endpoints. The gas phase axial velocity profile is superimposed on the top view (dashed lines).

to capture the spread in the burn out points is needed. The successful modeling of the spread of these rogue droplets would provide a significant contribution to further the design of incineration facilities to assure the wastes be disposed of in a safe and efficient manner.

The novel One Dimensional Turbulence model (ODT) developed by Kerstein (1999) seemed uniquely suited to fulfilling the need to predict accurately and simply this turbulent gas flow. (ODT is described in detail in Chapter 2.) The kiln has the requisite one homogeneous dimension $(\theta)$ and the gas flow is predominantly down the axis of the reactor yet the flow is 
highly turbulent. The Reynolds number (Re) based on the gas fuel nozzle diameter is of order 10,000 .

Other methods such as Large Eddy Simulation (LES) and Direct Numerical Simulation (DNS) could probably be applied successfully to solve this problem. Unlike LES, ODT would resolve the scales necessary to capture the smallest eddies. Albeit only in the one dimension (transverse to the flow) which ODT keeps track of. In addition LES needs a subgrid model to account for energy losses due to such things as viscosity. Since ODT keeps track of the smallest eddy scales, the standard viscous dissipation equation takes care of this. An additional consideration is that the LES method would need a subgrid model to account properly for the two-phase flow portion of the model. Because ODT captures the smallest scales, no additional assumptions are needed for implementation into ODT.

The state-of-the-art DNS modeling of turbulent flows is restricted by the maximum Re allowable due to the memory and speed of the fastest computers available. The memory requirement for DNS goes as the number of grid points needed to resolve fully the turbulent (Kolmogorov) scale raised to the third power. Since ODT keeps track of only one dimension, the required memory scales directly with the number of grid points needed to 
resolve the turbulent scale. Though progress to bring DNS to the high Re regime continues on all fronts, there is still much work to be done in order to use DNS for practical industrial applications. Since the ODT model capitalizes on the symmetry of the flow and utilizes innovative methods to model three-dimensional turbulent behavior on a one-dimensional domain, it is the model of choice for this highly turbulent flow configuration.

The existing ODT formulations did not have the option for two-phase flow computations already built in. Consequently a two-phase flow submodel to ODT needed to be developed for this work.

The development of two-phase flow models with a turbulent gas phase is traditionally broken into three categories, 1-way coupling, 2-way coupling, and 4-way coupling. The simplest is 1-way coupling, in this case the turbulent contribution of the dispersed phase is negligible. The turbulent field is much more likely to gain kinetic energy from the gas flow rather than from the flow of the dispersed phase (the liquid droplets in this case). Here, the fluid affects the particle, but the particle does not affect the fluid. This is often symbolized as: fluid $\rightarrow$ particle (one arrow).

The 2-way coupling flows are the next complicated. In this case the volume fraction of the dispersed phase is high enough that the mean flow of 
the particles can induce turbulent motion in the fluid. (Also as in the 1-way coupling the turbulent fluid motion induces motion in the particles. Hence the title, 2-way coupling.) This is symbolized as fluid $\leftarrow \rightarrow$ particle (two arrows).

The most complicated of the flows is referred to as 4-way coupling. In this case, the volume fraction of the dispersed phase is so large, that the dispersed phase particles not only affect the fluid flow, but also affect each other by way of collisions or near collisions (wake or boundary layer interactions). This is symbolized as fluid $\leftarrow \rightarrow$ particle $\leftarrow \rightarrow$ particle (four arrows). Elghobashi $(1991,1994)$ shows the range in which the three types of coupling are of importance.

This model utilizes the 1-way coupling assumption. A Lagrangian framework for the particle is used with the key forces on the particle considered to be a modified Stokes drag and gravity. Sirignano (1999) states that good engineering analysis can be performed using only modified stokes drag and the gravitational force. A majority of the state-of-the-art publications in the area of two-phase flow use the 1-way coupling assumption along with a drag force to compute the equation of motion of particles. 
In this thesis, the salient features of Kerstein's one-dimensional turbulence model are described in Chapter2. This is followed by a description of how two-phase flow can be added to the ODT model. Chapter 4 designates the droplet burning model also necessary to describe experimental droplet trajectory endpoints. While the following chapter the results and comparisons with Mulholland et al.'s data. Chapter 6 shows additional results showing the effects of the empirical parameter $\beta$, which appears in the formulation of the ODT two-phase flow submodel. The conclusions are presented in Chapter 7. 


\section{Chapter 2}

\section{One Dimensional Turbulence Model (ODT)}

\subsection{Introduction}

Kerstein (1999) developed a one-dimensional Monte Carlo modeling technique for turbulent mixing of velocity and scalar fields. He develops both time developing and spatially developing models, but only the time developing model will be discussed here since that is what was used in this application. The ODT approach represents turbulent advection by a random sequence of "eddy" maps applied to a one-dimensional computational domain. Profiles of the advected velocity component $(u)$ and the advected scalars evolve on this domain. Equations for the turbulent flow field are not solved explicitly, rather the viscous and diffusive equations are solved,

$$
\begin{aligned}
& \frac{\partial u}{\partial t}=v \frac{\partial^{2} u}{\partial y^{2}}-\frac{1}{\rho} \frac{\partial p}{\partial x} \\
& \frac{\partial \theta}{\partial t}=\kappa \frac{\partial^{2} \theta}{\partial y^{2}}
\end{aligned}
$$

where $\theta$ can be any advected scalar (e.g. temperature, species concentration, etc.) and $\kappa$ is the corresponding diffusion coefficient. In this thesis the last term in Equation 2-1 was set to zero, see Section 2.9 for a discussion of this. 
In order for ODT to be used there must be a minimum of one homogeneous direction. There should be a predominantly streamwise direction. The one dimension in the One Dimensional Turbulence model is transverse to the mean flow. See Figure 2.1 for an example flow configuration. It is informative to note that the application of Equation 2-1 under the given conditions would yield the well known laminar fluid flow solution without the intervention of triplet maps. The ODT model implements triplet maps or eddies as instantaneous rearrangements of the velocity $u(y, t)$ field.

\subsection{Definition of the triplet map}

The triplet map has a starting point $y_{\mathrm{o}}$ and a length $l$ which is sampled randomly from an eddy distribution rate. The mapping y $\rightarrow \overline{\mathrm{y}}$ rule for a triplet map is given by:

$$
\bar{y}=\left\{\begin{array}{cl}
y_{o}+1 / 3\left(y-y_{o}\right) & y_{o}<\bar{y}<y_{o}+1 / 3 l \\
y_{o}+2 / 3 l-1 / 3\left(y-y_{o}\right) & y_{o}+1 / 3 l<\bar{y}<y_{o}+2 / 3 l \\
y_{o}+2 / 3 l+1 / 3\left(y-y_{o}\right) & y_{o}+2 / 3 l<\bar{y}<y_{o}+l \\
y & \text { otherwise }
\end{array}\right.
$$

Where $\bar{y}$ is the y profile after the instantaneous rearrangement. 


\section{One-dimensional turbulence}

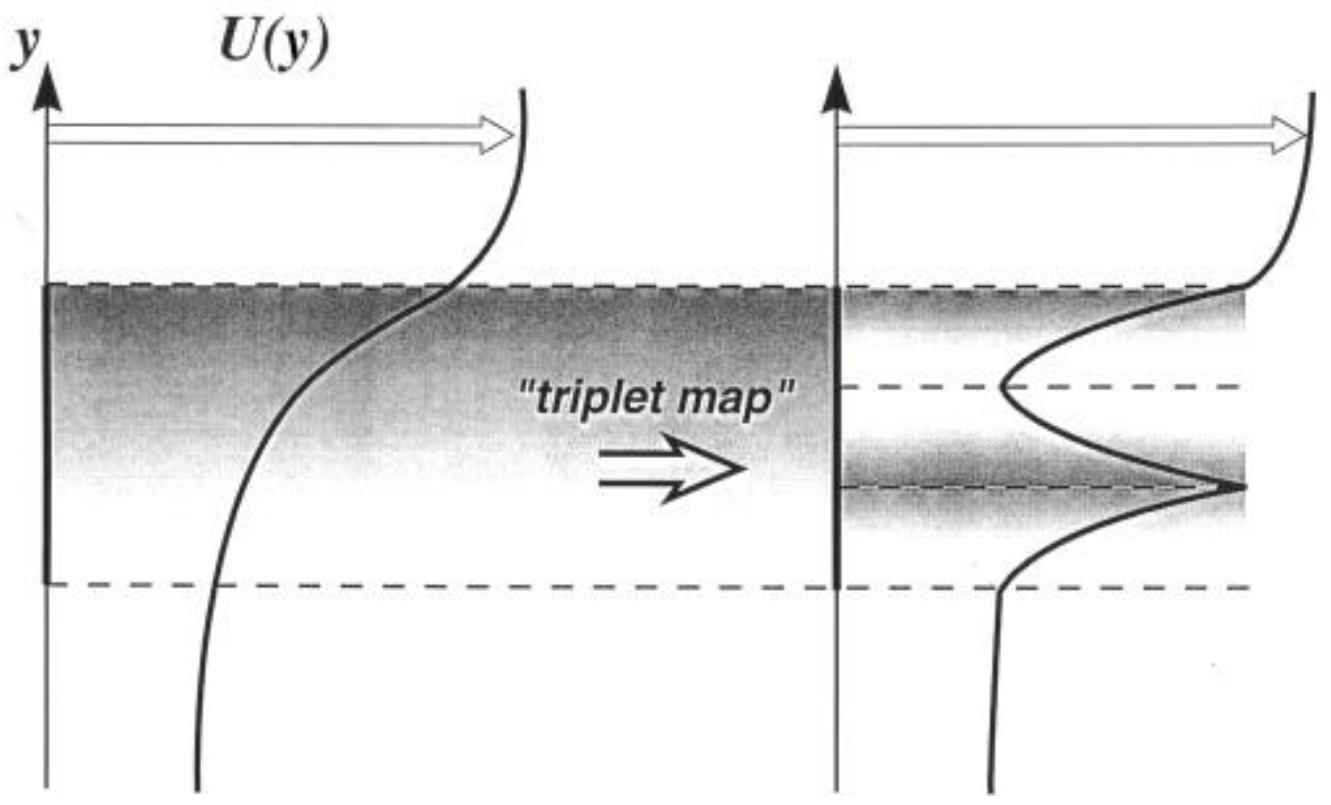

Figure 2.1: A sample velocity profile undergoing a triplet map "eddy".

The desired attribute of the triplet map is to provide a means of mimicking the increase in strain intensity, the decrease in strain length scale and the increase in mixing due to eddies in actual turbulent flow. This mapping rule assures that closest neighbors after the mapping event were no more then 3 cells apart before the mapping event. Hence the increased strain rate and shortening length scale is attained without undue introduction of discontinuities. As a matter of fact, in the limit as the discretized cell size 
becomes infinitesimally small, so that the velocity field becomes continuous, the resulting velocity profile $u(y, t)$ has no discontinuities, only discontinuous derivatives. This can be seen in Figure 2.1 as well as Figure 2.2 (A) and (B). Using the continuous analog to describe the triplet map: the original $u$ profile is reduced by a factor of three, and a copy is placed on both the top third and the bottom third of the eddy domain. For the middle third, the reduced image is inverted. The added benefit of the triplet map can be seen in Figure 2.2 (C) where neighboring cells (or fluid elements) before a triplet map can be separated by more than one half the eddy size after the mapping, thereby achieving the desired increase in mixing which is evidenced in reallife eddies.

Fluid parcels or elements are moved instantaneously during the triplet map from one $y$ location to another. The momentum and passive scalar properties of each fluid particle or cell is preserved and remains unaffected by the instantaneous rearrangement. Though this may seem counterintuitive, this method is also used in Prandtl's Mixing Length theory for turbulent transport. Also Monin and Yaglom (1971) note that all mixing length theories transfer momentum as a passive scalar, neglecting pressure-induced momentum changes within the fluid element during exchange. A recent 

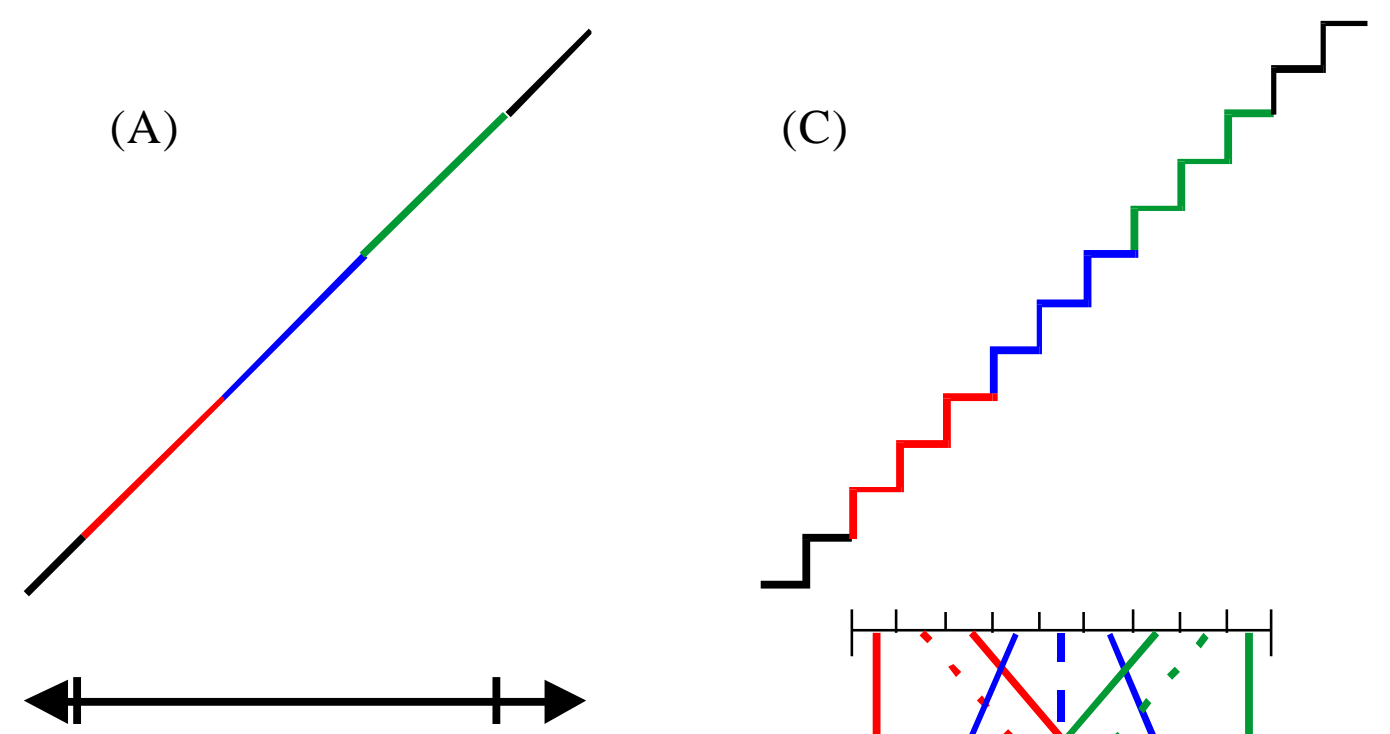

(B)
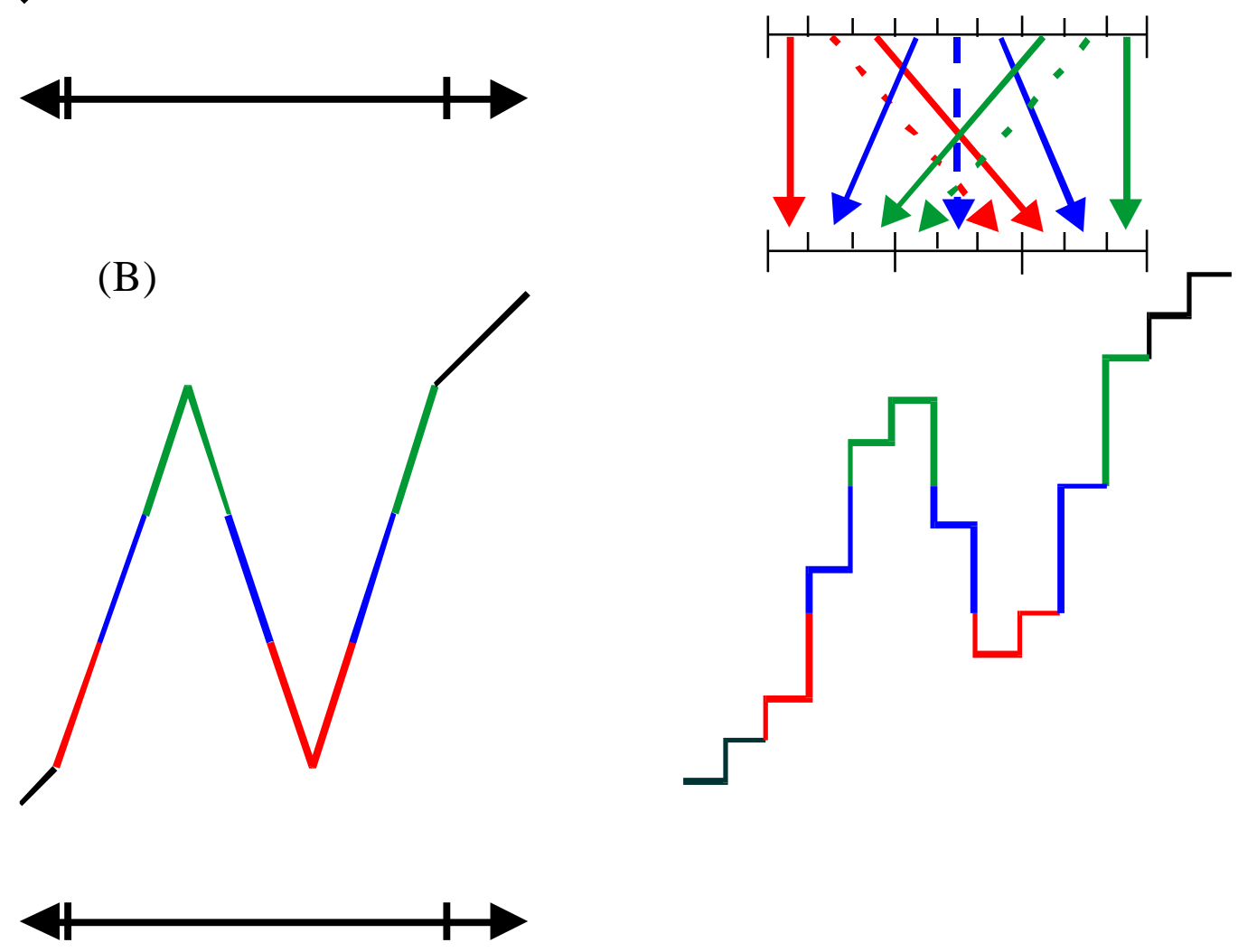

Figure 2.2: Effect of the triplet map on an initially linear velocity profile $u(y, t)$. (A) Initial profile. (B) Velocity profile after applying the triplet map to the interval denoted by ticks. (C) Discrete representation of the initial profile and illustration of the effect of a triplet map on an interval consisting of nine cells. For clarity, arrows indicating formation of the central of the three images of the original interval are dashed. Redrawn from Kerstein (1999). 
development in ODT keeps three velocity components, this in turn allows pressure scrambling (Kerstein et al., 2000)

\subsection{Eddy rate distribution}

The next challenge in the ODT model is deciding how to compute the eddy rate distribution $\lambda(l ; y, t)$, where $\lambda(l) \mathrm{d} l \mathrm{~d} y$ is the frequency of events in the size range $[l, l+\mathrm{d} l]$ per unit length along the $y$ coordinate in the range $[y$, $y+\mathrm{d} y$ ], which gives $\lambda$ units of (length ${ }^{2} \mathrm{x}$ time $^{-1}$. It is defined such that the total event rate $\mathrm{R}(t)$ (the total number of events per second) is given by

$$
R(t)=\int_{l_{\min }}^{Y} \int_{y_{\min }}^{y_{\max }} \lambda\left(l ; y_{o}, t\right) d y d l \quad \cdot \quad(2-4 A)
$$

An event is taken to be the occurrence of a triplet map. It describes the idealization of an eddy on the ODT domain. By dimensional arguments,

$$
\lambda(l)=\frac{1}{l^{2} \tau(l)} \quad(2-4 B)
$$

where $\tau(l)$ is an eddy time scale. Kerstein (1999) treats $\tau(l)$ as a local instantaneous time scale denoted $\tau(l ; y, t)$ whose evaluation is based on $u(y, t)$,

$$
\tau\left(l ; y_{o}, t\right)=\frac{l}{A \Delta u\left(y_{o}, l\right)} \quad(2-5)
$$


where $\Delta u\left(y_{0}, l\right)$ represents the velocity difference across the interval

$\left[y_{0}, y_{\mathrm{o}}+l\right]$ and $\mathrm{A}$ is a free parameter. Specifically

$\Delta u\left(y_{o}, l\right)=2\left|u_{l}\left(y_{o}+\frac{l}{2}, t\right)-u_{l}\left(y_{o}, t\right)\right|$

where

$u_{l}(y, t)=\frac{2}{l} \int_{y}^{y+l / 2} u\left(y^{\prime}, t\right) d y^{\prime} \quad . \quad(2-7)$

Hence the $\Delta u$ in Equation 2-5 is based on the mixing cup average velocity (Equation 2-7) of each half of the eddy. If these averages are shown to differ greatly (hence there is a large strain rate across the eddy) then $\tau\left(l ; y_{0}, t\right)$ is small and $\lambda\left(l ; y_{0}, t\right)$ is large. Equations (2-4) - (2-6) give

$$
\lambda\left(l ; y_{o}, t\right)=\frac{2 A\left|u_{l}\left(y_{o}, t\right)-u_{l}\left(y_{o}+\frac{l}{2}, t\right)\right|}{l^{3}} .
$$

The free parameter $\mathrm{A}$ is the only free parameter in the ODT model proper. The free parameter A was introduced to compensate for the fact that $\Delta u\left(y_{0}, l\right)$ was chosen as a matter of preference. This arbitrary choice of Equation 2-6 may in turn affect the numerical results, hence A allows fine adjustments to the eddy rate distribution. The upshot is that $\mathrm{A}$ becomes a 
turbulence intensity knob. Decreasing A decreases the level of turbulent intensity, and increasing A increases the turbulent intensity.

\subsection{Dynamic feedback}

At this point the eddy rate distribution is completely defined by the local position $\left[y_{0}, y_{0}+l\right]$ and velocity distribution $u(y, t)$. Areas of high strain rate will have a higher $\lambda\left(l ; y_{0}, t\right)$ therefore becoming more likely to have an eddy event (triplet map) than an identical area with a low strain rate. The onset of a triplet map increases the strain rate locally and hence increases the probability of spawning other eddies. Experiments have shown that this phenomenon of an eddy spawning other eddies occurs in physical flows. Kerstein (1999) has demonstrated in a number of flow simulations that a large triplet map will spawn a family of smaller triplet maps.

This is a powerful advantage of the ODT model. Flow properties (e.g. velocity variations) effect the eddy rate distribution through Equation 2-8 and the successful eddy events affect the velocity distribution (and passive scalars). This creates a feedback by increasing the strain rate which allows more triplet maps to occur. 


\subsection{Computing the eddy rate}

With the eddy rate distribution, $\lambda\left(l ; y_{0}, t\right)$ fully defined it would be possible to form the event rate,

$$
R(t)=\int_{l_{\min }}^{Y} \int_{y_{\min }}^{y_{\max }} \lambda\left(l ; y_{o}, t\right) d y d l \quad . \quad(2-9)
$$

From this, one could then obtain the time between stirring events (or eddies) by inverting the rate,

$$
\Delta t_{\text {stir }}=[R(t)]^{-1}=\left[\iint \lambda\left(l ; y_{o}, t\right) d y d l\right]^{-1} \cdot \quad(2-10)
$$

However since $\lambda$ is a function of starting position of the eddy, $y_{0}$, the length of the eddy, $l$, and the instant in time, $t$, this calculation is very expensive. As an alternative Kerstein (1999) proposes the following equivalent method based on the probability theory of a Poisson process.

At successive time increments $\Delta t$, sample the eddy parameters $y_{\mathrm{o}}$ and $l$ from specified pdfs $\mathrm{g}\left(y_{\mathrm{o}}\right)$ and $\mathrm{f}(l)$. respectively. Compute the corresponding value of $\lambda\left(l ; y_{0}, t\right)$ based on the current $u(y, t)$ profile. Then apply a triplet map to $\left[y_{0}, y_{0}+l\right]$ with probability 
$P\left(l ; y_{o}, t, \Delta t\right)=\frac{\lambda\left(l ; y_{o}, t\right) \Delta t}{f(l) g\left(y_{o}\right)} \quad, \quad(2-11)$

Which is the probability that the eddy at $y_{0}$ with length $l$ will occur. Provided that $\mathrm{f}$ and $\mathrm{g}$ are nonzero for all allowed values of $y_{\mathrm{o}}$ and $l$, Kerstein (1999) showed that this procedure is equivalent to direct sampling of the eddy rate distribution $\lambda\left(l ; y_{0}, t\right)$ in the limit as $\Delta t \rightarrow 0$.

Here $\mathrm{g}\left(y_{\mathrm{o}}\right)$ is chosen to be a pdf which gives a uniform probability of the entire $y$ domain $[0, \mathrm{Y}]$, because, there is no reason to assume there would be a preference in the eddy location. Similarly, $\mathrm{f}(l)$ is the pdf which gives the eddy length or domain. The form $\mathrm{f}(l)=\mathrm{B} l^{-2}$ is used where $\mathrm{B}$ is the pdf normalization constant when $l^{-2}$ is integrated over all possible eddy sizes. Kerstein (1991) defines the minimum triplet map as six discretized cells. The maximum allowable eddy size is taken to be any eddy which fits into the physical domain $[0, \mathrm{Y}]$. Theoretically the largest possible eddy would start at $y_{\mathrm{o}}=0$ and have a length of $l=\mathrm{Y}$. Any triplet map whose domain $y_{\mathrm{o}}$ $+l$ exceeds $\mathrm{Y}$ is suppressed on physical grounds. An eddy cannot pass through the chamber walls. 


\subsection{Small eddy suppression}

In real physical flows, the presence of viscosity dampens small scale $u$ fluctuations. This phenomenon is captured in the viscous evolution equation (Equation 2-1). However, no mechanism exists for preventing a small triplet mapping event from being driven by a large scale fluctuation. One of the very purposes of the triplet map is to increase the strain intensity (Section 2.2). The possibility exists of another (random) eddy event just after a large scale eddy, in which the smaller eddy is entirely or largely contained in just one of the previously defined eddies reduced images (the top third, the middle third, or the bottom third). Left to its own accord, this scenario would produce a plethora of small scale eddies. Note that this dynamic feedback is similar to that discussed in section 2.4 and is one of the real strengths of ODT over previous methods like LEM [Kerstein (1988)]

The small scale eddies do little to enhance advection because of their small scale. Kerstein (1999) has demonstrated this numerically, by allowing $\Delta y \rightarrow 0$ and confirming that the results became independent of $\Delta y$. This multiplication of small scale eddies is recognised as an artifact of the ODT phenomenology. (Separating eddies out as random events.) As an artifact of the model with little effect on the resulting $u$ profiles, small scale eddies 
are suppressed in order to save computaional time and mimic the role of viscosity in eddy events. Kerstein (1999) laboriously derives a relation for a viscous suppression time scale $\tau_{\mathrm{d}}$.

$\tau_{d}=l^{2} / 16 \mathrm{v}$

This is achieved by an analogy to the energy dissipation rate of a real eddy in ODT. He provides an analogy and not a derivation. As such there could be another free parameter inserted into Equation 2-12, but this is not done in order to keep empiricism down to a minimum. Using the model developed thus far, any eddy in which $\tau\left(l ; y_{0}, t\right)>\tau_{\mathrm{d}}$ is suppressed.

However, further development by Kerstein and Dreeben (2000) provides an energy relationship in which the square of $l / \tau_{\mathrm{d}}$ is taken to represent the viscous damping of eddy turnovers. The time scale $\tau\left(l ; y_{0}, t\right)$ can now be derived from this new relationship,

$$
\left(\frac{l}{\tau\left(l ; y_{o}, t\right)}\right)^{2}=\left[A\left|u_{l}\left(y_{o}, t\right)-u_{l}\left(y_{o}+\frac{l}{2}, t\right)\right|\right]^{2}-\left(\frac{l}{\tau_{d}}\right)^{2} \cdot \quad(2-13)
$$

The left-hand side is a representation, based on dimensional reasoning, of the kinetic energy per unit mass of a size $l$ eddy. While the first term on the 
right-hand side is taken to be the total amount of shear kinetic energy per unit mass which is available to drive the eddy. Under the improved energy formulation (which is used in this work) any event in which the viscous dampening estimate (the square of $l / \tau_{\mathrm{d}}$ ) exceeds the available shear kinetic energy, the negative right-hand side is interpreted as reflecting the suppression of small, dissipation-range, eddies and is therefore forbidden.

\subsection{Numerical implementation}

ODT requires the running of an ensemble of individual realizations. Using the ergodic hypothesis, the statistical averaging of the ensemble yields the mean flow properties of the turbulent flow. Consequently an individual realization can be viewed as a numerical experiment. All such realizations have identical initial conditions, but because of the randomness of triplet map implementation each realization develops in time uniquely under a variety of different processes. These processes include, but are not restricted to, turbulent motion and diffusion. This is pictorially represented in Figure 2.3 where the processes of diffusion, eddies, statistics, and droplet motion (which is discussed in Chapter 3) are given their own time lines with discrete tick marks representing events on that time line. A clock sweeps from left to right dictating the ordering of the process tasks. 


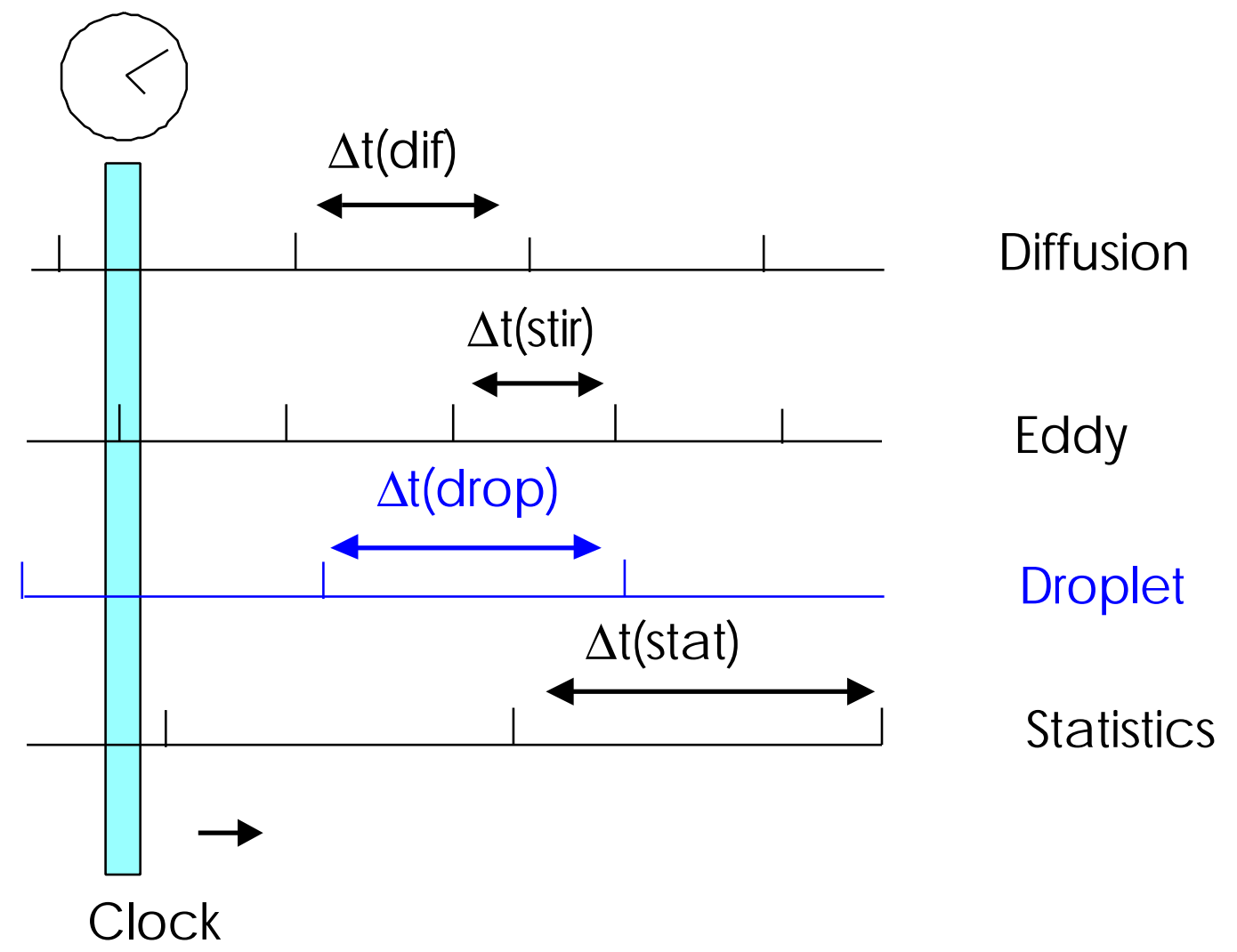

Figure 2.3: Illustration of a pointer, "clock" choosing the next logical process code to execute. Here the eddy process is chosen.

When the clock pointer reaches a tick mark on the stirring time line (as depicted in Figure 2.3) the program checks the possibility of an eddy event. The pdfs $g$ and $f$ are sampled by drawing one random number each. With $y_{\mathrm{o}}$ and $l$ chosen, $\lambda\left(l ; y_{0}, t\right)$ can be computed for that particular choice of eddy, and with a $\Delta \mathrm{t}$ given from the turbulent motion time line $\left(\Delta \mathrm{t}_{\mathrm{stir}}\right)$ the probability of that particular eddy at that particular time can be computed 
from Equation 2-11. This probability has two important uses. The first deals with whether or not the eddy is implemented. This process is executed by selecting another random number. If the probability, $\mathrm{P}\left(l ; y_{0}, t, \Delta t\right)$, computed from Equation 2-11 is less than or equal to this random number then the triplet map is implemented.

Second, the basis for using this technique (a Poison process) to determine the occurrence of triplet maps is valid only for a $\Delta \mathrm{t}_{\text {stir }}$ small enough so that the probability $\mathrm{P}\left(l ; y_{0}, t, \Delta t\right)$ is much less then 1 . This leads to the requirement that there be many more attempts at eddies than actual triplet map implementations. At first glance this may seem wasteful. However, it is still much cheaper to evaluate $\mathrm{P}\left(l ; y_{0}, t, \Delta t\right)$, with $\lambda\left(l ; y_{0}, t\right)$ at one pair of $y_{\mathrm{o}}, l$ values, many times without an eddy event taking place than evaluating Equation 2-9 over all possible combinations of $y_{\mathrm{o}}$ and $l$.

There is however one complication since $\Delta \mathrm{t}_{\text {stir }}$ is chosen in advance of the actual calculations. The possibility exists of choosing a $\Delta \mathrm{t}_{\text {stir }}$ too long and thereby generating a multitude of large $\mathrm{P}\left(l ; y_{0}, t, \Delta t\right)$ values. A large $\mathrm{P}\left(l ; y_{0}, t, \Delta t\right)$ value indicates that more then one stirring event should have occurred in that particular $\Delta \mathrm{t}_{\text {stir. }}$ This can be catastrophic since the 
probability argument which allows the use of $\mathrm{P}\left(l ; y_{0}, t, \Delta t\right)$ (Equation 2-11) to determine eddy events rather than the computing of costly pdf reconstruction (Equation 2-9) insists that the probability be small. To insure this, records are kept of the percentage of high $\mathrm{P}\left(l ; y_{0}, t, \Delta t\right)$ values. If this percentage goes above a certain acceptance level then $\Delta \mathrm{t}_{\text {stir }}$ must be decreased and the simulation re-run.

Typically the time between possible eddy events is shorter than the time between diffusion events. However the triplet maps are not always implemented, but the diffusion rate occurs predictably and therefore the diffusion events have ample opportunity to begin smoothing out the velocity and passive scalar profiles before the next eddy event. Since diffusion processes are often the most time consuming a brief discussion of the numerical method needed to solve Equations 2-1 and 2-2 is in order.

These equations were discretized using the forward Euler difference method in time $(t)$ and the central difference method in space (y). A stability analysis readily shows, \{Carnahan et al. (1969)\}

$$
\Delta t_{d i f}=\frac{\alpha(\Delta y)^{2}}{D}, \quad(2-14)
$$

where $\mathrm{D}$ is either $v$ or $\kappa$ from Equations $2-1$ or $2-2$, and $\alpha$ must be $<=0.5$. 
Intuitively one would suspect that going to an implicit method in time, such as backward Euler or the trapezoidal rule, would be beneficial since the rather stringent condition of $\alpha<=0.5$ would be relaxed and much larger time steps could be taken for the diffusion processes (which are the most time consuming calculations of the code). However, stability is not the dominant constraint on $\alpha$, but rather $\alpha$ is constrained by the frequency of the triplet map implementations. As mentioned earlier, the $\Delta \mathrm{t}_{\text {dif }}$ should be chosen so that diffusion occurs often enough to start smoothing the velocity profiles between actual eddy implementations. Therefore the ease of programming the forward Euler method and the speed of computation of that method as compared to the implicit methods make the explicit formulation the form of choice.

A general logic flow diagram for a basic ODT sub program (with a droplet model timeline included) is given in Figure 2.4. Modifications to the eddy event time line $\left(\Delta \mathrm{t}_{\text {stir }}\right)$ are indicated by a “*” in the diagram. The droplet model is the subject of chapter 3. The subprogram shown in Figure 2.4 would be executed once for each realization desired. 


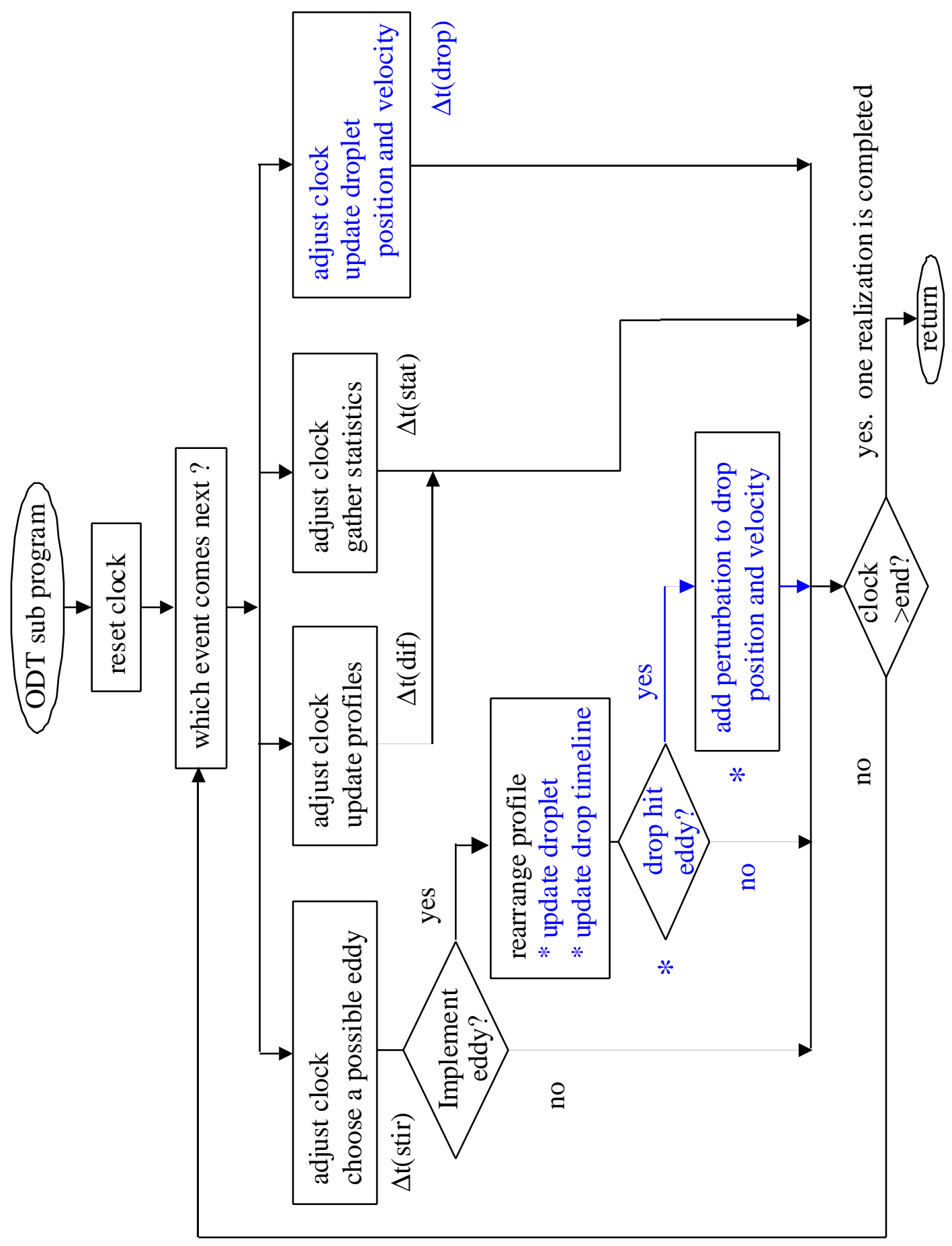

Figure 2.4: General logic flow for an ODT sub program with the droplet sub model added. This flow accounts for a single realization. 


\subsection{Space-time eddy history}

A pictorial rendering of the triplet maps, which will prove instrumental in understanding the development of the droplet sub model for ODT, is given in Figure 2.5. In this diagram the vertical spatial axis is the ODT $y$ domain and the abscissa is time. At each instant in time (each vertical line) there exists a complete (instantaneous) velocity profile and complete profiles for all the passive scalars, none of which are shown in the diagram. Whenever a triplet map is implemented at a certain point in time, the domain of the eddy event $\left[y_{0}, y_{0}+l\right]$ is recorded by placing a darkened line segment over said domain. Therefore the figure gives an overview of the eddy event history. One can see the time evolution of eddy events. This includes the spawning of smaller eddies from larger eddies. The pictorial in Figure 2.5 is an actual space-time history diagram from the first member of the ensemble used to generate the data presented in this paper.

\subsection{Specific Application of ODT}

In the time developing version of ODT, the flow develops at the rate of the bulk mean fluid velocity. To convert ODT time development to distance traveled downstream use 
$\Delta x_{O D T}=\Delta t_{O D T} \bar{u}_{b} \quad . \quad(2-15)$

In order to have ODT generate the time developing fluid velocity and temperature fields, the mean velocity and temperature profile as reported in Mulholland et al. (1991) were input as an initial condition into the ODT program. This ODT program is a constant density flow between two infinite parallel plates, so a point had to be chosen for the initial condition far enough downstream from the burner such that the constant density velocity profiles would match up well with those measured in the paper. Yet the desire was to have it as close to the burner as possible. Several initial conditions were tried, with the one decided upon being five $\mathrm{cm}$ down the $x$ coordinate (axial distance). With these initial conditions and a value of the one free parameter A equal to 0.169 , ODT was able to match the published velocity and temperature profiles well using a decaying turbulence assumption. (That is $\mathrm{dp} / \mathrm{dx}$ was set to zero.) With this value of $\mathrm{A}$, the ending time of Figure 2.5 ( 0.4 seconds) is equivalent to 0.32 meters down the $x$-axis of the reactor. The ODT simulation was run for a maximum of 0.7 seconds, this is equivalent to one channel height down the reactor. The approximation of using decaying turbulence rather than a non-zero $\mathrm{dp} / \mathrm{dx}$ 


\section{Space-Time History of Events:}

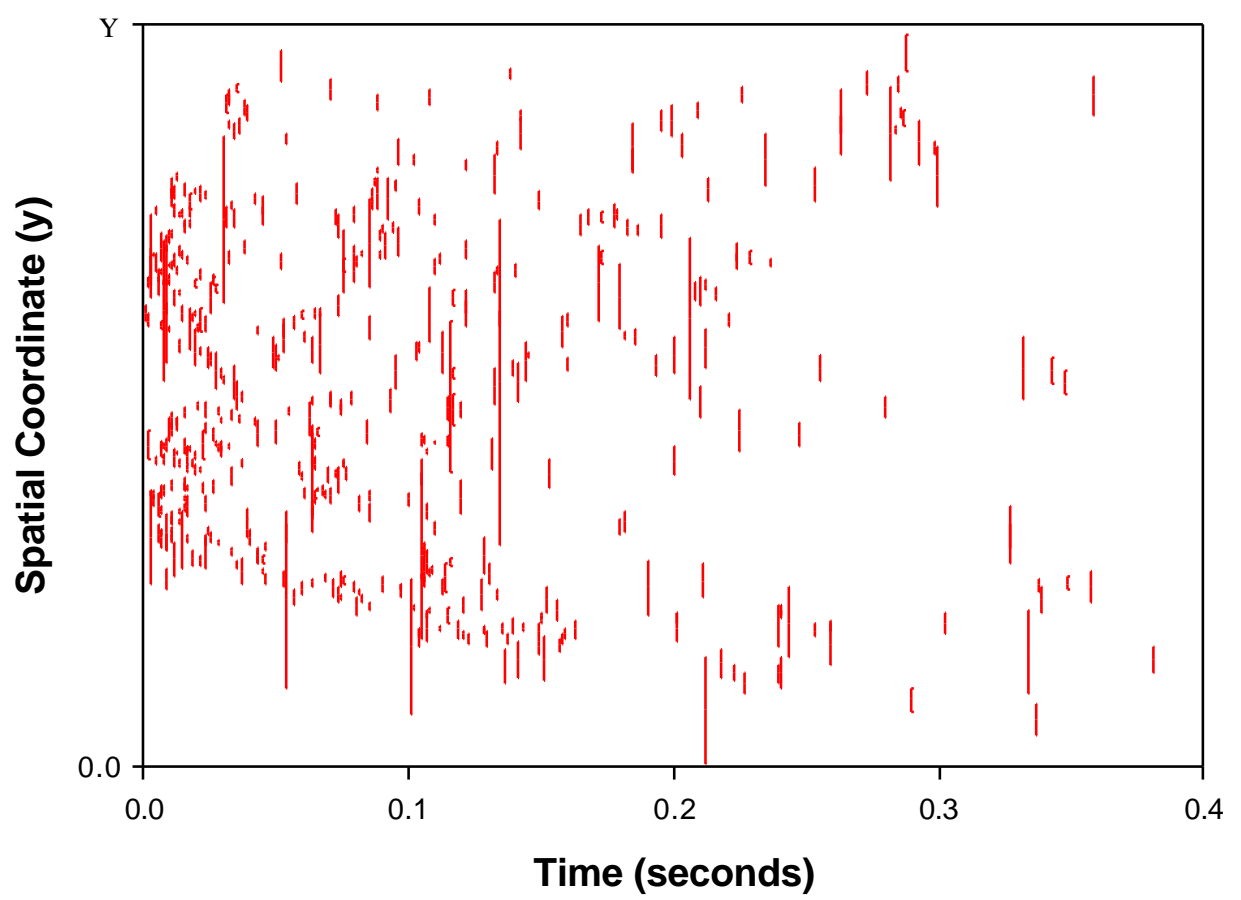

Figure 2.5: Pictorial representing the triplet maps in space-time coordinates. Darkened vertical lines represent eddies. Presented here is the space-time history of an actual realization used in this paper.

causes only a negligible error. Also, a numerical investigation with a nonzero $\mathrm{dp} / \mathrm{dx}$ was tried. The resultant eddy distribution was not significantly different for the time period in question. Hence for one channel length, the decaying turbulence resembles pressure driven flow. 
For comparison, the contour plots showing axial velocity and temperature profiles given in Srivastava et al. (1988) are reproduced in Figure 2.6.

They state that due to the turbulent nature of the flow, standard deviations of the velocity ranged from 1 to $5 \mathrm{~m} / \mathrm{s}$, often exceeding $50 \%$ of the mean velocity. Figure 2.7 shows selected plots with the computed mean axial velocity $(u)$ at selected distances from the burner. Keeping in mind the large standard deviation in the values of Mulholland et al., the comparison of the computed velocities, based on the model input of the mean measured velocity and temperature profiles at $5 \mathrm{~cm}$ downstream, shows good agreement. Figure 2.8 shows selected plots of the computed mean temperature profiles, the agreement is good. Figure 2.9 show a sample mean velocity profile (smooth curve) superimposed on an instantaneous velocity profile. Figure 2.10 shows a sample mean temperature profile (smooth curve) superimposed on an instantaneous temperature profile.

\subsection{Conclusion}

The ODT model is able to capture the relevant physics in this application of a highly turbulent bench scale furnace despite seemingly gross simplifications. The meticulous derivation of ODT in translating the threedimensional physical effects of a streamwise turbulent flow into a one- 
dimensional analogy with random rearrangements adequately matches the tubular reactor's velocity and temperature profile.
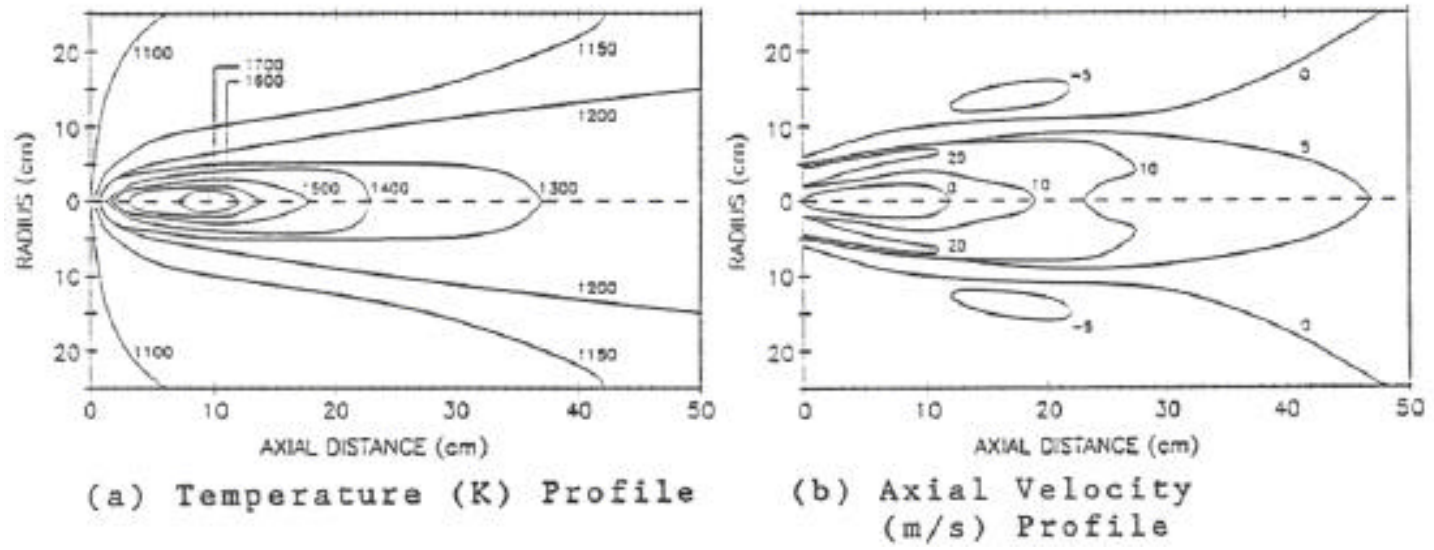

Figure 2.6: Measured mean temperature and axial velocity profiles. These are the near burner proflies for the Type $\mathrm{C}$ flame condition. Taken from mulholland et al. (1988) 
Axial distance $\sim 0.06 \mathrm{~m}$

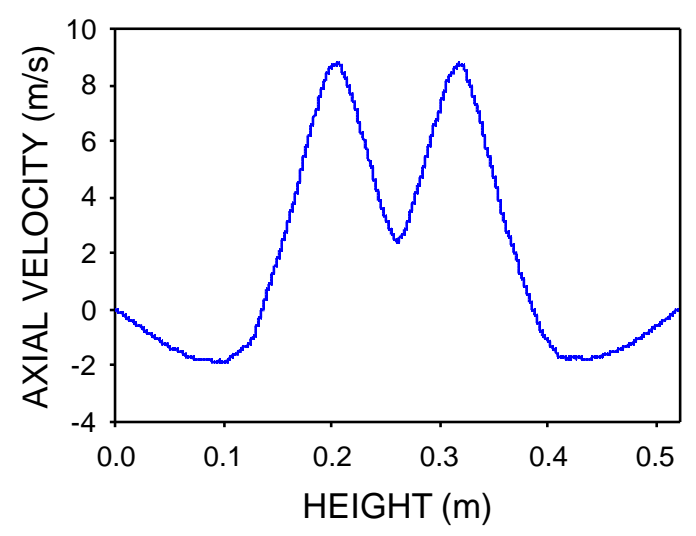

Axial distance $\sim 0.26 \mathrm{~m}$

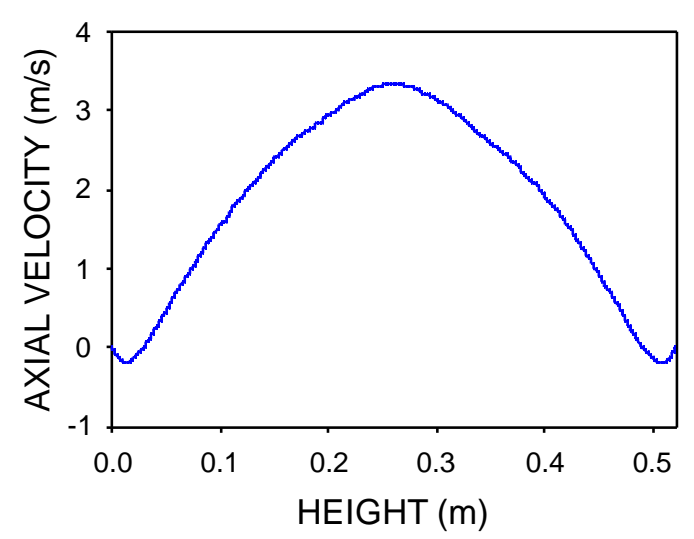

Axial distance $\sim 0.15 \mathrm{~m}$

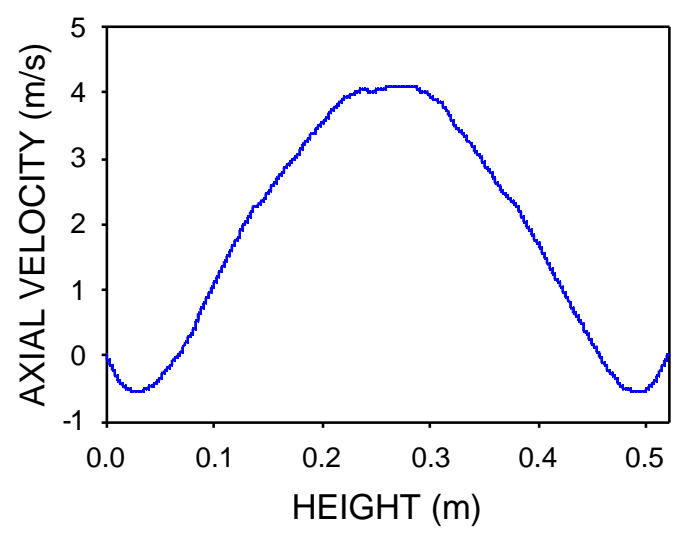

Axial distance $\sim 0.53 \mathrm{~m}$

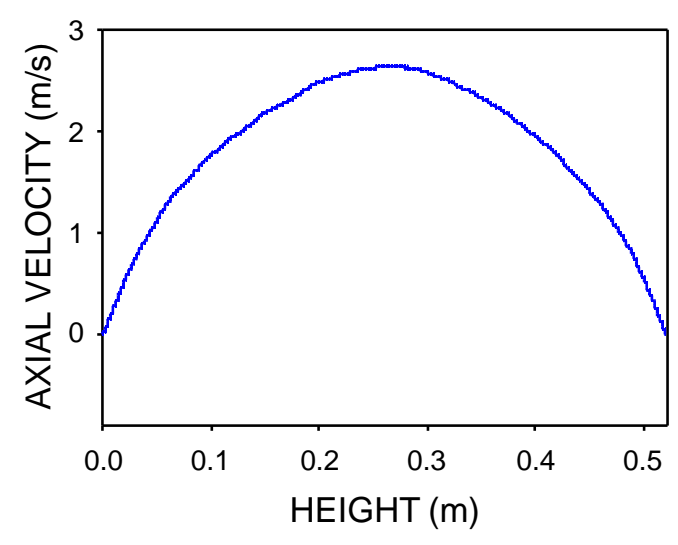

mean velocity

Figure 2.7: Selected mean velocity profiles. Four mean velocity profiles were chosen to show the mean axial velocity development over the range of the axial distance. These can be compared to Figure 2.6 to confirm agreement between the ODT model and the measured mean velocities. 
Axial distance $\sim 0.06 \mathrm{~m}$

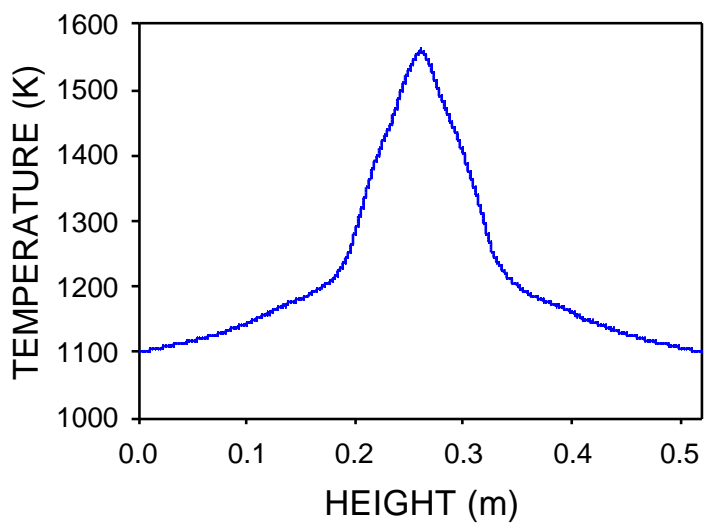

Axial distance $\sim 0.26 \mathrm{~m}$

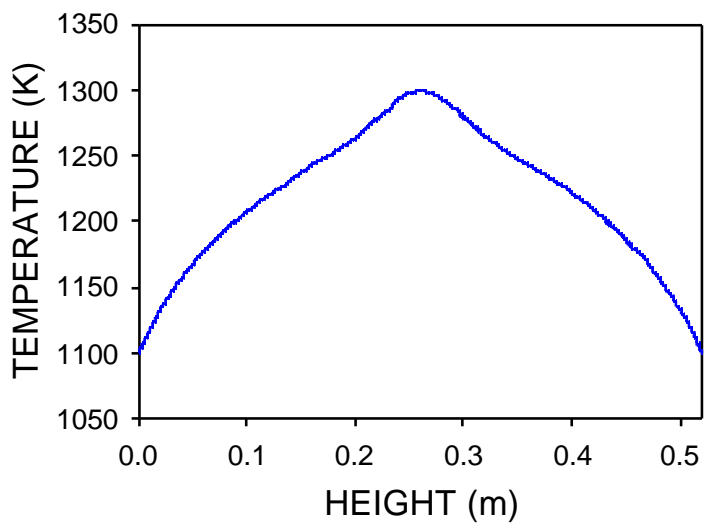

Axial distance $\sim 0.15 \mathrm{~m}$

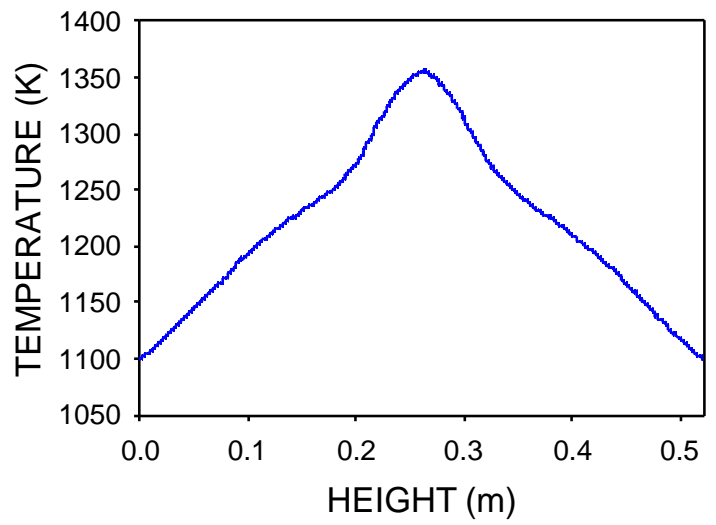

Axial distance $\sim 0.53 \mathrm{~m}$

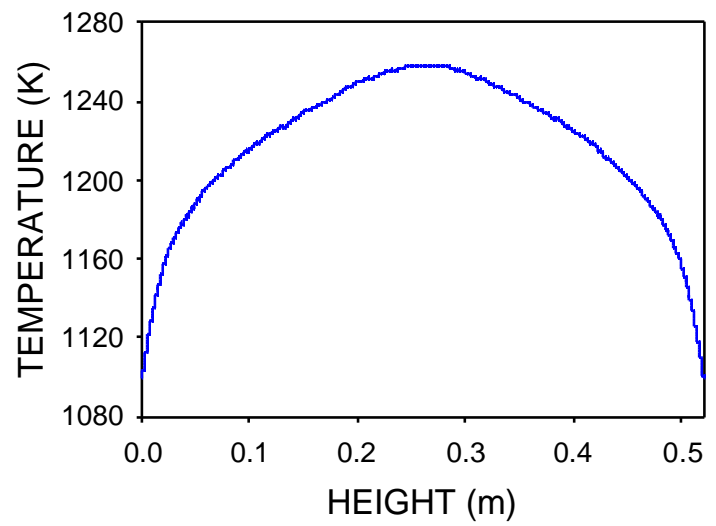

mean temperature

Figure 2.8: Selected mean temperature profiles. Four mean temperature profiles were chosen to show the mean temperature development over the range of the axial distance. These can be compared to Figure 2.6 to confirm agreement between the ODT model and the measured mean temperatures. 
Axial distance $\sim 0.19 \mathrm{~m}$

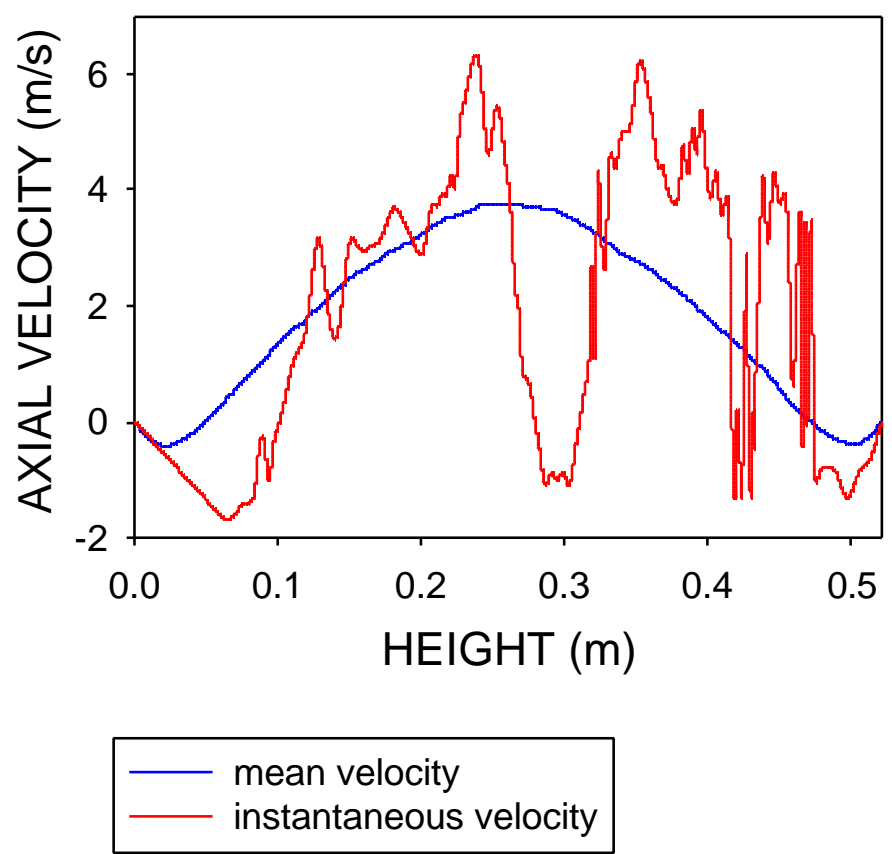

Figure 2.9: Mean and instantaneous velocity profiles. The data shown was randomly picked to demonstrate the difference between the mean velocity, computed for an ensemble size of 2000, and an arbitrary instantaneous velocity profile for an axial distance of $\sim 0.19 \mathrm{~m}$. The mean velocity is the smooth one. 
Axial distance $\sim 0.19 \mathrm{~m}$

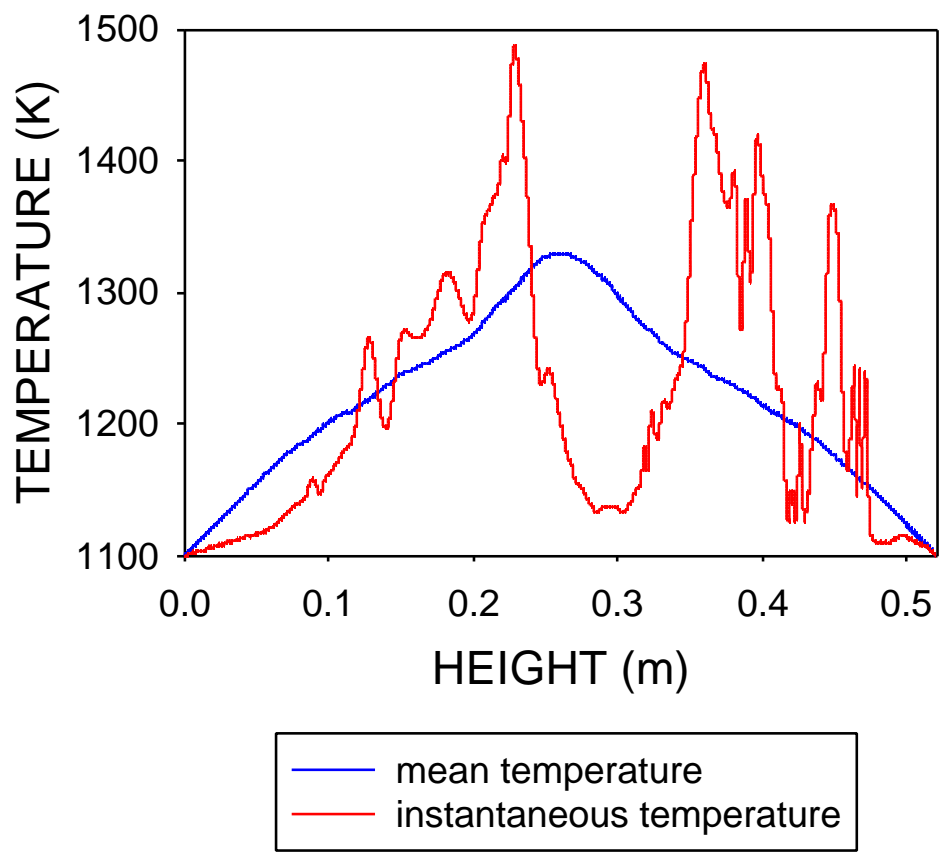

Figure 2.10: Mean and instantaneous temperature profiles. The data show was randomly picked to demonstrate the difference between the mean temperature, computed for an ensemble size of 2000, and an arbitrary instantaneous temperature profile for an axial distance of $\sim 0.19 \mathrm{~m}$. The mean temperature is the smooth one. 


\section{Chapter 3}

\section{Two-Phase Flow Addition to the ODT Model}

\subsection{Introduction}

An area of ongoing research is particle trajectories in turbulent flows. The development and validation of a two-phase flow submodel for ODT would prove advantageous for the advancement of one-dimensional (inexpensive) modeling. As a submodel to ODT, the particle-drag law must be in the same spirit as ODT. That is to say that the particle drag law should be linear, or better yet linearized in its application. As a linear equation it can easily be broken into $\mathrm{x}$ - and $\mathrm{y}$-components. The $\mathrm{y}$-component is identified with the spatial coordinate $y$ in the ODT formulation and the $\mathrm{x}$ component is identified with the streamwise spatial development. In this derivation, the flow will affect the particle trajectory, but the particle will not affect the fluid flow.

The modeling approach is exemplified by the special case of a tracer particle (zero inertia) traversing the ODT space-time domain. Since ODT idealizes the transverse velocity $v$ to be zero everywhere except at the triplet maps, the tracer particle will have a zero $y$ velocity $(\mathrm{V}=0)$ everywhere in the ODT space-time where $u(y, t)$ is undergoing continuous time evolution. 
In ODT, when $y$ fluid motion occurs, it is an instantaneous jump induced by a triplet map, hence a tracer particle must jump also. (See Figure 3.1 tracer path 2.) The goal of this chapter is to modify this picture to include particle inertia greater than zero.

The interaction between a particle and an eddy event is here defined as both the particle and the triplet map occupying the same space-time. Figure 3.1 shows a space-time history diagram similar to that introduced in Chapter 2, Figure 2.4, in which the representation of triplet maps are darkened vertical lines on the space-time domain. In Figure 3.1 we see an imagined representative detail of the eddy event history diagram (Figure 2.4). Only a magnified portion is chosen to simplify the chart so that particle trajectories can be seen in detail. Tracer particle path (1) transverses the eddy event history diagram without once encountering a triplet map. The challenge, then, is to develop a particle-drag interaction scheme for particle-eddy interactions consistent with the ODT phenomenology. When tracer particle path (2) intersects a triplet map it undergoes a position and velocity adjustment. The specifics of this adjustment are to be discussed in section 3.5 and following.

A particle with finite inertia would experience the net effects of its own 


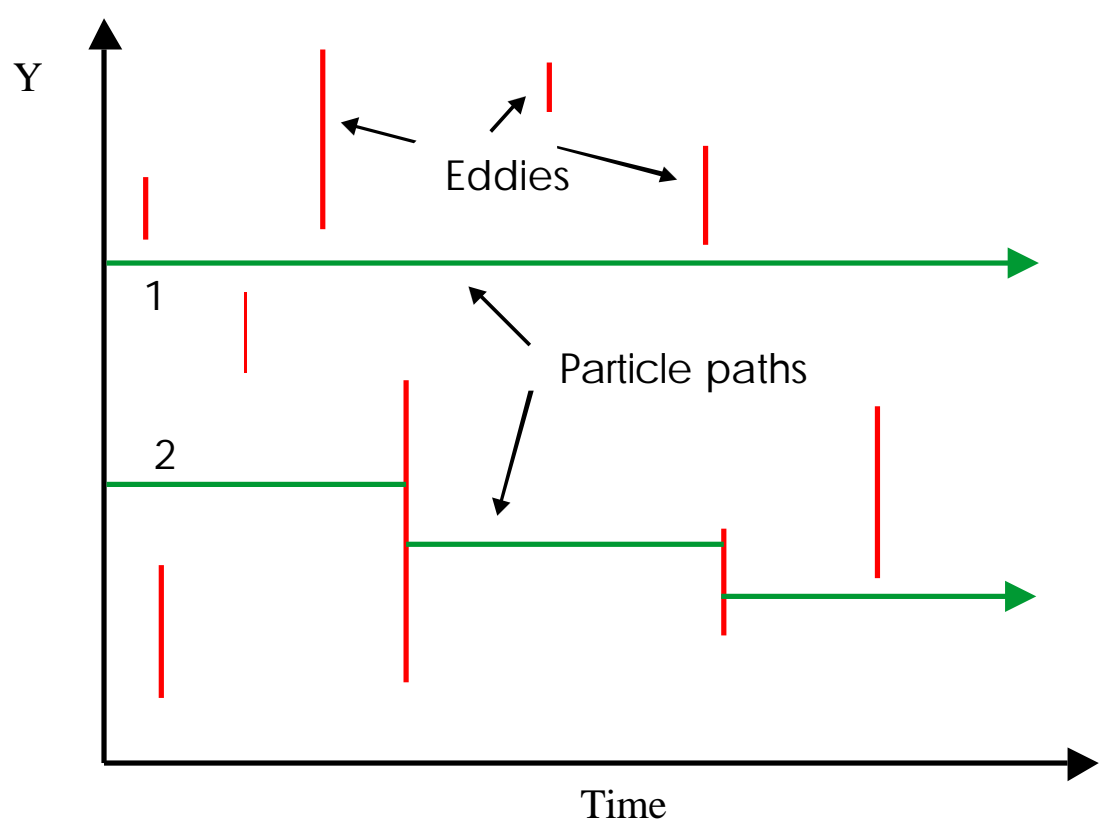

Figure 3.1: Tracer particle paths, 1 and 2, are represented as horizontal lines with arrowheads. Triplet maps are represented as darkened vertical lines. Areas of the plot without triplet maps are referred to as continuous time. Path 1 has no interactions with any triplet map. Path 2 interacts with two eddies.

inertia, the gravitational force, and the drag force. Any combination of these could give a curved trajectory to the particle path. The burden of this chapter is to derive the equations of motion to predict the particle trajectory in continuous time and to devise a model to correctly handle particle interactions with the instantaneous eddy events. 
To derive the equation of motion of a particle of mass $m_{p}$, a force balance is performed on the particle, which in vector form can be written as

$$
m_{p} \frac{d \tilde{V}}{d t}=\sum_{i} \tilde{F}_{i}, \quad(3-1)
$$

where " $\sim$ " denotes a vector quantity, and the sum, $\mathrm{i}$, is over all the forces acting on the particle. For this application, the only forces on the particle are gravity and the drag force. Hence Equations 3.2 and 3.3 give the equation of motion of the particle in vector form.

$$
\begin{aligned}
& m_{p} \frac{d \tilde{V}}{d t}=m_{p} \tilde{F}_{G}+\frac{3 \pi \mu D_{p}}{C_{c}}(\tilde{v}-\tilde{V}) \\
& \frac{d \tilde{X}}{d t}=\tilde{V} .
\end{aligned}
$$

These equations are correct only for linear (Stokes) drag force. Nonlinear drag laws do not decompose exactly into $x$ and $y$ components, however, a non-linear drag force results for a Reynolds number greater than one. This requires an empirical drag law for our application. The empirical nonlinear drag law $(f)$ of Rowe (1961) is used: 


$$
\frac{d \tilde{V}}{d t}=\tilde{F}_{G}+(\tilde{v}-\tilde{V}) \frac{f}{\tau_{p}} \quad(3-4)
$$

where the aerodynamic response time, $\tau_{\mathrm{p}}$, the nonlinear correction factor, $f$, and the particle Reynolds number are given by:

$$
\begin{aligned}
& \tau_{p}=\frac{\rho_{p} C_{c} D_{p}^{2}}{18 \mu} \\
& f=\left\{\begin{array}{cc}
1 & \operatorname{Re} \leq 1 \\
1+0.15 \operatorname{Re}^{0.687} & 1 \leq \operatorname{Re} \leq 1000
\end{array}\right. \\
& \operatorname{Re}=\frac{\rho_{g} r_{d}\left|\tilde{V}_{d}-\tilde{v}_{g}\right|}{\mu_{g}} .
\end{aligned}
$$

Derivation of this two-phase flow model is necessarily complicated by its attention to detail. This must be done to adequately capture the relevant physics of particle-eddy interactions. In order to assist the reader, the derivation is broken into several levels of complexity. First will come a discussion of the decomposing of the equation of motion (Equation 3-4) into y and x components for "conventional" fluid flow. Conventional fluid flow would be that ODT fluid flow which is not reshuffled by triplet maps. (See 
Chapter 2, specifically section 2.2 for a discussion on triplet maps.) This is followed in the later part of the chapter by a discussion of implementation of the equation of motion for fluid flow which undergoes triplet map rearrangements. Next a summary of the two-phase flow model is presented. Finally sections on two-phase flow model extensions and numerical implementation strategies are presented. For each component, levels of complexity are developed from simplest to most difficult, with the more complicated levels building on the concepts of the previous levels. Liberal use of diagrams and equations illustrates the points made in the various sections.

\subsection{Y component of the particle-drag law}

First a detailed look at the $y$ component. With the particle $y$-velocity denoted $\mathrm{V}$ and the fluid $y$-velocity by $v$, the linearized particle-drag law in the $y$ direction is

$$
\frac{d V}{d t}=-\frac{(V-v)}{\tau_{p}} f+F_{G}, \quad(3-8)
$$


where $\tau_{\mathrm{p}}$ and $\mathrm{f}$ are as defined above. When $\mathrm{f}=1$, the first term on the right hand side Equation 3-8 reduces to the well known Stokes drag force on a particle.

Examination of Equation 3-8 shows that the effect of the particle-drag law is to relax the velocity of the particle to the velocity of the fluid parcel which surrounds it with a particle time constant $\tau_{\mathrm{p}}$ corrected by a factor $\mathrm{f}$ which is a function of the Reynolds number.

This particle-drag law fits well into the framework of ODT's time developing formulation (see Chapter 2). In the ODT idealized flow, $v=0$ outside of eddy events. Hence the equation of motion (3-8) in continuous time is

$$
\frac{d V}{d t}=-(V) \frac{f}{\tau_{p}}+F_{G} .
$$

Two important opposite limits must be considered in the two-phase flow submodel. One is a particle with low inertia (small $\tau_{\mathrm{p}}$ ) and the other is a particle with high inertia (large $\tau_{\mathrm{p}}$ ). Emphasis here is on small inertia. The large inertia particles remain mostly unaffected by the forces of drag or gravity. 
In the limit of vanishing inertia, a particle would act in an identical manner as a fluid tracer particle. This can be seen by letting $\tau_{\mathrm{p}} \rightarrow 0$ in Equation 3-9. That is, it would conform instantaneously in velocity and position to its host fluid parcel. A fluid tracer particle in the idealized turbulent flow of ODT must not undergo translation in the $y$ direction unless it interacts with a triplet map. Indeed, Equation 3-9 readily reproduces the desired particle path behavior during times in which the particle does not interact with an eddy event.

\subsection{X component of the drag law}

By direct analogy, the particle-drag law (Equation 3-4) can be decomposed in the $x$ direction with $\mathrm{U}$ denoting the $x$ velocity of the particle and $u$ denoting the $x$ velocity of the fluid element containing the particle.

$$
\frac{d U}{d t}=\frac{-(U-u) f}{\tau_{p}}, \quad(3-10)
$$

where $\tau_{\mathrm{p}}$ and $\mathrm{f}$ are as defined in Equations 3-5 and 3-6. Here, the force of gravity is perpendicular to the $x$ direction and there are no additional body forces. However, the model is readily adapted to situations in which there is a streamwise body-force component. In an identical manner to that noted 
previously, with $\mathrm{f}=1$, the right hand side of Equation 3-10 reduces to the well known Stokes drag force on a particle.

Taking the limit of Equation 3-10 for vanishing inertia $\left(\tau_{p} \rightarrow 0\right)$ requires that $\mathrm{U}=u$. Tracer particles always match the local velocity of the fluid. Looking at the other extreme, the limit of Equation 3-10 as $\tau_{\mathrm{p}}$ tends to infinity, is $\mathrm{U}=\mathrm{U}_{\mathrm{o}}$. The infinite inertia particle $x$ velocity is unaffected by the particle drag.

Since the ODT $x$ direction develops in time with the mean $u$ velocity of the fluid, denoted $\overline{\mathrm{u}}_{\mathrm{b}}$, there is a problem when computing the particle trajectory in the $\mathrm{x}$ direction. The particle proceeds with its own $x$ velocity this is generally different from the gas $u$ velocity. The particle is subject to time-varying $u$ velocity both because $u$ evolves in time and because particle motion in the y direction induces time variation of $u$ in the particle reference frame. Due to finite inertia, $\mathrm{U}$ generally differs from $u$ at the particle location, and both these quantities differ from $\overline{\mathrm{u}}_{\mathrm{b}}$. Thus, there is typically a difference between the speed at which the particle traverses the $x$ direction and that at which the bulk mean velocity $\overline{\mathrm{u}}_{\mathrm{b}}$ traverses the $x$ direction. This inconsistency can best be fixed by computing the time, $\Delta t_{\mathrm{p}}$ it would take that 
particle surrounded by that fluid parcel to traverse the distance which the mean bulk flow traverses in a given time $\Delta t_{\mathrm{ODT}}$.

$\Delta t_{p}=\frac{\overline{u_{b}} \Delta t_{O D T}}{U\left(t_{p}\right)} \quad(3-11)$

This equation becomes complicated because $\mathrm{U}$ is a function of $t_{\mathrm{p}}$ due to the equation of motion. The introduction of $t_{\mathrm{p}}$ defines a particle time coordinate for integrating the drag law. Now the continuous time process, as modified to give the correct particle time, gives the means to faithfully represent the particles streamwise trajectory evolution.

\subsection{Particle-eddy interactions in ODT}

The discussion and the particle equation of motion up until this point reflect conventional approaches to drag law implementation in laminar flow, subject to two major caveats: (1) only the u component of gas velocity is nonzero, and (2) interpretation of time evolution in ODT as streamwise spatial evolution required the introduction of a particle time coordinate, $t_{\mathrm{p}}$, distinct from the fluid time coordinate. Particle interaction with a triplet map requires a novel approach. As defined in Section 3.1, this interaction occurs when the particle and triplet map occupy the same space-time. The 
particle drag law as written is not applicable to ODT particle-eddy interactions because triplet maps are instantaneous in time yet the particledrag law requires an interaction time to cause a change in the particle velocity or position.

Before discussing in detail possible solutions to this type of particle-eddy interaction (when they occupy the same space-time), there is yet another problem which arises due to the ODT formulation of eddies as instantaneous in time.

To understand this, first recall that in Figure 3.1 the particle path was that of a tracer particle and as such had no $y$ velocity, reflecting the corresponding feature of ODT gas-phase evolution. However, particles with finite inertia will generally have a finite, nonzero, $y$ velocity. Figure 3.2 depicts an eddy event history diagram consisting of four particle trajectories with varying initial $y$ velocities $(\mathrm{V})$. Particle trajectory 1 has no initial $y$ velocity component. After the particle interacts with eddy A, it obtains a positive $y$ velocity and a positive $y$ displacement, as explained shortly (Section 3.4.2). Trajectories 2, 3, and 4 have increasing (in magnitude) negative $y$ velocity components. Trajectory 2 , shows the effect of the gravitational force, (curved line) on the drag law, prior to the eddy 


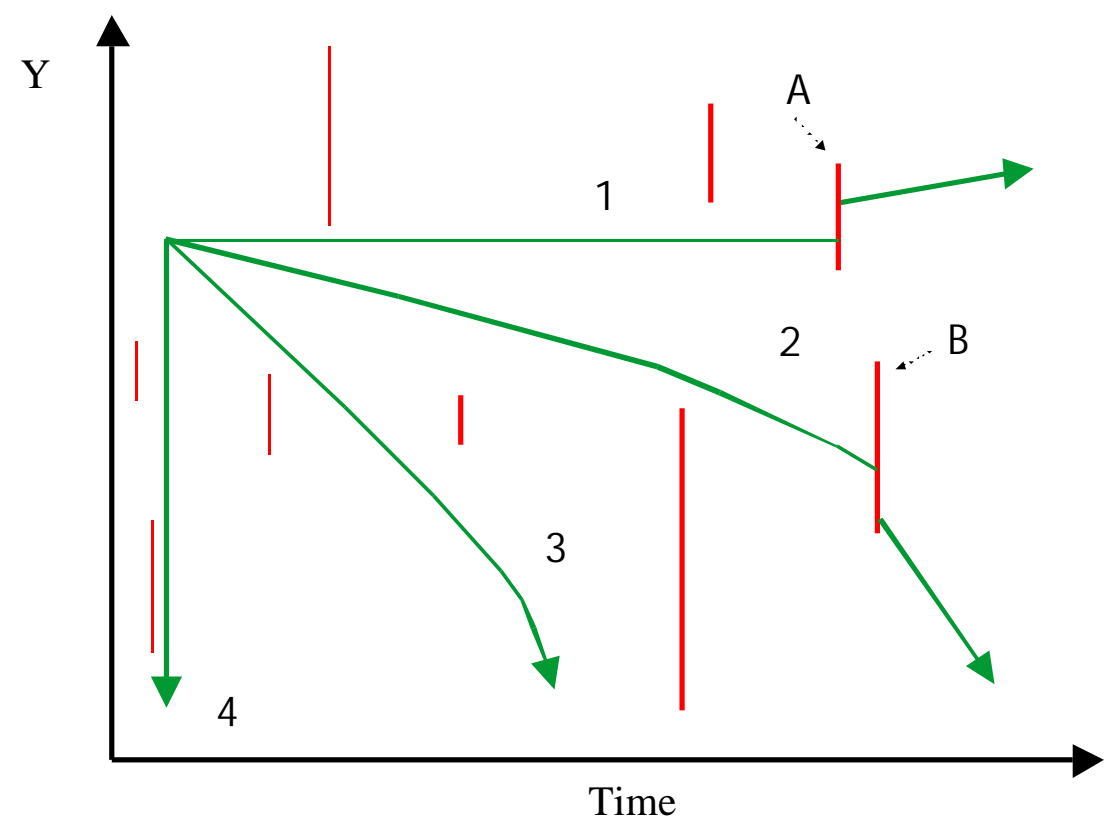

Figure 3.2: Particles with an initial velocity may transverse the ODT space-time domain without interacting with an eddy.

interaction. This particle-eddy interaction with eddy B gives the particle an negative $y$ displacement and increases the negative $y$ velocity of the particle. In the scenario set forth in Figure 3.2 both particle trajectories 1 and 2 eventually intersect an eddy event. At the intersection points the particle positions and velocities are changed instantaneously. (This process will be discussed in detail in the following section.) However the larger (in magnitude) the $y$ velocity becomes, the less likely a particle is to intersect an eddy event. In the limit (Figure 3.2, path 4) as the $y$ velocity component becomes infinite, the probability of an eddy interaction becomes zero 
because both the triplet maps and the particle trajectories are vertical lines. This phenomenon is an artifact of the ODT formulation since particles with large y velocities should interact with eddies. There needs to be a way for particles to interact with eddies even if the particles do not intersect the triplet map. This can be achieved by allowing the eddies to exist for an as yet undefined length of time. The essence of this would be to "pretend" that the ODT instantaneous eddies existed for a finite time. This would be represented as a box (not just a line) in the eddy event history diagram. Interactions of the type in which a particle enters the space time box associated with an eddy event through either of the two horizontal edges are referred to as type- $\mathrm{C}$ interactions because the particle is acted upon in a continuous manner by the eddy event as long as the particle is inside the box.

Type-C interactions are not developed further in this model application since the overwhelming majority of the Mulholland et al. data falls into the category of low $y$ velocity data. The low $y$ velocity data leads to mostly particle-eddy interactions in which the particle enters the space time box through the left vertical edge (i.e., the vertical line that represents an eddy in Figure 3.2), so there exists little need for type-C interactions in this model 
application. However, the concept of Type-C interactions is explained in detail in Section 3.11, entitled Model Extensions, type-C interactions. There are therefore, only two possibilities in this model for particle-eddy interactions. Either the particle path misses the vertical surface (representing the triplet map) of the eddy and therefore there is no interaction, or the particle path strikes the vertical surface and the particle undergoes an interaction of the type to be discussed next. These will be called type-I interactions.

Since this application of the two-phase flow submodel to ODT is mainly concerned with particle-eddy interactions occurring when particles intersect triplet maps, a major effort was put into capturing as much of the physics of this interaction onto the ODT model framework.

A zeroth order approximation of this interaction might be to simply adjust the $y$ position of the particle $\left(y_{\mathrm{p}}\right)$ by an amount equal to the mappinginduced displacement of the fluid element containing the particle just prior to the eddy event. \{A detailed description of triplet maps and their instantaneous jumps are given in Chapter 2 section 2.2.\} Even though this approximation will yield the desired solution for one special limiting case 
(zero inertia limit, illustrated in Figure 3.1), it fails for all other cases. Hence it is abandoned.

\subsection{Particle-eddy interaction model}

The logical solution to the difficulty of applying the particle drag law to particle-eddy interactions (due to the instantaneous nature of the triplet map) is to assign an interaction time to the particle-eddy interaction. For now, we will denote the interaction time coordinate as $T$, and the duration of the interaction as $T_{1}$. The latter quantity is specified in Section 3.9. The interaction time coordinate will be referred to as pseudotime because it is not a real time, just a time made up so that the particle drag law can have an effect on the particle. Another way to say this is since $T$ has units of time, but is not interpreted as real time due to the fact that eddy events are instantaneous in real time, it is referred to as pseudo (or fictitious) time. The latter quantity will be developed and defined later in the discussion. Particle evolution during this interaction is governed by the $y$ and $x$ components of the particle drag law (Equations 3-8 and 3-10) adjusted for the pseudotime coordinate. 


$$
\begin{aligned}
& \frac{d V}{d T}=-\frac{(V-v)}{\tau_{p}} f+F_{G} \\
& \frac{d U}{d T}=\frac{-(U-u) f}{\tau_{p}}
\end{aligned}
$$

where $v$ (defined later) is the fluid $\mathrm{y}$ component velocity induced by the triplet map, $T$ is the interaction time coordinate, $u$ is the velocity of the fluid cell which surrounds the particle, and all other terms are as previously defined. In this formulation tracer particles behave correctly, that is, they mimic the $u$ and $v$ velocities of the fluid cell which surrounds it during pseudotime. This can be verified by taking the limit of Equations 3-12 and $3-13$ as $\tau_{\mathrm{p}} \rightarrow 0$.

\subsection{Effect of pseudotime integration in the particle time domain}

A problem arises when comparing the integration done in pseudotime over a time period $T_{1}$ (Equation 3-12) and the conventional continuous-time integration of the particle-drag law (Equation 3-9, both duplicated here for clarity) subsequent to the particle-eddy interaction, over a comparable time interval $\Delta t=T_{1}$ and $\Delta T=T_{1}$.

$$
\frac{d V}{d t}=-(V) \frac{f}{\tau_{p}}+F_{G}
$$




$$
\frac{d V}{d T}=-\frac{(V-v)}{\tau_{p}} f+F_{G}
$$

Comparison of Equations 3-12 and 3-9 indicates that the equations contain similar terms and therefore the resultant actions of integrating them over the same time period are in fact double counting the effect of the gravitational force, once during the pseudotime integration from $T=0$ to $T=\mathrm{T}_{1}$ and once during the real time integration from the birth time of the eddy to a time increment of $T_{1}$. The particle experiences the gravitational force first for a period of $T_{1}$ in pseudotime and again for a period of $T_{1}$ in continuous time. This method represents a double counting of the effects of gravity and could lead to erroneous results.

To overcome this problem, which occurs when the particle and the triplet map occupy the same space-time, the particle trajectories with and without the effect of the eddy are computed. This is achieved by integrating (first to get velocity and then again to get position) both Equations 3-12 and 3-9 concurrently over the period $\mathrm{T}_{1}$, and determining the difference in the ending $y_{\mathrm{p}}$ position (defined in Equation 3-14). Similarly, the difference in the ending $\mathrm{V}$ velocities (defined in Equation 3-15) is also computed. These computations define the perturbations felt by the particle due to the eddy, 


$$
\begin{aligned}
& \Delta y_{p}=y_{p}^{e}\left(T_{1}\right)-y_{p}^{c}\left(T_{1}\right) \\
& \Delta V=V^{e}\left(T_{1}\right)-V^{c}\left(T_{1}\right), \quad(3-15)
\end{aligned}
$$

where the superscript e (for eddy) denotes the results based on Equation 312 and the superscript c (for continuous) denotes using Equation 3-9.

This procedure is pictorially represented in Figure 3.3 (A). The perturbation distance, $\Delta y_{\mathrm{p}}$, [demarcated with a " " in Figure 3.3] and the perturbation velocity, $\Delta \mathrm{V}$, are then added to the particle's original position and velocity (respectively) at the instant it first encounters the triplet map. The particle is then allowed to proceed in continuous time without the worry of double counting the gravity effects. The results of this procedure are shown in Figure 3.3 (B) as they would appear in Figure 2.4, a space-time history diagram.

In Figure $3.3(\mathrm{~A})$, the trajectory with the eddy interaction is based on pseudotime integration. It is convenient to define the shaded region as an eddy interaction box, whose vertical span is the $y$ range of the eddy and whose horizontal span is the time duration $T_{1}$ of the interaction.

With the eddy interaction box so defined in space (y) and time, the duration of a particle-eddy interaction is now idealized as the overlap of the 

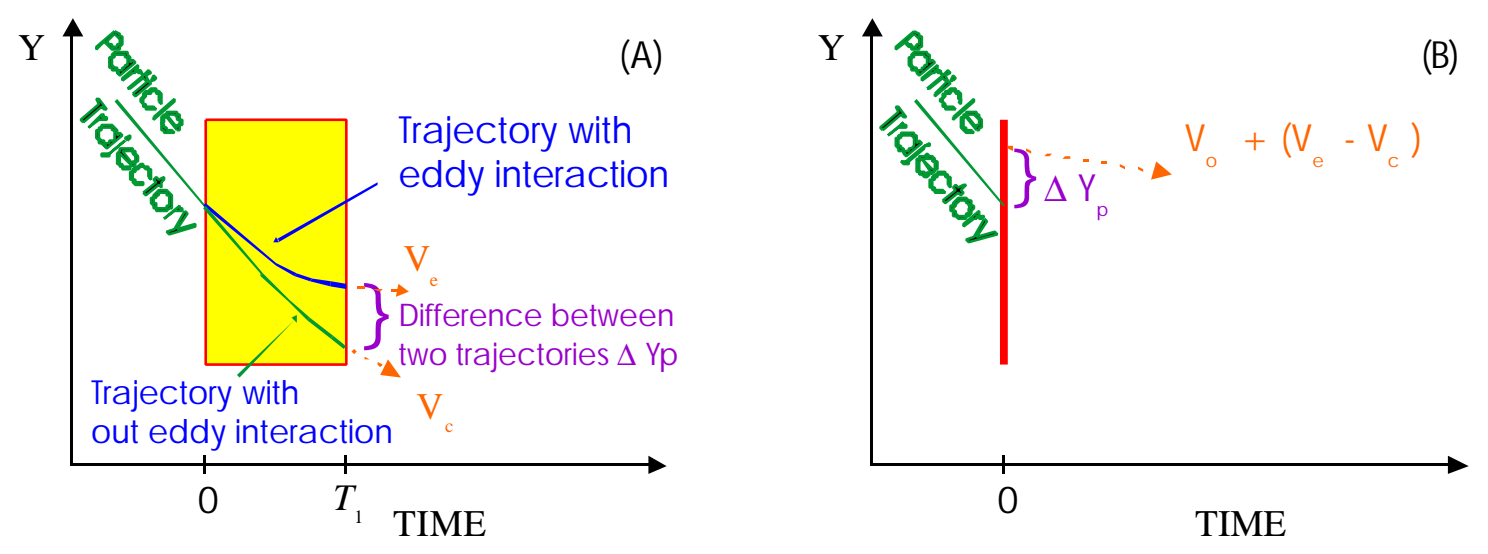

Figure 3.3: The procedure for implementing particle-eddy interactions which avoids double counting. Find $\Delta y_{\mathrm{p}}$ and $\Delta \mathrm{V}$ then add them to the original position and velocity. The resultant position and velocity are shown in B.

particle trajectory $\{$ under the influence of the eddy (Equation 3-12)\} and the eddy interaction box. The right hand side of the interaction box marks the end of the eddy lifetime. A particle with an initial $y$ velocity, under the effect of gravity, under the effect of drag, or any combination of these can exit the eddy interaction box from either of the two horizontal surfaces. This is visualized by understanding that the particle has left the space in which the eddy occupies before the eddy lifetime has expired.

The intended goal of introducing the particle-eddy interaction box and defining a position and velocity perturbation, which do not double count the gravitational effects, was to try to accommodate the particle-eddy interaction model to accurately fit variations in eddy size, particle speed and entry point 
into the eddy. As long as the correct interaction time, $T_{1}$, can be determined, this perturbation technique will give velocity and position jumps commensurate with the orientation of the particle trajectory and the eddy size.

A non-zero fluid velocity imposed by the eddy event (Equation 3-17) may act in the same direction as the particle's initial trajectory or in an opposing direction. The resultant particle path will either be bent upwards or downwards from the trajectory the particle would have taken if it did not interact with the eddy. For an example see Figure 3.4. A (the dotted line) is the path with no eddy interaction. All other trajectories $(\mathrm{B}, \mathrm{C}, \mathrm{D})$ include an imposed $v$ velocity from the eddy event. The particle-drag law, Equation 312 , is used to define the path from the moment the particle first encounters the eddy $(T=0)$ to the end of the eddy lifetime $\left(T=\mathrm{t}_{\mathrm{e}}\right.$, where $t_{\mathrm{e}}$ is defined in Section 3.7). For path $\mathrm{B}$, the $v$ component due to the triplet map is negative. For this particular case, the particle leaves the eddy interaction box by the bottom horizontal surface before time $t=\mathrm{T}_{1}$ has elapsed. For comparison, Path $\mathrm{C}$ has a positive $v$, the particle trajectory curves upward and exits the eddy interaction box through the back vertical surface. This path exits precisely at $T=t_{\mathrm{e}}$. A larger positive $v$ component is used in the calculation 


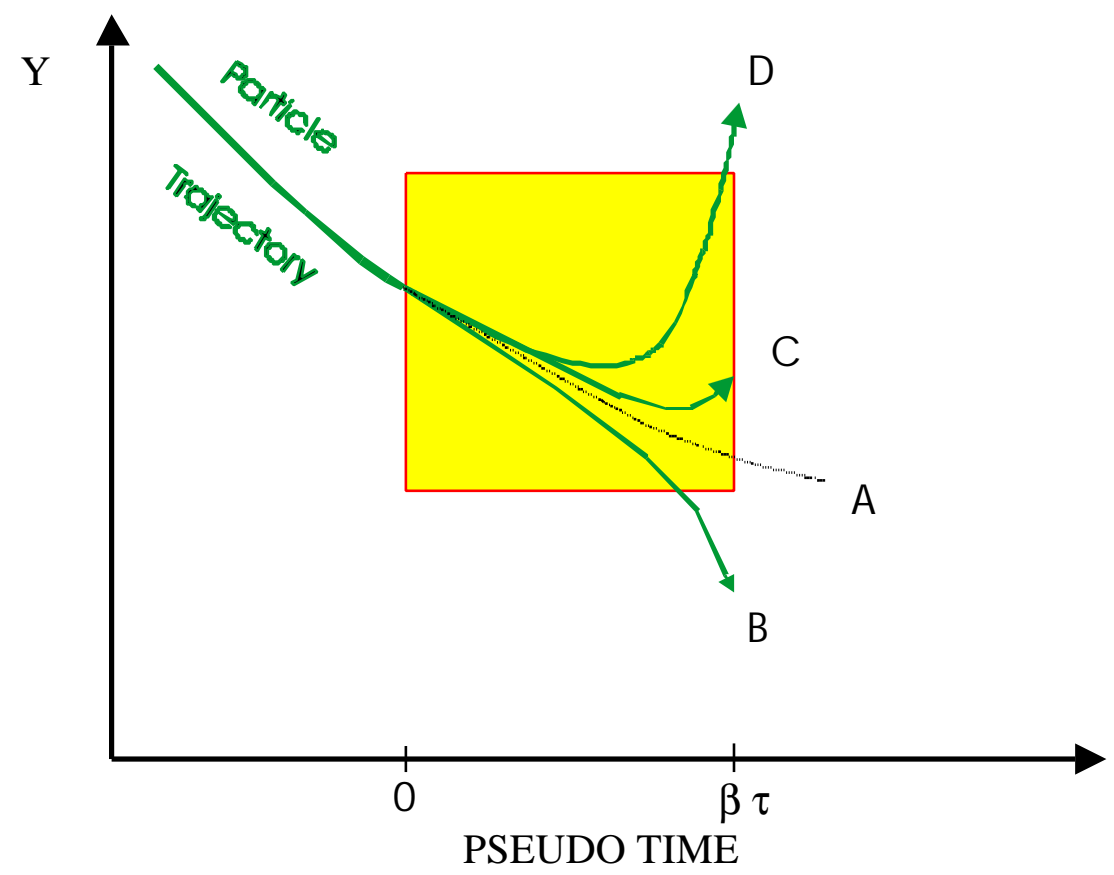

Figure 3.4: Particle-eddy interactions cause the path to deviate and may exit the interaction box by one of the horizontal surfaces before the eddy lifetime has expired

of path D. Note that there is an extremum (minimum) in the trajectory (as well as case C) and in a manner similar to path B, path D leaves the eddy interaction box by the horizontal (top) surface before the pseudotime interval $t_{\mathrm{e}}$ has elapsed. Care must be taken when computing particle-eddy interactions that these interactions stop the when a trajectory leaves the interaction box for the first time. This is because the eddy is "seen" by the particle as occupying that particular space-pseudotime and a particle path which leaves the box should no longer "feel" that eddy. 
Finding the correct interaction time $T_{1}$ is no easy matter, so we begin by considering only particles which interact with the eddy for the entire eddy lifetime. This will give insight into the behavior of particles while they interact with eddies without the added concern of computing the correct interaction time. This motivates the qualitative illustration of interaction regimes shown in Figure 3.5.

Figure 3.5 (A) shows the trajectory of a particle tracer as it enters an eddy interaction box (from the left of the box) to after it leaves (from the right of the box) in pseudotime. Figure 3.5 (B) presents the equivalent diagram as it appears in real space-time coordinates, just as it would if the trajectory were rendered in Figure 2.4. The particle is displaced in the $y$ direction the identical amount which the fluid parcel surrounding it is displaced in the $y$ direction by the triplet map. Figure 3.5 (C) represents the trajectory of a particle with medium inertia (neither low nor high) in pseudo time. Figure 3.5 (D) gives the same trajectory in real space-time coordinates. One can readily see that the particle is displaced upwards by the eddy event but only a fraction of the distance the fluid cell travels. Note carefully that the velocity vector in Figure 3.5 (D) is adjusted instantaneously in the manner defined by Equation 3-15. For completeness 

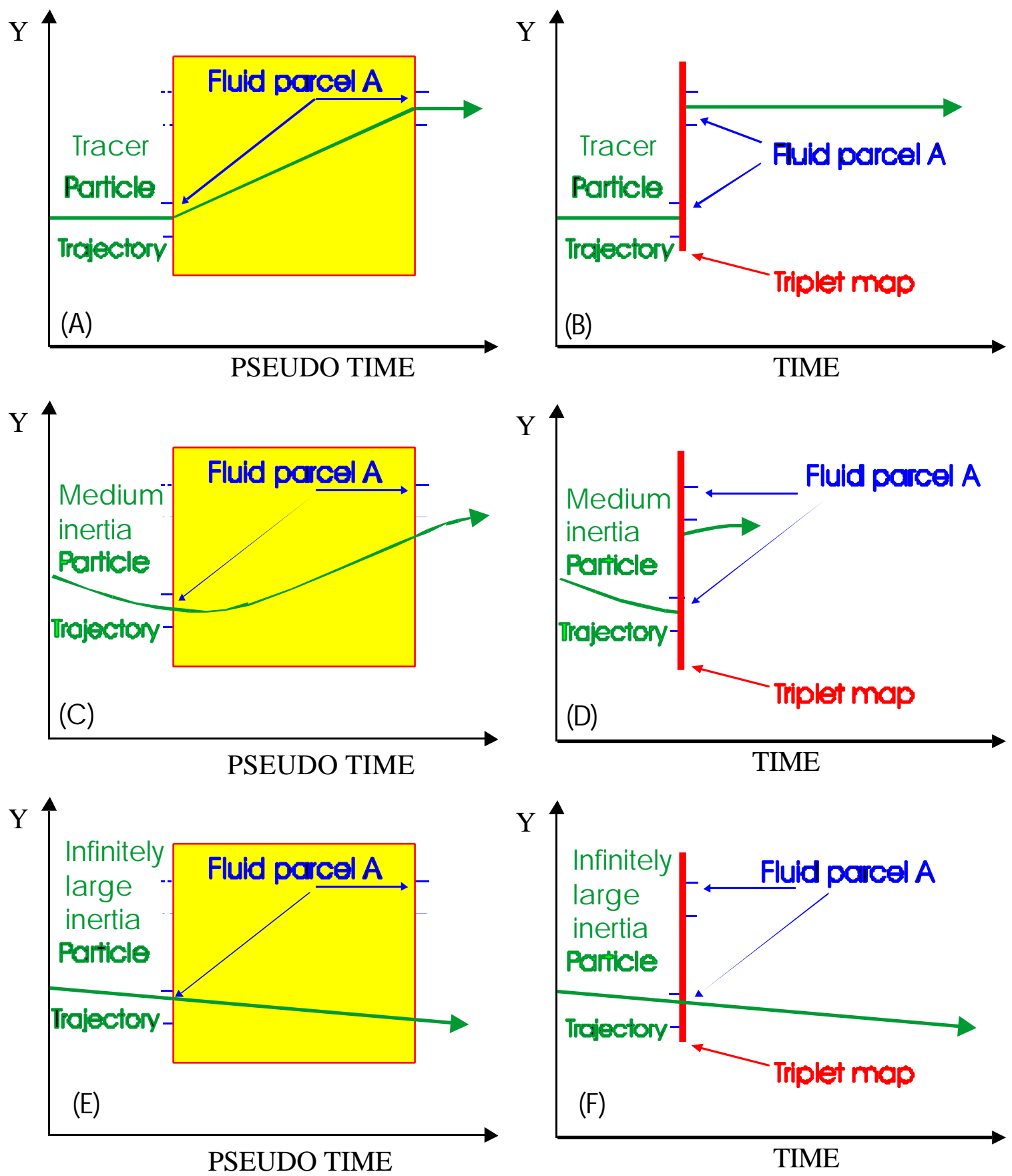

Figure 3.5: The trajectories of particles with varying inertia (low to high) are shown in pseudo time during particle-eddy interactions (A), (C), and (E). The paths in real time are shown for comparison, paths (B), (D), and (F) respectively. 
Figure 3.5 (E) shows the trajectory of a particle with infinitely large inertia in pseudotime. The corresponding trajectory in real space-time coordinates is shown in Figure 3.5 (F). As expected the particle-eddy interaction has no effect on the particle trajectory of an infinitely large inertia particle.

\subsection{Alternative representations of particle-eddy interactions}

There exist other possibilities for the design of a particle-eddy interaction model which include time, in other words procedures other than inventing pseudotime, computing the particle path, then projecting this difference back to the initial time. The most worthy of consideration is simply to allow the triplet map induced velocity profile $(v)$ to act upon the particle for an amount of real time equivalent to the amount of pseudotime $T$ which is the eddy lifetime t. This has the effect of reproducing the particle trajectories of Figure $3.5(\mathrm{~A}),(\mathrm{C})$, and (E) with the pseudo time axis (abscissa) replaced with real time. This is indeed a simplification in model design and implementation, so some justification needs to be made for choosing the more complicated model. Three arguments present themselves. The first and weakest argument is the heuristic argument that since the eddy events are instantaneous in time, so their effects (particle velocity/position changes) should be instantaneous also. The reason this argument is weak is that the 
instantaneous triplet maps are an artifact of the ODT model and are only an idealization of the physical reality in which eddies have a finite lifetime. This argument by itself cannot justify choosing the more complicated model.

The second argument relies on the physical principle that a particle with low enough inertia will exactly mimic a fluid tracer parcel. As is depicted (for example) in Figure $3.5(\mathrm{~B}, \mathrm{D}$, and $\mathrm{F}$ ) the fluid parcel has a discontinuous jump in the y axis (the ordinate) so a particle would need to have a discontinuous jump in order to mimic the fluid particle. Here the counter argument can be made that this discontinuous jump is also an artifact of the ODT model and that using the simpler model the particle path does not deviate from the fluid parcel for a very long time. After that "short" time period, the particle and the fluid parcel which surrounded it are reunited. So the simpler model still reasonably mimics a tracer particle and deserves consideration.

This leads to the final argument. In order to follow the logic, the following representative scenario is proposed, see Figure 3.6. A tracer particle is traveling along with its fluid parcel (denoted fluid parcel A). It encounters an eddy event denoted 1. Assuming the simple model, Figure 3.6 (A) results in: the particle's adjustment to the eddy interaction occurs over a 

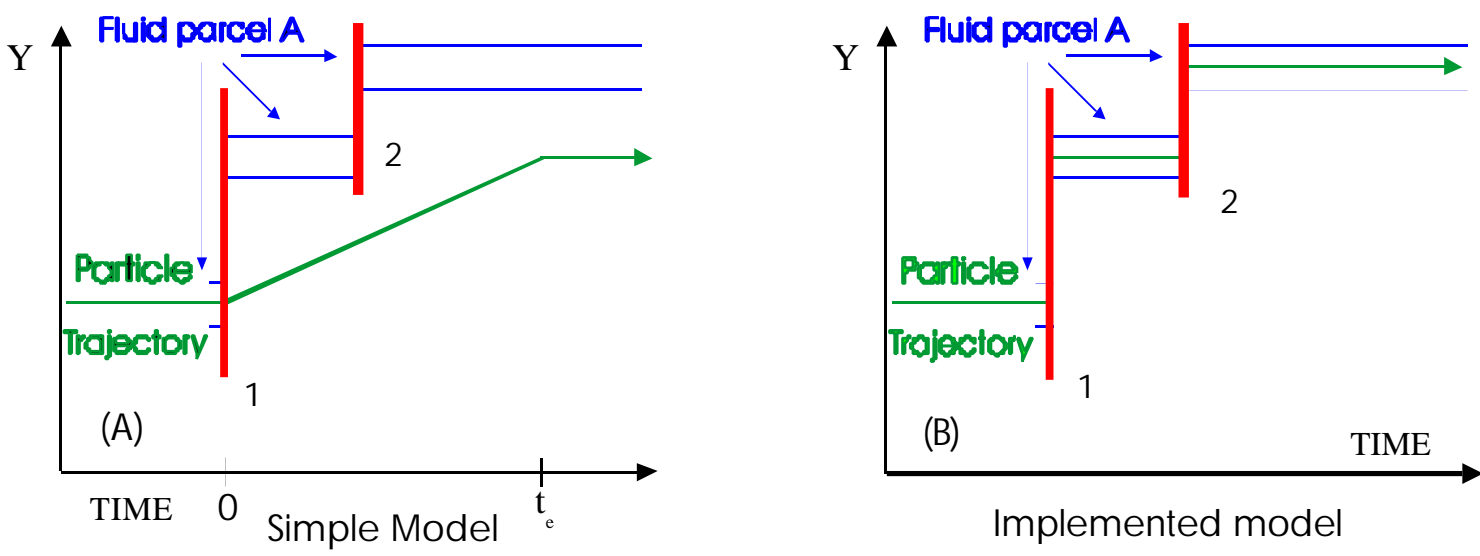

(B)

TIME

Implemented model

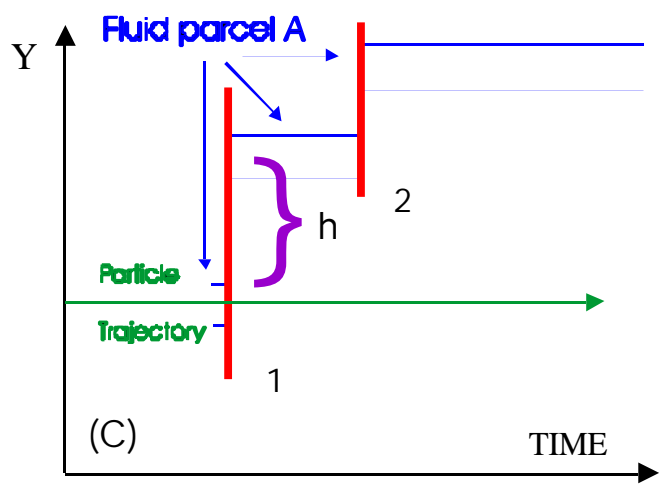

Implemented or Simple Model

Figure 3.6: Diagram A shows the trajectory of a tracer particle for a particle-eddy interaction using the simple model. B shows the implemented model for a tracer particle. Diagram $\mathrm{C}$ shows the path for a particle with very large inertia. Diagram $\mathrm{C}$ is the same for either the simple or the implemented model. In this example, there is no continuous time $v$ velocity.

time period $t_{\mathrm{e} 1}$, while the fluid parcel A undergoes the discontinuous jump $\mathrm{h}$. Before the particle has time to rejoin the fluid parcel A another eddy event (denoted 2) occurs and fluid parcel A is displaced farther away on the $y$ axis. At this point any correlation between the fluid parcel $\mathrm{A}$ and the tracer 
particle is lost. The simpler model does not reproduce the small inertia limit for this scenario. Even though this is only a plausible scenario and does not have to occur, the dynamic feedback property of ODT makes this scenario likely. Only the implemented (more complicated) particle-eddy interaction model \{see Figure 3.6 (B) \} correctly matches the fluid parcel A with its tracer particle.

It is interesting to note that for the other extreme, a particle of infinitely high inertia, both the simple and implemented particle-eddy interaction models give the correct result $\{$ see Figures $3.6(\mathrm{C})\}$. In both formulations, the eddy has no effect on the particle.

Even though it is impossible to precisely capture the particle-eddy interactions for the entire inertia range in a one-dimensional model, it is our goal to be as accurate as possible. The implemented model fits exactly for the two extremes and therefore should do a reasonable job fitting the values in between. Because the implemented model redirects the particles instantaneously when a particle-eddy interaction occurs, this type of particleeddy interaction is referred to as a type-I interaction, where $\mathrm{I}$ is for Instantaneous effect. 


\subsection{Eddy lifetime}

As will be shown in Section 3.8, determination of the particle-eddy interaction time, $T_{1}$, requires a definition of the lifetime $\mathrm{t}_{\mathrm{e}}$ of an ODT eddy. The eddy lifetime is also used to determine $v$ in Equation 3-17.

The ODT local instantaneous time scale, $\tau\left(l ; y_{0}, t\right)$, is discussed in detail in Chapter 2. $\tau\left(l ; y_{0}, t\right)$ is best interpreted as the characteristic time of the eddy event if it were to happen over a time period and not instantaneously. $\tau\left(l ; y_{0}, t\right)$ is however an artifact of the ODT model development and not an actual or physical time. Even though parameter dependencies of $\tau\left(l ; y_{0}, t\right)$ are based on solid reasoning there is no compelling reason to believe that it actually corresponds to the eddy lifetime. Therefore it is justifiable to introduce a particle model free parameter called $\beta$. The eddy lifetime is now given by Equation 3-16

$t_{e}=\beta \tau\left(l ; y_{o}, t\right)$

Ideally $\beta$ is a number close to unity since $\tau\left(l ; y_{0}, t\right)$ was based on good reasoning and logically should not be too far off. $\beta=1$ was used for all runs reported in this paper, except for a set of sensitivity runs designed to investigate the model's response to changes in $\beta$. The additional runs were 
for $\beta=0.5$ and $\beta=2.0$. Decreasing $\beta$ causes the particle trajectory to deviate more from its original path due to the particle-eddy interaction. Increasing $\beta$ causes less of a disturbance. This may seem counterintuitive, but a detailed discussion on the subject can be found in Chapter 6, along with the results from the sensitivity runs on burning droplets.

Now that we have a suitable choice for an eddy lifetime, $t_{\mathrm{e}}=\beta \tau$, it follows that the velocity $v$ of a fluid cell as it travels in pseudotime from its starting position (the one it occupied before the triplet map) to its ending position (that which it occupies after the eddy event) is the $y$ distance traveled by the cell (h) divided by $t_{\mathrm{e}}$, $v=h / t_{e} \quad \cdot \quad(3-17)$

It is interesting to note that Kerstein (1999) uses this definition, with $\beta=1$, to define $v^{\prime}$ in order to compute the ODT "Reynolds stress" profile $\left\langle u^{\prime} v^{\prime}\right\rangle$.

With $v$ thus defined, Equation 3-9 governing the particle-eddy interaction is fully defined, though the time duration $T_{1}$ of the interaction is as yet undetermined. 


\subsection{Duration of the particle-eddy interaction}

A real-life eddy would exist in a defined space (three-dimensional) and exist for a defined (measurable) lifetime. Particle-eddy interactions would occur while the particle occupies the same space-time $(x, y, z, t)$ as the real life eddy. It is the goal of this part of the two-phase flow submodel to discuss in detail the analysis required to assure that the particle-eddy interaction ceases the first time the particle leaves the ODT space time $(x, y$, t) which the eddy is idealized to occupy in this model.

\subsubsection{Particle-eddy interactions in the $y$ direction}

For clarity in discussion, Figure 3.5 purposefully omitted instances in which the particle exited the eddy interaction box by one of the horizontal surfaces. Here we revisit type-I interactions with particles which do exit by the top or bottom of the space-pseudotime interaction box.

As long as the particle exits the interaction box and remains outside of the interaction box for the duration of the eddy lifetime, no added complications occur in determining the eddy interaction time $T_{1}$. Any number of root finding techniques can be implemented to determine the correct interaction time to for a given accuracy. 
However, not all particle trajectories will remain out side of the eddy interaction box once it has exited. Figure 3.7 shows a trajectory in which the particle path (as calculated for the complete lifetime of the eddy) shows an extremum occurring outside of the eddy interaction box and the particle reenters the box. In cases like these checking particle $y$ location only the end point $t_{\mathrm{e}}$ of the particle path would give the false impression that the particle first leaves the box by the back side at time $T=t_{\mathrm{e}}$, when actually the particle first leaves the box on the bottom at time $T=T_{1}$. Here the particle-eddy interaction should cease at $T=T_{1}$.

\subsubsection{Particle-eddy interactions in the $x$ direction}

No adjustments are made to the $x$ position, $x_{\mathrm{p}}$, or the $x$ velocity, $\mathrm{U}$, of the particle due to a particle-eddy interaction. The nature of the time developing ODT model does not lend itself readily to sudden jumps in the $x$ domain which is related to the ODT time by Equation 2-14. So no reasonable method to account for instantaneous movement backward or forward in time could be developed. A jump in $x$ for this time developing flow is equivalent to a jump in time. There would have to be some provision for allowing movement backward in time, which seems more trouble than it is worth. However, in the analysis of the particle-eddy interactions it is 


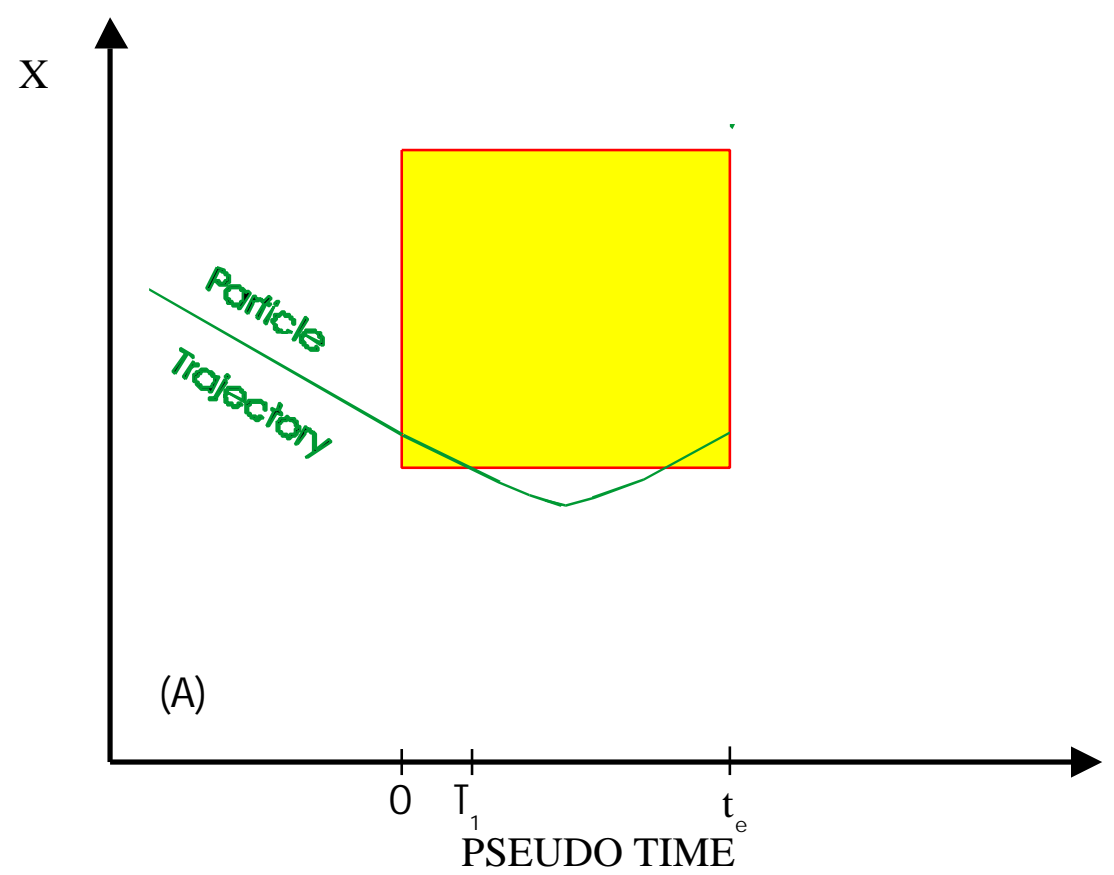

Figure 3.7: Particle paths can exit the interaction box and reenter the box due to particle eddy interactions. The particle-eddy interaction should cease when the path exits the box for the first time at T1.

important to determine whether and when the particle leaves the eddy ODT space-time in the $\mathrm{x}$ dimension in order to correctly determine the duration of the interaction.

The lifetime of the eddy in the $\mathrm{x}$ direction is identical to the lifetime $t_{\mathrm{e}}$ for an eddy as viewed in the y direction. (There is no reason why the lifetime should change for the different directions.)

$0 \leq T \leq t_{e} \quad(3-18)$ 
The y direction has a characteristic length, $l$, associated with the eddy in the ODT formulation, however, the $\mathrm{x}$ direction does not have any special length dimension associated with it. The logical choice then would be to use the distance of order $l$ in the $x$ direction. Since there is no connection between $l$ and the $x$ direction, there is nopredetermined relation between the position of the particle, $x_{\mathrm{p}}$, and the eddy interval $l$ on the $x$ coordinate. The formulation used to define the eddy in the $x$ direction is given in Equation 319 , in which $x_{\mathrm{o}}$ is defined as the $x$ position of the particle, $x_{\mathrm{p}}$, at the moment the particle-eddy interaction begins:

$x_{o}+u_{e d d y} T-l \leq x_{e} \leq x_{o}+u_{e d d y} T+l \quad(3-19)$

defines the range of $x$ values, $x_{\mathrm{e}}$, contained within the eddy, where $u_{\text {eddy }}$ is defined shortly.

Even though the parameter, $l$, is based on solid reasoning there is no compelling reason to believe that it actually corresponds to the eddy length. Therefore it would be possible to introduce another particle model free parameter called $\delta$. The length of the particle-eddy interaction box would be given by Equation 3-20. 


$$
x_{o}+u_{e d d y} T-\delta l \leq x_{e} \leq x_{o}+u_{e d d y} T+\delta l
$$

The use of $\delta$ as a free parameter is not implemented in the model. The author wishes to keep empiricism down to a minimum, that is, to keep the number of two-phase flow model free parameters to the one, $\beta$. There is a sound argument to implement the model with $\delta=1 / 2$. This would allow the total length of the eddy interaction box in the $x$ direction to be identical to the total length of the eddy interaction box in the $y$ direction. This is aesthetically pleasing to those who view real life eddies as circles, circles having the same height as width. The author however resisted this argument and refused to add yet another free parameter.

Particle-eddy interactions are more complicated in the $\mathrm{x}$ direction because the fluid parcels usually have a $u$ velocity. This can clearly be seen for the particular case depicted in Figure 2.1, where these particular fluid parcels all have a positive $u$ velocity. One would naturally expect that the eddy in that figure would have a net positive $u$ velocity since all of its elements have a positive $u$. A logical definition for the average $u$ velocity of 
an eddy event is the average of the $u$ velocities of the individual cells in the domain of the triplet map, $\left[y_{\mathrm{o}}, y_{\mathrm{o}}+l\right]$, at the time, $t_{\mathrm{eo}}$, of the eddy event.

$$
u_{\text {eddy }}=\left\langle u_{\text {cells }}\right\rangle=\frac{\int_{y_{o}}^{y_{o}+l} u\left(y, t_{e o}\right) d y}{l}
$$

Since turbulent eddies have a wide variation in their eddy velocities, $u_{\text {eddy }}$, one would expect a wide variety of interaction times between eddies and particles due to this relative motion.

The effect of a non-zero $x$ component eddy velocity $\left(\mathrm{u}_{\text {eddy }}\right)$ on the particle-eddy interaction time can be seen in Figure 3.8. This figure is a different rendering of the space-pseudotime eddy interaction box already introduced in Figure 3.4. Figure 3.8 has the $x$ direction associated with the ordinate (rather than the $y$ direction). The height of the interaction box is as defined in Equation 3-19 (2l), but the particle is centered in the height at the moment the particle eddy interaction begins. In all other ways, the new $x$ pseudotime eddy interaction box is the same as the previously defined interaction box. In Figure 3.8 diagram A, the eddy velocity is zero, the eddy does not move in time, and this particular particle trajectory is contained in the eddy for the duration of the eddy lifetime, $t_{\mathrm{e}}$. In diagram $\mathrm{B}$, the eddy has 


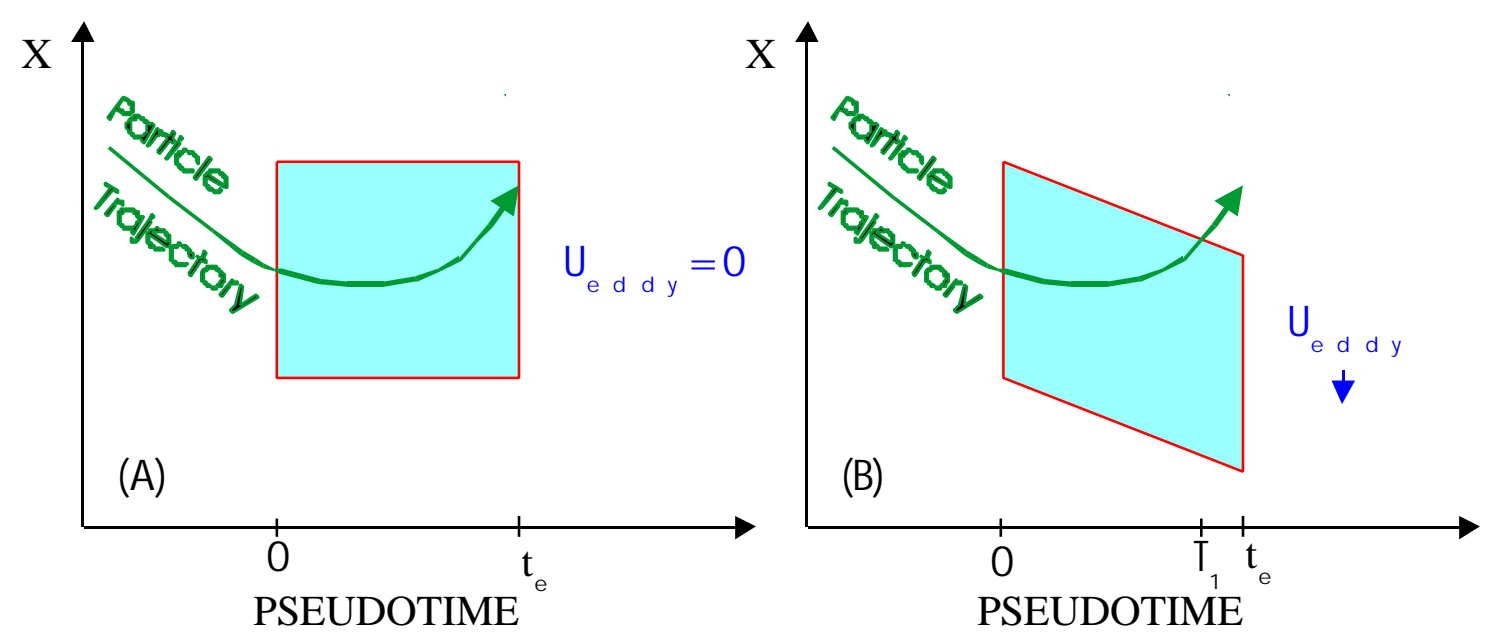

Figure 3.8: Effect of non-zero $x$ component eddy velocity on the particle-eddy interaction time. In figure $A$, the eddy velocity $\left(U_{\text {eddy }}\right)$ is zero, and the particle trajectory is contained in the eddy for the duration of the eddy lifetime. In figure B, the eddy has a negative velocity hence the eddy moves down as the particle curves up. This causes the particle to leave the eddy interaction box before $t_{\mathrm{e}}$ at time $=T_{1}$.

a negative velocity hence the eddy moves down as the particle curves up.

This causes the particle to leave the eddy interaction box at time equal to $T_{1}$, before the eddy lifetime expires at time $t_{\mathrm{e}}$, which it would not do if the eddy were not moving (diagram A). Therefore the correct end point for the particle eddy interaction is at time $T_{1}$ not time $t_{\mathrm{e}}$.

The proper ending time for the eddy-particle interaction must be the first time the particle leaves the eddy in either the $x$ or the $y$ direction, or the eddy lifetime, whichever comes first. The space-time eddy interaction box has 
been very useful in understanding the interaction time based on one spatial dimension (for example $y$ ) and one time dimension (pseudotime). The duration of the particle-eddy space-time overlap is governed by two spatial dimensions and time. The flipping between $x$ and $y$ on the ordinate tends to be confusing so that the intuitive understanding of the diagrams can easily be lost. Hence the introduction of a new diagram.

\subsubsection{Spatial ( $x$ and $y$ ) particle-eddy interaction box}

A more intuitive two-dimensional pictorial would be to place the spatial dimensions on the same graph. This would be equivalent to seeing a snapshot of the eddy and the particle at an instant in time. Subsequent times could be viewed in a series of snapshots. The new pictorial for the particleeddy interaction box in two spatial dimensions $(x$ and $y)$ is given in Figure 3.9 (B), with the $x$ direction used as the abscissa. Consequently the length ( $x$ dimension) is twice the height ( $y$ dimension) of the spatial eddy interaction box. Figure 3.9 (A) depicts the particle trajectory in $x-y$ space just prior to a particle-eddy event. Figure 3.9 (B) depicts the instant in time when the particle intersects the triplet map. The newly defined "spatial eddy interaction box" is demarcated as a box, that horizontally extends a distance $l$ in each direction from the particle $\mathrm{x}$ location, $x_{\mathrm{o}}$, at the instant of the type-I 
interaction. (This is consistent with Equation 3-19.) The box extends vertically with a starting point and length as defined by the eddy parameters:

$$
y_{o} \leq y_{e} \leq y_{o}+l \quad(3-22)
$$

The $y$ position of the particle in relation to the spatial eddy interaction box is the actual $y_{\mathrm{p}}$ of the particle. The box itself has its own $u$ velocity, $u_{\text {eddy }}$, which may be positive, negative, or zero. The relative motion of the particle and the eddy, $\mathrm{U}-u_{\text {eddy }}$, can cause a drastic shortening of the interaction time as discussed in Section 3.8.2 for the $x$ dimension only. In order to better understand these variations in $(x, y, t)$ space, three particular test cases will be explored using the new pictorial. The three test cases shall consist of a particle with finite inertia and modest $U$ velocity interacting with three different eddies. All the eddies will have the same eddy interaction box, but the mean eddy velocities will vary as follows: $u_{\text {eddy }} \gg \mathrm{U}, u_{\text {eddy }}=\mathrm{U}$, and $u_{\text {eddy }} \ll \mathrm{U}$. All three cases will therefore share the same initial conditions, as shown in Figure 3.9 A and B. In diagram A, the particle trajectory just prior to the type-I interaction is shown. Diagram B shows the moment the type I interaction begins. This corresponding to $T=0$. Also, $\mathrm{x}_{\mathrm{o}}$ is defined as the particle $x$ position at this instant. This is used to define the 
horizontal center of the eddy as defined in Equation 3-19. The $u_{\text {eddy }}$ depicted by an arrow is as yet undefined. (It can be positive, negative or zero.) In the first case $\left(u_{\text {eddy }}>>\mathrm{U}\right)$ the mean velocity of the eddy will carry the eddy much faster in the $x$ direction than the particle can travel (because the particle has finite inertia) so the eddy leaves the particle behind. This can be visualized in time by viewing Figure 3.9 in the sequence diagram A, B, and then C. For diagram C, a characteristic time increment, $t_{\mathrm{ch}}$, was chosen such that $u_{\text {eddy }}$ travels a distance just a little bit more than $l$. Implicit in the discussion of all three cases is the assumption that the eddy lifetime $t_{\mathrm{e}}$ is greater then $t_{\mathrm{ch}}$. There is little effect of the eddy on the particle trajectory in case $\mathrm{C}$ (its $\mathrm{U}$ velocity is increased slightly) because of the short interaction time.

In the second case $\left(u_{\text {eddy }}=\mathrm{U}\right)$ the particle and eddy travel in unison. This can be visualized in time by viewing Figure 3.9 in the sequence diagram A, $\mathrm{B}$, and then D. The characteristic time is significantly longer then either of the other two cases, since $t_{\mathrm{ch}}$ is defined as the amount of time for the eddy to travel 1 plus a little more. Note that we are still assuming that the eddy lifetime. $t_{\mathrm{e}}$, is greater than the characteristic time shown in D. However the mean eddy velocity in this case is much smaller than the mean eddy velocity 

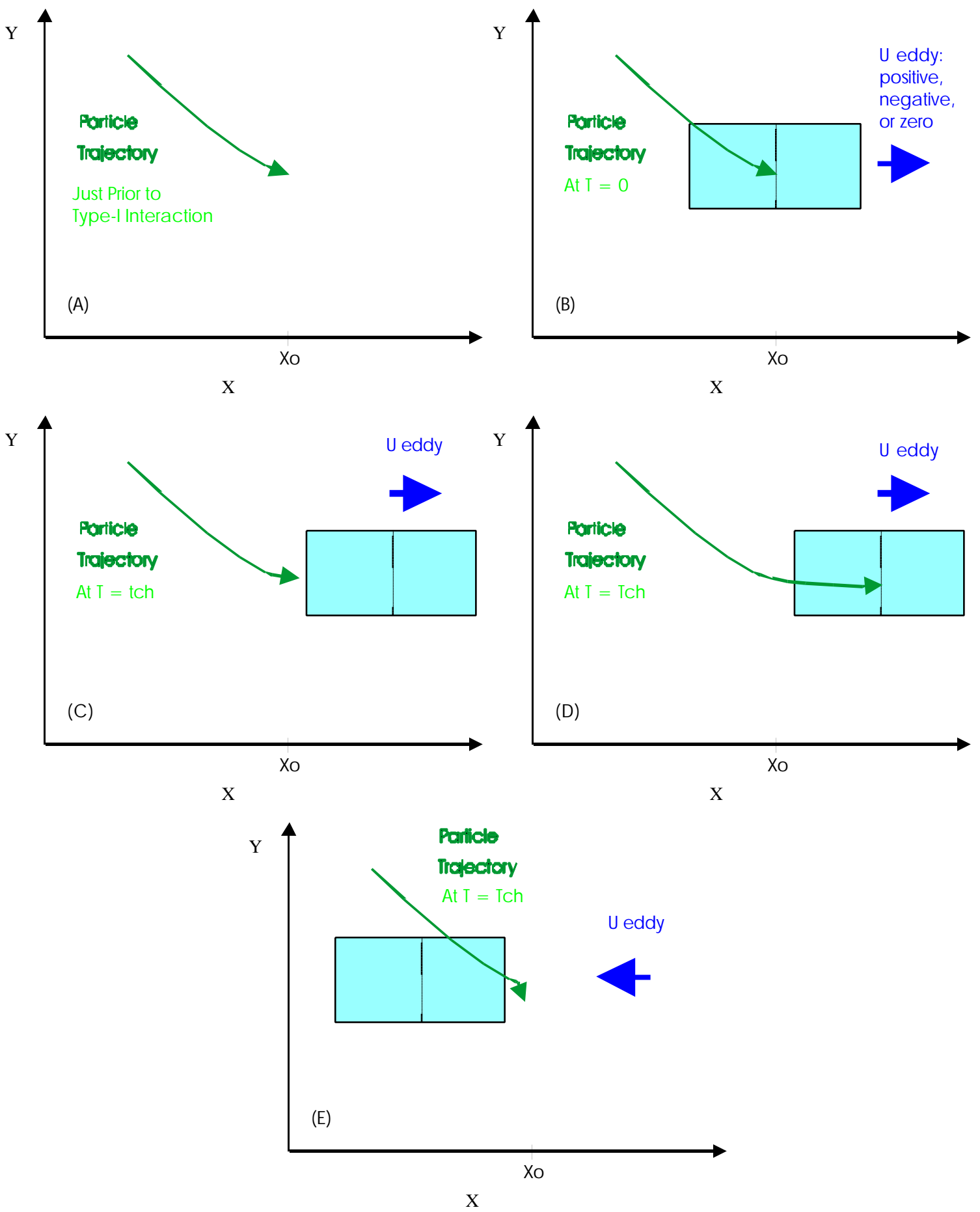

Figure 3.9: Time series qualitatively demonstrating the various affects of eddy velocities on particle trajectories. Diagram A and B show the initial conditions and are common to all trajectories. $\mathrm{U}_{\text {eddy }} \gg \mathrm{U}_{\mathrm{p}}$ is shown in $\mathrm{C}$, $\mathrm{U}_{\text {eddy }}=\mathrm{U}_{\mathrm{p}}$ is shown in $\mathrm{D}$, and $\mathrm{U}_{\text {eddy }} \ll \mathrm{U}_{\mathrm{p}}$ is shown in $\mathrm{E}$. 
for either of the other two cases, hence the time is much longer. Analysis of Figure 3.9 (D) shows the particle-eddy interaction has had time to significantly change the particle trajectory. In this case, the eddy and particle travel together to the right a distance slightly larger than $l$, and the particle's U velocity is increased slightly.

In the third case $\left(u_{\text {eddy }}<<\mathrm{U}\right)$ the particle leaves the eddy behind. This can be visualized in time by viewing Figure 3.9 in the sequence diagram A, $\mathrm{B}$, and then $\mathrm{E}$. The characteristic time is the same as case 1 since $u_{\text {eddy }}$ for case 1 is equal to the negative of $u_{\text {eddy }}$ for case 3. Analysis of Figure 3.9 (E) shows the same trend as the particle-eddy interaction depicted in diagram $\mathrm{C}$, that is the interaction time was too short to change significantly the particle trajectory. In diagram $\mathrm{E}$, the eddy passes to the left of the particle and the particle velocity, $\mathrm{U}$, is decreased slightly. In order to handle all combinations of $\mathrm{U}$ and $u_{\text {eddy }}$ correctly, the model must keep track of the relative positions of the particle and the eddy and must end the type-I interaction at the first instant the particle leaves the eddy.

The particle trajectory, under the influence of the eddy, has the possibility of leaving the eddy and then re-entering the eddy in either the $x$ or $y$ direction. This event is not easily depicted in this particular rendering of the 
spatial eddy interaction box since both the particle and the eddy move in time but the representation is at a given instant in time. This problem is solved by a simple coordinate transform. A switch from the fixed reference frame to the moving reference frame of the eddy provides a simple and informative pictorial of the particle trajectory as compared to the stationary spatial interaction box. To achieve this new reference frame only one transformation is needed, that is for the abscissa.

$$
x^{*}=x-u_{e d d y} T \quad(3-23)
$$

With $\mathrm{U}^{*}$ and $\mathrm{X}^{*}$ denoting the resulting transformed particle velocity and position respectively, the resulting drag law equation is:

$$
\frac{d U^{*}}{d T}=-\frac{f}{\tau_{p}}\left(U^{*}-u+u_{e d d y}\right)
$$

The horizontal dimension of the spatial eddy interaction box in the moving reference frame is defined in Equation 3-25. The vertical dimension is unchanged in this transformed reference frame and is still given by Equation $3-22$,

$$
x_{o}-l \leq x_{e}^{*} \leq x_{o}+l \quad . \quad(3-25)
$$


Figure 3.10 shows two particle trajectories in the reference frame of the eddy. In these particular examples there are extrema in both the $x^{*}$ and $y$ directions. In these representations the particle trajectory is traced in $x^{*}-y$ space from the time of birth of the eddy until the lifetime of the eddy, $t_{\mathrm{e}}$, elapses. As can be seen in Figure $3.10(\mathrm{~A})$, during that time period $\left(t_{\mathrm{e}}\right)$ the particle exits and reenters the eddy twice. The first time it leaves the vertical (right) face of the box. The second time it exits the bottom surface. Lastly the particle leaves the spatial interaction box for the last time by the left vertical surface. The particle equation of motion (based on the particle-drag laws Equations 3-12 and 3-24) evaluated in the $x^{*}$ and $y$ direction allow for a maximum of one extremum in each direction. Hence Figure 3.10 (A) is a worst case scenario which exits the interaction box three times. Figure 3.10 (B) also has two extrema, but only exits the interaction box once and that is well after both extrema are achieved. Not all particles will exit the spatial interaction box before the lifetime of the eddy expires. As explained in the beginning of Section 3.9 the actual particle interaction time, $T_{1}$, is taken to be the first time the particle leaves the spatial interaction box or the eddy lifetime, $t_{\mathrm{e}}$, whichever is sooner. This value of $T_{1}$ is plugged into Equations 3-14 and 3-15 to obtain the perturbations caused by the particle eddy 

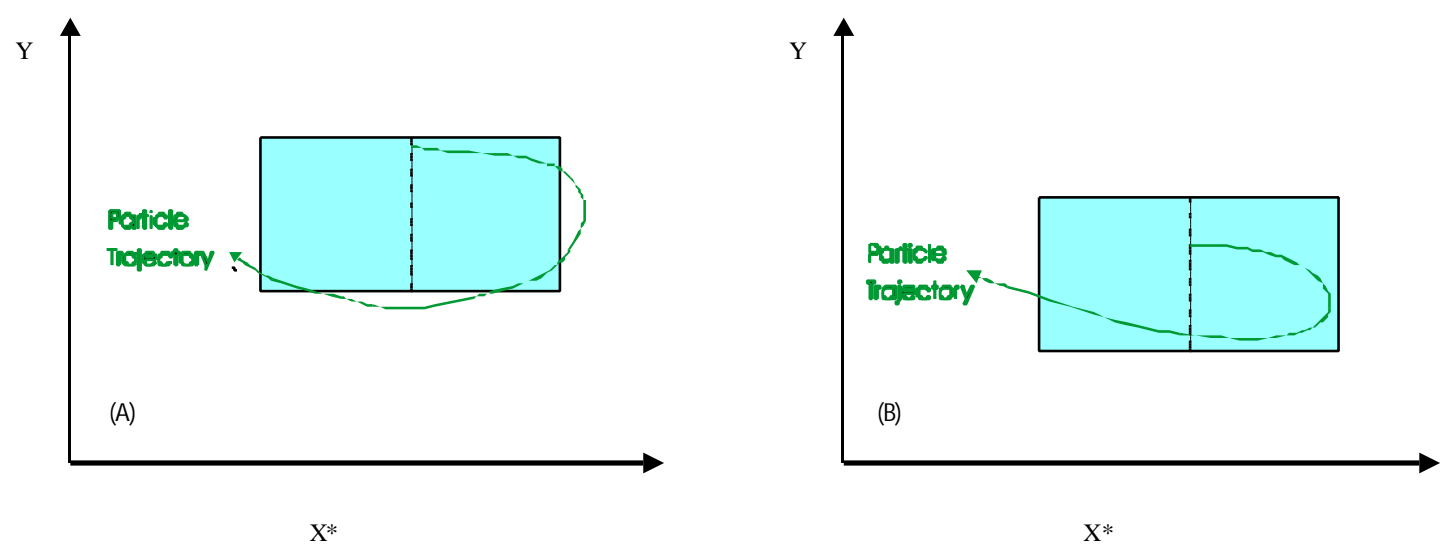

Figure 3.10: Particle trajectories in the moving reference frame of the eddy. This provides a clear view for the ending point of the particle-eddy interaction. The ending time of the interaction should be the first moment it leaves the spatial interaction box.

interaction. The particle position, $y_{\mathrm{p}}$, and velocity, $\mathrm{V}$, are adjusted instantaneously and the particle is allowed to proceed under the particledrag law (Equations 3-9 and 3-10) in continuous time until the particle encounters another eddy. There are no adjustments made to the $x$ position, $x_{\mathrm{p}}$, or the $x$ velocity, $\mathrm{U}$, of the particle due to the particle-eddy interaction. The nature of the time developing ODT model does not lend itself readily to sudden jumps in the $x$ domain. As noted in Section 3.3, the continuous-time process provides an adequate representation of streamwise particle evolution. 


\subsection{Conclusion and summary}

The two phase flow submodel for ODT is now complete. It reflects the key (Stokes) particle-drag interactions for regimes in which the fluid motion affects the particle but the particle does not effect the fluid flow. This model does so in a manner which captures the relevant physics in terms of the ODT phenomenology.

This derivation started with the basics and worked up to the final twophase flow submodel for ODT. There were many places in which the reader could have lost track of which equations were actually used in the submodel and which were used to derive the model or to illustrate a point. The model equations are summarized here to help clarify the two-phase flow model.

A particle is born into the ODT domain with an initial position $\left(x_{\mathrm{p}}\right.$ and $\left.y_{\mathrm{p}}\right)$ and velocity ( $\mathrm{U}$ and $\mathrm{V})$. The particle moves in "particle time" during which it follows the equations of motion defined by the particle-drag laws in $x$ and $y$ components given in Equations 3-9 and 3-10 modified to reflect the introduction of the particle time, $t_{\mathrm{p}}$, with $\tau_{\mathrm{p}}, f$, and Re defined in Equations 3-

5, 3-6, and 3.7:

$$
\frac{d V}{d t_{p}}=-(V) \frac{f}{\tau_{p}}+F_{G} \quad, \quad\left(3-9^{*}\right)
$$




$$
\frac{d U}{d t_{p}}=\frac{-(U-u) f}{\tau_{p}}, \quad\left(3-10^{*}\right)
$$

with the particle time, $\Delta t_{\mathrm{p}}$, computed by Equation 3-11. Pseudotime is not adjusted for the difference in particle and gas velocities since it is a fictitious time.

The particle follows the empirical nonlinear drag law until it occupies the same space-time as a triplet map, at which time it undergoes a type-I interaction. Type-I interactions are realized by the integrating the particle drag law components (Equations 3-12 and 3-24) in pseudotime, $T$, for a duration in pseudotime not exceeding $t_{\mathrm{e}}$ as defined in Equation 3-18. There is no adjustment of $\mathrm{U}$ for the particle-eddy interaction. The $\mathrm{x}$ component of the particle-drag law has been transformed to the constant velocity, $\mathrm{u}_{\mathrm{eddy}}$, coordinate system of the moving eddy as defined in Equation 3-23. The transformed coordinate is indicated by a *.

$$
\begin{aligned}
& \frac{d V}{d T}=-\frac{(V-v)}{\tau_{p}} f+F_{G} \\
& v=h / t_{e} \\
& \frac{d U^{*}}{d T}=-\frac{f}{\tau_{p}}\left(U^{*}-u+u_{\text {eddy }}\right)
\end{aligned}
$$


with $\mathrm{u}_{\text {eddy }}$ defined in Equation 3-21. The initial conditions $y_{\text {To }}, X_{\mathrm{o}}, \mathrm{V}_{\text {To }}$, and $\mathrm{U}_{\mathrm{o}} *$ are given by the particle position and velocity at the moment the particle intersects the eddy event. The form of the pseudotime equation of motion shows that the particle may exit and re-enter the eddy interaction box in either the $x^{*}$ or $y$ direction. The box is defined in the $y$ direction by Equation 3-22 and in the $x^{*}$ direction by Equation 3-25. Hence, merely evaluating the equation of motion at the end of pseudotime (letting $T_{1}=t_{\mathrm{e}}$ ) and verifying the particle is still in the eddy interaction box is not sufficient to assure that the particle-eddy interaction ceases the moment the particle first leaves the eddy interaction box. To overcome this added complication, one merely needs to evaluate the extrema in $x_{\mathrm{p}}$ and $y_{\mathrm{p}}$. If both extrema reside in the eddy interaction box, and the position of the particle, evaluated at $T=t_{\mathrm{e}}$ also resides in the eddy interaction box then the particle assuredly has remained inside the eddy interaction box for the entire time $t_{\mathrm{e}}$. If any one of these evaluations the particle exists outside of the eddy interaction box then a search of the $x^{*}, y, t$ space is necessary in order to find the first time in which the particle leaves the eddy. Care must be taken to ensure that $T_{1}$ is the first time at which the particle leaves the box, or if it does not leave the box, that $T_{1}$ is set to $t_{\mathrm{e}}$. 
The instantaneous change in the particle velocity, $\Delta y$, and position, $\Delta \mathrm{V}$ can then be calculated from Equations 3-14 and 3-15. These changes are then added to the particle's position and velocity at the instant of the eddy, $y_{\mathrm{To}}$ and $\mathrm{V}_{\mathrm{To}}$. The particle is then allowed to continue on in continuous time with the new values of $y_{\mathrm{p}}$ and $\mathrm{V}$.

\subsection{Two-phase Flow Model Extension - type-C interactions}

As explained in Section 3.4.1, the idealization of eddy events as instantaneous in time causes a model artifact. The larger (in magnitude) the $y$ velocity becomes, the less likely a particle is to intersect an eddy event. In the limit as the $y$ velocity component becomes infinite, the probability of an eddy interaction becomes zero because both the triplet maps and the particle trajectories are vertical lines. Since particles with large y velocities should interact with eddies in some way, the concept was put forward to allow the eddies to exist for a set length of time, this time would be the adjusted eddy lifetime $t_{\mathrm{e}}$. The essence of this would be to "pretend" that the ODT instantaneous eddies existed for a finite time. This would be represented as a box (not just a line) in the eddy event history diagram. Interactions of the type in which a particle enters the space time box associated with an eddy event through either of the two horizontal edges are referred to as type-C 
interactions because the particle is acted upon in a continuous manner by the eddy event as long as the particle is inside the box. This particular application did not require the added complication of type-C interactions. However, a brief outline of the conceptual design of type-C interactions is in order.

In contrast to type-I interactions, there are no instantaneous jumps from a type-C interaction. Type-C interactions can only occur in the y direction, hence, the pictorial with the most insight is the y space-time diagram. In this case the eddy is allowed exist in real time for the duration of the eddy lifetime $t_{\mathrm{e}}$. (See Figure 3.11)

Any particle which enters an eddy by the left vertical surface interacts with the eddy event in a type-I fashion and is not allowed to interact with the same eddy in a type-C fashion even if the particle leaves the eddy interaction box and comes back into the box by one of the horizontal surfaces. This is because the type-I interaction already takes into account the entire lifetime of the eddy. Conversely a particle which first enters an eddy interaction box from one of the horizontal surfaces may not undergo a type-I interaction with that eddy, but may interact with that eddy in a type-C manner as long as it is in the interaction box and may interact with that same eddy as many 
times as it re-enters the box. As soon as the particle exits the box by either one of the horizontal or the right vertical side the particle-eddy interaction ceases. Because of the random nature of the ODT triplet maps, virtually any conceivable combination of eddies may occur in a turbulent flow simulation. Once eddies are allowed to exist in time for the eddy lifetime, $t_{\mathrm{e}}$, it is quite probable that a particle trajectory may intersect in a type-C fashion with two or more eddies at the same time. While undergoing a type-C interaction a particle may interact with a different eddy in a type-I fashion. There is no possibility that two type-I interactions can occur simultaneously because of the ODT formulation implements triplet maps only one at a time.

One scenario is presented in Figure 3.11. Here a particle intersects a large eddy event denoted 1 , the particle continues to interact in a type-C fashion as long as the particle is inside of the eddy interaction box. While in the box, it also enters the interaction box of the eddy denoted 2. The particle feels both eddies \# 1 and \# 2 in a type-C manner until the particle leaves the interaction box of eddy number 2 . The particle continues feeling eddy \# 1 only, until it also enters eddy number 3 . At this point, it feels both \# 1 and \# 3. While still in eddy number 3 , the particle leaves the interaction box of 


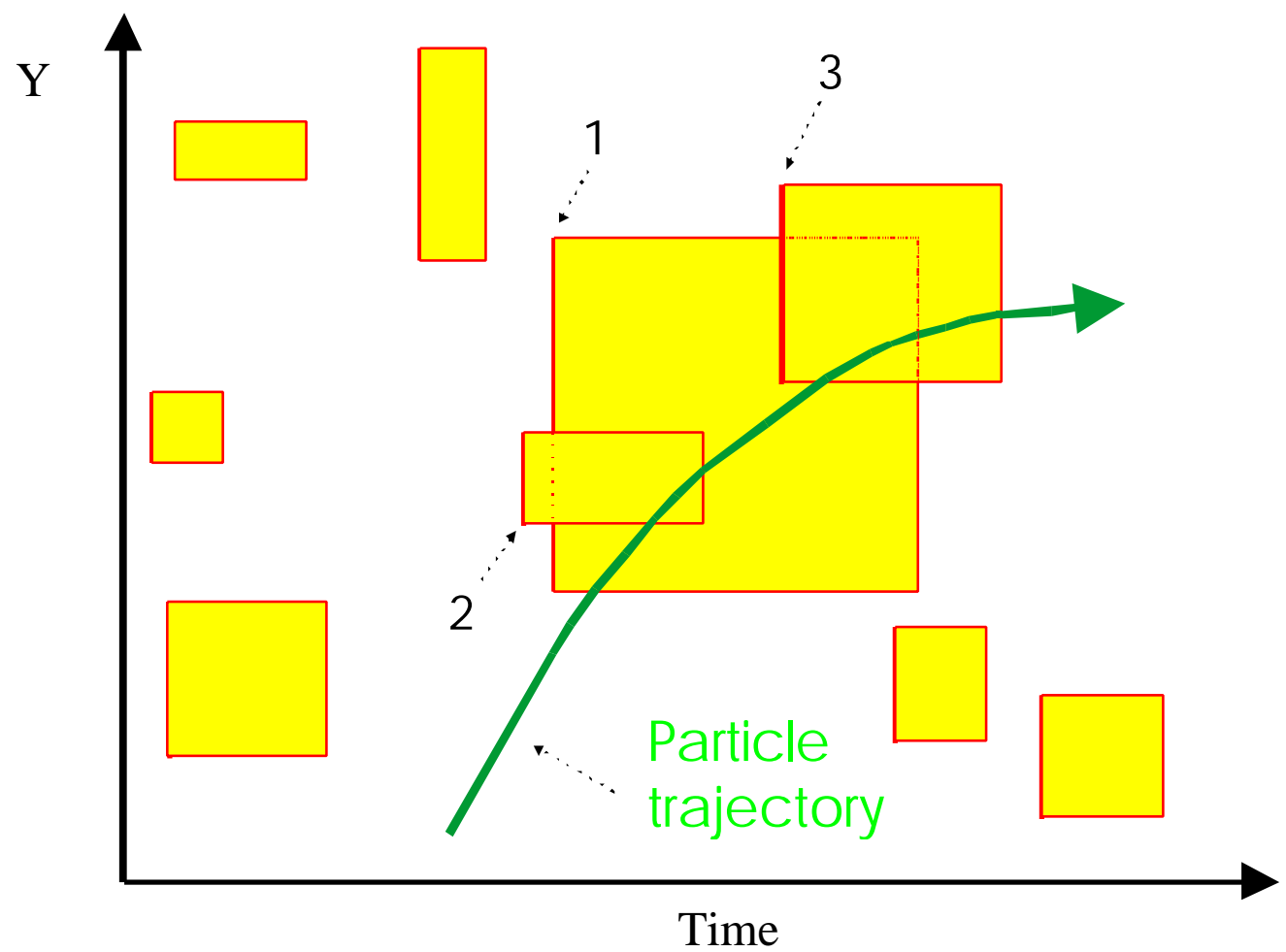

Figure 3.11: Multiple type-C interactions may occur at the same time. Here the particle interacts in a type- $\mathrm{C}$ fashion first with eddy number 1 , then with both eddies 1 and 2 , then with eddy 1 only, the with eddy 1 and 3 , and finally with eddy number 3 only.

eddy number one. After this, the particle continues in a type-C interaction with eddy \# 3 only, until the particle leaves the last interaction box.

Figure 3.12 gives a space-time history diagram with two possible particle paths in the same eddy sequence. In Figure 3.12 (A) the particle undergoes a type-I interaction with eddy number 1 , this is demarcated on the abscissa with a tick mark and is labeled I1 (for type-I interaction with eddy \#1). 

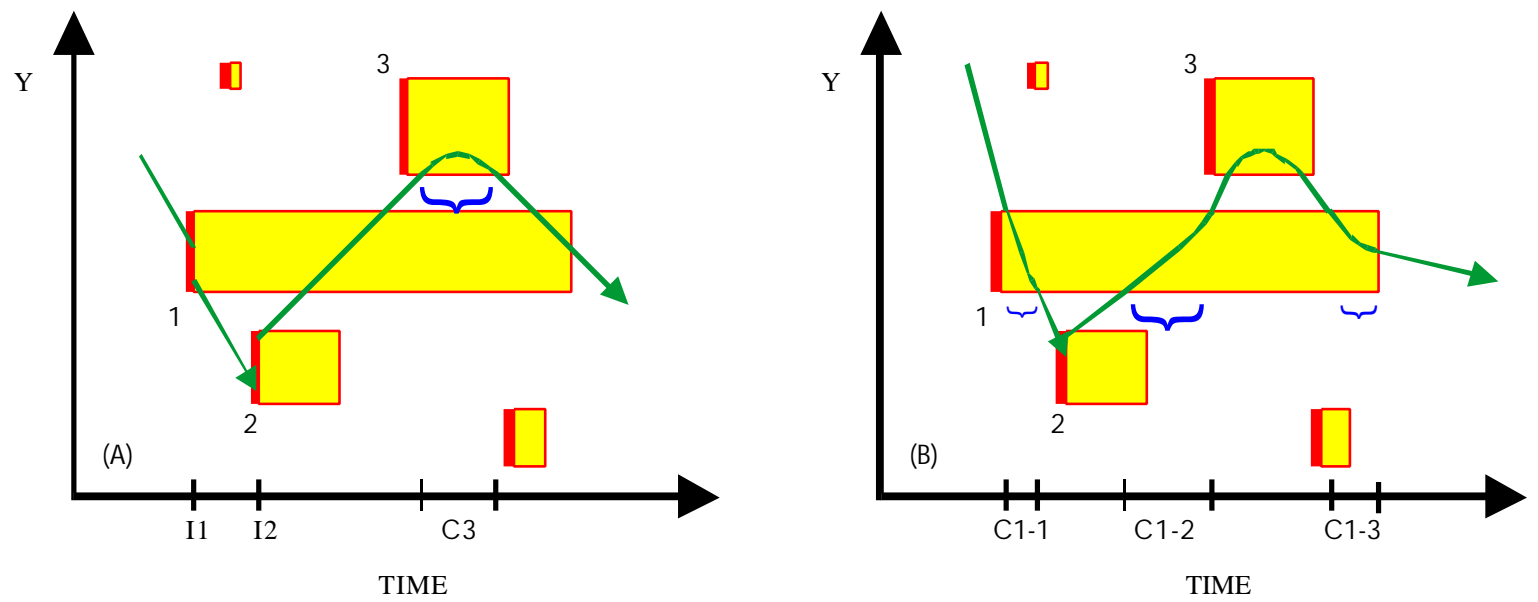

Figure 3.12: Possible type-I and type-C interactions for two different initial velocities. In (A) the particle re-enters eddy \#1 twice, but there is no additional interaction. In (B) the particle interacts with eddy \#1 three distinct times labeled C1-1, C1-2, and C1-3.

Then the particle undergoes a type-I interaction with eddy \#2, labeled I2. Finally it undergoes a type-C interaction with eddy \#3, this is demarcated with " $\{$ " and the time interval on the abscissa is marked with a $\mathrm{C} 3$ (for a Continuous interaction with eddy \#3). Even though the particle crosses the eddy \#1 interaction box two more times it does not interact with the eddy since it first experienced a type-I interaction. Figure 3.12 (B) depicts a different particle which enters eddy \#1 from the top and undergoes a type-C interaction for a time interval labeled C1-1 (for Continuous interaction with eddy \#1 - $1^{\text {st }}$ occurrence). It then experiences a type-I interaction with eddy \#2. It changes course and re-enters eddy \#1 and again experiences a type-C 
interaction labeled C1-2. It then undergoes a type-C interaction with eddy \#3 (not marked on the abscissa) and gets turned around once more where it re-enters eddy \#1's interaction box a third and final time. The particle then experiences a type-C interaction labeled C1-3.

Implementation of type-C interactions onto the ODT domain requires keeping track of the positions of all eddies from the time each eddy is born until that individual eddy's lifetime has expired. Any particle entering any of these y positions during that particular eddy's lifetime would interact continuously with that eddy as long as it had not undergone a type-I interaction with that same eddy previously. The result of allowing eddies to exist in the time domain is that for the duration of $t_{\mathrm{e}}$ the $y$ domain of the eddy $\left[y_{0}, y_{0}+l\right]$ is allowed to have a nonzero gas phase velocity $v$. Recall that in the ODT formulation turbulent flows are idealized as having a zero $v$ component, and the instantaneous triplet maps implement the eddy caused phenomena. Though the ODT program proper would be unaffected by the addition of the nonzero $v$ velocities, they would effect the particle time evolution as defined by the particle-drag law. Equation 3-9* would now have a gas phase component only in the $y$ interval $\left[y_{0}, y_{0}+l\right]$ and from the instant of the triplet map until the ODT time increment $t_{\mathrm{e}}$ elapsed. 


$$
\frac{d V}{d t_{p}}=-\left(V-\sum_{j} v\right) \frac{f}{\tau_{p}}+F_{G} \quad, \quad(3-9 * *)
$$

Here the summation over $j$ includes only the eddy events whose interaction box occupy the same space-time as the particle trajectory. There exists latitude in the specific mechanics to the type-C implementation, but the eddies would have either a positive or negative $v$ velocity (this could be chosen randomly). As such, the summation in Equation 3-9** would have to be a vector sum over all overlapping eddy events. Hence the affects of eddies could cancel each other out. There is also latitude in the definition of what the correct gas velocity is of the eddy. Some type of weighted average displacement of all the cells in the triplet map could be used as the distance, that with the eddy lifetime, $t_{\mathrm{e}}$, would then define the gas phase velocity of that particular eddy.

\subsection{Numerical implementation}

At this point the two-phase flow model is complete and a discussion of assumptions, simplifications, and numerical implementation techniques is in order. 
Rather than computing the Reynolds number as defined in Equation 3-7, the following numerical implementation simplifications were made for the $\mathrm{x}$ and y direction.

$$
\begin{aligned}
& \operatorname{Re}_{x}=\frac{\rho_{g} r_{d}\left|U-u_{g}\right|}{\mu_{g}} \\
& \operatorname{Re}_{y}=\frac{\rho_{g} r_{d}|V-v|}{\mu_{g}}
\end{aligned}
$$

These Reynolds numbers were then plugged into the Equation 3-6 (the empirical nonlinear drag law, f ) to predict the variation from Stokes drag for the respective particle-drag laws (in particle time, Equations 3-10* and 3-9*; in pseudotime modified to the moving coordinate system, Equations 3-24 and 3-12).

Integration of the 4 particle-drag laws $(x$ and $y$ components for particle time and pseudo time) is a key issue in efficient numerical implementation of the two-phase flow submodel. As discussed in detail in Section 2.7, the design of ODT is such that the greater the turbulence intensity (the larger the free parameter A) the shorter the eddy time step, $\Delta t_{\text {stir }}$, and hence a shorter the diffusion time step, $\Delta t_{\mathrm{dif}}$, is required to start smoothing the velocity 
profiles between implemented eddies. For highly turbulent flows, the values of eddy time-step and the diffusion time-step are constrained to be small.

Letting the droplet time step, $\Delta t_{\text {drop }}$, be approximately equal to the diffusion time step, allows the assumption that the coefficients in the particle-drag laws, $\tau_{\mathrm{p}}$ and $f$, are constant or nearly constant over the time period of integration of Equations $3-10^{*}$ and 3-9*. An added benefit is that $\Delta t_{\text {drop }}$ is in ODT time and must be adjusted to particle time, $\Delta t_{\mathrm{p}}$, by equation 3-11. On average the particle $\mathrm{U}$ velocity is greater then the bulk mean gas $u$ velocity hence the particle time increment is smaller then the prescribed ODT time difference, $\Delta t_{\text {drop. }}$. This gives more of a comfort zone to the assurance that the particle time increment is small enough to assure that the coefficients of the particle-drag law components are constant. An added level of comfort comes from the way in which the droplet submodel is implemented into the ODT structure. (See the general logic flow chart Figure 2.4, the pointer "clock" in Figure 2.3 and the corresponding discussion in Section 2.7.) The model implementation uses a fixed $\Delta t_{\text {drop }}$. Hence in the absence of an implementation of an eddy event the particle time is updated in proportion to the fixed $\Delta t_{\text {drop. }}$ However, whenever an eddy is implemented in the ODT time domain, the position of all particles at 
that instant must be computed in order to be assured that the particle and the triplet map occupy the same space time. This changes the time interval for the calculations from the maximum $\Delta t_{\text {drop }}$ to only some fraction of the maximum time interval. The time interval for the next update for the droplet is then adjusted to be $\Delta t_{\text {drop }}$. This corresponds to a shift in the droplet time line shown Figure 2.3. In the absence of another implementation of an eddy event during that time interval, $\Delta t_{\text {drop }}$, the particle position and velocity is not updated until the end of that time interval. If however, another eddy event is implemented, the particle is udated at the time of the eddy implementation and the time line is again adjusted as before.

Figure 3.13 is a pictorial continuation of Figure 2.3. The eddy event in Figure 2.3 was implemented hence the droplet position was computed at the identical time (using the much shorter particle time interval) and the droplet time line is adjusted (shifted left) so that the maximum time interval for integration of the particle-drag law is never more then the maximum $\Delta t_{\mathrm{drop}}$.

Assuming a small enough time interval such that the coefficients of Equation 3-9* $\left(\tau_{\mathrm{p}}\right.$ and $\left.f\right)$ are considered constant, integrating yields first the velocity, V, (Equation 3-28) and then integrating gives the position $\mathrm{y}_{\mathrm{p}}$ 


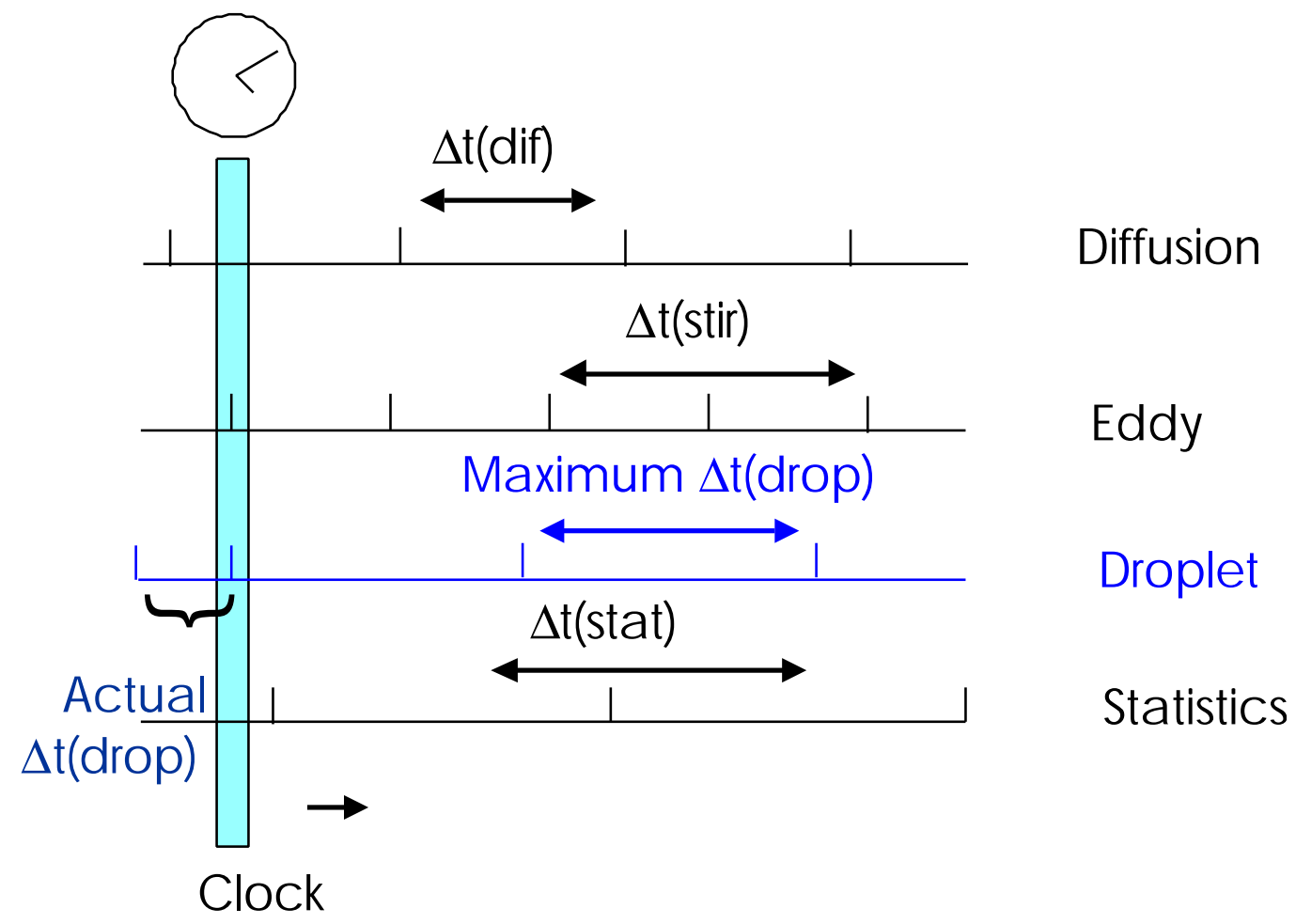

Figure 3.13: Actual $\Delta t_{\text {drop }}$ is only a fraction of the maximum $\Delta t_{\text {drop }}$ due to the implementation strategy of the two-phase flow submodel. The droplet time line is shifted in order to assure that the maximum time between droplet updates does not exceed the time interval $\Delta t_{\mathrm{drop}}$.

(Equation 3-29) in continuous time. The standard boundary conditions were used: at $t_{\mathrm{p}}=0, \mathrm{~V}=\mathrm{V}_{\mathrm{o}}$ and $y_{\mathrm{p}}=y_{\mathrm{o}}$.

$$
\begin{aligned}
& V=\frac{\tau_{p}}{f} F_{G}+\left(V_{o}-\frac{\tau_{p}}{f} F_{G}\right) \exp \left(-\frac{f}{\tau_{p}} t_{p}\right) \\
& y_{p}=y_{o}+\frac{\tau_{p}}{f} F_{G} t_{p}+\frac{\tau_{p}}{f}\left(V_{o}-\frac{\tau_{p}}{f} F_{G}\right)\left(1-\exp \left[-\frac{f}{\tau_{p}} t_{p}\right]\right)
\end{aligned}
$$


As discussed earlier, in continuous time there is no fluid $y$ velocity component, so, a fluid tracer particle (zero inertia) must not undergo translation in the $y$ direction. This can be verified easily by taking the limit as the particle inertia tends to zero of either Equation 3-28 or 3-29.

$$
\begin{aligned}
& \lim _{\tau_{p} \rightarrow 0} y_{p}=y_{o} \\
& \lim _{\tau_{p} \rightarrow 0} V=0
\end{aligned}
$$

The infinite inertia limit of Equation 3-28 shows that the particle continues to proceed unaffected by the particle drag law.

$$
\lim _{\tau_{p} \rightarrow \infty} V=V_{o}
$$

The continuous time particle trajectory in the $x$ direction is achieved by integrating Equation 3-10* twice with the standard initial conditions (at $t_{\mathrm{p}}=$ $0 ; x_{\mathrm{p}}=x_{\mathrm{o}}$, and $\mathrm{U}=\mathrm{U}_{\mathrm{o}}$ ). Again, assuming a small enough time interval such that the coefficients $\left(u, \tau_{\mathrm{p}}\right)$ of Equation 3-10* are considered constant, 


$$
\begin{aligned}
& U=u+\left(U_{o}-u\right) \exp \left[-\frac{f}{\tau_{p}} t_{p}\right] \\
& x_{p}=x_{o}+u t_{p}+\frac{\tau_{p}}{f}\left(U_{o}-u\right)\left\{1-\exp \left[-\frac{f}{\tau_{p}} t_{p}\right]\right\}
\end{aligned}
$$

As expected in the vanishing inertia limit, the particle matches the fluid parcel which surrounds it,

$$
\begin{gathered}
\lim _{\tau_{p} \rightarrow 0} U=u \\
\lim _{\tau_{p} \rightarrow 0} x_{p}=x_{o}+u t
\end{gathered}
$$

In the limit of infinite inertia Equation 3-33 gives the expected result that the velocity is unaffected by the particle drag,

$$
\lim _{\tau_{p} \rightarrow \infty} U=U_{o}
$$

The analytical solutions (Equations 3-33 and 3-34) had the added assumption that the particle does not leave the fluid parcel it is in during the particle time interval. This is an assumption since during continuous 
(particle) time the particles are allowed to move from one cell to another. This is a good assumption only for short time periods.

We now have a closed form solution for the particle velocity $\mathrm{U}\left(t_{\mathrm{p}}\right)$ for short times. With this the particle time can be computed using Equation 311 assuming the Re and diameter are constant and the particle does not leave the particular fluid cell it is in. This is only an approximation but gives the best estimate for the actual time the particle sees during the time the ODT streamwise evolution (at the mean $u$ velocity of the fluid). An alternate method to compute the time the particle actually experiences while the ODT time develops would be to actually iterate on the whole variable coefficient Equation (3-34) until convergence. This however would be very costly.

To compute the change in time, $\Delta t_{\mathrm{p}}$, which the particle experiences as the ODT time domain develops, $\Delta t_{\mathrm{ODT}}$, the ODT time is multiplied by at the mean $u$ velocity of the fluid, $\overline{\mathrm{u}}_{\mathrm{b}}$, to get the distance traveled. This resulting distance, given in Equation 3-38, is assigned to the difference in which the particle travels in Equation 3-34.

$$
x_{p}-x_{o}=\Delta x_{p}=\Delta t_{O D T} \bar{u}_{b} \quad(3-38)
$$

The resulting transcendental equation is 


$$
0=\Delta x_{p}+u \Delta t_{p}+\frac{\tau_{p}}{f}\left(U_{o}-u\right)\left\{1-\exp \left[-\frac{f}{\tau_{p}} \Delta t_{p}\right]\right\} \quad \cdot(3-39)
$$

Equation 3-39 could be solved, using any root finding technique, for the time the particle would take to traverse the distance $\Delta x_{\mathrm{p}}$ while experiencing drag. However, since the equation is only approximate, the exponential term in Equation 3-39 is expanded in a series with $\Delta t_{\mathrm{p}}$ and only the terms up to $\Delta t_{\mathrm{p}}^{2}$ are kept, giving

$$
H O T+\frac{(U-u) f}{2 \tau_{p}}\left(\Delta t_{p}\right)^{2}-U \Delta t_{p}+\Delta x_{p}=0
$$

All Higher Order Terms (HOT) are thrown out since $\Delta t_{\mathrm{p}}$ is small, and the resulting equation is solved using the standard equation to find the roots of a constant coefficient quadratic equation, where the particle time increment used is taken to be the smallest in magnitude, real root of the quadratic equation given in Equation 3-40. The ending particle position and velocity for the current step are taken as the initial conditions for the next step. Once the best estimate of the $\Delta t_{\mathrm{p}}$ for the particle is obtained, application of the continuous time particle-drag law is straightforward. This time increment is used to calculate actual variations in the particle position $\left(x_{\mathrm{p}}\right.$ and $\left.y_{\mathrm{p}}\right)$; particle 
velocity ( $\mathrm{U}$ and $\mathrm{V}$ ); and adjustments to the particle properties, e.g. diameter and temperature as discussed in detail in Chapter 4.

The next challenge is implementing the particle-drag laws for pseudotime. \{ In pseudotime, there are no adjustments made to the particle properties (diameter, temperature, etc.) since this would cause double counting once the particle re-enters particle time. The cause of this double counting is the analogous to the cause explained in Section 3.6. A brief discussion on alternative ways to take into account changing droplet properties during particle-eddy interactions is given in Chapter 4.\} In a manner similar to the particle time, it is assumed that during the pseudotime interval, $T_{1}$ : the eddy imposed $v$ velocity is constant (true in the limit for tracer particles) and the coefficients $\tau_{\mathrm{p}}$ and $f$ are constants, resulting in the ability to analytically integrate Equation 3-12. Integrating yields first the $y$ velocity (Equation 3-41) and then integrating again gives the position $y_{\mathrm{Tp}}$ (Equation 3-42) as a function of $T$. The particle's velocity and position at the instant of the particular particle-eddy interaction are taken as the boundary conditions: at $T=0, \mathrm{~V}_{\mathrm{T}}=\mathrm{V}_{\mathrm{To}}$ and $y_{\mathrm{Tp}}=y_{\mathrm{To}}$. Pseudotime integration gives 


$$
\begin{aligned}
& V_{T}=v+\frac{\tau_{p}}{f} F_{G}+\left(V_{T o}-v-\frac{\tau_{p}}{f} F_{G}\right) \exp \left(-\frac{f}{\tau_{p}} T\right) \\
& y_{T p}=y_{T o}+\left(v+\frac{\tau_{p}}{f} F_{G}\right) T+\frac{\tau_{p}}{f}\left(V_{T o}-v-\frac{\tau_{p}}{f} F_{G}\right)\left(1-\exp \left[-\frac{f}{\tau_{p}} T\right]\right)
\end{aligned}
$$

Note that a tracer particle behaves as it should (Equations 3-43 and 3-44).

$$
\begin{gathered}
\lim _{\tau_{p} \rightarrow 0} V_{T}=v \\
\lim _{\tau_{p} \rightarrow 0} y_{T p}=y_{T o}+v T
\end{gathered}
$$

Also, an infinitely large inertia particle is unaffected by the particle-eddy interaction (Equation 3-45).

$$
\lim _{\tau_{p} \rightarrow \infty} V_{T}=V_{o}
$$

In the $x^{*}$ direction, we also make the assumptions that during the pseudotime interval, $T_{1}$ : the fluid imposed $u$ velocity is a constant equal to the $u$ value of the cell initially containing the particle, (true in the limit for tracer particles) and the coefficients $\tau_{\mathrm{p}}$ and $f$ are constants, resulting in the ability to analytically integrate Equation 3-24. Integrating yields first the $x^{*}$ velocity (Equation 3-46) and then integrating again gives the position $\mathrm{X}^{*}$ (Equation 3-47) as a function of $T$. The particle velocity and position at the 
instant of the particular particle-eddy interaction are taken as the boundary conditions: at $T=0, \mathrm{U}^{*}=\mathrm{U}^{*}{ }_{\mathrm{o}}$ and $\mathrm{X}^{*}=\mathrm{X}_{\mathrm{o}}$. Pseudotime integration gives

$$
\begin{aligned}
& U^{*}=u-u_{e d d y}+\left(U_{o}^{*}-u+u_{e d d y}\right) \exp \left\{-\frac{f}{\tau_{p}} T\right\} \\
& X^{*}=X_{o}+\left(u-u_{e d d y}\right) T-\frac{\tau_{p}}{f}\left(U_{o}^{*}-u+u_{e d d y}\right) \exp \left[-\frac{f}{\tau_{p}} T\right]
\end{aligned}
$$

The infinitesimally small and infinitely large limits of particle inertia behave properly:

$$
\begin{aligned}
& \lim _{\tau_{p} \rightarrow 0} U^{*}=u-u_{\text {eddy }} \\
& \lim _{\tau_{p} \rightarrow 0} X^{*}=X_{o}+\left(u-u_{e d d y}\right) T \\
& \lim _{\tau_{p} \rightarrow \infty} U^{*}=U_{o}^{*} .
\end{aligned}
$$

With the pseudotime particle position $\mathrm{X}^{*}$ and $y_{\mathrm{Tp}}$ known analytically, determining the correct particle-eddy interaction time $T_{1}$, is quite easy. First. set Equation 3-41 to zero and solve for the time, if any, at which the $y$ extrema, $T_{\text {ey }}$, exists. Second, checking to see if this time, $T_{\text {ey }}$, is less than the eddy lifetime $t_{\mathrm{e}}$. If the extremum occurs during the eddy lifetime then the particle position $y_{\mathrm{Tp}}$, is evaluated using Equation 3-42. If this value is 
between $y_{\mathrm{o}}$ and $y_{\mathrm{o}}+l$ then there is no danger of the particle exiting the interaction box and reentering as depicted in Figure 3.7. In this case where the extremum is not outside the interaction box, $T_{1}$ is set equal to $t_{\mathrm{e}}$. If the particle exits the interaction box, for example, from the bottom, $y_{\mathrm{Tp}}\left(T_{\mathrm{ey}}\right)$ is less than $y_{\mathrm{o}}$, then in Equation 3-42 $y_{\mathrm{Tp}}$ is set equal to $y_{\mathrm{o}}$ and this is solved by a root finding technique to determine the time $T_{1 \mathrm{y}}$ at which the particle first leaves in the $y$ direction. For a particle which exits the top surface, $y_{\mathrm{p}}$ is set equal to $y_{0}+l$, and the first exit time is determined in the same fashion as above, after which $T_{1}$ is set to $T_{1 \mathrm{y}}$.

The next step in finding the correct particle-eddy interaction time is analogous to the procedure in determining $T_{1 \mathrm{y}}$. To determine whether and when the particle leaves the interaction box in the $x^{*}$ direction, Equation 346 is set to zero and the time $T_{\mathrm{ex}}$ of the occurrence of an extremum (if any) is calculated. If $T_{\mathrm{ex}}$ is less than $T_{1}$ then $\mathrm{X}^{*}\left(T_{\mathrm{ex}}\right)$ is evaluated using Equation 347. If $X^{*}$ is inside the range $[-l,+l]$ then the particle does not leave the interaction box in the $x$ direction. There is no danger of the particle exiting the interaction box in the $x^{*}$ direction and returning as shown in Figure 3.10.

However, if a particle leaves the interaction box by one of the vertical sides (say the right), $X^{*}$ in Equation $3-47$ is set equal to $l$, \{if the particle 
exits on the left hand side of the box, $\mathrm{X}^{*}$ is set equal to $-l$. Equation 3-47 is then solved for the time $T_{1 \mathrm{x}}$ at which the particle exits the interaction box. The interaction time $T_{1}$ is then set to $T_{1 \mathrm{x}}$. If the extrema of the particle trajectory exited the interaction box by either the $y$ or $x^{*}$ directions before the eddy lifetime expired, then $T_{1}$ is set to the correct interaction time at this point.

However, if neither the $y$ nor $x^{*}$ direction extrema exited the interaction box before the eddy lifetime expired, then $y_{\mathrm{Tp}}$ and $\mathrm{X}^{*}$ are evaluated at $t_{\mathrm{e}}$. If $y_{\mathrm{Tp}}\left(t_{\mathrm{e}}\right)$ and $\mathrm{X}^{*}\left(t_{\mathrm{e}}\right)$ are inside the interaction box, then the correct value of $T_{1}$ is $t_{\mathrm{e}}$. If the particle trajectory endpoint $\left(\mathrm{X}^{*}\left(t_{\mathrm{e}}\right), y_{\mathrm{Tp}}\left(t_{\mathrm{e}}\right)\right)$ falls outside the interaction box, then one or both of Equations 3-47 and 3-42 has to be solved in an analogous manner to the technique explained above for finding the first time that the particle exits the eddy. This is the correct value for $T_{1}$.

This completes the major simplification (Re), the assumptions (constant coefficients, etc.), and the numerical implementation techniques necessary to fully implement the two-phase flow submodel in an analogous manner used by the author to produce the numerical data which are presented in this paper. 


\section{Chapter 4}

\section{Droplet Burning Model}

\subsection{Introduction}

In order to understand the model for vaporization of the droplets, the reader requires a discussion of the principal physical phenomena which the droplets experienced during the experiment. Great effort was made to inject streams of droplets with uniform size, $\mathrm{D}_{0}$; uniform spacing, $\mathrm{s} / \mathrm{D}_{\mathrm{o}}$; injection velocity, $\mathrm{V}_{\mathrm{o}}$; and injection angle, $\theta$. Droplets were injected by way of a vibrating orifice device from a reservoir at room temperature into a $100 \mathrm{~kW}$ furnace. Sirignano (1999) states that a significant amount of the lifetime of this type of burning droplet is used in heating the droplet from room temperature to its dew point. This is accompanied by a significant ignition delay time, after which the droplet will ignite, provided there is an ignition source. After the droplet is burning, the heat release from the surrounding flame is a significant added heat source for the evaporating droplet. These phenomena were also observed by Mulholland et al. Hence a droplet heating/burning model is needed which includes the significant variables and predicts the heating rate is necessary. The flame is hot enough (maximum mean temperature is $1750 \mathrm{~K}$ ) to serve as an ignition source for the droplets. 
The ignition delay time was measured for each case. This measured ignition delay was used in the model to determine the onset of droplet ignition.

Another significant variable is the initial droplet spacing s/ $\mathrm{D}_{\mathrm{o}}$. A droplet that is part of a stream of droplets travels farther into a turbulent reactor than it would if it were injected alone. The droplet travels in the wake of the droplets in front of it. Therefore the drop experiences less drag and less evaporation and can travel farther into the reactor. Mulholland et al. (1991) observed that the measured droplet stream, once injected into the reactor, travels in a straight line until the droplets reach the high shear layer of the gas flow, at which point the droplet stream breaks up into random droplets.

Unfortunately the ODT model with its two-phase flow sub model does not lend itself to modeling droplet streams. The idea of a stream of droplets traveling in unison along a trajectory is hard to conceive of in a onedimensional turbulence model which develops in time and treats eddies as instantaneous mappings of the velocity and scalar fields. The main difficulty arises when one tries to model what happens to a droplet in the stream which is suddenly tossed about by an eddy. Does it leave the stream and go off by itself leaving the other droplets in the stream continuing in a linear manner? What effects would this one missing droplet out of a stream 
of droplets have on the drag and evaporation of the rest of the stream? No logically consistent model presents itself for a one-dimensional time developing flow.

Besides the conceptual difficulty of having a stream of droplets in ODT and the many complications any model would have to have in order to handle suddenly allowing a single droplet to leave instantaneously the droplet line, this type of behavior was not observed experimentally in the reactor. Hence a different approach to modeling the initial linear droplet trajectory is in order.

One of the main objectives of this work was to see whether the ODT model could reproduce the spread in the burn out points of the burning droplets observed in Mulholland et al. (1991). A reasonable plan then would be to reproduce the droplet burning model and droplet trajectory model used in the original paper as closely as possible for the initial linear trajectory of the droplets. Once the droplet stream reached the high shear layer of the fluid flow the droplet position, diameter, velocity and temperature computed from that model would be used as initial conditions for the ODT two-phase flow model. The only remaining addition needed for the ODT two-phase flow model would be that of a heating/burning model, for which the same 
model used for the initial conditions is utilized with the exception that the correction factor $\left(\eta_{\mathrm{s}}\right)$ for inter droplet spacing is assumed unity. In the ODT simulations, the droplets were assumed to act independently of each other. In the spirit of ODT each case would be modeled as an ensemble of droplets all having the same initial conditions. The computed spread of the droplet burnout points would be compared to the experiment.

\subsection{Droplet heating model used by Mulholland et al.}

A brief recapitulation of the droplet transient heating/burning model given in Mulholland et al. follows. The reader is referred to that publication for more details. The liquid droplet is assumed to be spherically symmetric with infinite heat diffusivity ("rapid mixing limit"). Therefore the droplet temperature is allowed to change with time, but there is no need to solve any of the fluid flow equations inside the droplet since the temperature is assumed constant across the droplet. The liquid phase properties (e.g. density and heat capacity) are constant. The heating time of the droplet is slow compared to the gas diffusion time. Mulholland et al. idealizes the droplet fuel mixture to be one representative (unnamed) chemical component. They give a table with mean properties of the liquid density, liquid heat capacity, boiling temperature, the heat of vaporization, the heat 
of combustion and the stoichiometric fuel-to-oxygen mass ratio for this idealized one component fuel. The concentration of this component in the gas phase (assuming equilibrium) is determined by the Clausius-Clapeyron equation:

$$
X_{f s}=\frac{1}{p} \exp \left[\frac{L}{R}\left(\frac{1}{T_{b}}-\frac{1}{T}\right)\right]
$$

where $\mathrm{X}_{\mathrm{fs}}$ is the gas phase mole fraction of the fuel species, $\mathrm{f}$, at the interface. The mass fraction, $\mathrm{Y}$, and mole fraction, $\mathrm{X}$, are related by the standard equations

$$
\begin{aligned}
& Y_{n}=\frac{X_{n} W_{n}}{\sum_{i} X_{i} W_{i}} \\
& X_{n}=\frac{Y_{n} / W_{n}}{\sum_{i} Y_{i} / W_{i}}
\end{aligned}
$$

with the sum $i$ being over all the species in the gas phase.

The gas phase is assumed to be in a quasi-steady state, with heat and mass diffusion rate controlling. With the assumption of $\mathrm{Le}=1$, the Sherwood number is equal to the Nusselt number. Ambient gas phase conditions are sub critical and the gas phase mixture is assumed to behave as an ideal gas mixture. Infinitely fast kinetics are assumed, so the flame sheet surrounds the burning droplet. (In other 
words, the reaction time is shorter than the diffusion time and a flame surrounds the droplet after it has ignited.) This was experimentally verified by Mulholland et al.. The droplets would ignite (glow) a short time before they would completely burn out.

The authors derive [following Law (1976)] the following set of equations. An energy balance around the drop results in

$$
m c_{p l}\left(\frac{d T_{d}}{d t}\right)=\left(H-\Delta H_{v}\right) \frac{d m}{d t} \quad(4-4)
$$

where $\mathrm{H}$ is the total heat transfer from the gas to the droplet. A mass balance yields

$$
\frac{d m}{d t}\left[=-\left(\pi \rho D^{2} / 2\right)(d D / d t)\right]=\pi \bar{\rho} \bar{D}_{A B} D S h^{*} \ln (1+B) \eta_{s}
$$

with $\eta_{\mathrm{s}}$, representing the effect of the interaction of the droplets in a linear array on vaporization. This has been quantified by Labowsky [(1980)] as

$$
\eta_{s}=1-\left(2 \frac{s}{D}\right)^{-1}+\left(2 \frac{s}{D}\right)^{-2}, \quad(4-6)
$$


The Spalding mass transfer number, $\mathrm{B}$, is given by

$$
B=\left\{\bar{c}_{p}\left(T_{\infty}-T_{d}\right)+Y_{0 \infty} f \Delta H_{c}\right\} / H \quad(4-7)
$$

and the total heat transfer from the gas to the droplet, $\mathrm{H}$, is given by

$$
H=\left(1-Y_{F s}\right)\left\{c_{p}\left(T_{\infty}-T_{d}\right)+Y_{O \infty} f \Delta H_{c}\right\}\left(Y_{F s}+Y_{O \infty} f\right)^{-1}
$$

The gas-phase density and other mean gas properties in the film layer are evaluated at the 'one-third law' temperature [Hubbard et al. (1975)]. Hence, the gas phase components of the Re, $\mathrm{Pr}$, and the Sc are computed at the representative temperature:

$$
\bar{T}=T_{d}+\frac{1}{3}\left(T_{f}-T_{d}\right)
$$

Assuming the adiabatic flame temperature for the flame sheet temperature, $\mathrm{T}_{f}$ becomes:

$$
T_{f}=T_{\infty}+Y_{O \infty} f\left\{\Delta H_{c}-H-\bar{c}_{p}\left(T_{f}-T_{d}\right)\right\} / c_{p}
$$

where $\bar{c}_{p}$ is the heat capacity of the fuel in the gas phase.

Clift et al. [1978] gives the following correlation for the Sherwood number without any vaporization: 


$$
\begin{gathered}
S h_{0}=1+(1+\operatorname{Re} S c)^{1 / 3} f(\operatorname{Re}) \quad(4-11) \\
f(\operatorname{Re})=\left\{\begin{array}{cc}
1 & \operatorname{Re} \leq 1 \\
\operatorname{Re}^{0.077} & 1<\operatorname{Re} \leq 400
\end{array}\right.
\end{gathered}
$$

The droplet Re,).

$$
\operatorname{Re}=\frac{\rho_{g} r_{d}\left|\tilde{V}_{d}-\tilde{v}_{g}\right|}{\mu_{g}},
$$

is based on the relative velocity between the droplet and the fluid, droplet radius, and gas properties (the same as in Chapter 3

They use the analysis of Abramzon and Sirignano [(1987)] to account for forced convection and vaporization effects on convective heat and mass transfer. They consider a spherical vaporizing droplet inducing a radial Stefan flow. The vapor convects radially outward while the heat conducts radially inward against the flow of the vapor toward the liquid-gas interface. The energy of the heat conducted in is divided between heating the liquid and the phase change of the liquid at the surface into the gas phase. This newly evaporated fluid in turn convects radially outward. The Sherwood number with Stefan type flow vaporization becomes 


$$
S h^{*}=2+\left(S h_{0}-2\right)\left\{(1+B)^{0.7} \ln (1+B) / B\right\}^{-1}
$$

\subsection{Introduction of the burning rate parameter}

The phenomenon of droplet burning is very complex and the many simplifying assumptions used in the preceding analysis caused Mulholland et al. to introduce a measured parameter $\lambda$ for the burning rate. They chose a parameter that could be measured in a laboratory-scale reactor. They defined $\lambda$ as

$$
\frac{d D}{d t}=-\lambda\left(\frac{S h^{*}}{2}\right) \eta_{s} / 2 D
$$

By the integration of Equation 4-15 for a constant $\lambda$ and both Sh* equal to two and $\eta_{\mathrm{s}}$ equal to unity, the familiar $\mathrm{d}^{2}$ law is reproduced

$$
D_{o}^{2}-D^{2}=\lambda t \quad(4-16)
$$

The Spalding mass transfer number is easily calculated from $\lambda$ from Equation 4-17 or 4-18. 


$$
\begin{aligned}
\lambda & =\left\{8 \bar{\rho} \bar{D}_{A B} / \rho_{l}\right\} \ln (1+B) \\
& =\left\{8 \bar{k} /\left(\bar{c}_{p} \rho_{l}\right)\right\} \ln (1+B)
\end{aligned}
$$

B is calculated independently, using Equation 4-7, from the point of injection until the droplet reaches its wet bulb temperature. This constitutes the major portion of the droplet lifetime. The wet bulb temperature is achieved as $\mathrm{dT}_{\mathrm{d}} / \mathrm{d} t$ approaches zero and simultaneously the total heat transfer $\mathrm{H}$ is used to cause a phase change in the liquid at the surface. After this, the measured burning rate, $\lambda$, is utilized in the model to compute $\mathrm{B}$ for the remainder of the droplet lifetime.

\subsection{Review of droplet-drag law used in Mulholland et al.}

As mentioned previously, the ideal initial conditions for the ODT twophase flow submodel would be to reproduce the droplet trajectory model from Mulholland et al. They develop a three-dimensional model which allows them to compute the $\mathrm{x}, \mathrm{y}$, and $\mathrm{z}$ variations of the drop. They use the mean measured gas velocity and temperature profiles to compute droplet trajectories. Mulholland et al. (1988) relate the drag of nonvaporizing droplets to interdroplet spacing as follows 


$$
\begin{aligned}
& \left\{C_{D}\left(\operatorname{Re}, \frac{s}{D}\right)\right\}^{-0.678}=\left\{C_{D}^{0}\left(\operatorname{Re}, \frac{s}{D}\right)\right\}^{-0.678}+\left\{C_{D}^{\infty}(\operatorname{Re})\right\}^{-0.678} \\
& C_{D}^{0}\left(\operatorname{Re}, \frac{s}{D}\right)=C_{D}^{00}(\operatorname{Re})+43 \operatorname{Re}^{-1}\left\{\frac{s}{D}-1\right\} \\
& C_{D}^{00}=0.755 / \operatorname{Re} \\
& C_{D}^{\infty}(\operatorname{Re})=\{24 / \operatorname{Re}\}\left\{1+0.11 \operatorname{Re}^{0.687}\right\}
\end{aligned}
$$

They use the model of Ayers et al. (1980) for the prediction of isolated droplets and modify it to include effects of droplet interaction on drag. Drag and gravitation are assumed the only external forces and they come up with

$$
\begin{aligned}
& \left|F_{D 1}\right|=C_{D}^{*} \rho A v_{r 1}^{2} / 2 \\
& \left|F_{D 2}\right|=C_{D}^{\infty *} \rho A v_{r 2}^{2} / 2
\end{aligned}
$$

where the $*$ denotes mass transfer droplet drag coefficient with evaporation, they define, $v_{\mathrm{g}}$ is the gas velocity, $\mathrm{V}$ is the droplet velocity, $v_{\mathrm{r} 1}$ is the relative droplet-gas velocity aligned in the direction in which the droplet is proceeding and $v_{\mathrm{r} 2}$ is the resultant vector of the difference of $v_{\mathrm{r}}$ and $v_{\mathrm{r} 1}$.

$$
\tilde{v}_{r}=\tilde{V}-\tilde{v}_{g} \quad(4-25)
$$




$$
\tilde{v}_{r 2}=\tilde{v}_{r}-\tilde{v}_{r 1} \quad(4-26)
$$

Eisenklam et al. (1967) found a correlation between the mass transfer droplet drag coefficient with and without evaporation

$$
\begin{aligned}
& C_{D}^{*}=\eta_{e} C_{D} \\
& \eta_{e}=1 /(1+B) \cdot(4-27)
\end{aligned}
$$

They (Mulholland et al.) do a force balance and get:

$$
\begin{aligned}
& \sum F_{x}=m a_{x}=F_{D 1 x}+F_{D 2 x} \\
& \sum F_{y}=m a_{y}=F_{D 1 y}+F_{D 2 y}+F_{G}(=-m g) \\
& \sum F_{z}=m a_{z}=F_{D 1 z}+F_{D 2 z}
\end{aligned}
$$

\subsection{Modification of trajectory model for this application}

This three-dimensional model is unnecessary for this particular application since they mentioned that the droplets travel in a straight line during the initial "droplet stream" phase of the trajectory. Also, an initial three-dimensional trajectory model would not make much sense feeding a 
one-dimensional model. Therefore the three-dimensional model is simply projected onto the straight linear path expressed in Equation 4-32.

$$
\sum F_{D 1}=m \frac{d V_{D 1}}{d t}=F_{G D 1}+C_{D}^{*} \rho A\left(v_{D 1}-V_{D 1}\right)^{2}
$$

where $V_{D 1}$ is the (scalar) velocity of the droplet in the D1 direction and $v_{D 1}$ is the component of the velocity of the gas in the direction the drop is traveling. The D1 direction is defined as the direction the droplet is traveling, so this is precisely the injection angle, $\theta$, measured down from the horizon, so

$$
F_{G D 1}=-m g \sin (\theta) \quad(4-33)
$$

Equation 4-32 is the non-linear droplet-drag law. When Equations 4-19, 420, 4-21, 4-22, 4-27, 4-28, 4-32 and 4-33 are used in conjunction with

$$
\operatorname{Re}=\frac{\rho_{g} r_{d}\left|V-v_{D 1}\right|}{\mu_{g}} \quad(4-34)
$$

and Equations 4-1, 4-4, 4-5, 4-6, 4-8, 4-9, 4-10, 4-11, 4-12, 4-14, and 4-7 the total set of equations determine the model to compute the boundary 
conditions for the ODT two-phase submodel, from initial droplet injection up until the point that the droplet appears in ODT space.

Since the initial conditions are only required to be computed once for each of the 12 cases (and are identical for all the ensembles) in this specific application of these equations the time steps were taken to be very small, of order $10^{-5}$ seconds. Hence the coefficients in Equation 4-32 are considered constant over that short time and the non-linear droplet-drag law is solved analytically over a given $\Delta t$. Then the droplet diameter is adjusted for that time period as per the droplet transient heating model.

Equations 4-1; 4-4; 4-5; 4-6; 4-8; 4-9; 4-10; 4-11; 4-12; 4-14; and 4-7 are used to determine the transient droplet heating/evaporation model for use in ODT space except that $\eta_{\mathrm{s}}$ is set equal to one, hence, there is no dropletdroplet interaction or to put it another way the s/D effect becomes nonexistant for the lone drop. After ignition has been achieved, the last equation (4-7) is replaced by Equations $4-15$ and $4-17$ or $4-18$ to give the transient droplet heating/evaporation model used, until complete burnout is achieved. In a manner similar to Mulholland et al. the published gas mean velocities and temperature profiles were used for calculating the drag. 
Once in the ODT space the simplifications of the Re number as given in Equations 3-26 and 3-27 are implemented (where needed) for all calculations of the transient heating/evaporation model.

No droplet diameter adjustments are made during a type-I eddy event no matter how long the droplet-eddy interaction event persists in pseudotime. This is because eddy events in ODT are considered instantaneous events and would lead to double counting the evaporation (loss in mass) and particle heating (increase in temperature) during the time $T_{1}$ of the droplet-eddy interaction once the droplet returned to particle time. There is the possibility of doing a subtraction method directly analogous to that used to avoid double counting of the gravitational force, as explained in Section 3.6. That is to compute the particle trajectories and corresponding mass and temperature changes with and without the presence of the eddy induced velocity $v$. After computing the loss in mass and the increase in temperature both ways, subtract the differences in mass and temperature in the same manner as the velocity and position of the particle are changed in Equations 3-14 and 315. This was not done in this thesis because of the added complexity involved. 


\subsection{Conclusion}

The end result is that the initial conditions used for the droplets in the ODT two-phase flow model are as close as possible to the droplet diameter, temperature, and position used in the numerical model of Mulholland et al. Only access to the original droplet trajectory code used by them would yield a more precise initial condition for the ODT droplet sub model. Application of their droplet heating/evaporation model to the ODT droplet sub model provided consistency between their work and this work and more importantly consistency between the initial conditions to this model and the model itself. This method has proved to provide insight into the prediction of the spread in end points for the burning of rogue droplets. (See Chapter 5.) 


\section{Chapter 5}

\section{Numerical Simulation Results}

\subsection{Introduction}

The results discussed in this chapter use the existing ODT model to predict turbulent fluid flow behavior (Chapter 2) along with the two-phase flow submodel developed in Chapter 3 and the droplet heating/evaporation model given in Chapter 4. Simulations were done to mimic the 12 cases (See Table 5.1) done by Mulholland et al. (1991) in which they varied the initial diameter, initial velocity, initial droplet spacing and the injection angle of a stream of droplets into a $100 \mathrm{~kW}$ combustor. According to Mulholland (2000) the amount of data used to compute the statistics in the 1991 paper was on the order of 100 droplets. Consequently, three cases of ensemble sizes are investigated for comparisons with the published experimental data. They are 200, 750, 2000 in number respectively. We believe that this is a wide enough range to capture any variation due to ensemble size. Pertinent figures will be given in all three ensemble sizes so the reader may compare them at his/her leisure.

As mentioned earlier, experimental observation revealed that the droplets traveled in a straight line trajectory until they reached the high fluid shear 
layer. After this point the droplet stream appeared to break up into individual droplets. This point is taken to be the point at which the individual droplets are born into the ODT simulation. In order to properly account for droplet heating and vaporization from the point of droplet injection to the point in which the droplets are born into ODT, the droplet drag law and droplet heating/vaporization scheme put forth in Chapter 4 were calculated for each case to determine the best possible estimate of the droplet diameter, and temperature. These Initial Conditions (ICs) were used for each case for all 2000 realizations. The computer code was modified such that all twelve cases (maximum of 15 cases possible) were computed simultaneously. Hence all twelve cases saw the same flow realization. Another way to say this is that a single ODT realization contained within it twelve different droplets of varying diameter, velocity, position, and temperature, which represented the twelve different cases being simulated. This was repeated 2000 times in order to get the total data set. The 200 realization set was taken to be the first 200 realizations, and the 750 realization set was taken to be the first 750 realizations. 


\subsection{Droplet trajectories}

As discussed in Chapter 3, the two-phase flow model allows instantaneous jumps in the $y$ position of the droplet to account for eddyparticle interactions. To demonstrate this visually, a thorough (but not exhaustive) search was done of Case 1 (the baseline case used in investigating variations in initial diameter, initial velocity, initial droplet spacing and the injection angle of a stream of droplets) for trajectories which would exemplify the extremes in the spread $(x$ and $y)$ in the burn out points of the 2000 size ensemble. The burn out point is taken to be the point at which the last amount of liquid is vaporized. This occurs after the droplet has been ignited, therefore the vapor is assumed combusted in the surrounding flame sheet.

Figure 5.1 shows twenty-one individual trajectories chosen from Case 1. The random nature of the submodel can be seen by examining the different trajectories. Individual trajectories can proceed to the end of their paths (the burn out point) without much visible effect of the eddy interaction. (See the center trajectory.) Trajectories may also jump up or down (or both) randomly as demonstrated in Figure 5.1. These instantaneous jumps cause variation in the $y$ position of the burn out point. Note also that the ODT 
imposed variations in fluid velocity and temperature cause a wide variation in the $x$ burn out position. Recall that these trajectories were purposefully chosen to show extremes and are not typical trajectories (nor are they a random sample).

\subsection{Burn out scatter plots}

To provide a clearer understanding of the simulated effects of the different varied parameters (i.e. initial diameter, initial velocity, initial droplet spacing and the injection angle) scatter plots of the burn out points are grouped together. (Scatter plots are $x-y$ plots where $x$ is the axial distance penetrated by the droplet and $\mathrm{y}$ is the height. The $y=0$ datum plane is defined as 0.25 meters below the burner. The burner is on the central axis of the tubular combustor.)

In Figure 5.2 the scatter plots for the variations in initial diameter: Case 1 (base case with $\left.D_{0}=234 \mu\right)$ diagram $(A)$, Case $2\left(D_{o}=306 \mu\right)$ diagram (B), and Case $3\left(D_{o}=371 \mu\right)$ diagram $(C)$ are grouped together for easy comparison of the 200 realization. Figure 5.3 gives the same grouping for the 750 realization and Figure 5.4 gives the variation in initial diameter for the 2000 realization. Analysis of the scatter plots in each of the figures, gives the conclusion that the model predicts that larger droplets (more mass) 
travel farther down the reactor. This is exemplified in both the onset of burnout (leftmost points) and in the completion of burnout (rightmost points). Analysis also shows that the larger the droplet (greater inertia) the less scatter (in the $y$ direction) from the mean droplet trajectory. The mean axial penetration of the droplets increase as the diameter increases. This is evident from the plots, however, the calculated mean values are presented in Figures 5.14, 5.15, and 5.16 (to be discussed later).

Figure 5.5 gives the scatter plots which account for the model's prediction of the variation due to the initial velocity: Case $4\left(\mathrm{~V}_{\mathrm{o}}=5.3 \mathrm{~m} / \mathrm{s}\right)$ diagram (A), Case $1\left(\mathrm{~V}_{\mathrm{o}}=8.5 \mathrm{~m} / \mathrm{s}\right)$ diagram $(\mathrm{B})$, and Case $5\left(\mathrm{~V}_{\mathrm{o}}=10.6 \mathrm{~m} / \mathrm{s}\right)$ diagram (C) for an ensemble size of 200. Figure 5.6 gives the same grouping for an ensemble size of 750. Figure 5.7 gives the grouping for an ensemble size of 2000. The trend, visible in all three figures, is that the simulation predicts the onset of burnout (leftmost points) occurs at shorter axial distances as the initial velocity decreases. Figures 5.6 and 5.7 readily show that the simulation predicts the completion of burnout that penetrates farther axially as the initial velocity increases. The 200 ensemble size comparison however does not exemplify this trend everywhere. Figure 5.5 (A) shows a single droplet which penetrates just a little bit farther axially 
than the right most point of the faster initial velocity case shown in Figure 5.5 (B). This anomaly is taken to be a function of the sample size because both the 750 and 2000 ensemble size comparisons give the correct trend, and that the mean penetration in all three ensemble sizes increase as the initial velocity increases.

Figure 5.8 gives the scatter plots which account for the model's prediction of the variation due to the initial droplet spacing: Case 6 (s/D $=$ 2.8) diagram (A), Case $7(\mathrm{~s} / \mathrm{D}=5.6)$ diagram (B), Case $8(\mathrm{~s} / \mathrm{D}=11.2)$ diagram $(\mathrm{C})$, Case $9(\mathrm{~s} / \mathrm{D}=22)$ diagram $(\mathrm{D})$, Case $10(\mathrm{~s} / \mathrm{D}=45)$ diagram $(\mathrm{E})$, and Case $1(\mathrm{~s} / \mathrm{D}=180)$ diagram $(\mathrm{F})$ for an ensemble size of 200. Figure 5.9 gives the same grouping for an ensemble size of 750 . Figure 5.10 gives the grouping for an ensemble size of 2000. The model predict a marked difference in the behavior of the burn out points below s/D $=11.2$ and the behavior of s/D at 11.2 and above. The first two s/D cases travel farther axially before burn out than any of the other 4 cases. This is consistent with the expected behavior.

There is no detectable trend inside of either of these two groupings (small s/D, large s/D). The model does not seem to be able to differentiate the effects of initial droplet spacing other than to say that a spacing of 11.2 or 
greater causes the droplets to behave independently. This result is consistent with that published by Mulholland et al. The failure of this model to predict a trend for the variation in initial droplet spacing occurs because the ODT droplet model does not have any s/D affects in it. The s/D affects are confined to the initial conditions and once the droplets are born into ODT space they are assumed to be independent of each other. In implementation of the simulation, all six cases for studying variations in initial droplet spacing, were assumed to enter the ODT domain at the same point. This reflects that the point at which the droplet stream is assumed to break into individual droplets is when the stream trajectory reaches the high shear layer of the gas phase. This was the only assumption possible given the statements by Mulholland et al. Hence s/D effects were investigated by variations in the droplet initial conditions only (diameter, velocity, and temperature). The lack of an overall trend would indicate that the point at which the droplets break free of the stream as a function of the initial spacing is not constant for all six cases. No avenue based on published experimental observation was available to adjust the position of the droplet stream break up. Any adjustment to the birth time into ODT would have been only a guess and therefore this avenue was not pursued. 
Figure 5.11 gives the scatter plots which account for the model's prediction of the variation due to the injection angle: Case $10(\theta=0$ degrees $)$ diagram (A), Case $1 \theta=30$ degrees) diagram (B), and Case $12 \theta=45$ degrees) diagram (C) for an ensemble size of 200. Figure 5.12 gives the same grouping for an ensemble size of 750 . Figure 5.13 gives the grouping for an ensemble size of 2000. The trend, visible in all three figures, is that the model predicts that smaller injection angles travel farther down the reactor. This is exemplified in both the onset of burn out (leftmost points) and in the completion of burn out (rightmost points). Also, the model predicts that the $y$ scatter is reduced as the injection angle increases. Based on physical grounds, the model predictions seem reasonable. The hottest part of the furnace (mean temperature of $1750 \mathrm{~K}$ ) occurs at a height of .25 meters and an axial distance of 0.05 meters. As the mean droplet path travels closer to the mean highest gas phase temperatures (as it does as the injection angle increases) the lifetime of the droplets gets shorter. The zero degree injection angle on average sees a less defined gas phase $u$ velocity which leads to more $y$ scatter. The gas phase velocity is less defined in the sense that the $u$ velocity tends to be smaller in magnitude and changing in direction. There is a re-circulation zone directly in the path of the zero 
degree droplet stream. The ODT representation of this recirculation zone is achieved by imputing negative axial velocities as the initial condition. These were the actual negative velocities measured.

Therefore the model predictions for injection angle variation are consistent with the physics captured in the model. However, the experimental observations of Mulholland et al do not agree with the model. The probable cause for the observed discrepancy is the lack of type-C interactions in the two-phase flow submodel. Type-C interactions were introduced in Chapter 3 to account for the ODT artifact that as the particle $y$ velocity increases (in magnitude) the probability of interacting with a triplet map becomes zero. As the injection angle increases the $y$ velocity increases and the number of eddy interactions decrease. Using a rough estimate (viewing eddy events as boxes, the 45 degree injected droplet would be just as likely to strike the eddy event on the horizontal surface as on the front surface) the number of eddy interactions for a particle injected at 45 degrees is approximately half as many as it would be if type-C interactions were included in the model. This could cause more variation in the $y$ scatter and might lead to longer droplet lifetimes. Another contributing factor is that the 45 degree stream will hit the high shear layer of the gas phase the earliest of 
all the cases. As such, the particle Re will be the largest, and the assumptions used to derive the equations in Chapter 4 are therefore the weakest. There would be a tendency for the droplets not to be spherical and drops are more likely to spin, causing lift forces, which are not accounted for in this model.

\subsection{Summary of burn out points by method varied}

Figure 5.14 (shows for an ensemble size of 200) in condensed form the maximum, average, and minimum axial burn out points for the four variations on initial conditions, namely diameter, velocity, droplet spacing, and injection angle. Also shown in open symbols are the published experimental data for maximum, average, and minimum axial burn out points. Figure 5.15 gives the same form for an ensemble size of 750 . Figure 5.16 gives the same form for an ensemble size of 2000. Analysis reveals that for all ensemble sizes and for all cases the model tends to under predict the axial penetration for the maximum, average and minimum distance traveled. The cause for this discrepancy is probably due to the idealization of the droplet fuel [which is a combination of hydrocarbons, predominantly aliphatic $\mathrm{C}_{7}$ hydrocarbons (40\%); Aromatic solvents \{xylene\} (29\%); and 
fuel distillate \{consisting of $\mathrm{C}_{10}-\mathrm{C}_{23}$ hydrocarbons (31\%)] as a single component unnamed pure fuel and the use of the burning rate parameter $\lambda$.

Variations in initial diameter and velocity show trends that match the observed experimental data. Variation in initial droplet spacing shows only a qualitative agreement with the first grouping (the two smallest s/D trials) penetrating significantly farther than the second grouping. Variation of the injection angle predicts a trend which is counter to the observed experimental trend. The model predicts that the penetration of the droplets decreases as the injection angle increases. The observed experimental data indicates that it remains the same for the zero and 30 degree trials. For the 45 degree trial it penetration distance increases. These observations can be found true for all ensemble sizes.

A major objective in this paper was to try to predict the spread in the burn out points due to the turbulent fluctuations. The spread was considered key since there were numerous simplifying assumptions in the model development. Figure 5.17 shows, for an ensemble size of 200, the predicted axial spread for the four variations on initial conditions, namely diameter, velocity, droplet spacing and injection angle. Analysis of Figure 5.18 shows excellent agreement of the predicted spread to the measured spread for the 
variation in initial diameter. The agreement with the variation in initial velocity is also very good with both the simulated and experimental data showing a downward trend as the initial velocity increases. There is also very good agreement in the second grouping (the last four cases) of the spread as function of the initial droplet spacing. The first grouping is not a good fit. As mentioned previously, the lack of s/D parameterization in the droplet submodel and the choice of the birth point into ODT is probably the cause of this discrepancy. The agreement for the zero and thirty degree injection angles is excellent. The forty-five degree case is significantly different from the measured data. This is probably due to the lack of type-C interactions and the larger particle Re.

The figures also show the experimental spread using open circles. Figure 5.18 gives the same form for an ensemble size of 750 . Figure 5.19 gives the same form for an ensemble size of 2000. Analysis of Figure 5.17 reveals a good agreement with spread over the entire range. The agreement for velocity is good, but the predicted trend is opposite that of the experimental trend. The predicted spread for the s/D variation is virtually constant. The measured spread approaches the predicted spread for the last three cases. This would indicate that the s/D effects were not important for these cases. 
Hence the modeling approach, for these last three cases, of confining the s/D affects to the boundary conditions was successful. The predicted spread for variation in injection angle was good for the zero and thirty degree case. It was contrary to the experimental results for the 45 degree case.

Analysis of Figure 5.18 shows excellent agreement of the predicted spread to the measured spread for the variation in initial diameter. The agreement with the variation in initial velocity is also very good with both the simulated and experimental data showing a downward trend as the initial velocity increases. There is also very good agreement in the second grouping (the last four cases) of the spread as function of the initial droplet spacing. Qualitatively the shape of the curve even matches. The first grouping is not a good fit. As mentioned previously, the lack of s/D parameterization in the droplet submodel and the choice of the birth point into ODT is probably the cause of this discrepancy. The agreement for the zero and thirty degree injection angles is excellent. The forty-five degree case is significantly different from the measured data. This is probably due to the lack of type-C interactions and the larger particle Re.

Analysis of Figure 5.19 shows good agreement of the predicted spread with the measured spread for the variation in initial diameter. Both the size 
200 and the size 750 ensembles are a better match however. The agreement with the variation in initial velocity is also very good with both the simulated and experimental data showing a downward trend as the initial velocity increases. The size 2000 ensemble set is a slight improvement to the agreement in the size 750 ensemble set. There is also good agreement in the second grouping (the last four cases) of the spread as function of the initial droplet spacing. However, the qualitative agreement found in the size 750 ensemble set is no longer seen. The predicted spread for last grouping has become a horizontal line, meaning that the model cannot tell them apart at 2000 iterations. The first grouping is a poor fit. As mentioned previously, the lack of s/D parameterization in the droplet submodel and the choice of the birth point into ODT is probably the cause of this discrepancy. The agreement for the zero and thirty degree injection angles is not as good as for the size 750 set, but better than the size 200 ensemble set. The forty-five degree case is significantly different from the measured data. This is probably due to the lack of type-C interactions and the larger particle Re.

Based on Figures 5.17, 5.18 and 5.19, the closest match to the experimental data of order 100 droplets is the size 750 ensemble set. The size 200 ensemble set seems to be too small. Random variations are playing 
too much of a role in the outcome of the model. With a sample size of order 100, the ensemble size of 2000 seems to be overkill. The results from the experiment may have shifted if the data set were order 1000 so it is best not to use an ensemble size of 2000.

Mulholland et al. published only one set (maximum and minimum) of data points concerning the spread in the $y$ direction of all the data collected (the twelve cases). This one set of data points was for the base case which occurs in all the variations of initial conditions. Strictly for closure, the $y$ variation (height in the reactor) of each case is plotted. The form of the plot shows variations based on initial diameter, velocity, droplet spacing, and injection angle. The plots show the simulated maximum, average, minimum $y$ value for the burn out point along with the predicted standard deviation. These are all shown in closed symbols. The one set of experimental data points are shown in open symbols, an open circle for the maximum experimental data point and an open square for the minimum experimental data point. Figure 5.20 gives the results for the size 200 ensemble. Figure 5.21 gives the results for the 750 ensemble set. Figure 5.22 gives the results for the size 2000 ensemble.

Certain trends can be correctly gleaned from the plots in Figures 5.20, 
Selected droplet trajectories for Case 1

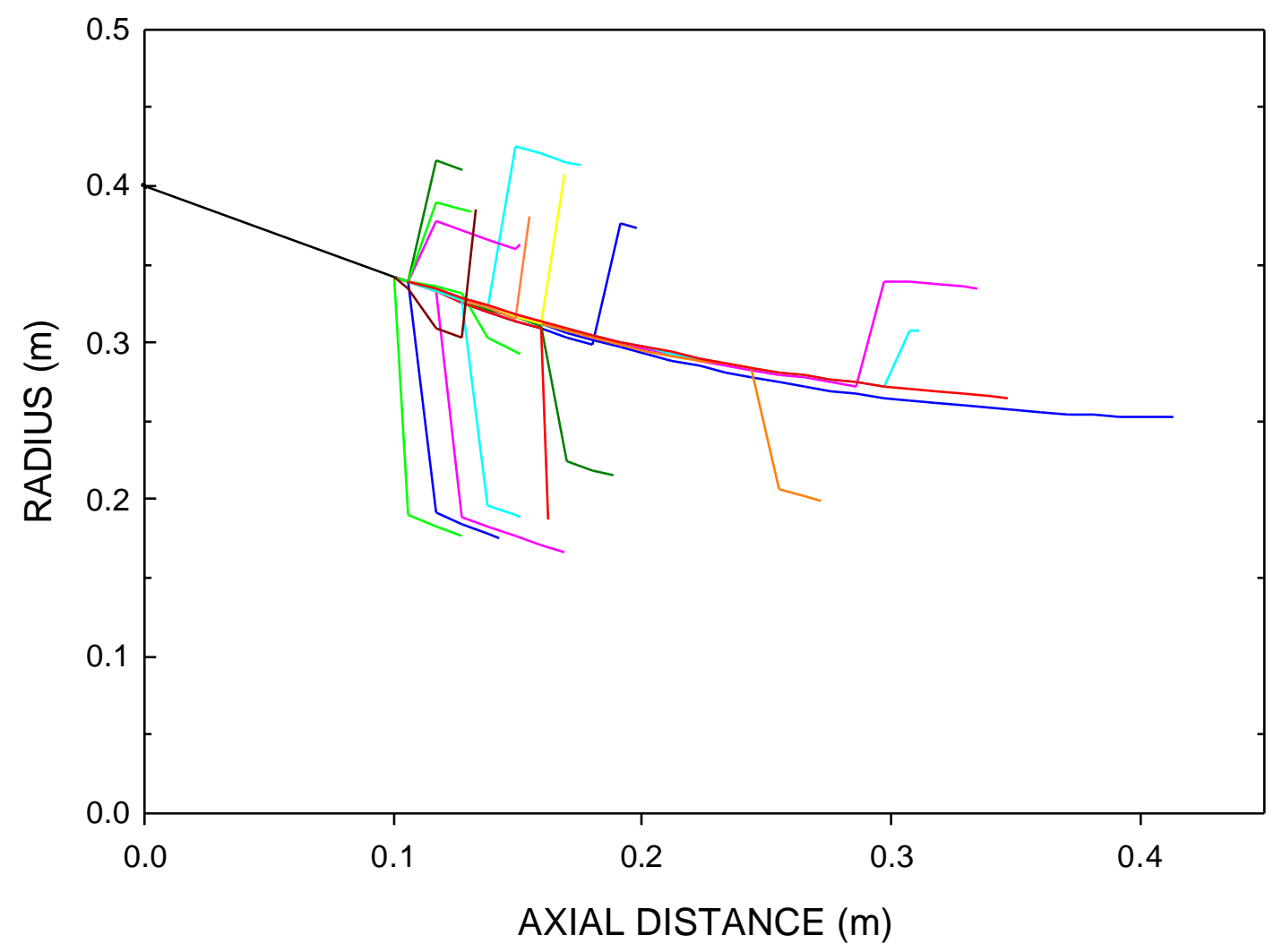

Figure 5.1: Trajectories from Case 1 chosen to exemplify the extremes of spread in the $x$ and $y$ directions of the burn out points.

5.21, and 5.22, such as the decrease in the $y$ scatter due to an increase in initial diameter. However, other properties are not so easily recognized. The scatter plots Figures 5.2 - 5.13 give a better feeling for trends. 
Table 5.1 Droplet Stream Test Conditions*

\begin{tabular}{l|lllll}
\hline Title & $\begin{array}{l}\text { Parameter } \\
\text { Varied }\end{array}$ & $\begin{array}{l}D^{0} \\
(\text { microns })\end{array}$ & $\begin{array}{l}V_{d}^{0} \\
(\mathrm{~m} / \mathrm{s})\end{array}$ & $\begin{array}{l}\mathrm{s}^{0} / D^{0} \\
(\mathrm{deg})\end{array}$ \\
\hline Case 1 & Nominal & 234 & 8.5 & 180 & 30 \\
Case 2 & Size & 306 & 8.5 & 140 & 30 \\
Case 3 & & 371 & 8.5 & 115 & 30 \\
Case 4 & Velocity & 216 & 5.3 & 120 & 30 \\
Case 5 & & 250 & 10.6 & 210 & 30 \\
Case 6 & Spacing & 234 & 8.5 & 2.8 & 30 \\
Case 7 & & 234 & 8.5 & 5.6 & 30 \\
Case 8 & & 234 & 8.5 & 11.2 & 30 \\
Case 9 & & 234 & 8.5 & 22 & 30 \\
Case 10 & & 234 & 8.5 & 45 & 30 \\
Case 11 & Angle & 234 & 8.5 & 180 & 0 \\
Case 12 & & 234 & 8.5 & 180 & 45 \\
\hline
\end{tabular}

* taken from Mulholland et al. (1991) 

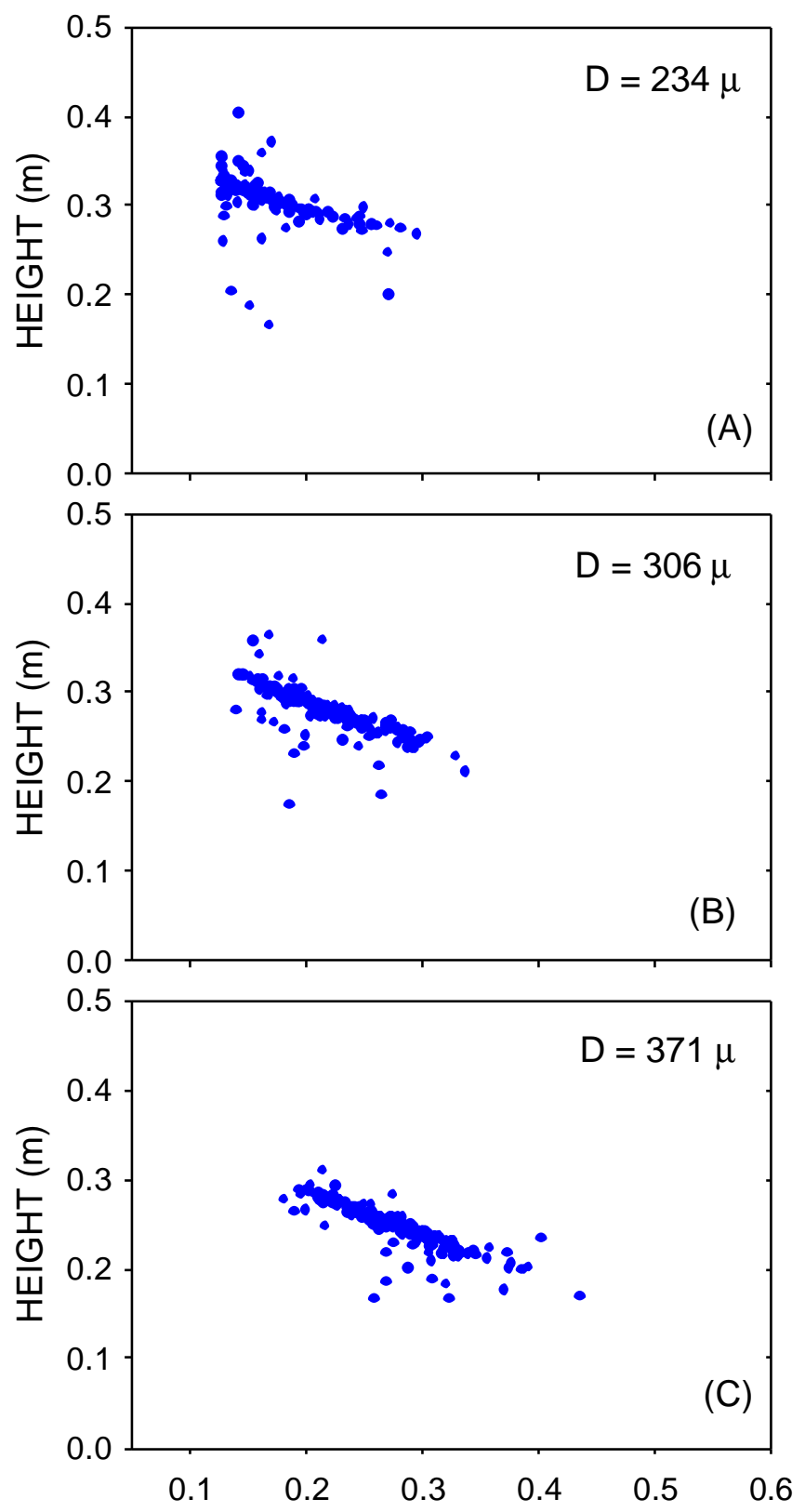

- burn out AXIAL DISTANCE (m)

Figure 5.2: Burn out points for variation in initial diameter, D. Various scatter plots of the burn out points for different initial diameter are shown in increasing order. An ensemble size of 200 was used to generate these plots. Larger droplets travel further down stream and resist being jostled around as much in the y direction (height). 

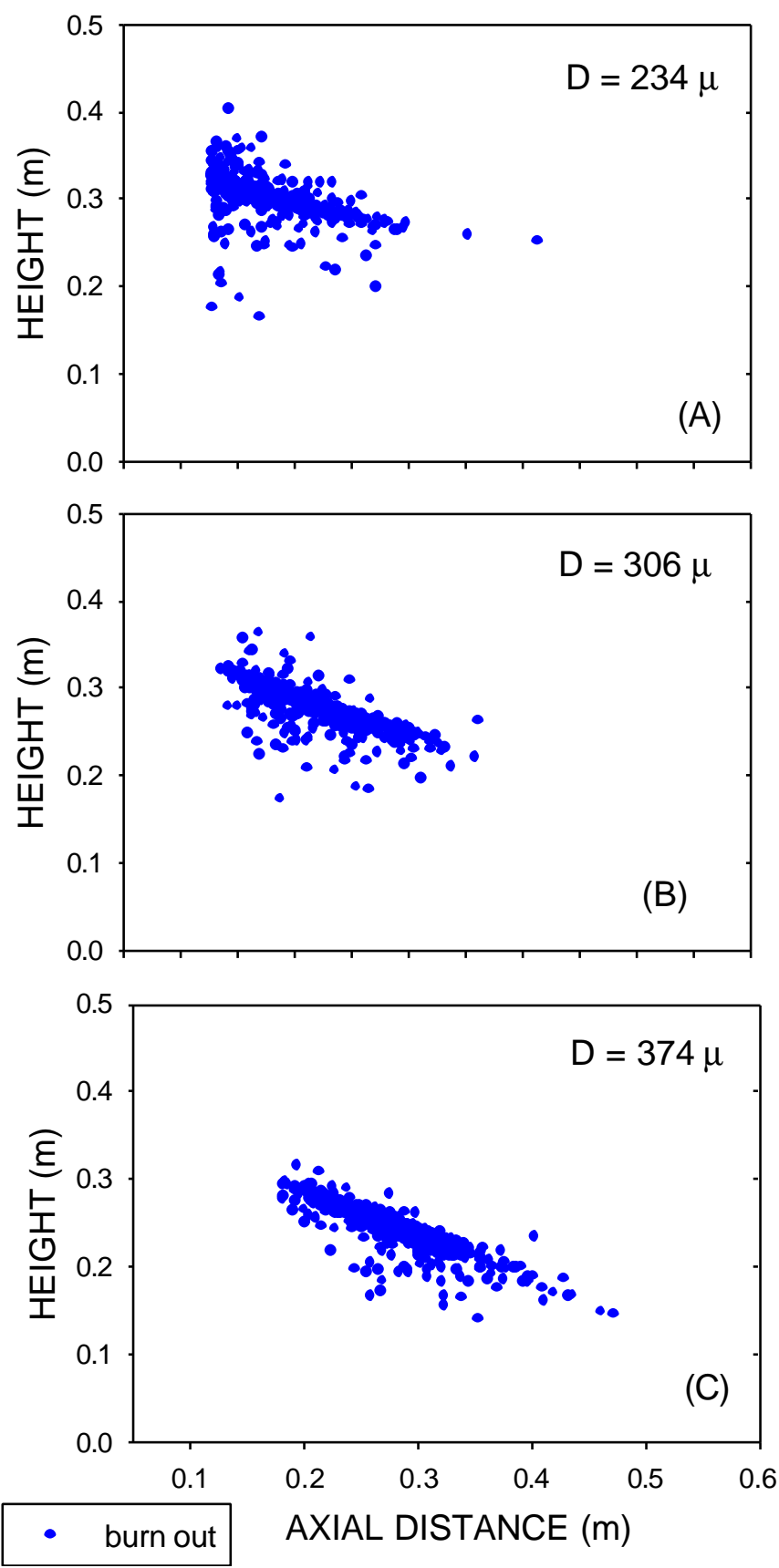

Figure 5.3: Burn out points for variation in initial diameter, D. Various scatter plots of the burn out points for different initial diameter are shown in increasing order. An ensemble size of 750 was used to generate these plots. Larger droplets travel further down stream and resist being jostled around as much in the y direction (height). 

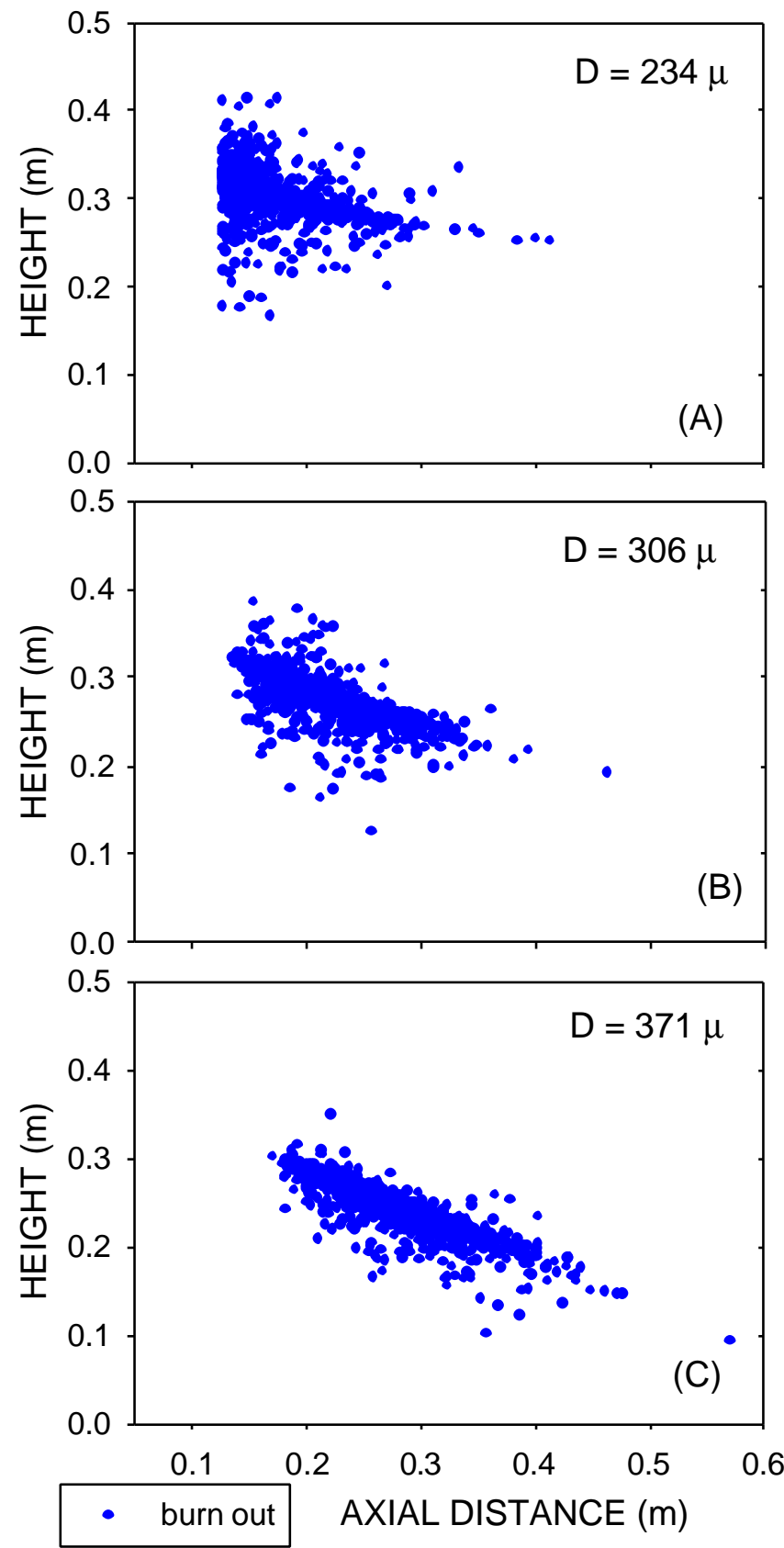

Figure 5.4: Burn out points for variation in initial diameter, D. Various scatter plots of the burn out points for different initial diameter are shown in increasing order. An ensemble size of 2000 was used to generate these plots. Larger droplets travel further down stream and resist being jostled around as much in the y direction (height). 

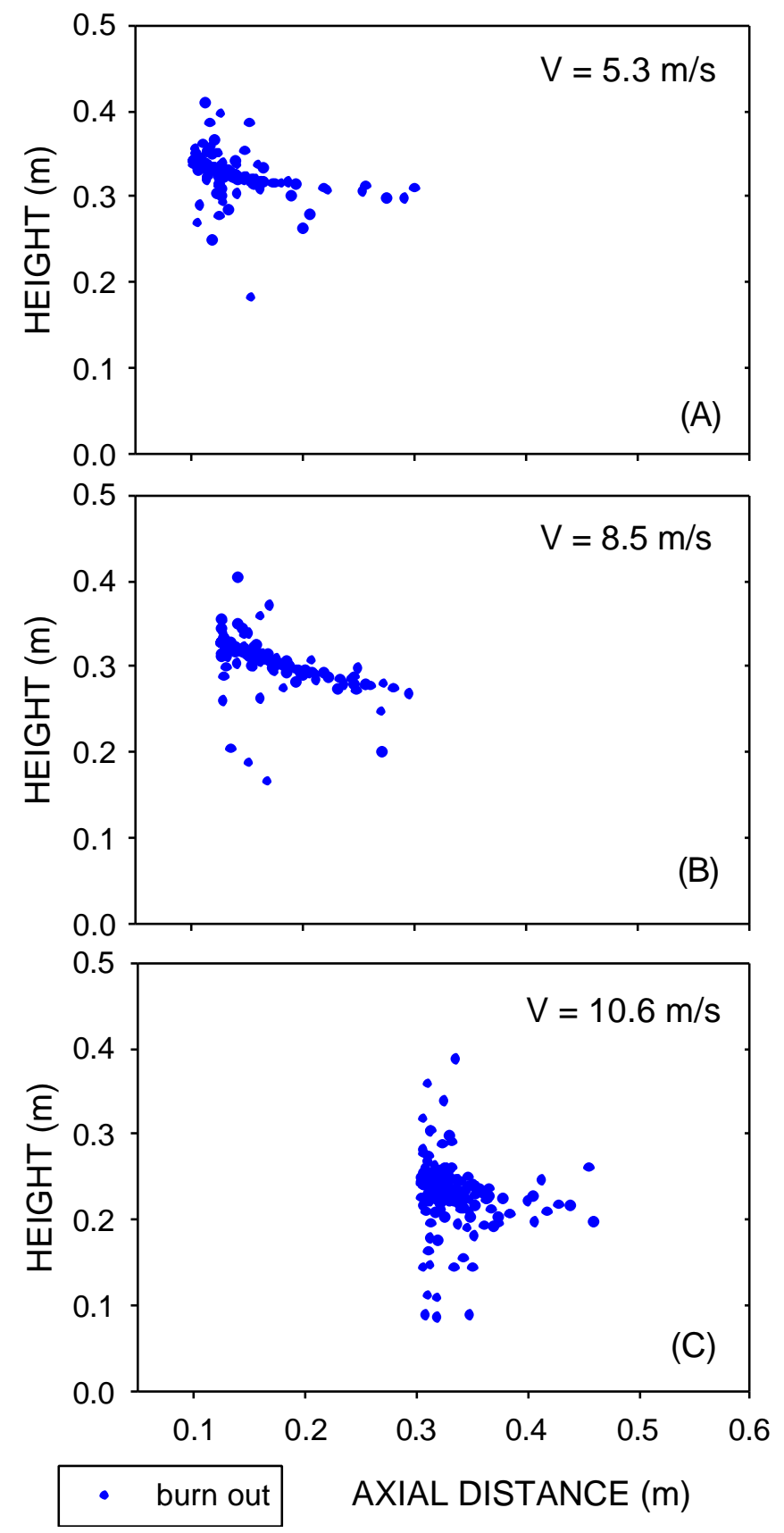

Figure 5.5: Burn out points for variation in initial velocity, V. Various scatter plots of the burn out points for different initial velocities are shown in increasing order. An ensemble size of 200 was used to generate these plots. Faster droplets travel further down stream and resist being jostled around as much in the y direction (height). However the further the particles travel downstream the more they are jostled. 

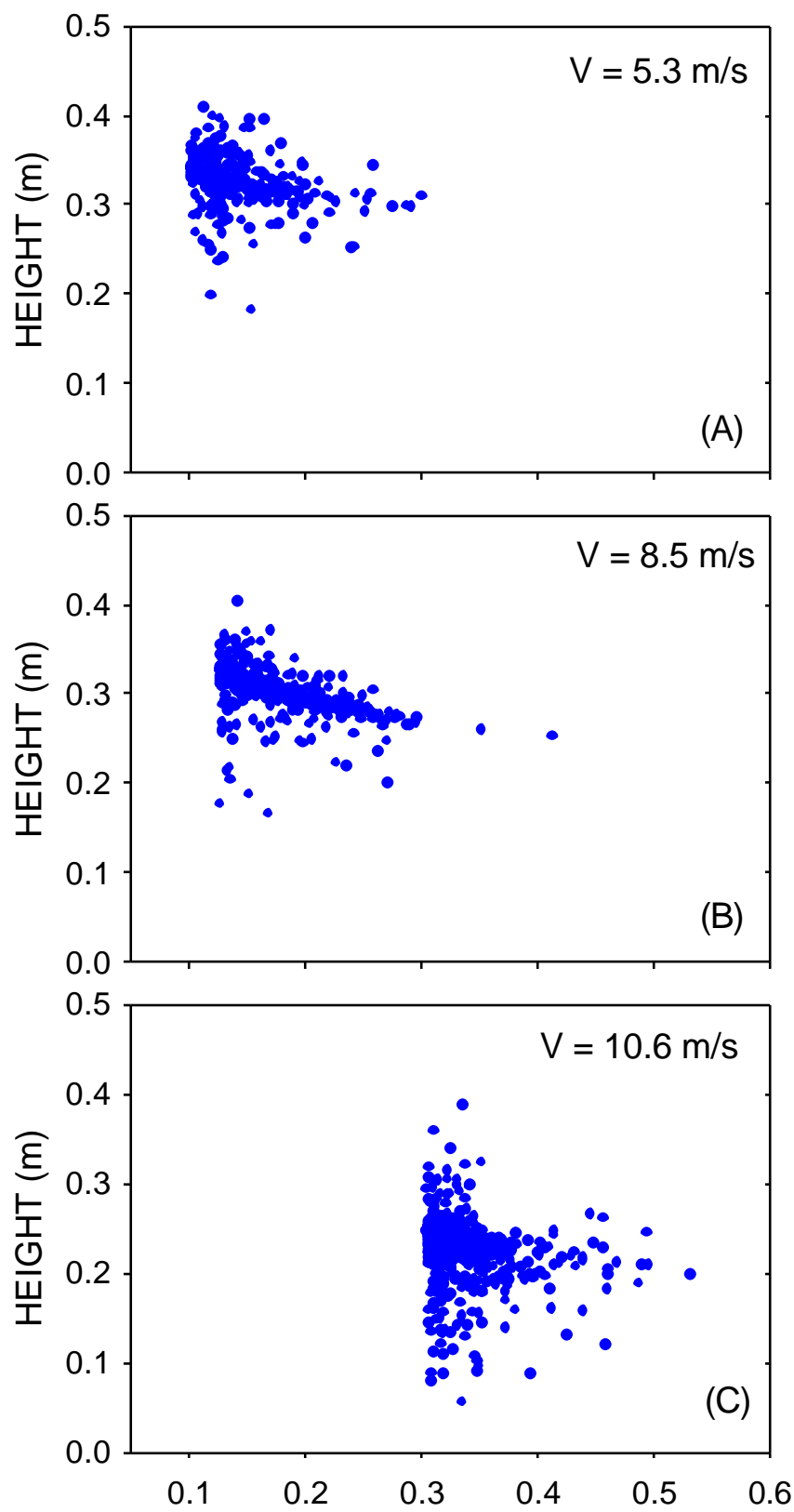

- burn out AXIAL DISTANCE (m)

Figure 5.6: Burn out points for variation in initial velocity, V. Various scatter plots of the burn out points for different initial velocities are shown in increasing order. An ensemble size of 750 was used to generate these plots. Faster droplets travel further down stream and resist being jostled around as much in the y direction (height). However the further the particles travel downstream the more they are jostled. 

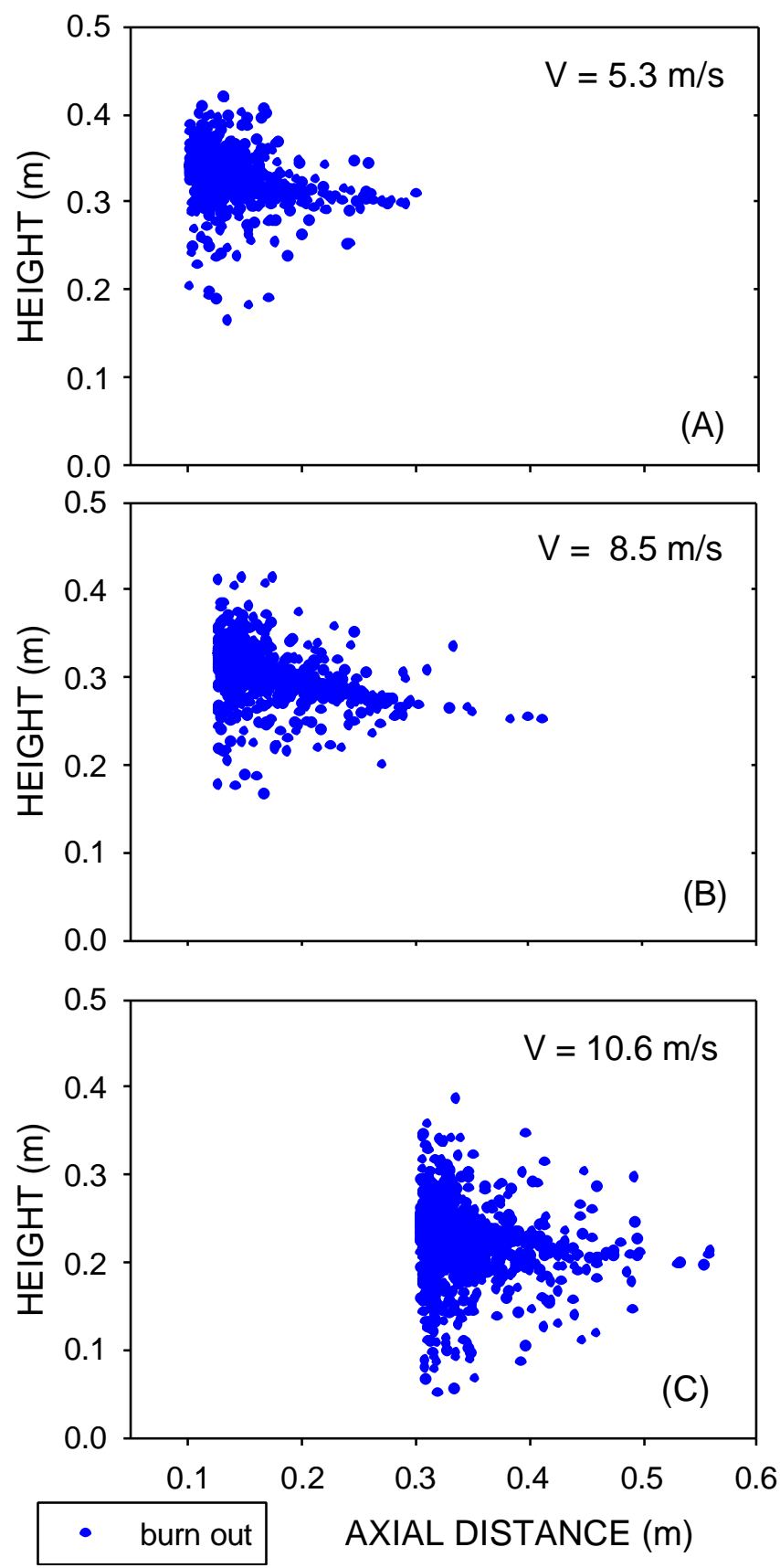

Figure 5.7: Burn out points for variation in initial velocity, V. Various scatter plots of the burn out points for different initial velocities are shown in increasing order. An ensemble size of 2000 was used to generate these plots. Faster droplets travel further down stream and resist being jostled around as much in the y direction (height). However the further the particles travel downstream the more they are jostled. 

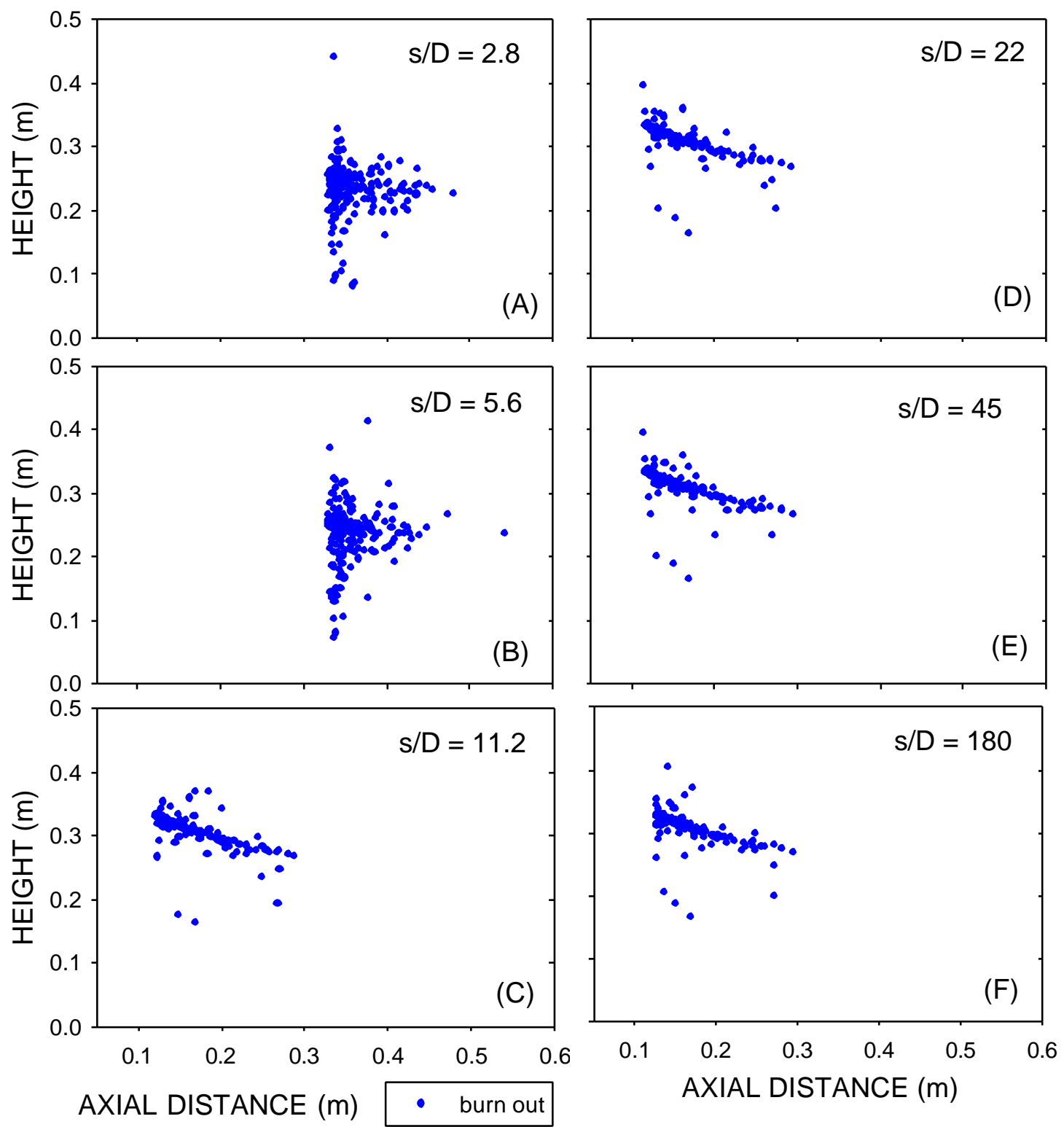

Figure 5.8: Burn out points for variation in initial droplet spacing, s/D. Various scatter plots of the burn out points for different initial droplet spacing are shown in increasing order. An ensemble size of 200 was used to generate these plots. A marked difference exists between the small s/D group (diagrams a and b) and the large s/D group (diagrams c, d, e, and f). No significant differences occur within either group. 

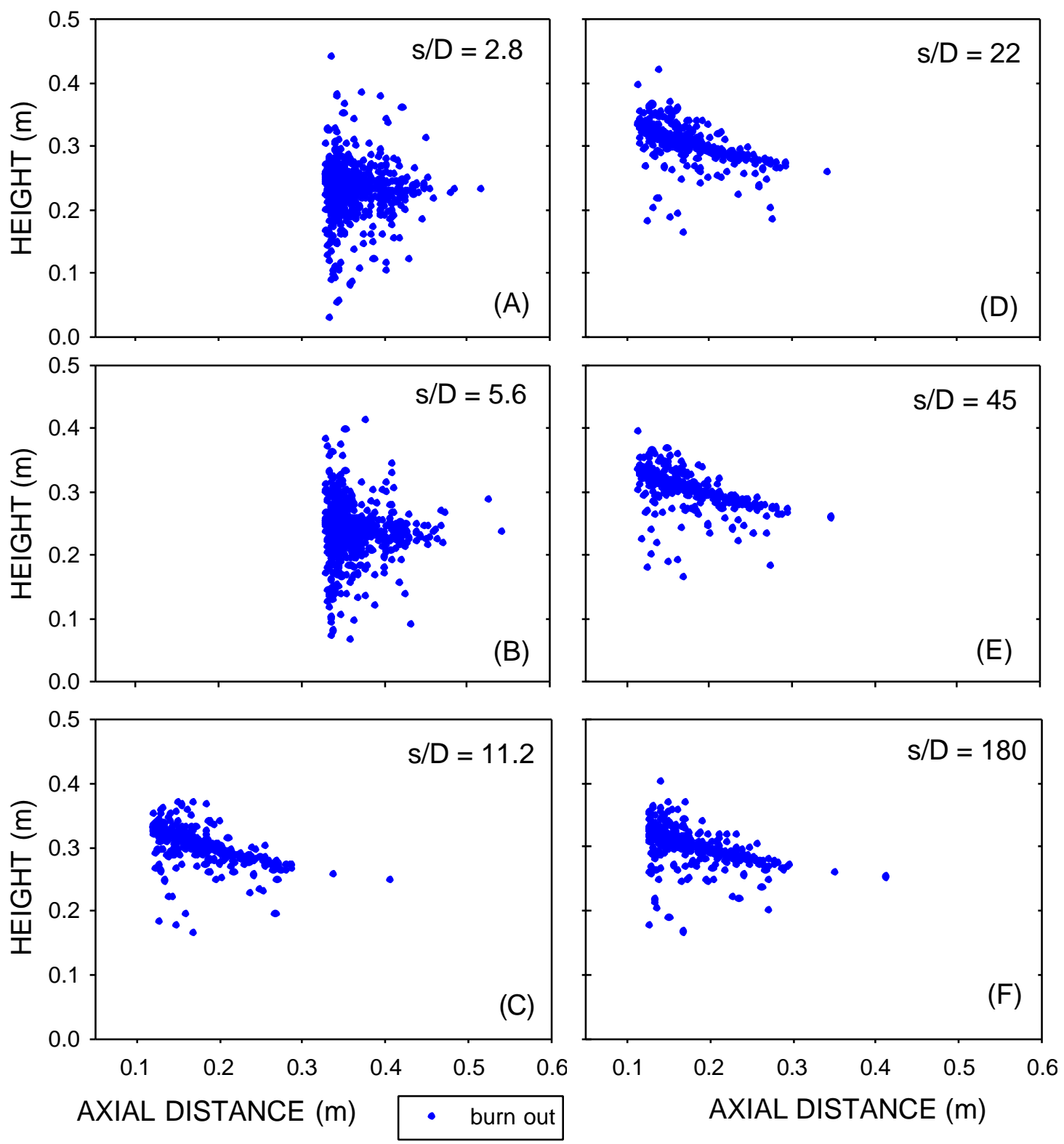

Figure 5.9: Burn out points for variation in initial droplet spacing, s/D. Various scatter plots of the burn out points for different initial droplet spacing are shown in increasing order. An ensemble size of 750 was used to generate these plots. A marked difference exists between the small s/D group (diagrams a and b) and the large s/D group (diagrams c, $\mathrm{d}, \mathrm{e}$, and $\mathrm{f}$ ). No significant differences occur within either group. 

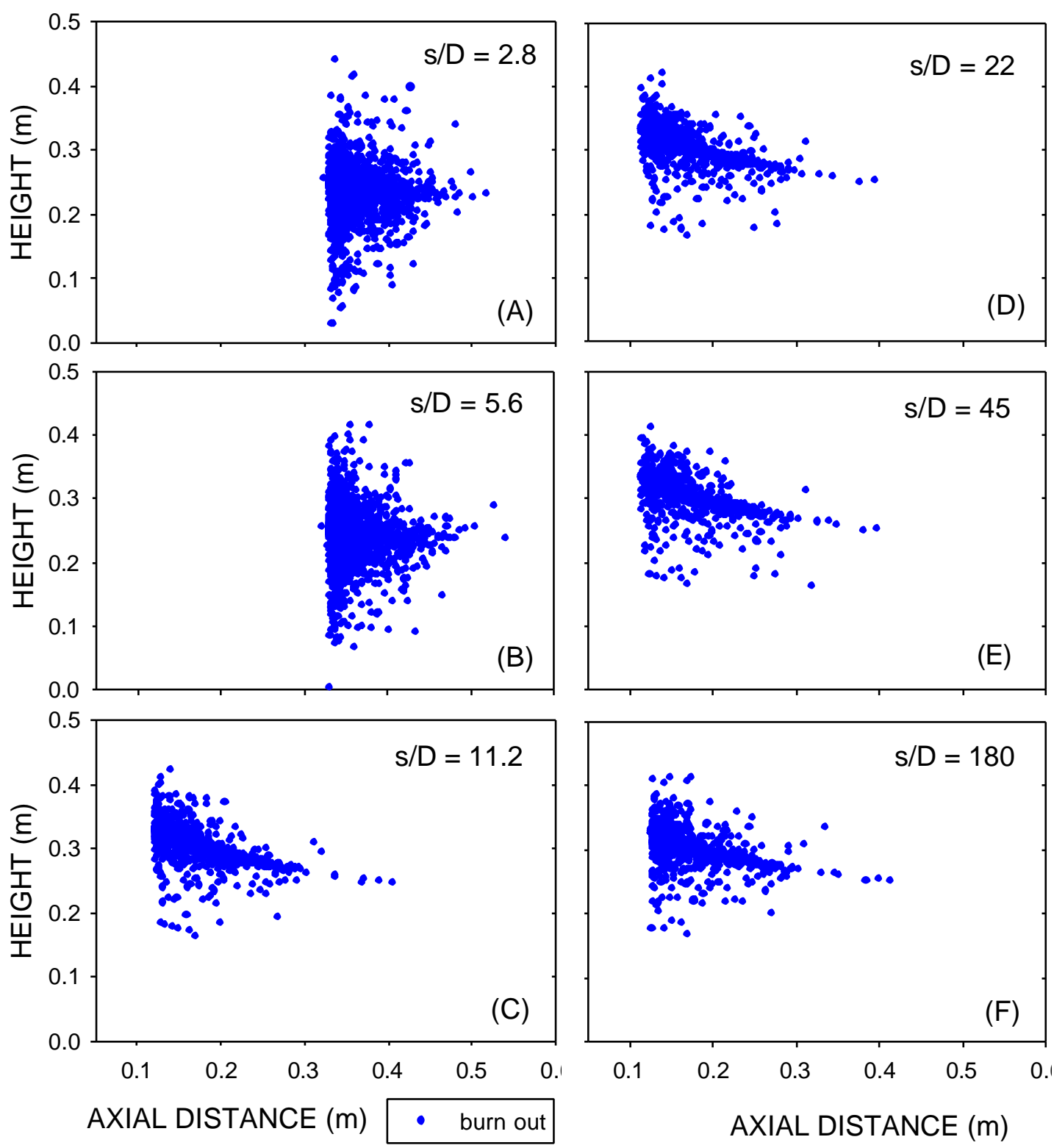

Figure 5.10: Burn out points for variation in initial droplet spacing, s/D. Various scatter plots of the burn out points for different initial droplet spacing are shown in increasing order. An ensemble size of 2000 was used to generate these plots. A marked difference exists between the small s/D group (diagrams a and b) and the large s/D group (diagrams $\mathrm{c}$, d, e, and f). No significant differences occur within either group. 


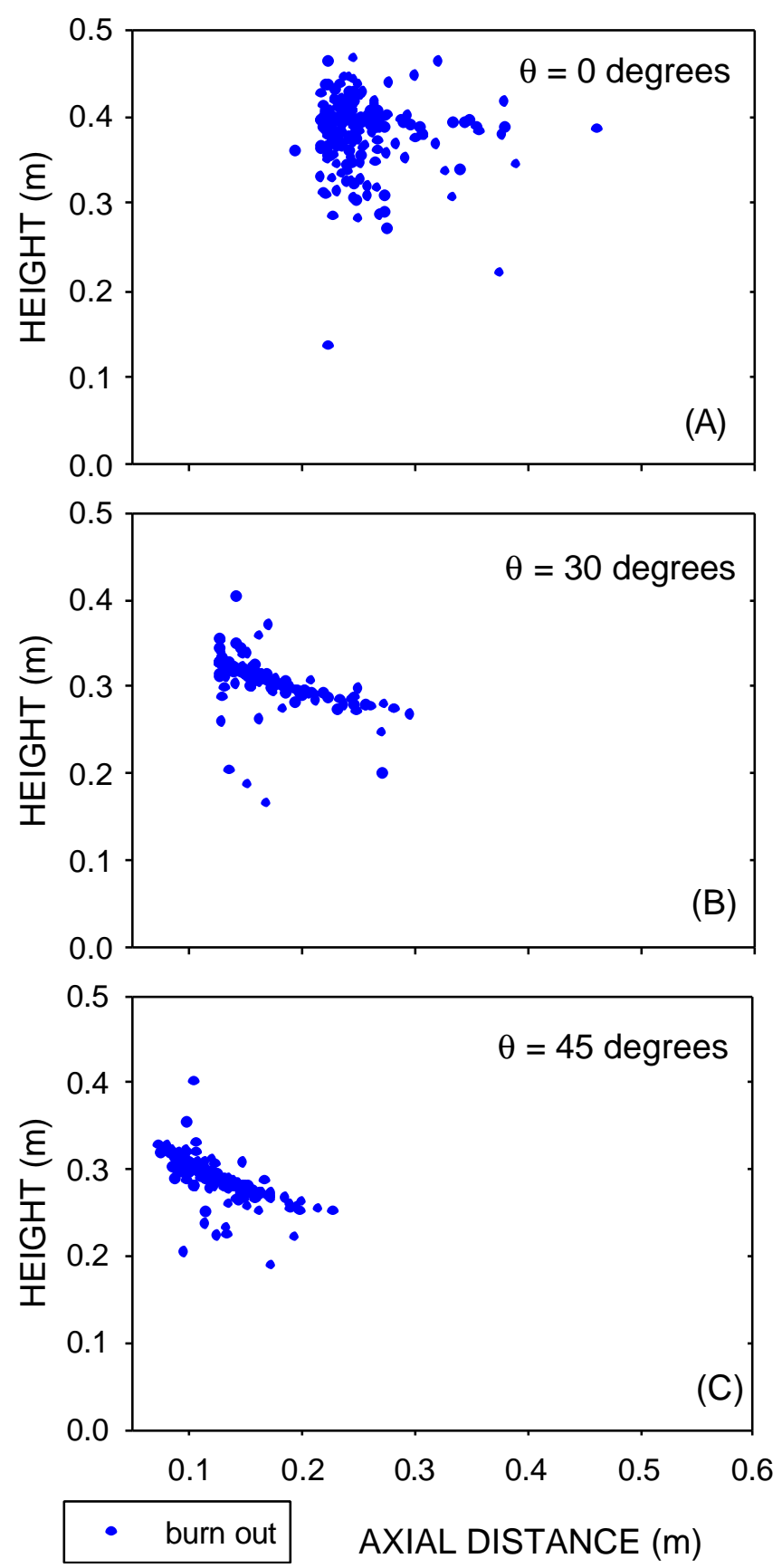

Figure 5.11: Burn out points for variation in initial injection angle, $\theta$. Various scatter plots of the burn out points for different initial injection angles are shown in increasing order. An ensemble size of 200 was used to generate these plots. Higher injection angles cause the droplets to travel closer to the flame and experience higher mean temperatures and larger mean down stream velocities. 

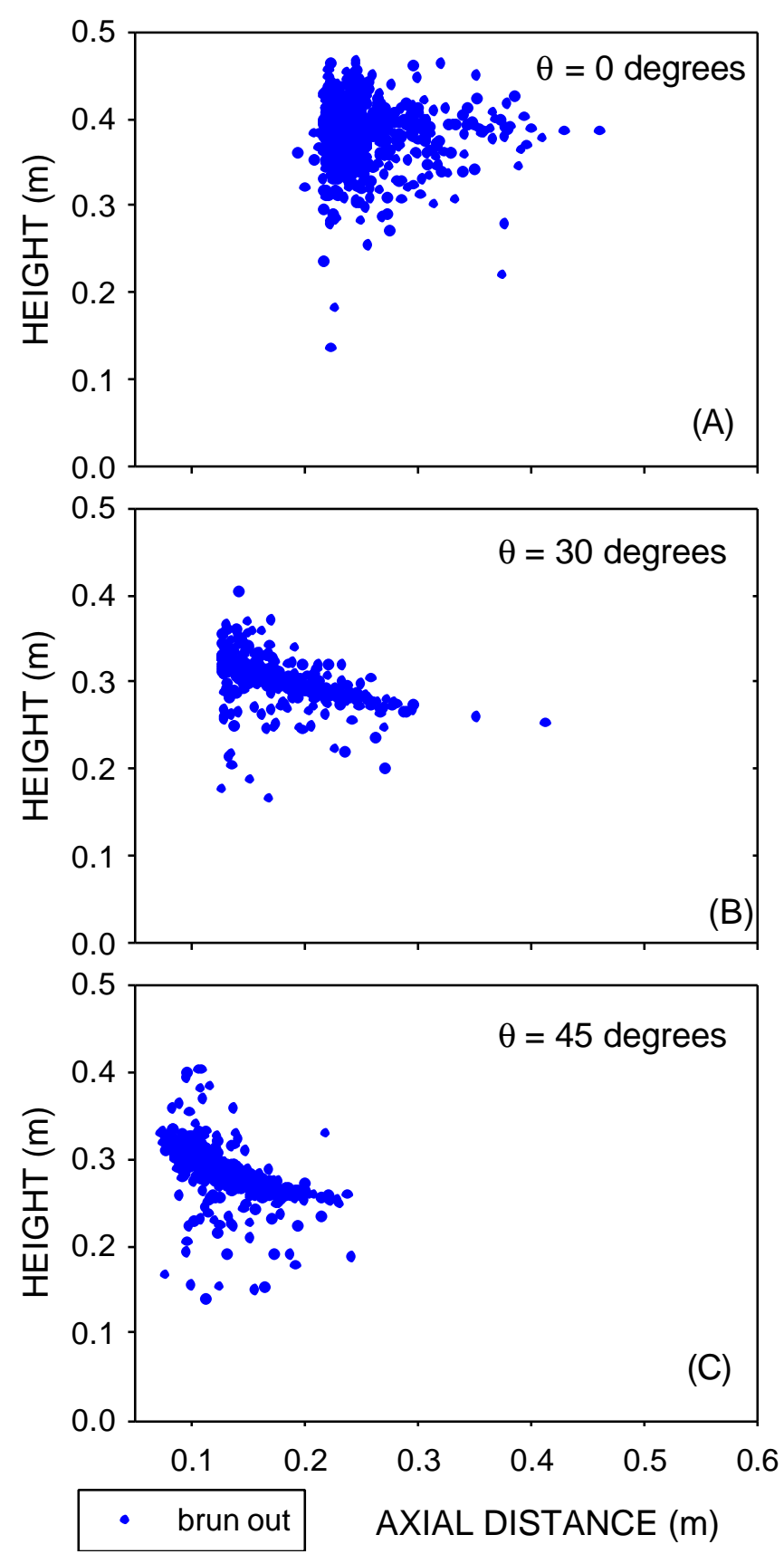

Figure 5.12: Burn out points for variation in initial injection angle, $\theta$. Various scatter plots of the burn out points for different initial injection angles are shown in increasing order. An ensemble size of 750 was used to generate these plots. Higher injection angles cause the droplets to travel closer to the flame and experience higher mean temperatures and larger mean down stream velocities. 

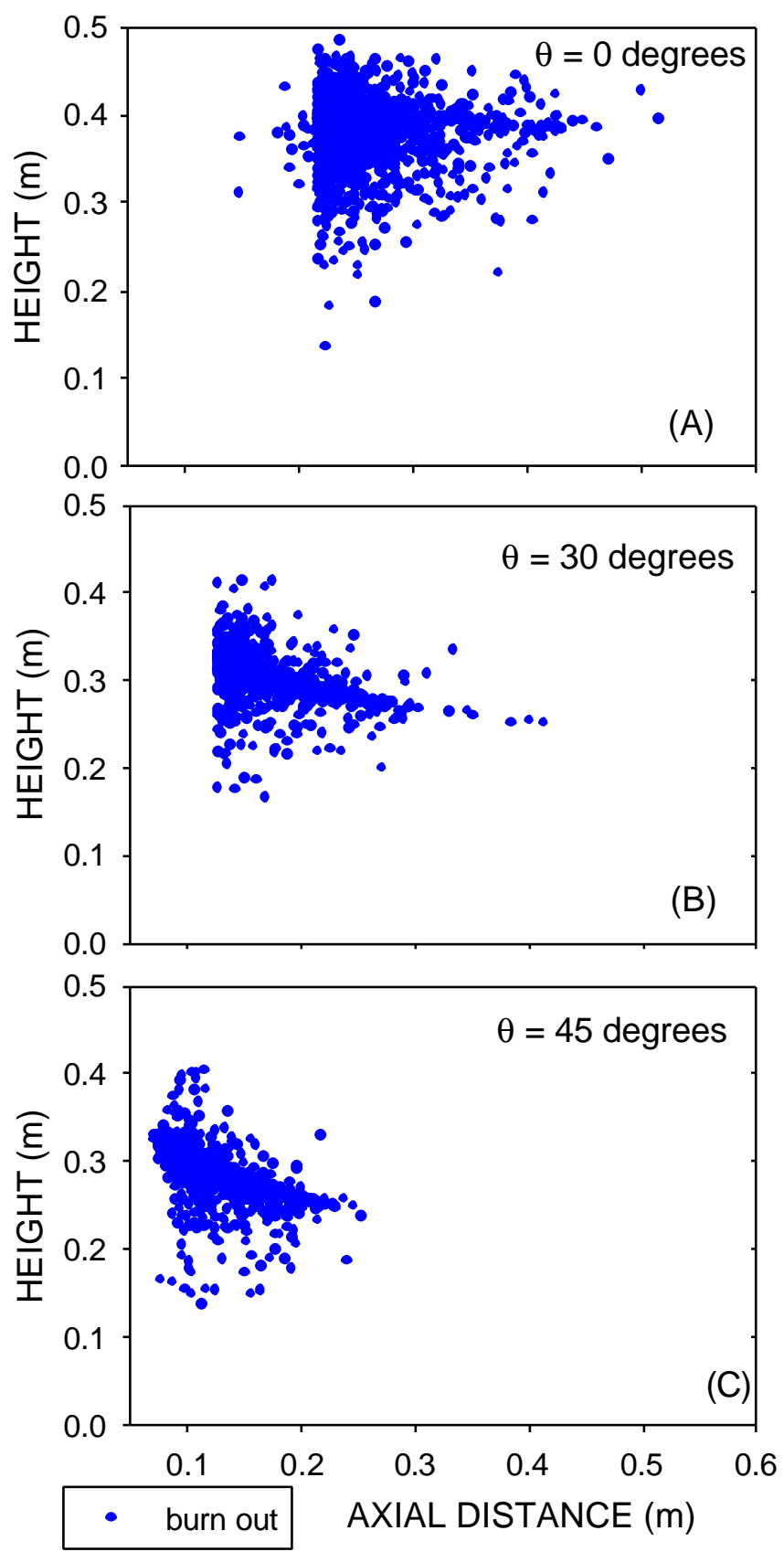

Figure 5.13: Burn out points for variation in initial injection angle, $\theta$. Various scatter plots of the burn out points for different initial injection angles are shown in increasing order. An ensemble size of 2000 was used to generate these plots. Higher injection angles cause the droplets to travel closer to the flame and experience higher mean temperatures and larger mean down stream velocities. 

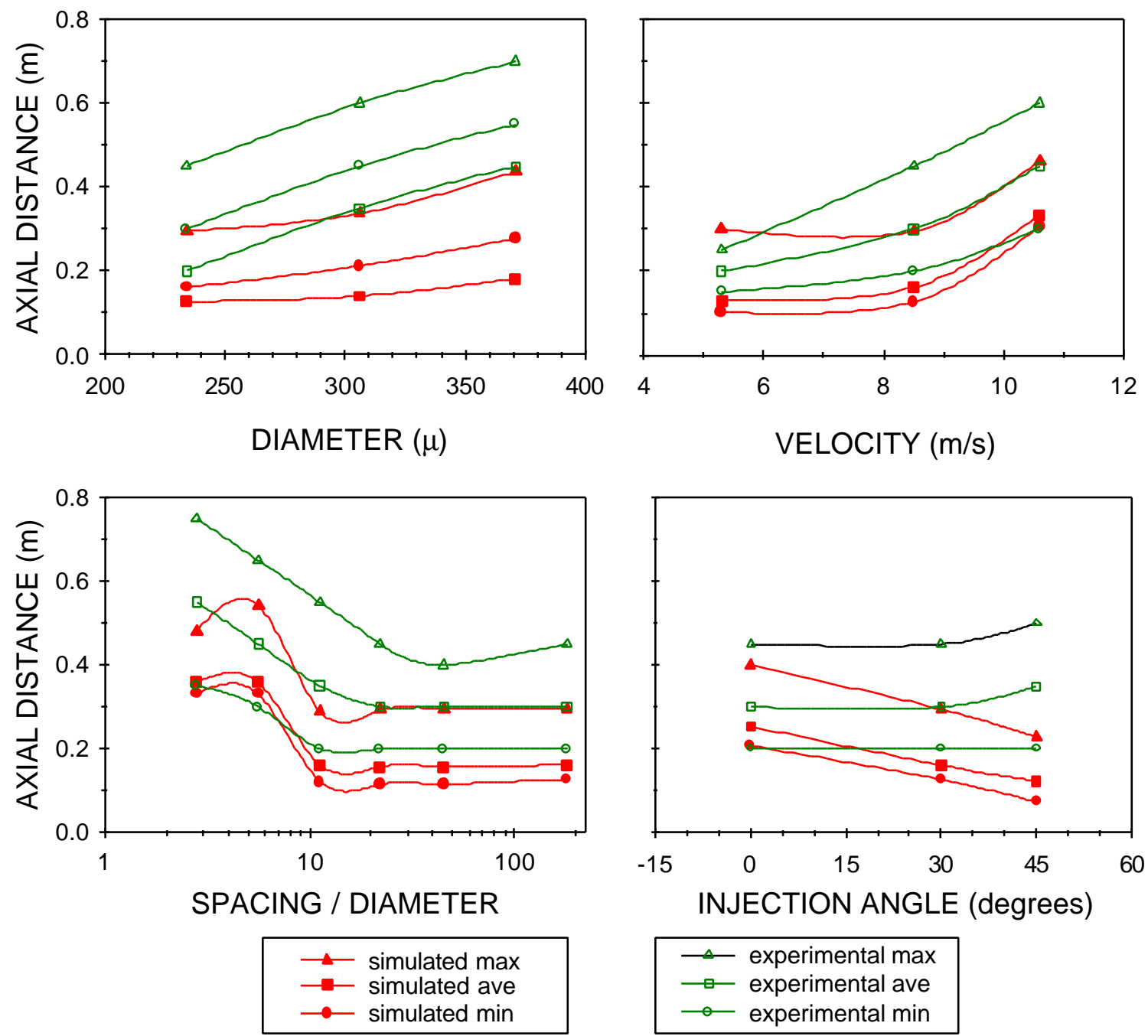

Figure 5.14: Comparison of experimental measurements and simulated burning droplets. The closed symbols represent the predicted maximum (triangle) average (square) and minimum (circle). The experimental data are shown in open symbols. An ensemble size of 200 was used to generate these plots. 

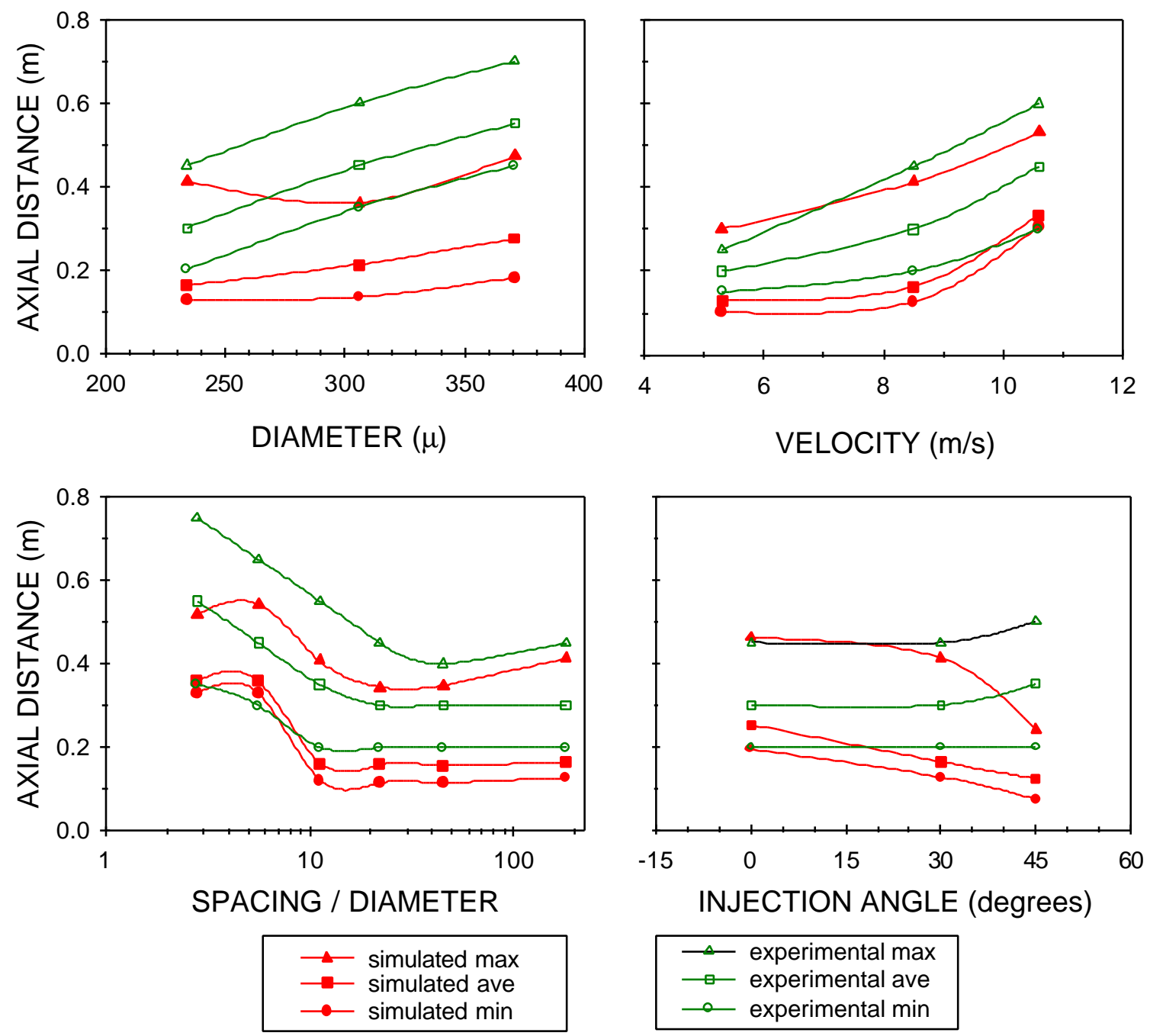

Figure 5.15: Comparison of experimental measurements and simulated burning droplets. The closed symbols represent the predicted maximum (triangle) average (square) and minimum (circle). The experimental data are shown in open symbols. An ensemble size of 750 was used to generate these plots. 

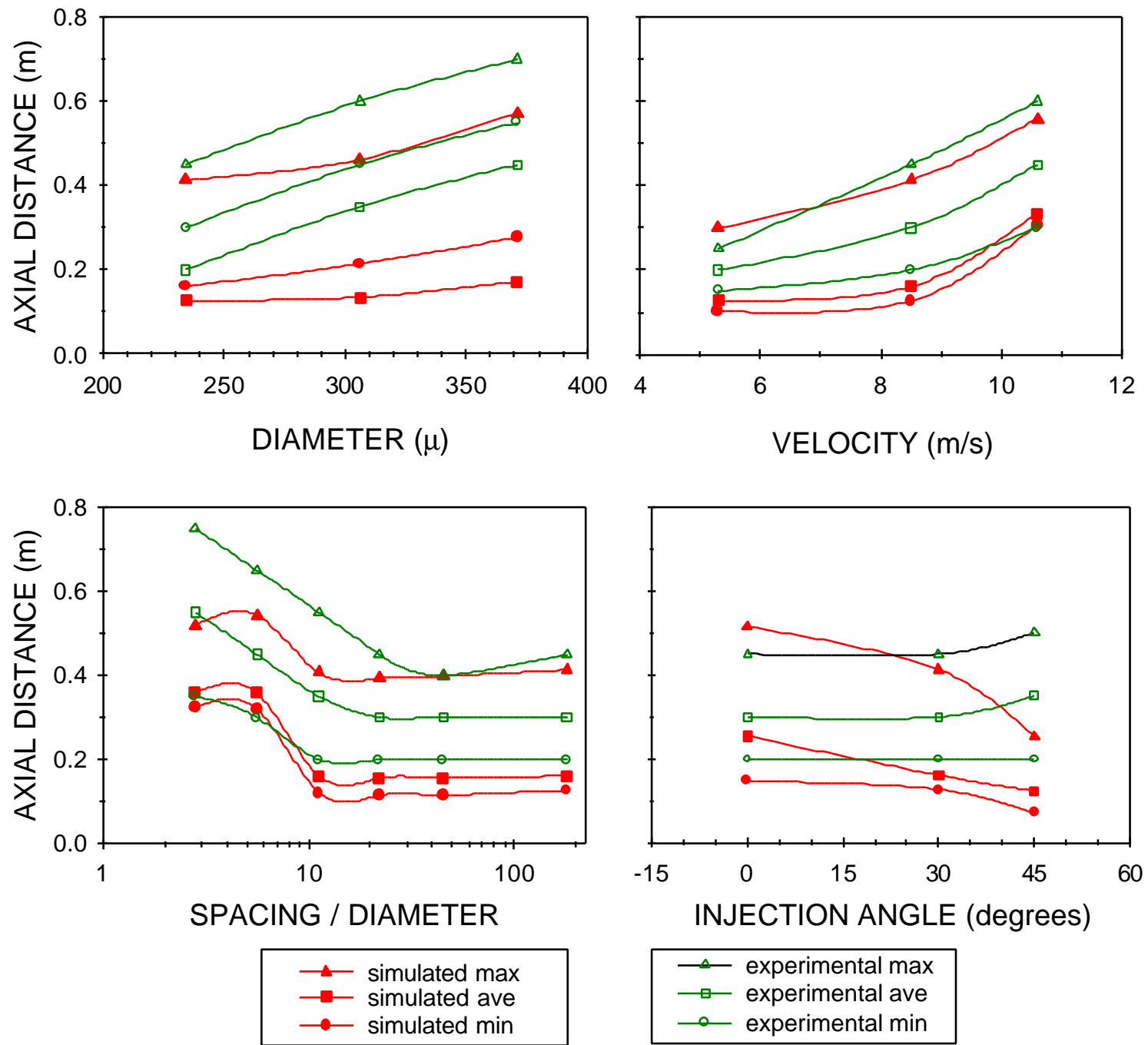

Figure 5.16: Comparison of experimental measurements and simulated burning droplets. The closed symbols represent the predicted maximum (triangle) average (square) and minimum (circle). The experimental data are shown in open symbols. An ensemble size of 2000 was used to generate these plots. 

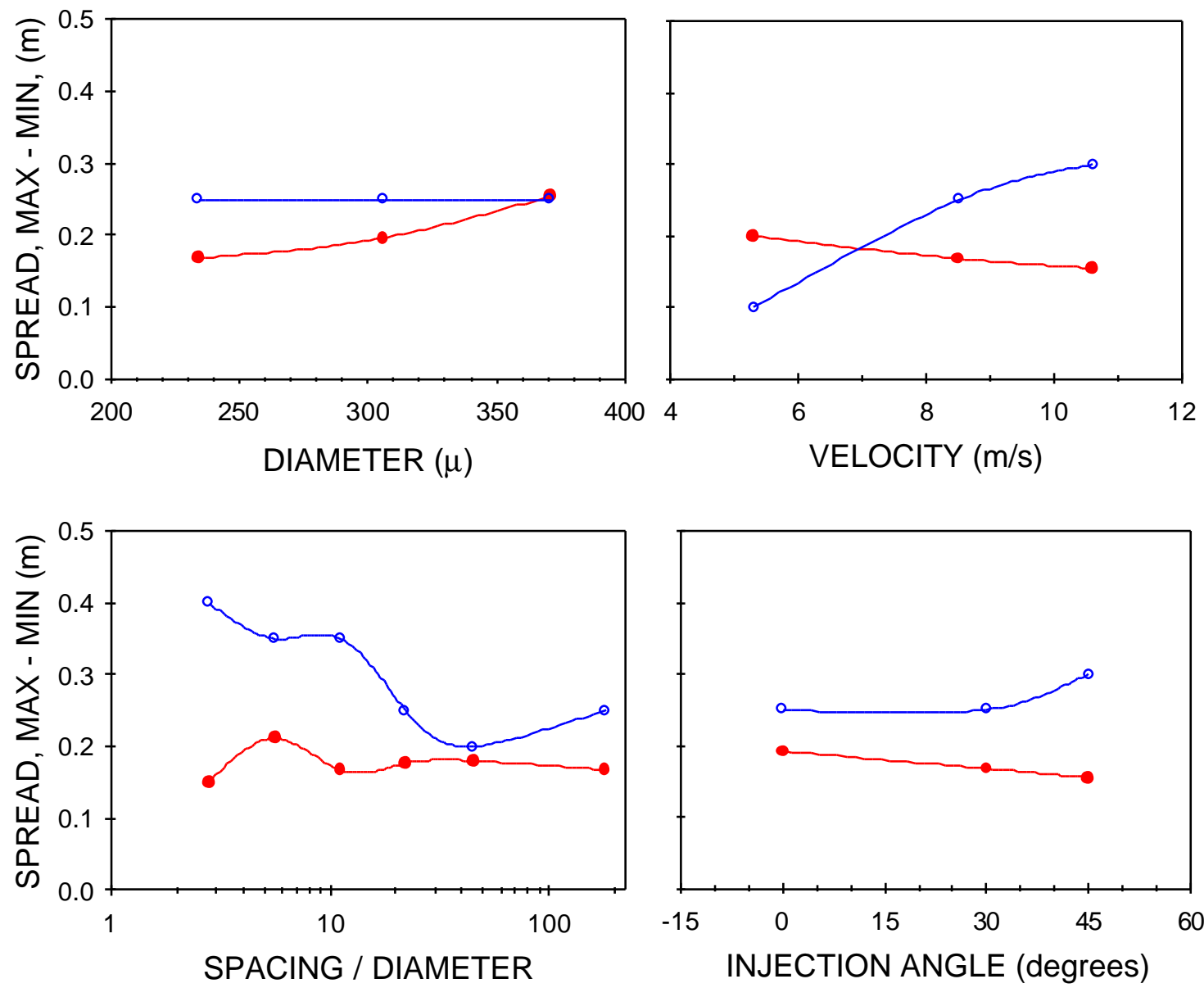

$\multimap$ simulated $\quad$ experimental

Figure 5.17: Comparison between the experimentally measured spread in burn out end points and the spread predicted by the simulation. This simulated data comes from an ensemble size of 200. 

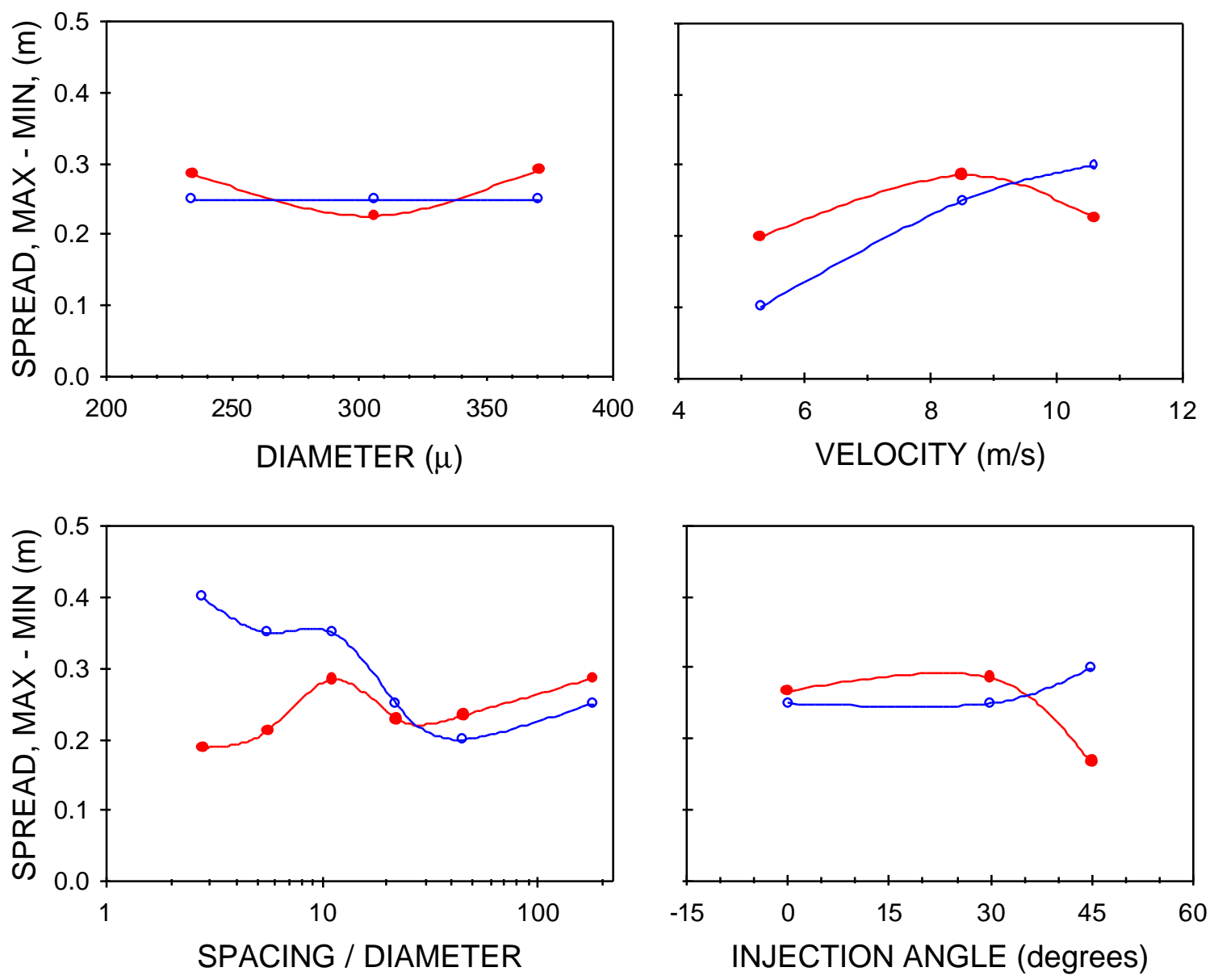

$\multimap$ simulated $\multimap$ experimental

Figure 5.18: Comparison between the experimentally measured spread in burn out end points and the spread predicted by the simulation. This simulated data comes from an ensemble size of 750 . 

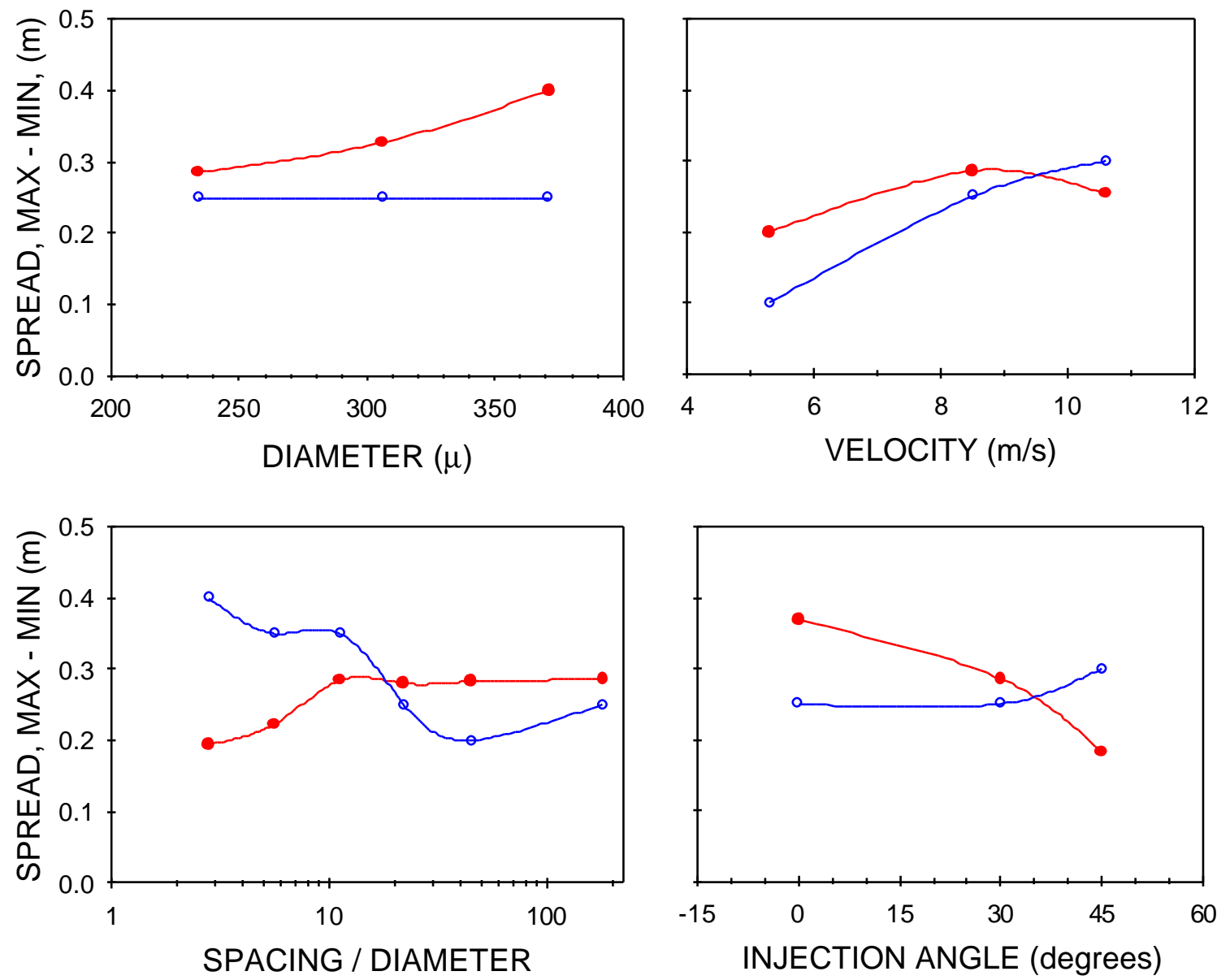

$\multimap$ simulated $\quad$ experimental

Figure 5.19: Comparison between the experimentally measured spread in burn out end points and the spread predicted by the simulation. This simulated data comes from an ensemble size of 2000 . 

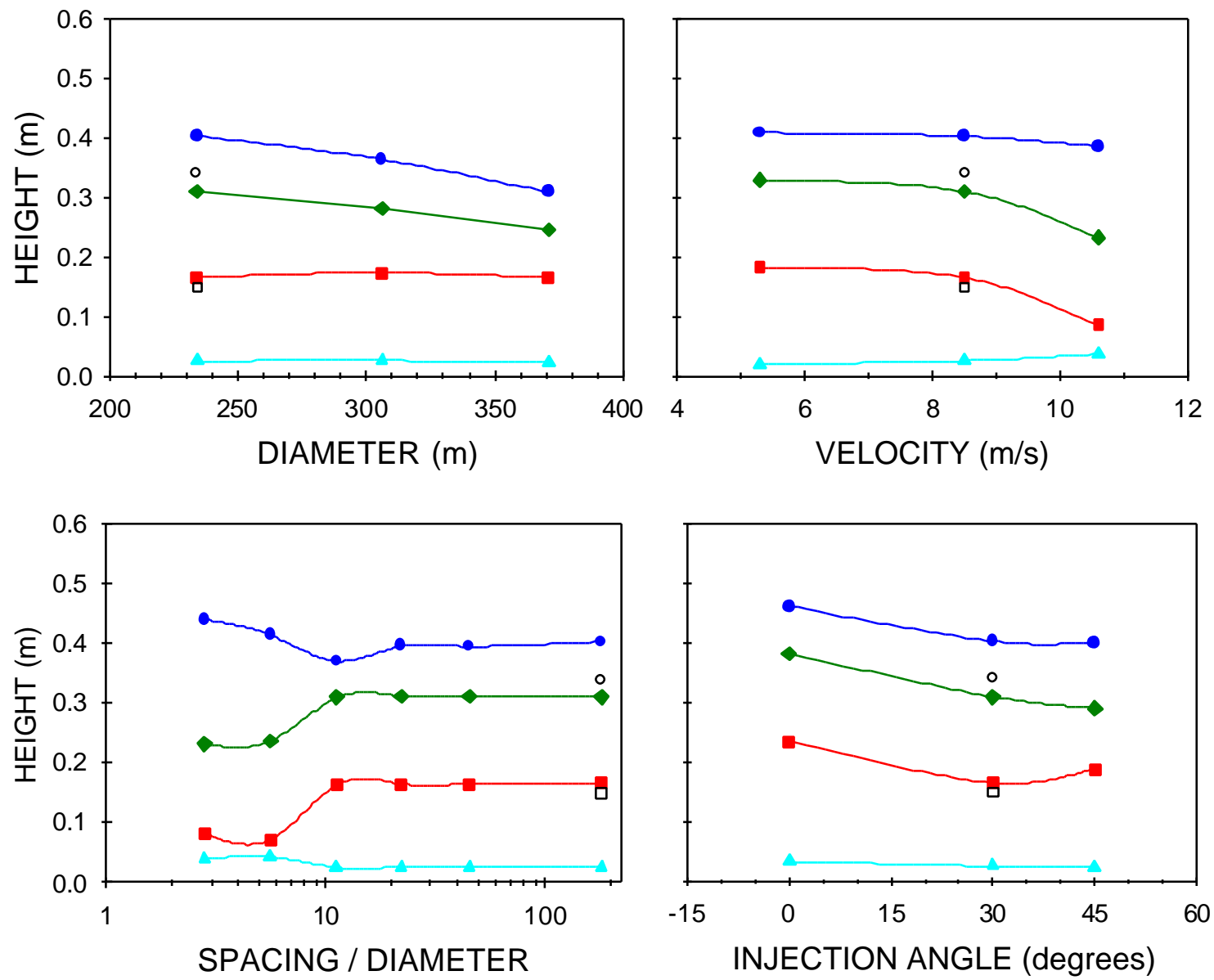

$$
\begin{aligned}
& \longrightarrow y \max \\
& -y \text { ave } \\
& \longrightarrow y \min
\end{aligned}
$$

$$
\begin{aligned}
- & \text { std dev } \\
\circ & \text { data max } \\
\square & \text { data min }
\end{aligned}
$$

Figure 5.20: Variation in the $Y$ component of the droplet position as a function of the input conditions. The only pair of measured variation in the $\mathrm{Y}$ position available is shown with an open circle for the maximum and an open square for the minimum. An ensemble size of 200 was used to generate these plots. 

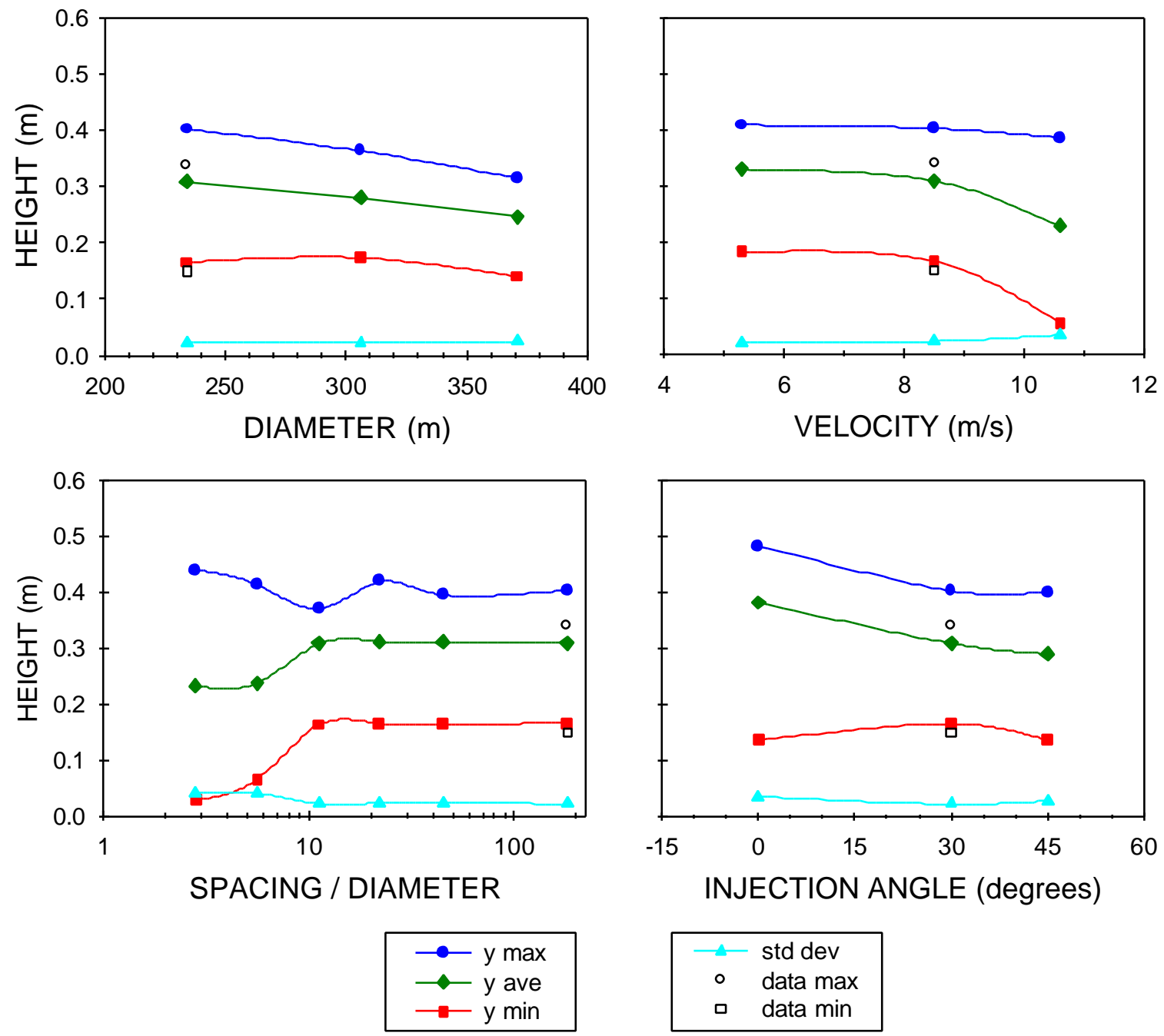

\begin{tabular}{|cl|}
\hline- & std dev \\
$\circ$ & data max \\
$\square$ & data min
\end{tabular}

Figure 5.21: Variation in the $Y$ component of the droplet position as a function of the input conditions. The only pair of measured variation in the $\mathrm{Y}$ position available is shown with an open circle for the maximum and an open square for the minimum. An ensemble size of 750 was used to generate these plots. 

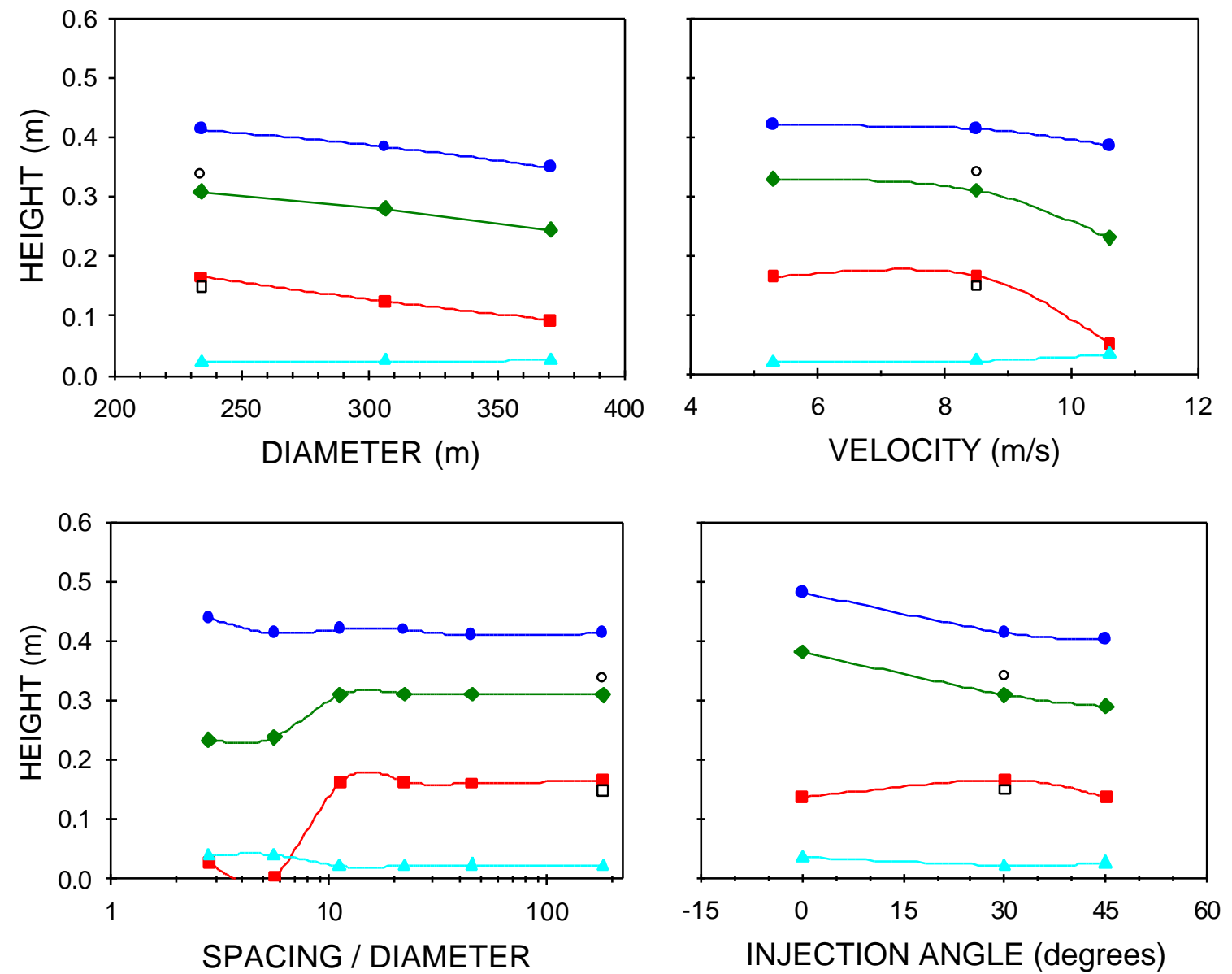

$$
\begin{aligned}
& \longrightarrow y \max \\
& -y \text { ave } \\
& \multimap y \min
\end{aligned}
$$

\begin{tabular}{|cl}
\hline- & std dev \\
$\circ$ & data max \\
$\square$ & data min
\end{tabular}

Figure 5.22: Variation in the $Y$ component of the droplet position as a function of the input conditions. The only pair of measured variation in the $\mathrm{Y}$ position available is shown with an open circle for the maximum and an open square for the minimum. An ensemble size of 2000 was used to generate these plots. 


\section{Chapter 6}

\section{Effects of the Two-phase Flow Parameter $\beta$}

\subsection{Droplet model free parameter $\beta$}

As discussed in Section 3.7, the internal ODT local instantaneous time scale $\tau\left(l ; y_{0}, t\right)$ is not a real physical time and therefore a droplet model free parameter called $\beta$ was introduced into the model to account for discrepancies between the actual eddy lifetime and the ODT instantaneous time scale $\tau\left(l ; y_{0}, t\right)$. This free parameter should ideally be a number close to unity since $\tau\left(l ; y_{0}, t\right)$ was based on good reasoning and logically should not be too far off. $\beta=1$ was used for all runs reported in this paper outside of this chapter. Here additional runs were made with $\beta=0.5$ and $\beta=2.0$. The eddy lifetime, $t_{\mathrm{e}}$, was defined in Chapter 3 as:

$$
t_{e}=\beta \tau\left(l ; y_{o}, t\right)
$$

$t_{\mathrm{e}}$ has two important and somewhat competing effects on the droplet-eddy interaction. The first effect is that it adjusts the end time of the eddy interaction box which either lengthens or shortens the maximum amount of time during which the droplet relaxes to the fluid velocity. The second is that it adjusts the fluid phase $y$ velocity, Equation 3-17: 
$v=h / t_{e} \quad \cdot \quad(3-17)$

A value of $\beta<1$ increases $v$, as compared to $v$ computed with $\beta=1$. A $\beta>$ 1 decreases $v$. The burden of the droplet-drag law (Equation 3-25) is to relax the particle to the surrounding gas flow $v$. With $\beta>1$, the maximum relaxation time $t_{\mathrm{e}}$ increases, but the velocity $v$, to which the particle is being relaxed to, is being decreased.

\subsection{Effect of $\beta$ on a tracer particle, $\tau_{p} \rightarrow 0$}

For the limit of infinitely low inertia, the net $y$ displacement caused by the particle-eddy interaction with $\beta>1$ is identical to the $y$ displacement which would be achieved with $\beta=1$ or $\beta<1$. However, the $y$ velocity of the particle, $\mathrm{V}$, at the end of the pseudo time calculations are all different. The particle velocity, $\mathrm{V}$, for $\beta>1$ would be the smallest in Magnitude, followed by $\mathrm{V}$ for $\beta=1$ and ending with the largest magnitude velocity $\mathrm{V}$ for $\beta<1$.

The tracer particle paths for the three cases $\beta=2, \beta=1, \beta=1 / 2$, are shown in Figure 6.1 with the arrows as shown in (B) now indicating velocity 

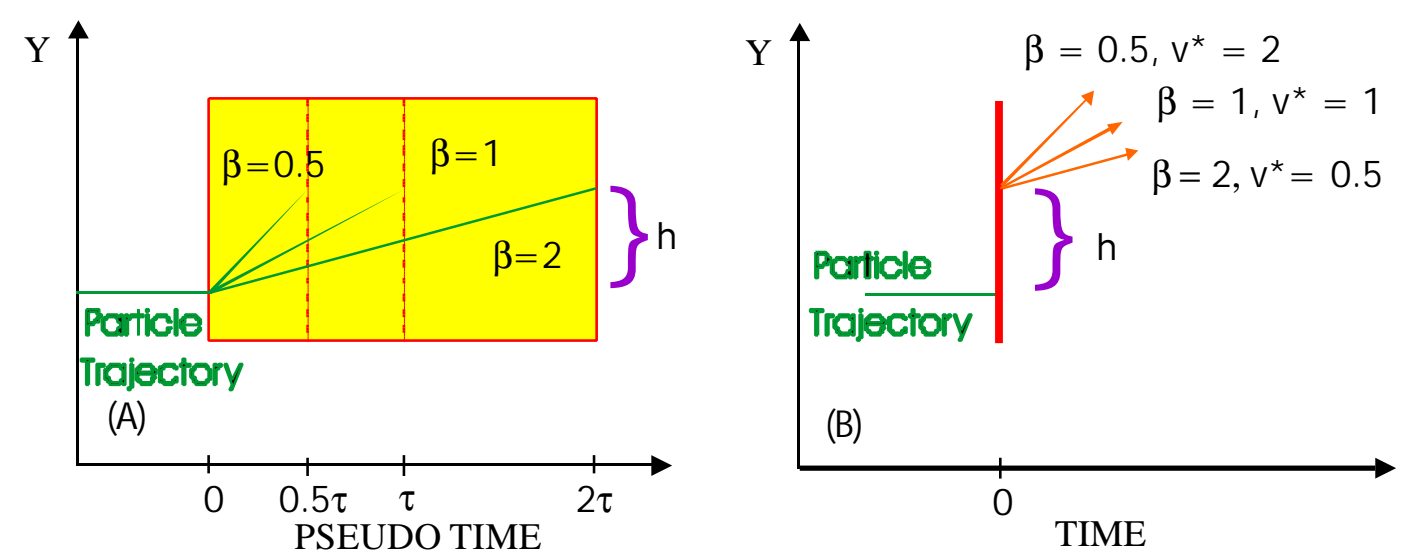

Figure 6.1: Effects of the free parameter $\beta$ on the eddy interaction box (A) and on the particle velocity (B) at the moment of the eddy interaction.

vectors, rather than trajectories as in previous diagrams. The velocity of the particle relative to the velocity of the particle with $\beta=1$ is given by:

$$
v^{*}=v(\beta) / v(\beta=1) \quad(6-1)
$$

The velocity of the particle path with $\beta=1 / 2$ will wish to deviate (by virtue of its velocity vector) the furthest from the particles original path. Conversely the path with $\beta=2$ will wish to deviate the least. Even though this analysis holds exactly only for the infinite drag droplet-eddy interaction $\left(\tau_{p} \rightarrow 0\right)$ at the instant of the interaction, the trend is identical for particles with finite drag in both the particle-eddy interaction and afterwards. The smaller the $\beta$ 
the greater the tendency of a real particle to deviate from its original course after the particle-eddy interaction is completed.

\subsection{Effect on a finite inertia particle exiting the eddy event early}

An additional complication is encountered when a particle leaves the eddy interaction box by one of the horizontal surfaces. The particular situation varies depending on the relative sign of the particle velocity, $\mathrm{V}$, and the gas velocity, $v$, but suffice it to say that: if the particle eddy interaction times $t_{\beta=2}, t_{\beta=1}$, and $t_{\beta=0.5}$ do not vary much (are similar in duration) then the interaction with the largest relative velocity, $v^{*}(\beta=1 / 2)$, will produce the largest $y$ deviation from the original trajectory. One particular case where this is true is shown in Figure 6.2.
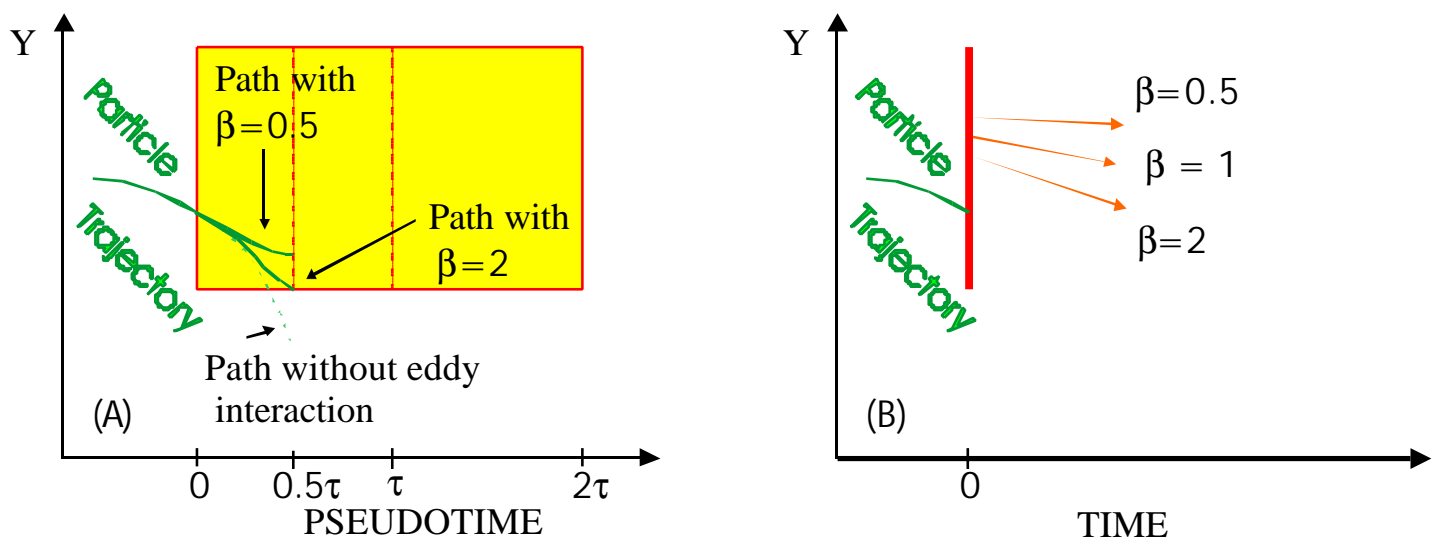

Figure 6.2: Effects of $\beta$ on one real particle with an initial y velocity. The smaller value of $\beta$ tends to cause a greater deviation from the particles original path. Path for $\beta=1$ is not shown. The arrows in (B) are velocities at the time just after the eddy event, not particle paths. 
In Figure 6.2 (A) the dotted line represents the path the particle would take if it did not interact with the illustrated eddy. The fluid cell which surrounds the particle at the point of the particle-triplet map interaction has an upward velocity (a positive $v$ ). This positive velocity will have varying effects depending on the value of $\beta$ used. Careful examination of the illustration shows that for all values of $\beta$, the velocity adjustment is positive since the $v$ velocity is positive. The smaller the value of beta used the greater the velocity difference compared to the velocity just before $\left(0^{-}\right)$the particleeddy interaction. Refer to Section 3.6 for details on how the change in position and velocity is computed.

\subsection{Particles with infinitely large inertia}

A brief discussion of the effects of $\beta$ on the other limiting case, infinite particle inertia is added for completeness. There is no scatter from the original path whatsoever for any value of $\beta$. No matter what the choice of $\beta$ the infinite inertia particle is not deflected by it, with the possible exception of $\beta=0$, in which $v^{*}$ tends to infinity and might than deflect the infinite inertia particle. However, since in the introduction of $\beta$ was made with the supposition that $\beta$ be a number close to one, no provision was added to account for a vanishing $\beta$. 


\subsection{Numerical results for 12 cases studied}

Figures $6.3-6.14$ give the variations in $\beta$ for each of the 12 cases studied, 1 - 12 respectively, using an ensemble size of 750 . See Table 5.1 for details on the initial diameter, initial velocity, initial droplet spacing and the injection angle of the stream of droplets for each particular case. All cases show the same trend, the smaller $\beta(0.5)$ causes more of a scatter in the $y$ direction than the unity $\beta$ and there is even less scatter on average for the large $\beta$ (2.0). There are however outliers in all of cases even the large $\beta$ due to the random nature of the ODT formulation. For the cases shown in this chapter (an ensemble size of 750) there is no appreciable change in the scatter in the $x$ direction due to changes in $\beta$. Though not shown in this thesis, the results for the ensemble size of 2000 yielded the same results as that of the 750 size. That is there was no appreciable change in the scatter in the $x$ direction due to $\beta$ and the scatter in the $y$ direction increased as $\beta$ decreased. The reason $\beta$ has an effect in the $y$ direction is that the idealized eddy events cause instantaneous displacements in the $y$ direction and adjustments to the $y$ velocity of the particle. No adjustments are made to the $x$ direction, see Section 3.9.2 for a discussion on this point. Since there is no direct effect of $\beta$ on motion in the $x$ direction the only influence that $\beta$ can 
have in the scatter in the $x$ direction is a secondary affect. For example, a smaller value of $\beta$ may cause the droplet to deviate from its path to a fluid zone which is significantly hotter (or colder) which in turn would cause the droplet to vaporize quicker (or more slowly). This change in vaporization rate of the droplet would result in more scatter in the $x$ direction. Hence this is only a secondary influence on the $x$ scatter.

This same (secondary) influence can be achieved by allowing the ODT program to run more realizations. That is the random nature of the ODT program (with a value of $\beta=1$ ) will, after a sufficient number of realizations, jostle the droplets enough to sample the range of temperature variations which influence the scatter in the $x$ direction. The runs with ensemble sizes of 750 and 2000 are large enough so that the $x$ direction scatter is not influenced by adjustments to $\beta$.

The results for an ensemble size of 200 (not shown in this thesis) show variation in the $x$ scatter due to changes in $\beta$, however, these variations are attributed to the small number of realizations and not to any physical phenomenon.

Figure 6.15 gives the variation as a function of $\beta$ (top, middle, bottom diagrams) of the variation in height (the $y$ direction) of the numerical data as 
a function of the initial diameter $\mathrm{D}_{\mathrm{o}}$ based on an ensemble size of 750 . The only available data points, for the variation in the $y$ direction, from the Mulholland et al. paper are shown as open symbols (circle for the maximum and a square for the minimum). This data is also included in Figures 6-16 6-18. The trend of larger $\beta$ giving less of a spread is detectable from these diagrams. However, the scatter plots given in Figures $6.3-6.14$ give a much more intuitive feeling for this conclusion since even for large $\beta$ there are outliers.

Figure 6.16 gives the variation in the $y$ direction as both a function of $\beta$ and initial velocity based on an ensemble size of 750 . The trend of larger $\beta$ producing less $y$ spread is also evident here. Figure 6.17 gives the variation in the $y$ direction as a function of $\beta$ and the initial s/D based on an ensemble size of 750. Figure 6.18 gives the variation in the $y$ direction as a function of $\beta$ and the initial injection angle based on an ensemble size of 750 .

Also included here are comparisons between the variation in the $x$ direction as a function of $\beta$ and initial diameter for an ensemble size of 750 in Figure 6.19. Figures 6.20 gives the variations in the $x$ direction variation as a function of initial velocity and $\beta$. Figures 6.21 gives the $x$ direction 
spread as a function of initial droplet spacing and $\beta$. Figure 6.22 gives the $x$ direction variation as function of injection angle and $\beta$. No definite trends could be derived from the variations in the axial direction burn out points as a function of $\beta$.

\subsection{Conclusions}

Consistent in all 12 cases, which included variations in initial diameter, initial velocity, initial droplet spacing and injection angle, the smaller value of $\beta$, produced the larger variation in the $y$ (transverse direction) from the droplets original trajectory. The larger value of $\beta$ produced less scatter in the $y$ direction. There were no detectable trends in the variation in the axial $(x)$ direction due to variation in $\beta$ in runs with an ensemble size of 750 . 

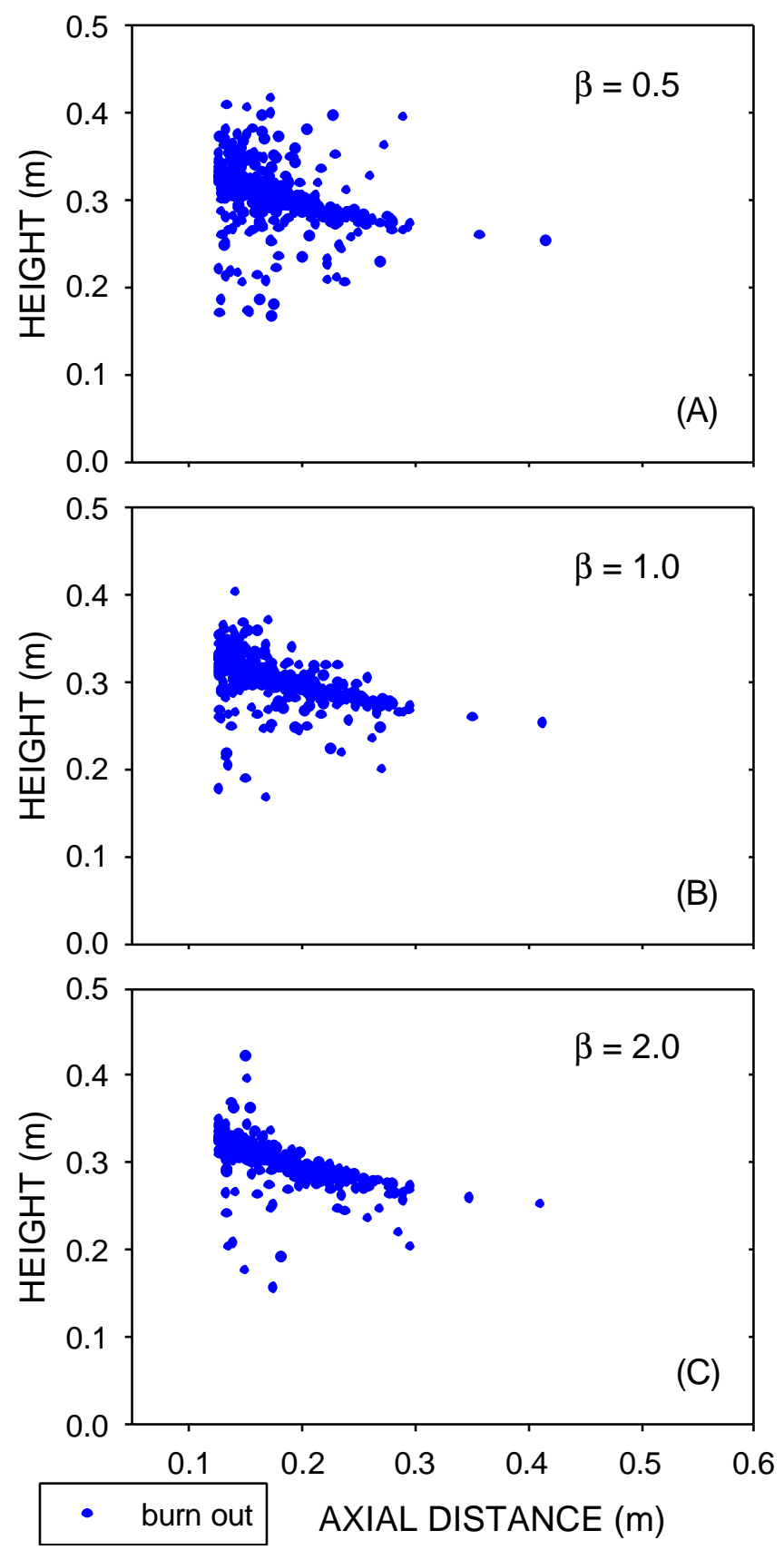

Figure 6.3: Variation of case 1 as a function of $\beta$. As beta decreases from a value of 1.0 the vertical $y$ spread increases. As beta increases from a value of 1.0, the vertical spread decreases. 

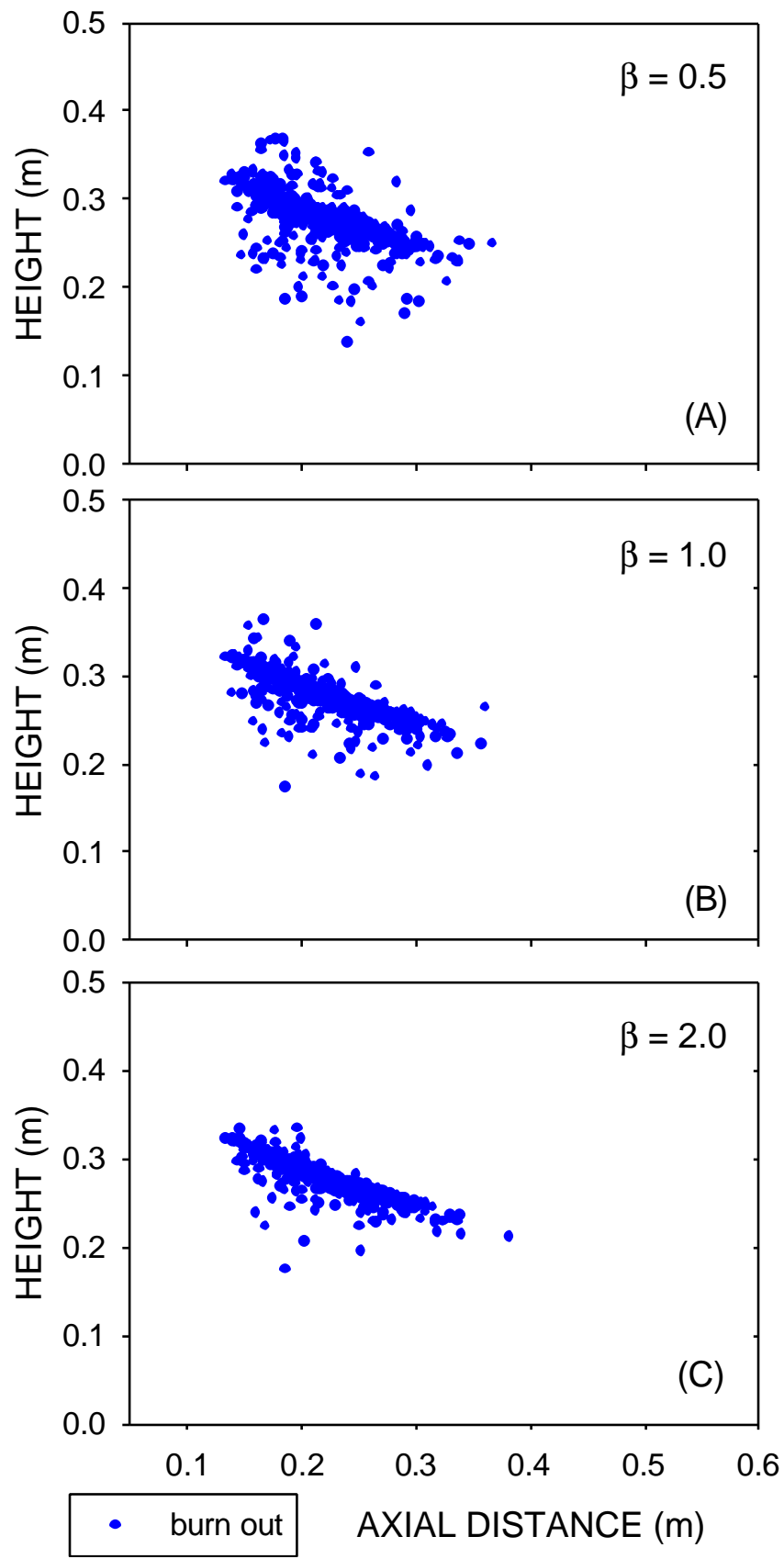

Figure 6.4: Variation of case 2 as a function of $\beta$. As beta decreases from a value of 1.0 the vertical $y$ spread increases. As beta increases from a value of 1.0, the vertical spread decreases. 

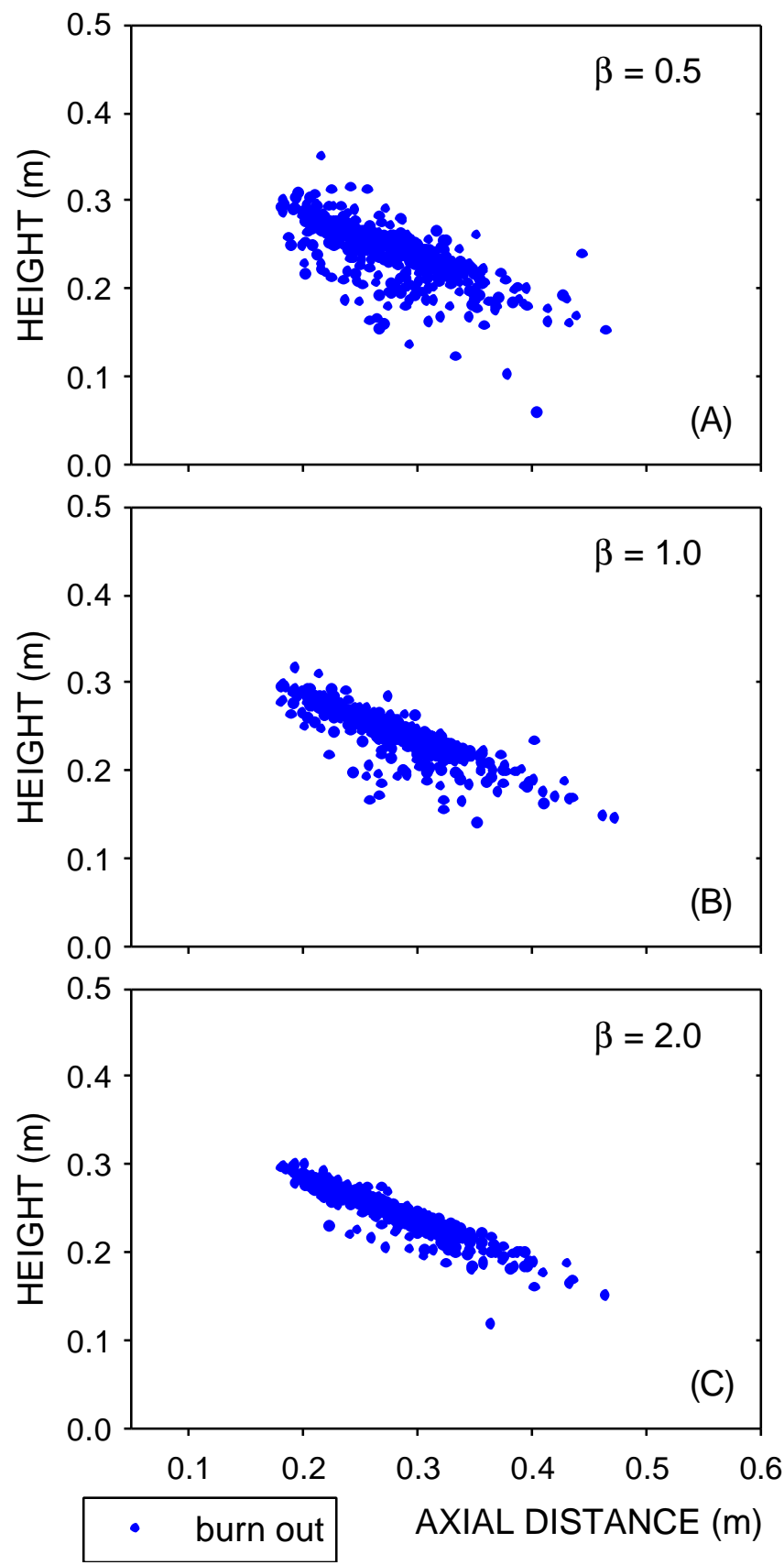

Figure 6.5: Variation of case 3 as a function of $\beta$. As beta decreases from a value of 1.0 the vertical $y$ spread increases. As beta increases from a value of 1.0, the vertical spread decreases. 

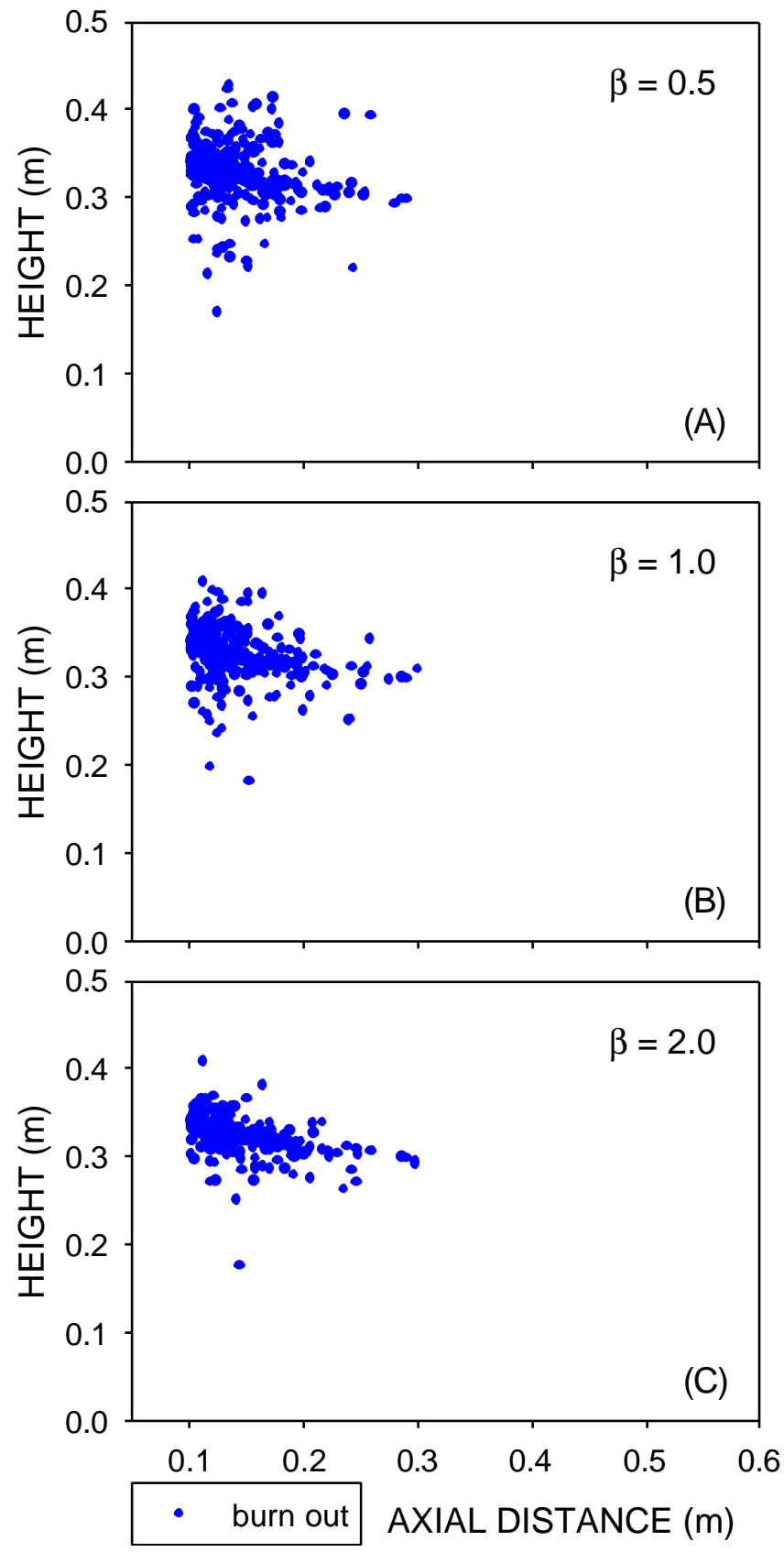

Figure 6.6: Variation of case 4 as a function of $\beta$. As beta decreases from a value of 1.0 the vertical $y$ spread increases. As beta increases from a value of 1.0 , the vertical spread decreases. 

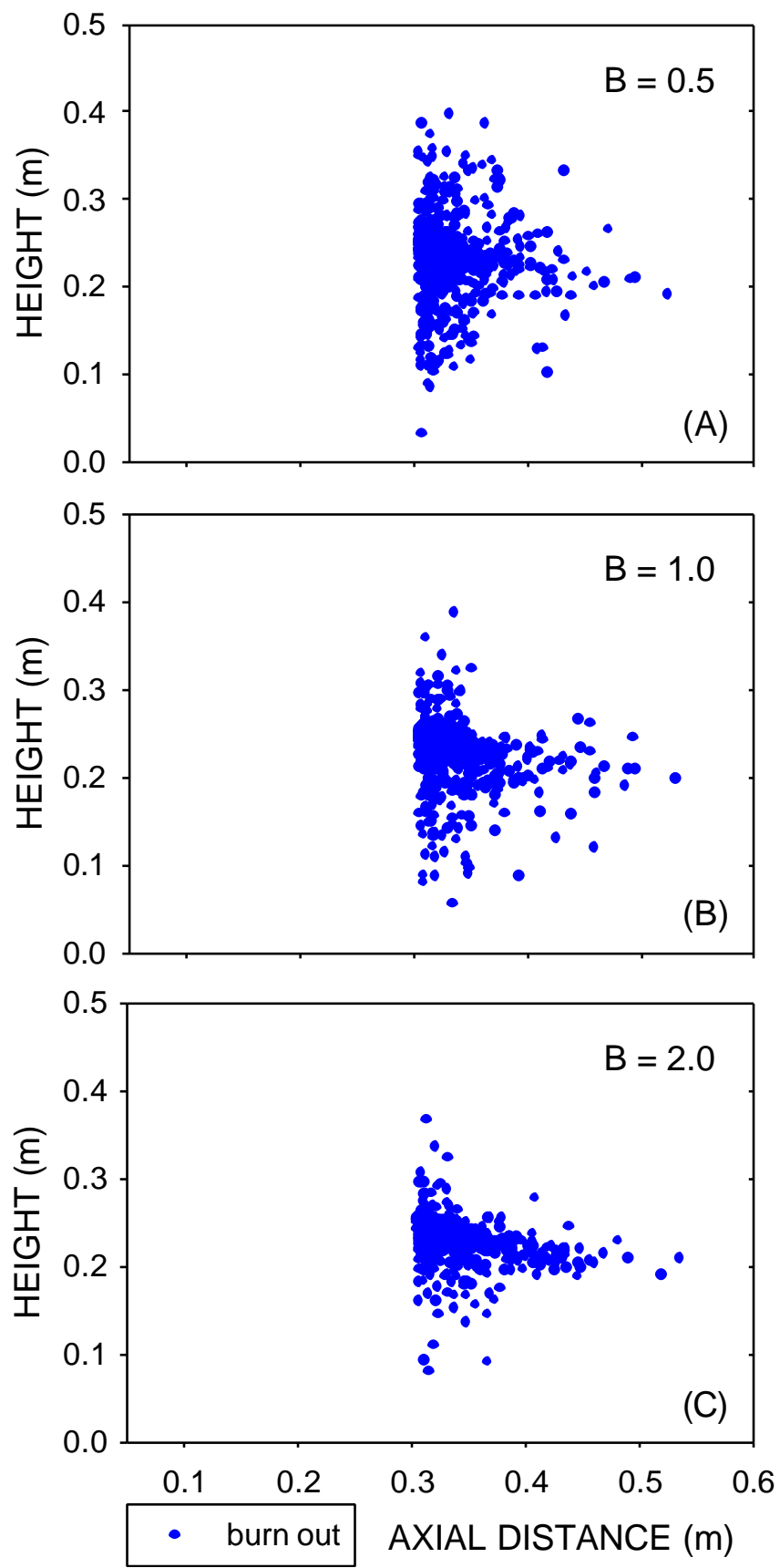

Figure 6.7: Variation of case 5 as a function of $\beta$. As beta decreases from a value of 1.0 the vertical $y$ spread increases. As beta increases from a value of 1.0, the vertical spread decreases. 

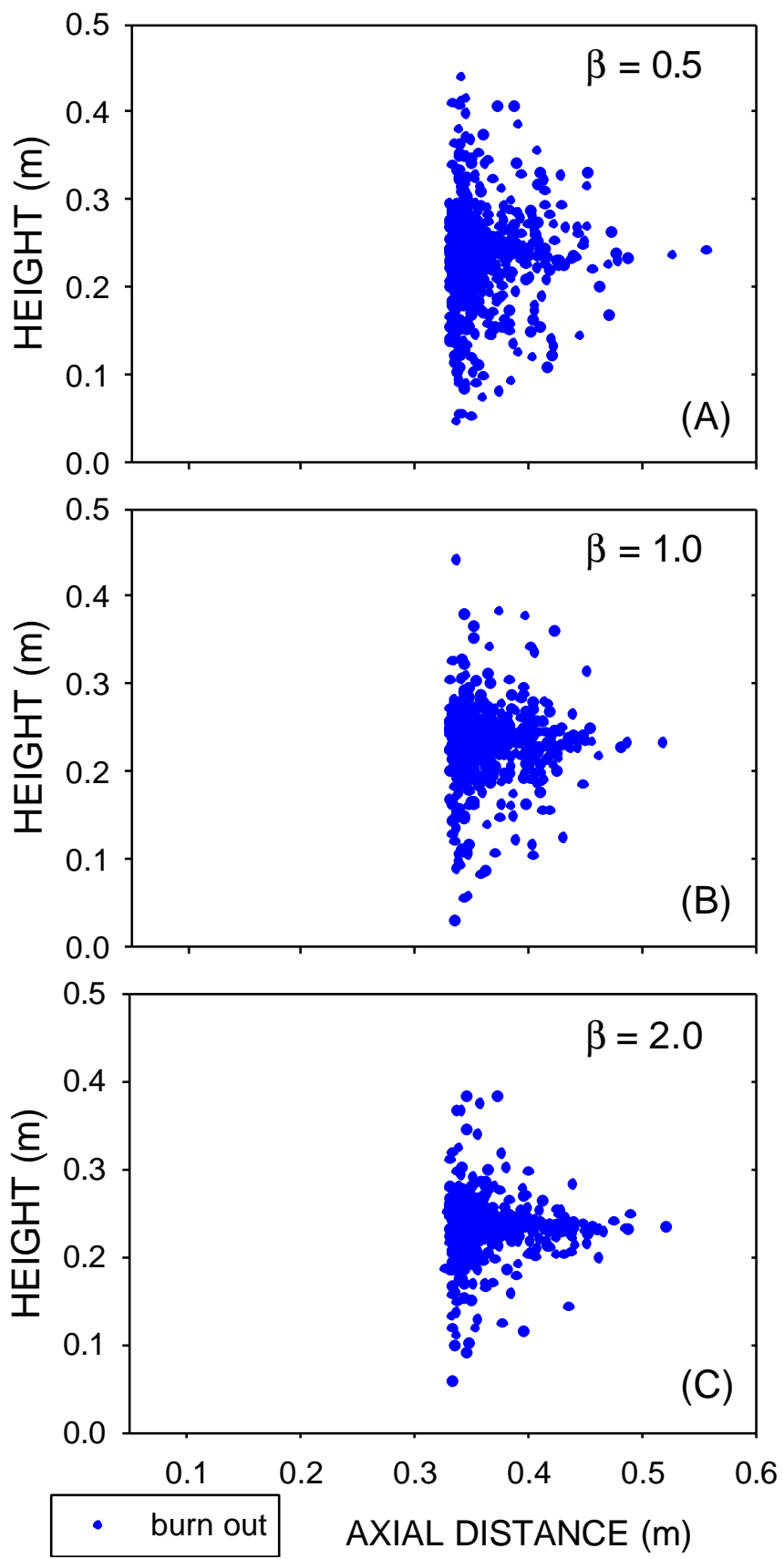

Figure 6.8: Variation of case 6 as a function of $\beta$. As beta decreases from a value of 1.0 the vertical $y$ spread increases. As beta increases from a value of 1.0, the vertical spread decreases. 

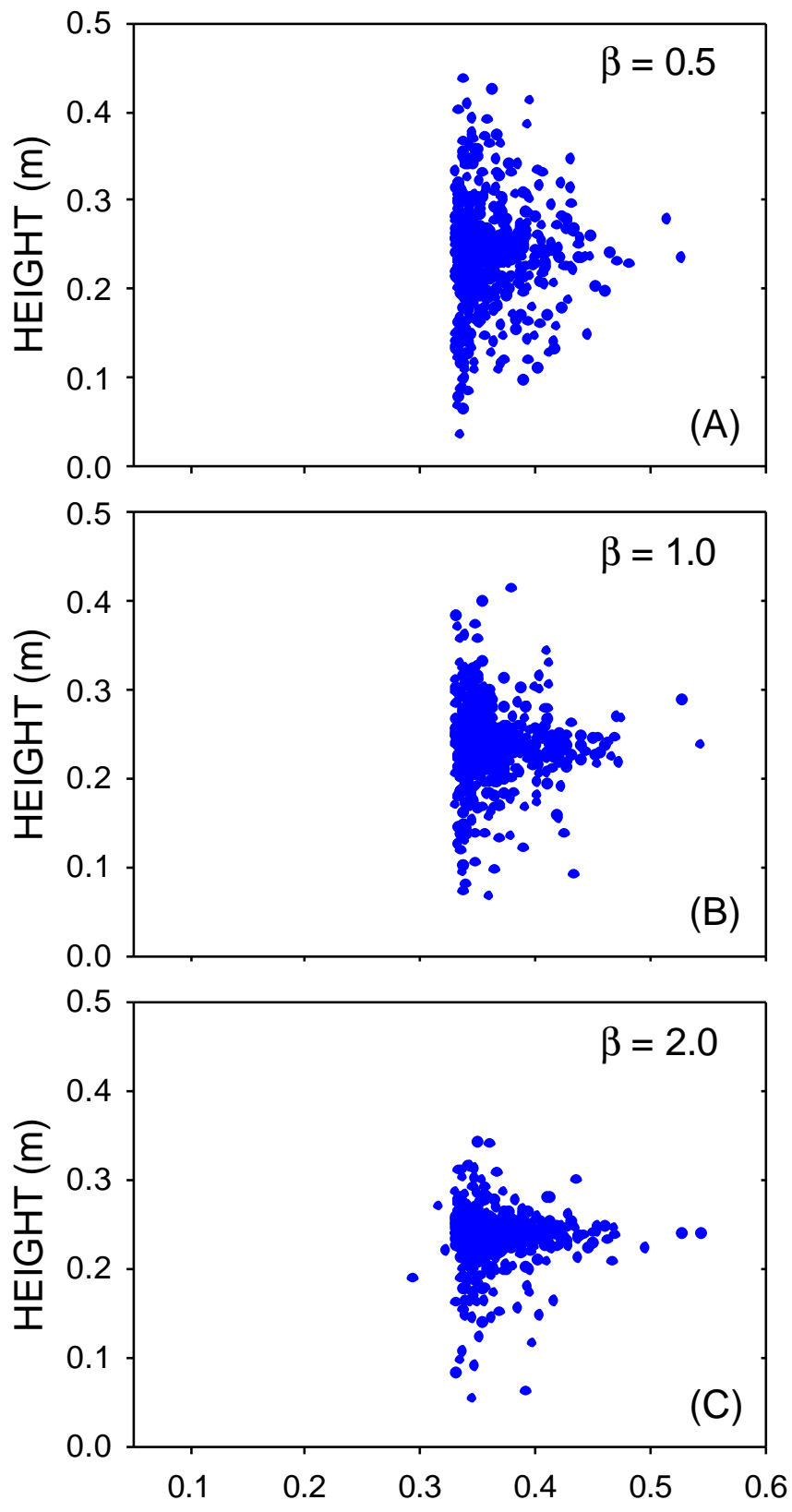

- $\quad$ burn out AXIAL DISTANCE (m)

Figure 6.9: Variation of case 7 as a function of $\beta$. As beta decreases from a value of 1.0 the vertical $y$ spread increases. As beta increases from a value of 1.0, the vertical spread decreases. 

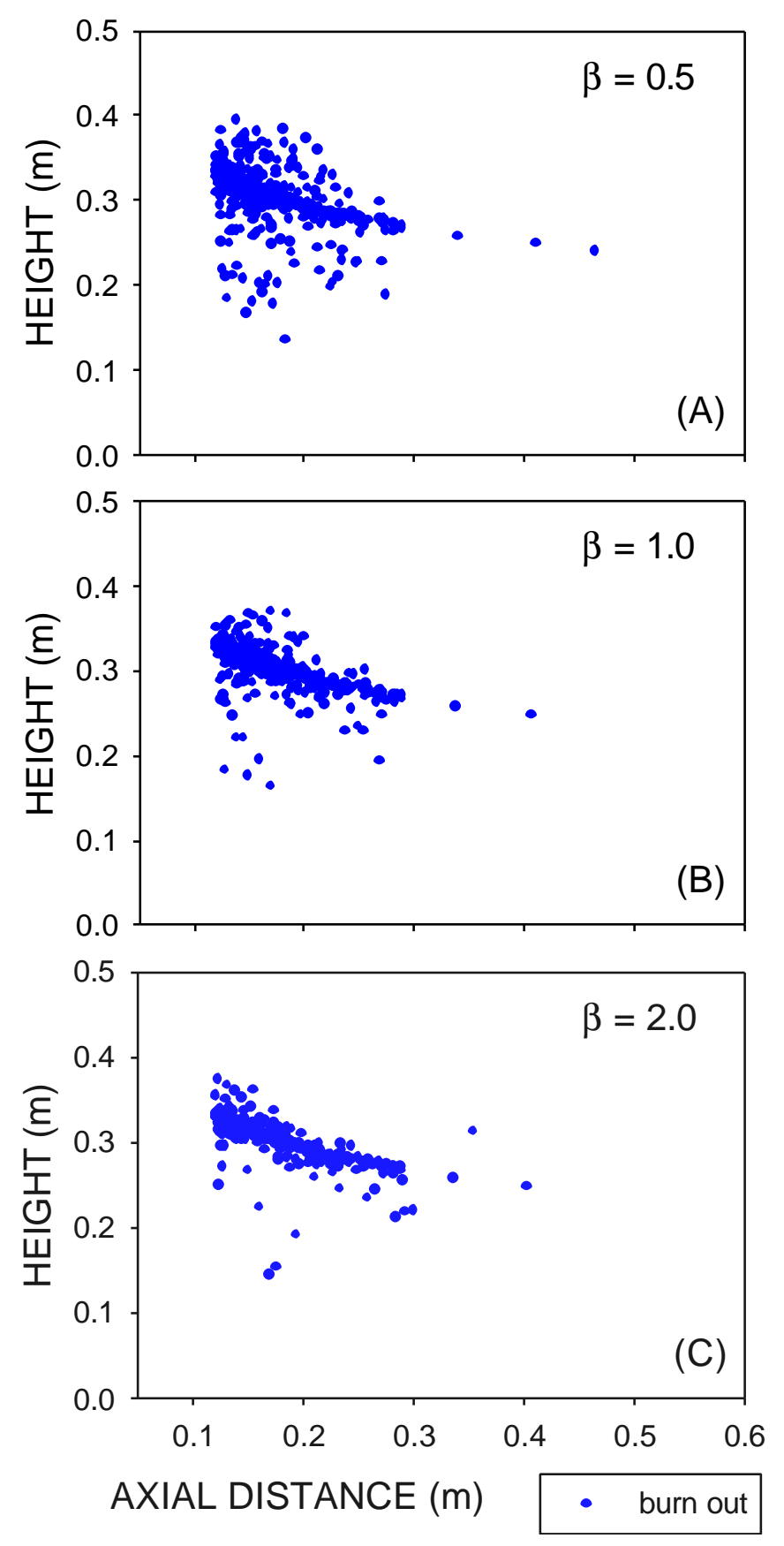

Figure 6.10: Variation of case 8 as a function of $\beta$. As beta decreases from a value of 1.0 the vertical $y$ spread increases. As beta increases from a value of 1.0, the vertical spread decreases. 

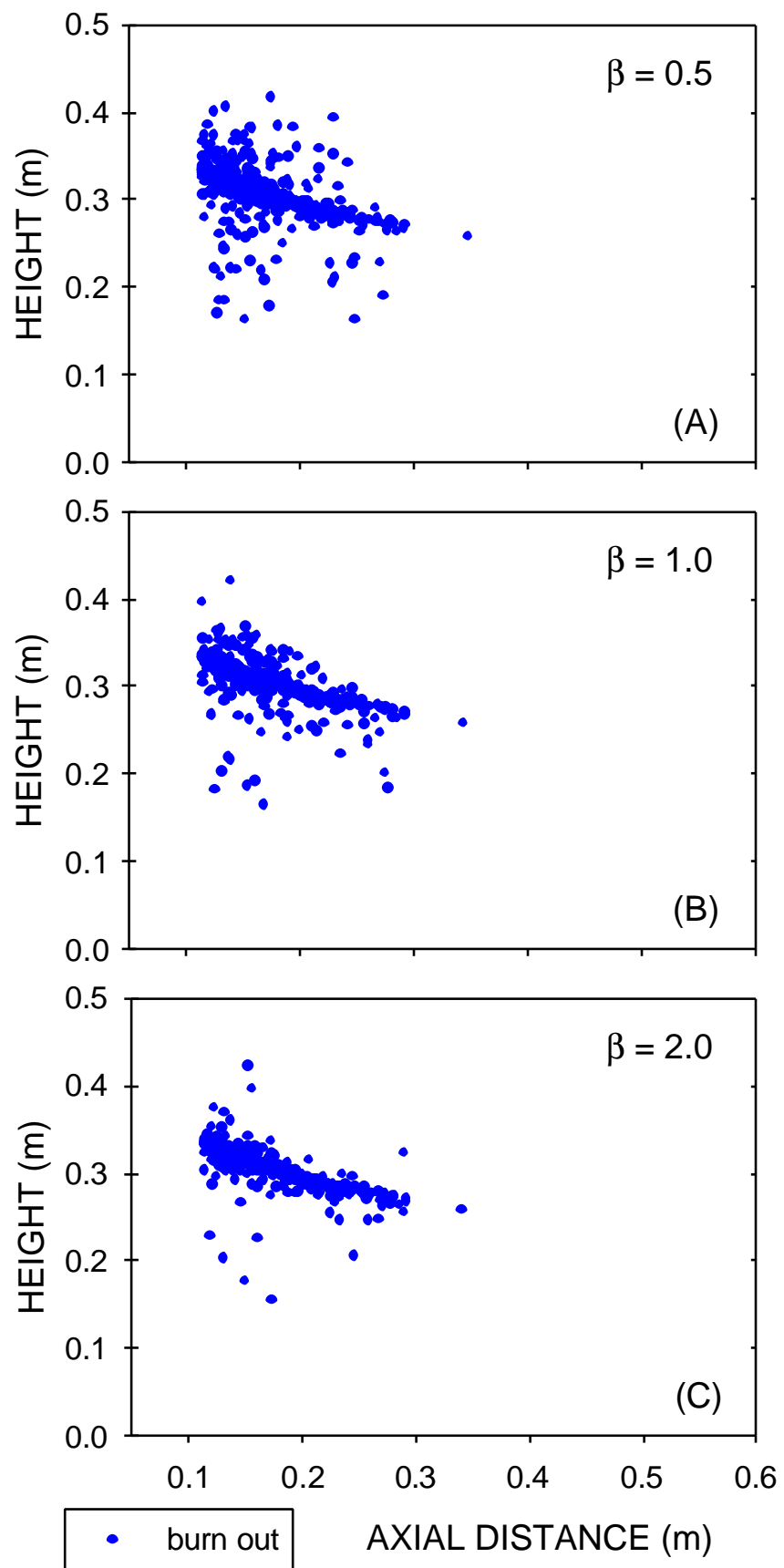

Figure 6.11: Variation of case 9 as a function of $\beta$. As beta decreases from a value of 1.0 the vertical $y$ spread increases. As beta increases from a value of 1.0, the vertical spread decreases. 

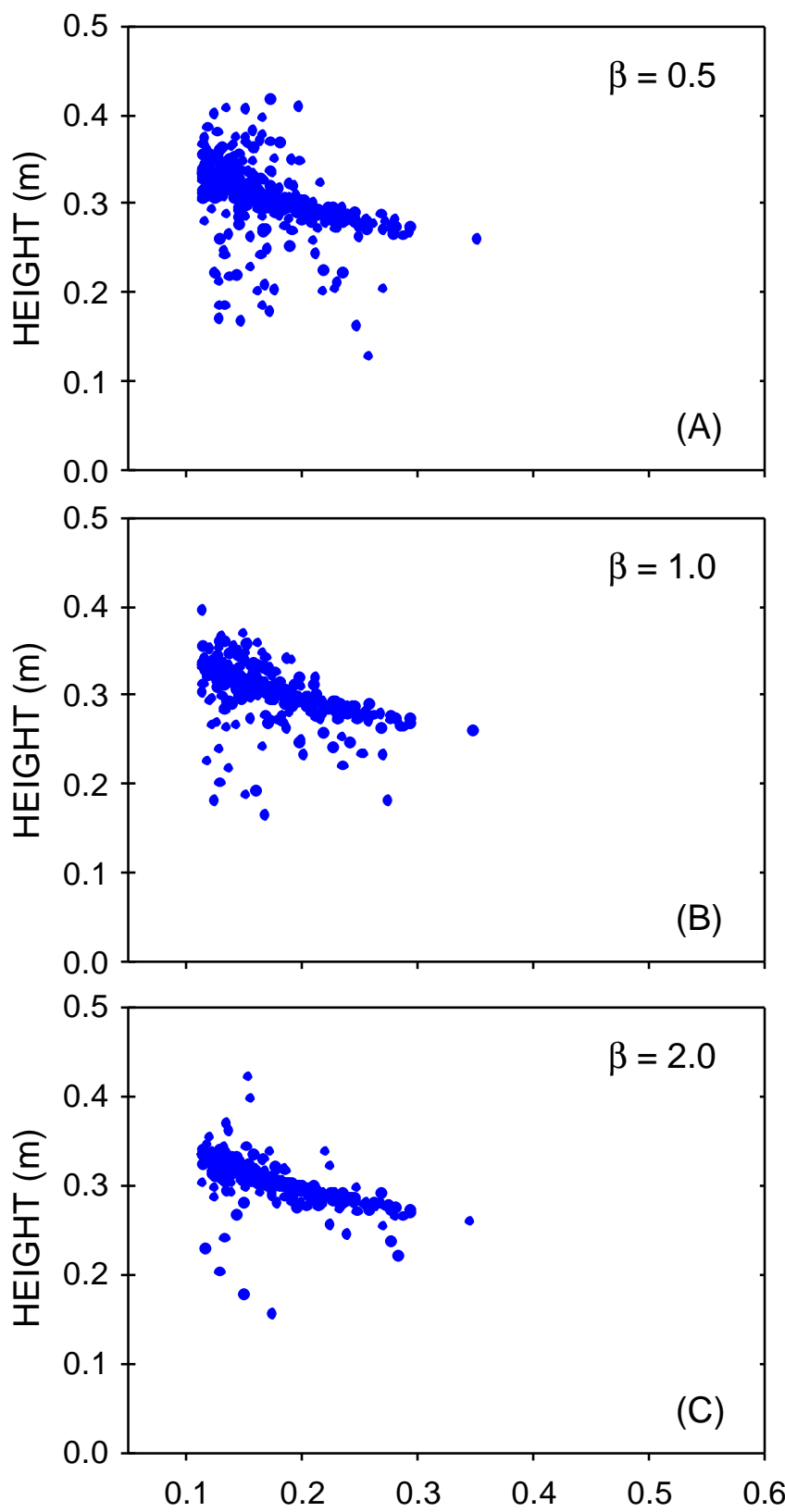

- burn out AXIAL DISTANCE (m)

Figure 6.12: Variation of case 10 as a function of $\beta$. As beta decreases from a value of 1.0 the vertical $y$ spread increases. As beta increases from a value of 1.0, the vertical spread decreases. 

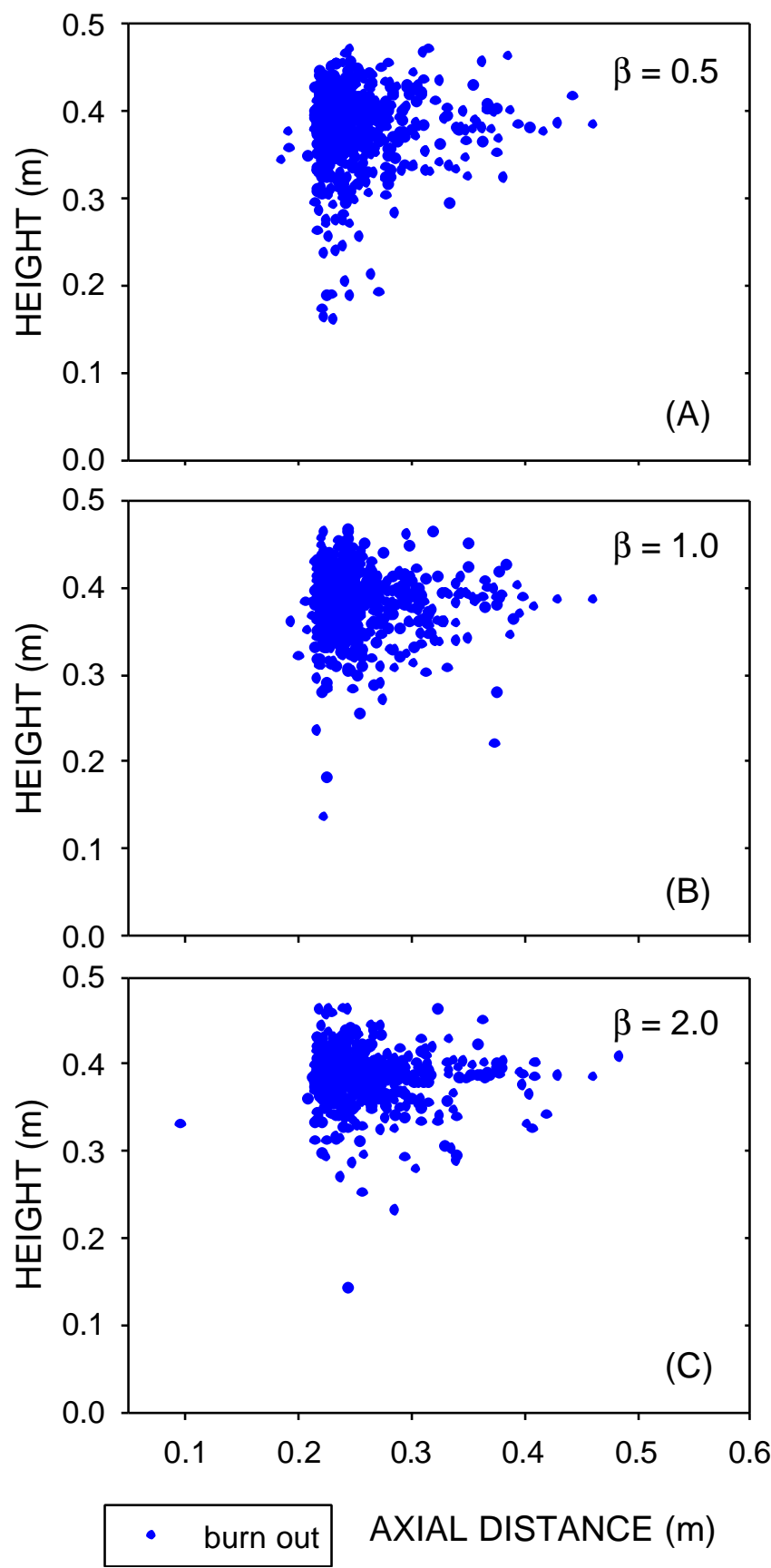

Figure 6.13: Variation of case 11 as a function of $\beta$. As beta decreases from a value of 1.0 the vertical $y$ spread increases. As beta increases from a value of 1.0, the vertical spread decreases. 

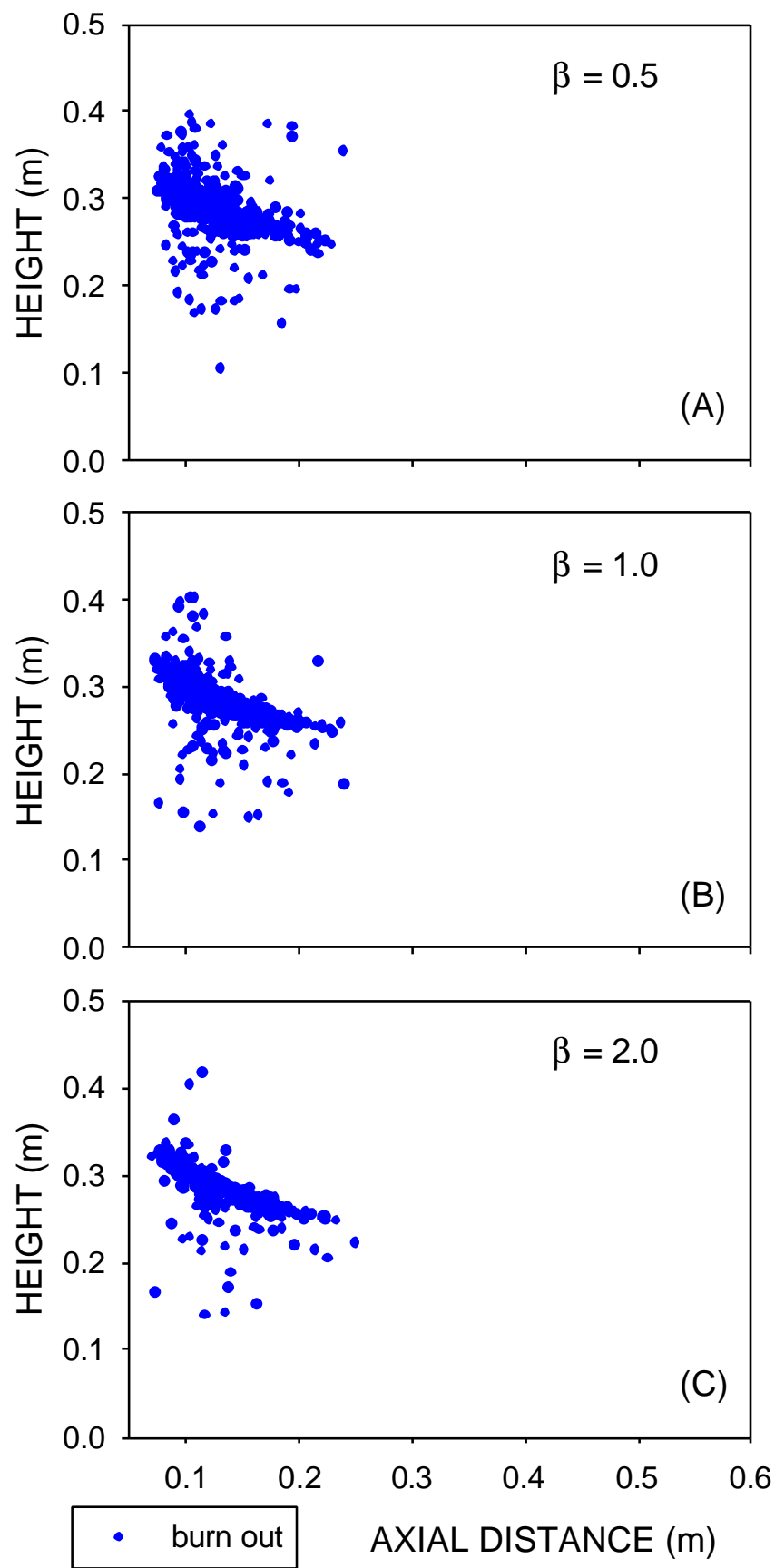

Figure 6.14: Variation of case 12 as a function of $\beta$. As beta decreases from a value of 1.0 the vertical $y$ spread increases. As beta increases from a value of 1.0, the vertical spread decreases. 

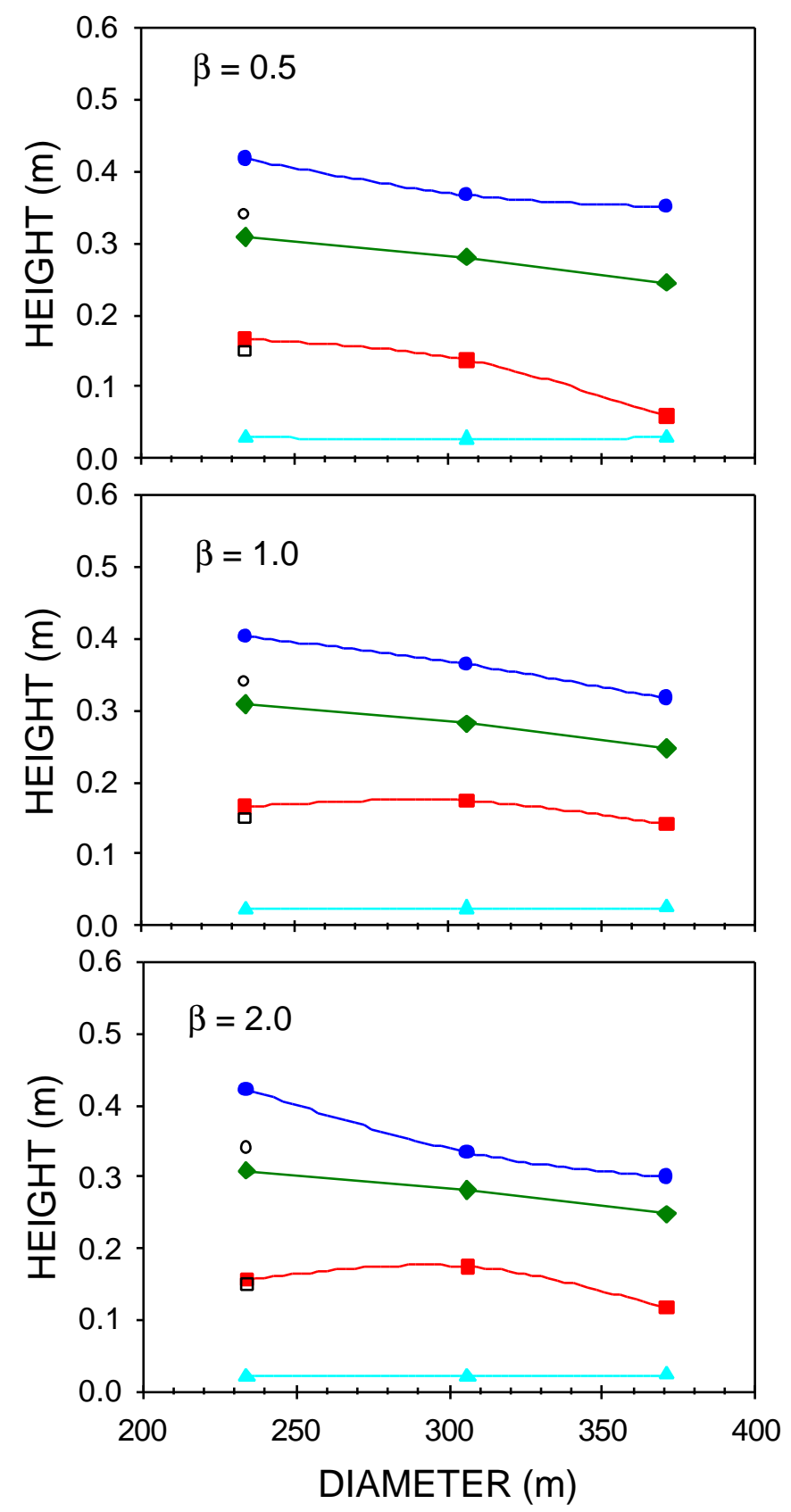

Figure 6.15: Variation in the $Y$ direction as a function of Diameter and $\beta$. As beta decreases from a value of 1.0 the vertical $y$ spread increases. As beta increases from a value of 1.0, the vertical spread decreases. 

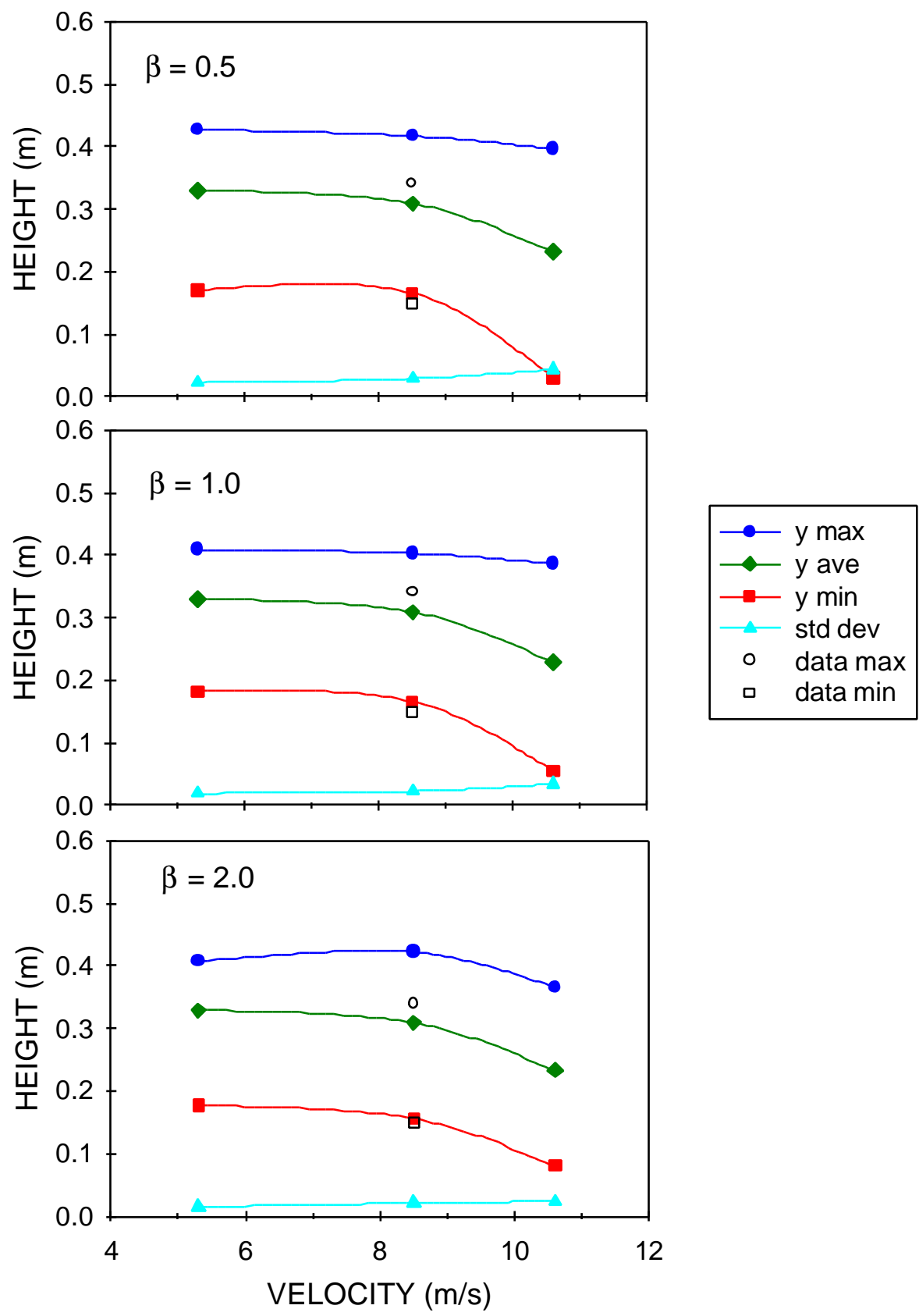

Figure 6.16: Variation in the $Y$ direction as a function of velocity and $\beta$. As beta decreases from a value of 1.0 the vertical $y$ spread increases. As beta increases from a value of 1.0, the vertical spread decreases. As $\beta$ decreases, so does the standard deviation. This is especially evident in the $10.5 \mathrm{~m} / \mathrm{s}$ data. 

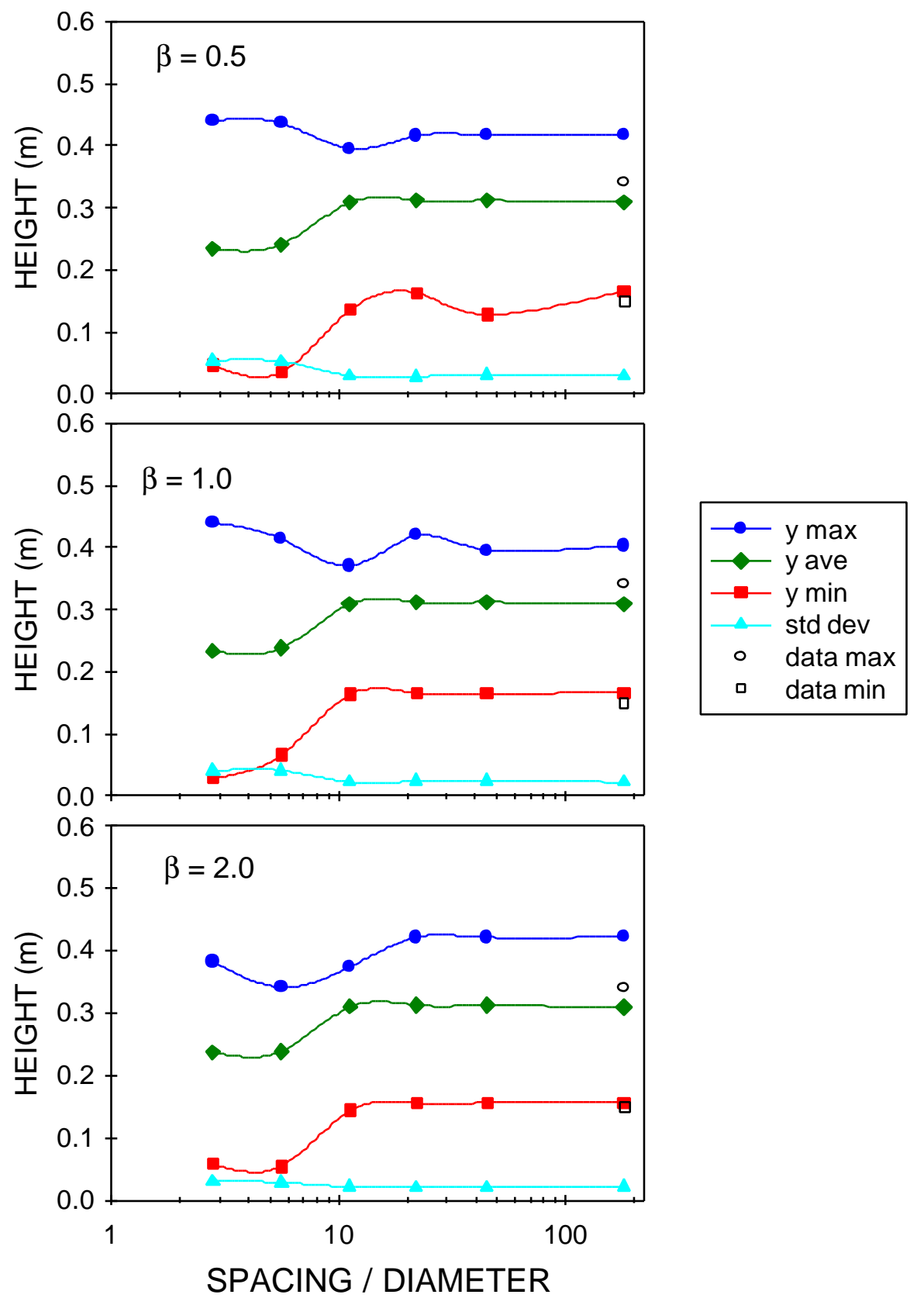

Figure 6.17: Variation in the $\mathrm{Y}$ direction as a function of initial interdroplet spacing and $\beta$. As beta decreases from a value of 1.0 the vertical $y$ spread increases. As beta increases from a value of 1.0, the vertical spread decreases. As $\beta$ decreases, so does the standard deviation. 

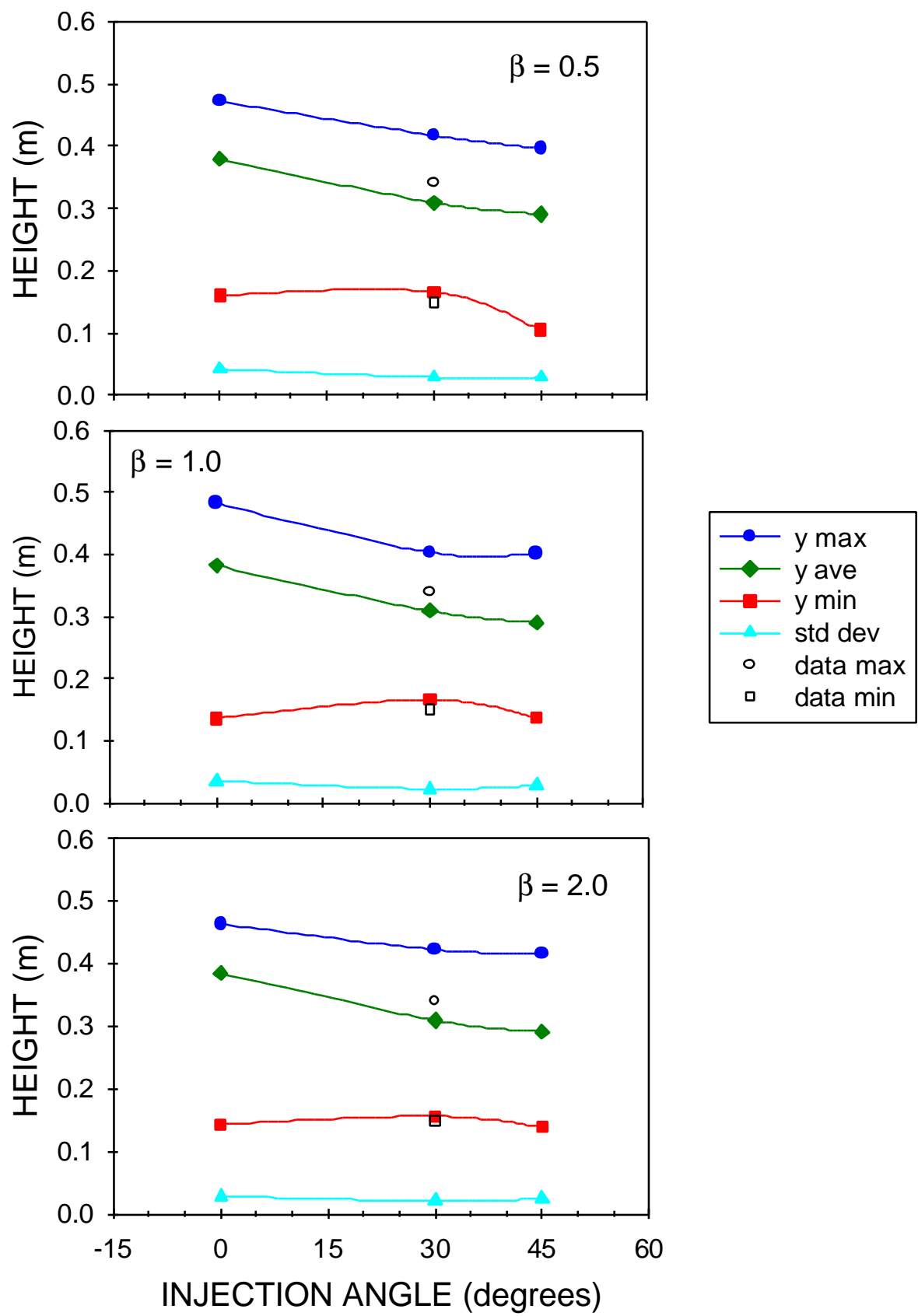

Figure 6.18: Variation in the $Y$ direction as a function of initial injection angle and $\beta$. As beta decreases from a value of 1.0 the vertical $y$ spread increases. As beta increases from a value of 1.0, the vertical spread decreases. 

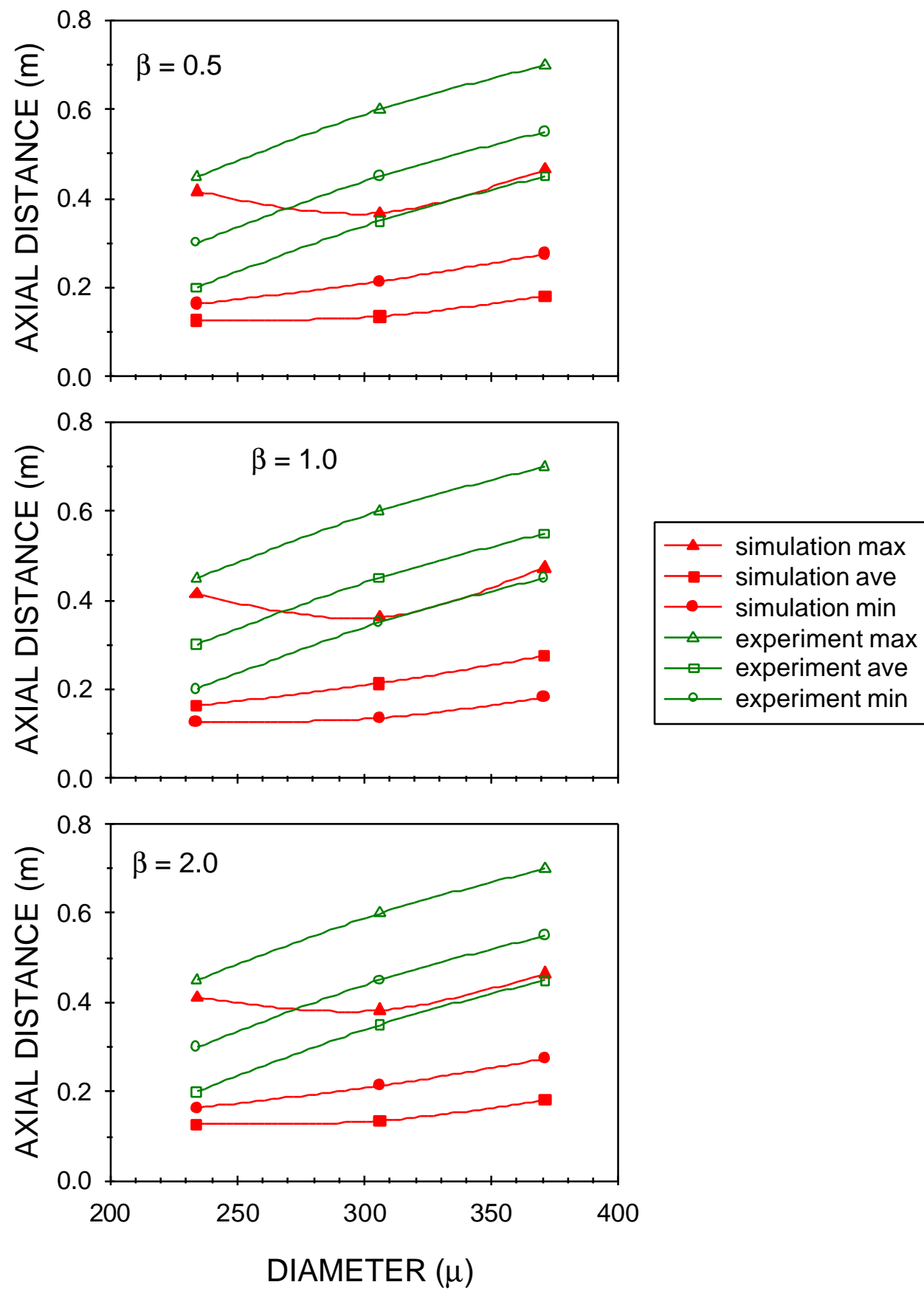

Figure 6.19: Variation in the axial direction as a function of initial diameter and $\beta$. Comparison of experimental measurements and simulated burning droplets. The closed symbols represent the predicted values. The experimental data are shown in open symbols. An ensenble size of 750 was used. No trend with beta variation was noted. 

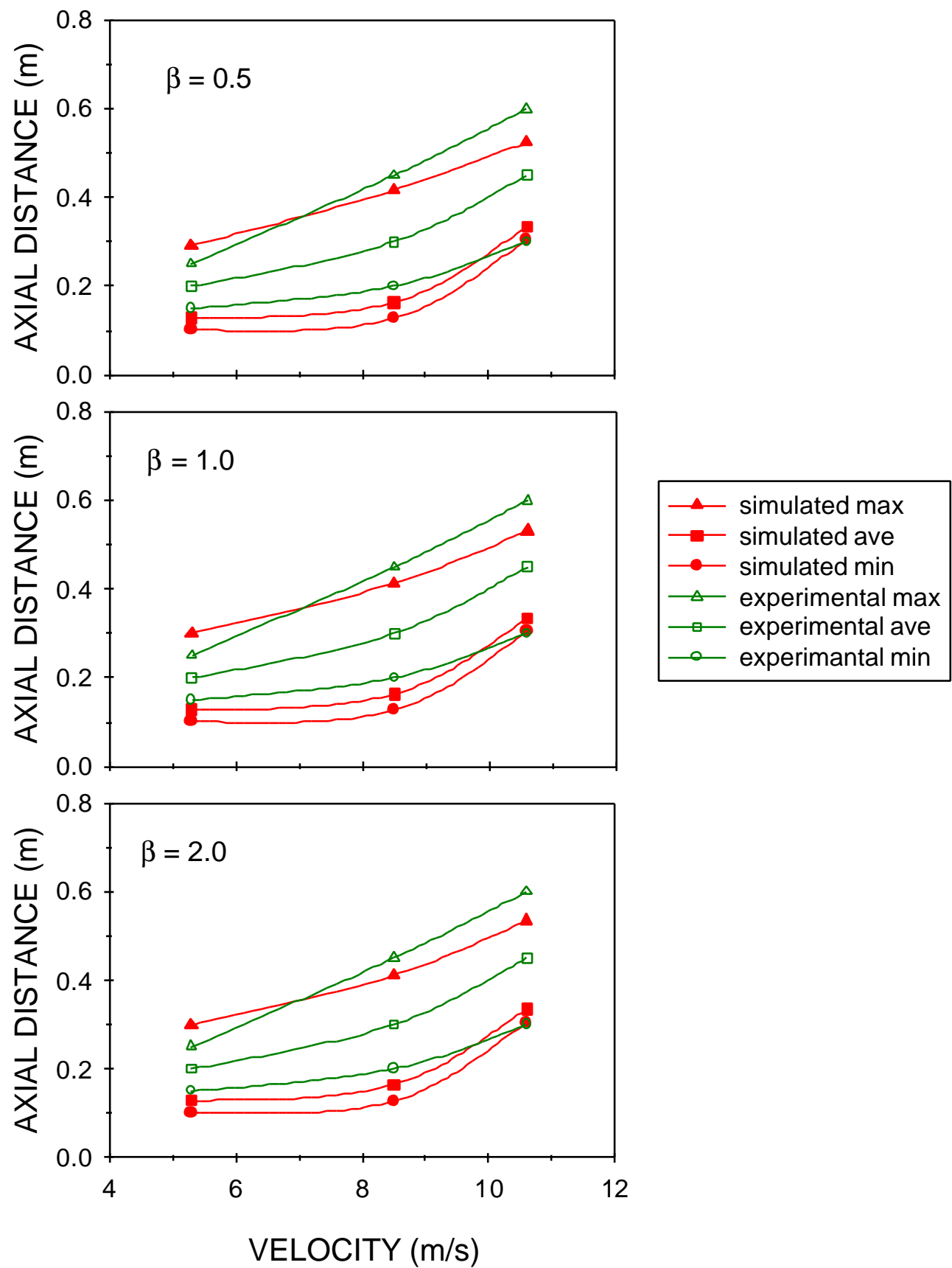

Figure 6.20: Variation in the axial direction as a function of initial velocity and $\beta$. Comparison of experimental measurements and simulated burning droplets. The closed symbols represent the predicted values. The experimental data are shown in open symbols. An ensemble size of 750 was used. No trend with beta variation was noted. 

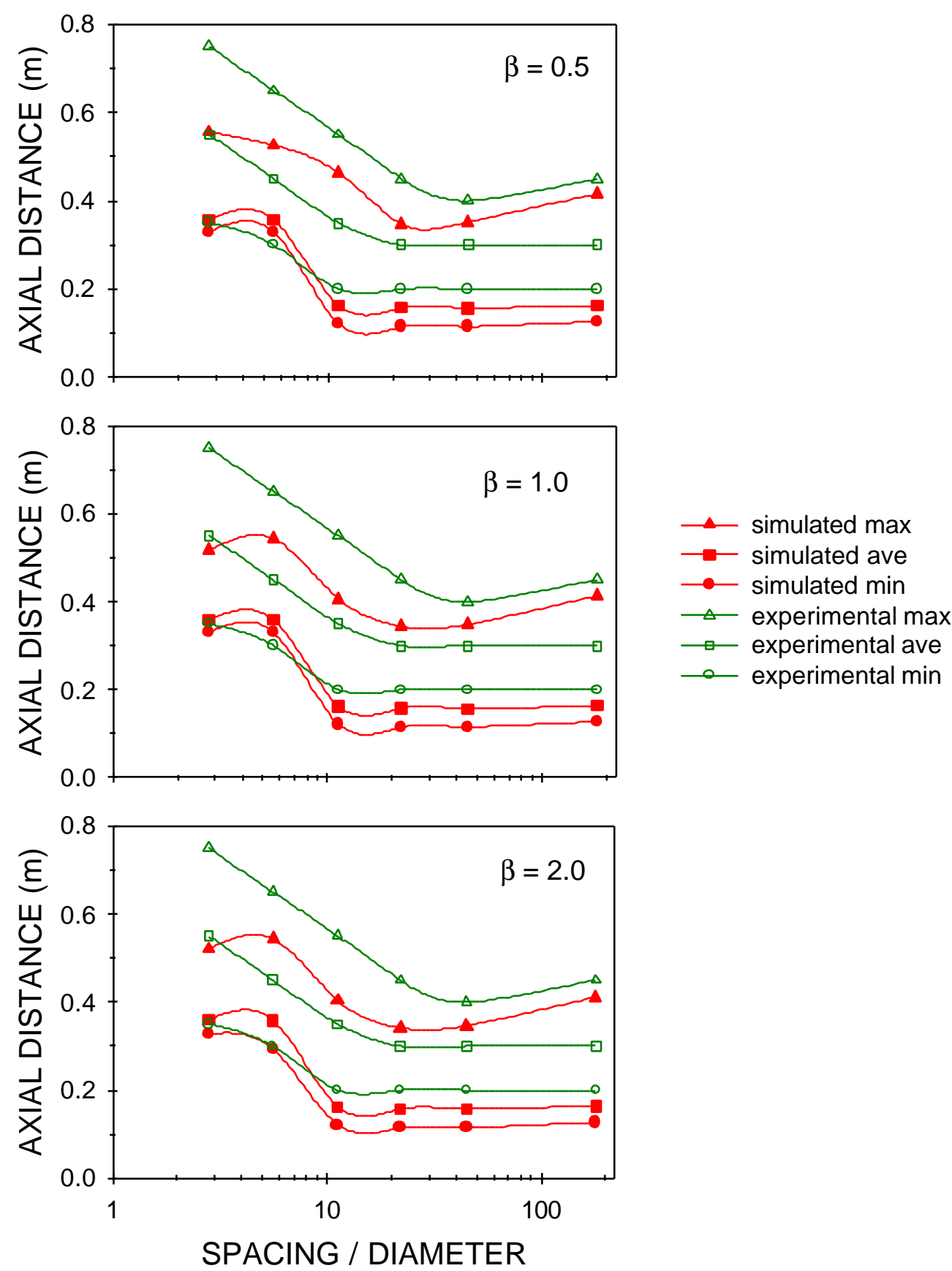

Figure 6.21: Variation in the axial direction as a function of initial droplet spacing and $\beta$. Comparison of experimental measurements and simulated burning droplets. The closed symbols represent the predicted values. The experimental data are shown in open symbols. An ensenble size of 750 was used. No trend with beta variation was noted. 

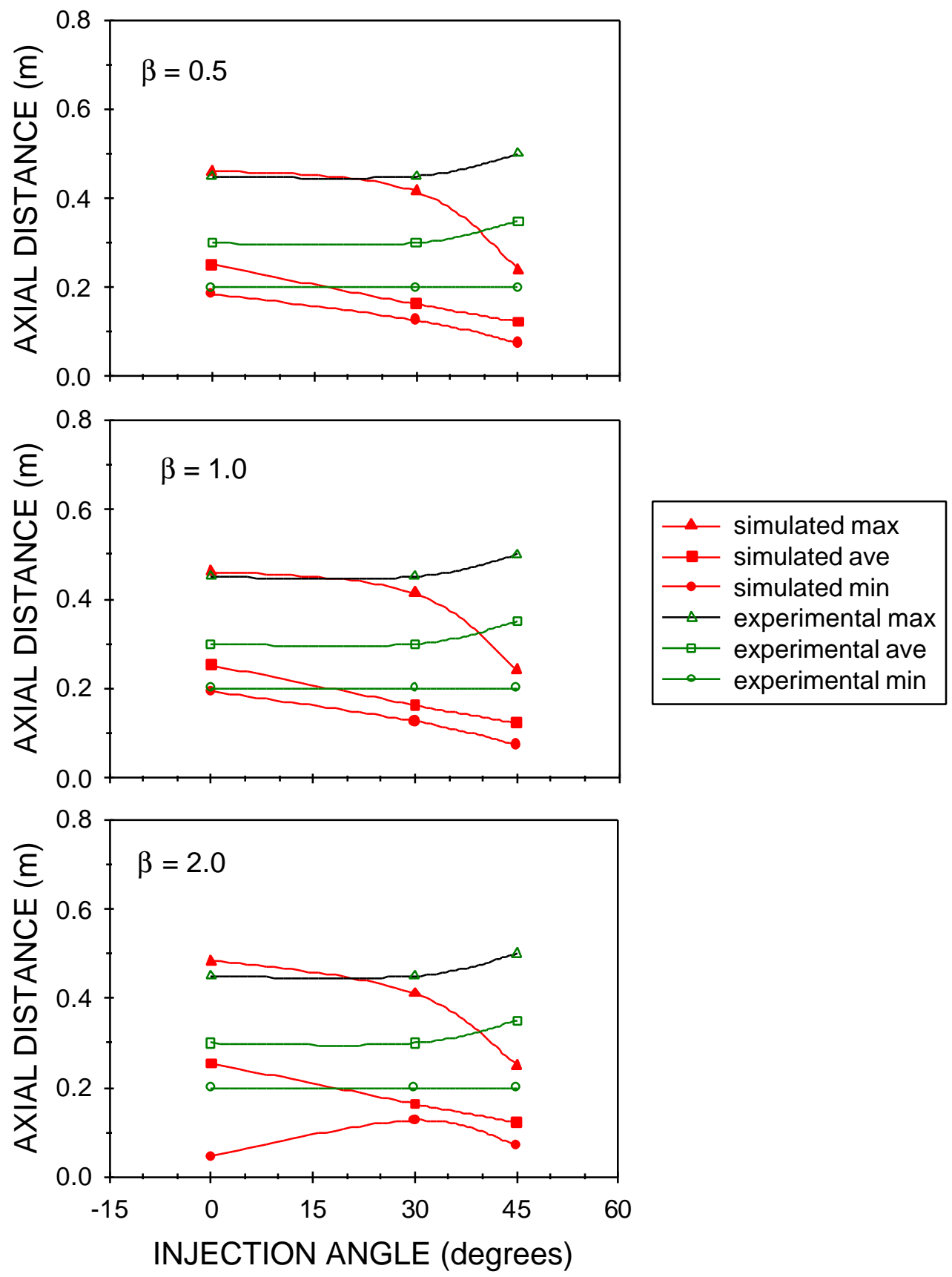

Figure 6.22: Variation in the axial direction as a function of initial injection angle and $\beta$. Comparison of experimental measurements and simulated burning droplets. The closed symbols represent the predicted values. The experimental data are shown in open symbols. An ensenble size of 750 was used. No trend with beta variation was noted. 


\section{Chapter 7}

\section{Conclusions and Future Work}

\subsection{Conclusions}

A One Dimensional Turbulence Model (ODT)-droplet trajectory model was developed to examine the performance and safety of a thermal treatment process for metal containing wastes. Results indicate that one can predict possible failure modes inherent in the incineration process due to rogue droplet penetration past the flame zone and subsequent failure to completely burn out.

The ODT model proper is able to reasonably predict the mean velocity and temperature profiles of a $100 \mathrm{~kW}$ combustor using as an initial condition the measured mean profiles (velocity and temperature) at a distance of $5 \mathrm{~cm}$ downstream of the burner.

The ODT two-phase flow model can accurately predict the spread of droplet burnout points due to the random variation of the velocity/temperature field (the high turbulence) without the cost of doing a 3-dimensional (DNS) calculation. Excellent agreement (using an ensemble size of 750) was found for predicting the spread due to variations in initial diameter. Good agreement was found for predicting the spread caused by 
variations in initial velocity and for the first two cases of droplet stream injection angle. (Those being the zero and thirty degree trials.) This was achieved without the computational burden of attempting a DNS calculation.

The 45 degree angle spread is underpredicted because type-C interactions were not considered in the ODT droplet model. Other important physics may also be missing from the model formulation.

The ODT two-phase flow model did not reproduce the spacing/diameter spread because no s/D effects were included inside the ODT droplet model and the point at which the droplet stream broke up into individual droplets was probably incorrect. Aside from a pure guess, there were no data available from the experiment to choose a droplet break up point other than the one which was presented in this paper.

\subsection{Future Work}

Future work should include the development and implementation of the type- $\mathrm{C}$ interactions. The theoretical groundwork for type- $\mathrm{C}$ interactions is mostly done. The implementation of type-C interactions may account for the error in the predicted values for the forty-five degree injection angle (Case 12) along with the error in the trend predicted of the axial spread 
decreasing as the angle increases. This error was attributed to the combination of the lack of type-C interactions and the possibility of other forces, such as the Saffmon lift force, causing the droplets to veer from the course predicted by the physics which are included in this model.

Hence an effort should be made to investigate other forces and their effect on the droplet trajectory. This could include thermophoresis.

The assumption of a spherical droplet is used often. However, during the initial part of the trajectory, the particle Re is such that the droplets are not likely to be spherical. Hence improvements in drag coefficients and droplet vaporization models should be investigated.

The failure of this model to predict a trend for the entire range of initial droplet spacing effects on the axial spread indicates that if this model is to be used to predict the droplet spacing variations, it must be modified to somehow include s/D effects. Because of the success the model had for the variations in diameter, velocity, and (to a lesser extent) injection angle a possible avenue of approach would be to design a different model to compute the initial conditions and the position at which the droplet stream breaks up and behaves as if it were a bunch of random, independent droplets. So rather than simply taking the point of stream break up to be the same for 
all six trials (Cases 1, 6, 7, 8, 9, 10) the new model would adjust this break up point to allow for variations in s/D. Intuitively the smaller the s/D the farther the stream should travel before breakup.

The ODT formulation proper used in this paper (which is constant density, a single velocity component, and between two parallel plates) has had a number of improvements done to it by Kerstein and co-workers. Formulations now exist which will provide variable density, three gas phase velocity components, and cylindrical geometrys. Implementation of any of these should prove an improvement to the present simulation.

As the ODT formulation stands, adding chemical reactions to the gas phase is straightforward. The major approximations made in the development of the droplet heating/vaporization/burning model were such that the author did not deem the introduction of chemistry, e.g. the depletion of oxygen in the fluid cell by the burning droplet, to be worth the trouble when the multi-component fuel was modeled as a single pure substance. If the model were to be redone with a better approximation of the phenomenon of multi-component droplet heating/vaporization, then also including some basic chemistry into the gas phase would be advantageous. 


\section{Appendix A}

\section{Source Code}

\section{SOURCE CODE FOR THE ODT - TWO-PHASE FLOW PROGRAM}

The main program (odtdriv) is listed first. Next comes the subprograms used for the two-phase flow portion of the program. This may be followed by the ODT proper code which was provided at the onset of this project. (See acknowledgements for details.) At this point only the code for the burning droplet submodel is included.

The second section of Appendix A lists the programs used to compute the initial conditions, of the 12 cases of droplets, used as input to the ODT two-phase flow program. 


\title{
A.1 Source code for ODT and droplet submodel
}

\author{
program odtdriv \\ C LAST MODIFIED BY JRS 04/28/00 @ 11:00 \\ C FOR EVAPORATING DROPS WITH PARAMETER SWITCH, multi drops \\ !! used to find droplet eddy interactions \\ C MODIFIED TO RUN WITH DP/DX NOT $=0.0$ \\ $\mathrm{C}$ Program to run the ensemble and time stepping of the iterative odt loop \\ implicit none \\ integer lout, lt, lmap, nts, nevents \\ integer nnsc, nnx, nnfld, nnflx, nnt, nnts \\ $\mathrm{C} \quad * * * * * * * * * * * * * * * * * * * * * * * *$ \\ C THE DROPLET TIME LINE WAS ADDED, NEVENTS CHANGED FROM 4 TO \\ 5 \\ C NNX = 4619 IS TOO BIG, 4431 good, 4333 bad \\ C MUST ALSO SET NNBIN TO NNX / 3 IN updfop.f AND mpdfop.f \\ $\mathrm{C} \quad * * * * * * * * * * * * * * * * * * * * * * * *$ \\ parameter $($ lout $=6$, nevents $=5$ ) \\ c for octane and flames use nnx $=1775$ \\ parameter $(\mathrm{nnsc}=2, \mathrm{nnx}=4431, \mathrm{nnfld}=19)$ \\ parameter $(\mathrm{nnflx}=3, \mathrm{nnt}=61, \mathrm{nnts}=8)$ \\ integer nx, nt, it, nens, nfld, nsc, nbin, nsam \\ integer ispec(20), minseg, $\mathrm{i}, \mathrm{j}$, iens, iskip \\ integer il, ke, indx(nnx), nesize(nnx) \\ integer neloc(nnx), neleft(nnx), neright(nnx), nopa \\ integer kuneu, ktneu \\ integer kounteddy \\ logical dtsreset, eddy \\ character*24 names(nnfld), tnames(nnts) \\ character* $(24 *$ nnfld +1$)$ wrkn \\ character*50 desc \\ real scal(nnsc,nnx), fld(nnx,nnfld,nnt), flux(nnx,nnflx,nnt) \\ real uvfl(nnx), tvfl(nnx), uuvfl(nnx) \\ real esize(nnx,nnt), emsd(nnx), edmid, edsize \\ real work(nnx*nnfld*nnt), wrk1(nnx), wrk2(nnx) \\ real rspec(30), a_eddy, p_a, refdens \\ real visc, tdiff, gbeta, dpdx \\ C DPDX IS DP/DX DIVIDED BY DENSITY (UNITS M/(S**2)) \\ C TDIFF IS THERMAL DIFFUSIVITY UNITS $\left(\mathrm{M}^{* * 2 / S}\right)$
}


C VISC IS KINEMATIC VISCOSITY UNITS (M**2/S)

real x1, xn, v1, vn, t1i, tni, t1f, tnf, t1, tn

real xsc, usc, vbulk, bulktemp

real scalinit(nnsc,nnx), rho(nnx), $x(n n x), d x(n n x)$, xdom, elmin

real time, dtm, dts, dta, t(nnt), tlast(nevents), tnext(nevents)

real tstat(nnt,nnts), accdts, accpa

real pa, pamax, etau, beta_eddy

real soonest

C VARIABLES FOR DROPLET MODEL:

$\mathrm{C}$ DELTA $\mathrm{V}=\mathrm{CHANGE}$ IN VELOCITY OF THE DROPLET

$\mathrm{C}$ DELTA $\mathrm{Y}=\mathrm{CHANGE}$ IN THE Y POSITION OF THE DROPLET

$\mathrm{C}$ DELTA $\mathrm{X}=\mathrm{CHANGE}$ IN THE $\mathrm{X}$ POSITION OF THE DROPLET

C D Y POSIT $=$ THE Y POSSITION OF THE DROPLET

C D Y PO I = INITIAL Y POSSITION OF THE DROPLET

C D X POS $=$ DROPLET POSITION IN THE X DIRECTION

C D X POS I $=$ INTIIAL DROPLET POSITION IN THE X DIRECTION

C D Y VELOC $=$ THE DROPLET V VELOCITY - VERTICLE

C D Y VEL I $=$ THE INITIAL DROPLET Y VELOCITY (USED IN ENSEMBLE)

C D U VEL $=$ DROPLET U VELOCITY - STREAMWISE

C D U VEL I = INITIAL DROPLET X VELOCITY

$\mathrm{C} \quad$ UPPLIMIT $=$ UPPER LIMIT FOR THE TIME INTEGRAL

$\mathrm{C} \quad$ DT DROP $=$ TIME STEP FOR THE DROP TIME LINE

$\mathrm{C}$ E LHS = EDDY LEFT HAND SIDE

$\mathrm{C}$ E RHS = EDDY RIGHT HAND SIDE

C OVRSHOOT $=$ DISTANCE OVER SHOOT WHEN DROP TRAVELS IN PSEUDO TIME

$\mathrm{C}$ PSEUDOY $=$ POSITON OF DROPLET AFTER INTEGRATION IN PSEUDO TIME

C D PARAM = THE D PARAMETER DEFINED FOR THE DROPLET MODEL

$\mathrm{C}$ D TAU $=$ D TIMES E TAU, THE DROPLET TAU

$\mathrm{C} \quad \mathrm{IR} \quad=$ RIGHT HAND NODE OF THE EDDY

$\mathrm{C}$ I D POS = INTEGER DROPLET POSITION (NODE) IN Y COORDINATE

$\mathrm{C}$ U BAR $=$ THE AVERAGE U VELOCITY OF THE EDDY

$\mathrm{C}$ U FLUID $=$ U VELOCITY OF THE FLUID ELEMENT WHICH HOLD THE DROPLET

C V END = PARTICLE Y VELOCITY AT THE END OF THE INTEGRATION

$\mathrm{C}$ U END = PARTICLE X VELOCITY AT THE END OF THE INTEGRATION

$\mathrm{C}$ DIA $=$ PARTICLE DIAMETER

$\mathrm{C}$ DIAI = INTIAL DROPLET DIAMETER

$\mathrm{C}$ TAU P $=$ THE DROPLET TIME CONSTANT

C TAU P I $=$ THE INITIAL DROPLET TIME CONSTANT

C BULK VEL $=$ BULK VELOCITY OF ALL THE FLUID

C DROP TIME $=$ DROPLET TIME WHICH IS DIFFERENT FROM DROPLET TIME LINE 


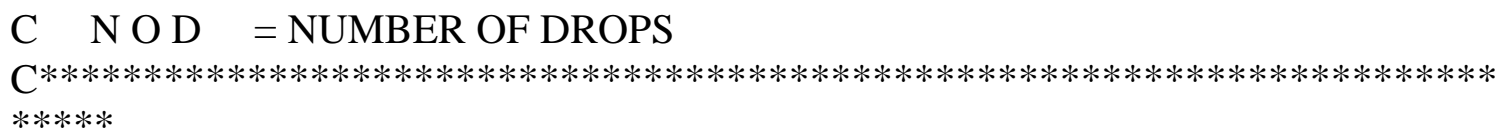

INTEGER NOD

PARAMETER $($ NOD $=15)$

REAL AIRNU, ALAMDA, BIRTHT(NOD), BLN, BMAST, BP1, BULKVEL

REAL CPFGAS

REAL DABZERO, DELTAT, DELTAX, DELTAY, DELTAV

REAL DIA(NOD), DIAI(NOD), DIANEW, DIGNIT(NOD), DPARAM, DPOSROOT

REAL DRMASS(NOD)

REAL DROPTIME(NOD), DTAU, DTDROP, DTDROPF, DTIMEI(NOD)

REAL DUVEL(NOD), DUVELI(NOD)

REAL DXPOS(NOD), DXPOSI(NOD)

REAL DYPOSIT(NOD), DYPOI(NOD), DYVELOC(NOD), DYVELI(NOD)

REAL ELHS, EPS, ERHS, GRAVAC

REAL HEATCAP, HEATCOM, HEATVAP, HEFF, HOLD

INTEGER ICOUNT, IDPOS, IG1, IG2

INTEGER IR, ISEED, INEW, ITT, J1, LDOUT, KDROP

INTEGER NODA

LOGICAL LALLIGN

LOGICAL LALLST, LDFINSH(NOD), LDROP, LDSTART(NOD), LDSTARTI(NOD)

LOGICAL LHEATUP(NOD), LITT, LLAMDA, LLONG, LMDIG(NOD)

INTEGER LMDOUT(NOD)

LOGICAL LMDROP(NOD), LMDROPI(NOD)

INTEGER LMDSOUT(NOD)

LOGICAL LTEST

CHARACTER *9 OUTFIL(NOD)

REAL OVRSHOOT, PEPS, PI, PSEUDOV, PSEUDOY, RHOGAS, RHOL

REAL SCHMIDT, STOICH, TAUP, TAUPI, TDARRAY (NOD, 10)

REAL TDROP(NOD), TDROPI(NOD), TEMPBP, TEMPZERO

REAL TFLAME(NOD), TINFIN

REAL TTHIRD, UBAR, UEND, UFLUID, UPPLIMIT, VEND, YOINFIN

OPEN (LOUT, FILE = 'screen.out')

call lunext(lt)

call lunext(lmap)

open (lt,file $=$ 'timelog')

open (lmap,file = 'ltmap')

C Read the specs in the input file 
$\mathrm{nsc}=\mathrm{nnsc}$

call rdkey(lout, nx, nt, nens, nnts, a_eddy, p_a, minseg,

1 ispec, rspec, refdens, nnfld, nfld, nts, names, tnames,

2 desc, iskip, kuneu, ktneu, beta_eddy)

C Memory check

call memck(lout, nt, nnt, nx, nnx, nfld, nnfld, nts, nnts)

C Set the problem-specific parameters

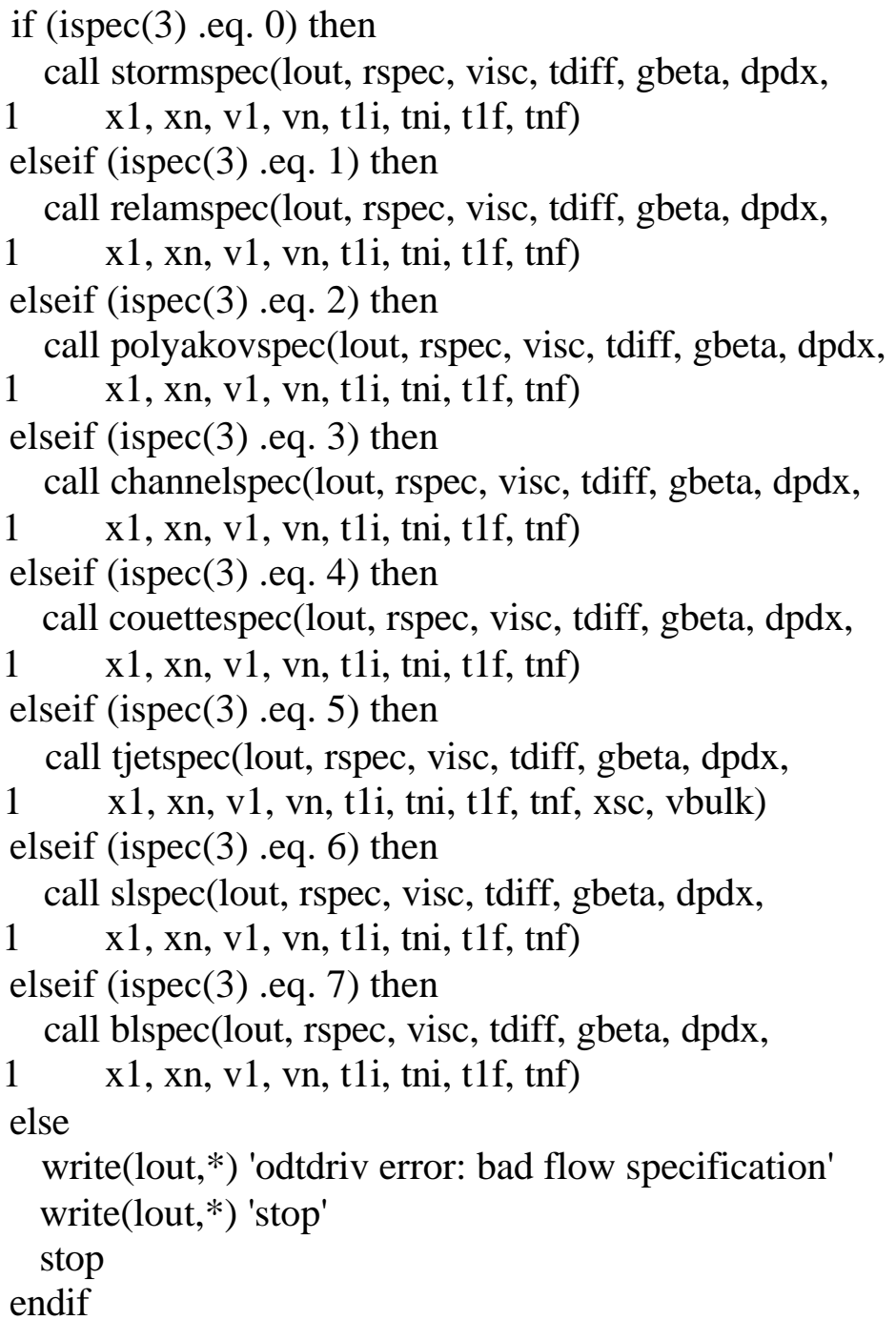

C Initialize the grid and specify the initial scalar fields 
call start(nnsc, nx, minseg,

$1 \mathrm{x} 1, \mathrm{xn}, \mathrm{v} 1, \mathrm{vn}, \operatorname{rspec}(16), \operatorname{rspec}(17), \mathrm{t} 1 \mathrm{i}$, tni, refdens,

2 xdom, $x, d x$, scalinit, rho, elmin)

C Specialized initial profiles

if (ispec(3) .eq. 5)

1 call tjetstart(lout, nnsc, nx, x, dx, xsc, vbulk, scalinit)

if (ispec(3) .eq. 6)

1 call slstart(lout, nnsc, nx, x, dx, v1, vn, scalinit)

C MODIFIED BY JRS FOR MULHOLLAND INITIAL CONDITIONS $* * * * * *$ IF (ISPEC(3) .EQ. 3) CALL INPVELOC (LOUT, NNSC, NX,

$\left.\mathrm{C}^{2} * * * * * * * * * * * * * * * * * * * * * *\right)$

C If desired, replace the initial scalar field with one that's read in

C from a file

if (ispec(6) .ge. 2)

1 call profrestart(lout, nnsc, nsc, nx, x1, xn, xdom,

$2 \quad \mathrm{x}, \mathrm{dx}, \operatorname{rspec}(16)$, rho, scalinit)

C Other initialization

call zfield(nnx, nnfld, nt, fld)

call zfield(nnx-1, nnflx, nt, flux)

call zorm(nnx, nt, esize)

call zorm(nnt, nts, tstat)

$\mathrm{C} \quad * * * * * * * * * * * * * * * * * * * * * * * * * * * * * * * * * * * * * * * * * * * * * * * * * * * * * * * * * * * * * * * * * *$

C INITIALIZE THE VARIABLES SPECIFIC TO THE DROPLET MODEL

CALL LUNEXT (LDOUT)

OPEN (LDOUT, FILE = 'jevap.out')

CALL LUNEXT (LMDSOUT (1))

OPEN (LMDSOUT(1), FILE = 'jshort1.out')

CALL DROPINIT(DX, NENS, NEVENTS, NOD, NNX, NX, ALAMDA, BIRTHT, 2 BULKVEL, DABZERO, DIAI, DIGNIT, DPARAM, DTIMEI, DYPOI, 3 DTDROPF, DUVELI, DXPOSI, DYVELI, EPS, GRAVAC, HEATCAP, 4 HEATCOM, HEATVAP, HOLD, LDOUT, LDROP, LDSTARTI, LLAMDA,

5 LLONG, LMDROPI, NODA, OUTFIL, PEPS, PI, RHOL,

6 STOICH, TDROPI, TEMPBP, TEMPZERO, TFLAME, YOINFIN) 
C1 ATY THIS POINT, THE SHORT OUTPUT IS NOT AVAILABLE !! 3/30/00 DO ICOUNT $=2$, NODA, 1

C IF (LLONG) THEN

CALL LUNEXT (LMDOUT(ICOUNT)) OPEN $($ LMDOUT $($ ICOUNT $)$, FILE $=$ OUTFIL $($ ICOUNT $))$

C END IF

CALL LUNEXT (LMDSOUT(ICOUNT)) OPEN $($ LMDSOUT $($ ICOUNT), FILE = 's' // OUTFIL $($ ICOUNT)

END DO

LMDOUT $(1)=$ LDOUT

C CALL LUNEXT (ISEED)

C OPEN (ISEED, FILE $=$ 'seed.out')

C

$\mathrm{C} \quad * * * * * * * * * * * * * * * * * * * * * * * * * * * * * * * * * * * * * * * * * * * * * * * * * * * * * * * * * * * * * * * * * *$

$\mathrm{C}$ Begin the ensemble averaging loop

do iens $=1$, nens

C Initialize the scalars and set the bulk temperature

call scalstart(nx, nnsc, nsc, scalinit, scal)

call temprefset(bulktemp, nnsc, nx, scal)

C Initialize the boundary conditions and time stepping

$\mathrm{t} 1=\mathrm{t} 1 \mathrm{i}$

$\operatorname{tn}=\operatorname{tni}$

call dtmol(visc, tdiff, dx, dtm, rspec(22))

call dtstir(lout, nnsc, nx, minseg, scal, x, dx, a_eddy,

1 elmin, visc, $\left.x d o m, p \_a, d t m, d t s\right)$

call tset(nt, $\operatorname{rspec}(20), \operatorname{rspec}(21), \mathrm{dta}, \mathrm{t})$

time $=0$.

tlast $(1)=0$.

tlast $(2)=0$.

tlast $(3)=0$.

tlast $(4)=0$.

$\operatorname{tnext}(1)=\mathrm{dtm}$

$\operatorname{tnext}(2)=\mathrm{dts}$

$\operatorname{tnext}(3)=\operatorname{rspec}(20)$

$\operatorname{tnext}(4)=\operatorname{rspec}(18)$

it $=0$ 
$\mathrm{C} \quad * * * * * * * * * * * * * * * * * * * * * * * * * * * * * * * * * * * * * * * * * * * * * * * * * * * * * * * * * * * * * * * * * *$

C JRS'S ADDITION FOR DROPLET SCALAR RESET

CALL DROPSTART (BIRTHT, DIAI, DTDROPF, DTIMEI, DTM, DUVELI, 2 DXPOSI, DYPOI, DYVELI, HOLD, IENS, LDOUT, LDSTARTI, 3 LLONG, LMDOUT, LMDROPI, NEVENTS, NOD, NODA, PI, RHOL, 4 TDROPI, DIA, DRMASS, DROPTIME, DTDROP, DUVEL, DXPOS, 5 DYPOSIT, DYVELOC, LALLIGN, LALLST, LDFINSH, LDROP, LDSTART, 6 LHEATUP, LMDIG, LMDROP, TDARRAY, TDROP, TLAST, TNEXT)

!! get trajectories stuff

C CLOSE (LDOUT)

C CLOSE (LMDSOUT(1))

C CALL GETRAJ (IENS, LDOUT, LMDSOUT(1))

C CALL RNUGET (IG1,IG2)

C WRITE (ISEED, 137 ) IENS, IG1, IG2

C 137 FORMAT (1X, I6, 1X, I20, 1X, I20)

$\mathrm{C} \quad * * * * * * * * * * * * * * * * * * * * * * * * * * * * * * * * * * * * * * * * * * * * * * * * * * * * * * * * * * * * * * * * * * * *$

C Time-stepping loop

do while (it .le. nt)

C Molecular loop

dtsreset $=$.false.

do while $(\operatorname{tnext}(1)$.eq. soonest(nevents, tnext))

C On the first pass, update the stirring time step

if (.not. dtsreset) then call dtstir(lout, nnsc, nx, minseg, scal, x, dx, a_eddy,

1 elmin, visc, xdom, p_a, dtm, dts) dtsreset $=$.true. endif

C Solve the diffusion equation

$\mathrm{C} \quad * * * * * * * * * * * * * * * * * * * * * * * * * * * * * * * * * * * * * * * * *$

C MODIFIED BY JRS SO THAT IN CHANNEL FLOW (MULHOLLAND DATA)

C TEMPERATURE ALSO GOES THROUGH DIFFUSION

c if (ispec(3) .ge. 3) then IF (ISPEC(3) .GT. 3) THEN 


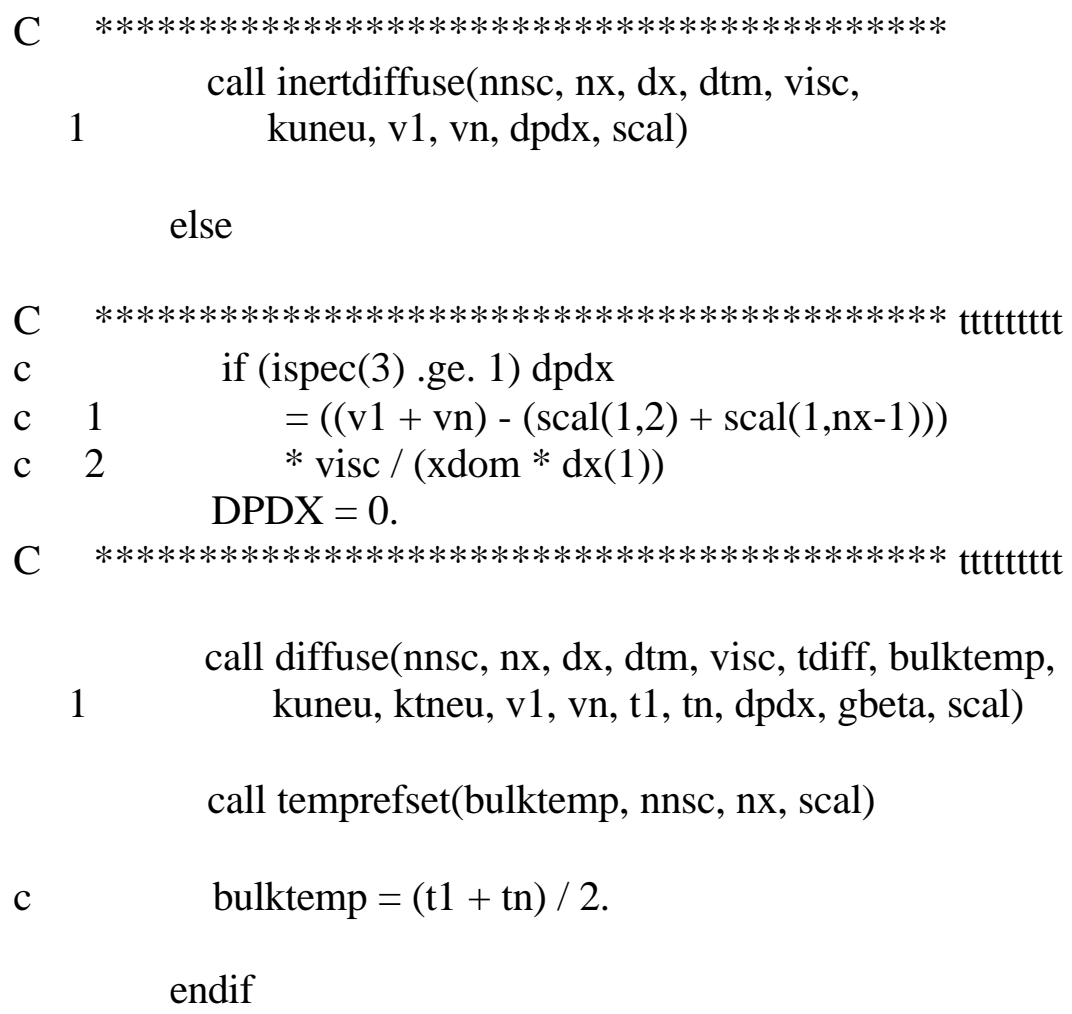

C Update the timing and the time itself

$$
\begin{aligned}
& \operatorname{tlast}(1)=\operatorname{tnext}(1) \\
& \operatorname{tnext}(1)=\operatorname{tnext}(1)+\mathrm{dtm} \\
& \text { time }=\text { time }+\mathrm{dtm} \\
& \text { enddo !End the molecular loop }
\end{aligned}
$$

C Stirring loop

do while (tnext(2) .eq. soonest(nevents, tnext))

C Select an eddy

call scottselodt(lout, visc, dts, nnsc, nx, scal, dx,

1 a_eddy, beta_eddy, minseg, elmin, x1, xn, xdom,

$2 \operatorname{rspec}(24), \operatorname{rspec}(25), i l, k e, \operatorname{tnext}(2)$, pamax,

3 eddy, edmid, edsize, pa, etau,IR)

C Update the eddy-specific statistics

if ( eddy ) then 
C Implement the eddy using Vebjorn's routines call tripmap(nx, il, ke, indx)

$\mathrm{C} \quad * * * * * * * * * * * * * * * * * * * * * * * * * * * * * * * * * * * * * * * * * * * * * * * * * * * * * * * * * * * * * * * * *$

C JRS'S ADDITION FOR DROPLET MODEL -- TYPE I INTERACTIONS

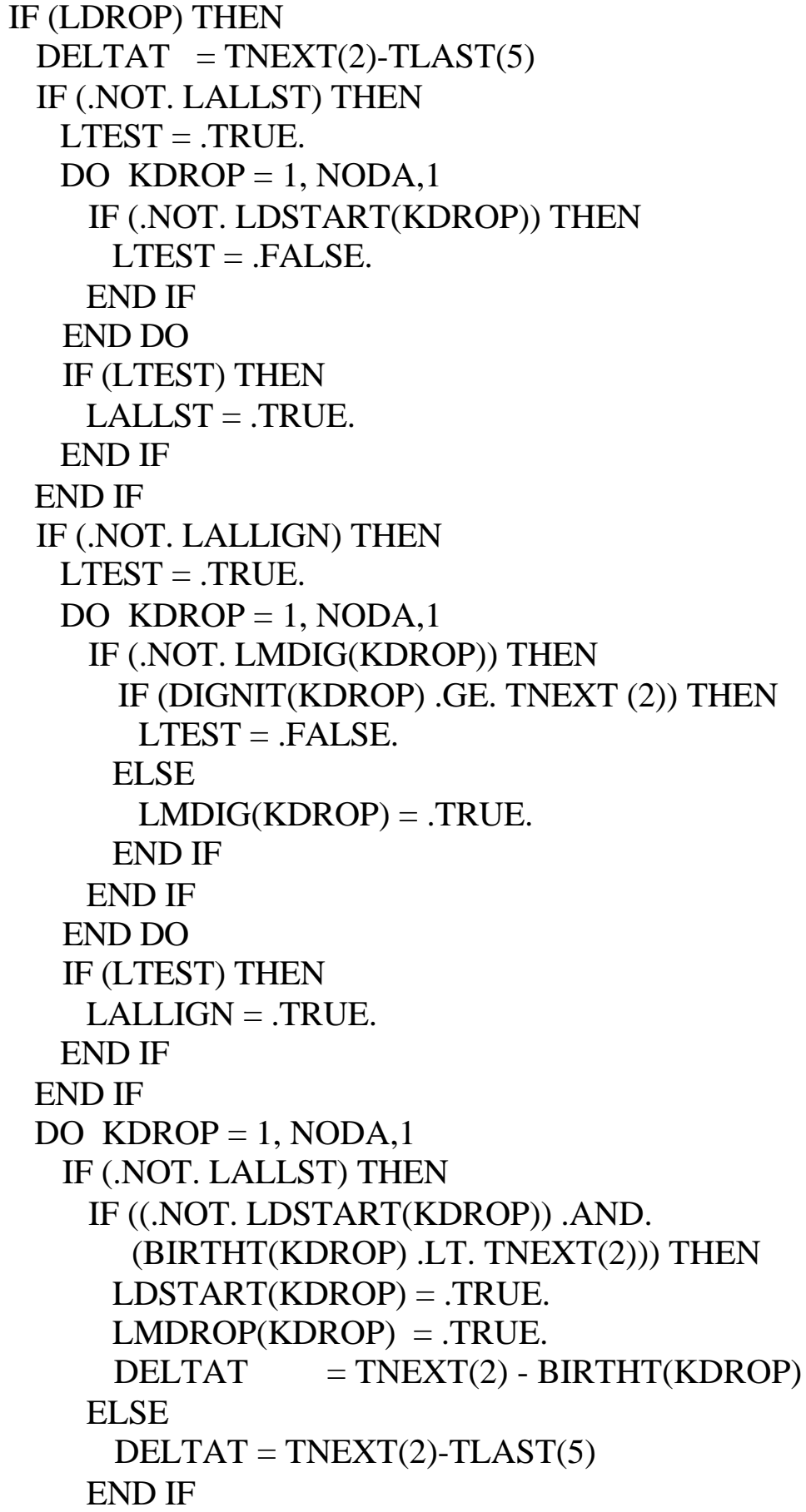


END IF

IF (LMDROP (KDROP)) THEN

$\mathrm{IDPOS}=(\mathrm{DYPOSIT}(\mathrm{KDROP})-\mathrm{X} 1) / \mathrm{DX}(1)+1.5$
UFLUID $=\operatorname{SCAL}(1, \mathrm{IDPOS})$

TINFIN $=$ SCAL(2,IDPOS)

IF (LALLIGN) THEN

CALL EFLAMET (DIA(KDROP), HEATCOM, KDROP,

2

3

$\mathrm{C}$ $\mathrm{C}$
LMDOUT(KDROP), .TRUE., LLONG, LOUT, RHOL, STOICH, TDROP(KDROP), TFLAME(KDROP), TINFIN, YOINFIN, AIRNU, CPFGAS, HEFF, RHOGAS, TAUP, TTHIRD)

ELSE

CALL EFLAMET (DIA(KDROP), HEATCOM, KDROP, LMDOUT(KDROP), LMDIG(KDROP), LLONG, LOUT, RHOL, STOICH, TDROP(KDROP), TFLAME(KDROP), TINFIN, YOINFIN, AIRNU, CPFGAS, HEFF, RHOGAS, TAUP, TTHIRD) END IF

UPPLIMIT = DPOSROOT(AIRNU, BULKVEL, DELTAT, DIA(KDROP), DUVEL(KDROP), LOUT, TAUP, UFLUID)

UPDATE THE POSITION \& VELOCITY OF THE PARTICLE ERE FIXING OUTPUT !!!!

IF (LMDIG(KDROP)) THEN

CALL DUPDATE (AIRNU, ALAMDA, CPFGAS, DABZERO, DIA(KDROP), DRMASS(KDROP), UPPLIMIT, GRAVAC, HEATVAP, HEATCAP, HEATCOM, HEFF, KDROP, LMDOUT(KDROP), LHEATUP(KDROP), LLAMDA, LOUT, NOD, PEPS, PI, RHOGAS, RHOL, STOICH, DUVEL(KDROP), DYVELOC(KDROP), TAUP, TDARRAY, TDROP(KDROP), TEMPZERO, TFLAME(KDROP), TINFIN, TTHIRD, UFLUID, YOINFIN, DELTAX,DELTAY,DIANEW,UEND, VEND) ELSE

CALL DUPDATE (AIRNU, ALAMDA, CPFGAS, DABZERO, DIA(KDROP), DRMASS(KDROP), UPPLIMIT, GRAVAC, HEATVAP, HEATCAP, HEATCOM, HEFF, KDROP, LMDOUT(KDROP), LHEATUP(KDROP),.FALSE., LOUT, NOD, PEPS, PI, RHOGAS, RHOL, STOICH, DUVEL(KDROP), 
DYVELOC(KDROP), TAUP,

TDARRAY, TDROP(KDROP), TEMPZERO, TFLAME(KDROP), TINFIN, TTHIRD, UFLUID, YOINFIN, DELTAX,DELTAY,DIANEW,UEND, VEND) END IF

DYPOSIT $($ KDROP $)=$ DYPOSIT $($ KDROP $)+$ DELTAY DXPOS $($ KDROP $)=$ DXPOS $($ KDROP $)+$ DELTAX DUVEL $($ KDROP $)=$ UEND DYVELOC $($ KDROP $)=$ VEND $\mathrm{DIA}(\mathrm{KDROP}) \quad=$ DIANEW DROPTIME $($ KDROP $)=$ DROPTIME $($ KDROP $)+$ UPPLIMIT

IF ((DIA(KDROP) .EQ. 0.0) .OR. (DYPOSIT(KDROP) .GE. XN) .OR. (DYPOSIT(KDROP) .LE. X1) ) THEN *******************************

FOR OUTPUTTING DROPLET POSITION \& TIME

CALL DROPOUT(DROPTIME(KDROP), DUVEL(KDROP), DYVELOC(KDROP), DXPOS(KDROP), DYPOSIT(KDROP), LMDOUT(KDROP), TNEXT(2))

CALL DROPOUT(DROPTIME(KDROP), DUVEL(KDROP), DYVELOC(KDROP), DXPOS(KDROP), DYPOSIT(KDROP), LMDSOUT(KDROP), TNEXT(2))

$* * * * * * * * * * * * * * * * * * * * * * * * * *$

IF (DIA(KDROP) .EQ. 0.) THEN

WRITE (LMDOUT(KDROP), 8)

FORMAT (' DROPLET EVAPORATED')

ELSE

WRITE (LMDOUT(KDROP),10)

FORMAT ( ' DROP HIT THE WALL')

WRITE (LMDSOUT(KDROP), 10)

END IF

LDFINSH $($ KDROP $)=$.TRUE.

LMDROP $($ KDROP $)=$. FALSE.

END IF

END IF

END DO

LTEST $=$.TRUE.

DO KDROP = 1, NODA, 1

IF ( .NOT. LDFINSH(KDROP)) THEN

LTEST $=$.FALSE. 


\section{END IF}

END DO

IF (LTEST) THEN

LDROP $=$. FALSE.

$\mathrm{TNEXT}(5)=\operatorname{TLAST}(5)+\mathrm{T}(\mathrm{NT})+.1$

ELSE

TLAST $(5)=$ TNEXT $(2)$

TNEXT $(5)=$ TLAST $(5)+$ DTDROP

END IF

UBAR $=0$.

DO J1 = IL,IR

$\mathrm{UBAR}=\mathrm{UBAR}+\mathrm{SCAL}(1, \mathrm{~J} 1)$

END DO

$\mathrm{UBAR}=\mathrm{UBAR} /(\mathrm{IR}-\mathrm{IL}+1)$

C NOTE, IF EVER USE A NON-UNIFORM GRID, MUST CHANGE THIS

ELHS $=(\mathrm{IL}-1.5) * \mathrm{DX}(1)$

ERHS $=(I R-0.5) * \mathrm{DX}(1)$

DO $\mathrm{KDROP}=1$, NODA, 1

IF (LMDROP (KDROP)) THEN

IF ((DYPOSIT(KDROP) .LE. ERHS) .AND.

2 (DYPOSIT(KDROP).GE. ELHS) ) THEN

C

PARTICLE IS INSIDE THE EDDY

C DTAU SHOULD NOT BE MULTIPLIED BY BULKVEL/DUVEL !

DTAU $=$ DPARAM $*$ ETAU

IDPOS $=(\mathrm{DYPOSIT}(\mathrm{KDROP})-\mathrm{X} 1) / \mathrm{DX}(1)+1.5$

UFLUID $=$ SCAL $(1$, IDPOS $)$

LITT $=$.TRUE.

$\mathrm{ITT}=1$

C CHECK INDEX () FOR FIRST \& LAST INDEX VALUES - SHOULD BE 1 \& NX.

C SINCE THE WAY INDX IS STORED IS DETERMINED BY THE AUTHOR OF THE MAIN

C PROGRAM THIS HAS TO BE REVISITED EACH TIME THE DROPLET MODEL IS PUT

C INTO A NEW PROGRAM.

DO WHILE (ITT .LE. (IR-IL+1) .AND. LITT)

IF (INDX(ITT) .EQ. IDPOS) THEN

INEW $=$ ITT

LITT $=$.FALSE.

END IF

$\mathrm{ITT}=\mathrm{ITT}+1$

END DO

$\mathrm{C} \quad * * * * * * * * * * * * * * * * * * * * *$

C TEMP DEBUG TERM 


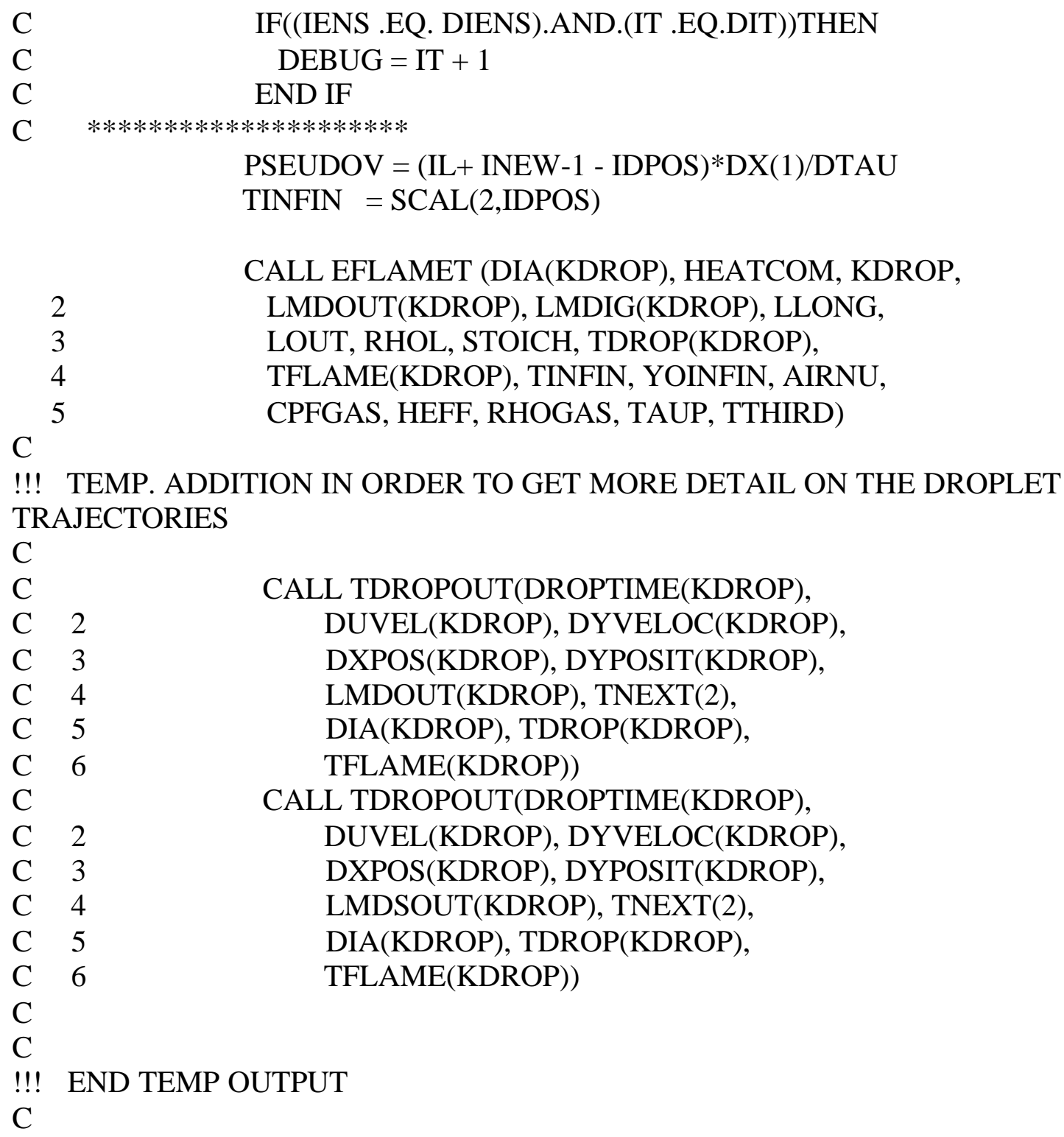

CALL DEUPDAT (AIRNU, DIA(KDROP), DTAU, ELHS, EPS, ERHS, IT, LMDOUT(KDROP), LOUT, DYPOSIT(KDROP), DUVEL(KDROP), DYVELOC(KDROP), TAUP, UBAR, UFLUID, PSEUDOV, DELTAY, DELTAV)

$\mathrm{C}$ INSTANTANEOUS ADJUSTMENT TO THE POSITION \&

C VELOCITY HERE. NOTE THIS EDDY - DROPLET

$\mathrm{C}$ INTERACTION DOES NOT EFFECT THE X VELOCITY OR POS. DYPOSIT $($ KDROP $)=$ DYPOSIT $($ KDROP $)+$ DELTAY DYVELOC $($ KDROP $)=$ DYVELOC $($ KDROP $)+$ DELTAV 
!!! TEMP. ADDITION IN ORDER TO GET MORE DETAIL ON THE DROPLET TRAJECTORIES

$\mathrm{C}$

C

C 2

C 3

C 4

C 5

C 6

C

C 2

C 3

C 4

C 5

C 6

$\mathrm{C}$

C 9137

C 2

CALL TDROPOUT(DROPTIME(KDROP), DUVEL(KDROP), DYVELOC(KDROP), DXPOS(KDROP), DYPOSIT(KDROP), LMDOUT(KDROP), TNEXT(2), DIA(KDROP), TDROP(KDROP), TFLAME(KDROP))

CALL TDROPOUT(DROPTIME(KDROP), DUVEL(KDROP), DYVELOC(KDROP), DXPOS(KDROP), DYPOSIT(KDROP), LMDSOUT(KDROP), TNEXT(2), DIA(KDROP), TDROP(KDROP), TFLAME(KDROP)) WRITE (LMDOUT(KDROP),9137) DELTAV, DELTAY FORMAT (2X, 'DELTAV FROM EDDY = ', E12.6, ' DELTAY = ', E12.6)

C

C

!!! END TEMP OUTPUT

IF ((DYPOSIT(KDROP) .GE. XN) .OR.

3

$\mathrm{C}$ 2

3

4

2

3

4

C
(DYPOSIT(KDROP) .LE. X1) ) THEN

FOR OUTPUTTING DROPLET POSITION \& TIME

CALL DROPOUT (DROPTIME(KDROP), DUVEL(KDROP),DYVELOC(KDROP), DXPOS(KDROP), DYPOSIT(KDROP), LMDOUT(KDROP), TNEXT(2))

CALL DROPOUT (DROPTIME(KDROP), DUVEL(KDROP),DYVELOC(KDROP), DXPOS(KDROP), DYPOSIT(KDROP), LMDSOUT(KDROP), TNEXT(2)) DROP HIT THE WALL WRITE (LMDOUT(KDROP),10)

WRITE (LMDSOUT(KDROP),10)

LDFINSH $($ KDROP $)=$.TRUE .

LMDROP $($ KDROP $)=$. FALSE .

END IF

END IF

END IF

END DO

LTEST $=$. TRUE. 


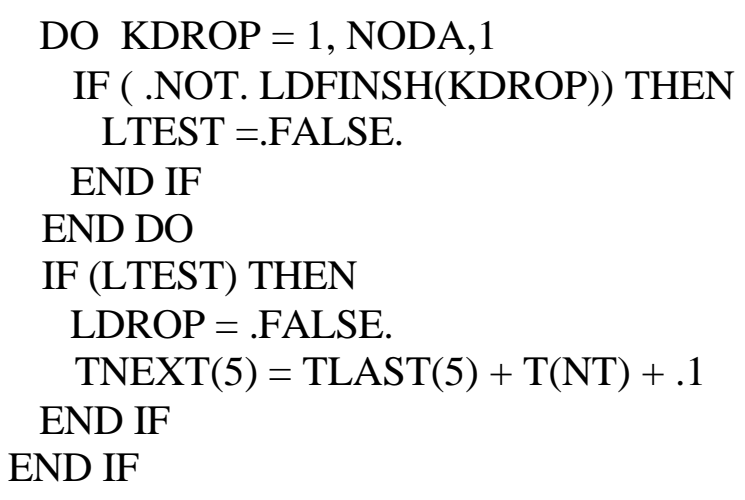

C2345678901234567892123456789312345678941234567895123456789612345678971

$\mathrm{C} \quad * * * * * * * * * * * * * * * * * * * * * * * * * * * * * * * * * * * * * * * * * * * * * * * * * * * * * * * * * * * * * * * * * * *$

call stirevent(nnsc, nx, nsc, il, ke, indx, wrk1,

1 scal)

C Accumulate eddy-based statistics

call eddyhist(il, ke, nnsc, nnx, nx, dx, dts,

1 pa, indx, scal, neloc, nesize, neleft, neright,

2 emsd, kounteddy, accdts, accpa, nopa,

3 uvfl, tvfl, uuvfl)

C Write out eddy map statistics

if (ispec(10) .eq. 1 .and. iens .eq. 1) then

write (lmap,103) tnext(2), edmid, edsize / 2.

103 format(3(1pe12.4))

endif

endif

C Update the timing

$$
\begin{aligned}
& \operatorname{tlast}(2)=\operatorname{tnext}(2) \\
& \operatorname{tnext}(2)=\operatorname{tnext}(2)+d t s
\end{aligned}
$$

enddo ! end of the stirring loop

C2345678901234567892123456789312345678941234567895123456789612345678971

2

$\mathrm{C} \quad * * * * * * * * * * * * * * * * * * * * * * * * * * * * * * * * * * * * * * * * * * * * * * * * * * * * * * * * * * * * * * * * * * *$ 
C JRS'S ADDITION OF A DROPLET TIME LINE

DO WHILE (TNEXT(5) .EQ. SOONEST (NEVENTS,TNEXT))

DELTAT $=$ TNEXT(5)-TLAST $(5)$

IF (.NOT. LALLST) THEN

LTEST $=$.TRUE .

DO KDROP = 1, NODA, 1

IF (.NOT. LDSTART(KDROP)) THEN

LTEST $=$.FALSE.

END IF

END DO

IF (LTEST) THEN

LALLST $=$.TRUE .

END IF

END IF

DO KDROP $=1$, NODA, 1

IF (.NOT. LALLST) THEN

IF ((.NOT. LDSTART(KDROP)) .AND. (BIRTHT(KDROP)

.LT. TNEXT(5))) THEN

LDSTART $($ KDROP $)=$.TRUE .

LMDROP $($ KDROP $)=$.TRUE.

DELTAT $\quad=$ TNEXT(5) - BIRTHT $($ KDROP $)$

ELSE

DELTAT $=$ TNEXT $(5)-T L A S T(5)$

END IF

END IF

IF (LMDROP (KDROP)) THEN

IF (.NOT. LMDIG(KDROP)) THEN

IF (DIGNIT(KDROP) .LT. TNEXT (5)) THEN

LMDIG $($ KDROP $)=$. TRUE .

END IF

END IF

IDPOS $=($ DYPOSIT $($ KDROP $)-\mathrm{X} 1) / \mathrm{DX}(1)+1.5$

UFLUID $=$ SCAL $(1$, IDPOS $)$

TINFIN $=\operatorname{SCAL}(2, \mathrm{IDPOS})$

CALL EFLAMET (DIA(KDROP), HEATCOM, KDROP, LMDOUT(KDROP), LMDIG(KDROP), LLONG, LOUT, RHOL, STOICH, TDROP(KDROP), TFLAME(KDROP), TINFIN, YOINFIN, AIRNU, CPFGAS, HEFF, RHOGAS, TAUP, TTHIRD)

UPPLIMIT = DPOSROOT(AIRNU, BULKVEL, DELTAT, DIA(KDROP), DUVEL(KDROP),

LOUT, TAUP, UFLUID) 


$\begin{array}{cc}\text { C } & \text { THE FIRST TIME THIS IS EXECUTED UPPLIMIT }=0 . \\ \text { C } & \text { HENCEFORTH UPPLIMIT IS ALWAYS DTDROP - NOT TRUE WITH } \\ \text { MULTIPLE DROPS } & \text { IF (LMDIG(KDROP)) THEN } \\ & \text { CALL DUPDATE (AIRNU, ALAMDA, CPFGAS, } \\ 2 & \text { DABZERO, DIA(KDROP), DRMASS(KDROP), } \\ 3 & \text { UPPLIMIT, GRAVAC, HEATVAP, HEATCAP, } \\ 4 & \text { HEATCOM, HEFF, KDROP, LMDOUT(KDROP), } \\ 5 & \text { LHEATUP(KDROP), LLAMDA, LOUT, NOD, PEPS, } \\ 6 & \text { PI, RHOGAS, RHOL, STOICH, DUVEL(KDROP), } \\ 7 & \text { DYVELOC(KDROP), TAUP, } \\ 8 & \text { TDARRAY, TDROP(KDROP), TEMPZERO, } \\ 9 & \text { TFLAME(KDROP), TINFIN, TTHIRD, UFLUID, } \\ 1 & \text { YOINFIN, DELTAX,DELTAY,DIANEW,UEND, VEND) } \\ & \\ & \text { ELSE } \\ 2 & \text { CALL DUPDATE (AIRNU, ALAMDA, CPFGAS, } \\ 3 & \text { DABZERO, DIA(KDROP), DRMASS(KDROP), } \\ 4 & \text { UPPLIMIT, GRAVAC, HEATVAP, HEATCAP, } \\ 5 & \text { HEATCOM, HEFF, KDROP, LMDOUT(KDROP), } \\ 6 & \text { LHEATUP(KDROP),.FALSE., LOUT, NOD, PEPS, } \\ 7 & \text { PI, RHOGAS, RHOL, STOICH, DUVEL(KDROP), } \\ 8 & \text { DYVELOC(KDROP), TAUP, } \\ 9 & \text { TDARRAY, TDROP(KDROP), TEMPZERO, } \\ 1 & \text { TFLAME(KDROP), TINFIN, TTHIRD, UFLUID, } \\ & \text { YOINFIN, DELTAX,DELTAY,DIANEW,UEND,VND) }\end{array}$

DYPOSIT $($ KDROP $)=$ DYPOSIT $($ KDROP $)+$ DELTAY

DXPOS $($ KDROP $)=$ DXPOS $($ KDROP $)+$ DELTAX

DUVEL $($ KDROP $)=$ UEND

DYVELOC $($ KDROP $)=$ VEND

$\mathrm{DIA}(\mathrm{KDROP}) \quad=$ DIANEW

DROPTIME $($ KDROP $)=$ DROPTIME $($ KDROP $)+$ UPPLIMIT

IF ((DIA(KDROP) .EQ. 0.0) .OR. (DYPOSIT(KDROP)

$\mathrm{C}$

.GE. XN) .OR. (DYPOSIT(KDROP) .LE. X1)) THEN

C

$* * * * * * * * * * * * * * * * * * * * * * * * * *$

FOR OUTPUTTING DROPLET POSITION \& TIME

CALL DROPOUT (DROPTIME(KDROP), DUVEL(KDROP), DYVELOC(KDROP), DXPOS(KDROP), DYPOSIT(KDROP), LMDOUT(KDROP), TNEXT(5))

CALL DROPOUT (DROPTIME(KDROP), DUVEL(KDROP), DYVELOC(KDROP), DXPOS(KDROP), 
C

IF (DIA(KDROP) .EQ. 0.) THEN

C

$\mathrm{C}$

$$
\text { DIA WENT TO ZERO }
$$

WRITE (LMDOUT(KDROP), 8)

ELSE

DROP HIT THE WALL WRITE (LMDOUT(KDROP),10)

WRITE (LMDSOUT(KDROP),10)

END IF

LDFINSH $($ KDROP $)=$.TRUE.

LMDROP $($ KDROP $)=$.FALSE.

END IF

END IF

END DO

LTEST $=$.TRUE.

DO KDROP = 1, NODA, 1

IF ( .NOT. LDFINSH(KDROP)) THEN

LTEST $=$.FALSE.

END IF

END DO

IF (LTEST) THEN

LDROP $=$. FALSE.

$\mathrm{TNEXT}(5)=\mathrm{TLAST}(5)+\mathrm{T}(\mathrm{NT})+.1$

\section{ELSE}

$\operatorname{TLAST}(5)=\operatorname{TNEXT}(5)$

TNEXT $(5)=$ TNEXT $(5)+$ DTDROP

LDROP $=$.TRUE.

\section{END IF}

END DO ! END DRPOLET LOOP

C

$* * * * * * * * * * * * * * * * * * * * * * * * * * * * * * * * * * * * * * * * * * * * * * * * * * * * * * * * * * * * * * * * *$

C Ensemble averaging

do while (tnext(3) .eq. soonest(nevents, tnext))

C On the first call, zero out the eddy accumulation variables

if (it .eq. 0) then call zoiv(nx, neloc)

call zoiv(nx, neleft)

call zoiv(nx, neright)

call zoiv(nx, nesize) 


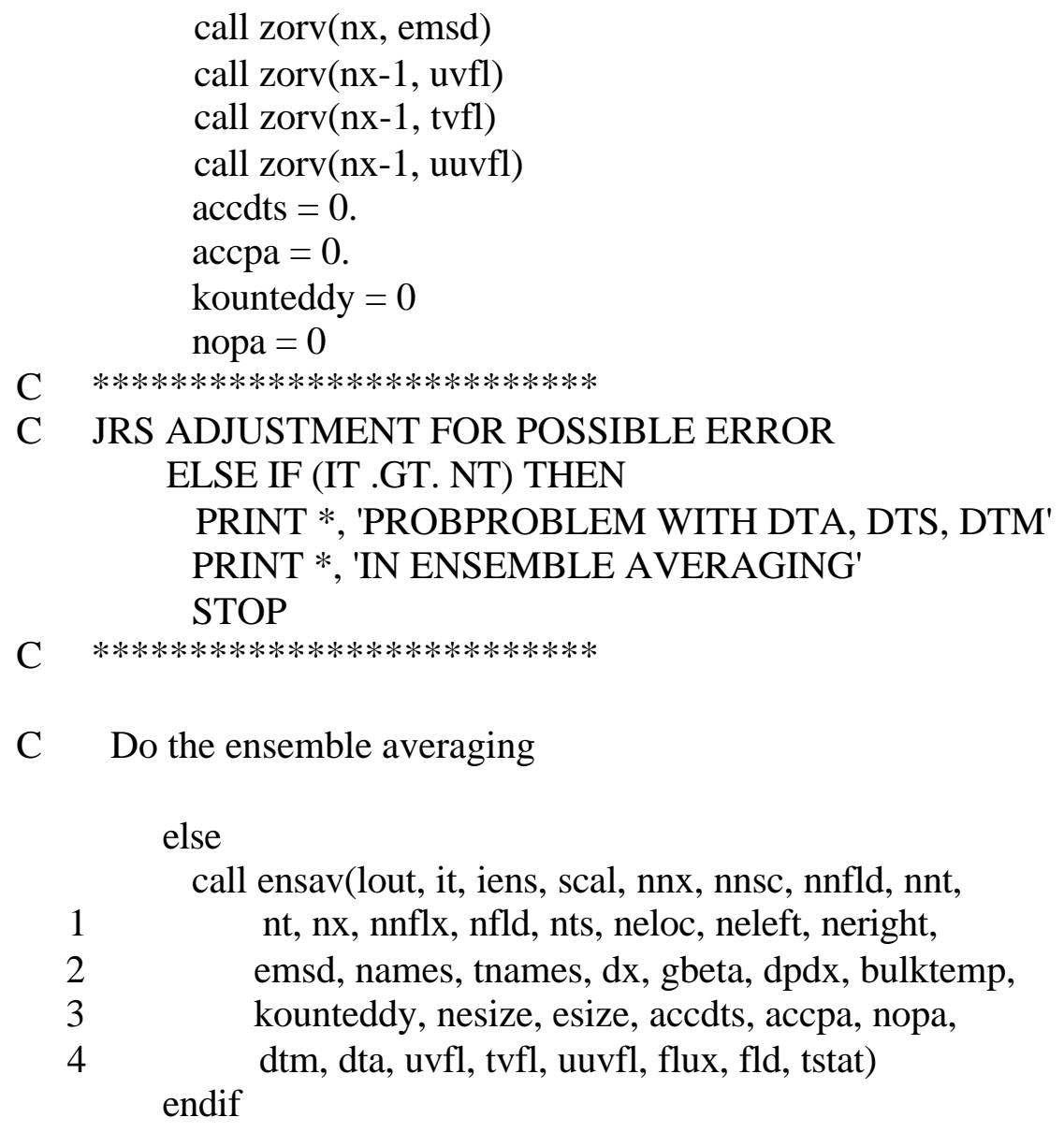

C Do the ensemble averaging

else

call ensav(lout, it, iens, scal, nnx, nnsc, nnfld, nnt, 1 nt, nx, nnflx, nfld, nts, neloc, neleft, neright,

C Update the timing

$$
\begin{aligned}
& \text { it }=\text { it }+1 \\
& \operatorname{tlast}(3)=\operatorname{tnext}(3) \\
& \operatorname{tnext}(3)=\operatorname{tlast}(3)+d t a
\end{aligned}
$$

$\mathrm{C} \quad * * * * * * * * * * * * * * * * * * * * * * * * * *$ may move this latter

C JRS ADJUSTMENT FOR OUTPUTTING DROPLET POSITION \& TIME IF (LDROP) THEN

DO KDROP = 1, NODA, 1

IF (LMDROP (KDROP)) THEN

CALL DROPOUT (DROPTIME(KDROP),

2 DUVEL(KDROP), DYVELOC(KDROP), DXPOS(KDROP), DYPOSIT(KDROP), LMDOUT(KDROP),TNEXT(5))

!!! temp addition to get more accurate data on traj 


\section{$\mathrm{C}$ \\ C 2 \\ C 3 \\ CALL TDROPOUT(DROPTIME(KDROP), DUVEL(KDROP), DYVELOC(KDROP), DXPOS(KDROP), DYPOSIT(KDROP), \\ C 4 \\ LMDSOUT(KDROP), TNEXT(2), \\ C 5 \\ DIA(KDROP), TDROP(KDROP), \\ C 6 \\ TFLAME(KDROP)) \\ !!! end traj}

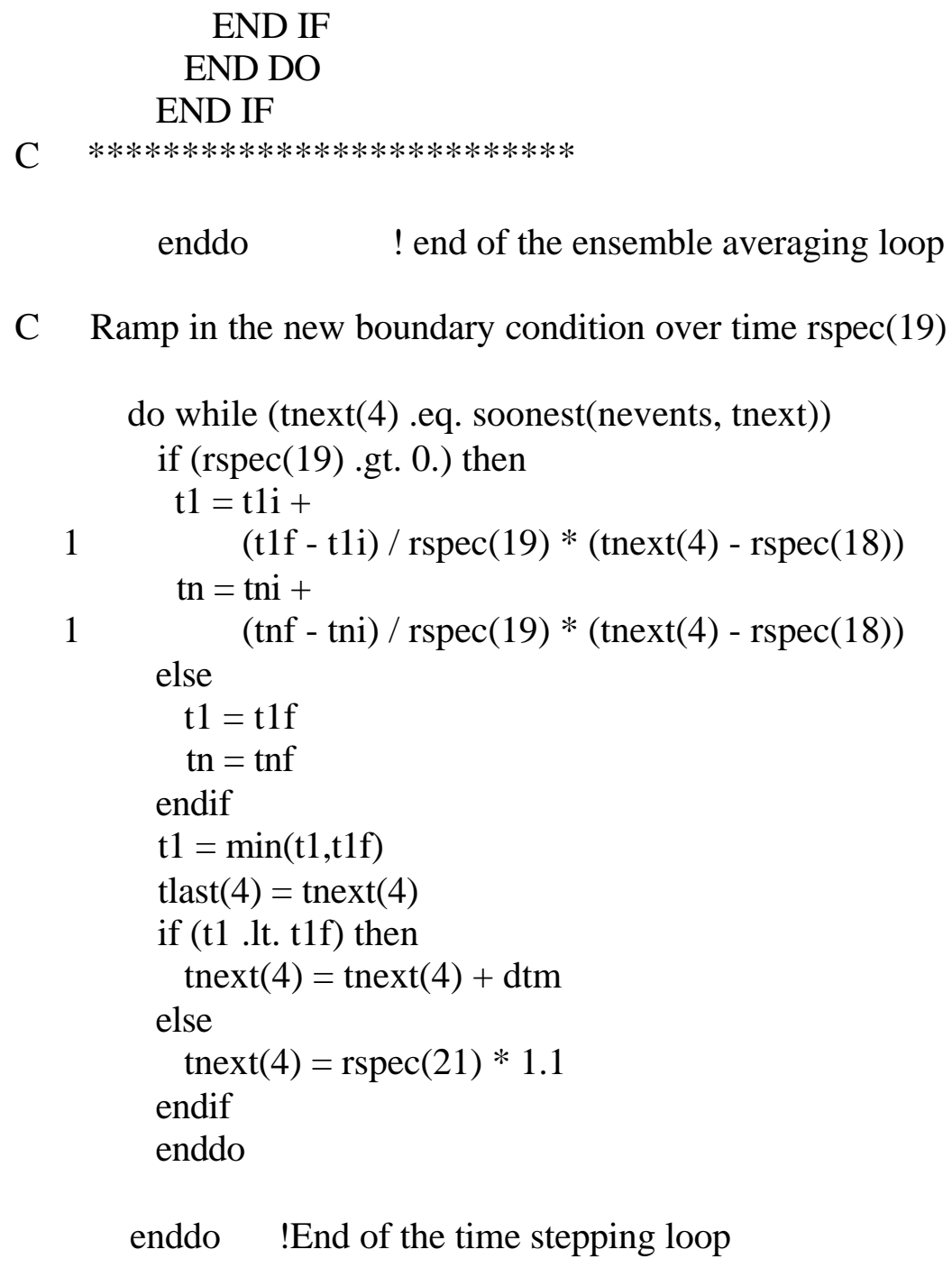

C Ramp in the new boundary condition over time rspec(19)

do while (tnext(4) .eq. soonest(nevents, tnext))

if (rspec(19) .gt. 0.) then

$$
\mathrm{t} 1=\mathrm{t} 1 \mathrm{i}+
$$$$
1 \quad(\mathrm{t} 1 \mathrm{f}-\mathrm{t} 1 \mathrm{i}) / \operatorname{rspec}(19) *(\operatorname{tnext}(4)-\operatorname{rspec}(18))
$$$$
\text { tn }=\text { tni }+
$$$$
1 \quad(\operatorname{tnf}-\operatorname{tni}) / \operatorname{rspec}(19) *(\operatorname{tnext}(4)-\operatorname{rspec}(18))
$$

else

$\mathrm{t} 1=\mathrm{t} 1 \mathrm{f}$

$\operatorname{tn}=\operatorname{tnf}$

endif

$\mathrm{t} 1=\min (\mathrm{t} 1, \mathrm{t} 1 \mathrm{f})$

tlast $(4)=\operatorname{tnext}(4)$

if (t1 .lt. t1f) then

$\operatorname{tnext}(4)=\operatorname{tnext}(4)+\mathrm{dtm}$

else

$\operatorname{tnext}(4)=\operatorname{rspec}(21) * 1.1$

endif

enddo

enddo !End of the time stepping loop

C Field and temporal output

if (mod(iens,iskip) .eq. 0 .or. iens .eq. nens) then include 'op.inc'

endif 


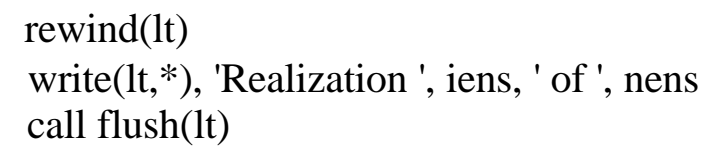

if (iens .eq. 1) close(lmap)

enddo !End of the ensemble loop

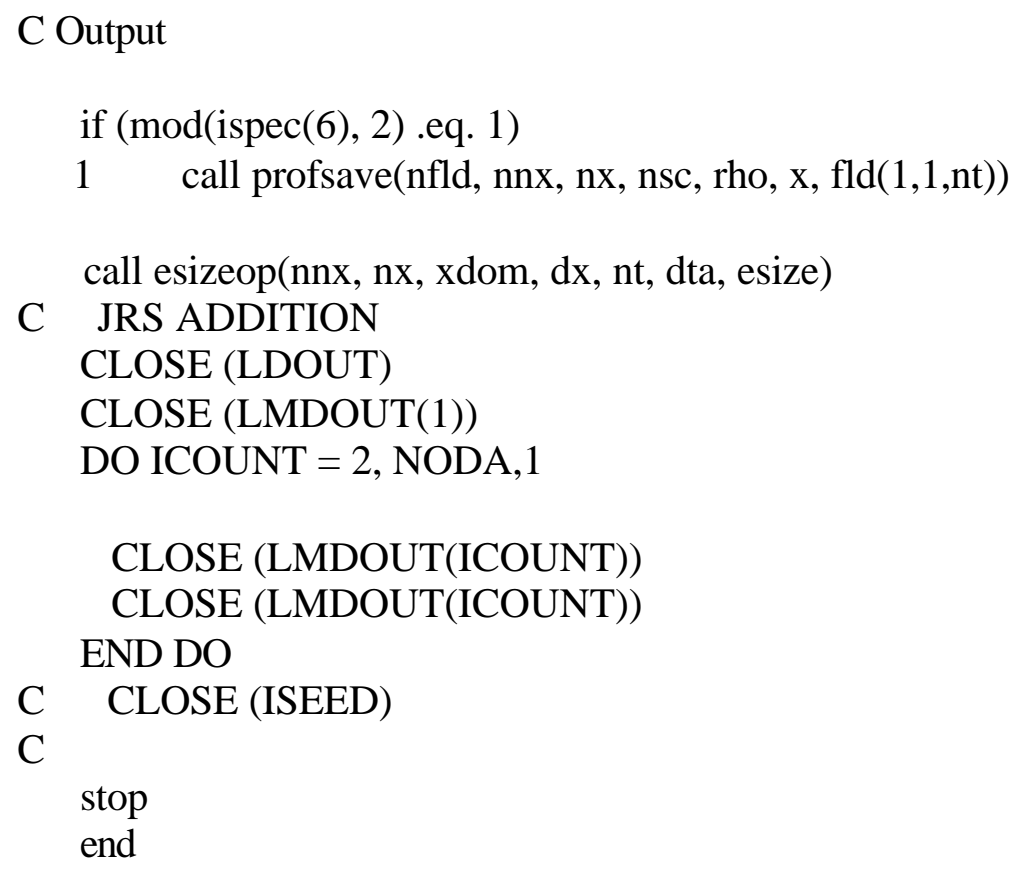


SUBROUTINE DEUPDAT (AIRNU, DIAP, DTDROP, ELHS, EPS, ERHS, ITOM, 2 LDOUT, LOUT, STRTPOS, STRTU, STRTVEL, TAUP, UBAR, 3 UFLUID, VFLUID, DELTAY, DELTAV)

$\mathrm{C} \quad * * * * * * * * * * * * * * * * * * * * * * * * * * * * * * * * * * * * * * * * * * * * * * * * * * * * * * * * * * * * * * * * * *$

!! modified by jrs on $5 / 29$ to get rid on deint stuff

C WRITTEN BY JRS FOR THE DROPLET MODEL. INTEGRATES THE DROPLET-

C DRAG LAW THE THE AMOUNT OF TIME STORED IN DELTA TIME.

C LAST UPDATED 04/28/00 AT 15:50

C LINEAR DROPLET-DRAG LAW WITH A CORRECTION FACTOR F

C NOTE: THIS SUBPROGRAM IS USED WHEN THE DROPLET IS IN AN EDDY

C HENCE, THERE IS A V VELOCITY OF THE FLUID, THERE IS GRAVITY

C SO THE DIFF. EQN. IS

C $\quad$ DV'/DT = F/TAUP *(VFLUID - V') - GRAV

C SINCE EDDIES OCCUR INSTANTANEOUS IN TIME STEP, F, VFLUID, \& TAUP

C ARE CONST. THE PARTICLE EXPERIENCES THE FLUID VELOCITY OF THE CELL

C WHICH CONTAINS IT DURING THE WHOLE TIME OF INTERACTION BETWEEN

C THE PARTICLE AND THE EDDY

C SIGNIFICANT VARIABLE DECLARATIONS:

$\mathrm{C} \quad$ STRT POS $=$ THE INITIAL DROPLET POSITION

$\mathrm{C} \quad$ STRT VEL $=$ THE INITIAL DROPLET VELOCITY

$\mathrm{C}$ STRT $\mathrm{U}=$ THE INITIAL DROPLET VELOCITY IN THE X DIRECTION

$\mathrm{C}$ DTDROP = TIME CHANGE OVER WHICH TO INTEGRATE

$\mathrm{C}$ DELTA $\mathrm{V}=\mathrm{CHANGE}$ IN VELOCITY OF THE PATICLE (VEND HERE)

$\mathrm{C}$ DELTA $\mathrm{Y}=\mathrm{CHANGE}$ IN THE POSITION OF THE PARTICLE

$\mathrm{C}$ E LHS = THE LEFT HAND SIDE (OR LOWER) EDDY END POINT

$\mathrm{C}$ E RHS = THE RIGHT (OR UPPER) EDDY END POINT

C CONST Y 3 = COEFFICIENT IN THE D.E.Q. MULTIPLYING VELOCIT ON RHS

C CONST X 3 = THE X DIRECTION EQUIVALENT TO CONSTY 3

C CONST1,5 $=$ CONVENIENT COEFFICIENT TO MAKE CALCULATIONS

FASTER

C F COEF $=$ CORRECTION FACTOR SO THE LINEAR DRAG LAW FITS BETTER

$\mathrm{C}$ TAU P = DROPLET TIME CONSTANT, RELAXATION TIME

$\mathrm{C}$ V FLUID = VELOCITY OF THE FLUID IN THE Y DIRECTION

$\mathrm{C}$ U FLUID = VELOCITY OF THE FLUID IN THE X DIRECTION

$\mathrm{C}$ U BAR = AVERAGE VELOCITY OF THE EDDY IN THE X DIRECTION

$\mathrm{C}$ REN = REYNOLDS NUMBER 


$$
\begin{array}{lll}
\text { C } & \text { DIA P } & =\text { DIAMETER OF THE PARTICLE } \\
\text { C } & \text { GRAV } & \text { = THE GRAVITATIONAL CONST. (+9.8 M/SS) } \\
\text { C } & \text { T HAT } & =\text { THE TIME FOR THE PARTICLE TO REACH THE EXTREMA } \\
\text { C } & \text { Y HAT } & \text { = THE Y POSITION AT THE EXTREMA TIME (T HAT) } \\
\text { C } & \text { X }=\text { A DUMMY VARIABLE REPRESENTING TIME IN THE FUNC. }
\end{array}
$$
STATEMENTS

$\mathrm{C}$ EPS = SMALLEST DISTANCE IN WHICH MATTERS (I.E. ZERO)

C HALF EDDY $=$ HALF THE EDDY LENGTH

C X NEW P $=$ X POSITION OF THE DROPLET IN THE MOVING COORDINATE SYSTEM

$\mathrm{C}$ T MIN = THE MINIMUM INTERACTION TIME BETWEEN THE EDDY 7 DROP

IMPLICIT NONE

REAL AIRNU, BC

REAL CON3, CON4, CON7

REAL CONST1, CONST2, CONST4, CONST5

REAL CONST6, CONST7, CONSTX3, CONSTY3

REAL DELTAT, DELTAY, DELTAV, DIAP

REAL DPOSIT, DTDROP, DVELOC

REAL ELHS, EPS, EPSDIS, ERHS

REAL FXCOEF, FYCOEF, FMID, FXL, FXR, FYL, FYR, GRAV

REAL HALFEDDY

INTEGER ITOM, ITT

LOGICAL LABOVE, LITT

INTEGER LDOUT, LOUT

REAL POSITION, RENX, RENY, STRTPOS, STRTU, STRTVEL, TAUP

REAL TMID, TMIN, TNEW

REAL TXHAT, TXL, TXR, TYHAT, TYL, TYR

REAL UBAR, UFLUID

REAL VELOCITY, VFL, VFLUID, $X$

LOGICAL XEXTREMA

REAL XHATP, XNEWP, XPOSITP

LOGICAL YEXTREMA

REAL YHAT, YNEW

!! TEMP ADDITON FOR MORE DETAILED OUTPUT

C REAL CY4, CY7

C INTEGER ITEMP

C REAL TDT, TDV, TDY, TTIME, TX, TY

C LOGICAL XEXTRIN, YEXTRIN

!! END TEMP ADDITON

C DEFINE FUNCTIONS FOR VELOCITY \& POSITION 
DVELOC $(\mathrm{VFL}, \mathrm{CON} 3, \mathrm{X})=\mathrm{VFL} *(1 .-\mathrm{EXP}(-\mathrm{CON} 3 * \mathrm{X}))$

DPOSIT(VFL, CON3, X) = VFL*X-VFL/CON3*(1. - EXP(-CON3*X))

POSITION $(\mathrm{BC}, \mathrm{CON} 3, \mathrm{CON} 4, \mathrm{CON} 7, \mathrm{X})=\mathrm{BC}+\mathrm{CON} 4 * \mathrm{X}+\mathrm{CON} 7 / \mathrm{CON} 3 *$

2 $(\mathrm{EXP}(-\mathrm{CON} 3 * \mathrm{X})-1)$

$\mathrm{XPOSITP}(\mathrm{CON} 3, \mathrm{CON} 4, \mathrm{CON} 7, \mathrm{X})=\mathrm{CON} 4 / \mathrm{CON} 3 *(1 .-\mathrm{EXP}(-\mathrm{CON} 3 * \mathrm{X}))-\mathrm{CON} 7 * \mathrm{X}$

$C$ USE THE ANALYTIC SOLUTION TO THE CONST. COEFICIENT

C DIFFERENTIAL EQUATION TO COMPUTE THE END POINT

C NOTE: GRAVITAIONAL ACCELERATION IN UNITS OF ---

!! TEMP. ADD A FLLAG TO SHOW THAT IT REACHED AN EXTREMA INSIDE THE EDDY

C $\quad$ YEXTRIN $=$.FALSE.

C $\quad$ XEXTRIN $=$.FALSE.

!! END TEMP ADDITION

C SINCE LINEAR, BREAKS DOWN INTO X \& Y COMPONENTS: Re CAN BE

C DIFFERENT IN THE X \& Y DIRECTIONS

RENY $=$ DIAP $*$ ABS(STRTVEL - VFLUID $) / A I R N U$

IF (RENY .LE. 1.0) THEN

C STOKES LAW

$\mathrm{FYCOEF}=1.0$

ELSE IF (RENY .LE. 1000) THEN

C CORRECTION FACTOR

$\mathrm{FYCOEF}=1.0+0.15 *(\mathrm{RENY}) * * 0.687$

ELSE

WRITE (LDOUT,*) ' PARTICLE ReY > 1000'

WRITE (LDOUT, *) ' STOPPED IN DEUPDAT', RENY

WRITE (LOUT, *) ' PARTICLE ReY > 1000'

WRITE (LOUT,*) ' STOPPED IN DEUPDAT', RENY

STOP

END IF

RENX $=$ DIAP $*$ ABS(STRTU - UFLUID) $/$ AIRNU

IF (RENX .LE. 1.0) THEN

C STOKES LAW

$\mathrm{FXCOEF}=1.0$

ELSE IF (RENX .LE. 1000) THEN

C CORRECTION FACTOR

$\mathrm{FXCOEF}=1.0+0.15 *(\mathrm{RENX}) * * 0.687$

ELSE

WRITE (LDOUT, *) ' PARTICLE ReX > 1000'

WRITE (LDOUT, *) ' STOPPED IN DEUPDAT', RENX

WRITE (LOUT, *) ' PARTICLE ReX > 1000' 
WRITE (LOUT, *) ' STOPPED IN DEUPDAT', RENX STOP

END IF

CONSTY $3=$ FYCOEF $/$ TAUP

CONSTX $3=$ FXCOEF $/$ TAUP

$\mathrm{C} * * * * * * * * * * * * * * * * * * * * * * *$

C THIS TAKES INTO ACCOUNT THE POSSIBILITY OF PARTICLES ON

C THE EDGE OF THE EDDY AND BEING TAKEN OUT BY ITS OWN VELOCITY

$\mathrm{C} * * * * * * * * * * * * * * * * * * * * * * *$

IF ((((ERHS-EPS) .LE. STRTPOS) .AND. (STRTVEL .GT. 0.)) .OR.

2 (((ELHS+EPS) .GE. STRTPOS) .AND. (STRTVEL .LT. 0.)) .OR.

3 (VFLUID .EQ. 0.)) THEN

C IF THE PARTICLE IS EPSILON CLOSE TO THE EDDY BORDER AND GOING

C IN THE DIRECTION TO TAKE IT OUT OF THE EDDY THEN THERE IS NO

C INSTANTANEOUS DROPLET-EDDY INTERATION. ALSO IF THERE IS NO

C INITIAL PERTURBATION VELOCITY (I.E. VFLUID) THEN THERE IS NO

C INTANTANEOUS EDDY EFFECT

DELTAY $=0.0$

DELTAV $=0.0$

$\mathrm{C} * * * * * * * * * * * * * * * * * * * * * * *$

C VELOCITY VARIATION AND POSITION CHANGE IS SET TO ZERO. RETURN TO

C CALLING ROUTINE

$\mathrm{C} * * * * * * * * * * * * * * * * * * * * * * *$

ELSE

C AT THIS POINT, PARTICLE IS "WELL" INSIDE EDDY. CHECK FOR

EXTREMA

C NEW ADDITION TO TAKE CARE OF EXTREMA - ADDED ON 10/4

YEXTREMA = .FALSE.

LITT $=$.TRUE.

TMIN $=$ DTDROP

CONST4 = VFLUID - GRAV / CONSTY3

CONST7 $=$ CONST4 - STRTVEL

C DO NOT LOOK FOR AN EXTREMA IF THE STARTING PARTICLE VELOCITY (STRTVEL)

C IS NEAR ZERO; THE STRTVEL MUST BE OF OPPOSITE SIGN OF CONST4 IN ORDER

C FOR THERE TO BE AN EXTREEMA IN REAL TIME. IF CONST4 IS NEAR ZERO THEN

C THERE IS VIRTUALLY NO DRIVING FORCE TO CHANGE THE PARTICLE VELOCITY 
IF ((ABS(STRTVEL) .GT. EPS/1000.) .AND.

2 (STRTVEL * CONST4 .LT. 0.)) THEN

IF (ABS(CONST4) .GT. EPS/1000.) THEN

CONST6 = ABS(CONST7 / CONST4)

IF (CONST6 .GT. 1.) THEN

!IS THIS ALWAYS TRUE????

TYHAT $=$ ALOG(CONST6) $/$ CONSTY3

YHAT $=$ POSITION(STRTPOS,CONSTY3,CONST4,CONST7,TYHAT)

IF (TYHAT .LE. DTDROP) THEN

YEXTREMA = .TRUE.

C YEXTREMA IS TRUE IF AND ONLY IF THE EXTREMA FALLS

C DURING THE LIFE TIME OF THE EDDY

END IF

END IF

END IF

END IF

IF (YEXTREMA .AND. (YHAT .GT. (ERHS+EPS))) THEN

C PARTICLE IS OUTSIDE OF THE EDDY BEFORE IT REACHES ITS EXTREMA

C (MAXIMUM POSITION)

C RE-ADJUST DELTA TIME FOR THE INTEGRAL USING REGULA FALSI METHOD

C NOTE: VELOCITY IS THE DERIVATIVE OF DISTANCE

LABOVE $=$.TRUE.

TYR = TYHAT

FYR $=$ YHAT - ERHS

TYL $=0.0$

FYL $=$ STRTPOS - ERHS

TMID $=-$ TYHAT $*$ FYL $/($ FYR - FYL $)$

ELSE IF (YEXTREMA .AND. (YHAT .LT. (ELHS-EPS))) THEN

$\mathrm{C}$ PARTICLE IS BELOW THE EDDY BEFORE THE EDDY ENDS

$$
\begin{aligned}
& \text { LABOVE }=. \text { FALSE. } \\
& \text { TYR }=\text { TYHAT } \\
& \text { FYR }=\text { YHAT }- \text { ELHS } \\
& \text { TYL }=0.0 \\
& \text { FYL }=\text { STRTPOS }- \text { ELHS } \\
& \text { TMID }=- \text { TYHAT } * \text { FYL } /(\text { FYR }- \text { FYL })
\end{aligned}
$$

ELSE

YNEW = POSITION(STRTPOS,CONSTY3,CONST4,CONST7,DTDROP) IF (((ERHS+EPS) .GE. YNEW).AND.((ELHS-EPS) .LE. YNEW))THEN 


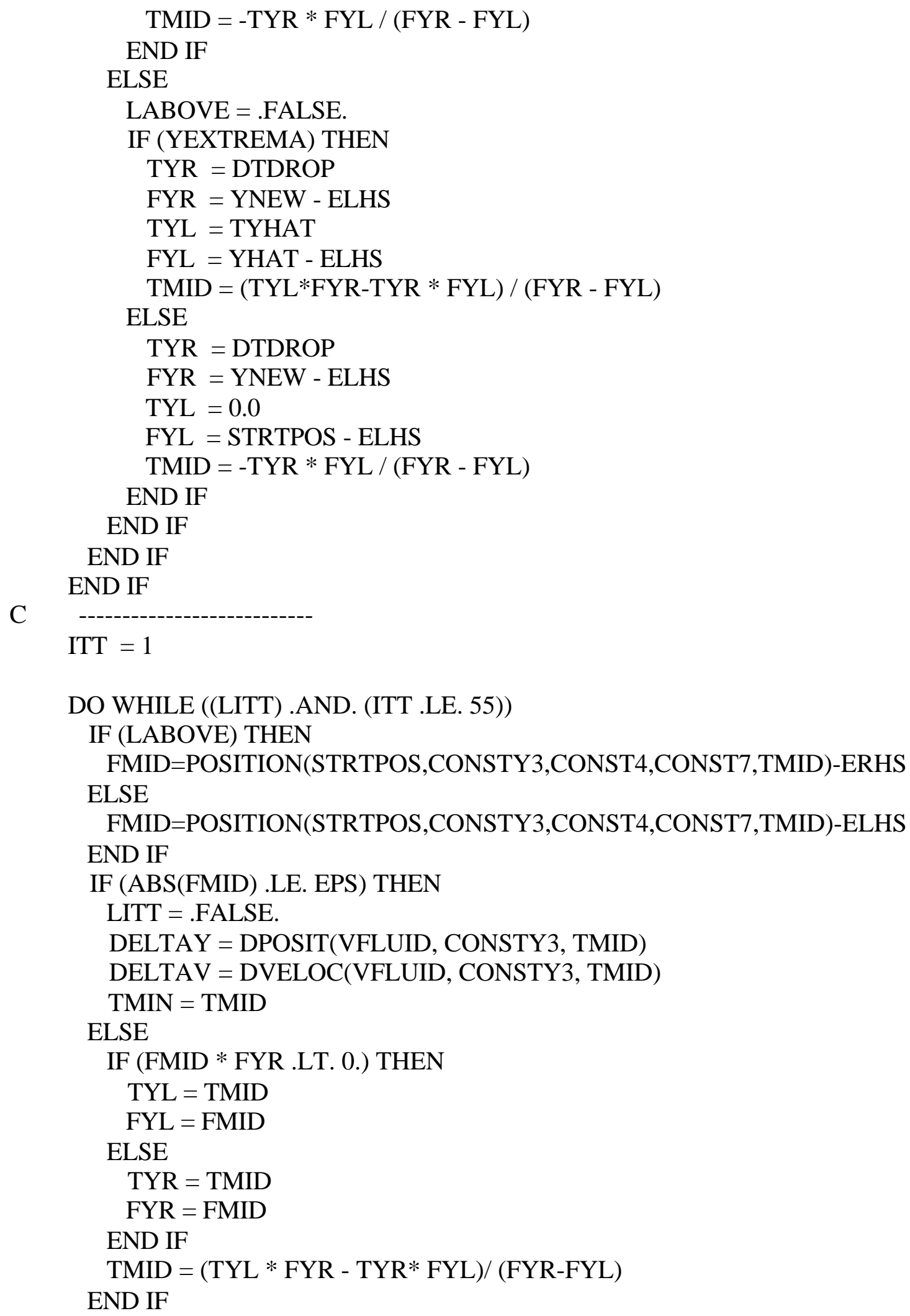


$\mathrm{ITT}=\mathrm{ITT}+1$

END DO

IF (LITT) THEN

WRITE (LDOUT,*) ' REGULA FALSI DID NOT CONVERGE (Y) IN 55 '

WRITE (LDOUT,*) 'ITTERATIONS, DROPLET IN DEUPDAT'

WRITE (LDOUT, *) TYL, TMID, TYR

WRITE (LDOUT,*) FYL, FMID, FYR

WRITE (LDOUT,*) YEXTREMA, LABOVE, ITOM

WRITE (LDOUT,*) TYHAT, YHAT, DTDROP, YNEW

WRITE (LOUT,*) ' REGULA FALSI DID NOT CONVERGE (Y) IN 55 '

WRITE (LOUT, *) 'ITTERATIONS, DROPLET IN DEUPDAT'

DELTAY $=$ DPOSIT(VFLUID, CONSTY3, TMID)

DELTAV $=$ DVELOC $($ VFLUID, CONSTY3, TMID $)$

TMIN $=$ TMID

END IF

$\mathrm{C} * * * * * * * * * * * * * * * * * * * * * * * * * * * * * * * * * * * * * * * * * * * * * * * * * * * * * * * * * * * * * * \mathrm{STOP}$

HERE

XEXTREMA $=$. FALSE.

LITT $=$.TRUE.

CONST4 $=$ STRTU - UFLUID

CONST7 $=$ UBAR - UFLUID

CONST2 $=$ UBAR - STRTU

CONST $1=$ CONST $2 *$ CONST7

C EDDY IS A DISTANCE L IN EACH DIRECTION (HENCE HALFEDDY IS L) HALFEDDY $=($ ERHS - ELHS $)$

C DO NOT LOOK FOR AN EXTREMA IF THE STARTING PARTICLE

VELOCITY (STRTU)

C RELATIVE TO THE U BAR IS NEAR ZERO OR IF THE U FLUID RELATIVE TO

C U BAR IS NEAR ZERO -- THERE IS NO DRIVING FORCE

C UBAR - STRTU MUST BE OF OPPOSITE SIGN OF UBAR - UFLUID IN ORDER

C FOR THERE TO BE AN EXTREEMA IN REAL TIME.

C !!!! WHAT TO DO ABOUT EPSILON ??????? EPS IS A DISTANCE MINIMUM !!

IF ((CONST1 .LT. 0.) .AND. (ABS(CONST7) .GT. EPS) .AND.

2 (ABS(CONST2) .GT. EPS) .AND. (ABS(CONST4) .GT. EPS)) THEN CONST6 $=$ CONST $4 /$ CONST7

IF (CONST6 .GT. 1.) THEN IIS THIS ALWAYS TRUE???

TXHAT $=$ ALOG(CONST 6$) /$ CONSTX3

XHATP $=$ XPOSITP $($ CONSTX3,CONST4,CONST7,TXHAT $)$

IF (TXHAT .LE. TMIN) THEN 
XEXTREMA $=$.TRUE.

C XEXTREMA IS TRUE IFF THE EXTREAMA FALLS BEFORE THE EDDY ENDS

END IF

END IF

END IF

IF (XEXTREMA .AND. ((XHATP-HALFEDDY) .GT. (EPS))) THEN

C PARTICLE IS OUTSIDE OF THE EDDY BEFORE IT REACHES ITS

EXTREMA

C (MAXIMUM POSITION)

C RE-ADJUST DELTA TIME FOR THE INTEGRAL USING REGULA FALSI METHOD

C NOTE: VELOCITY IS THE DERIVATIVE OF DISTANCE; ALSO LABOVE MEANS

C TO THE RIGHT OF THE EDDY IN THE X DIRECTION

LABOVE $=$.TRUE.

TXR $=$ TXHAT

FXR $=$ XHATP - HALFEDDY

$\mathrm{TXL}=0.0$

FXL $=-$ HALFEDDY

TMID $=-$ TXHAT $*$ FXL $/($ FXR - FXL $)$

ELSE IF (XEXTREMA .AND. ((XHATP+HALFEDDY) .LT. (-EPS))) THEN

LABOVE $=$.FALSE.

TXR $=$ TXHAT

FXR $=$ XHATP + HALFEDDY

$\mathrm{TXL}=0.0$

FXL $=$ HALFEDDY

TMID $=-$ TXHAT $*$ FXL $/($ FXR - FXL $)$

ELSE

C PARTICLE DOES NOT GO OUT OF THE EDDY IN THE X DIRECTION AT THE

C EXTREMA, BUT CAN STILL GO OUT BY THE END OF THE EDDY

LIFETIME

!! TEMP. ADD A FLLAG TO SHOW THAT IT REACHED AN EXTREMA INSIDE THE EDDY

C IF (XEXTREMA) THEN

C XEXTRIN $=$.TRUE.

CC XEXTRIN IS TRUE IFF THE X EXTREMA FALLS WITH IN THE EDDY LIFETIME

C IF (YEXTRIN) THEN

C WRITE (LDOUT,9137) STRTU, UBAR, UFLUID, CONSTX3,

C 2 CONST4, CONST7, TXHAT, XHATP, HALFEDDY

C 9137 FORMAT (1X, 'STRTU= ', E16.10, ' UBAR= ', E16.10, 


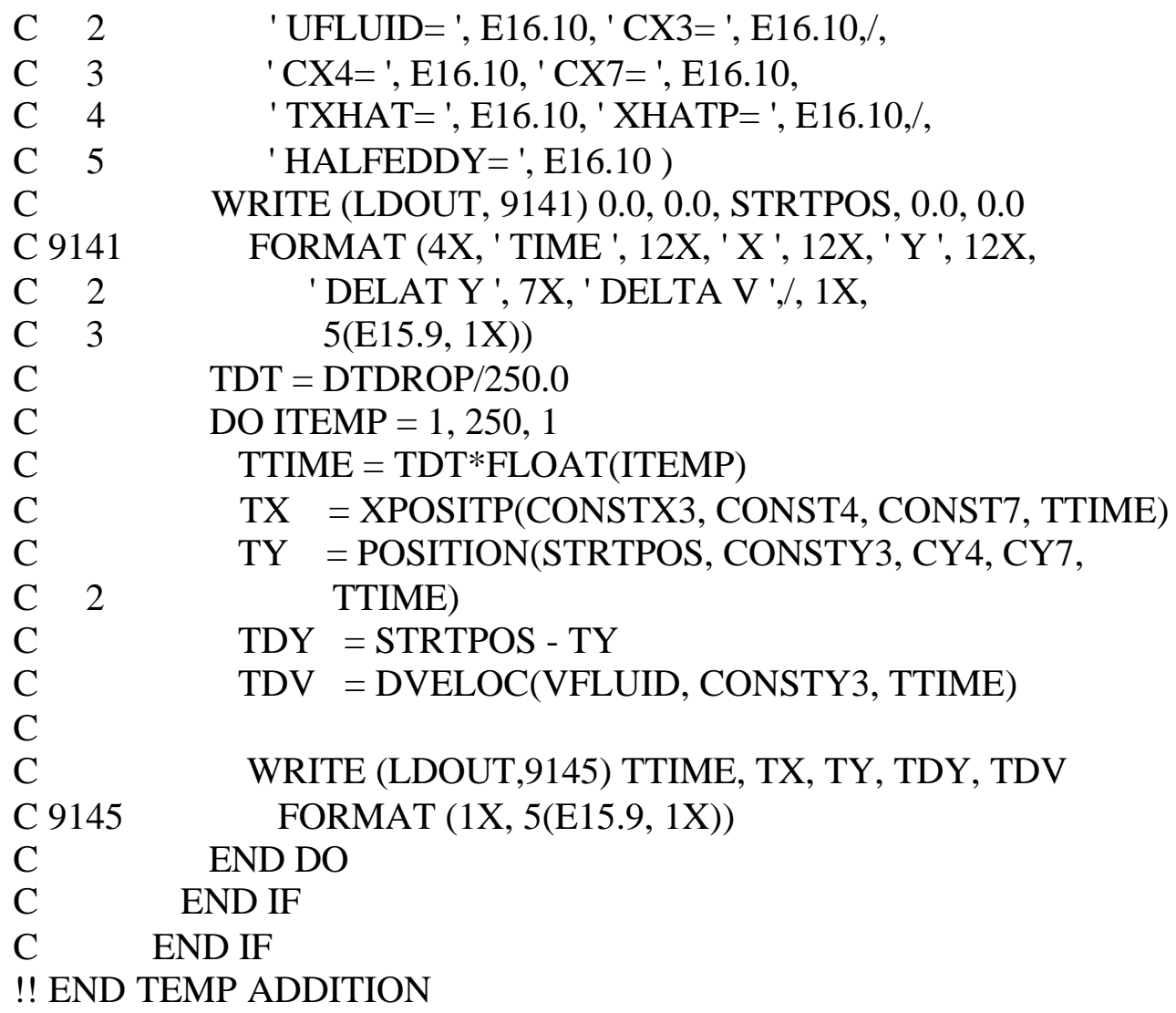

XNEWP $=$ XPOSITP $($ CONSTX3,CONST4,CONST7,TMIN $)$

IF ((XNEWP-HALFEDDY) .GT. EPS) THEN

LABOVE $=$.TRUE.

IF (XEXTREMA) THEN

TXR $=$ TMIN

FXR $=$ XNEWP - HALFEDDY

TXL $=$ TXHAT

FXL $=$ XHATP - HALFEDDY

$\mathrm{TMID}=(\mathrm{TXL} * \mathrm{FXR}-\mathrm{TXR} * \mathrm{FXL}) /(\mathrm{FXR}-\mathrm{FXL})$

ELSE

TXR $=$ TMIN

FXR $=$ XNEWP - HALFEDDY

$\mathrm{TXL}=0.0$

FXL $=-$ HALFEDDY

TMID $=-$ TXR $*$ FXL $/($ FXR - FXL $)$

END IF

ELSE IF((XNEWP+HALFEDDY) .LT. -EPS) THEN

LABOVE $=$.FALSE.

IF (XEXTREMA) THEN

TXR $=$ TMIN $\quad$ !NEEDS WORK HERE 


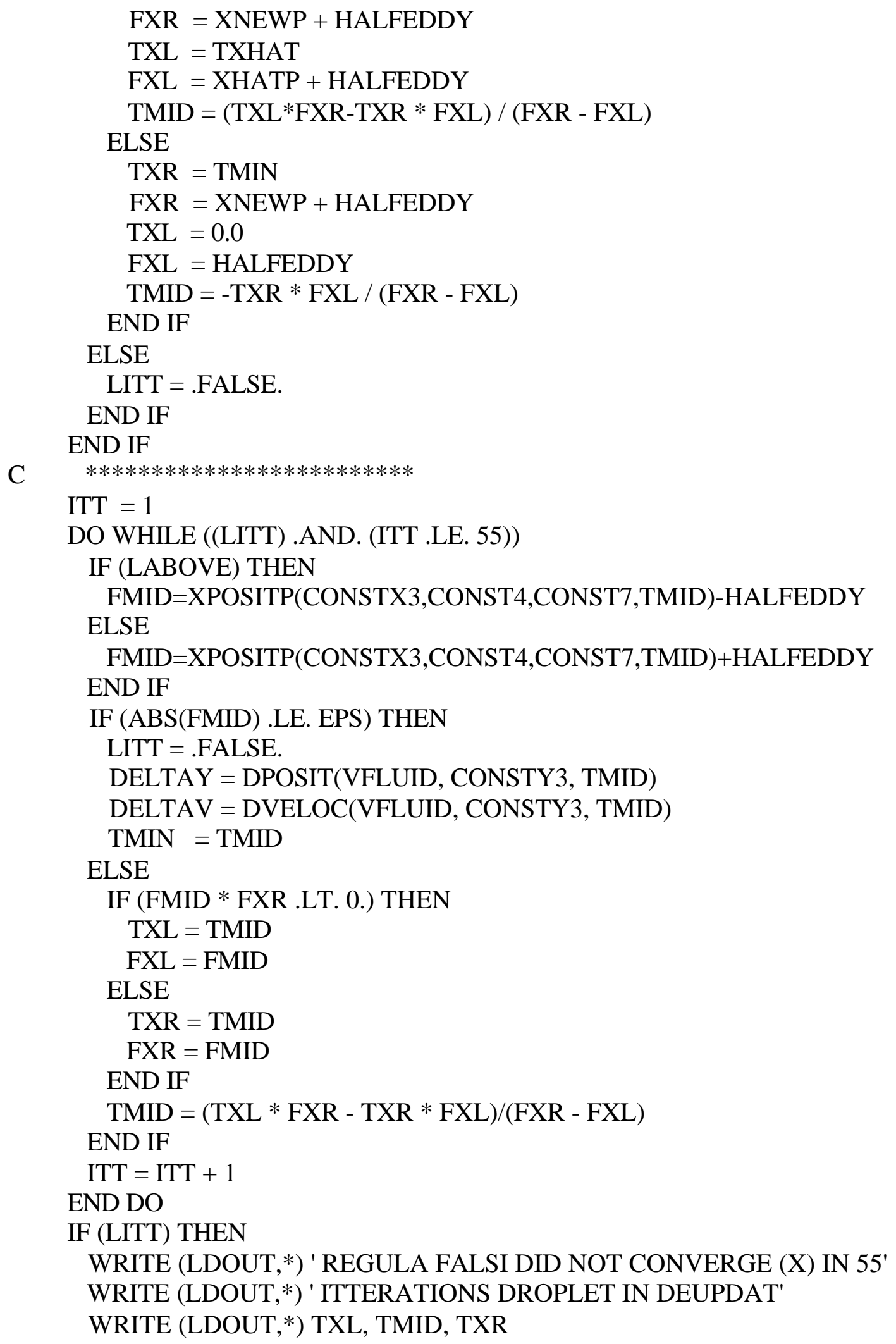


WRITE (LDOUT, *) FXL, FMID, FXR

WRITE (LDOUT,*) XEXTREMA, LABOVE, ITOM

WRITE (LOUT,*) ' REGULA FALSI DID NOT CONVERGE (X) IN 55 '

WRITE (LOUT, *) 'ITTERATIONS, DROPLET IN DEUPDAT'

DELTAY = DPOSIT(VFLUID, CONSTY3, TMID)

DELTAV = DVELOC(VFLUID, CONSTY3, TMID)

TMIN = TMID

END IF

END IF

!! TEMP. ADD A FLLAG TO SHOW THAT IT REACHED AN EXTREMA INSIDE THE EDDY

C IF (ITOM .EQ. 23) THEN

C WRITE (LDOUT,9159) TMIN

C 9159 FORMAT (1X, 'TMIN= ', E16.10)

C TY = POSITION(STRTPOS, CONSTY3, CY4, CY7, TMIN)

C TX $=$ XPOSITP(CONSTX3, CONST4, CONST7, TMIN)

C WRITE (LDOUT,9145) TMIN, TX, TY, DELTAY, DELTAV

C END IF

!! END TEMP ADDITION

RETURN

$\mathrm{C} * * * * * * * * * * * * * * * * * * * * * * * * * * * * * * * * * * * * * * * * * * * * * * * * * * * * * * * * * * * * * * \mathrm{STOP}$

HERE

END 
SUBROUTINE DROPINIT(DX, NENS, NEVENTS, NOD, NNX, NX, ALAMDA, 2 BIRTHT, BULKVEL, DABZERO, DIA, DIGNIT, DPARAM, DROPTIM, 3 DPOSIT, DTDROPF, DUVEL, DXPOS, STRTVEL, EPS, GRAVAC, 4 HEATCAP, HEATCOM, HEATVAP, HOLD, LDOUT, LDROP, LDSTART, LLAMDA,

5 LLONG, LMDROP, NODA, OUTFIL, PEPS, PI, RHOL, STOICH, TDROP,

6 TEMPBP, TEMPZERO, TFLAME, YOINFIN)

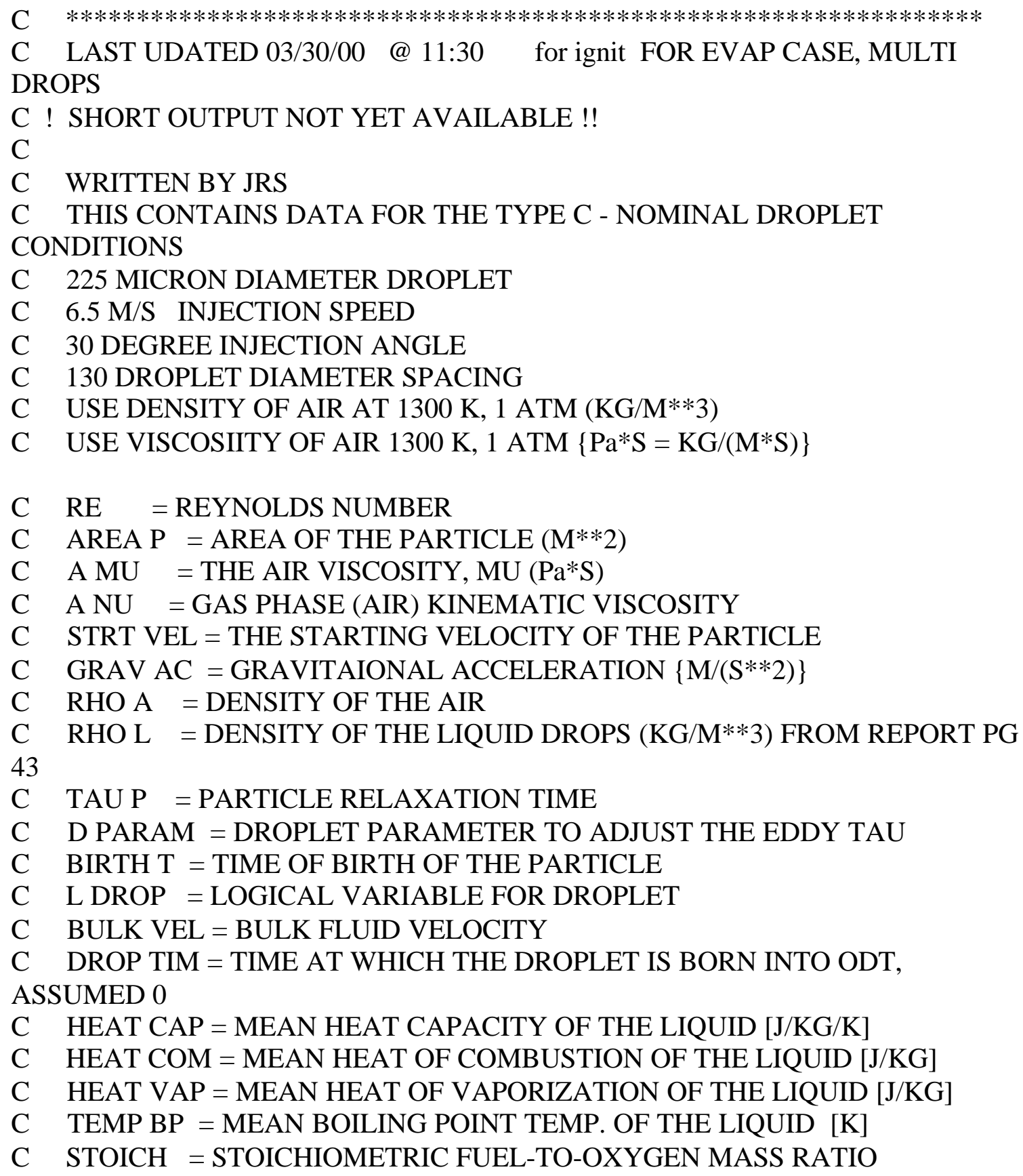


C D RATIO $=$ DENSITY RATIO OF GAS / LIQUID

C $\quad$ B MAS T $=$ MASS TRANSFER NO. CALLED B

C SCHMIDT $=$ THE SCHMIDT NUMBER FOR THE GAS PHASE

C YO INFIN = MASS FRACTION OF OXYGEN IN THE FLUE GASS

C T FLAME $=$ INITIAL GUESS FOR THE FLAME TEMPERATURE AROUND THE DROP

C T DROP $=$ INITIAL DROP TEMPERATURE

C N O D A $=$ ACTUAL NUMBER OF DROPS IN THIS SIMULATION

IMPLICIT NONE

REAL ALAMDA, AMU, BIRTHT(NOD)

REAL BULKVEL

REAL DABZERO, DIA(NOD)

REAL DIGNIT(NOD), DPARAM, DPOSIT(NOD)

REAL DROPTIM(NOD), DTDROPF, DTM, DUVEL(NOD)

REAL DX(NNX), DXPOS(NOD)

REAL EPS, EPSF

REAL GRAVAC, HEATCAP, HEATCOM, HEATVAP, HOLD

INTEGER I, IHOLD, ILAMDA, ILONG, INP, LDOUT

LOGICAL LDROP, LDSTART(NOD), LLAMDA, LLONG, LMDROP(NOD)

INTEGER NENS, NEVENTS, NOD, NODA, NNX, NX

CHARACTER $* 9$ OUTFIL(NOD)

REAL PEPS, PI

REAL RHOA, RHOL

REAL STOICH, STRTVEL(NOD)

REAL TEMPBP, TEMPZERO, TDROP(NOD), TFLAME(NOD)

REAL TLAST(NEVENTS), TNEXT(NEVENTS)

REAL VELOCITY, YOINFIN

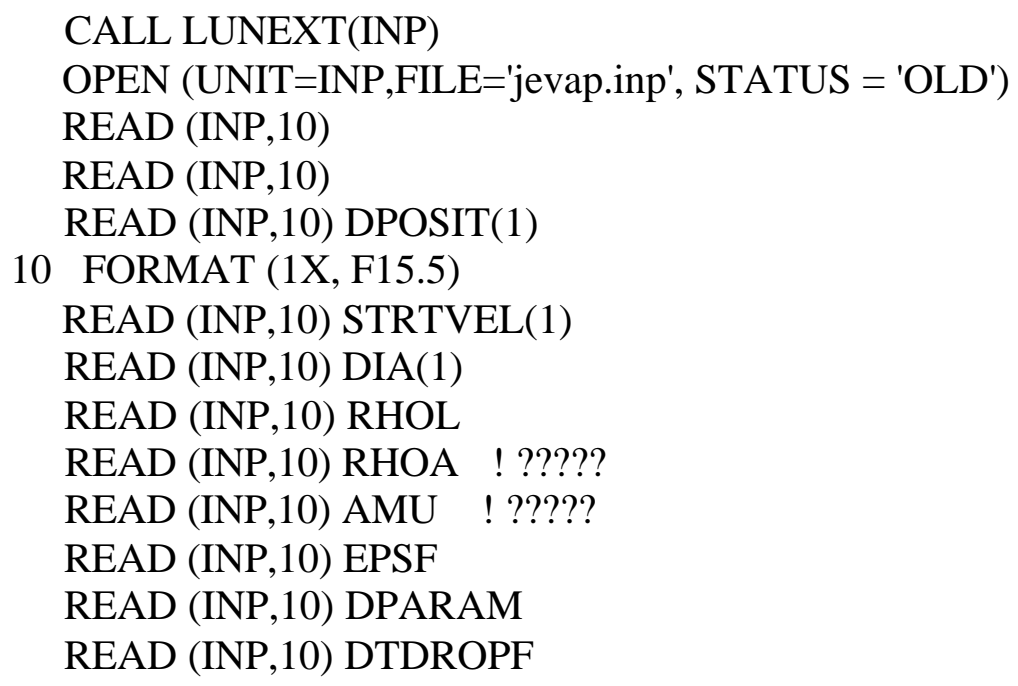


READ (INP,10) BIRTHT(1)

READ (INP,10) DUVEL(1)

READ (INP,10) DXPOS(1)

READ (INP,10) BULKVEL

READ (INP,10) DROPTIM(1)

READ (INP,10) GRAVAC

READ (INP,10) TEMPBP

READ (INP,10) HEATCAP

READ (INP,10) HEATVAP

READ (INP,10) HEATCOM

READ (INP,10) STOICH

READ (INP,10) ALAMDA

READ (INP,10) YOINFIN

READ (INP,10) TFLAME(1)

C DABZERO IS THE DIFFUSIVITY OF THE REPRESENTATIVE FUEL

C COMPONENT IN AIR AT THE TEMPERATURE [K] TEMPZERO

READ (INP,10) DABZERO

READ (INP,10) TEMPZERO

READ (INP,10) TDROP(1)

READ (INP,10) PEPS

READ (INP,20) NODA

20 FORMAT (1X, I2)

READ (INP,22) ILAMDA

22 FORMAT (1X,I1)

READ (INP,22) ILONG

READ (INP,10) DIGNIT(1)

C BULK VELOCITY $=0.8344 \mathrm{M} / \mathrm{S}$ AS COMPUTED FROM MULHOLAND ET. AL.

$\mathrm{C} \quad * * * * * * * * * * * * * * * * * * * * * * * * * * * * * * * * * * * * * * * * * * * * * * * * * * * * * * * * * * * * * * * * * *$

WRITE (LDOUT, 25) DIA(1), DROPTIM(1), BIRTHT(1), BULKVEL,

2 DUVEL(1), STRTVEL(1)

25 FORMAT (1X, 'INITIAL DIA = ', E10.4, ' Age of the drop when',

2 ' it is born into ODT = ', E10.4 ,/,1X, 'Time at which the',

3 ' droplet appears in the ODT domain = ', E12.6, /, 1X,

$4 \quad$ 'BULK FLUID VELOCITY = ', E12.6, ' U = ', E12.6,

$5 \quad$ ' $\mathrm{V}=$ ', E12.6)

WRITE (LDOUT, 30) DXPOS(1), DPOSIT(1)

30 FORMAT (1X,'INITIAL X POSITION = ',E12.6, ' INITIAL Y = ',E12.6)

WRITE (LDOUT, 40) RHOL, RHOA, AMU

40 FORMAT (1X, 'DENSITY OF LIQUID=',E10.4,' AIR: DENSITY = ',

2 E10.4, ' VISCOSITY = ',E10.4)

WRITE (LDOUT, 50) DPARAM, NENS, EPSF, DTDROPF

50 FORMAT (1X,'PARAMETER FOR ADJUSTING THE PSEUDO TIME TO

DROPLET', 
2 ' TIME IS ', E12.6, /, 1X,'No. of ensambles is ', I7, 1X,

3 'FACTOR FOR EPSILON,', ' SMALLEST DISTANCE IN WHICH' ,/, 1X,

4 ' THE ODT IS CONCERNED = ', E10.4, /, 1X, 'FACTOR FOR ',

5 'COMPUTING DROPLET TIME STEP FROM MOLECULAR STEP $(\mathrm{dtm})=$ ', 6 E10.4)

WRITE (LDOUT, 60) GRAVAC, TEMPBP, HEATCAP, HEATCOM, HEATVAP

60 FORMAT (1X, 'GRAVITATIONAL ACCELERATION = ', E12.6, ' BOILING',

2 ' PT. = ', E10.4, /, 1X, 'HEAT CAP. = ', E10.4, ' HEAT OF',

3 'COMBUST. = ', E10.4, ' HEAT OF VAPOR. = ', E10.4)

WRITE (LDOUT, 70) STOICH, ALAMDA, YOINFIN, TFLAME(1)

70 FORMAT (1X,'FUEL TO OXYGEN MASS RATIO = ', E10.4, ' BURNING ',

2 'PARAM. = ', E15.8, /,1X, 'MASS FRACTION OF O2 IN FLUE',

3 ' GAS = ', E10.4, ' GUESS FLAME SHEET ',

4 'TEMPERATURE ', E11.5)

WRITE (LDOUT,80) DABZERO, TEMPZERO

80 FORMAT (1X, 'DIFFUSIVITY OF FUEL INTO AIR IS ', E15.7, ' SQUARE',

2 ' METERS/SECOND' ,l, ' AT ', E12.6, ' KELVIN' )

WRITE (LDOUT,90) TDROP(1)

90 FORMAT (1X, 'STARTING TEMPERATURE OF THE LIQUID DROP = ', $2 \quad$ E15.7, ' KELVIN' )

WRITE (LDOUT,95) NX

95 FORMAT (1X,' NX=', I6)

PI $=\operatorname{ACOS}(-1$.

$\mathrm{C}$

IF (ILAMDA .EQ. 1) THEN

LLAMDA $=$.TRUE.

ELSE

LLAMDA $=$. FALSE

END IF

C !! FOR NOW, ONLY USE LONG OUTPUT !!!

C IF (ILONG .EQ. 1) THEN

LLONG $=$.TRUE.

C ELSE

$\mathrm{C} \quad \mathrm{LLONG}=$.FALSE.

C END IF

LDROP $=$.FALSE .

EPS $=$ EPSF $*$ DX $(1) \quad$ !ONE-TENTH OF A GRID POINT IS SMALL

C FOR EACH ADDITIONAL DROP, READ IN THE PERTENANT INFO.

DO I $=2$, NODA, 1

READ (INP,130) OUTFIL(I) 


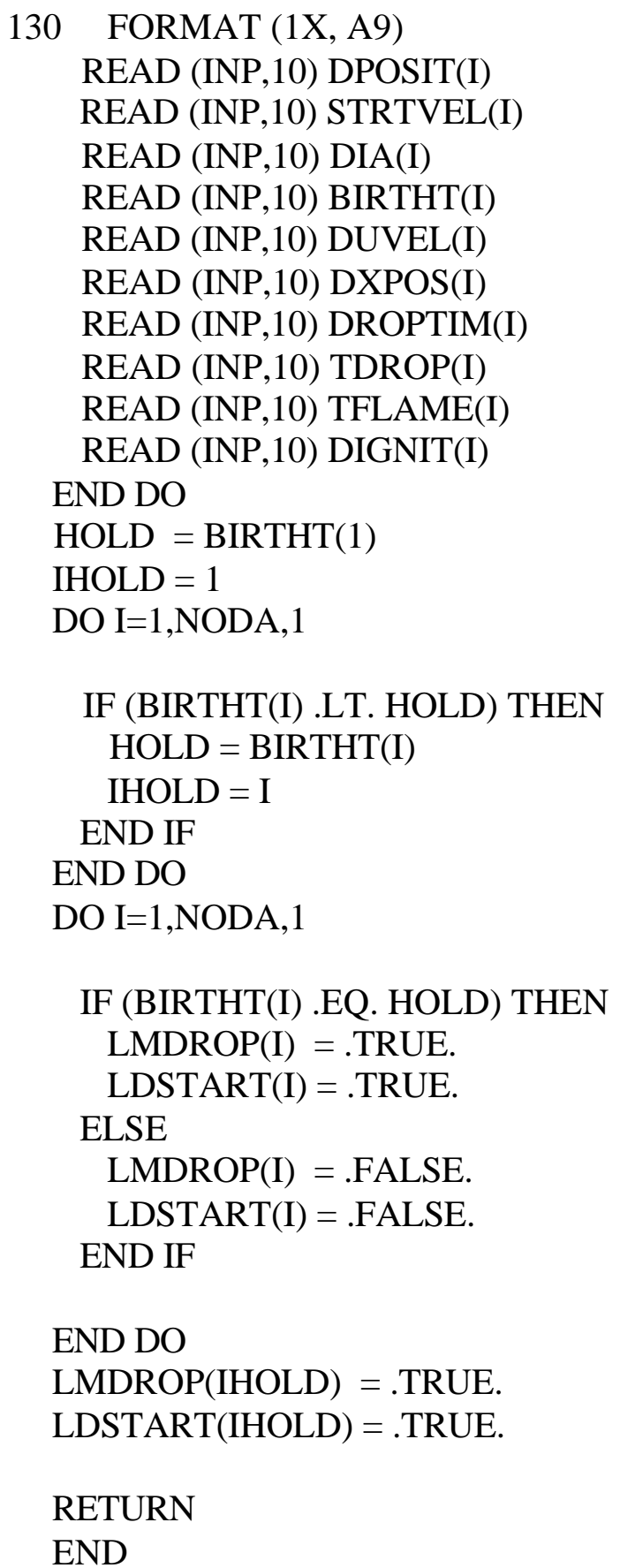


SUBROUTINE DROPSTART (BIRTHT, DIAI, DTDROPF, DTIMEI, DTM, DUVELI,

2 DXPOSI, DYPOSITI, DYVELOCI, HOLD, IENS, LDOUT, LDSTARTI, LLONG,

3 LMDOUT, LMDROPI, NEVENTS, NOD, NODA, PI, RHOL, TDROPI,

4 DIA, DRMASS, DROPTIME, DTDROP, DUVEL, DXPOS, DYPOSIT,

5 DYVELOC, LALLIGN, LALLST, LDFINSH, LDROP, LDSTART,

6 LHEATUP, LMDIG, LMDROP, TDARRAY, TDROP, TLAST, TNEXT)

$\mathrm{C} \quad * * * * * * * * * * * * * * * * * * * * * * * * * * * * * * * * * * * * * * * * * * * * * * * * * * * * * * * * * * * * * * * * * * *$

C LAST UPDATED 03/30/00 AT 11:30

C MODIFIED FOR MULTIPLE DROPS

C WRITTEN ON 11/16/99

C JRS'S PROGRAM TO RESET THE DROP VARIABLES FOR EACH

ENSEMBLE

C THE SUFFIX -I MEANS INITIAL VALUE TO BE RESET TO

IMPLICIT NONE

REAL BIRTHT(NOD), DIA(NOD), DIAI(NOD), DRMASS(NOD)

REAL DROPTIME(NOD), DUVEL(NOD)

REAL DTDROP, DTDROPF, DTIMEI(NOD), DTM, DUVELI(NOD), DXPOS(NOD)

REAL DXPOSI(NOD), DYPOSIT(NOD), DYPOSITI(NOD), DYVELOC(NOD)

REAL DYVELOCI(NOD), HOLD

INTEGER I, IENS

LOGICAL LALLIGN, LALLST, LDFINSH(NOD)

INTEGER LDOUT

LOGICAL LDROP, LDSTART(NOD), LDSTARTI(NOD), LHEATUP(NOD)

LOGICAL LLONG, LMDIG(NOD)

INTEGER LMDOUT(NOD)

LOGICAL LMDROP(NOD), LMDROPI(NOD)

INTEGER NEVENTS, NOD, NODA

REAL PI, RHOL, TDARRAY (NOD,10)

REAL TDROP(NOD), TDROPI(NOD), TLAST(NEVENTS), TNEXT(NEVENTS)

DO I=1,NODA, 1

$\mathrm{DIA}(\mathrm{I}) \quad=$ DIAI $(\mathrm{I})$

DYPOSIT $(\mathrm{I})=$ DYPOSITI $(\mathrm{I})$

DYVELOC $(\mathrm{I})=$ DYVELOCI $(\mathrm{I})$

DUVEL(I) = DUVELI (I)

DXPOS(I) $=$ DXPOSI $(\mathrm{I})$

DROPTIME(I) = DTIMEI (I)

TDROP(I) $=$ TDROPI $(\mathrm{I})$

DRMASS $(\mathrm{I})=$ RHOL $*$ PI $*$ DIA(I $* * 3 / 6.0$

TDARRAY $(\mathrm{I}, 1)=240.0$

TDARRAY $(\mathrm{I}, 2)=245.0$ 
TDARRAY $(\mathrm{I}, 3)=250.0$

TDARRAY $(I, 4)=255.0$

TDARRAY $(\mathrm{I}, 5)=260.0$

TDARRAY $(\mathrm{I}, 6)=265.0$

TDARRAY $(\mathrm{I}, 7)=270.0$

TDARRAY $(\mathrm{I}, 8)=275.0$

TDARRAY $(\mathrm{I}, 9)=280.0$

TDARRAY $(\mathrm{I}, 10)=285.0$

END DO

LDROP $=$.FALSE.

LALLST $=$.FALSE.

LALLIGN $=$.FALSE.

TLAST $(5)=$ HOLD

$\operatorname{TNEXT}(5)=$ HOLD

DO I=1,NODA, 1

LDFINSH $(\mathrm{I})=$.FALSE.

$\operatorname{LMDROP}(\mathrm{I})=\operatorname{LMDROPI}(\mathrm{I})$

$\operatorname{LDSTART}(\mathrm{I})=\operatorname{LDSTARTI}(\mathrm{I})$

LHEATUP $(\mathrm{I})=$.TRUE.

LMDIG(I) =.FALSE.

END DO

DTDROP $=$ DTDROPF $*$ DTM

WRITE (LDOUT,5) IENS

5 FORMAT (1X,'DROPLET NO. ', I7)

C $\quad * * * * * * * * * * * * * * * * * * * * * * * * * *$ ADD TEMP. TO DEBUG !!!

C WRITE (LDOUT, 20) DIA, DROPTIME, BIRTHT, DUVEL, DYVELOC

C 20 FORMAT (1X, 'INITIAL DIA = ', E10.4, ' Age of the drop when',

C 2 ' it is born into ODT = ', E10.4 ,/,1X, 'Time at which the',

C 3 ' droplet appears in the ODT domain = ', E12.6, /, 1X,

C $4 \quad$ ' U = ', E12.6, ' V = ', E12.6)

C WRITE (LDOUT, 30) DXPOS, DYPOSIT

C 30 FORMAT (1X,'INITIAL X POSITION = ',E12.6, ' INITIAL Y = ',E12.6)

C WRITE (LDOUT, 50) DTDROPF

C 50 FORMAT (1X, 'FACTOR FOR COMPUTING DROPLET TIME STEP FROM',

C 5 ' MOLECULAR STEP $(\mathrm{dtm})=$ ', E10.4)

$\mathrm{C} * * * * * * * * * * * * * * * * * * * * * * * * * *$ END OF TEMP DEBUG OUTPUT

WRITE (LDOUT,10)

10 FORMAT ( 4X, 'TIME',11X, 'U', 13X, 'V', 13X, 'X', 13X, 'Y',

2 13X, 'ODT TIME') 


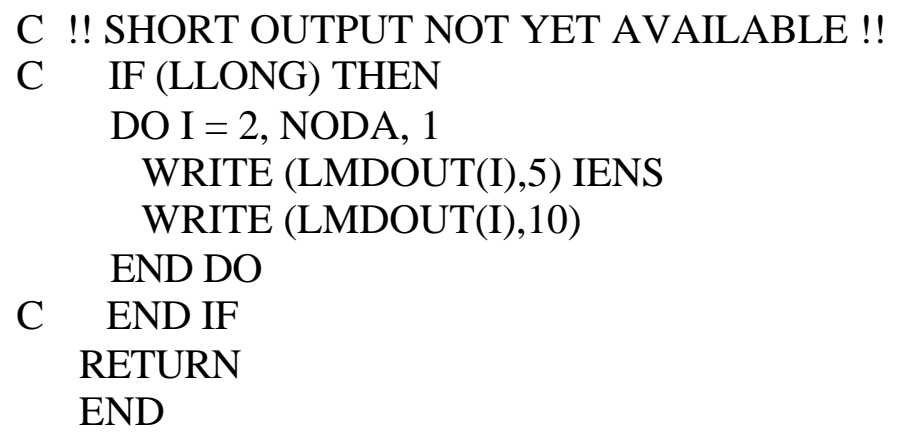


SUBROUTINE EFLAMET (DIAP, HEATCOM, KDROP, LDOUT, LIGNIT, LLONG,

2 LOUT, RHOL, STOICH, TDROP, TFLAME, TINFIN, YOINFIN, 3 AIRNU, CPFGAS, HEFF, RHOGAS, TAUP, TTHIRD)

$\mathrm{C} * * * * * * * * * * * * * * * * * * * * * * * * * * * * * * * * * * * * * * * * * * * * * * * * * * * * * * * * * * * * * * * * * * * * *$

C LAST UPDATED ON 02/03/00 AT 5:00 PM

C WRITTEN BY JRSCHMIDT ON 02/02/00

C T FLAME = FLAME SHEET TEMPERATURE, START WITH A GUESS

C $\quad$ T DROP $=$ DROP TEMP.

IMPLICIT NONE

REAL AIRMU, AIRNU, CPGAS, CPFGAS

REAL DIAP, HEATCOM, HEFF

INTEGER ITT, KDROP

LOGICAL LIGNIT, LITT, LLONG

INTEGER LDOUT, LOUT

REAL RHOGAS, RHOL, STOICH

REAL TAUP, TDROP, TFLAME, TFNEW, TINFIN, TTHIRD

REAL XFS, YCONST, YFS, YOINFIN

C CLAUSIUS-CLAPEYRON EQUATION. PRESSURE IS IN ATMOSPHERES

C GIVES MOLE FRACTION IN GAS AT THE SURFACE

C GAS CONSTANT R $=8.314 \mathrm{~J} / \mathrm{MOL} / \mathrm{K}$

C FROM EPA REPORT. AVE. MOLECULAR WEIGHT $=0.136 \mathrm{KG} / \mathrm{MOL}$,

C BOILING TEMP. $=400 \mathrm{~K}$, HEAT OF VAPORIZATION $=350,000 \mathrm{~J} / \mathrm{KG}$

$\mathrm{XFS}=\operatorname{EXP}(5725.283 *(0.0025-1 . / \mathrm{TDROP}))$

C $30 \%$ EXCESS AIR, ASSUMING 79\% N2, 21\% O2, COMPLETE BURNING.

C NATURAL GAS IS BETWEEN 95-98 \% METHANE (CH4)

YCONST $=1738.08 *$ XFS

YFS $=$ YCONST $/(353.866 *(1 .-X F S)+$ YCONST $)$

C !!!!! AIRNU MUST BE EVALUATED AT 1/3 RULE -SIRIGNANO

C YOINFIN WAS CALCULATED TO BE 5.1465342

LITT $=$.TRUE.

$\mathrm{ITT}=1$

DO WHILE ((LITT) .AND. (ITT .LE. 35))

TTHIRD $=$ TDROP $+($ TFLAME - TDROP $) / 3$.

CALL ETMPVAR (LDOUT, LLONG, TTHIRD, AIRMU, AIRNU, CPGAS,

2 CPFGAS, RHOGAS)

$\mathrm{HEFF}=(1 .-\mathrm{YFS}) *(\mathrm{CPGAS} *(\mathrm{TINFIN}-\mathrm{TDROP})$ 


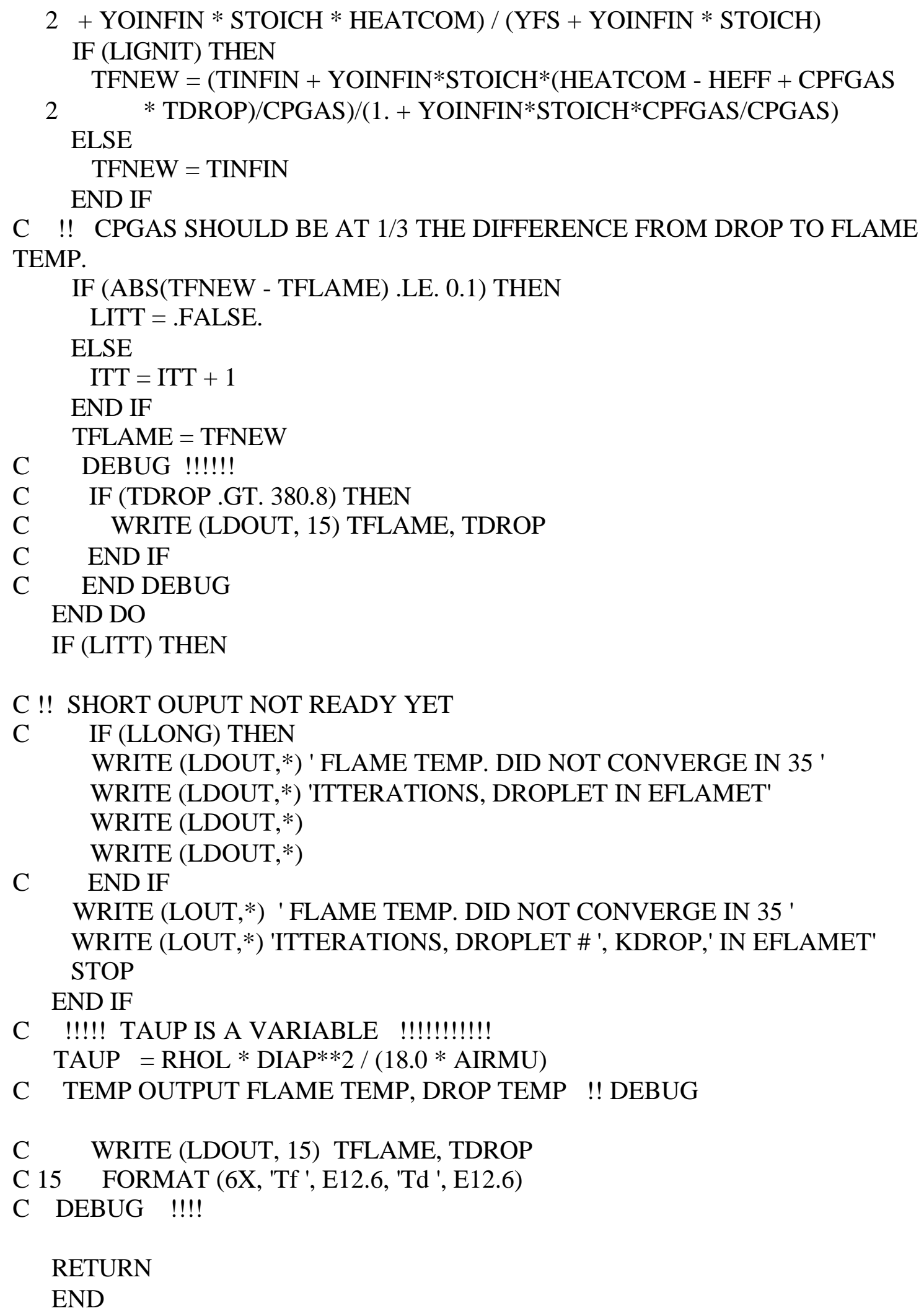


SUBROUTINE ETMPVAR (LDOUT, LLONG, TEMP, VISC, AIRNU, 2 CPGAS, CPFGAS, DENSITY)

$\mathrm{C} * * * * * * * * * * * * * * * * * * * * * * * * * * * * * * * * * * * * * * * * * * * * * * * * * * * * * * * * * * * * * * * * * * * * * *$

C LAST UPDATED 03/30/00 AT 7:30 PM

C !!!!! TEMPARARILY MODIFIED ON 02/17/00 TO TRY CPF = C10H21

C CREATED BY JOHN SCHMIDT ON 01/24/00

C THIS ROUTINE TAKES A TEMP AND FINDS THE CORESPONDING VISCOSITY (NU),

C THE HEAT CAPACITY OF THE GAS PHASE (CPGAS), AND THE HEAT CAPACITY OF

C THE GAS PHASE FUEL (CPFGAS) AT THAT TEMPERATURE

C R GAS $=$ MOLAR GAS CONSTANT UNITS J/G-MOL/K

IMPLICIT NONE

REAL AIRNU, CPFGAS, CPGAS, CP1, CP2, CP3

REAL DENSITY, EMW,EXMW, HMW

INTEGER LDOUT

LOGICAL LLONG

REAL SLOPE, TEMP, TMW, VISC

C FOR NOW ASSUME CPFGAS = CPGAS

C FIND TEMP RANGE AND LINEARLY INTERPOLATE

C IMPORTANT VARIABLES

C VISCOSITY OF AIR IN KG/S/M

C DENSITY OF AIR IN KG/M**3

C HEAT CAPACITY IN J/KG/K

IF (TEMP .LE. 299.8166) THEN

VISC $=0.000018602$

DENSITY $=1.175754964$

CPGAS $=1004.832$

ELSE IF (TEMP .LE. 533.15) THEN

SLOPE $=(0.000027696-0.000018602) / 233.3333$

VISC $=0.000018602+$ SLOPE $*($ TEMP -299.8166$)$

$\mathrm{SLOPE}=(0.659960552-1.175754964) / 233.3333$

DENSITY $=1.175754964+$ SLOPE $*($ TEMP -299.8166$)$

SLOPE $=(1013.2056-1004.832) / 233.3333$

CPGAS $=1004.832+\mathrm{SLOPE} *(\mathrm{TEMP}-299.8166)$

ELSE IF (TEMP .LE. 588.7055) THEN

SLOPE $=(0.000029763-0.000027696) / 55.5555$

$\mathrm{VISC}=0.000027696+\mathrm{SLOPE} *(\mathrm{TEMP}-533.15)$

$\mathrm{SLOPE}=(0.597488558-0.659960552) / 55.5555$ 


$$
\begin{aligned}
& \text { DENSITY }=0.659960552+\text { SLOPE } *(\text { TEMP }-533.15) \\
& \text { CPGAS }=1013.2056 \\
& \text { ELSE IF (TEMP .LE. 644.2611) THEN } \\
& \text { SLOPE }=(0.000031417-0.000029763) / 55.5555 \\
& \mathrm{VISC}=0.000029763+\mathrm{SLOPE} *(\mathrm{TEMP}-588.7055) \\
& \mathrm{SLOPE}=(0.546229486-0.597488558) / 55.5555 \\
& \text { DENSITY }=0.597488558+\text { SLOPE } *(\text { TEMP }-588.7055) \\
& \text { SLOPE }=(1017.3924-1013.2056) / 55.5555 \\
& \text { CPGAS }=1013.2056+\text { SLOPE } *(\text { TEMP }-588.7055) \\
& \text { ELSE IF (TEMP .LE. 699.8166) THEN } \\
& \text { SLOPE }=(0.00003307-0.000031417) / 55.5555 \\
& \text { VISC }=0.000031417+\mathrm{SLOPE} *(\mathrm{TEMP}-644.2611) \\
& \text { SLOPE }=(0.502979644-0.546229486) / 55.5555 \\
& \text { DENSITY }=0.546229486+\text { SLOPE } *(\text { TEMP }-644.2611) \\
& \mathrm{SLOPE}=(1021.5792-1017.3924) / 55.5555 \\
& \text { CPGAS }=1017.3924+\text { SLOPE } *(\text { TEMP }-644.2611) \\
& \text { ELSE IF (TEMP .LE. 755.3722) THEN } \\
& \text { SLOPE }=(0.000035137-0.00003307) / 55.5555 \\
& \mathrm{VISC}=0.00003307+\mathrm{SLOPE} *(\mathrm{TEMP}-699.8166) \\
& \text { SLOPE }=(0.47254457-0.502979644) / 55.5555 \\
& \text { DENSITY }=0.502979644+\text { SLOPE } *(\text { TEMP }-699.8166) \\
& \text { SLOPE }=(1025.766-1021.5792) / 55.5555 \\
& \text { CPGAS }=1021.5792+\text { SLOPE } *(\text { TEMP }-699.8166) \\
& \text { ELSE IF (TEMP .LE. 810.9277) THEN } \\
& \text { SLOPE }=(0.000036791-0.000035137) / 55.5555 \\
& \mathrm{VISC}=0.000035137+\mathrm{SLOPE} *(\mathrm{TEMP}-755.3722) \\
& \mathrm{SLOPE}=(0.44050765-0.47254457) / 55.5555 \\
& \text { DENSITY }=0.47254457+\text { SLOPE } *(\text { TEMP }-755.3722) \\
& \text { SLOPE }=(1029.9528-1025.766) / 55.5555 \\
& \text { CPGAS }=1025.766+\text { SLOPE } *(\text { TEMP }-755.3722) \\
& \text { ELSE IF (TEMP .LE. 922.0388) THEN } \\
& \text { SLOPE }=(0.000040098-0.000036791) / 111.1111 \\
& \text { VISC }=0.000036791+\text { SLOPE } *(\text { TEMP }-810.9277) \\
& \text { SLOPE }=(0.381239348-0.44050765) / 111.1111 \\
& \text { DENSITY }=0.44050765+\text { SLOPE } *(\text { TEMP }-810.9277) \\
& \text { SLOPE }=(1038.3264-1029.9528) / 111.1111 \\
& \text { CPGAS }=1029.9528+\text { SLOPE } *(\text { TEMP }-810.9277) \\
& \text { ELSE IF (TEMP .LE. 1033.15) THEN } \\
& \text { SLOPE }=(0.000043405-0.000040098) / 111.1111 \\
& \mathrm{VISC}=0.000040098+\mathrm{SLOPE} *(\mathrm{TEMP}-922.0388) \\
& \text { SLOPE }=(0.339591352-0.381239348) / 111.1111 \\
& \text { DENSITY }=0.381239348+\text { SLOPE } *(\text { TEMP }-922.0388) \\
& \text { SLOPE }=(1050.8868-1038.3264) / 111.1111 \\
& \text { CPGAS }=1038.3264+\operatorname{SLOPE} *(\text { TEMP }-922.0388)
\end{aligned}
$$


ELSE IF (TEMP .LE. 1144.2611) THEN

SLOPE $=(0.000046298-0.000043405) / 111.1111$

VISC $=0.000043405+$ SLOPE $*($ TEMP -1144.2611$)$

SLOPE $=(0.307554432-0.339591352) / 111.1111$

DENSITY $=0.339591352+$ SLOPE $*($ TEMP -1144.2611$)$

SLOPE $=(1063.4472-1050.8868) / 111.1111$

CPGAS $=1050.8868+$ SLOPE $*($ TEMP -1144.2611$)$

ELSE IF (TEMP .LE. 1255.3722) THEN

SLOPE $=(0.000049605-0.000046298) / 111.1111$

VISC $=0.000046298+$ SLOPE $*($ TEMP -1255.3722$)$

SLOPE $=(0.28032305-0.307554432) / 111.1111$

DENSITY $=0.307554432+$ SLOPE $*($ TEMP -1255.3722$)$

SLOPE $=(1076.0076-1063.4472) / 111.1111$

CPGAS $=1063.4472+$ SLOPE $*($ TEMP -1255.3722$)$

ELSE IF (TEMP .LE. 1366.4833) THEN

SLOPE $=(0.000052499-0.000049605) / 111.1111$

VISC $=0.000049605+$ SLOPE $*($ TEMP -1366.4833$)$

SLOPE $=(0.257897206-0.28032305) / 111.1111$

DENSITY $=0.28032305+$ SLOPE $*($ TEMP -1366.4833$)$

SLOPE $=(1088.5680-1076.0076) / 111.1111$

CPGAS $=1076.0076+$ SLOPE $*($ TEMP -1366.4833$)$

ELSE

VISC $=0.000052499$

DENSITY $=0.257897206$

CPGAS $=1088.5680$

C !! SHORT OUTPUT NOT YET INSTALLED

C IF (LLONG) THEN

WRITE (LDOUT, *) ' FLAME TEMPERATURE TOO HIGH '

WRITE (LDOUT, *) ' VISCOSITY IN ETMPVAR'

C END IF

END IF

C VALUES OF DENSITY, VISCOSITY, AND HEAT CAPACITY ARE

C FROM "AIR POLLUTION CONTROL, A DESIGN APPROACH"

C BY C.D. COOPER \& F.C. ALLEY. PAGE 654

$\mathrm{C}$

C $\quad \mathrm{NU}=$ VISCOSITY/DENSITY $\left[\mathrm{M}^{* * 2 / \mathrm{S}]}\right.$

AIRNU $=$ VISC $/$ DENSITY

C $\quad$ RGAS $=8.3144 \quad$ ! UNITS J/G-MOL/K

HMW $=100.204$

$\mathrm{EMW}=106.1674$ 
$\mathrm{TMW}=91.13258$

$\mathrm{EXMW}=141.27670$

$\mathrm{C} * * * * * * * * * * * * * * * * * * * * * * * * * * * * * * * * * * * * * * * * * * * * * * * * * * * * * * * * * * * * * * * * * * * * * * * *$

IF (TEMP .GE. 200.) THEN

IF (TEMP .LE. 1000.) THEN

C $\quad 200-1000 \mathrm{~K}$

C HEPTANE

CP1 $=11.15325-0.009494155 *$ TEMP $+0.0001955712 *$ TEMP $* * 2$

$2-0.0000002497525 * \mathrm{TEMP}^{*} * 3+9.848732 \mathrm{D}-11 * \mathrm{TEMP}^{*} * 4$

C ETHYLBENZENE

$\mathrm{CP} 2=3.51535+0.01781457 * \mathrm{TEMP}+0.000118934 * \mathrm{TEMP} * * 2$

$2 \quad-1.756398 \mathrm{D}-07 *$ TEMP $* * 3+7.320611 \mathrm{D}-11 *$ TEMP $* * 4$

C $\mathrm{C} 10 \mathrm{H} 21$

$\mathrm{CP} 3=3.089701+0.08411795 * \mathrm{TEMP}+1.590184 \mathrm{D}-05 * \mathrm{TEMP} * * 2$

$2-7.238793 \mathrm{D}-08 *$ TEMP**3 + 3.226693D-11*TEMP**4

ELSE IF (TEMP .LE. 6000) THEN

C $\quad 1000-6000 \mathrm{~K}$

C HEPTANE

$\mathrm{CP} 1=18.53547+0.03914205 * \mathrm{TEMP}-1.380303 \mathrm{D}-05 * \mathrm{TEMP} * * 2$

2 + 2.224039D-09* TEMP**3 - 1.334526D-13* TEMP**4

C ETHYLBENZENE

$\mathrm{CP} 2=15.57608+0.03230646 * \mathrm{TEMP}-1.190027 \mathrm{D}-05 * \mathrm{TEMP} * * 2$

$2+1.967925 \mathrm{D}-09 *$ TEMP $* * 3-1.199112 \mathrm{D}-13 *$ TEMP $* * 4$

$\mathrm{CP} 3=21.32213+0.06157352 *$ TEMP $-2.384948 \mathrm{D}-05 * \mathrm{TEMP}^{*} * 2$

$2+4.220912 \mathrm{D}-09 * \mathrm{TEMP} * * 3-2.788931 \mathrm{D}-13 * \mathrm{TEMP}^{*} * 4$

ELSE

PRINT*, ' TEMP. IS OVER 6000 K'

END IF

ELSE

PRINT**, ' TEMP. IS LESS THEN 200 K'

END IF

C DONE!! TEMPARARY TO CHECK OUT USING CP3 AS AN AVERAGE

$\mathrm{CPFGAS}=0.399 * \mathrm{CP} 1 / \mathrm{HMW}+0.289 * \mathrm{CP} 2 / \mathrm{EMW}+0.312 * \mathrm{CP} 3 / \mathrm{EXMW}$

C $\quad$ CPFGAS $=\mathrm{CP} 3 / \mathrm{EXMW}$

C $\quad$ RGAS * $\mathrm{CP} * 1000$

CPFGAS $=8314.4 *$ CPFGAS

RETURN

END 
REAL FUNCTION DPOSROOT (AIRNU, BULKVEL, DELTAT, DIAP, DUVEL, 2

LOUT, TAUP, UFLUID)

$\mathrm{C} * * * * * * * * * * * * * * * * * * * * * * * * * * * * * * * * * * * * * * * * * * * * * * * * * * * * * * * * * * * * * * * *$
$\mathrm{C}$ LAST MODIFIED ON $11 / 19 / 99 @ 11: 00$
$\mathrm{C}$
DIFFERENCES

C IN MAPPING THE LAGRANGIAN MODEL FOR THE PARTICL AND THE EULARIAN

C CONVERSION FACTOR IN THE ODT MODEL

C THIS PROGRAM FINDS THE 'POS'ITIVE 'ROOT' OF A QUADRADIC EQUATION

C AND RETURNS IT AS A REAL VALUE. IF THERE ARE TWO POSITIVE ROOTS,

C THE SMALLEST IS THE ONE OF INTEREST. IF THERE ARE NO POSITIVE

C ROOTS (I.E. ONLY NEGATIVE ROOTS, OR IMAGINARY ROOTS) THEN CHOOSE

C DELTAT AS THE CORRECT TIME.

C $\quad \mathrm{AX}^{* * 2} 2+\mathrm{BX}+\mathrm{C}=0$

C HOWEVER, THIS FUNCTION IS WRITTEN TO SPECIFICALLY SOVLE FOR THE

C POSITIVE ROOT OF THIS EQUATION

C (DUVEL - UFLUID)*FXCOEF*T**2/(2*TAUP) - DUVEL $* \mathrm{~T}+$ DELTAX $=0$.

C SIGNIFICANT VARIABLES

$\mathrm{C}$ ATTWO = 'A' 'T'IMES 'TWO' -- USEFUL IN NUMERIC IMPLEMENTATION

C TERM $=\mathrm{B} * * 2-2 *$ ATTWO $* \mathrm{C}$

C $\quad$ ROOT1 $=(-\mathrm{B}+\mathrm{SQRT}(\mathrm{TERM})) / \mathrm{ATTWO}$

C $\quad$ ROOT2 $=(-B-S Q R T(T E R M)) /$ ATTWO

IMPLICIT NONE

REAL AIRNU, ATTWO, BULKVEL, CONST6

REAL DELTAT, DELTAX, DIAP, DUVEL, FXCOEF

INTEGER LOUT

REAL RENX, ROOT1, ROOT2, TAUP, TERM, UFLUID

IF (DELTAT .GT. 0.0) THEN

C USE CORRRECTION FACTOR GIVEN BY WANG \& STOCK

CONST6 = DUVEL - UFLUID

RENX $=$ DIAP $*$ ABS(CONST6) $/$ AIRNU

IF (RENX .LE. 1.0) THEN

C STOKES LAW 
$\mathrm{FXCOEF}=1.0$

ELSE IF (RENX .LE. 1000) THEN

C CORRECTION FACTOR

$\mathrm{FXCOEF}=1.0+0.15 *(\mathrm{RENX}) * * 0.687$

ELSE

WRITE (LOUT, *) ' PARTICLE Re X > 1000'

WRITE (LOUT,*) ' STOPPED IN DPOSROOT', RENX

STOP

END IF

ATTWO $=$ CONST $6 *$ FXCOEF $/$ TAUP

DELTAX $=$ BULKVEL $*$ DELTAT

IF (ATTWO .EQ. 0.)THEN

IF (DUVEL .GT. 0.0) THEN

DPOSROOT $=$ DELTAX $/$ DUVEL

ELSE

DPOSROOT $=$ DELTAT

END IF

ELSE

TERM $=$ DUVEL $* * 2-2.0 *$ ATTWO $*$ DELTAX

IF (TERM .GE. 0.) THEN

C NOT IMAGINARY ROOTS, -- REAL ROOTS

IF (TERM .EQ. 0.) THEN

C

REAL REPEATED ROOTS

DPOSROOT $=$ DUVEL $/$ ATTWO

IF (DPOSROOT .LE. 0.0) THEN

DPOSROOT $=$ DELTAT

END IF

ELSE

ROOT1 $=($ DUVEL + SQRT $($ TERM $)) /$ ATTWO

ROOT2 $=($ DUVEL - SQRT $($ TERM $)) /$ ATTWO

IF (ROOT1*ROOT2 .LT. 0.0) THEN

C TWO REAL ROOTS, DIFFERENT SIGNS. WANT THE POSITIVE

ONE

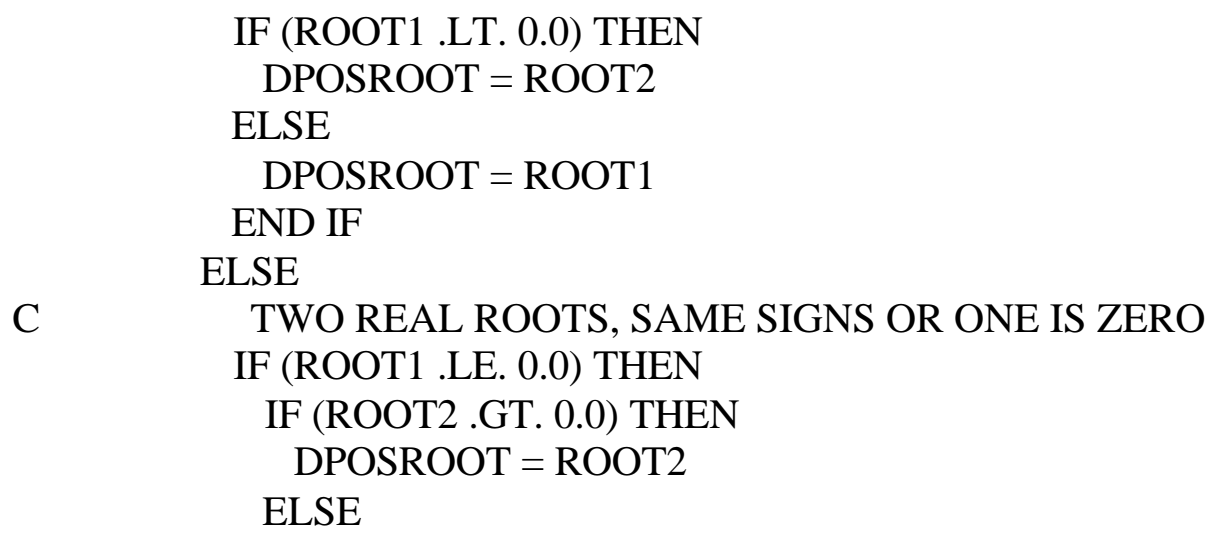




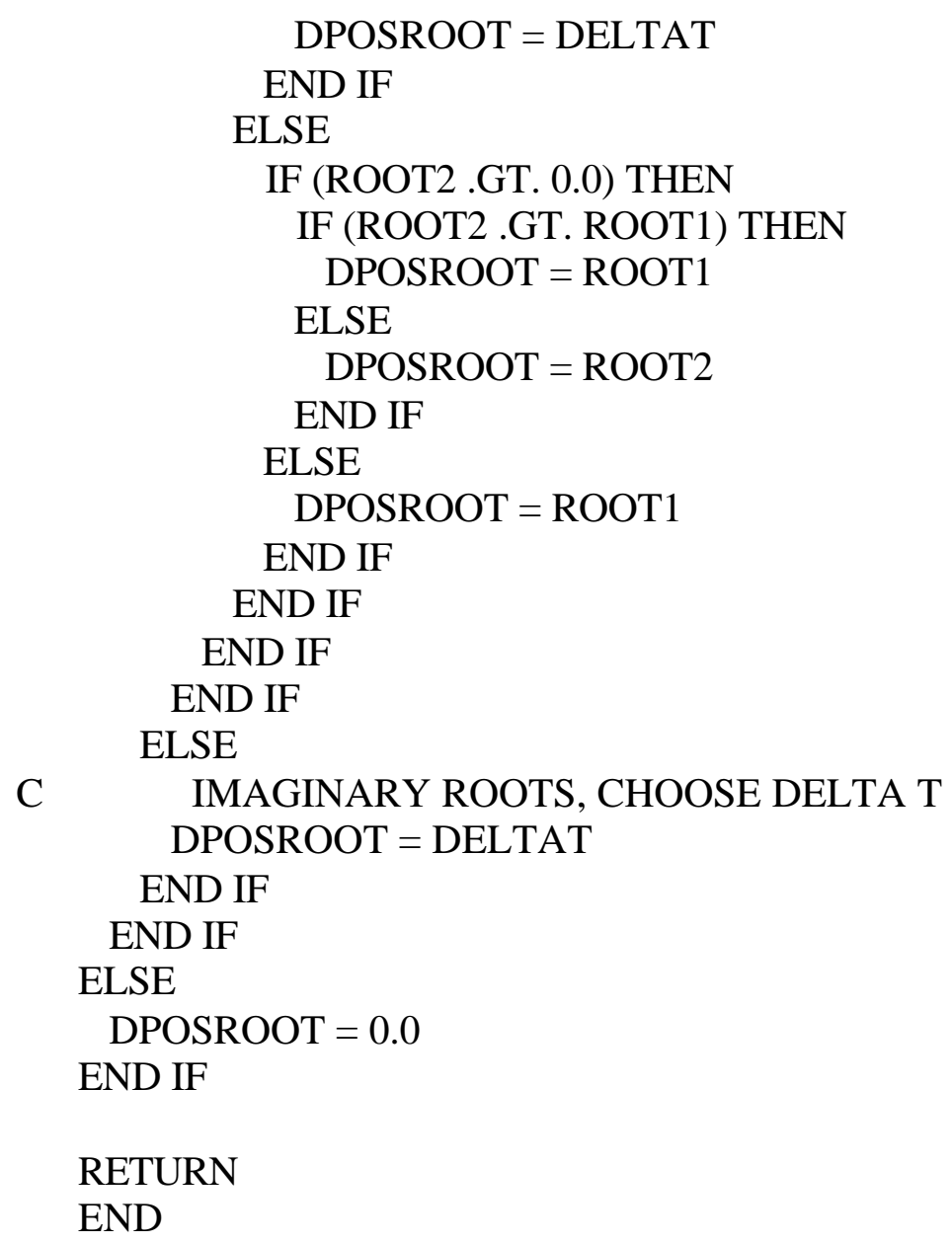


SUBROUTINE DUPDATE (AIRNU, ALAMDA, CPFGAS, DABZERO, DIAP, DRMASS,

2 DTDROP, GRAVAC, HEATVAP, HEATCAP, HEATCOM, HEFF, KDROP, LDOUT,

3 LHEATUP, LLAMDA, LOUT, NOD, PEPS, PI, RHOGAS, RHOL, STOICH,

4 STRTU, STRTVEL, TAUP, TDARRAY, TDROP, TEMPZERO, TFLAME, TINFIN,

5 TTHIRD, UFLUID, YOINFIN, DELTAX, DELTAY, DIANEW, UEND, VEND)

\footnotetext{
$\mathrm{C} * * * * * * * * * * * * * * * * * * * * * * * * * * * * * * * * * * * * * * * * * * * * * * * * * * * * * * * * * * * * * * * * * * *)$

C WRITTEM BY JRS FOR THE DROPLET MODEL. INTEGRATES THE DROPLET-

C DRAG LAW THE THE AMOUNT OF TIME STORED IN DELTA TIME.

C CALLED FROM MAIN ROUTINE ODTDRIV

C

C LAST UPDATED ON 03/03/00 AT 20:00

C

C UPDATED 02/02/00 MODIFIED FOR EVAPORATING DROPLETS

C LINEAR DROPLET-DRAG LAW WITH A CORRECTION FACTOR F

$C$ NOTE: THIS SUBPROGRAM IS USED WHEN THE DROPLET IS NOT IN AN EDDY

C HENCE, THERE IS NOT A V VELOCITY OF THE FLUID SO THE DIFF. EQN.

IS

C $\quad$ DV/DT $=-$ FY/TAUP $*(\mathrm{~V})-\mathrm{G}$

C DU/DT $=-$ FX/TAUP*(U-UFLUID)

C FOR THE SMALLEST TIME STEP, F, VFLUID, \& TAUP ARE CONST.

C SINCE WE ARE USING A LINEAR DROPLET DRAG LAW WITH A CORRECTION

C THE CORRECTION IN THE Y DIRECTION IS CONCIDERED INDEPENDENT OF THE

C CORRECTION IN THE X DIRECTION. F Y COEFF \& F X COEFF ARE INDEPENDENT

$\mathrm{C}$ STRT VEL $=$ THE INITIAL PARTICLE VELOCITY IN THE Y DIRECTION

$\mathrm{C}$ STRT U = INITIAL PARTICLE VELOCITY IN THE X DIRECTION

$\mathrm{C}$ DTDROP = TIME CHANGE OVER WHICH TO INTEGRATE

$\mathrm{C}$ DELTA Y $=$ CHANGE IN Y POSITION OF THE PARTICLE

$\mathrm{C}$ DELTA $\mathrm{X}=\mathrm{CHANGE}$ IN $\mathrm{X}$ POSITION OF THE PARTICLE

$\mathrm{C}$ V END = PARTICLE Y VELOCITY AT THE END OF THE INTEGRAL

$\mathrm{C}$ U END = PARTICLE $X$ VELOCITY AT END OF INTEGRAL

C CONST $4=$ TERM IN THE D.E.Q. NOT MULTIPLYING VELOCITY

$\mathrm{C}$ CONST $3=$ COEFFICIENT IN THE D.E.Q. MULTIPLYING VELOCIT ON RHS

C CONST1,8 $=$ CONVENIENT COEFFICIENT TO MAKE CALCULATIONS FASTER
} 
C F Y COEF $=$ CORRECTION FACTOR SO THE LINEAR DRAG LAW FITS BETTER

C F X COEF $=$ CORRECTION FACTOR IN THE X DIRECTION

$\mathrm{C}$ TAU P = DROPLET TIME CONSTANT, RELAXATION TIME

C U FLUID = VELOCITY OF THE FLUID IN THE X DIRECTION

C GRAV AC = GRAVITATIONAL ACCELERATION [UNITS] ----

$\mathrm{C}$ AIR NU $=$ KINEMATIC VISCOSITY OF THE GAS PHASE (AIR)

$\mathrm{C}$ DIA $\mathrm{P}=$ DIAMETER OF THE PARTICLE

C Y F S = MASS FRACTION OF THE FUEL AT DROPLET SURFACE

$\mathrm{C}$ X F S = MOLE FRACTION OF FUEL USING CLAUSIUS-CLAPEYRON EQN.

C Y O INFI = MASS FRACTION OF OXYGEN FAR FROM THE DROP

$\mathrm{C}$ T FLAME $=$ TEMPERATURE OF THE FLAME SHEET $[\mathrm{K}]$

$\mathrm{C}$ T DROP = TEMPERATURE OF THE DROP [K]

$\mathrm{C}$ T INFIN = TEMPERATURE FAR FROM THE DROP BUT IN THE COMPUTATIONAL CELL

C B MAS T $=$ THE SPALDING MASS TRANSFER NUMBER

$\mathrm{C} \quad \mathrm{D} \mathrm{AB}=$ GAS DIFFUSIVITY OF A IN B

$\mathrm{C}$ SCHMIDT $=$ SCHMIDT NUMBER OF GAS

$\mathrm{C} \quad \mathrm{RENT}=$ TOTAL REYNOLDS NO.

C $\mathrm{F}$ RE = FUNCTION OF REYNOLDS NO.

C BASE SH $=$ SHERWOOD NO. WITHOUT MASS TRANSFER (I.E. BASE)

$\mathrm{C}$ SH STAR $=$ MODIFIED SHERWOOD NO. FOR CALCULATIONS

$\mathrm{C} \quad$ PSI $=2 *$ DRATIO*DAB*LN $(1+\mathrm{B})$

$\mathrm{C}$ HEAT CAP $=$ THE LIQUID HEAT CAPACITY IN $[\mathrm{J} / \mathrm{KG} / \mathrm{K}]$

$\mathrm{C}$ CP GAS = AVERAGE GAS HEAT CAPACITY $[\mathrm{J} / \mathrm{KG} / \mathrm{K}]$

C CP F GAS = AVE. FUEL GAS HEAT CAPACITY $[\mathrm{J} / \mathrm{KG} / \mathrm{K}]$

C R GAS = MOLAR GAS CONSTANT, R $[\mathrm{J} / \mathrm{KG} / \mathrm{K}]$

C H EFF = THE ENTHALPY, H EFFECTIVE

C RHO GAS = DENSITY OF GAS

C $\quad$ E TMP VAR $=$ SUBROUTINE WHICH COMPUTES VARIATION AT $1 / 3$ TEMPERATURE

C Y CONST = VALUE FOR CONVERTING MOLE FRACTION TO MASS FRAC.

C DELTA D $=$ CHANGE IN DIAMETER OF THE DROP FOR DT DROP

$\mathrm{C}$ DELTA $\mathrm{M}=$ CHANGE IN MASS OF THE DROP

C DELTA T D = CHANGE IN TEMPERATURE OF THE DROP "

IMPLICIT NONE

REAL ALAMDA, AIRMU, AIRNU, BASESH, BLN, BMAST, BP1

REAL CONST1, CONST2, CONST4, CONST5

REAL CONST6, CONST7, CONST8

REAL CONSTX3, CONSTY3, CPFGAS, CPGAS

REAL DABAVE, DABZERO 
REAL DELTAD, DELTAM, DELTATD, DELTAX, DELTAY

REAL DIANEW, DIAP, DMNEW, DRMASS, DTDROP

REAL FRE, FXCOEF, FYCOEF, GRAVAC

REAL HEFF, HEATCAP, HEATCOM, HEATVAP

INTEGER ITT, KDROP, LDOUT

LOGICAL LHEATUP, LITT, LLAMDA

INTEGER LOUT, NOD

REAL PEPS, PI, RENT, RENX, RENY, RHOGAS, RHOL

REAL SCHMIDT, SHSTAR, STOICH, STRTU, STRTVEL

REAL TAUP, TDARRAY(NOD,10), TDNEW, TDROP, TEMPZERO, TFLAME, TFNEW

REAL TINFIN, TTHIRD, TVEL

REAL UEND, UFLUID, VEND

REAL XFS, YCONST, YFS, YOINFIN

C WHEN TIME IS ZERO, SET VELOCITIES AND DELTAS AND RETURN

IF (DTDROP .EQ. 0.0) THEN

DELTAY $=0.0$

DELTAX $=0.0$

VEND $=$ STRTVEL

UEND = STRTU

DIANEW $=$ DIAP

ELSE

C USE THE ANALYTIC SOLUTIONS TO THE CONST. COEFICIENT

DIFFERENTIAL

C EQUATIONS TO APPROZIMATE THE END POINT TO THE INTEGRAL

C USE CORRRECTION FACTOR GIVEN BY WANG \& STOCK

CONST6 $=$ STRTU - UFLUID

RENY $=$ DIAP $*$ ABS $($ STRTVEL $) /$ AIRNU

RENX $=$ DIAP $*$ ABS(CONST6) $/$ AIRNU

IF (RENY .LE. 1.0) THEN

C STOKES LAW

$\mathrm{FYCOEF}=1.0$

ELSE IF (RENY .LE. 1000) THEN

C CORRECTION FACTOR

$\mathrm{FYCOEF}=1.0+0.15 *(\mathrm{RENY}) * * 0.687$

ELSE

WRITE (LOUT, *) ' PARTICLE Re Y > 1000'

WRITE (LOUT,*) ' STOPPED IN DUPDATE', RENY

WRITE (LOUT, *) ' PARTICLE Re Y > 1000'

WRITE (LOUT,*) ' STOPPED IN DUPDATE', RENY

STOP 
END IF

IF (RENX .LE. 1.0) THEN

C STOKES LAW

$\mathrm{FXCOEF}=1.0$

ELSE IF (RENX .LE. 1000) THEN

C CORRECTION FACTOR

$\mathrm{FXCOEF}=1.0+0.15 *(\mathrm{RENX}) * * 0.687$

ELSE

WRITE (LOUT, *) ' PARTICLE Re X > 1000'

WRITE (LOUT, *) ' STOPPED IN DUPDATE', RENX

WRITE (LOUT, *) ' PARTICLE Re X > 1000'

WRITE (LOUT, *) ' STOPPED IN DUPDATE', RENX

STOP

END IF

C COMPUTE THE FINAL VELOCITY AND THE CHANGE IN POSITION IN THE Y

C DIRECTION OF THE PARTICLE

CONSTY3 $=$ FYCOEF $/$ TAUP

CONST4 $=$ GRAVAC $/$ CONSTY3

CONST $1=$ STRTVEL + CONST 4

CONST5 $=$ EXP $(-C O N S T Y 3 *$ DTDROP $)$

VEND $=$ CONST $1 *$ CONST5 - CONST4

DELTAY $=-$ CONST4 $*$ DTDROP $+($ CONST1 $/$ CONSTY3 $) *(1-$ CONST5 $)$

C COMPUTE THE FINAL VELOCITY AND CHANGE IN POSITION IN THE X

C DIRECTION OF THE PARTICLE

CONSTX3 $=$ FXCOEF $/$ TAUP

CONST8 $=$ EXP(-CONSTX3 $*$ DTDROP $)$

UEND $=$ UFLUID + CONST $6 *$ CONST 8

DELTAX $=($ CONST6 $/$ CONSTX3 $) *(1-$ CONST8 $)+$ UFLUID $*$ DTDROP

C ADJUST DROPLET DIAMETER AFTER MOVEMENT

$\mathrm{TVEL}=\mathrm{SQRT}((\mathrm{STRTVEL}) * * 2+(\mathrm{CONST} 6) * * 2)$

C AIRNU IS EVALUATED AT 1/3 RULE

RENT $=$ DIAP $*$ TVEL $/$ AIRNU

IF (RENT .LE. 1.0) THEN

C

$\mathrm{FRE}=1.0$ 
ELSE IF (RENT .LE. 400) THEN

C CORRECTION FACTOR

$\mathrm{FRE}=(\mathrm{RENT}) * * 0.077$

ELSE

WRITE (LOUT, *) ' PARTICLE Re T > 400'

WRITE (LOUT,*) ' STOPPED IN DUPDATE', RENT

WRITE (LOUT, *) ' PARTICLE Re T > 400'

WRITE (LOUT, *) ' STOPPED IN DUPDATE', RENT

STOP

END IF

BMAST $=($ CPFGAS $*($ TINFIN - TDROP $)+$

2 YOINFIN $*$ STOICH $*$ HEATCOM) / HEFF

$\mathrm{BP} 1=\mathrm{BMAST}+1$.

$\mathrm{BLN}=\mathrm{ALOG}(\mathrm{BP} 1)$

C FOR NOW RHOGAS IS THE DENSITY OF AIR AT THE 1/3 RULE TEMP. CALL ESCHMIDT(AIRNU, DABZERO, TTHIRD, TEMPZERO, 2 DABAVE, SCHMIDT)

$$
\begin{aligned}
& \text { BASESH }=1 .+\operatorname{FRE} *(1 .+\operatorname{RENT} * \operatorname{SCHMIDT}) * *(1 . / 3 .) \\
& \text { SHSTAR }=2 .+(\operatorname{BASESH}-2 .) * \operatorname{BMAST} /((\mathrm{BP} 1) * * 0.7 * \mathrm{BLN})
\end{aligned}
$$

IF (LHEATUP) THEN

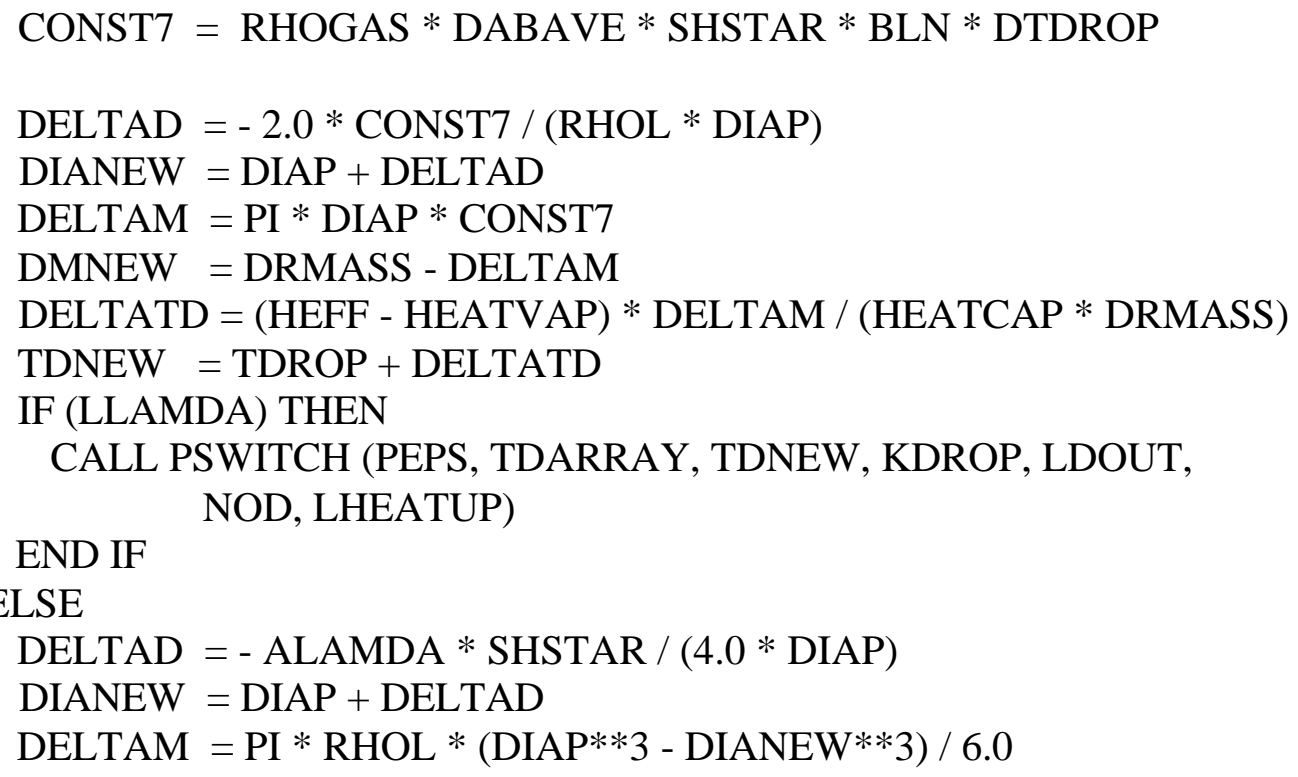
TEMP. 
C DOES NOT MATTER MUCH HERE BECAUSE IT HAS ACHIEVED THE C DEW POINT TEMPERATURE, AND THE MASS DOES NOT MATTER BECAUSE

C IT IS THE SAME AS DIAMETER

DMNEW $=$ DRMASS - DELTAM

DELTATD $=($ HEFF - HEATVAP $) *$ DELTAM $/($ HEATCAP $*$ DRMASS $)$

TDNEW $=$ TDROP + DELTATD

END IF

IF (DIANEW .LE. 0.0 .OR. DMNEW .LE. 0.0) THEN

DIANEW $=0.0$

DRMASS $=0.0$

TDROP $=$ TDNEW

ELSE

DRMASS $=$ DMNEW

TDROP $=$ TDNEW

END IF

C ALAMDA $=8$. $*$ RHOGAS $*$ DABAVE/RHOL $*$ BLN

$\mathrm{C}$ !! THIS BURNED TOO FAST SO DO DROPLET HEATING CASE !!

$\mathrm{C} \quad \mathrm{BASESH}=1 .+\mathrm{FRE} *(1 .+\mathrm{RENT} * \mathrm{SCHMIDT}) * *(1 . / 3$.

$\mathrm{C} \quad \mathrm{SHSTAR}=2 .+(\mathrm{BASESH}-2) /.((\mathrm{BP} 1) * * 0.7 * \mathrm{BLN} / \mathrm{BMAST})$

$\mathrm{C} \quad \mathrm{CONST} 2=\mathrm{DIAP} * * 2-\mathrm{PSI} * \mathrm{SHSTAR} *$ DTDROP

C IF (CONST2 .LE. 0.) THEN

$\mathrm{C} \quad$ DIANEW $=0$.

C ELSE

C $\quad$ DIANEW = SQRT(CONST2)

C END IF

\section{END IF}

$\mathrm{C} * * * * * * * * * * * * * * * * * * * * * * * * * * * * * * * * * * * * * * * * * * * * * * * * * * * * * * * * * * * * * * * * * * * * * *$

RETURN

END 


\author{
SUBROUTINE ESCHMIDT (AIRNU, DABZERO, TEMP, TEMPZERO,


SUBROUTINE PSWITCH (PEPS, TDARRAY, TDNEW, KDROP, LDOUT, 2 NOD, LHEATUP)

$\mathrm{C} \quad * * * * * * * * * * * * * * * * * * * * * * * * * * * * * * * * * * * * * * * * * * * * * * * * * * * * * * * * * * * * * * * * *$

C LAST UPDATED ON 2/25 AT 20:30

C MODIFIED FOR MULTIPLE DROPS

C WRITTEN BY JR SCHMIDT TO COMPUTE THE TIME TO SWITCH OVER TO THE

C PARAMETER LAMDA. WRITTEN ON 02/07/00

C CALLED FROM SUBROUTINE DUPDATE

$\mathrm{C}$

IMPLICIT NONE

REAL DELTA (10)

INTEGER ITEST, KDROP, LDOUT

LOGICAL LHEATUP, LTEST

INTEGER NOD, NON, NOP

REAL PEPS, TDARRAY(NOD,10), TDNEW

DELTA $(1)=$ TDARRAY $($ KDROP,2) - TDARRAY $(K D R O P, 1)$

$\operatorname{DELTA}(2)=$ TDARRAY $($ KDROP,3) - TDARRAY $(K D R O P, 2)$

DELTA(3) = TDARRAY $(\mathrm{KDROP}, 4)-$ TDARRAY $(\mathrm{KDROP}, 3)$

DELTA $(4)=$ TDARRAY $($ KDROP,5) - TDARRAY $(K D R O P, 4)$

DELTA $(5)=$ TDARRAY $(K D R O P, 6)-$ TDARRAY $(K D R O P, 5)$

$\operatorname{DELTA}(6)=$ TDARRAY $($ KDROP,7) - TDARRAY $($ KDROP,6)

$\operatorname{DELTA}(7)=$ TDARRAY $($ KDROP,8) - TDARRAY $(K D R O P, 7)$

DELTA $(8)=$ TDARRAY $($ KDROP,9) - TDARRAY $(K D R O P, 8)$

DELTA $(9)=$ TDARRAY $($ KDROP, 10) - TDARRAY $($ KDROP,9)

DELTA $(10)=$ TDNEW - TDARRAY $($ KDROP, 10$)$

$\mathrm{NON}=0$

NOP $=0$

LTEST $=$.TRUE.

DO ITEST $=1,10,1$

IF (DELTA (ITEST) .LT. 0.0) THEN

$\mathrm{NON}=\mathrm{NON}+1$

ELSE

$\mathrm{NOP}=\mathrm{NOP}+1$

END IF

IF (ABS(DELTA(ITEST)) .GT. PEPS) THEN

LTEST $=$.FALSE .

END IF

END DO

IF (LTEST .AND. ABS(NON - NOP) .LT.8) THEN

LHEATUP $=$.FALSE.

WRITE (LDOUT,10) 
10 FORMAT (1X, 'SWITCHED TO LAMDA')

END IF

C WHEN DONE, ADD TDNEW TO THE END OF THE LIST DO ITEST $=1,9,1$ TDARRAY $($ KDROP,ITEST $)=$ TDARRAY $($ KDROP,ITEST+1 $)$ END DO

TDARRAY $($ KDROP, 10$)=$ TDNEW

RETURN

END 
SUBROUTINE DROPOUT (DROPTIME, DUVEL, DVVELOC, DXPOS, 2 DYPOSIT, LDOUT, ODTTIME)

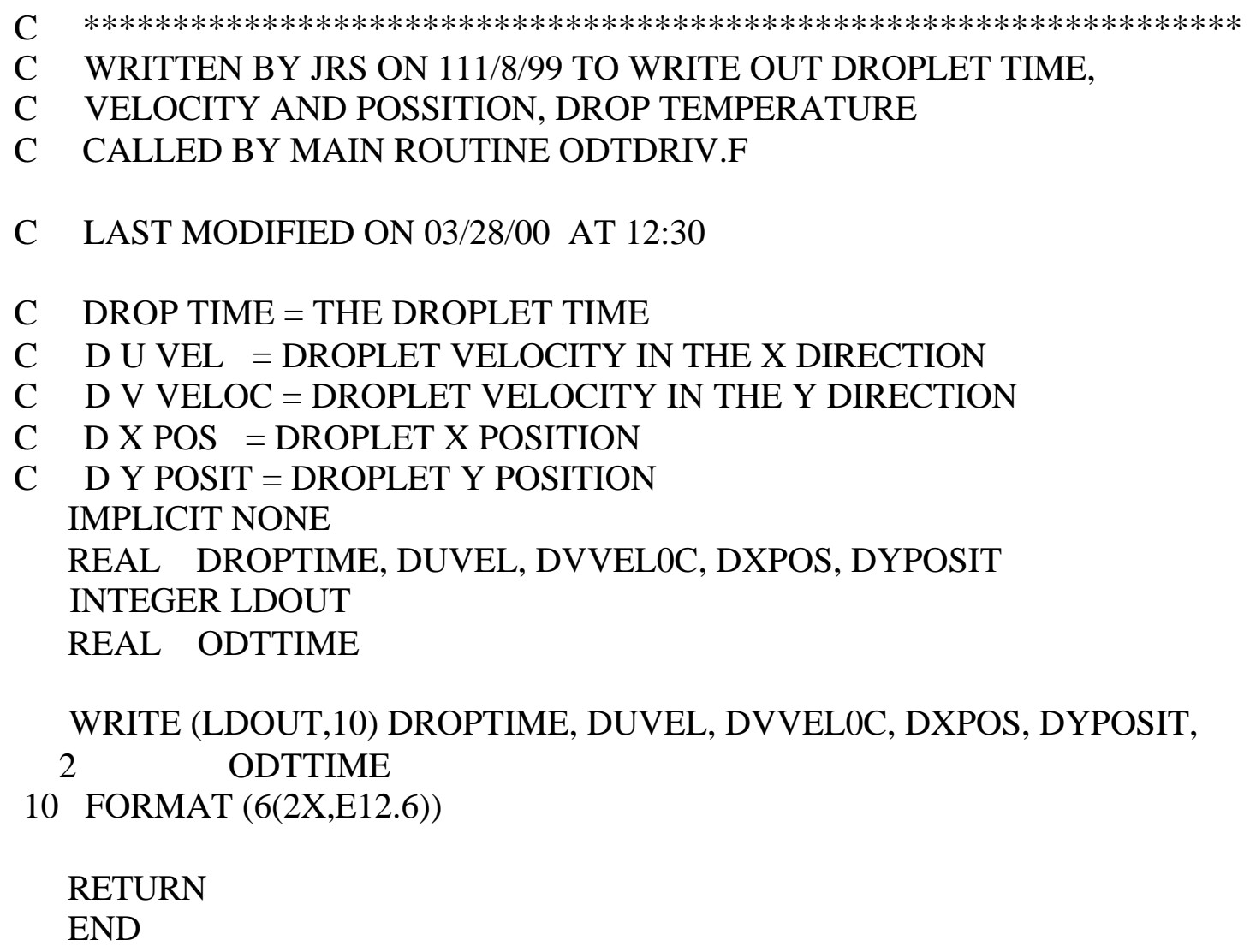


SUBROUTINE TDROPOUT (DROPTIME, DUVEL, DVVELOC, DXPOS, 2 DYPOSIT, LDOUT, ODTTIME, DIAP, TDROP, TFLAME)

\footnotetext{
$\mathrm{C} \quad * * * * * * * * * * * * * * * * * * * * * * * * * * * * * * * * * * * * * * * * * * * * * * * * * * * * * * * * * * * * * * * * * *$

C WRITTEN BY JRS ON 111/8/99 TO WRITE OUT DROPLET TIME,

C VELOCITY AND POSSITION, DROP TEMPERATURE

C CALLED BY MAIN ROUTINE ODTDRIV.F

C LAST MODIFIED ON 03/28/00 AT 12:30

C $\quad$ DROP TIME $=$ THE DROPLET TIME

$\mathrm{C}$ D U VEL = DROPLET VELOCITY IN THE X DIRECTION

C D V VELOC $=$ DROPLET VELOCITY IN THE Y DIRECTION

C $\quad$ D X POS $=$ DROPLET X POSITION

C D Y POSIT $=$ DROPLET Y POSITION

IMPLICIT NONE

REAL DIAP, DROPTIME, DUVEL, DVVELOC, DXPOS, DYPOSIT

INTEGER LDOUT

REAL ODTTIME, TDROP, TFLAME

WRITE (LDOUT,10) DROPTIME, DUVEL, DVVEL0C, DXPOS, DYPOSIT, 2 ODTTIME, DIAP, TDROP, TFLAME

10 FORMAT (6(2X,E12.6), 3(1X, E11.5) )

RETURN

END
} 
SUBROUTINE INPVELOC (LOUT, NNSC, NX, SCALINIT)

C CREATED ON 6/8/99 BY JRS LAST UPDATED 6/23/99

C THIS ROUTINE SETS UP THE VELOCITY INPUT FOR TOM DREEBEN'S

CODE FOR

C ENSEMBLE AVERAGING ODT.

C THE FOLLOWING DATA IS THAT GIVEN IN MULHOLLAND'S PAPER 1991

BASED ON

C EPA/600/7-88/006 PAGE E-4 DATA FOR *4* INCHES IN, AXIAL VELOCITY

C $* * *$ KNOWN MATCHES FOR NX NUMBER OF GRID POINTS ARE

C **** 795, 897, 1185, 1455, 1775

C VELOCITIES ARE IN METERS PER SECOND M/S

C TEMPERAURES ARE IN KELVIN K

C THE 16 VALUES (SYMMETRIC DATA POINTS) ARE FIT TO THE CHANNEL

C WIDTH ACCORDING TO THE 11 REGIONS GIVEN. A LINEAR FIT

BETWEEN DATA

C POINTS IS ASSUMED

C INPUTS ARE:

C LOUT $=$ THE DEVICE NUMBER FOR OUTPUT

C NNSC $=$ SPACE DIMENSIONED FOR THE NO. OF SCALARS (2)

$\mathrm{C} \quad \mathrm{NX}=$ NUMBER OF GRID NODES

C SCALINIT $()=$, THE INITIAL VELOCITY AND TEMP DISTRIBUTION

C NOG = ONE HALF OF (THE DOMAIN GRID POINTS -1)

$\mathrm{C} \quad \mathrm{REG}=\mathrm{REGION}$ OF INTEREST

C IREG = INTEGER VALUE OF THE REGION IN NODE POINTS

C TREG = THE REGION OF INTERSET FOR THE TEMPERATURE

$\mathrm{C}$ FREG $=$ FOLLOWING REGION

C IFREG $=$ INTEGER VALUE OF THE FOLLOWING REGION

C IREGAT $=$ INTEGER REGION AFTER THE FOLLOWING REGION

C IREGM $=$ IREG -1

C IPOSB = INTEGER NODE STARTING POSITION, BOTTOM

C IPOST $=$ INTEGER NODE STARTING POSITION, TOP

C TPOSB $=$ TEMPERARY INTEGER NODE POSITION, BOTTOM

C TPOSB $=$ TEMPERARY INTEGER NODE POSITION, TOP

C VELPOS $=$ VELOCITY AT POSITION OF INTEREST

C TEMPOS $=$ TEMPERATURE AT POSITION OF INTEREST

C TEMPTEM= TEMPARARY TEMPERATURE

C TSLOPE $=$ GRADIANT OF THE TEMPERATURE OVER THE REGION

C VSLOPE $=$ GRADIANT OF VELOCITY OVER REGION

IMPLICIT NONE

REAL FREG

INTEGER I, IFREG, IPOSB, IPOST, IREG, IREGAT, IREGM

INTEGER J, K 
INTEGER LOUT, NNSC, NOG, NX

REAL REG, SCALINIT(NNSC,NX), TEMPVEL, TEMPOS, TEMPTEM

INTEGER TPOSB, TPOST

REAL TSLOPE

REAL VELPOS, VSLOPE

C THIS DATA MUST HAVE AS AN ABSOLUTE MINIMUM NO. OF GRID POINTS 103

C AND IT SHOULD HAVE AN ODD NO. OF GRID POINTS

IF (NX .LT. 103 .OR. MOD(NX,2) .EQ. 0) THEN

WRITE (LOUT,*) ' ERROR IN INPUT VELOCITY: MUST HAVE AN ODD' WRITE (LOUT,*) ' NUMBER OF GRID POINTS WITH A MINIMUM OF 103' WRITE (LOUT,*) ' FOR THE MULHOLLAND DATA. 287 works well' STOP

END IF

$\mathrm{NOG}=\mathrm{NX} / 2$

C DATA EXISTS IN 12 REGIONS IN EACH HALF OF THE DOMAIN. REGION 1

$\operatorname{SCALINIT~}(1,1)=0.0$

SCALINIT $(1, \mathrm{NX})=0.0$

TEMPOS $=1100$.

SCALINIT $(2,1)=$ TEMPOS

SCALINIT $(2, \mathrm{NX})=$ TEMPOS

C THIS REGION IS .2484 THE HALF DISTANCE OF THE CHANNEL.

C THE CHANGE IN THE VELOCITY BETWEEN THE WALL AND THE FIRST DATA POINT

C IS -3.5 M/S AND THE CHANGE IN TEMPERATURE IS +50. K .

$$
\begin{aligned}
& \text { REG }=0.2484 * \text { NOG } * 2 . \\
& \text { IREG }=\text { REG }+0.49999 \\
& \text { FREG }=0.0536 * \text { NOG } * 2 . \\
& \text { IFREG }=\text { FREG }+0.49999 \\
& \text { VSLOPE }=-3.5 / \text { IREG } \\
& \text { TSLOPE }=50 . /(\text { IREG+IFREG }) \\
& \text { DO I }=1, \text { IREG- } 1,1=\text { VSLOPE } * \text { I } \\
& \text { TEMPVEL }=\text { TSLOPE } * \text { I }+ \text { TEMPOS } \\
& \text { TEMPTEM } \quad=\text { TEM } \\
& \text { SCALINIT }(1,1+\mathrm{I})=\text { TEMPVEL } \\
& \text { SCALINIT }(1, \text { NX-I }=\text { TEMPVEL } \\
& \text { SCALINIT }(2,1+\mathrm{I})=\text { TEMPTEM } \\
& \text { SCALINIT }(2, \text { NX-I }=\text { TEMPTEM } \\
& \text { END DO }
\end{aligned}
$$




$$
\begin{array}{ll}
\text { IPOSB } & =1+\text { IREG } \\
\text { IPOST } & =\mathrm{NX}-\text { IREG }
\end{array}
$$

VELPOS $=-3.5$

SCALINIT $(1$, IPOSB $)=$ VELPOS

SCALINIT $(1$, IPOST $)=$ VELPOS

TEMPOS $=$ TSLOPE $*$ IREG + TEMPOS

SCALINIT $(2, \mathrm{IPOSB})=$ TEMPOS

SCALINIT $(2$, IPOST $)=$ TEMPOS

C REGION 2

IREG = IFREG

IREGM $=$ IREG -1

VSLOPE $=3.5 /$ IREG

DO I = 1, IREGM, 1

TEMPVEL $=$ VSLOPE $*$ I + VELPOS

$\mathrm{J}=\mathrm{IPOSB}+\mathrm{I}$

$\mathrm{K}=$ IPOST $-\mathrm{I}$

SCALINIT $(1, \mathrm{~J})=$ TEMPVEL

SCALINIT $(1, \mathrm{~K})=$ TEMPVEL

TEMPTEM $=$ TSLOPE $* \mathrm{I}+$ TEMPOS

SCALINIT $(2, \mathrm{~J})=$ TEMPTEM

SCALINIT $(2, \mathrm{~K})=$ TEMPTEM

END DO

C REGION 3

VELPOS $=0$.

TEMPOS $=1150$.

IPOSB $=$ IPOSB + IREG

IPOST $=$ IPOST - IREG

SCALINIT $(1$, IPOSB $)=$ VELPOS

SCALINIT $(1$, IPOST $)=$ VELPOS

SCALINIT $(2$, IPOSB $)=$ TEMPOS

SCALINIT $(2$, IPOST $)=$ TEMPOS

REG $=0.0509 * \mathrm{NOG}^{*} 2$.

IREG $=$ REG +0.49999

FREG $=0.0105 * \mathrm{NOG} * 2$.

IFREG $=$ FREG +0.49999

IREGAT $=0.00915 *$ NOG $* 2 .+0.49999$

IREGM $=$ IREG -1

VSLOPE $=5 . /$ IREG

TSLOPE $=50 . /($ IREG + IFREG + IREGAT $)$ 


$$
\begin{aligned}
& \text { DO I = 1, IREGM, } 1 \\
& \text { TEMPVEL = VSLOPE } * \mathrm{I} \\
& \mathrm{J}=\mathrm{IPOSB}+\mathrm{I} \\
& \mathrm{K}=\text { IPOST-I } \\
& \operatorname{SCALINIT}(1, \mathrm{~J})=\text { TEMPVEL } \\
& \text { SCALINIT }(1, \mathrm{~K})=\text { TEMPVEL } \\
& \text { TEMPTEM }=\text { TSLOPE } * \text { I + TEMPOS } \\
& \text { SCALINIT }(2, \mathrm{~J})=\text { TEMPTEM } \\
& \text { SCALINIT }(2, \mathrm{~K})=\text { TEMPTEM } \\
& \text { END DO } \\
& \text { C REGION } 4 \\
& \text { IPOSB }=\text { IPOSB + IREG } \\
& \text { IPOST }=\text { IPOST }- \text { IREG } \\
& \text { VELPOS }=5 \text {. } \\
& \text { TEMPOS }=\text { TSLOPE } * \text { IREG + TEMPOS } \\
& \text { IREG = IFREG } \\
& \text { IFREG }=\text { IREGAT } \\
& \text { IREGM }=\text { IREG }-1 \\
& \text { SCALINIT }(1, \text { IPOSB })=\text { VELPOS } \\
& \text { SCALINIT }(1, \text { IPOST })=\text { VELPOS } \\
& \text { SCALINIT }(2, \text { IPOSB })=\text { TEMPOS } \\
& \text { SCALINIT }(2, \text { IPOST })=\text { TEMPOS } \\
& \text { VSLOPE }=15 . / \text { IREG } \\
& \text { DO I = 1, IREGM, } 1 \\
& \text { TEMPVEL }=\text { VSLOPE } * I+\text { VELPOS } \\
& \mathrm{J}=\mathrm{IPOSB}+\mathrm{I} \\
& \mathrm{K}=\text { IPOST-I } \\
& \operatorname{SCALINIT}(1, \mathrm{~J})=\text { TEMPVEL } \\
& \text { SCALINIT }(1, \mathrm{~K})=\text { TEMPVEL } \\
& \text { TEMPTEM }=\text { TSLOPE } * \text { I + TEMPOS } \\
& \operatorname{SCALINIT}(2, \mathrm{~J})=\text { TEMPTEM } \\
& \text { SCALINIT }(2, \mathrm{~K})=\text { TEMPTEM } \\
& \text { END DO }
\end{aligned}
$$


SCALINIT $(2$, IPOSB $)=$ TEMPOS

SCALINIT $(2$, IPOST $)=$ TEMPOS

IREG = IFREG

IREGM $=$ IREG -1

VSLOPE $=4 . /$ IREG

DO I = 1, IREGM, 1

TEMPVEL $=$ VSLOPE $* \mathrm{I}+$ VELPOS

$\mathrm{J}=\mathrm{IPOSB}+\mathrm{I}$

$\mathrm{K}=$ IPOST $-\mathrm{I}$

SCALINIT $(1, \mathrm{~J})=$ TEMPVEL

SCALINIT $(1, \mathrm{~K})=$ TEMPVEL

TEMPTEM $=$ TSLOPE $*$ I + TEMPOS

SCALINIT $(2, \mathrm{~J})=$ TEMPTEM

SCALINIT $(2, \mathrm{~K})=$ TEMPTEM

END DO

C REGION 6

IPOSB $=$ IPOSB + IREG

IPOST $=$ IPOST - IREG

VELPOS $=24$.

TEMPOS $=1200$.

SCALINIT $(1$, IPOSB $)=$ VELPOS

SCALINIT $(1$, IPOST $)=$ VELPOS

SCALINIT $(2$, IPOSB $)=$ TEMPOS

SCALINIT $(2$, IPOST $)=$ TEMPOS

REG $=0.01307 * \mathrm{NOG} * 2$.

IREG $=$ REG + 0.49999

FREG $=0.01706 *$ NOG $* 2$.

IFREG $=$ FREG +0.49999

IREGM $=$ IREG -1

VSLOPE $=-4 . /$ IREG

TSLOPE $=100 . /($ IREG + IFREG $)$

DO I = 1, IREGM, 1

TEMPVEL $=$ VSLOPE $* I+$ VELPOS

$\mathrm{J}=\mathrm{IPOSB}+\mathrm{I}$

$\mathrm{K}=$ IPOST $-\mathrm{I}$

SCALINIT $(1, \mathrm{~J})=$ TEMPVEL

SCALINIT $(1, \mathrm{~K})=$ TEMPVEL

TEMPTEM = TSLOPE* $\mathrm{I}+$ TEMPOS

SCALINIT $(2, \mathrm{~J})=$ TEMPTEM

SCALINIT $(2, \mathrm{~K})=$ TEMPTEM

END DO 


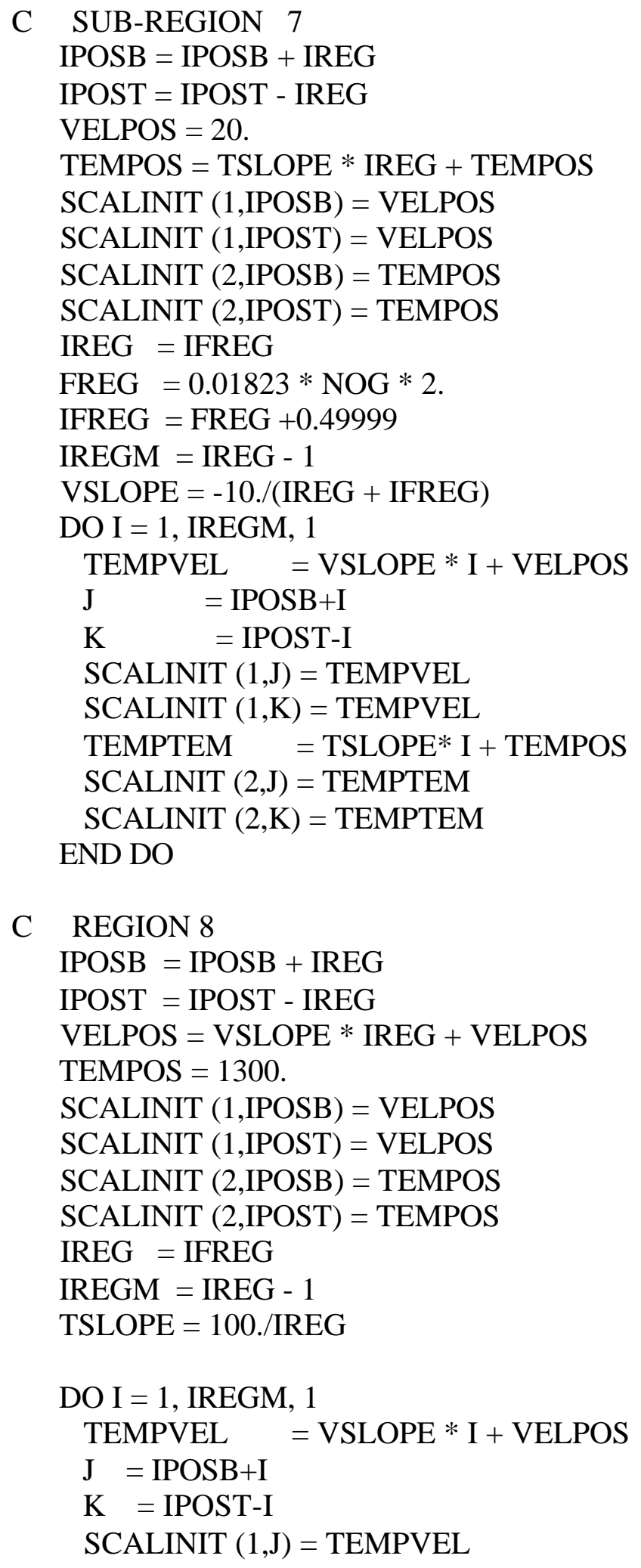


SCALINIT $(1, \mathrm{~K})=$ TEMPVEL

TEMPTEM $=$ TSLOPE $*$ I + TEMPOS

$\operatorname{SCALINIT}(2, \mathrm{~J})=$ TEMPTEM

SCALINIT $(2, \mathrm{~K})=$ TEMPTEM

END DO

C REGION 9

IPOSB $=$ IPOSB + IREG

IPOST $=$ IPOST - IREG

VELPOS $=10$.

TEMPOS $=1400$.

SCALINIT $(1$, IPOSB $)=$ VELPOS

SCALINIT $(1$, IPOST $)=$ VELPOS

SCALINIT $(2$, IPOSB $)=$ TEMPOS

SCALINIT $(2$, IPOST $)=$ TEMPOS

REG $=0.03086 * \mathrm{NOG} * 2$.

IREG $=$ REG +0.49999

FREG $=0.01228 *$ NOG $* 2$.

IFREG $=$ FREG +0.49999

IREGM $=$ IREG -1

VSLOPE $=-10 . /($ IREG + IFREG $)$

TSLOPE $=100 . /$ IREG

DO I = 1, IREGM, 1

TEMPVEL $=$ VSLOPE $* I+$ VELPOS

$\mathrm{J}=\mathrm{IPOSB}+\mathrm{I}$

$\mathrm{K}=$ IPOST-I

SCALINIT $(1, \mathrm{~J})=$ TEMPVEL

SCALINIT $(1, \mathrm{~K})=$ TEMPVEL

TEMPTEM $=$ TSLOPE $*$ I + TEMPOS

SCALINIT $(2, \mathrm{~J})=$ TEMPTEM

SCALINIT $(2, \mathrm{~K})=$ TEMPTEM

END DO

C REGION 10

IPOSB $=$ IPOSB + IREG

IPOST $=$ IPOST - IREG

VELPOS $=$ VSLOPE $*$ IREG + VELPOS

TEMPOS $=1500$.

SCALINIT $(1$, IPOSB $)=$ VELPOS

SCALINIT $(1$, IPOST $)=$ VELPOS

SCALINIT $(2$, IPOSB $)=$ TEMPOS

SCALINIT $(2$, IPOST $)=$ TEMPOS

IREG = IFREG

IREGM $=$ IREG -1 
TSLOPE $=100 . /$ IREG

DO I = 1, IREGM, 1

TEMPVEL $=$ VSLOPE $* I+$ VELPOS

$\mathrm{J}=\mathrm{IPOSB}+\mathrm{I}$

$\mathrm{K}=$ IPOST $-\mathrm{I}$

SCALINIT $(1, \mathrm{~J})=$ TEMPVEL

SCALINIT $(1, \mathrm{~K})=$ TEMPVEL

TEMPTEM = TSLOPE* $\mathrm{I}+$ TEMPOS

$\operatorname{SCALINIT}(2, \mathrm{~J})=$ TEMPTEM

SCALINIT $(2, \mathrm{~K})=$ TEMPTEM

END DO

C REGION 11

IPOSB $=$ IPOSB + IREG

IPOST $=$ IPOST - IREG

VELPOS $=0.0$

TEMPOS $=1600$.

SCALINIT $(1$, IPOSB $)=$ VELPOS

SCALINIT $(1$, IPOST $)=$ VELPOS

SCALINIT $(2$, IPOSB $)=$ TEMPOS

SCALINIT $(2$, IPOST $)=$ TEMPOS

REG $=0.01246 * \mathrm{NOG} * 2$.

IREG $=$ REG +0.49999

FREG $=0.02349 * \mathrm{NOG} * 2$.

IFREG $=$ FREG +0.49999

IREGM $=$ IREG -1

VSLOPE $=-2.5 /($ IREG + IFREG $)$

TSLOPE $=100 . /$ IREG

DO I = 1, IREGM, 1

TEMPVEL $=$ VSLOPE $* \mathrm{I}$

$\mathrm{J}=\mathrm{IPOSB}+\mathrm{I}$

$\mathrm{K}=$ IPOST $-\mathrm{I}$

SCALINIT $(1, \mathrm{~J})=$ TEMPVEL

SCALINIT $(1, \mathrm{~K})=$ TEMPVEL

TEMPTEM = TSLOPE* ${ }^{*}+$ TEMPOS

SCALINIT $(2, \mathrm{~J})=$ TEMPTEM

SCALINIT $(2, \mathrm{~K})=$ TEMPTEM

END DO

C REGION 12

IPOSB $=$ IPOSB + IREG

IPOST $=$ IPOST - IREG 


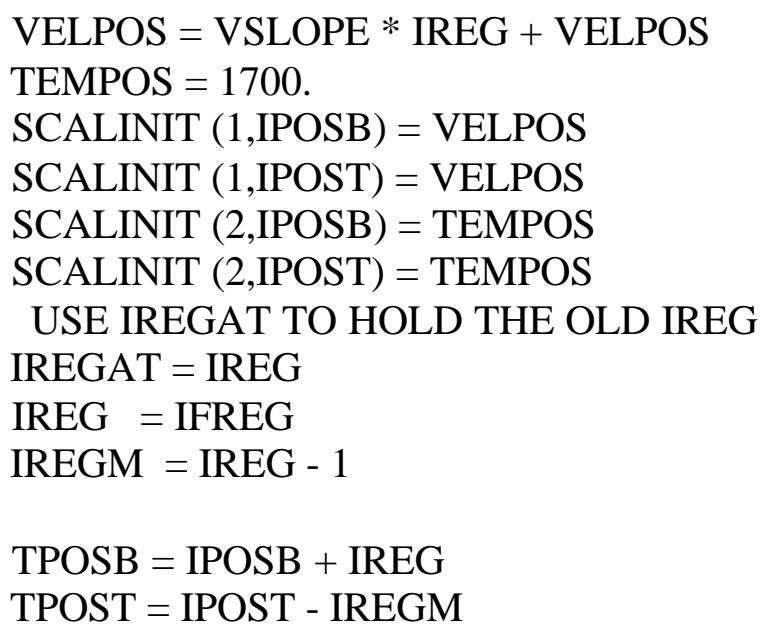

IF (TPOST .LE. TPOSB ) THEN

WRITE (LOUT,*) ' ROUNDOFF ERROR IN INPUT VELOCITY DISTRIBUTION'

WRITE (LOUT,*) ' NUMBER OF GRID POINTS MUST BE ADJUSTED.'

WRITE (LOUT, $*)^{\prime} \quad$ SHOULD BE ODD AND 103 OR > .'

STOP

END IF

IF (TPOST - 1 .EQ. TPOSB) THEN

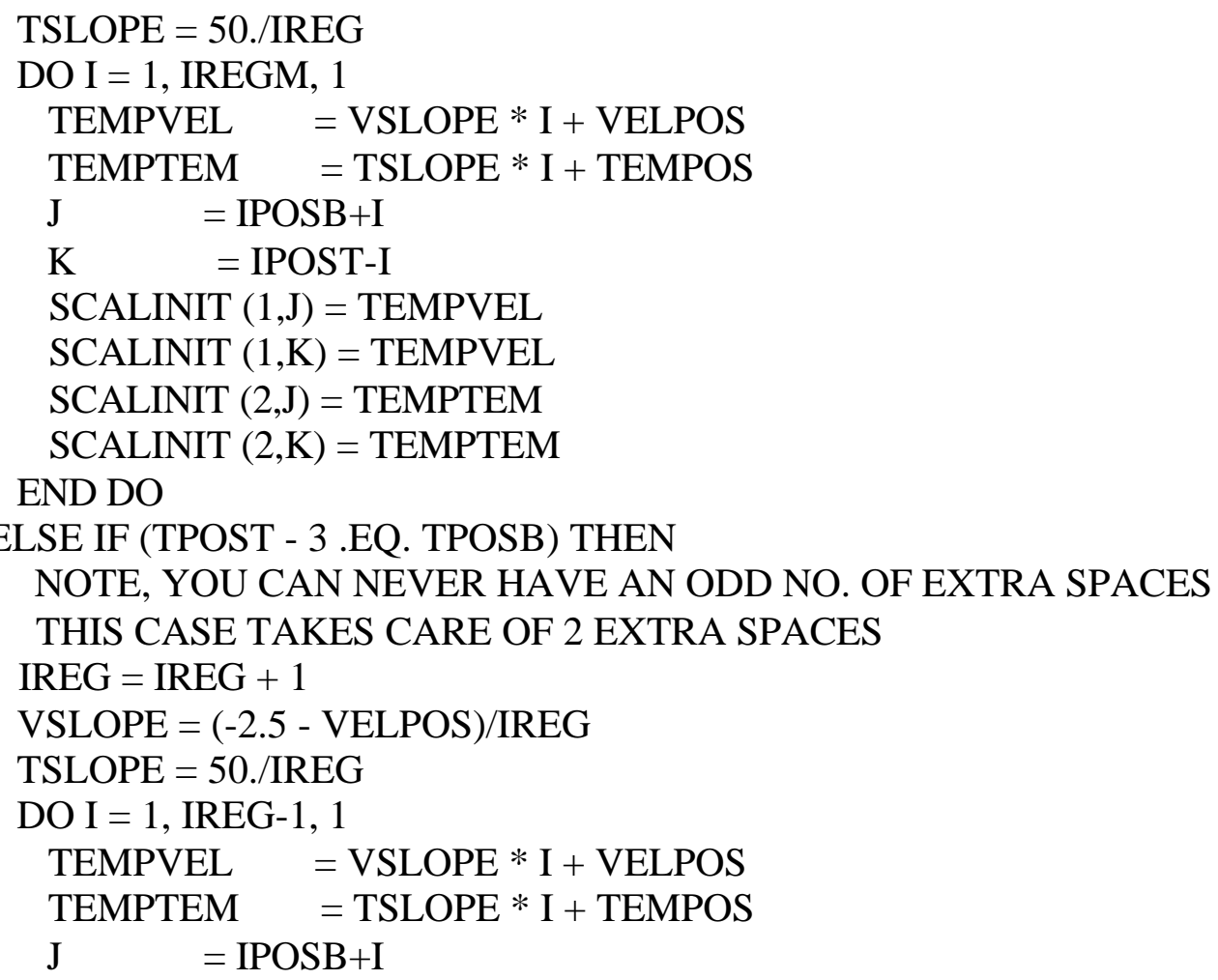


$\mathrm{K}=$ IPOST-I

$\operatorname{SCALINIT}(1, \mathrm{~J})=$ TEMPVEL

SCALINIT $(1, \mathrm{~K})=$ TEMPVEL

$\operatorname{SCALINIT}(2, \mathrm{~J})=$ TEMPTEM

SCALINIT $(2, \mathrm{~K})=$ TEMPTEM

END DO

ELSE

C IF THERE ARE MORE THEN 2 EXTRA SPACES, ABORT

WRITE (LOUT,*) ' ROUNDOFF ERROR IN INPUT VELOCITY

DISTRIBUTION'

WRITE (LOUT,*) ' THERE ARE MORE THEN TWO EXTRA SPACES '

WRITE (LOUT,*) ' NUMBER OF GRID POINTS MUST BE ADJUSTED.'

WRITE $\left(\right.$ LOUT, $\left.{ }^{*}\right)$ ' $\quad$ SHOULD BE ODD AND 103 OR > .'

STOP

END IF

$\operatorname{SCALINIT}(1, \mathrm{NOG}+1)=-2.5$

SCALINIT $(2$, NOG +1$)=1750$.

RETURN

END 


\section{A.2 Source code for the initial conditions (Mulholland et al. "like model)}

PROGRAM INITIALCON

$\mathrm{C} \quad * * * * * * * * * * * * * * * * * * * * * * * * * * * * * * * * * * * * * * * * * * * * * * * * * * * * * * * * * * * * * * * *$

C LAST MODIFIED ON 03/18/00 @ 10:00

!!!!! TFLAME $=$ TINFIN I.E. NO FLAME SHEET, OR NO IGNITION

C WRITTEN BY JRS ON 02/14/00 TO COMPUTE THE INPUT CONDITIONS TO

C THE ODT MODEL, USING A MODIFIED VERSION OF THE DROPLET

C TRAJECTORY MODEL IN MULHOLLAND ET AL. APPENDIX A IMPLICIT NONE

REAL AIRNU, ANGLE, AREA, ATTWO, BASESH, BMAST

REAL BLN, BP1, BULKVEL

REAL CDBAS, CDINF, CDO, CDOO, CDSTAR, CONST1

REAL CONST2, CONST3, CONST4, CONST5, CONST6

REAL CONST7, COSANG, CPFGAS

REAL DABAVE, DABZERO, DELTAD, DELTAM, DELTASD

REAL DELTAT, DELTATD, DELTAX, DIANEW, DIAP

REAL DMASS, DMNEW, DPOS

REAL EPSIL, EPSILON, ETAE, FGD, FRE, FXCOEF

REAL GAD, GAMMA, HEATCAP, HEATCOM, HEATVAP, HEFF

INTEGER IDEGRE, IINT

LOGICAL LCP3

INTEGER LOUT, NOI

REAL PI

REAL REN, RHOG, RHOL, ROOT1, ROOT2

REAL SCHMIDT, SHSTAR, SINANG, SOD, STOICH, TAUP, TDNEW, TDROP

REAL TEMPZERO, TERM, TFLAME, TIME, TINFIN

REAL TTHIRD

REAL VDNEW, VDROP, VGD

REAL XPOS, XVEL, YOINFIN, YPOS, YVEL

$\mathrm{C} \quad \mathrm{AX} * * 2+\mathrm{BX}+\mathrm{C}=0$

C SIGNIFICANT VARIABLES

C ATTWO = 'A' 'T'IMES 'TWO' -- USEFUL IN NUMERIC IMPLEMENTATION

$\mathrm{C} \quad$ TERM $=\mathrm{B} * * 2-2 * \mathrm{ATTWO}^{*} \mathrm{C}$

C $\quad$ ROOT1 $=(-\mathrm{B}+$ SQRT $($ TERM $)) /$ ATTWO

C $\quad$ ROOT2 $=(-\mathrm{B}-\mathrm{SQRT}($ TERM $)) /$ ATTWO

C $\quad$ V DROP $=$ VELOCITY OF THE DROP IN THE D DIRECTION [=] M/S

C V G D = VELOCITY OF THE GAS STREAM IN THE D DIRECTION [=] M/S

C $\quad F$ G D $=$ FORCE OF GRAVITY IN THE D DIRECTION $=\operatorname{SIN}(30)$ MASS * GRAVITY 
C GA D = GRAVITAIONAL ACCEL.(9.811 M/S**2) TIMES SIN(THETA) IN D DIRECT.

C $\quad$ S O D $=$ S/D THE INTERDROPLET SPACING

C RE N $=$ REYNOLDS NUMBER IN THE D DIRECTION

C $\quad$ CD INF $=$ DRAG COEFF. FOR INFINITELY SPACED DROPS

C CD BAS = BASE DRAG COEF, WITH OUR MASS TRANSFER

$\mathrm{C} \quad \mathrm{ETAE}=$ MASS TRANSFER CORRECTION TO CD

C ASSIGN SIGNIFICANT VALUES HERE

LOUT $=6$

OPEN (LOUT, FILE = 'noignc10cp3.out')

IDEGRE $=30$

DIAP $=0.000234$

VDROP $=8.5$

SOD $=45.0$

TDROP $=296.15$

LCP3 =.TRUE.

$\mathrm{DPOS}=0.0$

TFLAME $=1100$.

C NO FLAME IN THIS ROUTINE !!!!

C TFLAME $=1386.8877$

HEATCAP $=2000.0$

HEATCOM $=44000000.0$

HEATVAP $=350000.0$

$\mathrm{RHOL}=813.3$

STOICH $=0.317$

YOINFIN $=0.0515$

DABZERO $=0.0000088$

TEMPZERO $=303.15$

C $\quad$ GAD $=\operatorname{SIN}($ THETA) $*$ GRAV. ACC.

$\mathrm{GAD}=0.5 * 9.811$

RHOL $=813.3 \quad ![=] \mathrm{KG} / \mathrm{M} * * 3$

PI $=$ ACOS $(-1.0)$

ANGLE $=3 . / 18 . * \mathrm{PI}$

COSANG $=$ COS(ANGLE)

SINANG $=$ SIN $($ ANGLE $)$

NOI $=9000$

TIME $=0.14 / \mathrm{VDROP} / 4000.0$

DMASS $=$ PI $*$ DIAP $* * 3 *$ RHOL $/ 6.0$

WRITE (LOUT,5) DIAP, SOD, TDROP

5 FORMAT ( 2X, 'INITIAL DIAM = ', E14.7, ' SPACING = ', E14.7,

2 ' TEMP. =',E14.7, ' K')

IF (LCP3) THEN

WRITE (LOUT,10) IDEGRE, TIME, VDROP 
10 FORMAT (3X, I3, ' DEGREES, TIME STEP =', E14.7, ' VEL. =',

$2 \quad \mathrm{E} 14.7, \mathrm{e}^{\prime} \mathrm{cpf}=$ touluene, heptane, c10h21', /,

3 ' new corrected background velocity gradient')

ELSE

WRITE (LOUT,15) IDEGRE, TIME, VDROP

15 FORMAT (3X, I3, ' DEGREES, TIME STEP =', E14.7, ' VEL. =', $2 \quad \mathrm{E} 14.7,{ }^{\prime} \mathrm{cpf}=\mathrm{c} 10 \mathrm{~h} 211^{\prime}, /$,

3 ' new corrected background velocity gradient')

END IF

WRITE (LOUT,20)

20 FORMAT(1X,' DPOS XPOS YPOS',

2 ' DIAP TDROP VDROP')

DO IINT $=1$, NOI, 1

CALL VGDTHIRTY(DPOS, LOUT, TINFIN, VGD)

CALL EFLAMET (DIAP, HEATCOM, LCP3, LOUT, RHOL, 2 STOICH, TDROP, TFLAME, TINFIN, YOINFIN,

3 AIRNU, CPFGAS, HEFF, RHOG, TAUP, TTHIRD)

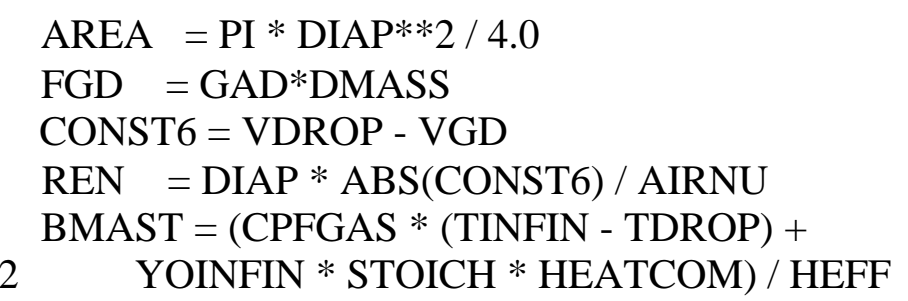

IF (CONST6 .GT. 0.0) THEN

C MULHOLLAND ET AL. MODEL WORKS FOR VDROP > VGD

$$
\begin{aligned}
& \text { CDINF }=24.0 / \text { REN } *\left(1+0.11 * \mathrm{REN}^{* *} 0.687\right) \\
& \mathrm{CDOO}=0.755 / \mathrm{REN} \\
& \mathrm{CDO}=\mathrm{CDOO}+43.0 / \mathrm{REN} *(\mathrm{SOD}-1)
\end{aligned}
$$

CDBAS $=\left(\mathrm{CDO}^{* *}(-0.678)+\mathrm{CDINF}^{* *}(-0.678)\right)^{* *}(-1 / 0.678)$

$\mathrm{ETAE}=1 . /(1.0+\mathrm{BMAST})$

$\mathrm{CDSTAR}=\mathrm{ETAE} * \mathrm{CDBAS}$

CONST $1=$ CDSTAR $*$ RHOG $*$ AREA

EPSIL $=$ CONST $1 /$ DMASS

IF (FGD .EQ. 0.0) THEN

C $\quad \mathrm{FGD}=0$ WHEN INCIDENT ANGLE IS 0.0

IF (VGD .EQ. 0.0) THEN

C SIMPLE FORM OF DVD/VD $* * 2=$ ACONST $*$ DT

CONST $2=$ VDROP $*$ CONST $1 *$ TIME

VDNEW $=$ DMASS $*$ VDROP $/($ CONST $2+$ DMASS $)$ 
CONST $3=$ CONST2 $/$ DMASS +1.0

IF (CONST3 .LE. 0.0) THEN

WRITE (LOUT, 90)

STOP

ELSE

DELTASD $=$ DMASS $/$ CONST $1 *$ LOG $($ CONST 3$)$

END IF

ELSE

CONST $2=$ CONST $6 *$ CONST $1 *$ TIME

VDNEW $=$ DMASS $*$ CONST $6 /($ DMASS + CONST 2$)+$ VGD

CONST $3=1.0+$ CONST $2 /$ DMASS

IF (CONST3 .LE. 0.0) THEN

WRITE (LOUT, 90)

STOP

ELSE

DELTASD $=$ DMASS $/$ CONST $1 *$ LOG $($ CONST3)

END IF

END IF

ELSE

GAMMA $=$ FGD $/$ CONST 1

EPSILON $=$ CONST $1 /$ DMASS

CONST2 $=$ SQRT $($ GAMMA)

ROOT $1=$ VGD + CONST2

ROOT2 = VGD - CONST2

CONST3 $=($ VDROP - ROOT2 $) /($ VDROP - ROOT1 $)$

CONST $4=\operatorname{EXP}($ EPSILON $* 2.0 *$ CONST2 $*$ TIME $)$

CONST $5=$ CONST $3 *$ CONST 4

CONST7 $=1.0-$ CONST5

VDNEW $=(-$ ROOT $1 *$ CONST5 + ROOT2 $) /$ CONST7

DELTASD $=$ LOG(CONST7 / (1.0 - CONST3 $)) /$ EPSILON

$2+\mathrm{ROOT} 2 * \mathrm{TIME}$

$\mathrm{C}$

$* * * * * * * * * * * * * * * * * * * * * * * * * * * * * * * * * * * * * * * * * * * * * * * * * * * * * * * * * * * * * * * * *$

END IF

ELSE IF ((CONST6 .EQ. 0.0) .AND. (FGD .NE. 0.0)) THEN

VDNEW $=$ GAD $*$ TIME + VDROP

DELTASD $=$ GAD $*$ TIME $* * 2 / 2.0+$ VDROP $*$ TIME

ELSE IF (FGD .EQ. 0.0) THEN

C MUST COMPUTE CD STAR SOMEHOW !!

$\mathrm{CDINF}=24.0 / \mathrm{REN} *\left(1+0.11 * \mathrm{REN}^{* *} 0.687\right)$

$\mathrm{CDOO}=0.755 / \mathrm{REN}$

$\mathrm{CDO}=\mathrm{CDOO}+43.0 / \mathrm{REN} *(\mathrm{SOD}-1)$

$\mathrm{CDBAS}=\left(\mathrm{CDO}^{* *}(-0.678)+\mathrm{CDINF}^{* *}(-0.678)\right)^{* * *}(-1 / 0.678)$ 
$\mathrm{ETAE}=1 . /(1.0+\mathrm{BMAST})$

CDSTAR $=$ ETAE $*$ CDBAS

CONST1 $=$ CDSTAR $*$ RHOG $*$ AREA

CONST $2=$ CONST $6 *$ CONST $1 *$ TIME

VDNEW $=$ VGD + DMASS $*$ CONST6 $/($ DMASS + CONST2 $)$

C !!! DO I NEED TO CHECK THE SIGN OF LOG ?

CONST $3=1.0+$ CONST2/DMASS

IF (CONST3 .LE. 0.0) THEN

WRITE (LOUT, 90)

90

FORMAT (1X, 'CONST3 IS NEGATIVE')

STOP

END IF

DELTASD $=$ VGD $*$ TIME + DMASS/CONST1 $*$ LOG(CONST3)

ELSE

WRITE (LOUT,95)

95 FORMAT (1X, 'ALWAYS IMAGINARY ROOTS!!')

STOP

END IF

DPOS $=$ DPOS + DELTASD

$\mathrm{C} * * * * * * * * * * * * * * * * * * * * * * * * * * * * * * * * * * * * * * * * * * * * * * * * * * * * * * * * * * * *$

IF (REN .LE. 1.0) THEN

C

$\mathrm{FRE}=1.0$

ELSE IF (REN .LE. 400) THEN

C CORRECTION FACTOR

$\mathrm{FRE}=(\mathrm{REN}) * * 0.077$

ELSE

WRITE (LOUT, *) ' PARTICLE Re T > 400'

WRITE (LOUT, *) ' STOPPED IN DUPDATE', REN

WRITE $($ LOUT, *) ' PARTICLE Re T > 400'

WRITE (LOUT, *) ' STOPPED IN DUPDATE', REN

STOP

END IF

$\mathrm{BP} 1=\mathrm{BMAST}+1$.

$\mathrm{BLN}=\mathrm{ALOG}(\mathrm{BP} 1)$

C FOR NOW RHOGAS IS THE DENSITY OF AIR AT THE 1/3 RULE TEMP.

CALL ESCHMIDT(AIRNU, DABZERO, TTHIRD, TEMPZERO, 2

DABAVE, SCHMIDT)

BASESH $=1 .+\operatorname{FRE} *(1 .+\operatorname{REN} * \operatorname{SCHMIDT}) * *(1 . / 3$. 


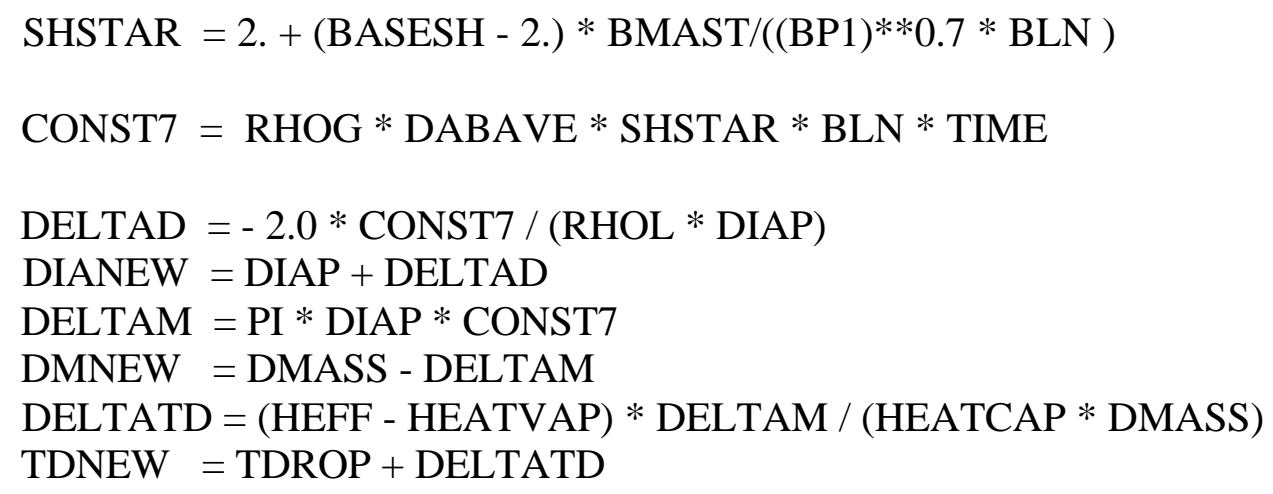




\author{
SUBROUTINE ESCHMIDT (AIRNU, DABZERO, TEMP, TEMPZERO,


SUBROUTINE EFLAMET (DIAP, HEATCOM, LCP3, LOUT, RHOL, 2 STOICH, TDROP, TFLAME, TINFIN, YOINFIN, 3 AIRNU, CPFGAS, HEFF, RHOGAS, TAUP, TTHIRD)

$\mathrm{C} * * * * * * * * * * * * * * * * * * * * * * * * * * * * * * * * * * * * * * * * * * * * * * * * * * * * * * * * * * * * * * * * * * * * *$

C LAST UPDATED ON 03/18/00 AT 10:00 AM

!!!!! TFLAME $=$ TINFIN

C WRITTEN BY JRSCHMIDT ON 02/02/00

C T FLAME $=$ FLAME SHEET TEMPERATURE, START WITH A GUESS

C $\quad$ T DROP $=$ DROP TEMP.

IMPLICIT NONE

REAL AIRMU, AIRNU, CPGAS, CPFGAS

REAL DIAP, HEATCOM, HEFF

INTEGER ITT

LOGICAL LCP3, LITT

INTEGER LOUT

REAL RHOGAS, RHOL, STOICH

REAL TAUP, TDROP, TFLAME, TFNEW, TINFIN, TTHIRD

REAL XFS, YCONST, YFS, YOINFIN

C CLAUSIUS-CLAPEYRON EQUATION. PRESSURE IS IN ATMOSPHERES

C GIVES MOLE FRACTION IN GAS AT THE SURFACE

C GAS CONSTANT R $=8.314 \mathrm{~J} / \mathrm{MOL} / \mathrm{K}$

C FROM EPA REPORT. AVE. MOLECULAR WEIGHT $=0.136 \mathrm{KG} / \mathrm{MOL}$,

C BOILING TEMP. $=400 \mathrm{~K}$, HEAT OF VAPORIZATION $=350,000 \mathrm{~J} / \mathrm{KG}$

$\mathrm{XFS}=\operatorname{EXP}(5725.283 *(0.0025-1 . / \mathrm{TDROP}))$

C $30 \%$ EXCESS AIR, ASSUMING 79\% N2, 21\% O2, COMPLETE BURNING.

C NATURAL GAS IS BETWEEN 95-98\% METHANE (CH4)

YCONST $=1738.08 *$ XFS

$\mathrm{YFS}=\mathrm{YCONST} /(353.866 *(1 .-\mathrm{XFS})+\mathrm{YCONST})$

C !!!!! AIRNU MUST BE EVALUATED AT 1/3 RULE -SIRIGNANO

C YOINFIN WAS CALCULATED TO BE 5.1465342

LITT $=$.TRUE

ITT $=1$

DO WHILE ((LITT) .AND. (ITT .LE. 35))

TTHIRD $=$ TDROP $+($ TFLAME - TDROP $) / 3$.

CALL ETMPVAR (LCP3, LOUT, TTHIRD, AIRMU, AIRNU, CPGAS,

CPFGAS, RHOGAS) 


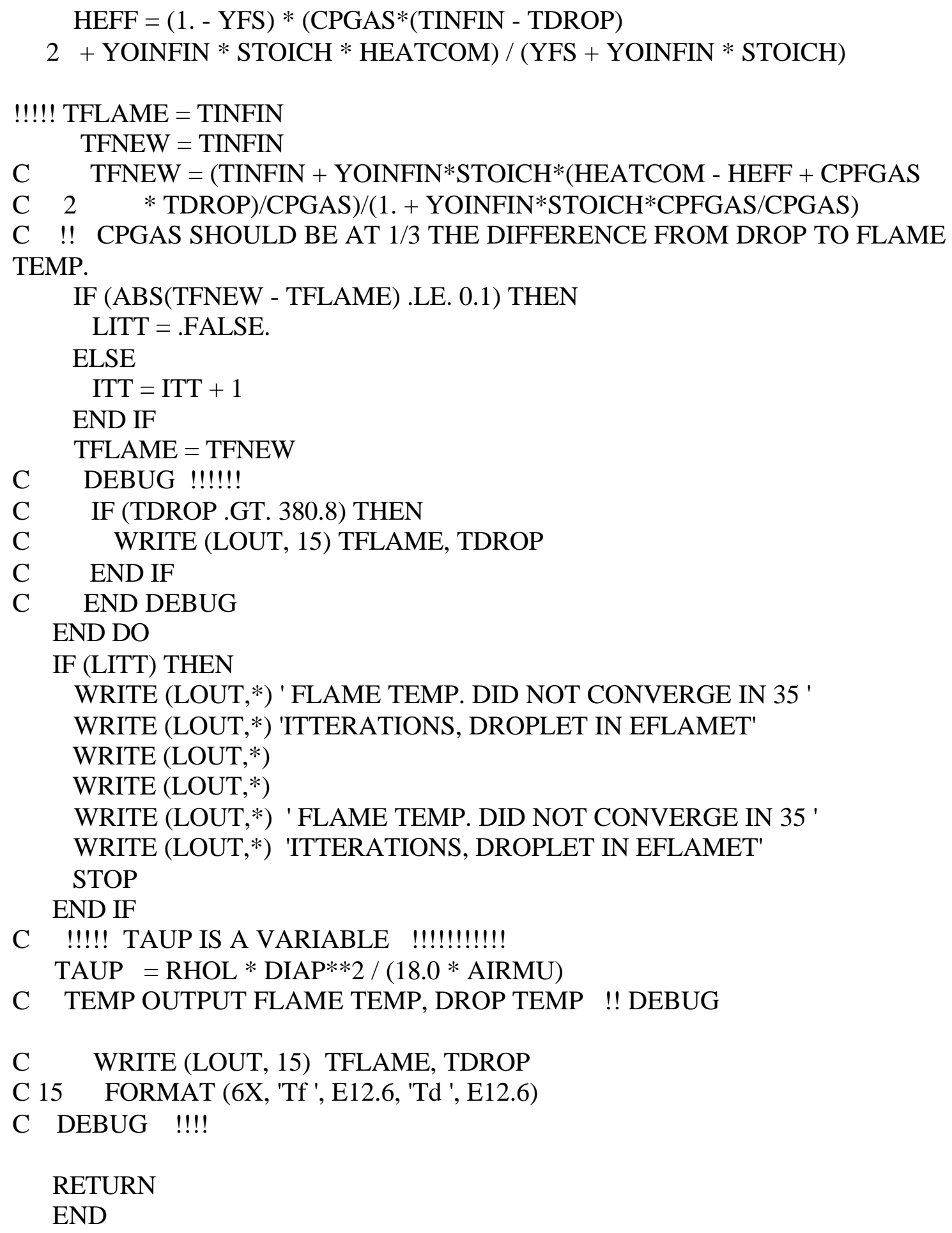


SUBROUTINE ETMPVAR (LCP3, LDOUT, TEMP, VISC, AIRNU, CPGAS, CPFGAS,

2

$\mathrm{C} * * * * * * * * * * * * * * * * * * * * * * * * * * * * * * * * * * * * * * * * * * * * * * * * * * * * * * * * * * * * * * * * * * * * * *$

C LAST UPDATED 02/03/00 AT 7:30 PM

C !!!!! TEMPARARILY MODIFIED ON 02/17/00 TO TRY CPF $=\mathrm{C} 10 \mathrm{H} 21$

C CREATED BY JOHN SCHMIDT ON 01/24/00

C THIS ROUTINE TAKES A TEMP AND FINDS THE CORESPONDING

VISCOSITY (NU),

C THE HEAT CAPACITY OF THE GAS PHASE (CPGAS), AND THE HEAT

CAPACITY OF

C THE GAS PHASE FUEL (CPFGAS) AT THAT TEMPERATURE

C R GAS $=$ MOLAR GAS CONSTANT UNITS J/G-MOL/K

IMPLICIT NONE

REAL AIRNU, CPFGAS, CPGAS, CP1, CP2, CP3

REAL DENSITY, EMW,EXMW, HMW

LOGICAL LCP3

INTEGER LDOUT

REAL SLOPE, TEMP, TMW, VISC

C FOR NOW ASSUME CPFGAS $=$ CPGAS

C FIND TEMP RANGE AND LINEARLY INTERPOLATE

C IMPORTANT VARIABLES

C VISCOSITY OF AIR IN KG/S/M

C DENSITY OF AIR IN KG/M**3

C HEAT CAPACITY IN J/KG/K

IF (TEMP .LE. 299.8166) THEN

VISC $=0.000018602$

DENSITY $=1.175754964$

CPGAS $=1004.832$

ELSE IF (TEMP .LE. 533.15) THEN

SLOPE $=(0.000027696-0.000018602) / 233.3333$

VISC $=0.000018602+$ SLOPE $*($ TEMP -299.8166$)$

SLOPE $=(0.659960552-1.175754964) / 233.3333$

DENSITY $=1.175754964+$ SLOPE $*($ TEMP -299.8166$)$

SLOPE $=(1013.2056-1004.832) / 233.3333$

CPGAS $=1004.832+$ SLOPE $*($ TEMP -299.8166$)$

ELSE IF (TEMP .LE. 588.7055) THEN

SLOPE $=(0.000029763-0.000027696) / 55.5555$

VISC $=0.000027696+$ SLOPE $*($ TEMP -533.15$)$ 


$$
\begin{aligned}
& \text { SLOPE }=(0.597488558-0.659960552) / 55.5555 \\
& \text { DENSITY }=0.659960552+\text { SLOPE } *(\text { TEMP }-533.15) \\
& \text { CPGAS }=1013.2056 \\
& \text { ELSE IF (TEMP .LE. 644.2611) THEN } \\
& \text { SLOPE }=(0.000031417-0.000029763) / 55.5555 \\
& \mathrm{VISC}=0.000029763+\mathrm{SLOPE} *(\mathrm{TEMP}-588.7055) \\
& \text { SLOPE }=(0.546229486-0.597488558) / 55.5555 \\
& \text { DENSITY }=0.597488558+\text { SLOPE } *(\text { TEMP }-588.7055) \\
& \text { SLOPE }=(1017.3924-1013.2056) / 55.5555 \\
& \text { CPGAS }=1013.2056+\text { SLOPE } *(\text { TEMP }-588.7055) \\
& \text { ELSE IF (TEMP .LE. 699.8166) THEN } \\
& \text { SLOPE }=(0.00003307-0.000031417) / 55.5555 \\
& \mathrm{VISC}=0.000031417+\mathrm{SLOPE} *(\mathrm{TEMP}-644.2611) \\
& \text { SLOPE }=(0.502979644-0.546229486) / 55.5555 \\
& \text { DENSITY }=0.546229486+\text { SLOPE } *(\text { TEMP }-644.2611) \\
& \mathrm{SLOPE}=(1021.5792-1017.3924) / 55.5555 \\
& \text { CPGAS }=1017.3924+\text { SLOPE } *(\text { TEMP }-644.2611) \\
& \text { ELSE IF (TEMP .LE. 755.3722) THEN } \\
& \text { SLOPE }=(0.000035137-0.00003307) / 55.5555 \\
& \mathrm{VISC}=0.00003307+\mathrm{SLOPE} *(\mathrm{TEMP}-699.8166) \\
& \mathrm{SLOPE}=(0.47254457-0.502979644) / 55.5555 \\
& \text { DENSITY }=0.502979644+\text { SLOPE } *(\text { TEMP }-699.8166) \\
& \text { SLOPE }=(1025.766-1021.5792) / 55.5555 \\
& \text { CPGAS }=1021.5792+\text { SLOPE } *(\text { TEMP }-699.8166) \\
& \text { ELSE IF (TEMP .LE. 810.9277) THEN } \\
& \text { SLOPE }=(0.000036791-0.000035137) / 55.5555 \\
& \mathrm{VISC}=0.000035137+\mathrm{SLOPE} *(\mathrm{TEMP}-755.3722) \\
& \mathrm{SLOPE}=(0.44050765-0.47254457) / 55.5555 \\
& \text { DENSITY }=0.47254457+\text { SLOPE } *(\text { TEMP }-755.3722) \\
& \text { SLOPE }=(1029.9528-1025.766) / 55.5555 \\
& \text { CPGAS }=1025.766+\text { SLOPE } *(\text { TEMP }-755.3722) \\
& \text { ELSE IF (TEMP .LE. 922.0388) THEN } \\
& \text { SLOPE }=(0.000040098-0.000036791) / 111.1111 \\
& \text { VISC }=0.000036791+\operatorname{SLOPE} *(\text { TEMP }-810.9277) \\
& \text { SLOPE }=(0.381239348-0.44050765) / 111.1111 \\
& \text { DENSITY }=0.44050765+\text { SLOPE } *(\text { TEMP }-810.9277) \\
& \text { SLOPE }=(1038.3264-1029.9528) / 111.1111 \\
& \text { CPGAS }=1029.9528+\text { SLOPE } *(\text { TEMP }-810.9277) \\
& \text { ELSE IF (TEMP .LE. 1033.15) THEN } \\
& \text { SLOPE }=(0.000043405-0.000040098) / 111.1111 \\
& \text { VISC }=0.000040098+\text { SLOPE } *(\text { TEMP }-922.0388) \\
& \text { SLOPE }=(0.339591352-0.381239348) / 111.1111 \\
& \text { DENSITY }=0.381239348+\text { SLOPE } *(\text { TEMP }-922.0388) \\
& \mathrm{SLOPE}=(1050.8868-1038.3264) / 111.1111
\end{aligned}
$$


CPGAS $=1038.3264+$ SLOPE $*($ TEMP -922.0388$)$

ELSE IF (TEMP .LE. 1144.2611) THEN

SLOPE $=(0.000046298-0.000043405) / 111.1111$

VISC $=0.000043405+$ SLOPE $*($ TEMP -1144.2611$)$

SLOPE $=(0.307554432-0.339591352) / 111.1111$

DENSITY $=0.339591352+$ SLOPE $*($ TEMP -1144.2611$)$

SLOPE $=(1063.4472-1050.8868) / 111.1111$

CPGAS $=1050.8868+$ SLOPE $*($ TEMP -1144.2611$)$

ELSE IF (TEMP .LE. 1255.3722) THEN

SLOPE $=(0.000049605-0.000046298) / 111.1111$

VISC $=0.000046298+$ SLOPE $*($ TEMP -1255.3722$)$

SLOPE $=(0.28032305-0.307554432) / 111.1111$

DENSITY $=0.307554432+$ SLOPE $*($ TEMP -1255.3722$)$

SLOPE $=(1076.0076-1063.4472) / 111.1111$

CPGAS $=1063.4472+$ SLOPE $*($ TEMP -1255.3722$)$

ELSE IF (TEMP .LE. 1366.4833) THEN

SLOPE $=(0.000052499-0.000049605) / 111.1111$

VISC $=0.000049605+$ SLOPE $*($ TEMP -1366.4833$)$

SLOPE $=(0.257897206-0.28032305) / 111.1111$

DENSITY $=0.28032305+$ SLOPE $*($ TEMP -1366.4833$)$

SLOPE $=(1088.5680-1076.0076) / 111.1111$

CPGAS $=1076.0076+$ SLOPE $*($ TEMP -1366.4833$)$

ELSE

VISC $=0.000052499$

DENSITY $=0.257897206$

CPGAS $=1088.5680$

WRITE (LDOUT, *) ' FLAME TEMPERATURE TOO HIGH '

WRITE (LDOUT, *) ' VISCOSITY IN ETMPVAR'

END IF

C VALUES OF DENSITY, VISCOSITY, AND HEAT CAPACITY ARE

C FROM "AIR POLLUTION CONTROL, A DESIGN APPROACH"

C BY C.D. COOPER \& F.C. ALLEY. PAGE 654

$\mathrm{C}$

C NU $=$ VISCOSITY/DENSITY $\left[\mathrm{M}^{* * 2 / \mathrm{S}}\right]$

AIRNU $=$ VISC $/$ DENSITY

C $\quad$ RGAS $=8.3144$ ! UNITS J/G-MOL/K

$\mathrm{HMW}=100.204$

EMW $=106.1674$

TMW $=91.13258$

EXMW $=141.27670$ 
$\mathrm{C} * * * * * * * * * * * * * * * * * * * * * * * * * * * * * * * * * * * * * * * * * * * * * * * * * * * * * * * * * * * * * * * * * * * * * * *$

IF (TEMP .GE. 200.) THEN

IF (TEMP .LE. 1000.) THEN

C $\quad 200-1000 \mathrm{~K}$

C HEPTANE

CP1 $=11.15325-0.009494155 *$ TEMP $+0.0001955712 *$ TEMP $* * 2$

$2-0.0000002497525 *$ TEMP $* * 3+9.848732 \mathrm{D}-11 * \mathrm{TEMP}^{* * 4}$

C ETHYLBENZENE

$\mathrm{CP} 2=3.51535+0.01781457 *$ TEMP $+0.000118934 *$ TEMP $* * 2$

$2-1.756398 \mathrm{D}-07 *$ TEMP $* * 3+7.320611 \mathrm{D}-11 *$ TEMP $* * 4$

C $\quad \mathrm{C} 10 \mathrm{H} 21$

$\mathrm{CP} 3=3.089701+0.08411795 * \mathrm{TEMP}+1.590184 \mathrm{D}-05 * \mathrm{TEMP} * * 2$

$2-7.238793 \mathrm{D}-08 *$ TEMP $* * 3+3.226693 \mathrm{D}-11 *$ TEMP $* * 4$

C TEMPARARY TO CHECK OUT USING CP3 AS AN AVERAGE

C $\quad$ CPFGAS $=0.399 * \mathrm{CP} 1 / \mathrm{HMW}+0.289 * \mathrm{CP} 2 / \mathrm{EMW}+0.312 * \mathrm{CP} 3 / \mathrm{EXMW}$

C $\quad$ CPFGAS $=\mathrm{CP} 3 / \mathrm{EXMW}$

ELSE IF (TEMP .LE. 6000) THEN

C $\quad 1000-6000 \mathrm{~K}$

C HEPTANE

CP1 $=18.53547+0.03914205 *$ TEMP $-1.380303 \mathrm{D}-05 *$ TEMP $* * 2$

$2+2.224039 \mathrm{D}-09 *$ TEMP $* * 3-1.334526 \mathrm{D}-13 *$ TEMP $* * 4$

C ETHYLBENZENE

CP2 $=15.57608+0.03230646 *$ TEMP $-1.190027 \mathrm{D}-05 *$ TEMP $* * 2$

$2+1.967925 \mathrm{D}-09 *$ TEMP $* * 3-1.199112 \mathrm{D}-13 *$ TEMP $* * 4$

$\mathrm{CP} 3=21.32213+0.06157352 *$ TEMP $-2.384948 \mathrm{D}-05 *$ TEMP $* 2$

$2+4.220912 \mathrm{D}-09 *$ TEMP $* * 3-2.788931 \mathrm{D}-13 *$ TEMP $* * 4$

C TEMPARARY MODIFICATION TO CHECK OUT USING CP3 AS AN

AVERAGE

C $\quad$ CPFGAS $=0.399 * \mathrm{CP} 1 / \mathrm{HMW}+0.289 * \mathrm{CP} 2 / \mathrm{EMW}+0.312 * \mathrm{CP} 3 / \mathrm{EXMW}$

C $\quad$ CPFGAS $=$ CP3/EXMW

ELSE

PRINT*, ' TEMP. IS OVER 6000 K'

END IF

ELSE

PRINT*, ' TEMP. IS LESS THEN 200 K'

END IF

C TEMPARARY TO CHECK OUT USING CP3 AS AN AVERAGE IF (LCP3) THEN

$\mathrm{CPFGAS}=0.399 * \mathrm{CP} 1 / \mathrm{HMW}+0.289 * \mathrm{CP} 2 / \mathrm{EMW}+0.312 * \mathrm{CP} 3 / \mathrm{EXMW}$ 
ELSE CPFGAS $=\mathrm{CP} 3 / \mathrm{EXMW}$

END IF

C $\quad \mathrm{RGAS} * \mathrm{CP} * 1000$

CPFGAS $=8314.4 *$ CPFGAS

RETURN

END 
SUBROUTINE VGDTHIRTY (DPOS, LOUT, TINFIN, VGD)

C SPECIFIC FOR THE 30 DEGREE DROPS

C UNIT NORMAL IN D DIRECTION IS $(-2.88675 \mathrm{i}+0 \mathrm{j}+5 \mathrm{k}) / 5.773502692$

IMPLICIT NONE

REAL DPOS

INTEGER LOUT

REAL RUN, SLOPE, TINFIN, VGD

RUN $=0.05773502692$

C 5 CM POSITION DPOS $=0.05773502692$ EQ. TO 5 CM IN Z DIRECTION

IF (DPOS .LE. RUN) THEN

SLOPE $=-1.116378713 /$ RUN

VGD $=$ SLOPE $*$ DPOS

SLOPE $=(1128.2-1090.0) /$ RUN

$\mathrm{TINFIN}=1090.0+\mathrm{SLOPE} * \mathrm{DPOS}$

ELSE IF (DPOS .LE. $2.0 *$ RUN) THEN

SLOPE $=(1.174872261+1.116378713) /$ RUN

$\mathrm{VGD}=-1.116378713+\mathrm{SLOPE} *(\mathrm{DPOS}-\mathrm{RUN})$

SLOPE $=(1170.0-1128.2) /$ RUN

$\mathrm{TINFIN}=1128.2+\mathrm{SLOPE} *(\mathrm{DPOS}-\mathrm{RUN})$

ELSE IF (DPOS .LE. 0.162) THEN

SLOPE $=(8.4-1.174872261) /(0.162-2.0 *$ RUN $)$

$\mathrm{VGD}=1.174872261+\mathrm{SLOPE} *(\mathrm{DPOS}-2.0 * \mathrm{RUN})$

SLOPE $=(1250-1170.0) /(0.162-2.0 *$ RUN $)$

$\mathrm{TINFIN}=1170.0+\mathrm{SLOPE} *(\mathrm{DPOS}-2.0 * \mathrm{RUN})$

ELSE

WRITE (LOUT, 10)

10 FORMAT (1X, ' WENT BEYOND $14 \mathrm{~cm}$ IN THE Z DIRECTION')

STOP

END IF

RETURN

END 
SUBROUTINE VGDZERO (DPOS, LOUT, TINFIN, VGD)

C SPECIFIC FOR THE 0 DEGREE DROPS

C UNIT NORMAL IN D DIRECTION IS $(2.88675 i+0 j+5 k) / 5.773502692$

IMPLICIT NONE

REAL DPOS

INTEGER LOUT

REAL RUN, SLOPE, TINFIN, VGD

RUN $=0.05$

C 5 CM POSITION DPOS $=5.773502692$ EQ. TO 5 CM IN Z DIRECTION

IF (DPOS .LE. RUN) THEN

SLOPE $=-3.149489357 /$ RUN

$\mathrm{VGD}=\mathrm{SLOPE} * \mathrm{DPOS}$

SLOPE $=(1125.4-1090$.$) / RUN$

TINFIN $=1090+$ SLOPE $*$ DPOS

ELSE IF (DPOS .LE. $2.0 *$ RUN) THEN

SLOPE $=(-5.267692907+3.149489357) /$ RUN

VGD $=-3.149489357+$ SLOPE $*($ DPOS - RUN $)$

SLOPE $=(1130.4-1125.4) /$ RUN

TINFIN $=1125.4+$ SLOPE $*($ DPOS - RUN $)$

ELSE

WRITE (LOUT,10)

10 FORMAT (1X, ' WENT BEYOND $10 \mathrm{~cm}$ IN THE Z DIRECTION')

STOP

END IF

RETURN

END 
SUBROUTINE VGDFORTYFIVE (DPOS, LOUT, TINFIN, VGD)

C SPECIFIC FOR THE 45 DEGREE DROPS

C UNIT NORMAL IN D DIRECTION IS $(2.88675 \mathrm{i}+0 \mathrm{j}+5 \mathrm{k}) / 5.773502692$

IMPLICIT NONE

REAL DPOS

INTEGER LOUT

REAL RUN, SLOPE, TINFIN, VGD

RUN $=0.03535533906$

C 5 CM POSITION DPOS $=5.773502692$ EQ. TO 5 CM IN Z DIRECTION

IF (DPOS .LE. RUN) THEN

SLOPE $=-2.866005 /$ RUN

$\mathrm{VGD}=\mathrm{SLOPE} * \mathrm{DPOS}$

SLOPE $=(1120 .-1090.0) /$ RUN

TINFIN $=1090.0+$ SLOPE $*$ DPOS

ELSE IF (DPOS .LE. $2.0 *$ RUN) THEN

SLOPE $=(4.330129+2.866005) /$ RUN

$\mathrm{VGD}=-2.866005+\mathrm{SLOPE} *(\mathrm{DPOS}-\mathrm{RUN})$

SLOPE $=(1155.0-1120.0) /$ RUN

TINFIN $=1120.0+$ SLOPE $*($ DPOS - RUN $)$

ELSE IF (DPOS .LE. $2.5 *$ RUN) THEN

SLOPE $=(24.150619-4.330129) /($ RUN/2.0)

$\mathrm{VGD}=4.330129+\mathrm{SLOPE} *(\mathrm{DPOS}-2.0 * \mathrm{RUN})$

SLOPE $=(1200.0-1155.0) /($ RUN/2.0 $)$

TINFIN $=1155.0+$ SLOPE $*($ DPOS $-2.0 *$ RUN $)$

ELSE IF (DPOS .LE. $3.0 *$ RUN) THEN

SLOPE $=(11.16023209-24.150619) /(\mathrm{RUN} / 2.0)$

$\mathrm{VGD}=24.150619+\mathrm{SLOPE} *(\mathrm{DPOS}-2.5 * \mathrm{RUN})$

SLOPE $=(1400.0-1200.0) /($ RUN/2.0 $)$

TINFIN $=1200.0+$ SLOPE $*($ DPOS $-2.5 *$ RUN $)$

ELSE IF (DPOS .LE. $4.0 *$ RUN) THEN

SLOPE $=(-3.46401323-11.16023209) /$ RUN note: $\mathrm{vZ}=17 \mathrm{~m} / \mathrm{s}$

$\mathrm{VGD}=11.16023209+\mathrm{SLOPE} *(\mathrm{DPOS}-3.0 * \mathrm{RUN}) \mathrm{vr}=5 \mathrm{~m} / \mathrm{s}$

SLOPE $=(1750.0-1400.0) /$ RUN

TINFIN $=1400.0+$ SLOPE $*($ DPOS $-3.0 *$ RUN $)$

ELSE

WRITE (LOUT,10)

10 FORMAT (1X, ' WENT BEYOND $10 \mathrm{~cm}$ IN THE Z DIRECTION')

STOP

END IF

RETURN

END 


\section{Appendix B}

\section{List of Random Number Seeds}

A complete list of all the initial values of the random number generator seeds is provided for all 2000 realizations. Any ensemble realization can be generated in any order by simply inputting the random number pair subsequent to the subroutine call DROPLINIT. 
The realization number is listed first. The next two numbers on the same line are the random number seeds which were used to start that particular ensemble.

\begin{tabular}{|c|c|c|c|c|c|}
\hline 1 & 12345 & 67890 & 50 & 1074312419 & 973785132 \\
\hline 2 & 1186279957 & 69005677 & 51 & 1352358419 & 1866928629 \\
\hline 3 & 1690624489 & 211101369 & 52 & 1161849866 & 1848858644 \\
\hline 4 & 1795846609 & 1456147840 & 53 & 258763620 & 1420724382 \\
\hline 5 & 868835522 & 1002735207 & 54 & 1589846737 & 553980043 \\
\hline 6 & 1749268059 & 2101811192 & 55 & 781044615 & 2048819304 \\
\hline 7 & 834384517 & 561404391 & 56 & 1401027045 & 666635467 \\
\hline 8 & 1305514941 & 728648880 & 57 & 520783586 & 601713317 \\
\hline 9 & 792686244 & 765654447 & 58 & 1230177439 & 693112874 \\
\hline 10 & 347183723 & 226715027 & 59 & 571499690 & 193053836 \\
\hline 11 & 1668624725 & 1879801423 & 60 & 2074082037 & 1059719827 \\
\hline 12 & 163682225 & 1663712837 & 61 & 484389688 & 1475716536 \\
\hline 13 & 1017370303 & 1162812113 & 62 & 1792330786 & 1218693923 \\
\hline 14 & 767732798 & 1025633173 & 63 & 1304128765 & 1571625624 \\
\hline 15 & 1027662748 & 1009212420 & 64 & 608921086 & 1680671271 \\
\hline 16 & 1912163172 & 780109556 & 65 & 231124642 & 488616410 \\
\hline 17 & 1377783800 & 1920041416 & 66 & 811240934 & 38371122 \\
\hline 18 & 1378625952 & 969054343 & 67 & 2143912096 & 1578998139 \\
\hline 19 & 1845178641 & 908365528 & 68 & 1580560513 & 1230095510 \\
\hline 20 & 1593358618 & 1298840694 & 69 & 127643294 & 479959950 \\
\hline 21 & 112840350 & 2050693735 & 70 & 910459273 & 204478471 \\
\hline 22 & 537145081 & 2003939625 & 71 & 1712130301 & 713880291 \\
\hline 23 & 1940986384 & 1592407989 & 72 & 1138982360 & 406132529 \\
\hline 24 & 978291229 & 274351680 & 73 & 1724368929 & 1128109168 \\
\hline 25 & 688766542 & 834298067 & 74 & 236064501 & 142341261 \\
\hline 26 & 872317613 & 41335488 & 75 & 1146520950 & 66430823 \\
\hline 27 & 1443058074 & 1743631118 & 76 & 448997168 & 1518677961 \\
\hline 28 & 209917579 & 1843240682 & 77 & 317971091 & 1424366250 \\
\hline 29 & 1054574241 & 896994182 & 78 & 147076255 & 973730810 \\
\hline 30 & 1929434401 & 964193217 & 79 & 2080901098 & 573600355 \\
\hline 31 & 151269297 & 1232499654 & 80 & 1278264254 & 1683255589 \\
\hline 32 & 850760451 & 1253440098 & 81 & 396599059 & 939800107 \\
\hline 33 & 1665953462 & 123270931 & 82 & 1007419940 & 159327419 \\
\hline 34 & 529469208 & 1603084778 & 83 & 1274622524 & 2046320806 \\
\hline 35 & 1735167252 & 1467960639 & 84 & 841789059 & 1530640285 \\
\hline 36 & 752369616 & 1666067347 & 85 & 2020013400 & 322719301 \\
\hline 37 & 371162873 & 1408293313 & 86 & 1146258361 & 482829452 \\
\hline 38 & 1729251049 & 545886262 & 87 & 554133148 & 1298393944 \\
\hline 39 & 2004147156 & 1308833762 & 88 & 620488237 & 2009709776 \\
\hline 40 & 1256550714 & 2027121577 & 89 & 1038115902 & 1125642409 \\
\hline 41 & 520326295 & 180485227 & 90 & 1573361580 & 568873943 \\
\hline 42 & 523361037 & 112185635 & 91 & 2103335868 & 370564251 \\
\hline 43 & 303061974 & 1613251527 & 92 & 1332799398 & 440259673 \\
\hline 44 & 1829406235 & 483678039 & 93 & 174263239 & 1691150618 \\
\hline 45 & 2132986265 & 1606122815 & 94 & 1400518741 & 1576932835 \\
\hline 46 & 1263111895 & 915201664 & 95 & 1202142930 & 257492718 \\
\hline 47 & 549605900 & 1551385008 & 96 & 2091923457 & 1727583184 \\
\hline 48 & 1826782150 & 613338377 & 97 & 2066878349 & 1454862013 \\
\hline 49 & 1235913031 & 101860716 & 98 & 2037982505 & 81592728 \\
\hline
\end{tabular}




\begin{tabular}{|c|c|c|c|c|c|}
\hline 99 & 1534364503 & 681484694 & 152 & 399995428 & 1033245690 \\
\hline 100 & 1353505990 & 139281425 & 153 & 335577628 & 1884165922 \\
\hline 101 & 316379665 & 692645865 & 154 & 772281295 & 1771293824 \\
\hline 102 & 1422911596 & 1741027341 & 155 & 441688430 & 12318293 \\
\hline 103 & 1617028093 & 1829046090 & 156 & 73914492 & 1946787956 \\
\hline 104 & 1966322753 & 207031741 & 157 & 1339330664 & 127576599 \\
\hline 105 & 53734522 & 2037336266 & 158 & 516180202 & 1171799564 \\
\hline 106 & 88769184 & 81586106 & 159 & 1930013724 & 367768973 \\
\hline 107 & 1395653044 & 2039121254 & 160 & 1273049744 & 418710975 \\
\hline 108 & 1632373712 & 1142068965 & 161 & 996299189 & 1021557839 \\
\hline 109 & 1236202080 & 1532557227 & 162 & 1344004690 & 202249753 \\
\hline 110 & 1545390256 & 774071936 & 163 & 1156017218 & 1658756808 \\
\hline 111 & 1254291175 & 1098568345 & 164 & 790232100 & 423933585 \\
\hline 112 & 352860717 & 1396712198 & 165 & 192790345 & 1689739541 \\
\hline 113 & 456785419 & 776943970 & 166 & 1012187731 & 39989808 \\
\hline 114 & 1864286288 & 1451667994 & 167 & 303299331 & 1596314286 \\
\hline 115 & 792936520 & 1004288999 & 168 & 1684373187 & 884488773 \\
\hline 116 & 1123236698 & 946461362 & 169 & 596442472 & 235922311 \\
\hline 117 & 524359377 & 1227551022 & 170 & 372273911 & 1435804340 \\
\hline 118 & 2073931531 & 1087975091 & 171 & 1188363758 & 1087559656 \\
\hline 119 & 1565281358 & 1793670829 & 172 & 452239311 & 348247400 \\
\hline 120 & 899460129 & 1870790834 & 173 & 1978041431 & 2051348194 \\
\hline 121 & 1295715191 & 1685490796 & 174 & 2013668233 & 1377576452 \\
\hline 122 & 671990602 & 2114634167 & 175 & 1171951946 & 1153887003 \\
\hline 123 & 871619689 & 1310564649 & 176 & 128965577 & 1405234734 \\
\hline 124 & 1562528074 & 999706816 & 177 & 534164264 & 650825098 \\
\hline 125 & 1187179865 & 1436748136 & 178 & 1334634475 & 308715443 \\
\hline 126 & 948841502 & 1603541180 & 179 & 1699993048 & 1438193574 \\
\hline 127 & 584867317 & 1363710252 & 180 & 10730975 & 1364719394 \\
\hline 128 & 959414535 & 2029311870 & 181 & 776808177 & 1670425591 \\
\hline 129 & 1064108851 & 1273179556 & 182 & 1128669691 & 971388479 \\
\hline 130 & 2052490821 & 45183121 & 183 & 23959463 & 656730208 \\
\hline 131 & 1792503598 & 944769387 & 184 & 464634325 & 38449253 \\
\hline 132 & 2000346834 & 1958982655 & 185 & 577209206 & 588259153 \\
\hline 133 & 1116973781 & 1089595402 & 186 & 75664259 & 813127169 \\
\hline 134 & 176212546 & 1489154479 & 187 & 1236337863 & 1528500151 \\
\hline 135 & 149088988 & 1002114439 & 188 & 986945543 & 220659907 \\
\hline 136 & 532627125 & 1416866238 & 189 & 1392159857 & 896950143 \\
\hline 137 & 1328588014 & 1109935707 & 190 & 1162693271 & 1915425509 \\
\hline 138 & 1760100544 & 1441532886 & 191 & 1926942905 & 2011323288 \\
\hline 139 & 986701072 & 1540828410 & 192 & 716339850 & 351856912 \\
\hline 140 & 2028151563 & 1114816938 & 193 & 2048812503 & 1526686547 \\
\hline 141 & 1203944694 & 255992726 & 194 & 139912267 & 166848355 \\
\hline 142 & 1601584413 & 402916159 & 195 & 774183112 & 1603613346 \\
\hline 143 & 1608768230 & 820162894 & 196 & 40314461 & 1760945339 \\
\hline 144 & 1272212662 & 1302061686 & 197 & 1862375087 & 1547467728 \\
\hline 145 & 539630487 & 1783425599 & 198 & 922931048 & 2138231185 \\
\hline 146 & 1680993430 & 643328599 & 199 & 1700016462 & 1612700865 \\
\hline 147 & 736799359 & 692751124 & 200 & 1503895733 & 1734490578 \\
\hline 148 & 1998268385 & 867881526 & 201 & 335518060 & 1139189603 \\
\hline 149 & 344773061 & 1787059558 & 202 & 475031609 & 2063342745 \\
\hline 150 & 1245899065 & 347316868 & 203 & 653670677 & 1274118940 \\
\hline 151 & 530817802 & 1175506073 & 204 & 2112262468 & 2107575910 \\
\hline
\end{tabular}




\begin{tabular}{|c|c|c|c|c|c|}
\hline 205 & 1388897175 & 887646311 & 258 & 1312050776 & 584330139 \\
\hline 206 & 886197835 & 1775987183 & 259 & 2141418935 & 1398863571 \\
\hline 207 & 2006041520 & 1824202878 & 260 & 1245332147 & 428275452 \\
\hline 208 & 1846114640 & 1839752023 & 261 & 77248785 & 1625464370 \\
\hline 209 & 371991841 & 1004869923 & 262 & 257281739 & 1504607338 \\
\hline 210 & 256628332 & 2059976090 & 263 & 795698235 & 1384183848 \\
\hline 211 & 577656443 & 290848225 & 264 & 1410756273 & 616749127 \\
\hline 212 & 1365552684 & 1217853554 & 265 & 890523787 & 1182825539 \\
\hline 213 & 607713362 & 1342318006 & 266 & 1089934493 & 439981218 \\
\hline 214 & 1397905833 & 337706462 & 267 & 464975597 & 1056165456 \\
\hline 215 & 143774410 & 409414900 & 268 & 1061469282 & 784067942 \\
\hline 216 & 630149976 & 1712888800 & 269 & 1153064820 & 407439828 \\
\hline 217 & 1583053866 & 1593364008 & 270 & 1792871357 & 1204555552 \\
\hline 218 & 1774160020 & 509281924 & 271 & 1831981870 & 1357224187 \\
\hline 219 & 1734859660 & 1451198654 & 272 & 739784997 & 1036309182 \\
\hline 220 & 585981359 & 1864951180 & 273 & 2093605854 & 404570353 \\
\hline 221 & 1705182038 & 1690070250 & 274 & 1412483300 & 1602066603 \\
\hline 222 & 957493071 & 159922051 & 275 & 391868626 & 1084343251 \\
\hline 223 & 738594063 & 980700862 & 276 & 402704287 & 2115655586 \\
\hline 224 & 174901762 & 404177469 & 277 & 1826026332 & 811650417 \\
\hline 225 & 166210468 & 2060540112 & 278 & 160925558 & 186631783 \\
\hline 226 & 861134986 & 488164062 & 279 & 794586115 & 1396457137 \\
\hline 227 & 312739712 & 1964185534 & 280 & 1224132106 & 676928970 \\
\hline 228 & 813244032 & 1203996675 & 281 & 1709409215 & 1743151030 \\
\hline 229 & 1880141482 & 1693463447 & 282 & 711319873 & 2030582758 \\
\hline 230 & 362715101 & 71119974 & 283 & 873195913 & 1757582611 \\
\hline 231 & 32149084 & 971940061 & 284 & 1897770831 & 1965090892 \\
\hline 232 & 1663458951 & 969846069 & 285 & 1992952803 & 1767653300 \\
\hline 233 & 1191365238 & 340512777 & 286 & 1109382742 & 1921344734 \\
\hline 234 & 1885115048 & 890899598 & 287 & 1310298138 & 1116155526 \\
\hline 235 & 1087553149 & 489297179 & 288 & 1884079626 & 1509816114 \\
\hline 236 & 1956380645 & 1928957525 & 289 & 1937854990 & 1299229875 \\
\hline 237 & 99061688 & 386125290 & 290 & 632510679 & 1318644629 \\
\hline 238 & 758455947 & 701689653 & 291 & 2112788786 & 275193112 \\
\hline 239 & 1512184138 & 1024141839 & 292 & 1668150123 & 1684866830 \\
\hline 240 & 1079550676 & 1837141807 & 293 & 572478429 & 1608740710 \\
\hline 241 & 1369084569 & 787698450 & 294 & 1576714825 & 861953555 \\
\hline 242 & 1253859466 & 2100861590 & 295 & 1211724637 & 438833654 \\
\hline 243 & 260937365 & 1436882719 & 296 & 1755010733 & 1260701146 \\
\hline 244 & 2019058373 & 557806386 & 297 & 737310543 & 1536793247 \\
\hline 245 & 2145843711 & 965209034 & 298 & 22833584 & 1652960674 \\
\hline 246 & 93541056 & 33960827 & 299 & 571289681 & 1003187391 \\
\hline 247 & 164196467 & 967178080 & 300 & 1147652666 & 550064331 \\
\hline 248 & 1313894758 & 2119569182 & 301 & 1678854998 & 1999054875 \\
\hline 249 & 2005656967 & 677078626 & 302 & 1141305352 & 164483214 \\
\hline 250 & 1195268110 & 591293112 & 303 & 302982268 & 752151102 \\
\hline 251 & 1172126242 & 1290875013 & 304 & 76792022 & 836895694 \\
\hline 252 & 1832417435 & 625671069 & 305 & 2008528172 & 314418418 \\
\hline 253 & 602873955 & 1232365359 & 306 & 1963340227 & 1970360475 \\
\hline 254 & 1899092396 & 1348621896 & 307 & 438441826 & 2016903336 \\
\hline 255 & 653180388 & 1549822722 & 308 & 1071097714 & 661776208 \\
\hline 256 & 643956001 & 662759856 & 309 & 423018578 & 1886988651 \\
\hline 257 & 1666152564 & 176649168 & 310 & 1467243631 & 1960091671 \\
\hline
\end{tabular}




\begin{tabular}{|c|c|c|c|c|c|}
\hline 311 & 1260314185 & 1141813464 & 364 & 1289309791 & 1332978770 \\
\hline 312 & 1781629685 & 1574814653 & 365 & 1716568058 & 90638482 \\
\hline 313 & 74134126 & 1559308155 & 366 & 588541615 & 107437509 \\
\hline 314 & 1341521548 & 1235505970 & 367 & 931730704 & 836539238 \\
\hline 315 & 199829188 & 1498587635 & 368 & 1628898897 & 1544899367 \\
\hline 316 & 968663515 & 1172596928 & 369 & 12272696 & 1944202921 \\
\hline 317 & 579330971 & 1376214753 & 370 & 295831688 & 463979418 \\
\hline 318 & 2103306756 & 1074232198 & 371 & 1840992523 & 78581409 \\
\hline 319 & 1263334214 & 639802697 & 372 & 1141199566 & 264976046 \\
\hline 320 & 861802515 & 490656795 & 373 & 1923283252 & 751905082 \\
\hline 321 & 1205740578 & 2054658401 & 374 & 1661121380 & 9390369 \\
\hline 322 & 2042445825 & 838066546 & 375 & 1385885036 & 809635876 \\
\hline 323 & 1155418198 & 30581044 & 376 & 293949725 & 1115675222 \\
\hline 324 & 1790888635 & 761425294 & 377 & 430075440 & 795328467 \\
\hline 325 & 646682360 & 97650985 & 378 & 1729348402 & 1794485016 \\
\hline 326 & 1190011586 & 656509781 & 379 & 1672717171 & 809390695 \\
\hline 327 & 1266053413 & 946719240 & 380 & 1510143305 & 1094020983 \\
\hline 328 & 1070040516 & 11444575 & 381 & 184679140 & 421739853 \\
\hline 329 & 1172797462 & 1408911572 & 382 & 1623747814 & 680327039 \\
\hline 330 & 1866270297 & 434108840 & 383 & 2140264317 & 417130778 \\
\hline 331 & 811899425 & 1883559192 & 384 & 987539049 & 1313470759 \\
\hline 332 & 206399276 & 1347342406 & 385 & 36863841 & 1007246464 \\
\hline 333 & 33669685 & 687877178 & 386 & 65210880 & 1222349785 \\
\hline 334 & 1264504465 & 1023809338 & 387 & 1007202930 & 1356605371 \\
\hline 335 & 2091923581 & 1260295466 & 388 & 1340426627 & 83304061 \\
\hline 336 & 1141209719 & 161513616 & 389 & 981583416 & 94758727 \\
\hline 337 & 2117289644 & 796907663 & 390 & 1780429097 & 1307408932 \\
\hline 338 & 1804403569 & 1296707796 & 391 & 1312155787 & 1248679280 \\
\hline 339 & 286600807 & 1016685880 & 392 & 1035884489 & 619508063 \\
\hline 340 & 1989726528 & 1560102813 & 393 & 125135950 & 642719154 \\
\hline 341 & 127062184 & 1251712400 & 394 & 742740429 & 1693890731 \\
\hline 342 & 1347058183 & 832210430 & 395 & 288825255 & 1955118230 \\
\hline 343 & 1409340522 & 1996018650 & 396 & 2014156709 & 1254279496 \\
\hline 344 & 1189227405 & 2104331372 & 397 & 1214307410 & 1018694086 \\
\hline 345 & 1899480715 & 426715196 & 398 & 641148392 & 822438719 \\
\hline 346 & 1711723894 & 1694565484 & 399 & 325387496 & 54414209 \\
\hline 347 & 753680565 & 1239192032 & 400 & 2055056277 & 613449976 \\
\hline 348 & 1032991156 & 1717081230 & 401 & 776442765 & 2076486058 \\
\hline 349 & 1324267489 & 1234983203 & 402 & 885102022 & 1149796441 \\
\hline 350 & 1228710464 & 1146948653 & 403 & 927275849 & 752844039 \\
\hline 351 & 1018802434 & 21813410 & 404 & 1334327393 & 917679585 \\
\hline 352 & 1911244789 & 337532525 & 405 & 1675453773 & 1281629760 \\
\hline 353 & 1983157251 & 2074171422 & 406 & 555294696 & 2133108384 \\
\hline 354 & 1539339023 & 1829805786 & 407 & 800783442 & 1587803903 \\
\hline 355 & 1060082626 & 1972231738 & 408 & 1740720889 & 1731544993 \\
\hline 356 & 276201572 & 1097008267 & 409 & 1730609842 & 1096128219 \\
\hline 357 & 149880558 & 934481177 & 410 & 1058241085 & 1846766848 \\
\hline 358 & 714121826 & 298183567 & 411 & 1785146475 & 1531785678 \\
\hline 359 & 1843325261 & 1610219570 & 412 & 740357912 & 1787941284 \\
\hline 360 & 631523153 & 1248389806 & 413 & 1147500 & 670262593 \\
\hline 361 & 391848137 & 1371569011 & 414 & 575200385 & 896487560 \\
\hline 362 & 551329533 & 1212483377 & 415 & 1471808410 & 1736230883 \\
\hline 363 & 938989005 & 2073840280 & 416 & 1941068257 & 824995758 \\
\hline
\end{tabular}




\begin{tabular}{|c|c|c|c|c|c|}
\hline 417 & 1160856161 & 1475446798 & 470 & 1514213203 & 313779669 \\
\hline 418 & 921806729 & 2018804778 & 471 & 1920775260 & 102539627 \\
\hline 419 & 1788098369 & 574313877 & 472 & 64724571 & 994457135 \\
\hline 420 & 133789132 & 721445202 & 473 & 1329349426 & 128968179 \\
\hline 421 & 285803709 & 2032694861 & 474 & 704512771 & 1362969883 \\
\hline 422 & 1864846469 & 1255385842 & 475 & 716389172 & 359259411 \\
\hline 423 & 1394368802 & 1770979060 & 476 & 1350390463 & 898274290 \\
\hline 424 & 428096424 & 717390469 & 477 & 994640427 & 820580723 \\
\hline 425 & 1530011961 & 1810685406 & 478 & 1990487275 & 168796960 \\
\hline 426 & 87720417 & 1487356173 & 479 & 2016449080 & 1385624087 \\
\hline 427 & 1688620286 & 570471055 & 480 & 406198312 & 501737421 \\
\hline 428 & 1772635608 & 1720849654 & 481 & 1291306112 & 349413705 \\
\hline 429 & 305629937 & 171137503 & 482 & 1936895553 & 1081844744 \\
\hline 430 & 2019319262 & 1003907006 & 483 & 804007878 & 360746069 \\
\hline 431 & 1245837848 & 455090054 & 484 & 1216908435 & 949843423 \\
\hline 432 & 1723936450 & 2004813525 & 485 & 1149783014 & 1314830419 \\
\hline 433 & 1254214291 & 21663349 & 486 & 525669499 & 1204688427 \\
\hline 434 & 988806309 & 1762791285 & 487 & 1451733570 & 115820452 \\
\hline 435 & 1486257176 & 1938951816 & 488 & 2057456263 & 1729063416 \\
\hline 436 & 528722329 & 265523341 & 489 & 1589818680 & 1016661611 \\
\hline 437 & 1615987471 & 97000370 & 490 & 1172308231 & 767976867 \\
\hline 438 & 1968848052 & 1095217691 & 491 & 173470663 & 1408412475 \\
\hline 439 & 1762887491 & 1448510334 & 492 & 2030571242 & 548906642 \\
\hline 440 & 1746948900 & 1585256823 & 493 & 2099487725 & 1429979261 \\
\hline 441 & 561257488 & 674453006 & 494 & 100802875 & 777346498 \\
\hline 442 & 1998544889 & 1241774042 & 495 & 465831687 & 1369568087 \\
\hline 443 & 1713155272 & 805169418 & 496 & 1213006582 & 1820983059 \\
\hline 444 & 412354136 & 835982966 & 497 & 377599565 & 211694992 \\
\hline 445 & 259673874 & 319769302 & 498 & 2113968442 & 1861292489 \\
\hline 446 & 1343565228 & 488852680 & 499 & 1511528650 & 3552113 \\
\hline 447 & 259687812 & 154668440 & 500 & 825573620 & 123790919 \\
\hline 448 & 434487406 & 579378249 & 501 & 1347209343 & 1617019942 \\
\hline 449 & 673432385 & 712056863 & 502 & 1903684402 & 354805716 \\
\hline 450 & 1891005503 & 1597995846 & 503 & 1950131213 & 1712557288 \\
\hline 451 & 373288012 & 2104548073 & 504 & 341162418 & 613338376 \\
\hline 452 & 399213769 & 582725001 & 505 & 2079196026 & 1900108003 \\
\hline 453 & 1570447985 & 703904093 & 506 & 1518743748 & 1011881568 \\
\hline 454 & 519758726 & 1897977421 & 507 & 1573726427 & 1149059810 \\
\hline 455 & 1190907461 & 1910726428 & 508 & 1837760687 & 378753279 \\
\hline 456 & 1532578229 & 850986986 & 509 & 1267943539 & 610960024 \\
\hline 457 & 1277169775 & 589096147 & 510 & 487332958 & 1125294995 \\
\hline 458 & 2139137135 & 1701648196 & 511 & 1481239760 & 1672399164 \\
\hline 459 & 2124965063 & 1199338723 & 512 & 2017502195 & 2100767980 \\
\hline 460 & 1901408148 & 2136019460 & 513 & 1611145466 & 974135938 \\
\hline 461 & 1422303415 & 851151583 & 514 & 1037537336 & 1145478759 \\
\hline 462 & 1643821410 & 659871253 & 515 & 2060906356 & 696408018 \\
\hline 463 & 27764346 & 723946608 & 516 & 468250002 & 734552037 \\
\hline 464 & 1541873143 & 1762908967 & 517 & 849754097 & 1306150353 \\
\hline 465 & 788749927 & 170784860 & 518 & 128249084 & 1745004428 \\
\hline 466 & 940612682 & 1982347087 & 519 & 1047323917 & 263322818 \\
\hline 467 & 1766382927 & 822715884 & 520 & 1599611436 & 1908238055 \\
\hline 468 & 1164806076 & 1529267999 & 521 & 1661573927 & 1346063841 \\
\hline 469 & 1303892614 & 1206018921 & 522 & 1273082375 & 403707125 \\
\hline
\end{tabular}




\begin{tabular}{|c|c|c|c|c|c|}
\hline 523 & 1161870917 & 798157817 & 576 & 1339606827 & 726148332 \\
\hline 524 & 1028014402 & 2040008576 & 577 & 192757058 & 190237815 \\
\hline 525 & 965692614 & 1123093686 & 578 & 2139692563 & 983684706 \\
\hline 526 & 1301831077 & 1961133565 & 579 & 610527028 & 129719695 \\
\hline 527 & 599559324 & 1207646506 & 580 & 2145025782 & 1503214604 \\
\hline 528 & 1992628552 & 947715100 & 581 & 1064988541 & 1382331839 \\
\hline 529 & 1080469780 & 703778413 & 582 & 950519526 & 363563736 \\
\hline 530 & 1695439219 & 2112417841 & 583 & 891672657 & 1198966942 \\
\hline 531 & 1865232798 & 1708678058 & 584 & 365479037 & 1387126662 \\
\hline 532 & 793907253 & 1523254806 & 585 & 840933783 & 915419050 \\
\hline 533 & 287507413 & 485785884 & 586 & 1000880066 & 291922389 \\
\hline 534 & 1307090439 & 1872828210 & 587 & 1914633538 & 221919535 \\
\hline 535 & 94153252 & 1112253058 & 588 & 2053263396 & 1778512038 \\
\hline 536 & 248329167 & 1047300490 & 589 & 1662710058 & 1406062067 \\
\hline 537 & 1255891655 & 352462819 & 590 & 869750392 & 567102530 \\
\hline 538 & 1342578140 & 200436258 & 591 & 219651550 & 1465116750 \\
\hline 539 & 1128811393 & 342371565 & 592 & 1561773292 & 1099916667 \\
\hline 540 & 684059411 & 157196518 & 593 & 1338666098 & 1421016852 \\
\hline 541 & 192843790 & 1806905158 & 594 & 817153290 & 355010092 \\
\hline 542 & 757911484 & 909088767 & 595 & 1936112939 & 41303893 \\
\hline 543 & 2094877972 & 2076198489 & 596 & 840070149 & 116194216 \\
\hline 544 & 1521617173 & 2015626315 & 597 & 1937918451 & 1430041003 \\
\hline 545 & 1690423289 & 1191445148 & 598 & 469772241 & 81175569 \\
\hline 546 & 123475452 & 1180625450 & 599 & 378877641 & 393179972 \\
\hline 547 & 1739410881 & 1067896 & 600 & 626982170 & 110597988 \\
\hline 548 & 1971415905 & 1999500895 & 601 & 1876933435 & 1013399843 \\
\hline 549 & 1570584314 & 604058701 & 602 & 979590664 & 2139972938 \\
\hline 550 & 616688930 & 481652737 & 603 & 417997964 & 1022364432 \\
\hline 551 & 1452964214 & 1322152946 & 604 & 1142674231 & 1585582419 \\
\hline 552 & 1648154184 & 2020474349 & 605 & 1344799876 & 2132203958 \\
\hline 553 & 536179518 & 518029535 & 606 & 1175283465 & 939327522 \\
\hline 554 & 210413213 & 107832165 & 607 & 918845489 & 810255574 \\
\hline 555 & 1316873234 & 309834618 & 608 & 384410385 & 1421795304 \\
\hline 556 & 1239237104 & 64705243 & 609 & 1172884838 & 1741183620 \\
\hline 557 & 1408572747 & 2132748931 & 610 & 1785355789 & 251750292 \\
\hline 558 & 1105206813 & 540136846 & 611 & 1351351695 & 1469998520 \\
\hline 559 & 1296675788 & 1633953662 & 612 & 1194887870 & 183384099 \\
\hline 560 & 311157571 & 1270892397 & 613 & 1021117843 & 878963463 \\
\hline 561 & 1913180375 & 579551341 & 614 & 1192134034 & 1951724719 \\
\hline 562 & 361965394 & 2086164853 & 615 & 606079214 & 560163047 \\
\hline 563 & 678733435 & 1909407061 & 616 & 1228009572 & 934018601 \\
\hline 564 & 1900749209 & 717500834 & 617 & 1304360428 & 903678870 \\
\hline 565 & 473353117 & 1008284359 & 618 & 532618540 & 384910313 \\
\hline 566 & 502618513 & 1096527811 & 619 & 763194726 & 1083662416 \\
\hline 567 & 1111210760 & 353441376 & 620 & 2005129761 & 2012245814 \\
\hline 568 & 1073204170 & 152385830 & 621 & 1051824616 & 1787130897 \\
\hline 569 & 139114258 & 2062720897 & 622 & 1342290614 & 1273077750 \\
\hline 570 & 665212036 & 573523094 & 623 & 1767308920 & 151459158 \\
\hline 571 & 78958400 & 2140940992 & 624 & 2137393023 & 1292664392 \\
\hline 572 & 1340026313 & 2015106063 & 625 & 1563910114 & 1782904264 \\
\hline 573 & 1070741205 & 639731493 & 626 & 315984262 & 1266144854 \\
\hline 574 & 1359904212 & 1553901877 & 627 & 799137468 & 683057050 \\
\hline 575 & 1976633724 & 1246559812 & 628 & 322897380 & 1465603872 \\
\hline
\end{tabular}




\begin{tabular}{|c|c|c|c|c|c|}
\hline 629 & 879858557 & 1413396637 & 682 & 569281496 & 734547835 \\
\hline 630 & 1971649187 & 590308456 & 683 & 1735141825 & 1157136743 \\
\hline 631 & 1389296742 & 73619211 & 684 & 1708392198 & 1019516856 \\
\hline 632 & 2038977772 & 398365551 & 685 & 1656370257 & 2052569863 \\
\hline 633 & 1296434108 & 575532750 & 686 & 1172510227 & 1089671946 \\
\hline 634 & 1904958378 & 265434147 & 687 & 1590082616 & 1518321161 \\
\hline 635 & 1296663931 & 314956556 & 688 & 1995076949 & 1599008707 \\
\hline 636 & 406016541 & 855648898 & 689 & 1866960302 & 1222045472 \\
\hline 637 & 1341667012 & 1348502326 & 690 & 539799296 & 568133759 \\
\hline 638 & 509163858 & 441630926 & 691 & 214609086 & 1905441655 \\
\hline 639 & 295920320 & 1423921723 & 692 & 467034919 & 405874259 \\
\hline 640 & 228147726 & 1988516544 & 693 & 1454904655 & 13325141 \\
\hline 641 & 246819730 & 1860030987 & 694 & 2061774327 & 1837628716 \\
\hline 642 & 805798880 & 1097770896 & 695 & 245517089 & 1727188799 \\
\hline 643 & 1887353965 & 469122319 & 696 & 651610502 & 2067230783 \\
\hline 644 & 1601281967 & 1489735074 & 697 & 2055813165 & 2038309543 \\
\hline 645 & 184005517 & 2122041340 & 698 & 299770526 & 457119092 \\
\hline 646 & 2000714164 & 1224644294 & 699 & 1265885164 & 1460272465 \\
\hline 647 & 1050614131 & 1843543104 & 700 & 390285975 & 183594145 \\
\hline 648 & 18450969 & 1359449769 & 701 & 614863030 & 983652313 \\
\hline 649 & 2037301791 & 1649015603 & 702 & 1352039806 & 14472227 \\
\hline 650 & 1257762843 & 387605029 & 703 & 1688912960 & 309400019 \\
\hline 651 & 270012966 & 1918109143 & 704 & 1475277001 & 905212637 \\
\hline 652 & 856282854 & 337120581 & 705 & 623059165 & 1211174887 \\
\hline 653 & 1199135876 & 925971046 & 706 & 677060625 & 1103838023 \\
\hline 654 & 590401654 & 667177332 & 707 & 1304767863 & 723211517 \\
\hline 655 & 2050507837 & 1597371560 & 708 & 1494313604 & 1727687760 \\
\hline 656 & 1792561151 & 1671581440 & 709 & 655143619 & 1557309125 \\
\hline 657 & 1473180888 & 48801129 & 710 & 999643726 & 453822693 \\
\hline 658 & 695488413 & 325196499 & 711 & 103913624 & 1234437237 \\
\hline 659 & 2034065102 & 2005216717 & 712 & 214456222 & 1255338286 \\
\hline 660 & 321163104 & 1612979587 & 713 & 183974463 & 410297043 \\
\hline 661 & 2033109981 & 390164981 & 714 & 1425324918 & 1904058094 \\
\hline 662 & 1810705409 & 1441368155 & 715 & 576041230 & 1088409460 \\
\hline 663 & 1297300985 & 1100855845 & 716 & 1854564475 & 2040310744 \\
\hline 664 & 1313524075 & 82105216 & 717 & 537212291 & 674819486 \\
\hline 665 & 23690604 & 1630506869 & 718 & 44896068 & 1357067059 \\
\hline 666 & 860521510 & 10517671 & 719 & 285218316 & 547710224 \\
\hline 667 & 427163761 & 900411361 & 720 & 1888222388 & 907621948 \\
\hline 668 & 699930254 & 254299936 & 721 & 1844541679 & 62768446 \\
\hline 669 & 770467990 & 1891744976 & 722 & 714996056 & 1444344573 \\
\hline 670 & 316108179 & 1146877270 & 723 & 2024396968 & 465597618 \\
\hline 671 & 105445804 & 899337523 & 724 & 1476443427 & 1349425341 \\
\hline 672 & 1723634978 & 821249886 & 725 & 269167998 & 563222022 \\
\hline 673 & 2050168568 & 1427817043 & 726 & 2073651537 & 1464025231 \\
\hline 674 & 882768091 & 746760020 & 727 & 1552624594 & 643051815 \\
\hline 675 & 328033928 & 1211952506 & 728 & 904200976 & 1767094852 \\
\hline 676 & 362468427 & 129311401 & 729 & 591911588 & 408395929 \\
\hline 677 & 1337265389 & 1286281020 & 730 & 193603012 & 104309716 \\
\hline 678 & 1083793641 & 1669994540 & 731 & 1635276234 & 2118616635 \\
\hline 679 & 30744239 & 1361978712 & 732 & 269534072 & 586413346 \\
\hline 680 & 838457806 & 883335016 & 733 & 495111194 & 2031149907 \\
\hline 681 & 386833515 & 847860458 & 734 & 652104434 & 1861053426 \\
\hline
\end{tabular}




\begin{tabular}{|c|c|c|c|c|c|}
\hline 735 & 1549621890 & 606932006 & 788 & 603260299 & 1016609620 \\
\hline 736 & 568973961 & 2137667037 & 789 & 1942866583 & 1450131526 \\
\hline 737 & 813132436 & 502816034 & 790 & 1966881205 & 1194334402 \\
\hline 738 & 1336017158 & 1139558464 & 791 & 98940130 & 1834786196 \\
\hline 739 & 2071596236 & 657046668 & 792 & 938591094 & 1501288250 \\
\hline 740 & 779393390 & 903207919 & 793 & 1788669 & 548906223 \\
\hline 741 & 1804773814 & 2124651837 & 794 & 818078991 & 550709219 \\
\hline 742 & 708944123 & 2140742153 & 795 & 831741665 & 470953378 \\
\hline 743 & 1358121740 & 1578318555 & 796 & 678750261 & 930639951 \\
\hline 744 & 416587614 & 1428486717 & 797 & 1214630979 & 867876881 \\
\hline 745 & 1862714134 & 2079535032 & 798 & 706969436 & 683791455 \\
\hline 746 & 1904973701 & 744502297 & 799 & 1665822559 & 1469770788 \\
\hline 747 & 753773950 & 729850485 & 800 & 1636359045 & 1101520784 \\
\hline 748 & 1362418469 & 51398739 & 801 & 1245658947 & 728733088 \\
\hline 749 & 1791080455 & 355894745 & 802 & 770895483 & 1562862259 \\
\hline 750 & 668747570 & 1493311534 & 803 & 1081488056 & 1189116736 \\
\hline 751 & 717405743 & 788579588 & 804 & 1788263704 & 1226936635 \\
\hline 752 & 1194224249 & 844393016 & 805 & 2091086118 & 234809488 \\
\hline 753 & 1524471741 & 1283354336 & 806 & 465814633 & 2117571834 \\
\hline 754 & 1645308077 & 480110849 & 807 & 1202601672 & 90141784 \\
\hline 755 & 1554335652 & 1172575103 & 808 & 1163546797 & 176308674 \\
\hline 756 & 1372719286 & 1478188318 & 809 & 8265669 & 536367546 \\
\hline 757 & 1598828487 & 310507642 & 810 & 2991910 & 97011034 \\
\hline 758 & 987406440 & 429478546 & 811 & 286259077 & 777332244 \\
\hline 759 & 985929029 & 823824422 & 812 & 957041779 & 339752025 \\
\hline 760 & 335700720 & 2113853784 & 813 & 1676807412 & 1748657593 \\
\hline 761 & 376527438 & 1730824654 & 814 & 492236217 & 755848555 \\
\hline 762 & 242664657 & 1021906877 & 815 & 841182624 & 665732276 \\
\hline 763 & 2121735020 & 1354728910 & 816 & 1536190781 & 1813340910 \\
\hline 764 & 1327897940 & 889006596 & 817 & 1333552019 & 440861906 \\
\hline 765 & 1970662657 & 1666245602 & 818 & 379388092 & 1954600911 \\
\hline 766 & 1920201445 & 963733081 & 819 & 1261210682 & 246599731 \\
\hline 767 & 422442582 & 1453461343 & 820 & 1665503721 & 1180613087 \\
\hline 768 & 2003863729 & 1087830245 & 821 & 1229258734 & 393611108 \\
\hline 769 & 858447002 & 401645133 & 822 & 253932038 & 1306172048 \\
\hline 770 & 971578456 & 1117501101 & 823 & 1350335177 & 1287498924 \\
\hline 771 & 1466895876 & 77309022 & 824 & 196617278 & 790302179 \\
\hline 772 & 602919921 & 1283663176 & 825 & 1056505297 & 203703749 \\
\hline 773 & 1509114735 & 987869867 & 826 & 805750730 & 1225116269 \\
\hline 774 & 129801316 & 118904973 & 827 & 138628040 & 1204408318 \\
\hline 775 & 1842398258 & 677888047 & 828 & 388136030 & 1730164170 \\
\hline 776 & 1217344316 & 254015901 & 829 & 2132211527 & 1362304872 \\
\hline 777 & 1049631999 & 1369824110 & 830 & 1523763470 & 1139975127 \\
\hline 778 & 1688162090 & 1130079100 & 831 & 429024224 & 634610725 \\
\hline 779 & 360492337 & 1870281605 & 832 & 448141959 & 1156182458 \\
\hline 780 & 2125255252 & 1244503369 & 833 & 1544434358 & 2003315747 \\
\hline 781 & 1800605788 & 501136467 & 834 & 412055400 & 1898528373 \\
\hline 782 & 806777465 & 876529528 & 835 & 1796085439 & 1961689965 \\
\hline 783 & 833746762 & 1609891137 & 836 & 2130378651 & 1507702451 \\
\hline 784 & 1512511171 & 937635328 & 837 & 621144941 & 1924077274 \\
\hline 785 & 772547761 & 1355321984 & 838 & 576013353 & 168345174 \\
\hline 786 & 1994543238 & 1163413333 & 839 & 278474290 & 1558910692 \\
\hline 787 & 1502625202 & 1862705312 & 840 & 1007588450 & 297873961 \\
\hline
\end{tabular}




\begin{tabular}{|c|c|c|c|c|c|}
\hline 841 & 189659492 & 1426282628 & 894 & 569580565 & 2063797248 \\
\hline 842 & 1549386342 & 1063133883 & 895 & 145860577 & 557743849 \\
\hline 843 & 1464432368 & 2134358053 & 896 & 1749343170 & 595182200 \\
\hline 844 & 1622360294 & 1730282467 & 897 & 1742151508 & 2098281232 \\
\hline 845 & 1216360874 & 1069569045 & 898 & 1986870148 & 168627864 \\
\hline 846 & 2085590591 & 1905369354 & 899 & 1240381461 & 1591418276 \\
\hline 847 & 100518245 & 609923737 & 900 & 1443411677 & 2065876470 \\
\hline 848 & 1041681347 & 1844072131 & 901 & 358853212 & 738999234 \\
\hline 849 & 1154855117 & 205597261 & 902 & 1792679753 & 1799807991 \\
\hline 850 & 1952228254 & 520852702 & 903 & 781577791 & 153018955 \\
\hline 851 & 1868904127 & 1597836043 & 904 & 557896040 & 865359883 \\
\hline 852 & 1805018889 & 1347893542 & 905 & 720342174 & 1578966858 \\
\hline 853 & 730595367 & 14939763 & 906 & 1258784072 & 480563479 \\
\hline 854 & 2081787660 & 2129063590 & 907 & 1795507439 & 28300839 \\
\hline 855 & 24613594 & 842975978 & 908 & 958276197 & 1935246820 \\
\hline 856 & 1550320980 & 1134481207 & 909 & 1573576131 & 1667150276 \\
\hline 857 & 1554010854 & 2023196023 & 910 & 245836596 & 715750058 \\
\hline 858 & 1338021463 & 1503531824 & 911 & 507875401 & 1137066463 \\
\hline 859 & 834930214 & 1518739221 & 912 & 1468637288 & 1808826700 \\
\hline 860 & 4980078 & 1613109128 & 913 & 1369369289 & 617022592 \\
\hline 861 & 978701636 & 1401488204 & 914 & 505211999 & 1854850944 \\
\hline 862 & 925310659 & 1868170601 & 915 & 1076179717 & 1826618842 \\
\hline 863 & 2018606654 & 62991103 & 916 & 79430661 & 1842071310 \\
\hline 864 & 784361996 & 1801737675 & 917 & 914700893 & 974260565 \\
\hline 865 & 2129457043 & 83500459 & 918 & 1270092358 & 1038533406 \\
\hline 866 & 187564438 & 1432183519 & 919 & 387115672 & 320042913 \\
\hline 867 & 1435766986 & 1853684108 & 920 & 424082943 & 526976964 \\
\hline 868 & 1977391683 & 339192316 & 921 & 1959930884 & 1091249810 \\
\hline 869 & 1739709675 & 496353298 & 922 & 1657875177 & 2004252113 \\
\hline 870 & 1990149958 & 444868210 & 923 & 894408965 & 583144855 \\
\hline 871 & 736848787 & 1586686049 & 924 & 1712552436 & 1362601167 \\
\hline 872 & 93756849 & 1103683426 & 925 & 122317987 & 1586944482 \\
\hline 873 & 94732634 & 2136275555 & 926 & 1722724504 & 711966500 \\
\hline 874 & 1920896178 & 992018922 & 927 & 1535475814 & 84120780 \\
\hline 875 & 692906723 & 807012742 & 928 & 1128584846 & 1342600984 \\
\hline 876 & 778618281 & 74445502 & 929 & 1442293122 & 1152685195 \\
\hline 877 & 2070573599 & 656993496 & 930 & 105545458 & 1707021128 \\
\hline 878 & 101702110 & 1075047423 & 931 & 1253655175 & 1350852019 \\
\hline 879 & 306370154 & 2027947707 & 932 & 627925321 & 244115220 \\
\hline 880 & 123744277 & 1288720685 & 933 & 1649426210 & 1923757599 \\
\hline 881 & 569137163 & 914593389 & 934 & 371040301 & 1999879482 \\
\hline 882 & 1741957404 & 730510090 & 935 & 139792537 & 1862953037 \\
\hline 883 & 1935580948 & 2103553901 & 936 & 39621123 & 2083634411 \\
\hline 884 & 1221799271 & 1118323098 & 937 & 1911007337 & 863097028 \\
\hline 885 & 1765428079 & 1387127800 & 938 & 1057580664 & 824001308 \\
\hline 886 & 593719119 & 1702251275 & 939 & 1672122228 & 215559997 \\
\hline 887 & 2023344700 & 825202023 & 940 & 1407765945 & 293020814 \\
\hline 888 & 995099800 & 1819831391 & 941 & 924687022 & 400463614 \\
\hline 889 & 1032466845 & 1409798426 & 942 & 262059125 & 260626 \\
\hline 890 & 1425425981 & 1521001800 & 943 & 1202250847 & 1727287195 \\
\hline 891 & 1470116259 & 311496099 & 944 & 1807707702 & 987766565 \\
\hline 892 & 1224198037 & 905630039 & 945 & 416008863 & 778290102 \\
\hline 893 & 1980939658 & 2006556013 & 946 & 1212716291 & 62468831 \\
\hline
\end{tabular}




\begin{tabular}{|c|c|c|c|c|c|}
\hline 947 & 1550540293 & 2131980504 & 1000 & 2098403047 & 1795647722 \\
\hline 948 & 690231262 & 1624073243 & 1001 & 582087394 & 1547430139 \\
\hline 949 & 1859824510 & 1682450350 & 1002 & 1500319924 & 1942832978 \\
\hline 950 & 466562081 & 1309247970 & 1003 & 268274790 & 63396443 \\
\hline 951 & 1117866127 & 484114791 & 1004 & 1984269740 & 1483307004 \\
\hline 952 & 350981795 & 1664953465 & 1005 & 620974345 & 521298334 \\
\hline 953 & 328681929 & 1472610359 & 1006 & 1694340223 & 1648875271 \\
\hline 954 & 1154065668 & 931914891 & 1007 & 417069438 & 2129744694 \\
\hline 955 & 389671481 & 717419913 & 1008 & 1631261200 & 1070044228 \\
\hline 956 & 1557342903 & 1380380811 & 1009 & 1869621444 & 1973064398 \\
\hline 957 & 929715374 & 1143982011 & 1010 & 978049678 & 1211821264 \\
\hline 958 & 632080275 & 2085128401 & 1011 & 302621674 & 574139566 \\
\hline 959 & 196989854 & 1127122649 & 1012 & 643989471 & 373799674 \\
\hline 960 & 257630756 & 126142490 & 1013 & 2119762287 & 876550333 \\
\hline 961 & 1481731973 & 932681075 & 1014 & 936815450 & 630510843 \\
\hline 962 & 1131443818 & 352190985 & 1015 & 740092235 & 2039312854 \\
\hline 963 & 2076472275 & 1431371651 & 1016 & 754191525 & 1361151262 \\
\hline 964 & 306579967 & 1481130136 & 1017 & 2081853691 & 210297209 \\
\hline 965 & 873292095 & 1591482248 & 1018 & 397319817 & 64288361 \\
\hline 966 & 1241167652 & 965114288 & 1019 & 1391778438 & 1955714290 \\
\hline 967 & 652734589 & 231120903 & 1020 & 281532932 & 947865039 \\
\hline 968 & 1962764836 & 981921390 & 1021 & 550322248 & 723713558 \\
\hline 969 & 1452097313 & 824435113 & 1022 & 764706808 & 2082865872 \\
\hline 970 & 262778656 & 1384069562 & 1023 & 348668959 & 1514432369 \\
\hline 971 & 636552735 & 2124099112 & 1024 & 168889412 & 1689678414 \\
\hline 972 & 1947705943 & 1634584048 & 1025 & 256252265 & 1691896028 \\
\hline 973 & 1298751990 & 202254118 & 1026 & 1479544459 & 280147193 \\
\hline 974 & 1227483540 & 1565701938 & 1027 & 205174393 & 1209896060 \\
\hline 975 & 1953350015 & 2044981894 & 1028 & 2095041788 & 116415974 \\
\hline 976 & 692120442 & 12482561 & 1029 & 1572481731 & 1015057200 \\
\hline 977 & 842407569 & 249165249 & 1030 & 463816515 & 321718374 \\
\hline 978 & 1429529937 & 1045438384 & 1031 & 626894492 & 2122868719 \\
\hline 979 & 481923120 & 549681535 & 1032 & 1898401020 & 186100333 \\
\hline 980 & 1816585128 & 1121120383 & 1033 & 591768014 & 337928454 \\
\hline 981 & 915499934 & 1551347515 & 1034 & 1812087409 & 709107099 \\
\hline 982 & 1489811660 & 1651059116 & 1035 & 1844629067 & 1113482128 \\
\hline 983 & 2131870244 & 186713544 & 1036 & 130120520 & 1208402300 \\
\hline 984 & 486435599 & 1728204306 & 1037 & 1046217973 & 1518664124 \\
\hline 985 & 1906099470 & 960011795 & 1038 & 1435979737 & 1774595890 \\
\hline 986 & 1004313051 & 2104941270 & 1039 & 1543805024 & 241798858 \\
\hline 987 & 1409786269 & 673315085 & 1040 & 1224658984 & 1275132934 \\
\hline 988 & 1280745264 & 399403599 & 1041 & 1832984530 & 385336709 \\
\hline 989 & 421301336 & 1450703063 & 1042 & 1701341982 & 1717570761 \\
\hline 990 & 2109527744 & 848504545 & 1043 & 1207370730 & 1179292286 \\
\hline 991 & 1762301116 & 638250731 & 1044 & 1501895721 & 578363805 \\
\hline 992 & 900403581 & 325744433 & 1045 & 474919743 & 1158489530 \\
\hline 993 & 558590037 & 1936112959 & 1046 & 1867392781 & 853045475 \\
\hline 994 & 1044612017 & 1604227016 & 1047 & 967204169 & 825374626 \\
\hline 995 & 2011860500 & 752824607 & 1048 & 154003684 & 912327532 \\
\hline 996 & 1337852760 & 2118038800 & 1049 & 1427953300 & 187906156 \\
\hline 997 & 872199193 & 633659178 & 1050 & 1054901256 & 1047785038 \\
\hline 998 & 763627693 & 1371335136 & 1051 & 636401898 & 166767561 \\
\hline 999 & 1170365 & 111278425 & 1052 & 214993172 & 1583558954 \\
\hline
\end{tabular}




\begin{tabular}{|c|c|c|c|c|c|}
\hline 1053 & 868299589 & 1197536786 & 1106 & 1561561886 & 1467155860 \\
\hline 1054 & 1792740545 & 2120208810 & 1107 & 897755166 & 970145126 \\
\hline 1055 & 1685382318 & 2041318244 & 1108 & 1902784514 & 1231126019 \\
\hline 1056 & 1009035642 & 2081498441 & 1109 & 836749758 & 1443874738 \\
\hline 1057 & 207560370 & 558817466 & 1110 & 1281129768 & 1965782274 \\
\hline 1058 & 321214659 & 1177611750 & 1111 & 1117983213 & 1075080572 \\
\hline 1059 & 2127076312 & 1181311227 & 1112 & 643620225 & 1026663782 \\
\hline 1060 & 767089852 & 1085142492 & 1113 & 1987965234 & 1676398385 \\
\hline 1061 & 529383580 & 356082840 & 1114 & 2055191896 & 793256707 \\
\hline 1062 & 406885819 & 1175277902 & 1115 & 877649402 & 1009877247 \\
\hline 1063 & 1155844352 & 925874004 & 1116 & 1616648970 & 2052475573 \\
\hline 1064 & 2144600136 & 561830683 & 1117 & 862683430 & 510397079 \\
\hline 1065 & 1929871872 & 28234600 & 1118 & 962970537 & 115316105 \\
\hline 1066 & 136788723 & 1604508149 & 1119 & 1867496430 & 2383432 \\
\hline 1067 & 896624785 & 1636078253 & 1120 & 1835236572 & 2135629675 \\
\hline 1068 & 1207508341 & 1579436594 & 1121 & 1785647116 & 430054039 \\
\hline 1069 & 96769002 & 1050595949 & 1122 & 1013462286 & 272517198 \\
\hline 1070 & 1614190925 & 660439219 & 1123 & 843454157 & 1324766538 \\
\hline 1071 & 1767888610 & 1834768326 & 1124 & 429879478 & 1262795367 \\
\hline 1072 & 915865899 & 370252052 & 1125 & 1891042870 & 119117564 \\
\hline 1073 & 1421475999 & 1839307170 & 1126 & 1231462285 & 476267077 \\
\hline 1074 & 1702587975 & 2057459659 & 1127 & 450060242 & 1339256504 \\
\hline 1075 & 1832407942 & 1517842216 & 1128 & 185193432 & 428064020 \\
\hline 1076 & 747775129 & 1759294109 & 1129 & 1878518777 & 993819015 \\
\hline 1077 & 1416956813 & 78600056 & 1130 & 241727672 & 250208465 \\
\hline 1078 & 527465959 & 2032796221 & 1131 & 1204768748 & 1020145378 \\
\hline 1079 & 1928054640 & 1630502065 & 1132 & 1669864168 & 1535573382 \\
\hline 1080 & 285220964 & 1476468977 & 1133 & 1885989678 & 371896841 \\
\hline 1081 & 76865213 & 1649608038 & 1134 & 980271600 & 2016005578 \\
\hline 1082 & 1265594525 & 1136301041 & 1135 & 1664705284 & 1000388424 \\
\hline 1083 & 658254486 & 624707655 & 1136 & 1541882087 & 1078641602 \\
\hline 1084 & 376202886 & 92021559 & 1137 & 477031320 & 1898387598 \\
\hline 1085 & 474421297 & 751786175 & 1138 & 1855602349 & 2084505716 \\
\hline 1086 & 1038018472 & 34053094 & 1139 & 1425499653 & 893801664 \\
\hline 1087 & 1944134447 & 957761032 & 1140 & 981643853 & 1280626221 \\
\hline 1088 & 1595252131 & 930056987 & 1141 & 530512249 & 2135530807 \\
\hline 1089 & 713615469 & 1116571139 & 1142 & 785201287 & 918354651 \\
\hline 1090 & 1270616884 & 1957639212 & 1143 & 239962919 & 22905477 \\
\hline 1091 & 1267504172 & 1266172043 & 1144 & 1596997586 & 2080505082 \\
\hline 1092 & 741784997 & 1083525613 & 1145 & 169756353 & 362178019 \\
\hline 1093 & 1537921804 & 1297915386 & 1146 & 1070838109 & 808584087 \\
\hline 1094 & 19884794 & 1460730661 & 1147 & 328731231 & 1760225818 \\
\hline 1095 & 2005357805 & 1579081866 & 1148 & 1546726314 & 1570685433 \\
\hline 1096 & 284527880 & 1851720820 & 1149 & 1850764169 & 1186517317 \\
\hline 1097 & 838870868 & 1909169784 & 1150 & 349979449 & 241728099 \\
\hline 1098 & 1023337363 & 523691363 & 1151 & 1373662011 & 1723517284 \\
\hline 1099 & 1868958795 & 213510211 & 1152 & 327078266 & 31420573 \\
\hline 1100 & 651454838 & 1167380421 & 1153 & 1757786491 & 1343095587 \\
\hline 1101 & 1007757679 & 1079181010 & 1154 & 1697327676 & 1337094674 \\
\hline 1102 & 341236870 & 762552158 & 1155 & 2133924836 & 720818544 \\
\hline 1103 & 1629149734 & 955104776 & 1156 & 641581381 & 696728336 \\
\hline 1104 & 1364868264 & 1778291437 & 1157 & 1998340175 & 770440155 \\
\hline 1105 & 1861171722 & 2086044347 & 1158 & 1398921164 & 2046415629 \\
\hline
\end{tabular}




\begin{tabular}{|c|c|c|c|c|c|}
\hline 1159 & 1910209335 & 1505205457 & 1212 & 1777979964 & 2038985312 \\
\hline 1160 & 1458107992 & 1160312546 & 1213 & 2003691470 & 2031886042 \\
\hline 1161 & 1797064346 & 723104862 & 1214 & 269222088 & 126881735 \\
\hline 1162 & 2092569710 & 1223769806 & 1215 & 1101827210 & 1788596132 \\
\hline 1163 & 1882208369 & 1978041597 & 1216 & 708024830 & 1480249642 \\
\hline 1164 & 1929900211 & 1601063895 & 1217 & 1737691587 & 1909144722 \\
\hline 1165 & 1275769318 & 355889868 & 1218 & 386535782 & 296082022 \\
\hline 1166 & 1751858318 & 1665589870 & 1219 & 358082626 & 408825339 \\
\hline 1167 & 245864857 & 680582327 & 1220 & 758382098 & 723008060 \\
\hline 1168 & 1138034801 & 2112164428 & 1221 & 388579217 & 414116955 \\
\hline 1169 & 1100529193 & 1979094441 & 1222 & 800185491 & 847998358 \\
\hline 1170 & 2076307694 & 1887361948 & 1223 & 844976677 & 1617721815 \\
\hline 1171 & 1448510299 & 1438071286 & 1224 & 242023418 & 896925141 \\
\hline 1172 & 51226700 & 392405371 & 1225 & 1875625375 & 1686990085 \\
\hline 1173 & 737831467 & 379824665 & 1226 & 2014545064 & 1469623711 \\
\hline 1174 & 2019806740 & 603473755 & 1227 & 99167929 & 365069460 \\
\hline 1175 & 856869603 & 1958580050 & 1228 & 1733908232 & 466453227 \\
\hline 1176 & 1788521570 & 627443410 & 1229 & 463755464 & 1244759281 \\
\hline 1177 & 1836297514 & 2069130506 & 1230 & 985599674 & 1401301252 \\
\hline 1178 & 568894273 & 1592358924 & 1231 & 1073113567 & 783229836 \\
\hline 1179 & 318125386 & 1405194149 & 1232 & 1036534905 & 379424021 \\
\hline 1180 & 1197370120 & 1621462708 & 1233 & 631908101 & 1254274978 \\
\hline 1181 & 1613036358 & 1290440049 & 1234 & 729715207 & 329385847 \\
\hline 1182 & 575873955 & 1982734674 & 1235 & 1962317503 & 1765240913 \\
\hline 1183 & 288949564 & 1424425693 & 1236 & 318391149 & 2106637806 \\
\hline 1184 & 691674655 & 243987534 & 1237 & 1602996274 & 1865597943 \\
\hline 1185 & 412549865 & 1172275184 & 1238 & 554667495 & 520199028 \\
\hline 1186 & 193191117 & 413290620 & 1239 & 1039163916 & 1887722665 \\
\hline 1187 & 643447277 & 781536306 & 1240 & 1419238370 & 483889898 \\
\hline 1188 & 463154112 & 1717494035 & 1241 & 2018128348 & 191003587 \\
\hline 1189 & 2084021046 & 251342995 & 1242 & 1608020696 & 713625003 \\
\hline 1190 & 420714434 & 1519442349 & 1243 & 1375627361 & 1783593992 \\
\hline 1191 & 949526110 & 1732454167 & 1244 & 513853217 & 772038322 \\
\hline 1192 & 1443723058 & 2029295492 & 1245 & 100646437 & 1905103025 \\
\hline 1193 & 1610704389 & 599166942 & 1246 & 260861899 & 268667561 \\
\hline 1194 & 1262757904 & 354155117 & 1247 & 409854814 & 498358450 \\
\hline 1195 & 1709132089 & 239929171 & 1248 & 706010569 & 1610830423 \\
\hline 1196 & 1447940720 & 627850537 & 1249 & 351796218 & 1037696108 \\
\hline 1197 & 1623966753 & 1642262311 & 1250 & 279082267 & 1708735884 \\
\hline 1198 & 1459711516 & 1647957567 & 1251 & 1727732814 & 65550379 \\
\hline 1199 & 83596129 & 650714782 & 1252 & 1515247632 & 620173792 \\
\hline 1200 & 1534938822 & 734389925 & 1253 & 1250524944 & 2098005990 \\
\hline 1201 & 2082738379 & 1947067720 & 1254 & 101045652 & 443427211 \\
\hline 1202 & 751566322 & 1448540044 & 1255 & 525137331 & 753403639 \\
\hline 1203 & 1848191948 & 2052764727 & 1256 & 1308556452 & 978817008 \\
\hline 1204 & 466634813 & 1576263232 & 1257 & 1284562083 & 1715945392 \\
\hline 1205 & 1740827153 & 2004046254 & 1258 & 1755041538 & 845239706 \\
\hline 1206 & 1137742734 & 802626584 & 1259 & 2042811342 & 328784258 \\
\hline 1207 & 2118319632 & 1275112694 & 1260 & 1729499957 & 1910275969 \\
\hline 1208 & 445848475 & 1329945404 & 1261 & 954953258 & 1047142760 \\
\hline 1209 & 1463429558 & 1098286280 & 1262 & 1180370541 & 1850980086 \\
\hline 1210 & 1893399196 & 1269490369 & 1263 & 2056881194 & 223107510 \\
\hline 1211 & 1715024497 & 1714302367 & 1264 & 1805543824 & 2033209369 \\
\hline
\end{tabular}




\begin{tabular}{|c|c|c|c|c|c|}
\hline 1265 & 1938420262 & 1353309264 & 1318 & 1409758929 & 158944681 \\
\hline 1266 & 992085298 & 396881058 & 1319 & 1742350937 & 1504621840 \\
\hline 1267 & 917421339 & 579488859 & 1320 & 1420213232 & 300328550 \\
\hline 1268 & 1865858060 & 1335578259 & 1321 & 524176726 & 2009218748 \\
\hline 1269 & 1612663492 & 746542618 & 1322 & 2049490509 & 2003651343 \\
\hline 1270 & 1320979724 & 218517710 & 1323 & 783339800 & 1351019393 \\
\hline 1271 & 1019997975 & 152312250 & 1324 & 513801280 & 1696251248 \\
\hline 1272 & 1711467638 & 1909481400 & 1325 & 1546144252 & 317151514 \\
\hline 1273 & 206663354 & 946263647 & 1326 & 49945446 & 735914629 \\
\hline 1274 & 2012822912 & 1551154535 & 1327 & 1568635971 & 1477914470 \\
\hline 1275 & 1164835489 & 915717049 & 1328 & 1467909965 & 1560013729 \\
\hline 1276 & 248049990 & 187518163 & 1329 & 1614756973 & 1730712168 \\
\hline 1277 & 1870462824 & 2039279628 & 1330 & 1154453372 & 1637989555 \\
\hline 1278 & 1331306566 & 914770495 & 1331 & 403821014 & 1948856752 \\
\hline 1279 & 1052508853 & 1730208258 & 1332 & 831616594 & 681514890 \\
\hline 1280 & 2066698122 & 77438511 & 1333 & 1910353614 & 1713440227 \\
\hline 1281 & 150707201 & 1914185964 & 1334 & 1948268146 & 787042904 \\
\hline 1282 & 1858517078 & 1732585568 & 1335 & 1273461904 & 1160853984 \\
\hline 1283 & 1714770060 & 1918441435 & 1336 & 398986595 & 328835073 \\
\hline 1284 & 874756506 & 1047935655 & 1337 & 1036146182 & 1545178118 \\
\hline 1285 & 379518346 & 112644302 & 1338 & 394370164 & 276168173 \\
\hline 1286 & 697433801 & 1361816681 & 1339 & 201954031 & 613529063 \\
\hline 1287 & 747229901 & 1862478658 & 1340 & 1515125425 & 1670623429 \\
\hline 1288 & 227586173 & 1087724606 & 1341 & 1223203885 & 90664017 \\
\hline 1289 & 1491757751 & 162010870 & 1342 & 664756321 & 1188828699 \\
\hline 1290 & 123660261 & 540621925 & 1343 & 493355737 & 374254737 \\
\hline 1291 & 1950536909 & 1331979740 & 1344 & 226233839 & 1303404582 \\
\hline 1292 & 884866464 & 1003591253 & 1345 & 1791898414 & 994572529 \\
\hline 1293 & 1840691834 & 1272803288 & 1346 & 1039547237 & 1977611774 \\
\hline 1294 & 328045984 & 1876571130 & 1347 & 1368204898 & 1554691372 \\
\hline 1295 & 730102723 & 1235725791 & 1348 & 1664667114 & 562343187 \\
\hline 1296 & 222370351 & 254616855 & 1349 & 18110846 & 1445818558 \\
\hline 1297 & 2061557340 & 1819999707 & 1350 & 573551901 & 1745451631 \\
\hline 1298 & 8236789 & 1801386295 & 1351 & 1400203875 & 1593437271 \\
\hline 1299 & 425048583 & 1535308230 & 1352 & 663143260 & 2001230900 \\
\hline 1300 & 1387267976 & 1951319841 & 1353 & 98374216 & 1536292839 \\
\hline 1301 & 122959405 & 641157948 & 1354 & 1674499003 & 1081831757 \\
\hline 1302 & 897537819 & 1465546170 & 1355 & 662597223 & 1213846375 \\
\hline 1303 & 1277934398 & 185692243 & 1356 & 218370875 & 1123281894 \\
\hline 1304 & 2016032852 & 889670571 & 1357 & 682675452 & 275757716 \\
\hline 1305 & 521559394 & 34453155 & 1358 & 2005337826 & 2077728728 \\
\hline 1306 & 1971838314 & 1417844088 & 1359 & 727574283 & 1816934445 \\
\hline 1307 & 109850650 & 1060392380 & 1360 & 2083622008 & 1759843933 \\
\hline 1308 & 840148479 & 465104748 & 1361 & 1384264739 & 443649799 \\
\hline 1309 & 651072117 & 1244315140 & 1362 & 482744912 & 394778212 \\
\hline 1310 & 1723158873 & 577170208 & 1363 & 790114098 & 2062532151 \\
\hline 1311 & 263747052 & 518409543 & 1364 & 78132923 & 1091552367 \\
\hline 1312 & 2019672293 & 1272035079 & 1365 & 603369153 & 307000647 \\
\hline 1313 & 131727661 & 1877031666 & 1366 & 1853133011 & 189527760 \\
\hline 1314 & 1891476783 & 90756051 & 1367 & 438936344 & 1094627271 \\
\hline 1315 & 1192721542 & 694344656 & 1368 & 1562891840 & 1925163520 \\
\hline 1316 & 626632324 & 718714699 & 1369 & 712906628 & 1034108105 \\
\hline 1317 & 588763863 & 1304712452 & 1370 & 1696786231 & 2130030696 \\
\hline
\end{tabular}




\begin{tabular}{|c|c|c|c|c|c|}
\hline 1371 & 1067511231 & 226896065 & 1424 & 1849369336 & 58062118 \\
\hline 1372 & 1238154454 & 245686256 & 1425 & 692487155 & 69868631 \\
\hline 1373 & 839106473 & 1907725508 & 1426 & 1467030854 & 1105802823 \\
\hline 1374 & 579689833 & 1467282874 & 1427 & 1052313225 & 1394551129 \\
\hline 1375 & 1156352128 & 1310295605 & 1428 & 203490181 & 1231248510 \\
\hline 1376 & 1320418956 & 1798118060 & 1429 & 531567489 & 1203246554 \\
\hline 1377 & 188439104 & 890865388 & 1430 & 1420937752 & 1395727772 \\
\hline 1378 & 1002760163 & 2072421549 & 1431 & 162295878 & 1161748477 \\
\hline 1379 & 1020005900 & 1794453552 & 1432 & 1953669060 & 1087152115 \\
\hline 1380 & 1813268603 & 1653350832 & 1433 & 1116364788 & 1137459873 \\
\hline 1381 & 144169749 & 1612111171 & 1434 & 552094929 & 1244967974 \\
\hline 1382 & 155925237 & 502245907 & 1435 & 461450751 & 1067675964 \\
\hline 1383 & 162701019 & 261155961 & 1436 & 1772590148 & 787969626 \\
\hline 1384 & 267275303 & 1966520850 & 1437 & 680261088 & 2070430548 \\
\hline 1385 & 1900441308 & 112169760 & 1438 & 1325863534 & 893670744 \\
\hline 1386 & 1471085755 & 1297723388 & 1439 & 927711703 & 289464282 \\
\hline 1387 & 216940708 & 1696666429 & 1440 & 723702204 & 2072787405 \\
\hline 1388 & 678289762 & 1497494576 & 1441 & 1758918253 & 1340483054 \\
\hline 1389 & 970789776 & 344212284 & 1442 & 621602094 & 1459523535 \\
\hline 1390 & 1601503619 & 789939178 & 1443 & 50585949 & 1904810577 \\
\hline 1391 & 710516028 & 381045994 & 1444 & 1746929508 & 1150747983 \\
\hline 1392 & 685156785 & 1568705281 & 1445 & 1219685187 & 1410569582 \\
\hline 1393 & 231899923 & 1908179612 & 1446 & 1979807033 & 1852667180 \\
\hline 1394 & 703532568 & 774637291 & 1447 & 913429892 & 299566379 \\
\hline 1395 & 1941131970 & 906731478 & 1448 & 419233867 & 1474911437 \\
\hline 1396 & 1316626595 & 138684760 & 1449 & 1906222988 & 838793779 \\
\hline 1397 & 1189942080 & 1618769979 & 1450 & 1613546433 & 700746733 \\
\hline 1398 & 1609158362 & 381558212 & 1451 & 206421920 & 1044304267 \\
\hline 1399 & 1384155542 & 1099935013 & 1452 & 2126492072 & 13385301 \\
\hline 1400 & 2106301267 & 992131321 & 1453 & 658442822 & 1279336558 \\
\hline 1401 & 1205706960 & 1800168458 & 1454 & 1895790595 & 234219571 \\
\hline 1402 & 1185544025 & 1082162728 & 1455 & 957070019 & 437265791 \\
\hline 1403 & 2063245365 & 457804331 & 1456 & 477802472 & 1742854176 \\
\hline 1404 & 752054893 & 1549344061 & 1457 & 540573705 & 1395810474 \\
\hline 1405 & 1840414573 & 911709329 & 1458 & 1222456720 & 1159307918 \\
\hline 1406 & 907026916 & 1316189492 & 1459 & 642212302 & 1187444713 \\
\hline 1407 & 1679467553 & 870090934 & 1460 & 157249813 & 1643935894 \\
\hline 1408 & 78918077 & 146756508 & 1461 & 912436216 & 1804357297 \\
\hline 1409 & 602835818 & 1359573699 & 1462 & 262265168 & 1439690367 \\
\hline 1410 & 548931161 & 1071337716 & 1463 & 1044296175 & 1638592786 \\
\hline 1411 & 811711139 & 758856869 & 1464 & 1056080294 & 1056395500 \\
\hline 1412 & 2018260759 & 736538496 & 1465 & 434377688 & 2053846014 \\
\hline 1413 & 1293406275 & 2067445579 & 1466 & 1954761827 & 212747978 \\
\hline 1414 & 882763618 & 894721600 & 1467 & 48939873 & 1606014656 \\
\hline 1415 & 806404968 & 511911620 & 1468 & 1134632940 & 801715833 \\
\hline 1416 & 805173488 & 1949725221 & 1469 & 1578047672 & 1535095181 \\
\hline 1417 & 215661273 & 1183123899 & 1470 & 1553808636 & 481206529 \\
\hline 1418 & 249849771 & 181012435 & 1471 & 34987215 & 733976264 \\
\hline 1419 & 1660825868 & 763056551 & 1472 & 2034620008 & 937190206 \\
\hline 1420 & 883970330 & 439122188 & 1473 & 2067918340 & 953829687 \\
\hline 1421 & 618128196 & 1570885109 & 1474 & 1254150854 & 973846264 \\
\hline 1422 & 807595585 & 748936122 & 1475 & 1123453543 & 1635536669 \\
\hline 1423 & 2070558645 & 1008522829 & 1476 & 230060182 & 43308004 \\
\hline
\end{tabular}




\begin{tabular}{|c|c|c|c|c|c|}
\hline 1477 & 2038451632 & 1821339121 & 1530 & 215084298 & 761446539 \\
\hline 1478 & 2001538745 & 1833325638 & 1531 & 613287687 & 614022234 \\
\hline 1479 & 789860655 & 286519379 & 1532 & 357214632 & 165309066 \\
\hline 1480 & 1144262327 & 1679685550 & 1533 & 296969099 & 1850176102 \\
\hline 1481 & 694329156 & 1939116468 & 1534 & 1312192863 & 1964111157 \\
\hline 1482 & 1467572802 & 1633476986 & 1535 & 1286658066 & 278207145 \\
\hline 1483 & 1876958166 & 1733044426 & 1536 & 136306110 & 949965210 \\
\hline 1484 & 80379517 & 7465051 & 1537 & 1812180549 & 780142846 \\
\hline 1485 & 1123461358 & 1448126634 & 1538 & 26802958 & 489973314 \\
\hline 1486 & 208876641 & 432782878 & 1539 & 2093294327 & 865547657 \\
\hline 1487 & 2070858618 & 406033976 & 1540 & 850009775 & 921800402 \\
\hline 1488 & 134782047 & 24844577 & 1541 & 1948064373 & 1332757460 \\
\hline 1489 & 578409241 & 982412049 & 1542 & 540065011 & 838725039 \\
\hline 1490 & 644446952 & 412115337 & 1543 & 717505060 & 895563349 \\
\hline 1491 & 1402094809 & 1639941069 & 1544 & 2071390318 & 1748775424 \\
\hline 1492 & 539450474 & 1493590652 & 1545 & 1555366360 & 1474235196 \\
\hline 1493 & 1697100524 & 1036300414 & 1546 & 406005288 & 261313525 \\
\hline 1494 & 2091765856 & 1878499749 & 1547 & 1884722367 & 1721255051 \\
\hline 1495 & 1616272849 & 1026328813 & 1548 & 701961458 & 1224076944 \\
\hline 1496 & 105994637 & 1004116799 & 1549 & 740592677 & 718041110 \\
\hline 1497 & 632417914 & 1684159183 & 1550 & 1777317446 & 1804810357 \\
\hline 1498 & 1349000038 & 1708628918 & 1551 & 875964821 & 1275106976 \\
\hline 1499 & 1857809104 & 731644258 & 1552 & 882622926 & 1733120309 \\
\hline 1500 & 1837156669 & 1783980821 & 1553 & 1462038295 & 1850367188 \\
\hline 1501 & 300221221 & 1445121866 & 1554 & 1321385785 & 1294639395 \\
\hline 1502 & 376427501 & 1082636036 & 1555 & 267353760 & 2067125010 \\
\hline 1503 & 2006019893 & 480365275 & 1556 & 1000680096 & 1743847497 \\
\hline 1504 & 370254759 & 1859875442 & 1557 & 2062022113 & 330984932 \\
\hline 1505 & 1804628901 & 1347652249 & 1558 & 59970792 & 1317742791 \\
\hline 1506 & 30179183 & 1215765289 & 1559 & 393380620 & 1648349736 \\
\hline 1507 & 2044062195 & 701773181 & 1560 & 285139178 & 1234805632 \\
\hline 1508 & 926799341 & 972507671 & 1561 & 1390937816 & 779053924 \\
\hline 1509 & 1979323469 & 1002285173 & 1562 & 125859822 & 2135637575 \\
\hline 1510 & 1886446254 & 245600128 & 1563 & 560526994 & 479508850 \\
\hline 1511 & 503778972 & 1681082037 & 1564 & 794377064 & 710355063 \\
\hline 1512 & 207051730 & 490603596 & 1565 & 1701992626 & 70796908 \\
\hline 1513 & 809099143 & 1743936585 & 1566 & 1853615649 & 38719006 \\
\hline 1514 & 1308729369 & 1070395219 & 1567 & 1445723797 & 1211216627 \\
\hline 1515 & 2039882896 & 1365513389 & 1568 & 1289126292 & 545000793 \\
\hline 1516 & 1435787582 & 1719722837 & 1569 & 2056573209 & 236028951 \\
\hline 1517 & 1118625911 & 1293181155 & 1570 & 500909741 & 1281059857 \\
\hline 1518 & 732480930 & 211701878 & 1571 & 737907886 & 2120490974 \\
\hline 1519 & 640178312 & 997636023 & 1572 & 491250120 & 970242986 \\
\hline 1520 & 665498762 & 1865688627 & 1573 & 720278375 & 1329974003 \\
\hline 1521 & 1596749307 & 2139722124 & 1574 & 94690695 & 1052550732 \\
\hline 1522 & 899473051 & 4311708 & 1575 & 539829466 & 1762201433 \\
\hline 1523 & 1823191685 & 953848203 & 1576 & 479228934 & 823556077 \\
\hline 1524 & 2139989761 & 63455522 & 1577 & 1881792273 & 420415878 \\
\hline 1525 & 1634279271 & 529695388 & 1578 & 429329182 & 1826572568 \\
\hline 1526 & 407136064 & 1481663439 & 1579 & 1939722379 & 1377901483 \\
\hline 1527 & 1416530455 & 463571879 & 1580 & 688828388 & 1005763337 \\
\hline 1528 & 925748605 & 1823382493 & 1581 & 212077881 & 1196395858 \\
\hline 1529 & 961756782 & 1073019998 & 1582 & 1014836323 & 512048876 \\
\hline
\end{tabular}




\begin{tabular}{|c|c|c|c|c|c|}
\hline 1583 & 876546126 & 180481797 & 1636 & 431249618 & 1999197914 \\
\hline 1584 & 1347711917 & 953915981 & 1637 & 2034741426 & 1484298447 \\
\hline 1585 & 1508302185 & 151428300 & 1638 & 591554363 & 858740665 \\
\hline 1586 & 1341445146 & 703329600 & 1639 & 1465324057 & 645300975 \\
\hline 1587 & 1021246035 & 1967486497 & 1640 & 173688363 & 919744327 \\
\hline 1588 & 1486023329 & 1418491603 & 1641 & 1229835371 & 1487254812 \\
\hline 1589 & 1953436940 & 1213453256 & 1642 & 730687194 & 1228185712 \\
\hline 1590 & 688203245 & 374496755 & 1643 & 1687800117 & 1723246803 \\
\hline 1591 & 25111392 & 1756809754 & 1644 & 1113793410 & 1869628761 \\
\hline 1592 & 1472794911 & 777560974 & 1645 & 2059331170 & 1990061307 \\
\hline 1593 & 2023133413 & 520672388 & 1646 & 1164491572 & 1837936299 \\
\hline 1594 & 262584983 & 1878988298 & 1647 & 617642233 & 1886033992 \\
\hline 1595 & 1060140512 & 614949004 & 1648 & 862515667 & 64605081 \\
\hline 1596 & 148935871 & 56123734 & 1649 & 606568484 & 2138574144 \\
\hline 1597 & 489011080 & 92660441 & 1650 & 1833473262 & 1649473292 \\
\hline 1598 & 1561740635 & 65845048 & 1651 & 2113463498 & 1997247777 \\
\hline 1599 & 178795131 & 648794187 & 1652 & 349186629 & 1329869530 \\
\hline 1600 & 534502028 & 1754059675 & 1653 & 199649172 & 970072799 \\
\hline 1601 & 586626106 & 370529866 & 1654 & 19941015 & 52963773 \\
\hline 1602 & 1658081567 & 1515874044 & 1655 & 1602108658 & 42007318 \\
\hline 1603 & 1561834458 & 1560572233 & 1656 & 523106489 & 622263426 \\
\hline 1604 & 345924407 & 1478694919 & 1657 & 647290792 & 170505568 \\
\hline 1605 & 1380899969 & 1688678767 & 1658 & 118366782 & 1676828944 \\
\hline 1606 & 416569953 & 339695298 & 1659 & 483719292 & 591899398 \\
\hline 1607 & 1751888137 & 426464758 & 1660 & 670038979 & 1981353544 \\
\hline 1608 & 1043666171 & 364806681 & 1661 & 1108311707 & 782309292 \\
\hline 1609 & 2075929142 & 702238355 & 1662 & 628613195 & 1356700627 \\
\hline 1610 & 1848413938 & 1838866018 & 1663 & 361471736 & 1015794886 \\
\hline 1611 & 1481589063 & 1547185298 & 1664 & 775384055 & 306221215 \\
\hline 1612 & 2147055959 & 1369782442 & 1665 & 643724611 & 469529059 \\
\hline 1613 & 868263797 & 1741354369 & 1666 & 1308373560 & 754130153 \\
\hline 1614 & 2052673799 & 115459505 & 1667 & 1681302748 & 1293117910 \\
\hline 1615 & 262337902 & 663377826 & 1668 & 714330361 & 738708789 \\
\hline 1616 & 1006557838 & 1884609916 & 1669 & 1441704947 & 1729777674 \\
\hline 1617 & 1742006109 & 2008844677 & 1670 & 1986699746 & 1645471638 \\
\hline 1618 & 537970539 & 1892665765 & 1671 & 1643224930 & 924651135 \\
\hline 1619 & 666868866 & 150713997 & 1672 & 1190531046 & 68311585 \\
\hline 1620 & 1125492241 & 956617360 & 1673 & 1577701080 & 1391503172 \\
\hline 1621 & 678424091 & 132891748 & 1674 & 1689023741 & 849337610 \\
\hline 1622 & 2104667231 & 1452904458 & 1675 & 327935493 & 955608125 \\
\hline 1623 & 2113533278 & 1249797962 & 1676 & 297549730 & 682500623 \\
\hline 1624 & 145303559 & 1678325091 & 1677 & 1317725153 & 122646372 \\
\hline 1625 & 32231760 & 1593379386 & 1678 & 976700194 & 1124109085 \\
\hline 1626 & 1298134466 & 515704021 & 1679 & 1047816379 & 666357786 \\
\hline 1627 & 1019377240 & 268776521 & 1680 & 1983780330 & 836141739 \\
\hline 1628 & 638845910 & 2012179580 & 1681 & 1869612473 & 1712256322 \\
\hline 1629 & 1057271239 & 1857391397 & 1682 & 1618615231 & 1740346183 \\
\hline 1630 & 1024534661 & 1981472284 & 1683 & 1868572158 & 1880344442 \\
\hline 1631 & 1976467142 & 1864534694 & 1684 & 1595404837 & 1713205510 \\
\hline 1632 & 1540428786 & 1971352703 & 1685 & 2026124789 & 477717677 \\
\hline 1633 & 1007660759 & 1892353733 & 1686 & 1741026337 & 909038600 \\
\hline 1634 & 1368173572 & 1036670937 & 1687 & 1205304617 & 1236008235 \\
\hline 1635 & 1167548847 & 1773055044 & 1688 & 987574256 & 2075208950 \\
\hline
\end{tabular}




\begin{tabular}{|c|c|c|c|c|c|}
\hline 1689 & 1308372518 & 119812629 & 1742 & 1069554470 & 1596355049 \\
\hline 1690 & 600082453 & 566210255 & 1743 & 1224361565 & 953681367 \\
\hline 1691 & 1327566783 & 1811607522 & 1744 & 711158030 & 841827724 \\
\hline 1692 & 638837957 & 1202199922 & 1745 & 1943024177 & 1272366815 \\
\hline 1693 & 1508035404 & 263010759 & 1746 & 185559277 & 2061643304 \\
\hline 1694 & 494243902 & 1866801315 & 1747 & 1991866447 & 431670308 \\
\hline 1695 & 1043376571 & 2044667111 & 1748 & 1296265770 & 1398000555 \\
\hline 1696 & 833394966 & 1811836133 & 1749 & 776578403 & 1561326163 \\
\hline 1697 & 217151736 & 524850152 & 1750 & 1049895568 & 1226670373 \\
\hline 1698 & 1847733097 & 1451436386 & 1751 & 1156001895 & 806803418 \\
\hline 1699 & 469152080 & 755002090 & 1752 & 131653770 & 1997055620 \\
\hline 1700 & 46659778 & 1763572191 & 1753 & 652853715 & 1506316316 \\
\hline 1701 & 998358201 & 244005516 & 1754 & 478502904 & 620838913 \\
\hline 1702 & 2059588343 & 1015245243 & 1755 & 976405729 & 1231323247 \\
\hline 1703 & 476839069 & 1028111798 & 1756 & 5581098 & 3857214 \\
\hline 1704 & 479419698 & 464550976 & 1757 & 204426267 & 581687364 \\
\hline 1705 & 631698586 & 531428033 & 1758 & 498811960 & 1766253627 \\
\hline 1706 & 569351231 & 1818758233 & 1759 & 1283381396 & 76633146 \\
\hline 1707 & 1187678750 & 113512002 & 1760 & 2132069321 & 1779530873 \\
\hline 1708 & 714924146 & 789178792 & 1761 & 1943877497 & 1921584764 \\
\hline 1709 & 1599282007 & 1259162467 & 1762 & 1179798154 & 1870830937 \\
\hline 1710 & 1639512118 & 1368051774 & 1763 & 1535666351 & 1680444210 \\
\hline 1711 & 808573750 & 1122272718 & 1764 & 2128350095 & 1464511570 \\
\hline 1712 & 15222873 & 1589951453 & 1765 & 495109181 & 939836606 \\
\hline 1713 & 310033629 & 368568250 & 1766 & 292476398 & 18467322 \\
\hline 1714 & 1452841860 & 1469531810 & 1767 & 1445382233 & 107880247 \\
\hline 1715 & 1633688596 & 1254810525 & 1768 & 1682739627 & 1574423200 \\
\hline 1716 & 1849831264 & 951151408 & 1769 & 736273846 & 2102893710 \\
\hline 1717 & 1188844048 & 301389076 & 1770 & 660968201 & 1583311375 \\
\hline 1718 & 367362955 & 2076356397 & 1771 & 1687774859 & 1034509246 \\
\hline 1719 & 1611662750 & 2095211713 & 1772 & 231939627 & 2098962667 \\
\hline 1720 & 1796840336 & 763278883 & 1773 & 495628219 & 770901675 \\
\hline 1721 & 366530119 & 1426794320 & 1774 & 2078114357 & 1361205016 \\
\hline 1722 & 1243723756 & 1077422424 & 1775 & 1614463772 & 992179705 \\
\hline 1723 & 1582043817 & 1326527113 & 1776 & 1876608745 & 1959912108 \\
\hline 1724 & 852668531 & 1784821442 & 1777 & 1038355199 & 1437863995 \\
\hline 1725 & 878451286 & 713999956 & 1778 & 173240155 & 2008957222 \\
\hline 1726 & 1412916504 & 355051082 & 1779 & 562860926 & 1021743557 \\
\hline 1727 & 1542482620 & 1529313314 & 1780 & 601111396 & 1080956922 \\
\hline 1728 & 380052281 & 1998229824 & 1781 & 97355608 & 646222892 \\
\hline 1729 & 687714529 & 1388382474 & 1782 & 1799633484 & 398834182 \\
\hline 1730 & 1218217807 & 1053051419 & 1783 & 735281137 & 296082598 \\
\hline 1731 & 1867816589 & 1740975576 & 1784 & 1269870696 & 22823250 \\
\hline 1732 & 1056942787 & 1266963980 & 1785 & 786456593 & 1340278066 \\
\hline 1733 & 1082397513 & 682832675 & 1786 & 99699564 & 1131321837 \\
\hline 1734 & 1666624772 & 175079248 & 1787 & 731937277 & 52482941 \\
\hline 1735 & 727631750 & 1598682675 & 1788 & 713056018 & 332140384 \\
\hline 1736 & 1002090237 & 1854017388 & 1789 & 255970098 & 1060017487 \\
\hline 1737 & 974272299 & 1063793268 & 1790 & 1744802495 & 475100729 \\
\hline 1738 & 1027422326 & 216908857 & 1791 & 652768377 & 1147364499 \\
\hline 1739 & 329945564 & 1949216527 & 1792 & 1452958587 & 1790416718 \\
\hline 1740 & 1561618442 & 1270266368 & 1793 & 1248051164 & 103711286 \\
\hline 1741 & 1785110679 & 156859614 & 1794 & 967132713 & 558877578 \\
\hline
\end{tabular}




\begin{tabular}{|c|c|c|c|c|c|}
\hline 1795 & 1306723441 & 53613073 & 1848 & 22875089 & 750582650 \\
\hline 1796 & 1824370691 & 364856635 & 1849 & 916285985 & 80209179 \\
\hline 1797 & 193389047 & 822831030 & 1850 & 1823417359 & 668492830 \\
\hline 1798 & 1183964759 & 1383732981 & 1851 & 1895272191 & 247505247 \\
\hline 1799 & 1357209985 & 329083192 & 1852 & 702850088 & 1354531240 \\
\hline 1800 & 97688038 & 1964857607 & 1853 & 1065515611 & 987568694 \\
\hline 1801 & 468563867 & 1250859951 & 1854 & 614868891 & 1493171569 \\
\hline 1802 & 775618139 & 1605135118 & 1855 & 2004136650 & 2060745992 \\
\hline 1803 & 40300458 & 1876517308 & 1856 & 368873961 & 859157737 \\
\hline 1804 & 989792430 & 684964759 & 1857 & 106694018 & 567520440 \\
\hline 1805 & 1548277381 & 2101161528 & 1858 & 2115488155 & 81666276 \\
\hline 1806 & 1369011552 & 585602020 & 1859 & 1048067272 & 1277134316 \\
\hline 1807 & 1979359990 & 126987723 & 1860 & 1678132177 & 821415899 \\
\hline 1808 & 1097240894 & 1699017927 & 1861 & 993061920 & 1111379418 \\
\hline 1809 & 689957244 & 494513606 & 1862 & 1236549662 & 1766505289 \\
\hline 1810 & 1016424683 & 433027950 & 1863 & 1360986454 & 1860156407 \\
\hline 1811 & 116823138 & 1124548150 & 1864 & 882849355 & 2119253056 \\
\hline 1812 & 568035283 & 2069955488 & 1865 & 1889052503 & 1987079071 \\
\hline 1813 & 471609569 & 247115020 & 1866 & 522478249 & 577538295 \\
\hline 1814 & 2029709465 & 1553955346 & 1867 & 40829668 & 1127593261 \\
\hline 1815 & 1968216015 & 1350704733 & 1868 & 1968503125 & 1095241294 \\
\hline 1816 & 1208274464 & 439697573 & 1869 & 1802301951 & 228588316 \\
\hline 1817 & 1901972673 & 555741699 & 1870 & 1463393179 & 1554145933 \\
\hline 1818 & 1816727530 & 1670623207 & 1871 & 1077137170 & 211533566 \\
\hline 1819 & 518548484 & 433153365 & 1872 & 1376815498 & 1355369237 \\
\hline 1820 & 986043052 & 1946171399 & 1873 & 253774360 & 1314379716 \\
\hline 1821 & 1200097012 & 451633799 & 1874 & 1078287226 & 73919883 \\
\hline 1822 & 1262315631 & 1885573059 & 1875 & 2015641867 & 1511940540 \\
\hline 1823 & 153815567 & 471766143 & 1876 & 1358707656 & 1298703212 \\
\hline 1824 & 929924988 & 305319460 & 1877 & 153797305 & 1639810980 \\
\hline 1825 & 1277650126 & 1255170019 & 1878 & 1421623873 & 1728240283 \\
\hline 1826 & 1357710116 & 2027388581 & 1879 & 2001283298 & 115165003 \\
\hline 1827 & 489903484 & 1746813429 & 1880 & 1929625549 & 373615754 \\
\hline 1828 & 1937016308 & 1248733964 & 1881 & 1396319972 & 2070959700 \\
\hline 1829 & 877135982 & 71060835 & 1882 & 1059778787 & 1807494755 \\
\hline 1830 & 1854833774 & 1984997339 & 1883 & 933622833 & 1984623817 \\
\hline 1831 & 638067527 & 829649896 & 1884 & 800907079 & 1284537236 \\
\hline 1832 & 395385216 & 1693046344 & 1885 & 480099620 & 893384183 \\
\hline 1833 & 1503529189 & 809566997 & 1886 & 427703704 & 204927730 \\
\hline 1834 & 1909253905 & 741370905 & 1887 & 246294888 & 53753414 \\
\hline 1835 & 1026216765 & 855241168 & 1888 & 1873144346 & 828677657 \\
\hline 1836 & 1109326659 & 1199651300 & 1889 & 1214617885 & 28756969 \\
\hline 1837 & 1299417248 & 968818299 & 1890 & 1290481608 & 164626048 \\
\hline 1838 & 1190572028 & 746266402 & 1891 & 1085574712 & 1179233327 \\
\hline 1839 & 460925618 & 914987152 & 1892 & 65068031 & 180386129 \\
\hline 1840 & 1257883161 & 730366766 & 1893 & 538662057 & 1990219634 \\
\hline 1841 & 987217691 & 972608212 & 1894 & 1814075886 & 730916329 \\
\hline 1842 & 1562141661 & 908216238 & 1895 & 1066522421 & 53129262 \\
\hline 1843 & 899928330 & 2061039746 & 1896 & 1284743177 & 1687060144 \\
\hline 1844 & 10038845 & 896438726 & 1897 & 1246021432 & 1439224317 \\
\hline 1845 & 1504412052 & 2124415431 & 1898 & 1512534271 & 1938646287 \\
\hline 1846 & 771393248 & 472153032 & 1899 & 284453307 & 2117151015 \\
\hline 1847 & 843854766 & 1781802093 & 1900 & 742551961 & 1613045003 \\
\hline
\end{tabular}




\begin{tabular}{|c|c|c|c|c|c|}
\hline 1901 & 1194723552 & 730655350 & 1951 & 1979418008 & 2113983876 \\
\hline 1902 & 1911211132 & 848511829 & 1952 & 1676503446 & 578429934 \\
\hline 1903 & 72901757 & 528406025 & 1953 & 625690974 & 907413121 \\
\hline 1904 & 1590905915 & 1401525345 & 1954 & 410439111 & 1498304138 \\
\hline 1905 & 1267201976 & 1638438269 & 1955 & 166384413 & 200339130 \\
\hline 1906 & 1962579703 & 524047714 & 1956 & 394390800 & 439199991 \\
\hline 1907 & 24017781 & 789232588 & 1957 & 1919635857 & 914381559 \\
\hline 1908 & 983890213 & 1060899747 & 1958 & 82501826 & 1503851715 \\
\hline 1909 & 628398522 & 229771721 & 1959 & 1836984381 & 1699855878 \\
\hline 1910 & 1324515708 & 435278737 & 1960 & 1733004326 & 734767138 \\
\hline 1911 & 1302537823 & 1476102526 & 1961 & 1130638008 & 1803595063 \\
\hline 1912 & 983250281 & 519841596 & 1962 & 1817423469 & 2075266448 \\
\hline 1913 & 1611826098 & 1065243024 & 1963 & 532432583 & 960219862 \\
\hline 1914 & 120234928 & 1141880034 & 1964 & 462939494 & 1092363026 \\
\hline 1915 & 738873800 & 1174823969 & 1965 & 236555855 & 1302673698 \\
\hline 1916 & 156089648 & 1284220406 & 1966 & 222600077 & 513825655 \\
\hline 1917 & 781596009 & 1274559486 & 1967 & 1259149155 & 1206068277 \\
\hline 1918 & 90799661 & 1669380083 & 1968 & 1508868801 & 222250655 \\
\hline 1919 & 582770292 & 5978831 & 1969 & 286237667 & 1932387185 \\
\hline 1920 & 1675624057 & 1141470142 & 1970 & 810619978 & 84221489 \\
\hline 1921 & 2087058689 & 1982098128 & 1971 & 1968789342 & 3406300 \\
\hline 1922 & 1448244551 & 1399798419 & 1972 & 7143772 & 1624831848 \\
\hline 1923 & 985715519 & 836519657 & 1973 & 380876987 & 595910 \\
\hline 1924 & 2005542815 & 124097475 & 1974 & 806679797 & 532613858 \\
\hline 1925 & 1787481873 & 1015838230 & 1975 & 587829473 & 447324038 \\
\hline 1926 & 1962649666 & 457993605 & 1976 & 796580056 & 2092594215 \\
\hline 1927 & 574256785 & 5205523 & 1977 & 2017071437 & 345120951 \\
\hline 1928 & 1245341641 & 499807671 & 1978 & 993480643 & 297355686 \\
\hline 1929 & 959148918 & 1443054722 & 1979 & 2100308972 & 1130726449 \\
\hline 1930 & 93795773 & 861053684 & 1980 & 8559413 & 1933712031 \\
\hline 1931 & 477787846 & 936058506 & 1981 & 1815133990 & 743580341 \\
\hline 1932 & 1275479146 & 897987006 & 1982 & 778653742 & 608531008 \\
\hline 1933 & 1638044142 & 594515675 & 1983 & 1274600195 & 1836593135 \\
\hline 1934 & 305894613 & 1663580191 & 1984 & 270136916 & 1531912299 \\
\hline 1935 & 1643160383 & 2066966886 & 1985 & 1924921264 & 1022748 \\
\hline 1936 & 894207793 & 2047774362 & 1986 & 1028900827 & 216879682 \\
\hline 1937 & 1227921742 & 2019167879 & 1987 & 1792365310 & 2066941187 \\
\hline 1938 & 2121984515 & 270316059 & 1988 & 485292584 & 2117943793 \\
\hline 1939 & 574227789 & 1838241605 & 1989 & 2008367322 & 1901506479 \\
\hline 1940 & 1858561831 & 2137389464 & 1990 & 1986537433 & 764832 \\
\hline 1941 & 793322184 & 1514473341 & 1991 & 271885611 & 2138290558 \\
\hline 1942 & 1307712985 & 1527646462 & 1992 & 858517359 & 447504486 \\
\hline 1943 & 1508682543 & 1013356194 & 1993 & 1728487375 & 773684721 \\
\hline 1944 & 217022716 & 368548386 & 1994 & 1100561290 & 933912958 \\
\hline 1945 & 1401466633 & 713855090 & 1995 & 2129602216 & 447794448 \\
\hline 1946 & 707230345 & 685309479 & 1996 & 1132652774 & 1612006369 \\
\hline 1947 & 178991308 & 275064400 & 1997 & 459973107 & 652194731 \\
\hline 1948 & 170847806 & 1736248075 & 1998 & 1508472885 & 733305425 \\
\hline 1949 & 1794177750 & 378487995 & 1999 & 1776229888 & 1204581758 \\
\hline 1950 & 811317412 & 1654125795 & 2000 & 765822650 & 97111410 \\
\hline
\end{tabular}




\section{Appendix C}

\section{Sample Run Sheets}

First is given the input file required for the droplet portion of the ODT program. The file given is that used to generate all the $\beta=1$ data given in this thesis. This file should be in a file called "jevap.inp".

Second is given the input data required for the ODT proper. The input file here was used to generate all the data given in this thesis. This file should be called "input.data".

Third is given a sample long output for the base case (case number 1) for one realization. This data comes out in a file called "jevap.out". There is also a series of output files (for all the cases being run) called "scase\#.out". This stands for short output for case number _. The short output contains only the burn out point. Hence one line per realization. A sample of this output is not shown. 


\section{C.1 Sample input sheet for 12 cases two-phase flow submodel}

itial cond. first 11 cases at $10 \mathrm{~cm}$, for $\mathrm{a}=.169, \mathrm{bv}=.6833$ no ignition in $\mathrm{BC}$ delayed ignition, $\mathrm{cp} 3=$ heptane, toluene, $\mathrm{c} 10 \mathrm{~h} 21$, case 12 at $5.24 \mathrm{~cm}$

$0.3423097 \quad$ ! y possition of droplet

-3.232294 ! y velocity of droplet

0.0002137292 ! shpherical diameter of drop (standard 0.000234)

813.3 ! Density of the liquid drop $[\mathrm{kg} / \mathrm{m} 3]$

0.2715 ! density of the gas phase

0.0000493 ! viscosity of the gas phase

$0.1 \quad$ ! epsilon factor; fraction of a grid step

$1.0 \quad$ ! D parameter

1.5 ! drop time step factor

0.07318 ! birth time of droplet into the combustor

5.598498 ! u velocity of the drop

$0.1000091 \quad ! \mathrm{x}$ position of the drop

0.6833 ! bulk velocity of fluid

0.14635 ! age of the drop when it appears in ODT domain

9.81 ! gravitational acc. $[\mathrm{m} / \mathrm{s} 2]$

$400.0 \quad$ ! mean boiling point of liquid $[\mathrm{K}]$

2000.0 ! mean heat capacity $(\mathrm{Cp})$ of liquid $[\mathrm{J} / \mathrm{kg} / \mathrm{K}]$

$350000.0 \quad$ ! heat of vap. [J/kg]

$44000000.0 \quad$ ! heat of combustion $[\mathrm{J} / \mathrm{kg}]$

0.317 ! stoichiometric fluel-to-oxygen mass ratio (f)

0.00000066 ! burning rate param. asymptotic value of lamda [m2/s]

0.0515 ! mass frac. of $\mathrm{O} 2$ in the flue gas (EPAreportpgE-30)

1100. ! initial guess of the flame temp [K]

0.0000088 ! Diffusivity (toluene at $30 \mathrm{C}$ ) [m2/sec] (Perry's)

303.15 ! temp. at which Schmidt number for the gas [K]

378.9214 ! starting temp. of the liquid drop [K]

0.0025 ! epsilon for determining when to switch to lamda

13 ! number of drops in this simulation. Max 15

1 ! switch to lamda? 1 for yes, 2 for no

1 ! long output? 1 for yes, 2 for no - not working yet

0.112688 ! ignition time of drop 1 , must be >= birth time (5 in)

case2.out ! start a new drop, OUTPPUT FILE HAS THIS NAME

0.3423157 ! y possition of droplet for drop \#2

$-3.382890 \quad$ ! y velocity of droplet for drop \#2

0.0002939429 ! shpherical diameter of drop \#2 (standard 0.000234)

0.07318 ! birth time of droplet into the combustor

5.859337 ! u velocity of the drop for drop \#2

0.09999877 ! x position of the drop for drop \#2

0.14635 ! age of drop \#2 when it appears in ODT domain

374.6468 ! starting temp. of the liquid drop \#2 [K]

1100. ! initial guess of the flame sheet temperature $[\mathrm{K}]$ for drop \#2 


\begin{tabular}{|c|c|}
\hline 0.094102 & ! ignition time of drop 2 , must be $>=$ birth time $(4.5$ in $)$ \\
\hline case3.out & ! start a new drop, OUTPPUT FILE HAS THIS NAME \\
\hline 0.3423163 & ! y possition of droplet for drop \#3 \\
\hline-3.482738 & ! y velocity of droplet for drop \#3 \\
\hline 0.0003632376 & 6 ! shpherical diameter of drop \#3 (standard 0.000234) \\
\hline 0.07318 & ! birth time of droplet into the combustor \\
\hline 6.032278 & ! u velocity of the drop for drop \#3 \\
\hline 0.09999777 & ! x position of the drop for drop \#3 \\
\hline 0.14635 & ! age of drop \#3 when it appears in ODT domain \\
\hline 367.6673 & ! starting temp. of the liquid drop \#3 [K] \\
\hline 1100. & ! initial guess of the flame sheet temperature $[\mathrm{K}]$ for drop \#3 \\
\hline 0.112688 & ! ignition time of drop 3, must be $>=$ birth time $(5$ in) \\
\hline case4.out & ! start a new drop, OUTPPUT FILE HAS THIS NAME \\
\hline 0.3423116 & ! y possition of droplet for drop \#4 \\
\hline-1.902991 & ! y velocity of droplet for drop \#4 \\
\hline 0.0001778522 & 2 ! shpherical diameter of drop \#4 (standard 0.000234) \\
\hline 0.07318 & ! birth time of droplet into the combustor \\
\hline 3.296077 & ! u velocity of the drop for drop \#4 \\
\hline 0.1000059 & ! x position of the drop for drop \#4 \\
\hline 0.14635 & ! age of drop \#4 when it appears in ODT domain \\
\hline 379.5895 & ! starting temp. of the liquid drop \#4 [K] \\
\hline 1100. & ! initial guess of the flame sheet temperature $[\mathrm{K}]$ for drop \#4 \\
\hline 0.075516 & ! ignition time of drop $4,>=$ birth time (4 in)-no data \\
\hline case5.out & ! start a new drop, OUTPPUT FILE HAS THIS NAME \\
\hline 0.3423158 & ! y possition of droplet for drop \#5 \\
\hline-4.119725 & ! y velocity of droplet for drop \#5 \\
\hline 0.0002360611 & 1 ! shpherical diameter of drop \#5 (standard 0.000234) \\
\hline 0.07318 & ! birth time of droplet into the combustor \\
\hline 7.135573 & ! u velocity of the drop for drop \#5 \\
\hline 0.09999865 & ! x position of the drop for drop \#5 \\
\hline 0.14635 & ! age of drop \#5 when it appears in ODT domain \\
\hline 377.1597 & ! starting temp. of the liquid drop \#5 [K] \\
\hline 1100. & ! initial guess of the flame sheet temperature $[\mathrm{K}]$ for drop \#5 \\
\hline 0.37290 & ! ignition time of drop 5, must be $>=$ birth time ( 12 in) \\
\hline case6.out & ! start a new drop, OUTPPUT FILE HAS THIS NAME \\
\hline 0.3423099 & ! y possition of droplet for drop \#6 \\
\hline-3.795005 & ! y velocity of droplet for drop \#6 \\
\hline 0.0002160238 & 8 ! shpherical diameter of drop \#6 (standard 0.000234) \\
\hline 0.07318 & ! birth time of droplet into the combustor \\
\hline 6.573141 & ! u velocity of the drop for drop \#6 \\
\hline 0.1000088 & ! x position of the drop for drop \#6 \\
\hline & ! age of drop \#6 when it appears in ODT domain \\
\hline 378.5820 & ! starting temp. of the liquid drop \#6 [K] \\
\hline 1100. & ! initial guess of the flame sheet temperature $[\mathrm{K}]$ for drc \\
\hline
\end{tabular}




\begin{tabular}{|c|c|}
\hline 0.410069 & ! ignition time of drop 6 , must be $>=$ birth time (13 in) \\
\hline case7.out & ! start a new drop, OUTPPUT FILE HAS THIS NAME \\
\hline 0.3423162 & ! y possition of droplet for drop \#7 \\
\hline-3.603644 & ! y velocity of droplet for drop \#7 \\
\hline 0.0002153167 & $7 \quad$ ! shpherical diameter of drop \#7 (standard 0.000234) \\
\hline 0.07318 & ! birth time of droplet into the combustor \\
\hline 6.241694 & ! u velocity of the drop for drop \#7 \\
\hline 0.09999793 & ! x position of the drop for drop \#7 \\
\hline 0.14635 & ! age of drop \#7 when it appears in ODT domain \\
\hline 378.7016 & ! starting temp. of the liquid drop \#7 [K] \\
\hline 1100. & ! initial guess of the flame sheet temperature $[\mathrm{K}]$ for drop \#7 \\
\hline 0.410069 & ! ignition time of drop 7, must be $>=$ birth time (13 in) \\
\hline case8.out & ! start a new drop, OUTPPUT FILE HAS THIS NAME \\
\hline 0.3423081 & ! y possition of droplet for drop \#8 \\
\hline-3.466336 & ! y velocity of droplet for drop \#8 \\
\hline 0.0002147615 & 5 ! shpherical diameter of drop \#8 (standard 0.000234) \\
\hline 0.07318 & ! birth time of droplet into the combustor \\
\hline 6.003870 & ! u velocity of the drop for drop \#8 \\
\hline 0.1000119 & ! x position of the drop for drop \#8 \\
\hline 0.14635 & ! age of drop \#8 when it appears in ODT domain \\
\hline 378.7857 & ! starting temp. of the liquid drop \#8 [K] \\
\hline 1100. & ! initial guess of the flame sheet temperature $[\mathrm{K}]$ for drop \#8 \\
\hline 0.103395 & ! ignition time of drop 8, must be $>=$ birth time $(4.75$ in) \\
\hline case9.out & ! start a new drop, OUTPPUT FILE HAS THIS NAME \\
\hline 0.3423185 & ! y possition of droplet for drop \#9 \\
\hline-3.372172 & ! y velocity of droplet for drop \#9 \\
\hline 0.0002143662 & 2 ! shpherical diameter of drop \#9 (standard 0.000234) \\
\hline 0.07318 & ! birth time of droplet into the combustor \\
\hline 5.840773 & ! u velocity of the drop for drop \#9 \\
\hline 0.09999395 & ! x position of the drop for drop \#9 \\
\hline 0.14635 & ! age of drop \#9 when it appears in ODT domain \\
\hline 378.8405 & ! starting temp. of the liquid drop \#9 $[\mathrm{K}]$ \\
\hline 1100. & ! initial guess of the flame sheet temperature $[\mathrm{K}]$ for drop \#9 \\
\hline 0.094102 & ! ignition time of drop 9, must be $>=$ birth time ( 4.5 in) \\
\hline case10.ot & ! start a new drop, OUTPPUT FILE HAS THIS NAME \\
\hline 0.3423103 & ! y possition of droplet for drop \#10 \\
\hline-3.304122 & ! y velocity of droplet for drop \#10 \\
\hline 0.0002140602 & 2 ! shpherical diameter of drop \#10 (standard 0.000234) \\
\hline 0.07318 & ! birth time of droplet into the combustor \\
\hline 5.722907 & ! u velocity of the drop for drop \#10 \\
\hline 0.1000080 & ! x position of the drop for drop \#10 \\
\hline 0.14635 & ! age of drop \#10 when it appears in ODT domain \\
\hline 378.8805 & ! starting temp. of the liquid drop \#10 [K] \\
\hline 1100. & initial ouess of the flame sheet temnerature $[\mathrm{K}] \mathrm{f}$ \\
\hline
\end{tabular}




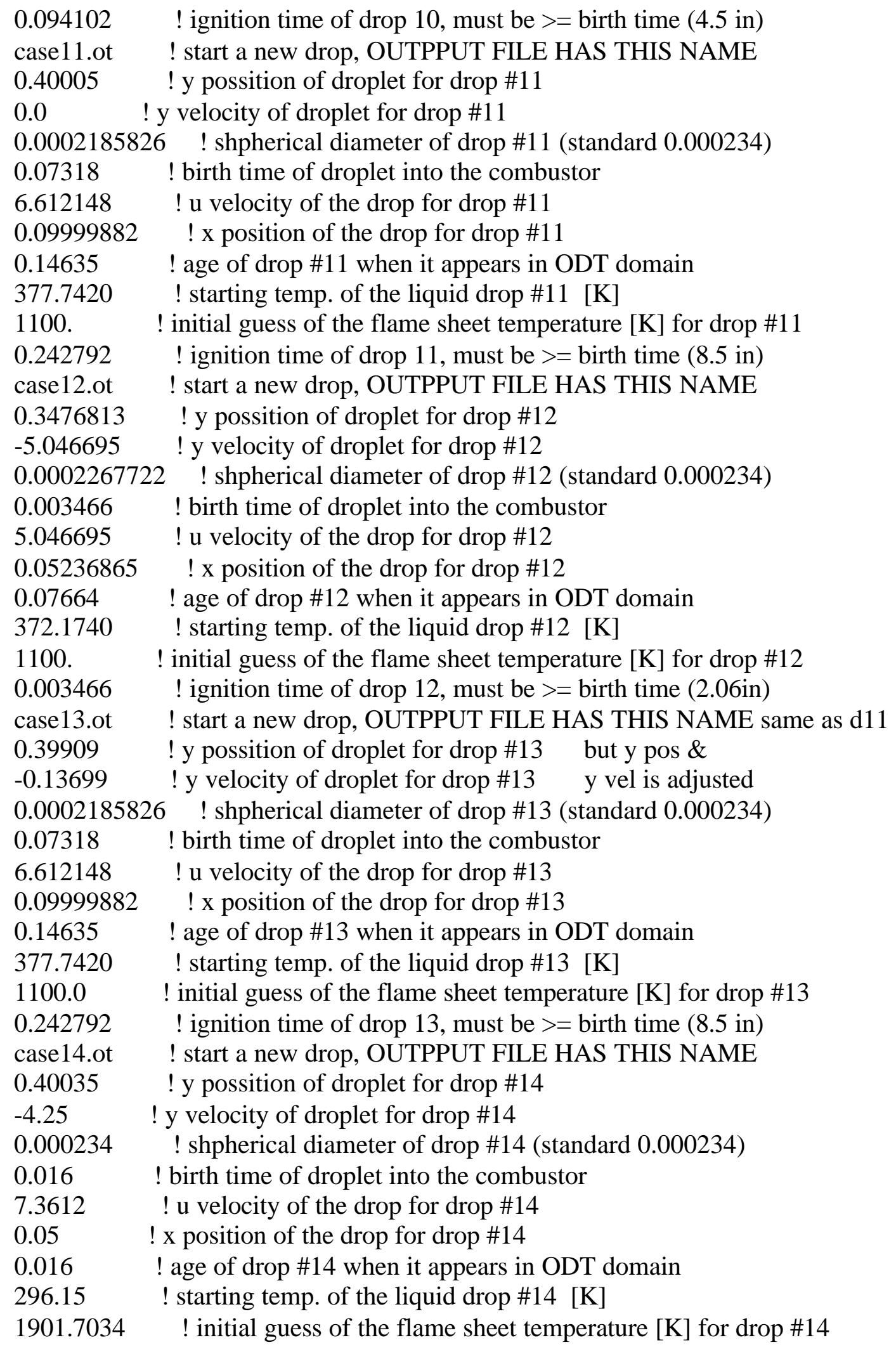


0.048846 ! ignition time of drop 14, must be >= birth time (5 in)

case15.ot ! start a new drop, OUTPPUT FILE HAS THIS NAME

0.40035 ! y possition of droplet for drop \#15

-4.25 ! y velocity of droplet for drop \#15

0.000234 ! shpherical diameter of drop \#15 (standard 0.000234)

0.016 ! birth time of droplet into the combustor

7.3612 ! u velocity of the drop for drop \#15

$0.05 \quad$ ! x position of the drop for drop \#15

0.016 ! age of drop \#15 when it appears in ODT domain

$296.15 \quad$ ! starting temp. of the liquid drop \#15 [K]

1901.7034 ! initial guess of the flame sheet temperature $[\mathrm{K}]$ for drop \#15

0.048846 ! ignition time of drop 15 , must be $>=$ birth time (5 in)

0.01 


\section{C.2 Sample input sheet for ODT program proper}

Input file: Each parameter is preceeded by its index in the line above 'channel search' DISCRETE SPECIFICATIONS: ispec

1 - Number of iterations

61

2 - Number of cells

4431

3 - Flow config: 0 - storm, 1 - relam, 2 - Polyakov, 3 - channel, 4 - Couette, 5 - jet 3

4 - Number of ensembles

2000

5 - Diffusion scheme: 0 for explicit, 1 for implicit

0

6 - Profile restart: 0 none; 1 just write; 2 just read; 3 read and write

0

7 - Rough number of intermediate field updates to output

1

8 - Boundary conditions on velocity: 0 for Dirichlet; 1 for Neumann (+flux in)

0

9 - Boundary conditions on temperature: 0 for Dirichlet; 1 for Neumann

0

10 - Tarek's eddy map

1

$-1$

CONTINUOUS SPECIFICATIONS: rspec

1 - Lower / left endpoint

0 .

2 - Upper / right endpoint

0.5207

3 - Speed of lower / left wall condition

0 .

4 - Speed of upper / right wall condition

0 .

5 - Initial lower / left temperature condition 1100.

6 - Initial upper / right temperature condition

1100.

7 - Final lower / left temperature condition

1100 .

8 - Final upper / right temperature condition 1100. 
9 - Eddy distribution parameter A

.169

10 - Eddy acceptance probability for the time step calculation

0.0014

11 - pressure gradient divided by $\operatorname{rho}(\mathrm{M} /(\mathrm{S} * \mathrm{~S}))$

$-2.759 \mathrm{e}-5$

12 - kinematic viscosity $(\mathrm{M} * \mathrm{M} / \mathrm{S}$ air at $1300 \mathrm{~K})$

$18.2 \mathrm{e}-5$

13 - thermal diffusivity $\{\mathrm{M} * \mathrm{M} / \mathrm{S}$ air at $1300 \mathrm{~K}\}$

$2.5798 \mathrm{e}-4$

14 - reference density; default $=1$.

1.

15 - Boussinesq coefficient $\mathrm{g}$ beta

0 .

16 - Initial bulk velocity

0 .

17 - Initial temperature

0 .

18 - Time to impose a new condition

0 .

19 - Time scale over which to ramp in the new boundary condition

0.

20 - Time at which to begin taking data

0.005

21 - Total run time

.95

22 - diffusion time step coefficient

.45

23 - beta for eddies

0 .

24 - size of the most frequent eddy, as a fraction of the whole domain 0.005

25 - Eddy cutoff parameter

16.

$-1$ 


\section{C.3 Sample long output sheet for one case, one realization}

INITIAL DIA $=0.2137 \mathrm{E}-03$ Age of the drop when it is born into ODT $=0.1464 \mathrm{E}+00$ Time at which the droplet appears in the ODT domain $=0.731800 \mathrm{E}-01$ BULK FLUID VELOCITY $=0.683300 \mathrm{E}+00 \mathrm{U}=0.559850 \mathrm{E}+01 \mathrm{~V}=-.323229 \mathrm{E}+01$ INITIAL X POSITION $=0.100009 \mathrm{E}+00$ INITIAL $\mathrm{Y}=0.342310 \mathrm{E}+00$ DENSITY OF LIQUID $=0.8133 \mathrm{E}+03$ AIR: DENSITY $=0.2715 \mathrm{E}+00$ VISCOSITY $=$ 0.4930E-04

PARAMETER FOR ADJUSTING THE PSEUDO TIME TO DROPLET TIME IS $0.100000 \mathrm{E}+01$

No. of ensambles is 2000 FACTOR FOR EPSILON, SMALLEST DISTANCE IN WHICH

THE ODT IS CONCERNED $=0.1000 \mathrm{E}+00$

FACTOR FOR COMPUTING DROPLET TIME STEP FROM MOLECULAR STEP $(\mathrm{dtm})=0.1500 \mathrm{E}+01$

GRAVITATIONAL ACCELERATION $=0.981000 \mathrm{E}+01$ BOILING PT.$=0.4000 \mathrm{E}+03$ HEAT CAP. $=0.2000 \mathrm{E}+04$ HEAT OF COMBUST.$=0.4400 \mathrm{E}+08$ HEAT OF VAPOR . $=0.3500 \mathrm{E}+06$

FUEL TO OXYGEN MASS RATIO $=0.3170 \mathrm{E}+00$ BURNING PARAM. $=$ $0.66000000 \mathrm{E}-06$

MASS FRACTION OF O2 IN FLUE GAS $=0.5150 \mathrm{E}-01$ GUESS FLAME SHEET

TEMPERATURE $0.11000 \mathrm{E}+04$

DIFFUSIVITY OF FUEL INTO AIR IS 0.8800000E-05 SQUARE METERS/SECOND

AT 0.303150E+03 KELVIN

STARTING TEMPERATURE OF THE LIQUID DROP $=0.3789214 \mathrm{E}+03 \mathrm{KELVIN}$ $\mathrm{NX}=4431$

DROPLET NO. 1

$\begin{array}{llllll}\text { TIME } & \mathrm{U} & \mathrm{V} & \mathrm{X} & \mathrm{Y} & \text { ODT TIME }\end{array}$

$0.147477 \mathrm{E}+00 \quad 0.561863 \mathrm{E}+01 \quad-.313522 \mathrm{E}+01 \quad 0.106332 \mathrm{E}+00 \quad 0.338728 \mathrm{E}+00$

$0.824699 \mathrm{E}-01$

$0.149355 \mathrm{E}+00 \quad 0.566447 \mathrm{E}+01 \quad-.299195 \mathrm{E}+01 \quad 0.116915 \mathrm{E}+00 \quad 0.332975 \mathrm{E}+00$

$0.979578 \mathrm{E}-01$

$0.151213 \mathrm{E}+00 \quad 0.572269 \mathrm{E}+01 \quad-.284016 \mathrm{E}+01 \quad 0.127504 \mathrm{E}+00 \quad 0.327629 \mathrm{E}+00$

$0.113454 \mathrm{E}+00$

$0.153076 \mathrm{E}+00 \quad 0.562871 \mathrm{E}+01 \quad-.298997 \mathrm{E}+01 \quad 0.138090 \mathrm{E}+00 \quad 0.313538 \mathrm{E}+00$

$0.128947 \mathrm{E}+00$

SWITCHED TO LAMDA

$\begin{array}{lllll}0.153751 \mathrm{E}+00 & 0.557510 \mathrm{E}+01 & -.224963 \mathrm{E}+01 \quad 0.141873 \mathrm{E}+00 \quad 0.403125 \mathrm{E}+00\end{array}$

$0.134447 \mathrm{E}+00$

DROPLET EVAPORATED 


\section{Appendix D}

\section{Nomenclature}

a acceleration

A free parameter in ODT proper

B Spalding mass transfer number

$\mathrm{c}_{\mathrm{p}} \quad$ specific heat capacity

$C_{D}^{\infty} \quad$ drag coefficient for isolated droplet

$\mathrm{C}_{\mathrm{D}}{ }^{0} \quad$ drag coeffiecint for closely spaced droplets

$\mathrm{C}_{\mathrm{D}}{ }^{00}$ skin friction drag coefficient of an infinite rod

D droplet diameter

$\mathrm{D}_{\mathrm{AB}} \quad$ gas diffusivity

$f \quad$ stoichiometric fuel-to-oxygen mass ratio or the empirical nonlinear drag law

F force

$\mathrm{F}_{\mathrm{G}} \quad$ gravitational force

g gravitational acceleration

$\mathrm{H}$ total heat transfer to the drop

$\Delta \mathrm{H}_{\mathrm{c}} \quad$ heat of combustion

$\Delta \mathrm{H}_{\mathrm{v}} \quad$ heat of vaporization

$\mathrm{k}$ thermal conductivity

$l \quad$ length of an eddy

m mass of the droplet

P probability of an eddy occurrence 
Pr Prandtl number

r droplet radius

Re Reynolds number

s interdroplet spacing

Sc Schmidt number

Sh Sherwood number

$\mathrm{t}$ time

T temperature

$\mathrm{u} \quad \mathrm{x}$ component of the fluid velocity

U x component of the droplet velocity

$\mathrm{v} \quad \mathrm{y}$ component of the fluid velocity

V y component of the particle velocity

$\mathrm{x}, \mathrm{y}, \mathrm{z} \quad$ Cartesian coordinates

$\mathrm{X}$ gas phase mole fraction

Y gas phase mass fraction

$Y_{O_{\infty}}$ oxygen mass fraction in the gas free stream

$\mathrm{Y}_{\mathrm{Fs}}$ fuel mass fraction at the droplet surface

\section{Greek Symbols}

$\alpha \quad$ stability coefficient for forward Euler differencing

$\beta \quad$ free parameter in the two-phase flow submodel

$\lambda$ burning rate constant

$\mu \quad$ viscosity 
$\eta_{\mathrm{e}} \quad$ evaporation factor on drag

$\eta_{\mathrm{s}}$ droplet interaction factor on vaporization rate

$\kappa \quad$ diffusion coefficient

$\rho$ density

$\theta \quad$ injection angle or any advected scalar

$\tau$ eddy time scale

$\tau_{\mathrm{p}} \quad$ particle aerodynamic response time

v kinematic viscosity

\section{Subscripts/Superscripts}

c using continuous time for computations

d droplet

e using pseudotime for computations

f flame

ODT belonging to the ODT domain

$\mathrm{p} \quad$ particle

$\mathrm{x}, \mathrm{y}, \mathrm{z} \quad$ Cartesian coordinates

1 droplet stream coordinate

0 initial condition

* with mass transfer or using the moving coordinate system

\section{Overbar}

mean quantity, in the droplet film layer
a vector quantity 


\section{References}

Abromzon, B., Sirignano, W.A., Approximate Theory of a Single Droplet Vaporization in a Convective Field: Effects of Variable Properties, Stefan Flow and Transient Liquid Heating, in 1987 ASME/JSME Thermal Engineering Conference - Volume one (P.J. Martor and I.

Tanasawa, Eds.), Book No. 10219A, American Society of Mechanical Engineers, New York, p. 11 - 18, (1987)

Ayers, W.H., Boysan, F., and Swithenbank, J., Droplet Trajectories in Three-Dimensional Gas Turbine Flow Fields, Air Force Report AFOSRTR-81-0543, September 1980.

Carnahan, B., Luther, H.A., Wilkes, J.O., Applied Numerical Methods. John Wiley \& Sons, New York (1969).

Clift, R., Grace, J.R., and Weber, M.E., Bubbles, Drops, and Particles, Academic Press, New York, p. 111 (1978)

Eisenklam, P., Arunachalam, S.A., and Weston, J.A., Evaporation Rates and Drag Resistance of Burning Drops, Eleventh Symposium (International) on Combustion, The Combustion Institute, Pittsburgh, pp. $715-728,(1967)$

Elghobashi, S., Particle-Laden Turbulent Flows: Direct Simulation and Closure Models, Appl. Sci. Res. 48, 301-314, (1991)

Elghobashi, S., On Predicting Particle-Laden Turbulent Flows, Appl. Sci. Res. 52, 309-329, (1994)

Hubbard, G.L., Denny. V.E., and Mills, A.F., Droplet Evaporation: Effects of Transients and Variable Properties, Int. J. Heat Mass Transf. 18: 1003 -1008 (1975)

Kerstein, Alan R., A Linear-eddy model of turbulent scalar transport and mixing, Combust. Sci. and Tech. 60: 391-421 (1988) 
Kerstein, Alan R., Linear-eddy modelling of turbulent transport. Part V: Geometry of scalar interfaces, Physics of Fluids A3: 1110-1114 (1991).

Kerstein, Alan R., One-dimensional turbulence: model formulation and application to homogeneous turbulence, shear flows, and buoyant stratified flows, J. Fluid Mech. 392: 277-334 (1999).

Kerstein, A.R., Ashurst, W.T., Nilsen, V. and Wunsch, S.E., manuscript in preparation, (2000).

Kerstein, A.R., and Dreeben, T.D., Prediction of turbulent free shear flow statistics using a simple stochastic model, Phys. Fluids 12: $418-424$ (2000)

Kramlich, J.C., Heap, M.P., Seeker, W.R., and Samuelsen, G.S., FlameMode Destruction of Hazardous Waste Compounds, Twentieth Symposium (International) on Combustion, p. 1991, The Combustion Institute, 1984.

Labowsky, M., Calculation of the Burning Rates of Interacting Fuel Droplets, Combust. Sci. Technol. 22: 217-226 (1980)

Law, C.K., Unsteady Droplet Combustion with Droplet Heating, Combust. Flame . 26: 17 - 22 (1976)

Monin, A.S. and Yaglom, A.M. Statistical Fluid Mechanics, vol. 1. MIT Press (1971).

Mulholland, J.A., Srivasta, R.K., Ryan, J.V., and Wendt, J.O.L., Influence of Droplet Spacing on Drag Coefficient in Nonevaporating, Monodisperse Streams, AIAA J. 26: 1231 - 1237 (1988)

Mulholland, J.A., Srivasta, R.K., Wendt, J.O.L., Agrawal, S.R., and Lanier, W.S., Trajectory and Incineration of Rogue Droplets in a Turbulent Diffusion Flame, Combust. Flame, 86: 297-310 (1991).

Mulholland, J.A., Private conversation. March 2000. 
Papoulis, Athanasios, Probability, Random Variables, and Stochastic Processes. McGraw-Hill Inc. (1991).

Sirignano, W.A., Fluid Dynamiccs and Transport of Droplets and Sprays, Cambridge University Press, United Kingdom (1999)

Srivastava, R.K., Ryan, J.V., Van Roy, J., The Role of Rogue Droplet Combustion in Hazardous Waste Incineration, Environmental Protection Agency Report EPA/600/7-88/006, April 1988.

Stock, D.E., Particle Dispersion in Flowing Gases - 1994 Freeman

Scholar Lecture, Transactions of the ASME, 118: 4 - 17 (1996)

Wang, Lian-Ping, and Stock, D.E., Dispersion of Heavy Particles by Turbulent Motion, J. Atmos. Sci., 50: 1897-1913 (1993).

Wendt, J.O.L., Combustion Science for Incineration Technology, Twentieth-Fifth Symposium (International) on Combustion, p. 277, The Combustion Institute, 1994. 


\title{
Appendix C
}

\author{
"Trajectories of Evaporating Droplets in a Turbulent \\ Combustor Using the One Dimensional Turbulence Model" \\ J . R. Schmidt, J .0.L. Wendt, and A.R. Kerstein \\ unpublished manuscript
}

(Self paginated) 


\title{
Trajectories of Evaporating Droplets in a Turbulent Combustor Using the One Dimensional Turbulence Model
}

\author{
J. R. Schmidt, J. O. L. Wendt*, \\ Department of Chemical and Environmental Engineering \\ University of Arizona, \\ Tucson, Arizona 85721 \\ wendt@u.arizona.edu \\ and A. R. Kerstein \\ Combustion Research Facility, Sandia National Laboratories, \\ Livermore, California 94550-0969
}

A full length article with the running title of

\section{Droplet Trajectories using ODT}

Corresponding author:

Jost O. L. Wendt

Department of Chemical and Environmental Engineering

University of Arizona,

P. O. Box 210011, Rm 108

Tucson, Arizona 85721-0011

(520) 621-2591

(520) 621-6048 FAX

wendt@u.arizona.edu 


\begin{abstract}
During the incineration of liquid hazardous wastes there may exist "rogue" droplets $(>300 \mu \mathrm{m}$ diameter) which penetrate past the flame zone and burn as isolated droplets in the postflame gases. The ability to predict individual, as distinct from average, droplet burnout points would enable establishment of a guarantee that no drops can exit past a certain point. This would facilitate the design of incinerators that are able to achieve destruction efficiency in excess of the 99.99\% as required by law. Previous work has shown that there is a large spread of droplet burnout endpoints in a combustor. This is due to turbulence and cannot be predicted from knowledge of the mean flow field alone.

In this paper the spread in trajectory endpoints of individual evaporating droplet streams injected into a turbulent combustor is investigated numerically. Correlations between the spread in the burnout points and initial droplet size, droplet velocity, interdroplet spacing, and droplet injection angle are examined. The numerical investigation utilizes the novel One Dimensional Turbulence Model (ODT) by Kerstein for the time-developing fluid velocity and temperature fields, and adds a new two-phase-flow model (one-way coupling) for predicting particle trajectories. The numerical investigation also includes a droplet heating/burning model. Results are in good agreement with the measurements available in the literature.
\end{abstract}




\section{NOMENCLATURE}

A free parameter in ODT proper

$\mathrm{C}_{\mathrm{p}} \quad$ heat capacity per unit mass

$\mathrm{C}_{\mathrm{D}} \quad$ drag coefficient

D diameter

$f \quad$ empirical nonlinear drag law

$\mathrm{F}_{\mathrm{G}} \quad$ gravitational force

$\mathrm{h}$ distance traveled by a fluid cell during a triplet map rearrangement

$\mathrm{k}$ thermal conductivity

$l \quad$ length of an eddy

$\mathrm{m} \quad$ mass of the droplet

$\mathrm{p} \quad$ pressure

$\mathrm{r} \quad$ droplet radius

Re Reynolds number

s interdroplet spacing

$\mathrm{t}$ time

$\mathrm{t}_{\mathrm{e}} \quad$ eddy lifetime

$\mathrm{T}_{\mathrm{p}} \quad$ particle time

$\mathrm{u} \quad \mathrm{x}$ component of the fluid velocity

$\mathrm{U} \quad \mathrm{x}$ component of the droplet velocity

$\mathrm{V} \quad \mathrm{y}$ component of the fluid velocity

$\mathrm{V} \quad \mathrm{y}$ component of the particle velocity

$\mathrm{x}, \mathrm{y}$ position in the respective Cartesian coordinate

\section{Greek Symbols}

$\beta \quad$ free parameter in the two-phase flow submodel

$\lambda \quad$ eddy rate distribution

$\mu \quad$ dynamic viscosity

$\kappa \quad$ diffusion coefficient

$\rho$ density

$\theta \quad$ injection angle or any advected scalar

$\tau \quad$ ODT eddy time scale

$\tau_{\mathrm{p}} \quad$ particle aerodynamic response time

$v \quad$ kinematic viscosity

\section{Subscripts/Superscripts}

b bulk

c using ODT time for computations

e using particle time for computations

g gas phase

ODT belonging to the ODT domain

$\mathrm{p}$ particle

$\mathrm{x}, \mathrm{y} \quad$ Cartesian coordinates

0 initial condition 


\section{Overbar}

- mean quantity

\section{INTRODUCTION}

There exists a large volume of liquid hazardous waste in virtually perpetual storage by the Department of Energy (DOE). This has motivated the DOE to look at other ways to handle, perhaps dispose of, these liquid hazardous wastes. A good method of disposing of these wastes is thermal reaction or incineration. The US regulations on emissions in many cases require a 99.99\% destruction of the organic hazardous material as demonstrated during test burns. In some cases (e.g. polychlorobiphenyls and dioxins) a destruction level of $99.9999 \%$ is required [1] during test burns. Studies of liquid injection incineration systems have shown the existence of large "rogue" droplets which penetrate the flame zone and burn as individual droplets [1]. Mulholland et al. [2] have shown that turbulence causes a surprisingly large spread (maximum axial distance traveled before complete burning minus the minimum axial distance traveled before complete burning) in the burnout points of droplet streams introduced into a combustor. These large spreads occurred even with streams of identical droplets injected with identical initial diameters, velocities, inter-droplet spacings, and injection angles into a $100 \mathrm{~kW}$ turbulent combustor. In twelve cases studied they varied each of these initial parameters (see Table 1) and measured the ensuing effect on the spread of the droplet burnout points. A fuel oil was used as the hazardous waste surrogate. The droplets passed through the flame zone and subsequently ignited and burned out and the locations of ignition and burnout were noted. The nominal droplet stream conditions, Case 1, consisted of $234 \mu \mathrm{m}$ initial droplet diameter, $8.5 \mathrm{~m} / \mathrm{s}$ initial droplet velocity, 180 diameter initial droplet spacing, and 30 degree stream injection angle. 
Mulholland et al. found that models predicting mean behavior were clearly inadequate in predicting the spread. Figure 1 shows the observed range of trajectory endpoints (location at which burnout was completed) for the nominal case (Case 1). The diagram represents a cylindrical combustor. The $100 \mathrm{~kW}$ burner is located on the centerline (radius $=0$ ) on the left hand side just outside the view of the diagram. The mean measured gas phase axial velocity is superimposed on the diagram. The experiments were done with a high swirl number (1.3) which caused the swirling turbulent jet diffusion flame to stabilize close to the burner. The large measured burnout spread was attributed solely to the highly turbulent nature of the flow inside the combustor. The purpose of the research described here was to predict quantitatively the spread in burnout points (in contrast to the average location). Prediction of maximum possible penetration of droplets in turbulent flow constitutes a step towards a procedure that helps establish a guarantee that each droplet is completely destroyed.

In turbulent flows, different fragments of waste that are introduced in an identical manner have different histories. A description of the mean time/temperature/environment history does not capture this. Rather it is necessary to employ a modeling method which captures the relevant physics of the turbulent fluid flow and the two-phase flow, in as simple a formulation as possible. Simplicity is required in order to reach an answer for a specific practical problem. This approach is in contrast to Direct Numerical Simulation (DNS) where all the turbulent fluctuations are modeled in detail. The state-of-the-art DNS modeling of turbulent flows is restricted by the maximum Reynolds number $(\mathrm{Re})$ allowable, due to the memory and speed of the fastest computers available. The memory requirement for DNS goes as the cube of the number of grid points needed to resolve fully the smallest (Kolmogorov) turbulent scale. Since ODT keeps track of only one dimension, the required memory of ODT scales directly with the 
number of grid points needed to resolve the Kolmogorov scale. Though progress to bring DNS to the high Re regime continues, it has a way to go before it can be used for practical industrial applications such as the one considered here. Since the ODT model capitalizes on the symmetry of the flow and utilizes innovative methods to model three-dimensional turbulent behavior on a one-dimensional domain, it is the model of choice for this highly turbulent flow configuration.

Other methods such as Large Eddy Simulation (LES) might also be applied successfully to solve this problem. However, unlike LES, ODT is able to resolve the scales necessary to capture the smallest eddies, albeit only in the one dimension (transverse to the flow). LES requires a subgrid model to account for energy losses due to such things as viscosity. Since ODT keeps track of the smallest eddy scales, the standard viscous dissipation equation handles this requirement. An additional consideration is that the LES method would need a subgrid model to account properly for the two-phase flow portion of the model. Because ODT captures the smallest scales, no additional assumptions are needed for two-phase flow implementation into ODT.

RANS based models have also been used to predict two-phase-flow problems. Call and Kennedy [3, 4] have shown that stochastic separated RANS models do a poor job in capturing the behavior of evaporating droplets in turbulent flow. They suggest that the treatment of droplet vaporization may be problematic. The multi-physics involved (the coupling between the droplet evaporation, the drag, and the turbulent eddies on the droplet trajectory) indicates that the instantaneous gas temperature profile is also of primary importance when droplets are undergoing vaporization since the variations in the gas phase temperature directly corresponds to variations in the droplet temperature profile. Since ODT keeps track of the instantaneous 
velocity and temperature profiles, it has a distinct advantage, for the present problem, over RANS which does not correlate the velocity and temperature fluctuations.

The existing ODT model framework had to be adapted to incorporate 1) a two-phase-flow model and 2) a model for predicting the droplet heating and evaporation.

\section{ODT MODEL}

The ODT model is well suited to address this problem. It has been applied to several different flows including decaying turbulence and wall bounded shear flows [5]. In essence, ODT is a one-dimensional Monte Carlo modeling technique to describe turbulent mixing of velocity and scalar fields. The ODT approach represents turbulent advection by a random sequence of "eddy" maps (or triplet maps) applied to a one-dimensional computational domain. Profiles of the advected velocity component $(u)$ and the advected scalars evolve on this domain. Equations for the turbulent flow field are thus not solved explicitly.

The viscous and diffusive equations are solved,

$$
\begin{aligned}
& \frac{\partial u}{\partial t}=v \frac{\partial^{2} u}{\partial y^{2}}-\frac{1}{\rho_{g}} \frac{\partial p}{\partial x} \\
& \frac{\partial \theta}{\partial t}=\kappa \frac{\partial^{2} \theta}{\partial y^{2}}
\end{aligned}
$$

where $v$ is the kinematic viscosity, $\theta$ is here taken to be any number of advected scalars, in this case temperature, and $\kappa$ is the corresponding molecular diffusion coefficient, in this case the molecular thermal diffusion coefficient $\left\{\mathrm{k} /\left(\rho_{\mathrm{g}} \mathrm{C}_{\mathrm{p}}\right)\right\}$. Kerstein [5] also developed a spatially developing version of ODT, but only the time-developing model was used.

In order for ODT to be used there must be a minimum of one homogeneous direction and there should be a predominantly streamwise direction. The single dimension in the ODT model is transverse to the mean flow. The ODT model implements triplet maps, or eddies, as 
instantaneous rearrange-ments of the velocity field $u(y, t)$. (See Fig. 2.) The triplet map has a starting point $y_{\mathrm{o}}$ and a length $l$ which are sampled randomly from a rate distribution. For this purpose, Kerstein develops an eddy rate distribution $\lambda(l ; y, t)$, where $\lambda(l) \mathrm{d} l \mathrm{~d} y$ is the frequency of events per unit distance along the $y$ coordinate in the size range $[l, l+\mathrm{d} l]$ with $y_{\mathrm{o}}$ in the range $[y$, $y+\mathrm{d} y$ ], which gives $\lambda$ units of (length ${ }^{2} \mathrm{x}$ time $^{-1}$. An event is taken to be the occurrence of a triplet map. It describes the idealization of an eddy on the ODT domain. By dimensional arguments,

$$
\lambda(l)=\frac{1}{l^{2} \tau(l)}
$$

where $\tau(l)$ is an eddy time scale. Kerstein [5] treats $\tau(l)$ as a local instantaneous time scale denoted $\tau(l ; y, t)$ whose evaluation is based on $u(y, t)$,

$$
\tau\left(l ; y_{o}, t\right)=\frac{l}{A \Delta u\left(y_{o}, l\right)}
$$

where $\Delta u\left(y_{0}, l\right)$ is a measure of velocity variation along the interval $\left[y_{0}, y_{0}+l\right]$ and $A$ is a free parameter. Specifically

$$
\Delta u\left(y_{o}, l\right)=2\left|u_{l}\left(y_{o}+\frac{l}{2}, t\right)-u_{l}\left(y_{o}, t\right)\right|
$$

where

$$
u_{l}(y, t)=\frac{2}{l} \int_{y}^{y+l / 2} u\left(y^{\prime}, t\right) d y^{\prime}
$$


Hence $\Delta u$ in Eq. 5 is based on the mixing-cup average velocity of each half of the eddy. If these averages are shown to differ greatly (hence there is a large strain rate across the eddy) then $\tau\left(l ; y_{0}, t\right)$ is small and $\lambda\left(l ; y_{0}, t\right)$ is large. Equations $3-5$ give

$$
\lambda\left(l ; y_{o}, t\right)=\frac{2 A\left|u_{l}\left(y_{o}, t\right)-u_{l}\left(y_{o}+\frac{l}{2}, t\right)\right|}{l^{3}} .
$$

The free parameter $A$ is the only free parameter in the ODT model proper. The free parameter $A$ was introduced to compensate for the fact that $\Delta u\left(y_{0}, l\right)$ was chosen as a matter of preference. This arbitrary choice of Eq. 6 may in turn affect the numerical results, hence $A$ allows fine adjustments to the eddy rate distribution. The upshot is that $A$ becomes a turbulence intensity knob. Decreasing $A$ decreases the level of turbulent intensity, and increasing $A$ increases the turbulent intensity.

A pictorial rendering of the triplet maps is given in Fig. 3. In this diagram the vertical spatial axis is the ODT $y$ domain and the abscissa is time. At each instant in time (each vertical line) there exist a complete (instantaneous) velocity profile and complete profiles for all the passive scalars, none of which are shown in the diagram. Whenever a triplet map is implemented at a certain point in time, the domain of the eddy event $\left[y_{0}, y_{0}+l\right]$ is recorded by placing a darkened line segment over said domain. Therefore the figure gives an overview of the eddy event history. One can see the time evolution of eddy events, including the clustering of eddies that emulates the turbulent cascade. The pictorial in Fig. 3 is an actual space-time history diagram from a member of the ensemble used to generate the data presented in this paper.

For the implementation of this cylindrical turbulent diffusion flame combustor the geometry was idealized as turbulent channel flow. The center of the channel corresponds to the zero radius 
position in Figs. 1 and 4. The bottom of the channel $(y=0$ on the ODT domain) corresponds to the bottom (maximum radius) of Fig. 1. The axial bulk mean flow velocity of $0.6 \mathrm{~m} / \mathrm{s}$ used in the simulation corresponds to the outflow bulk velocity as measured in the experiment [8].

The time development of the velocity and temperature fields is numerically computed for individual realizations, and statistics are extracted from an ensemble of realizations. To convert ODT time development to distance traveled downstream, we simply multiply the elapsed time by the bulk mean velocity. In order to have ODT generate the time developing fluid velocity and temperature fields, the mean measured velocity and temperature profiles at $5 \mathrm{~cm}$ downstream (as reported in [2]) were input as initial conditions. With these initial conditions, ODT was able to match trends in the published mean velocity and mean temperature profiles of [2], which are reproduced in Fig. 4. The simulated results for mean velocity and temperature profiles are shown in Fig. 5 A and B. The results presented in this paper were computed from 350 realizations, which roughly correspond to the order of the number of experimental observations used to compile Fig. 1 [7]. It is clear that the velocities predicted by ODT decay too rapidly for this flow. This reflects the combined effect of neglecting swirl, recirculation, and thermal expansion in this application. Computations were done using decaying turbulence ( $\mathrm{dp} / \mathrm{dx}$ set equal to zero) and repeated for $\mathrm{dp} / \mathrm{dx}$ nonzero. There was no discernable difference in the results between the two. This is due to the short downstream distance of interest, approximately one channel height.

A recent ODT model improvement [8] includes three velocity components. This would allow the capture of swirl (in cylindrical coordinates). Consequently it might improve the comparison, though there are other ODT simplifications that also affect the comparison. Variations of the one ODT parameter $A$ showed that both velocity and temperature profiles could not be matched 
simultaneously. Temperature has a more pronounced influence over vaporization, so temperature was chosen to calibrate the value of $A$ and therefore the bulk mean fluid velocity. Another consideration for choosing temperature over velocity was that there is no reported value of the measured velocity fluctuations [6], hence no way to measure the accuracy of the ODT prediction of velocity fluctuations for this flow, which matters more than the mean velocity for droplet dispersion (endpoint variability). Examples of the predicted mean and instantaneous velocity and temperature as shown in Fig. $6 \mathrm{~A}$ and B.

The existing ODT formulations were limited to single-phase flow. Consequently a twophase-flow submodel compatible with ODT is introduced here.

\section{TWO-PHASE-FLOW MODEL}

To derive the equation of motion of a particle of mass $m_{\mathrm{p}}$, a force balance is performed on the particle. For this application, the only forces on the particle are gravity and the drag force. The $y$ and $x$ components of the assumed particle drag law are given in Eqs. 8 and 9 with $U$ being the particle velocity in the $x$ direction and $V$ being the particle velocity in the $y$ direction.

$$
\begin{aligned}
& \frac{d V}{d t}=-(V) \frac{f}{\tau_{p}}+F_{G}, \\
& \frac{d U}{d t}=\frac{-(U-u) f}{\tau_{p}} .
\end{aligned}
$$

A complete derivation of these equations can be found in [9]. Note that ODT idealizes the gas $y$ velocity, $v$, by setting it equal to zero outside eddy events. Triplet maps do mimic a $v$ velocity within eddies during their implementation, as explained later. The aerodynamic response time, 
$\tau_{\mathrm{p}}$, the empirical nonlinear drag correction factor, $f$, of Rowe [10] and the particle Reynolds number components are given by:

$$
\begin{aligned}
& \tau_{p}=\frac{\rho_{p} C_{D} D_{p}^{2}}{18 \mu_{g}} \\
& f=\left\{\begin{array}{cc}
1 & \operatorname{Re} \leq 1 \\
1+0.15 \operatorname{Re}^{0.687} & 1 \leq \operatorname{Re} \leq 1000
\end{array}\right. \\
& \operatorname{Re}_{x}=\frac{\rho_{g} r_{d}\left|U-u_{g}\right|}{\mu_{g}} \\
& \operatorname{Re}_{y}=\frac{\rho_{g} r_{d}|V-v|}{\mu_{g}} .
\end{aligned}
$$

ODT evolves in time, with the time coordinate (here denoted $t_{\mathrm{ODT}}$ ) interpreted as streamwise (x) development based on the mean bulk velocity of the fluid, denoted $\bar{u}_{\mathrm{b}}$. However, each particle proceeds with its own $x$ velocity, $U$. The particle is subject to time-varying gas velocity, $u$, both because $u$ evolves in time, $t_{\mathrm{ODT}}$, and because particle motion in the $\mathrm{y}$ direction induces variation of $u$ in the particle reference frame. Due to the finite inertia of the particle, $U$ generally differs from $u$ at the particle location, and both these quantities differ from $\bar{u}_{\mathrm{b}}$. Thus, there is typically a difference between the speed at which the particle traverses the $x$ direction and that at which the bulk mean velocity $\overline{\mathrm{u}}_{\mathrm{b}}$ traverses the $x$ direction. The time $\Delta t_{\mathrm{p}}$ required for a particle to traverse the $x$ distance that the mean bulk flow traverses in a given time $\Delta t_{\mathrm{ODT}}$ is

$$
\Delta t_{p}=\frac{\overline{u_{b}} \Delta t_{O D T}}{U\left(t_{p}\right)}
$$

Integration of Eq. 14 operationally defines a particle time coordinate $t_{\mathrm{p}}$ whose dependence on ODT (gas-phase) time $t_{\mathrm{ODT}}$ is distinct for each particle trajectory. The dependence of $t_{\mathrm{p}}$ on $t_{\mathrm{ODT}}$ is complicated because $U$ is a function of $t_{\mathrm{p}}$ due to the equation of motion. Integration of Eqs. 8 and 9, with $t_{\mathrm{p}}$ substituted for $t$, concurrently with Eq. 1, in which $t$ corresponds to $t_{\mathrm{ODT}}$, and Eq. 
14, which relates the two time coordinates, determines particle streamwise trajectory evolution except when a particle interacts with an instantaneous eddy event (triplet map). A full discussion can be found in [9].

Particle interaction with a triplet map requires a novel approach. This interaction occurs when the particle and triplet map occupy the same space-time. Before discussing in detail particle-eddy interactions, there is a problem which arises due to the ODT formulation of eddies as instantaneous in time. Particles with finite inertia will generally have a finite, nonzero, $y$ velocity. Figure 7 depicts an eddy event history diagram which includes four possible particle trajectories with various initial $y$ velocities $(V)$. Particle trajectory 1 has no initial $y$ velocity component. Trajectories 2, 3, and 4 have increasing (in magnitude) negative $y$ velocity components. In the scenario set forth in Fig. 7 both particle trajectories 1 and 2 eventually intersect an eddy event. At the intersection points the particle positions and velocities are changed instantaneously. However the larger (in magnitude) the $y$ velocity becomes, the less likely a particle is to intersect an eddy event. In the limit (Fig. 7, path 4) as the $y$ velocity component becomes infinite, the probability of an eddy interaction becomes zero because both the triplet maps and the particle trajectories are vertical lines. This phenomenon is an artifact of the ODT formulation since particles with large $y$ velocities should be allowed to interact with eddies. This artifact becomes important when the particle $y$ velocity becomes large, for example as the initial injection angle deviates more from zero incidence.

The particle drag law as written is not applicable to ODT particle-eddy interactions because triplet maps are instantaneous in time yet the particle-drag law requires an interaction time to cause a change in the particle velocity or position. The logical solution to this difficulty is to assign an interaction time to the particle-eddy interaction. For now, we will denote the 
interaction time coordinate as $T_{\mathrm{p}}$, and the duration of the interaction as $T_{1}$. The interaction time coordinate $t_{\mathrm{p}}$, distinct from the simulation time coordinate $t_{\mathrm{ODT}}$, is a device to enable particleeddy interactions. The quantity $T_{1}$ will be developed and defined later in the discussion. Particle evolution during this interaction is governed by the $y$ and $x$ components of the particle drag law Eqs. 8 and 9 adapted for the interaction time coordinate,

$$
\begin{aligned}
& \frac{d V}{d T_{p}}=-\frac{(V-v)}{\tau_{p}} f+F_{G} \\
& \frac{d U}{d T_{p}}=-\frac{(U-u) f}{\tau_{p}},
\end{aligned}
$$

where $v$ (defined later) is the fluid y-component velocity induced by the triplet map, $T_{\mathrm{p}}$ is the interaction time coordinate, $u$ is the velocity of the fluid cell which contains the particle, and all other terms are as previously defined. In this formulation tracer particles behave correctly, that is, they remain within the fluid cells that contain them initially [9].

Determination of the particle-eddy interaction time, $T_{1}$, requires a definition of the lifetime $t_{\mathrm{e}}$ of an ODT eddy. The ODT local instantaneous time scale $\tau\left(l ; y_{0}, t\right)$ (see [5]) is best interpreted as the characteristic time of the eddy event if it were to happen over a time period and not instantaneously. $\tau\left(l ; y_{0}, t\right)$ is however an artifact of the ODT model development and not an actual or physical time. Even though parameter dependencies of $\tau\left(l ; y_{0}, t\right)$ are based on solid reasoning there is no compelling reason to believe that it necessarily corresponds to the eddy lifetime. Therefore it is justifiable to introduce a particle-model free parameter denoted $\beta$. The eddy lifetime is then

$$
t_{e}=\beta \tau\left(l ; y_{o}, t\right) .
$$


Ideally $\beta$ is a number close to unity since $\tau\left(l ; y_{0}, t\right)$ was based on good reasoning and logically should not be too far off. $\beta=1$ was used for all runs reported in this paper. A set of sensitivity runs designed to investigate the model's response to changes in $\beta$ were made for $\beta=0.5$ and $\beta=$ 2.0. Results can be found in [9]. Decreasing $\beta$ causes increasing deviation of the particle trajectory in the $y$ direction from its original path due to the particle-eddy interaction. Increasing $\beta$ causes less deviation. This may seem counterintuitive, but the free parameter $\beta$ has two important and somewhat competing effects on the particle-eddy interaction. The first effect is that it adjusts the maximum allowable end time of the particle-eddy interaction, Eq. 17, which either lengthens or shortens the amount of time during which the particle relaxes to the fluid velocity. The second is that it adjusts the fluid phase $y$ velocity, Eq. 18 below. A value $\beta<1$ increases $v$, while a value $\beta>1$ decreases $v$. The burden of the drag law (Eq. 15) is to relax the particle to the surrounding gas flow $v$. With $\beta>1$, the maximum relaxation time $t_{\mathrm{e}}$ increases, but the velocity $v$ to which the particle relaxes decreases. A detailed discussion on the subject can be found in [9], along with the results from the sensitivity runs on burning droplets.

As noted, the eddy lifetime is used to determine $v$ during the eddy event. The gas phase $y$ velocity is computed by dividing $h$, the $y$ distance traveled by the fluid element containing the particle just prior to the eddy event, by the eddy lifetime:

$$
v=h / t_{e} .
$$

A problem arises when comparing the integration done in the interaction time coordinate over a time period $T_{1}$ and the conventional continuous-time integration of the drag law subsequent to the particle-eddy interaction, over comparable time intervals $\Delta T_{\mathrm{p}}=T_{1}$ and $\Delta t_{\mathrm{p}}=T_{1}$. Comparison 
of Eqs. 8 and 15 indicates that the equations contain similar terms and therefore the resultant actions of integrating them over the same time period are in fact double-counting the effect of the gravitational force, once during the interaction time integration from $T_{\mathrm{p}}=0$ to $T_{\mathrm{p}}=\mathrm{T}_{1}$ and once during the particle time, $\mathrm{t}_{\mathrm{p}}$, time integration from the birth time of the eddy to a time increment of $\mathrm{T}_{1}$. In other words the particle experiences the gravitational force first for a period of $\mathrm{T}_{1}$ in interaction time, $T_{\mathrm{p}}$, and again for a period of $\mathrm{T}_{1}$ in particle time, $t_{\mathrm{p}}$. To overcome this problem, which occurs when the particle and the triplet map occupy the same space-time, the particle trajectories with and without the effect of the eddy are computed. This is achieved by integrating (first to get velocity and then again to get position) both Eqs. 8 and 15 concurrently over the period $\mathrm{T}_{1}$, and determining the difference in the ending $y_{\mathrm{p}}$ position (defined in Eq. 19). Similarly, the difference in the ending $V$ velocities (defined in Eq. 20) is also computed. These computations define the instantaneous perturbations felt by the particle due to the particle-eddy interaction,

$$
\begin{aligned}
& \Delta y_{p}=y_{p}^{e}\left(T_{1}\right)-y_{p}^{c}\left(T_{1}\right) \\
& \Delta V=V^{e}\left(T_{1}\right)-V^{c}\left(T_{1}\right),
\end{aligned}
$$

where the superscript $e$ (for eddy) denotes the results based on Eq. 15 and the superscript $c$ (for continuous) denotes using Eq. 8. Fig. 8 shows this procedure graphically. Figure 9 shows additional examples with varying particle inertia.

No adjustments are made to the $x$ position, $x_{\mathrm{p}}$, or the $x$ velocity, $U$, of the particle due to a particle-eddy interaction. The nature of the time developing ODT model does not lend itself readily to sudden jumps in the $x$ domain, which is related to the ODT time. No reasonable method to account for instantaneous movement backward or forward in time could be developed. 
A real-life eddy would exist in a defined space (three-dimensional) and exist for a defined (measurable) lifetime. Particle-eddy interactions would occur while the particle occupies the same space-time $(x, y, z, t)$. Hence this two-phase-flow model must assure that the particle-eddy interaction ceases the first time the particle leaves the ODT space time $(x, y, t)$ which the eddy is idealized to occupy. A complete definition of idealized eddy is in order. The $y$ dimension of the eddy is defined in the ODT model proper and those dimensions are also used in the two-phaseflow submodel. The eddy is bounded in the $y$ direction by: $y_{\mathrm{o}} \leq y_{\mathrm{e}} \leq y_{\mathrm{o}}+l$. The $x$ dimension of the idealized eddy is taken to be the centered around the $x$ position of the particle with an assigned length $l$ in each direction. Test runs were completed to determine the effect of using an assigned length of $l / 2$ in each direction. There was no discernable difference in the spread due to this change. Particle-eddy interactions are more complicated in the $x$ direction because the fluid parcels usually have a $u$ velocity. This velocity would usually be carrying the eddy at a different rate then the particle. The particle would see an eddy moving in time in the $x$ direction at a rate of the average $u$ velocity. A logical definition for the average $u$ velocity of an eddy event is the average of the $u$ velocities of the individual cells in the domain of the triplet map, [ $\left.y_{0}, y_{0}+l\right]$, at the time of the eddy event. Hence the eddy is defined in the $x$ direction as:

$$
x_{o}+u_{e d d y} T_{p}-l \leq x_{e} \leq x_{o}+u_{e d d y} T_{p}+l .
$$

With the $x, y$, and $t_{\mathrm{e}}$ of the idealized eddy completely defined, it is merely a matter of bookkeeping to keep track of the first time $T_{1}$ that the particle leaves the idealized eddy. This time is then plugged into Eqs. 19 and 20 to compute the instantaneous effect of the particle-eddy interaction. 


\section{DROPLET HEATING/BURNING MODEL}

A modified version of the burning model used by Mulholland et al. was used to predict droplet lifetimes. A brief summary of the droplet transient heating/burning model follows. (The reader is referred to [2] for more details of the burning model and to [9] for details on this specific application.) The liquid droplet is assumed to be spherically symmetric with infinite heat diffusivity ("rapid mixing limit"). Therefore the droplet temperature is allowed to change with time, but there is no need to solve any of the fluid-flow equations inside the droplet since the temperature is assumed constant in the droplet. The liquid-phase properties (e.g. density and heat capacity) are constant. The heating time of the droplet is slow compared to the gas diffusion time. Mulholland et al. idealize the droplet fuel mixture to be one representative (unnamed) chemical component. The concentration of this component in the gas phase (assuming equilibrium) is determined by the Clausius-Clapeyron equation. The gas phase is assumed to be in a quasi-steady state, with heat and mass diffusion rate controlling. With the assumption of unity Lewis number, the Sherwood number is equal to the Nusselt number. Ambient gas-phase conditions are subcritical and the gas-phase mixture is assumed to behave as an ideal-gas mixture. Infinitely fast kinetics are assumed, so the flame sheet surrounds the burning droplet. (In other words, the reaction time is shorter than the diffusion time and a flame surrounds the droplet after it has ignited.) This was experimentally verified by Mulholland et al.. The droplets would ignite (glow) a short time before they would completely burn out.

The ODT formulation used in this work utilizes a constant kinematic viscosity $\left(1.82 \times 10^{-5}\right.$ $\mathrm{m}^{2} / \mathrm{s}$ ) for air at $1300 \mathrm{~K}[11]$ in the gas-flow calculations. However, when computing the mean gas phase properties in the droplet film layer, for evaluation of the pertinent drag and heat and mass transfer rates, the "one-third rule" [12] for evaluating the temperature was utilized. 
Hubbard et al. [12] demonstrate that proper temperature choice is essential to obtain the correct heat and mass transfer rates. These temperatures were used to estimate values of viscosity, density and heat capacity of the fuel-air mixture in the resistance film surrounding the drop as needed (e.g. Eqs. $10-13)$.

In contrast, the values of $v$ and $\kappa$ used to compute the fluid phase flow (in ODT proper) only affect the details of the fine-scale structure of the velocity and temperature fields. Hence temperature variations in and of themselves are not crucial, but accurate temperature dependencies of the physical properties $\left(v, \rho, C_{p}\right)$ are essential to compute heat and mass transfer correctly and are therefore central to the aforementioned coupling between droplet evaporation and drag.

A distinguishing feature of ODT is that it fully captures these unsteady couplings and therefore provides higher fidelity representation of the multi-physics involved than conventional methods $[3,4]$.

\section{IMPLEMENTATION AND RESULTS}

Simulations were done to mimic the 12 published cases [2] in which the initial diameter, initial velocity, initial droplet spacing and the injection angle of a stream of droplets into a 100 $\mathrm{kW}$ combustor were varied. Experimental observation [2] revealed that the droplets traveled along a straight-line trajectory until they reached the high-fluid-shear layer. After this point the droplet stream appeared to disperse. This point is taken to be the point at which the individual droplets are born into the ODT simulation since the ODT model does not lend itself to modeling streams of droplets [9, p. 120]. In order to properly account for droplet heating and vaporization from the point of droplet injection to the point at which the droplets are born into ODT, the droplet drag law and droplet heating/vaporization scheme put forth in [2] were calculated for 
each case to determine the best possible estimate of the droplet diameter and temperature. Consistent with Mulholland et al. [2] the average measured ignition points, as reported in [6], were used in the simulation to initiate droplet flame sheet. Mulholland also alludes to the difficulties in predicting ignition points of droplets [2]. The burnout point is taken to be the point at which the last amount of liquid is vaporized. This occurs after the droplet has been ignited, so the vapor is assumed combusted in the surrounding flame sheet.

The two-phase-flow model allows instantaneous jumps in the $y$ position of the droplet to account for eddy-particle interactions. Fig. 10 shows trajectories from the nominal case (Case 1) which exemplify the extremes in the spread $(x$ and $y)$ in the burnout points. The random nature of the submodel can be seen by examining the different trajectories. Individual trajectories can jump up and down randomly.

To provide a clearer understanding of the simulated effects of the different varied parameters, scatter plots of the burnout points for the first three cases are grouped together in Fig. 11. Scatter plots are $x-y$ plots where $x$ is the axial distance penetrated by the droplet and $y$ is the height. The $y=0$ datum plane is defined as a quarter of a meter below the burner. The burner is on the central axis of the tubular combustor.

A major objective of this paper is to try to predict the spread in the burnout points due to the turbulent fluctuations. Fig. 12 shows, for an ensemble size of 350, the predicted axial spread for the four variations of initial stream conditions, namely diameter, velocity, droplet spacing, and injection angle. An ensemble size of 350 was used because it roughly corresponds to the order of the number of experimental observations used in [2]. Analysis shows excellent agreement of the predicted spread with the measured spread for the variation in initial diameter. Table 1 shows that in the experiments for size variation (Cases 1-3) the droplet spacing was also varied 
$\left(180 \geq s / D_{\mathrm{o}} \geq 115\right)$. These values of $s / D_{\mathrm{o}}$ are in the range where one would expect the droplets in the stream to behave independently of each other.

The agreement with the variation in initial velocity is reasonable, with both the simulated and experimental data bending downward as the initial velocity increases. As can be seen in Table 1, the runs for velocity variations (Cases 4,1,5) not only included variations in the initial velocity, but also in the initial spacing $\left(120 \leq s / D_{\mathrm{o}} \leq 210\right)$ and in initial diameter $\left(216 \leq \mathrm{D}_{\mathrm{o}} \leq 250 \mu \mathrm{m}\right)$. The $s / D_{\mathrm{o}}$ values were large enough so that there should be a negligible effect on the observed results.

The model shows a marked change in behavior of the spread as the initial spacing/diameter $\left(s / D_{\mathrm{o}}\right)$ changes. Above a value of $s / D_{\mathrm{o}}$ of 10 , the particles behave independently. There is very good agreement in the second grouping (the last three cases) of the spread as compared to the measured data. The first grouping (of three) is not a good match. The lack of $s / D_{\mathrm{o}}$ parameterization in the ODT droplet submodel is probably the cause of this discrepancy.

The agreement for the zero and thirty degree injection angles is good. The trend of the simulated data is significantly different from the measured data. This is probably due to the artifact created in ODT by the assumption that eddies are instantaneous in time. As the injection angle increases the $y$-component of the particle velocity increases and the error due to this artifact becomes more pronounced.

An additional objective of this paper is try and develop a guarantee that each droplet is completely destroyed. The results quoted so far were with a sample size of 350 iterations, a number chosen to be of the same order as the number of samples taken in the experiments of [2, 8]. To verify that the ODT two-phase-flow model is able to predict a maximum possible spread of burnout points, runs were made for much larger ensemble size, approximately 1400 iterations, and analyzed to determine spread for all the cases. Further analysis of a run of approximately 
2000 iterations was completed with no increase in the spread of droplet endpoints. This indicates that the spread in burnout points seems to peak at approximately 1400 iterations then stay stable from then on.

\section{CONCLUSIONS}

1) An ODT-droplet-trajectory model was developed to simulate incinerator performance attributes. Results indicate that one can predict possible failure modes inherent in the incineration process due to rogue droplet penetration past the flame zone and subsequent failure to completely burn out.

2) The ODT model proper is able to reasonably predict trends in the mean velocity and temperature profiles of a $100 \mathrm{~kW}$ combustor using as an initial condition the measured mean profiles (velocity and temperature) at a distance of $5 \mathrm{~cm}$ downstream of the burner. However, matching both velocity and temperature was not possible due to simplifications in the model. The choice of fitting temperature over velocity provided good results.

3) The ODT two-phase-flow model can accurately predict the spread of droplet burnout points due to the random variation of the velocity/temperature field. Excellent agreement (using an ensemble size of 350) was found for predicting the spread due to variations in initial diameter. Good agreement was found for predicting the spread caused by variations in initial velocity and for the first two cases of droplet stream injection angle.

4) The spread is underpredicted for the 45-degree-angle injection because of the artifact that results from assuming instantaneous eddy events.

5) The ODT two-phase-flow model did not reproduce the trends for variation in the spacing/diameter spread because no $s / D$ effects were included inside the ODT droplet model. 
6) Decreasing the eddy-lifetime coefficient $\beta$ causes the particle trajectory to deviate in the $y$ direction more from its original path due to the particle-eddy interaction. Increasing $\beta$ causes less of a disturbance.

7) The model demonstrated a maximum in spread for an ensemble size of approximately 1400.

\section{FUTURE WORK}

Rather than using the experimentally measured mean profiles as an initial condition (IC) for the ODT flow simulation, an alternative tested method either in ODT itself or using a CFD package could be used to obtain a better IC for this simulation which would include temperatures and velocities above the mean at the IC. Also since the experiments were done on a combustor with high swirl a method to implement swirl into ODT may help.

\section{ACKNOWLEDGEMENTS}

Financial support was provided by the DOE under project number DEFG0797ER14831 and through the Office of Science, Division of Chemical Sciences, Geosciences, and Biosciences. Dr. Tom Dreeben provided a FORTRAN code for ODT proper which served as the starting point of this work. The Authors are grateful to Dr. Mulholland for his help. 
References

1. Wendt, J. O. L., Twentieth-Fifth Symposium (International) on Combustion, The Combustion Institute, 1994, pp. 277-289.

2. Mulholland, J. A., Srivasta, R. K., Wendt, J. O. L., Agrawal, S. R., and Lanier, W. S., Combust. Flame, 86:297-310 (1991).

3. Call, C. J., Kennedy, I. M., Droplet dispersion, Velocity and Vaporization in Heated and Unheated Jets, AIAA 93-0904, $31^{\text {st }}$ Aerospace Sciences Meeting and Exhibit, Reno, NV (1993).

4. Call, C. J., Kennedy, I. M., AIAA Journal, 32:874-875 (1994).

5. Kerstein, A. R., J. Fluid Mech, 392:277-334 (1999).

6. Srivastava, R. K., Ryan, J.V., and Van Roy, J., The Role of Rogue Droplet Combustion in Hazardous Waste Incineration, Environmental Protection Agency Report EPA/600/7-88/006, April 1988.

7. Mulholland, J. A., Private conversation. March 2000

8. Kerstein, A. R., Ashurst, Wm. T., Wunsch, S., and Nilsen, V., One-dimensional turbulence: vector formulation and application to free shear flows, J. Fluid Mech, in press (2001).

9. Schmidt, J. R., Trajectories of Evaporating Droplets in a Turbulent Combustor Using the One Dimensional Trubulence Model, Masters Thesis, University of Arizona, 2000.

10. Rowe, P. N., Trans. Inst. Chem. Eng, 39:175-181 (1961).

11. Perry's Chemical Engineers' Handbook (R. H. Perry, D. W. Green, and J. O. Maloney, Eds.) McGraw Hill, New York, 1997.

12. Hubbard, G. L., Denny, V. E., and Mills, A. F., Int. J. Heat Mass Transf. 18:1003-1008 (1975). 
TABLE 1

Droplet Stream Test Conditions ${ }^{\mathrm{a}}$

\begin{tabular}{l|lllll}
\hline Title & $\begin{array}{l}\text { Parameter } \\
\text { Varied }\end{array}$ & $\begin{array}{l}D^{0} \\
(\mu \mathrm{m})\end{array}$ & $\begin{array}{l}V_{d}^{0} \\
(\mathrm{~m} / \mathrm{s})\end{array}$ & $s^{0} / D^{0}$ & $\begin{array}{l}\theta^{0} \\
(\mathrm{deg})\end{array}$ \\
\hline Case 1 & Nominal & 234 & 8.5 & 180 & 30 \\
Case 2 & Size & 306 & 8.5 & 140 & 30 \\
Case 3 & & 371 & 8.5 & 115 & 30 \\
Case 4 & Velocity & 216 & 5.3 & 120 & 30 \\
Case 5 & & 250 & 10.6 & 210 & 30 \\
Case 6 & Spacing & 234 & 8.5 & 2.8 & 30 \\
Case 7 & & 234 & 8.5 & 5.6 & 30 \\
Case 8 & & 234 & 8.5 & 11.2 & 30 \\
Case 9 & & 234 & 8.5 & 22 & 30 \\
Case 10 & & 234 & 8.5 & 45 & 30 \\
Case 11 & Angle & 234 & 8.5 & 180 & 0 \\
Case 12 & & 234 & 8.5 & 180 & 45 \\
\hline
\end{tabular}

${ }^{a}$ redrawn from Mulholland et al. by permission of the authors. 


\section{List of Figures}

Fig. 1: Measured trajectories for the nominal droplet stream condition (Case 1) in a turbulent flame. The shaded area represents the range of trajectory endpoints. The gas phase axial velocity profile is superimposed on the top view (dashed lines). Taken from Ref. [2] with permission of the authors.

Fig. 2: Effect of the trip let map on an initially linear velocity profile $u(y, t)$. (A) Initial profile. (B) Velocity profile after applying the triplet map to the interval denoted by ticks. (C) Discrete representation of the initial profile and illustration of the effect of a triplet map on an interval consisting of nine cells. For clarity, arrows indicating formation of the central of the three images of the original interval are dashed. Redrawn from Ref.[5] with permission of the author.

Fig. 3: Pictorial representing the eddy events in space-time coordinates. Vertical lines represent spatial ranges and times of occurrence of eddies. Presented here is the space-time history of an actual realization used in this paper.

Fig. 4: Measured mean temperature (a) and axial velocity (b) profiles. These are the near burner proflies for the Type $\mathrm{C}$ flame condition. Taken from Ref. [2] with permission of authors.

Fig. 5: Fig. 5. Simulated mean temperature and axial velocity profiles, based on an ensemble size of 750. From here on measurements made by Mulholland et al. [2] are transformed into the ODT $y$ coordinate as explained in the text. The ODT gas-phase simulation starts at $0.05 \mathrm{~m}$ into the reactor as explained in the text.

Fig. 6: Mean (solid curves) and instantaneous (dashed curves) velocity (A) and temperature (B) profiles at an axial distance of about $0.19 \mathrm{~m}$. The mean profiles are based on 2000 simulated realizations.

Fig. 7: Particles with high initial $y$ velocity are more likely to traverse the ODT space-time domain without interacting with an instantaneous eddy event.

Fig. 8: The procedure for implementing particle-eddy interactions which avoids double counting. Find $\Delta y_{\mathrm{p}}$ and $\Delta \mathrm{V}$ by integrating the drag law with and without eddy interaction (A) then add them to the original position and velocity (evaluated when the particle-eddy interaction begins). The resultant position and velocity based on the original velocity $V_{o}$, are shown in (B).

Fig. 9: The trajectories of particles with varying inertia (low to high) are shown in interaction time during particle-eddy interactions (A), (C), and (E). The paths in particle time are shown for comparison, paths (B), (D), and (F) respectively.

Fig. 10:Trajectories from Case 1 chosen to exemplify the extremes of spread in the $x$ and $y$ directions of the burnout points. 
Fig. 11:Burnout points for variation in initial diameter, D. Various scatter plots of the burn out points for different initial diameter are shown in increasing order. An ensemble size of 350 was used to generate these plots. Larger droplets travel further downstream and are less dispersed in the y direction (height).

Fig. 12:Comparison between the experimentally measured spread in burnout endpoints and the spread predicted by the simulation. This simulated data comes from an ensemble size of 350. 

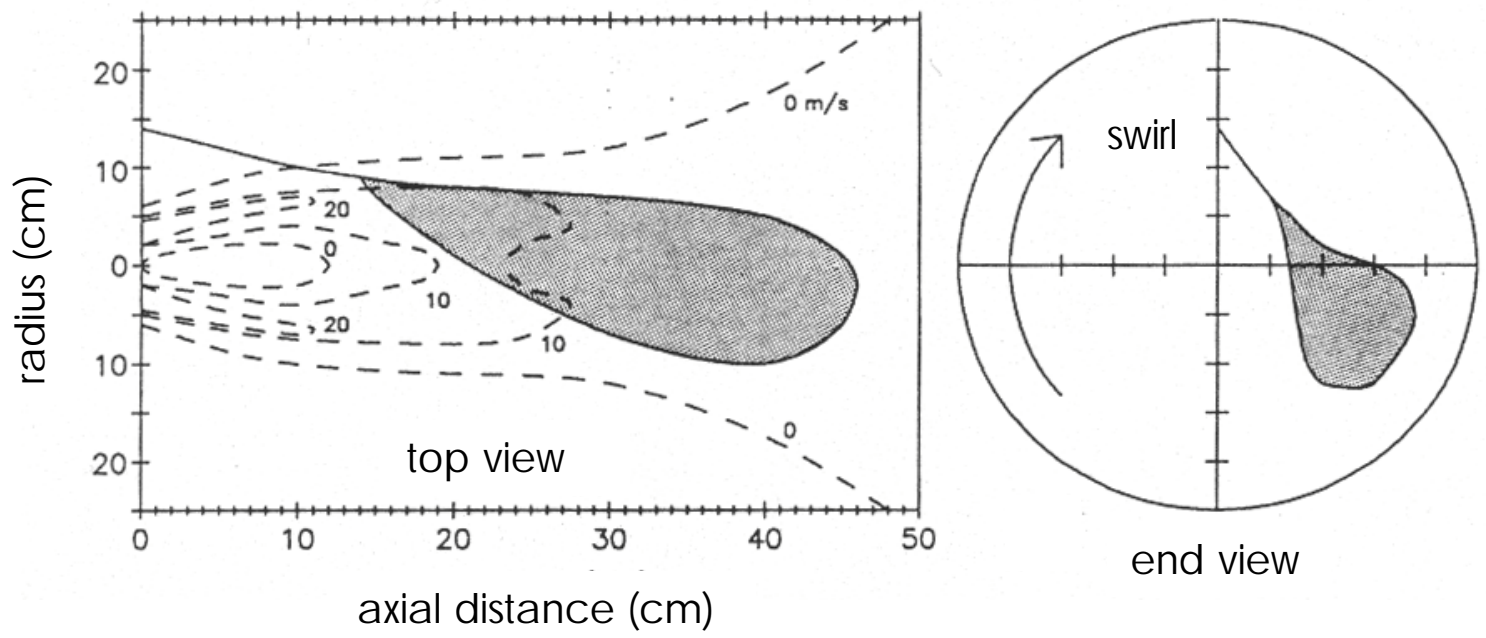

Fig. 1. Measured trajectories for the nominal droplet stream condition (Case 1) in a turbulent flame. The shaded area represents the range of trajectory endpoints. The gas phase axial velocity profile is superimposed on the top view (dashed lines). Taken from Ref. [2] with permission of the authors. 


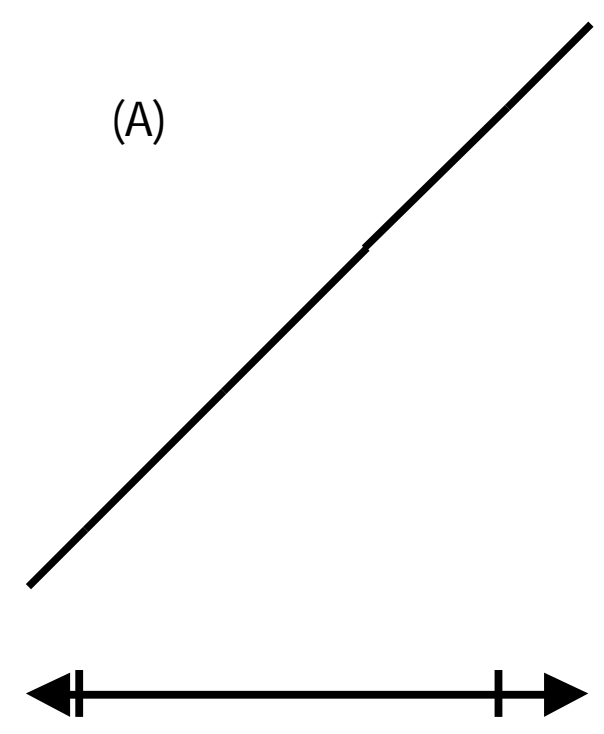

(B)

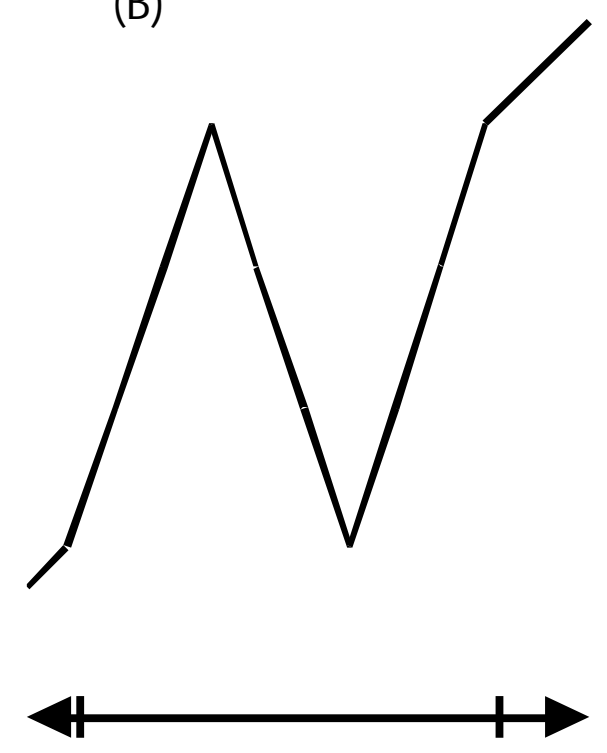

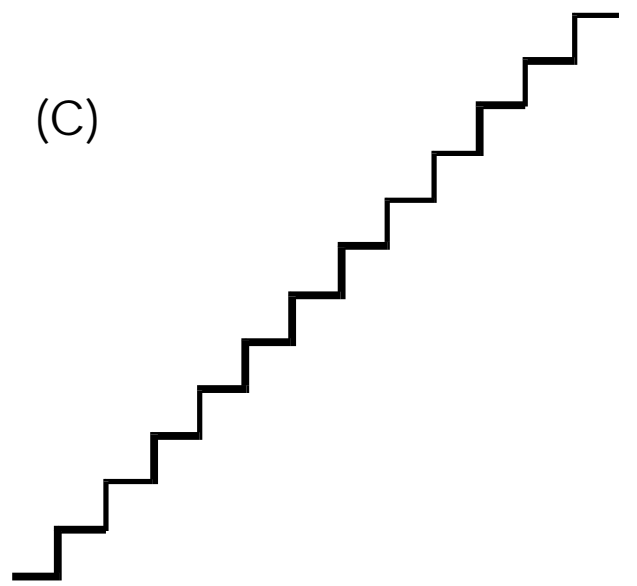
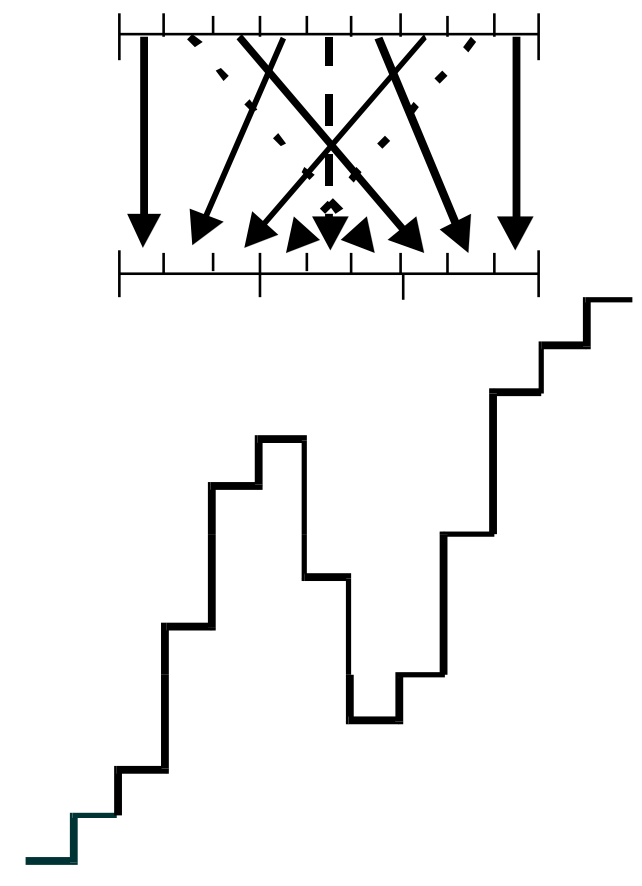

Fig. 2. Effect of the triplet map on an initially linear velocity profile $u(y, t)$. (A) Initial profile. (B) Velocity profile after applying the triplet map to the interval denoted by ticks. (C) Discrete representation of the initial profile and illustration of the effect of a triplet map on an interval consisting of nine cells. For clarity, arrows indicating formation of the central of the three images of the original interval are dashed. Redrawn from Ref. [5] with permission of the author. 


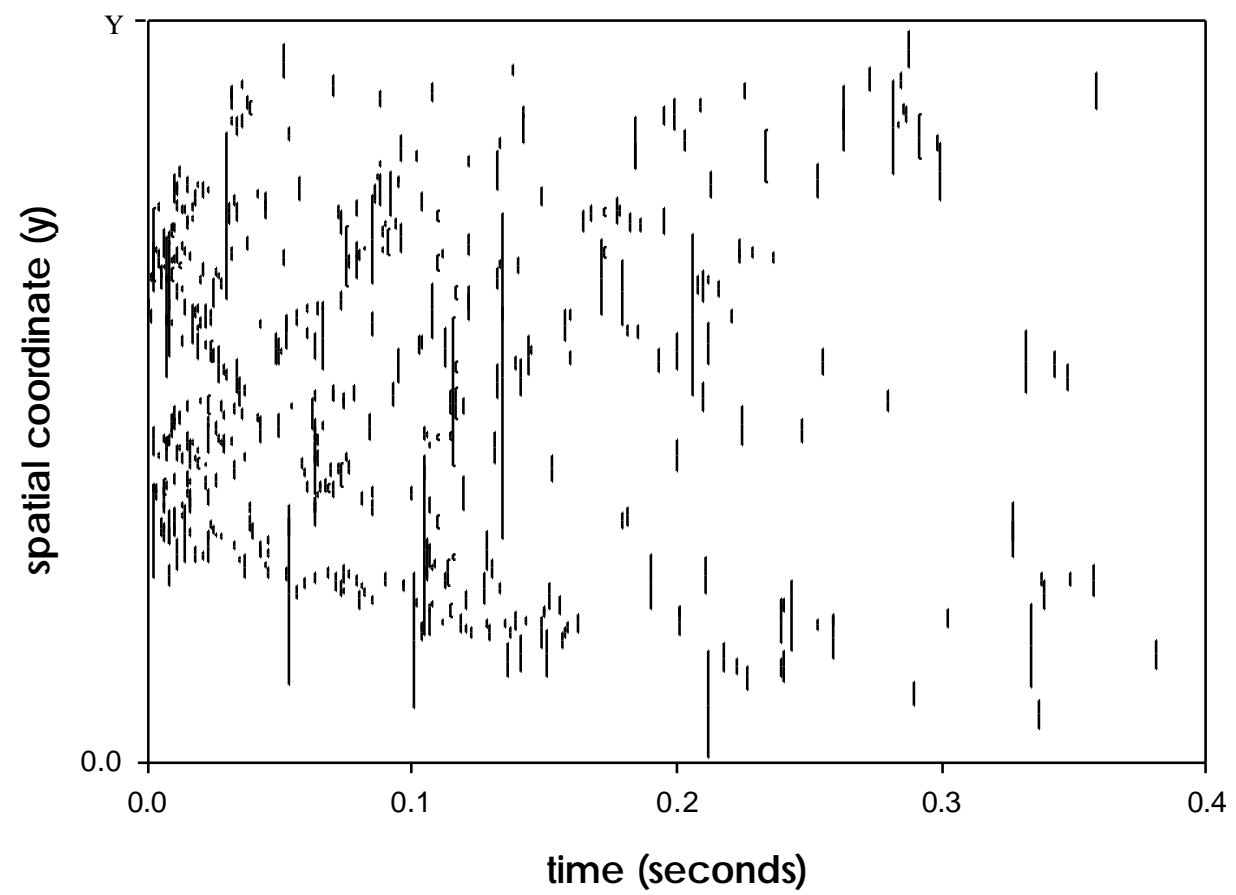

Fig. 3. Pictorial representing the eddy events in space-time coordinates. Vertical lines represent spatial ranges and times of occurrence of eddies. Presented here is the space-time history of an actual realization used in this paper. 


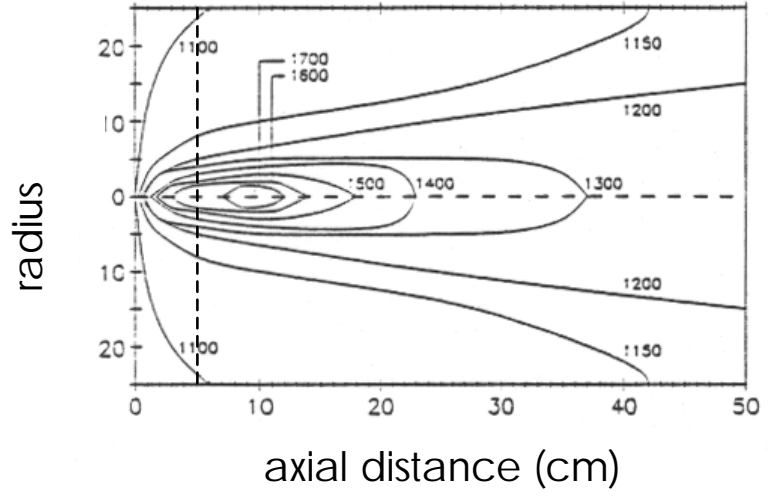

(a) temperature $(K)$ profile

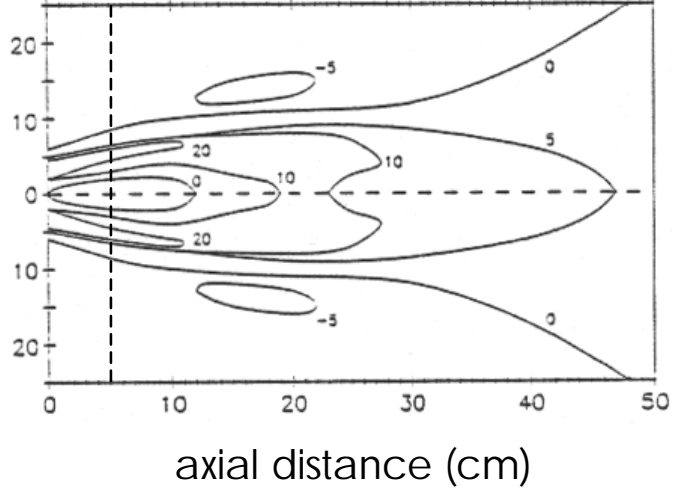

(b) axial velocity $(\mathrm{m} / \mathrm{s})$ profile

Fig. 4. Measured mean temperature (a) and axial velocity (b) profiles. These are the measured near-burner profiles. The dashed vertical lines were added to facilitate comparison with the simulated profiles shown in Fig. 5. Taken from Ref. [2] with permission of authors. 


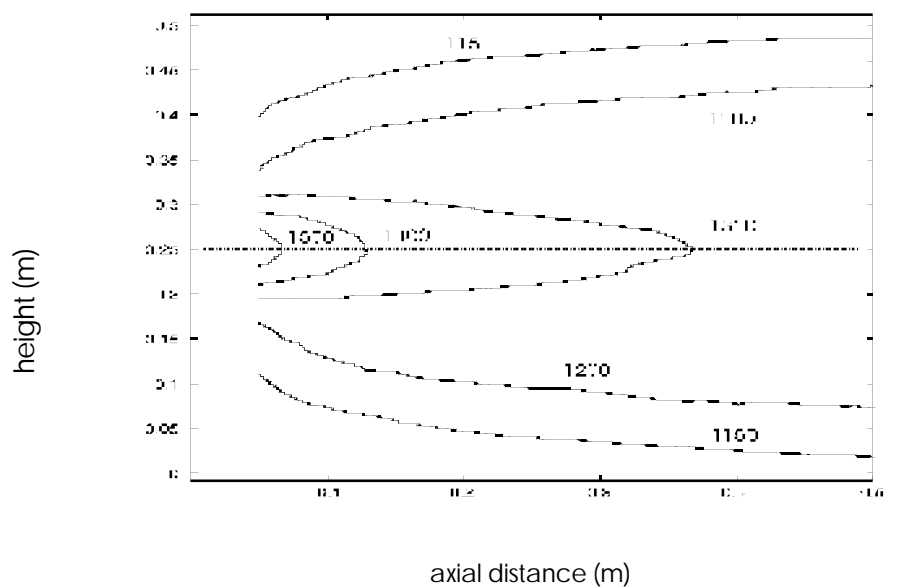

(a) temperature $(\mathrm{K})$ profile

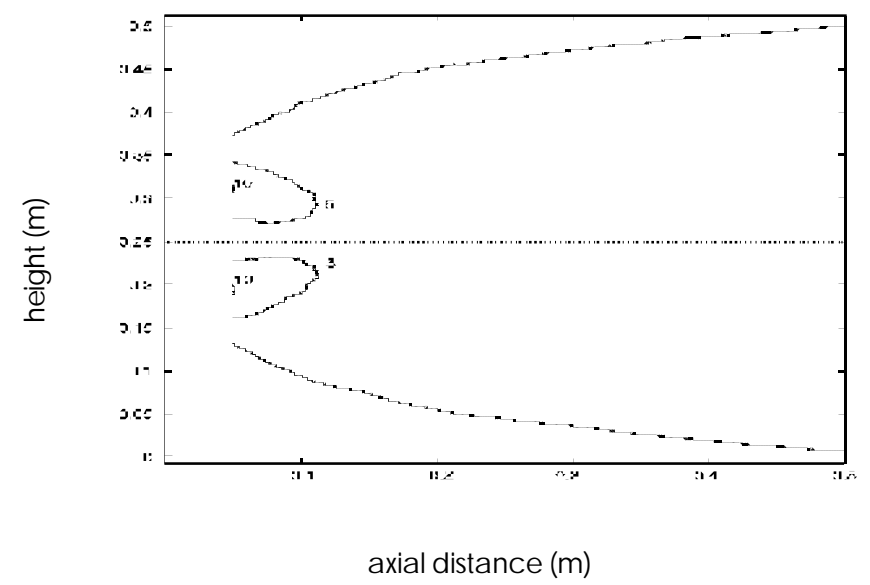

(b) axial velocity $(\mathrm{m} / \mathrm{s})$ profile

Fig. 5. Simulated mean temperature and axial velocity profiles, based on an ensemble size of 750. From here on measurements made by Mulholland et al. [2] are transformed into the ODT y coordinate as explained in the text. The ODT gas-phase simulation starts at $0.05 \mathrm{~m}$ into the reactor as explained in the text. 
(A)

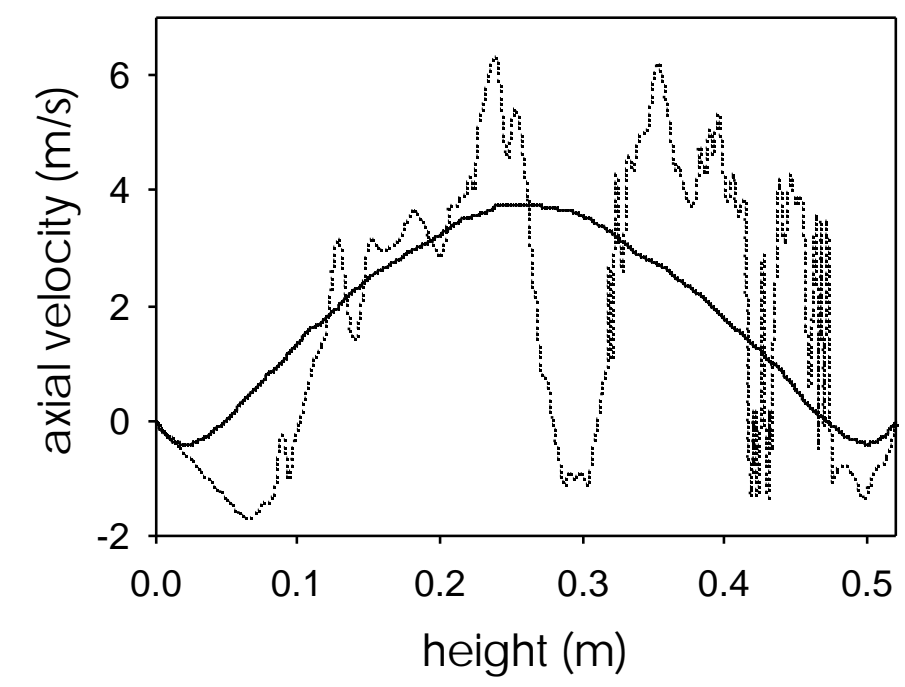

(B)

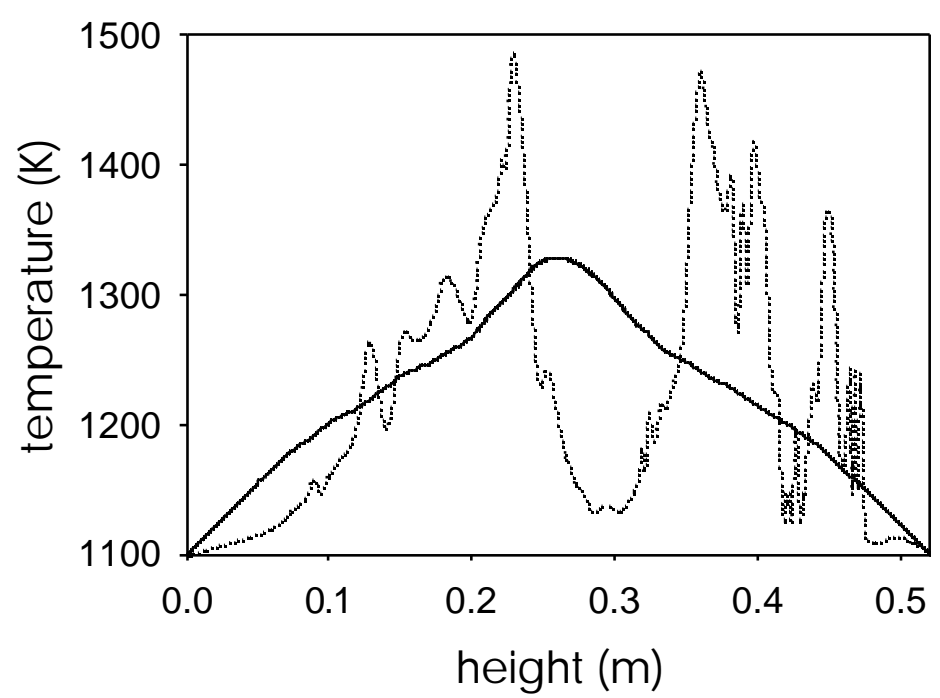

Fig. 6. Mean (solid curves) and instantaneous (dashed curves) velocity (A) and temperature (B) profiles at an axial distance of about $0.19 \mathrm{~m}$. The mean profiles are based on 2000 simulated realizations. 


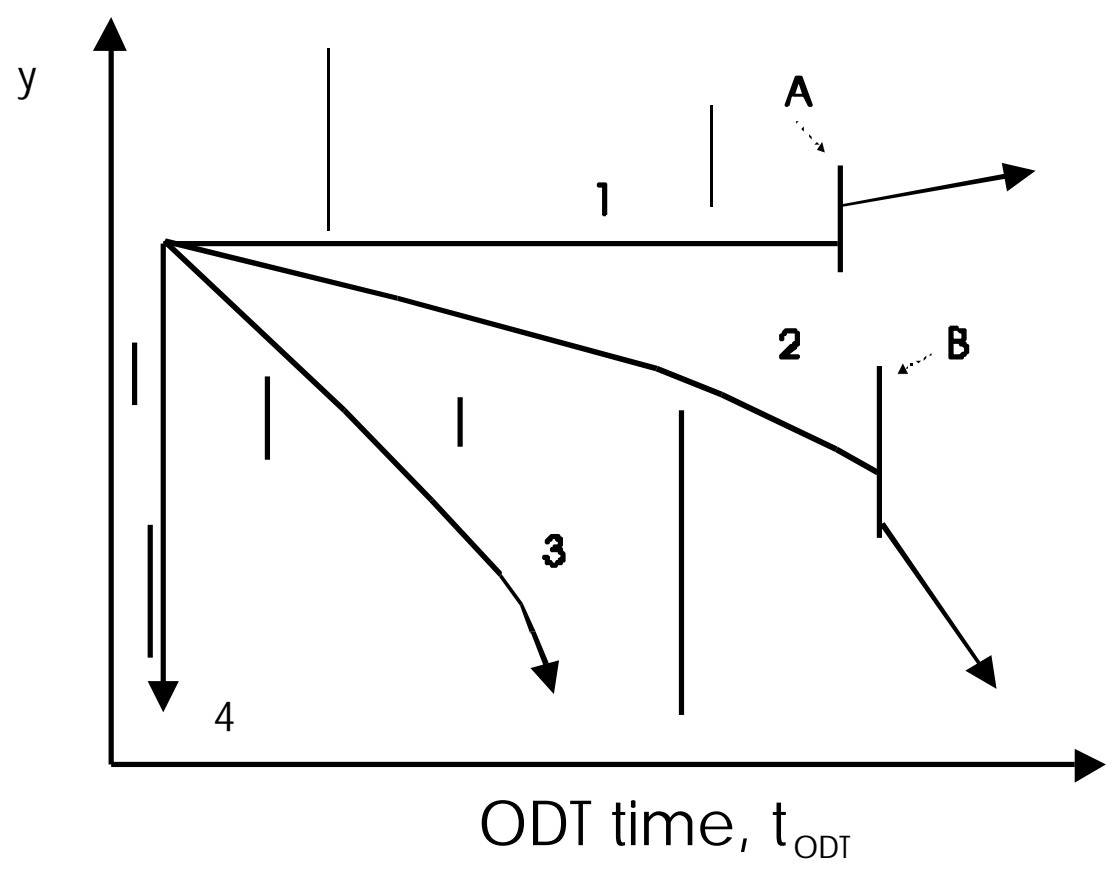

Fig. 7. Particles with high initial $y$ velocity are more likely to traverse the ODT space-time domain without interacting with an instantaneous eddy event. 

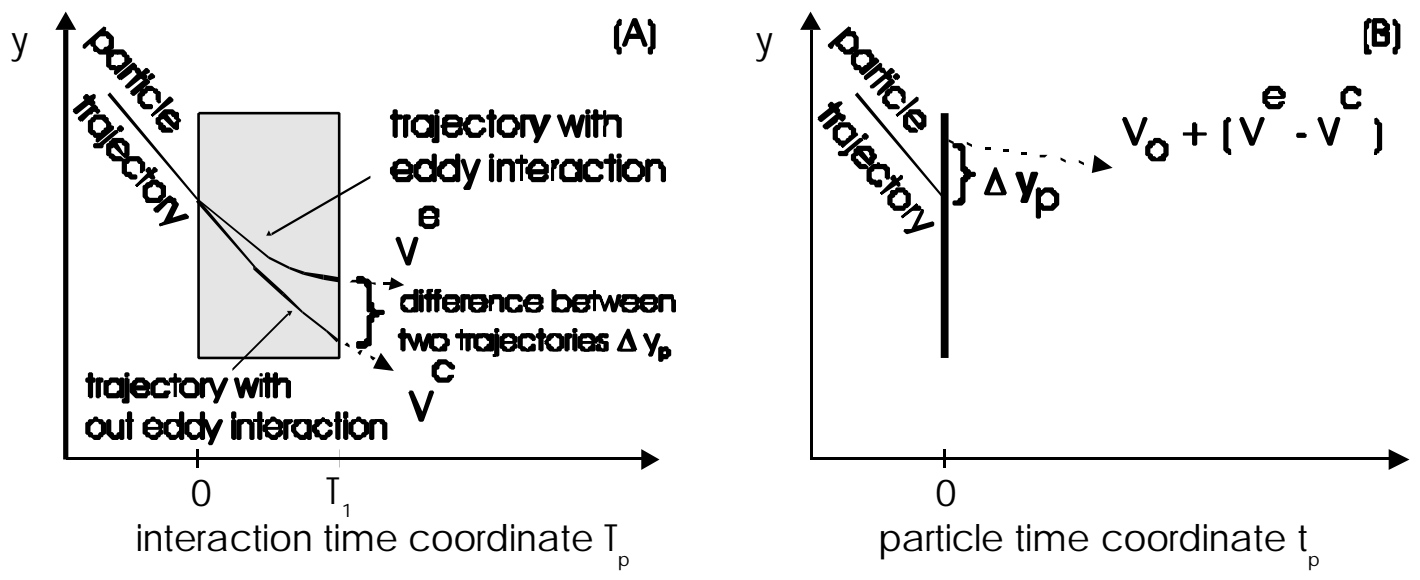

Fig. 8. The procedure for implementing particle-eddy interactions which avoids double counting. Find $\Delta y_{\mathrm{p}}$ and $\Delta \mathrm{V}$ by integrating the drag law with and without eddy interaction (A) then add them to the original position and velocity (evaluated when the particle-eddy interaction begins). The resultant position and velocity based on the original velocity $V_{0}$, are shown in (B). 

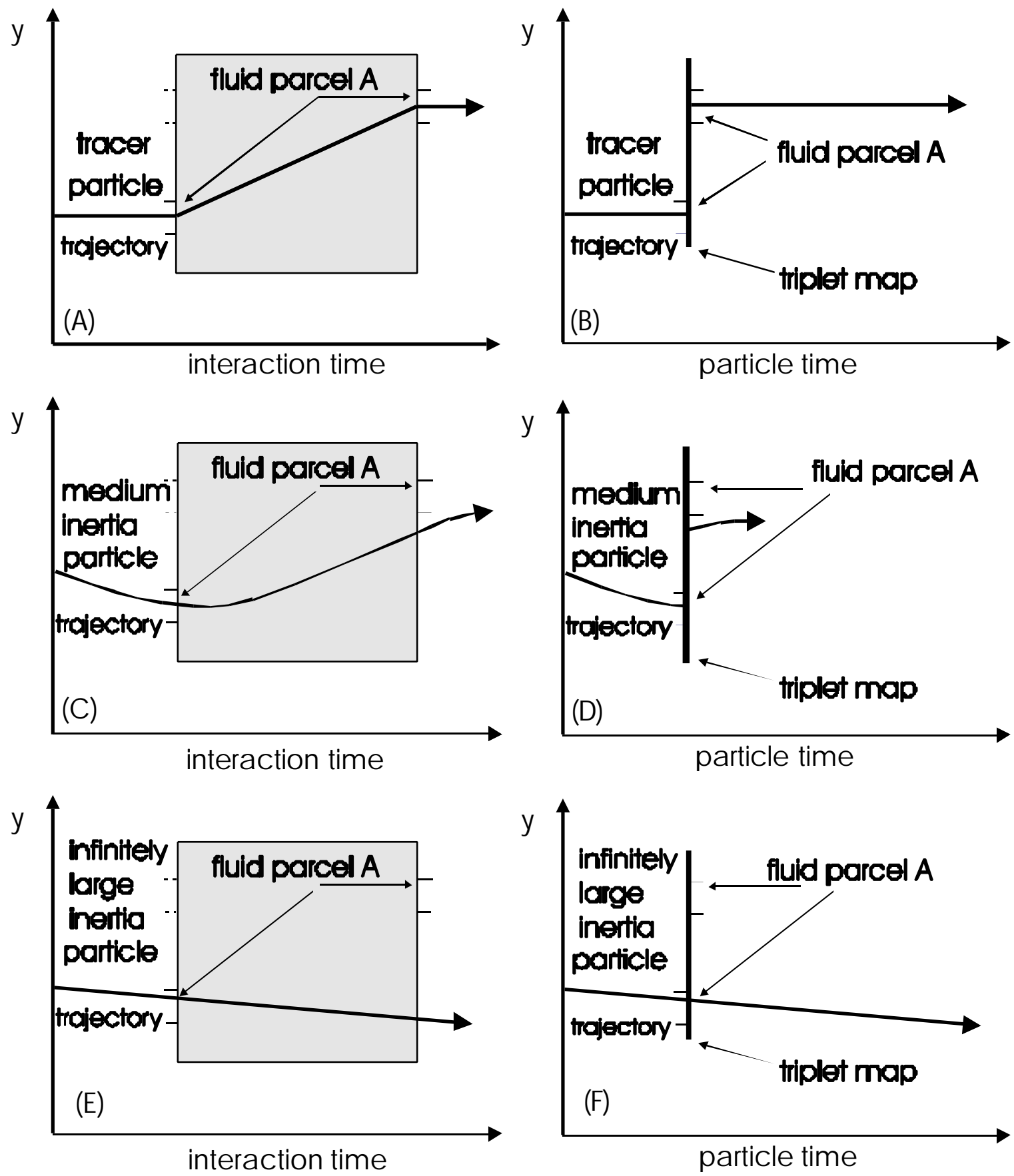

Fig. 9. The trajectories of particles with varying inertia (low to high) are shown in interaction time during particle-eddy interactions $(\mathrm{A}),(\mathrm{C})$, and $(\mathrm{E})$. The paths in particle time are shown for comparison, paths (B), (D), and (F) respectively. 


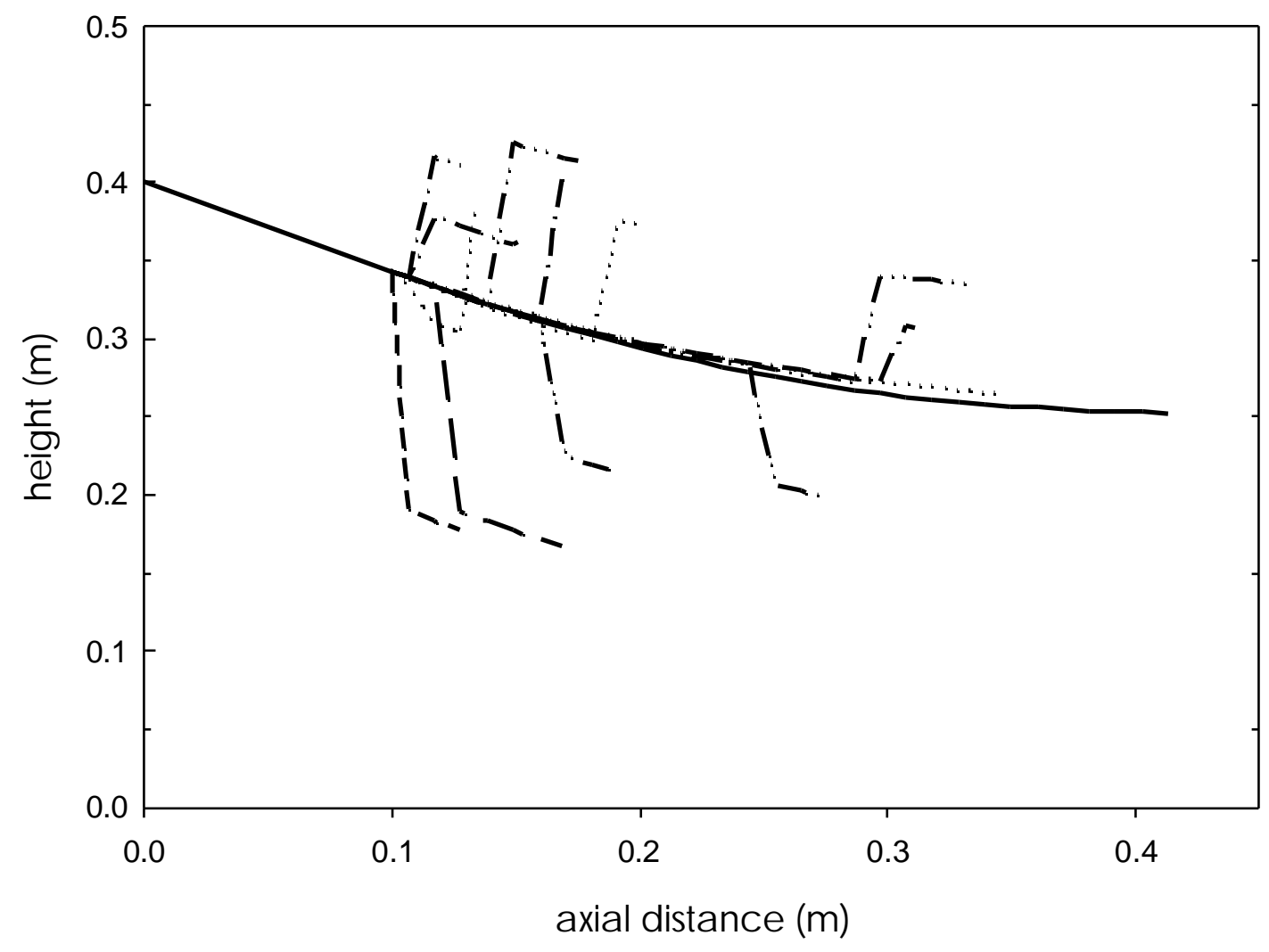

Fig. 10. Trajectories from Case 1 chosen to exemplify the extremes of spread in the $x$ and $y$ directions of the burnout points. 

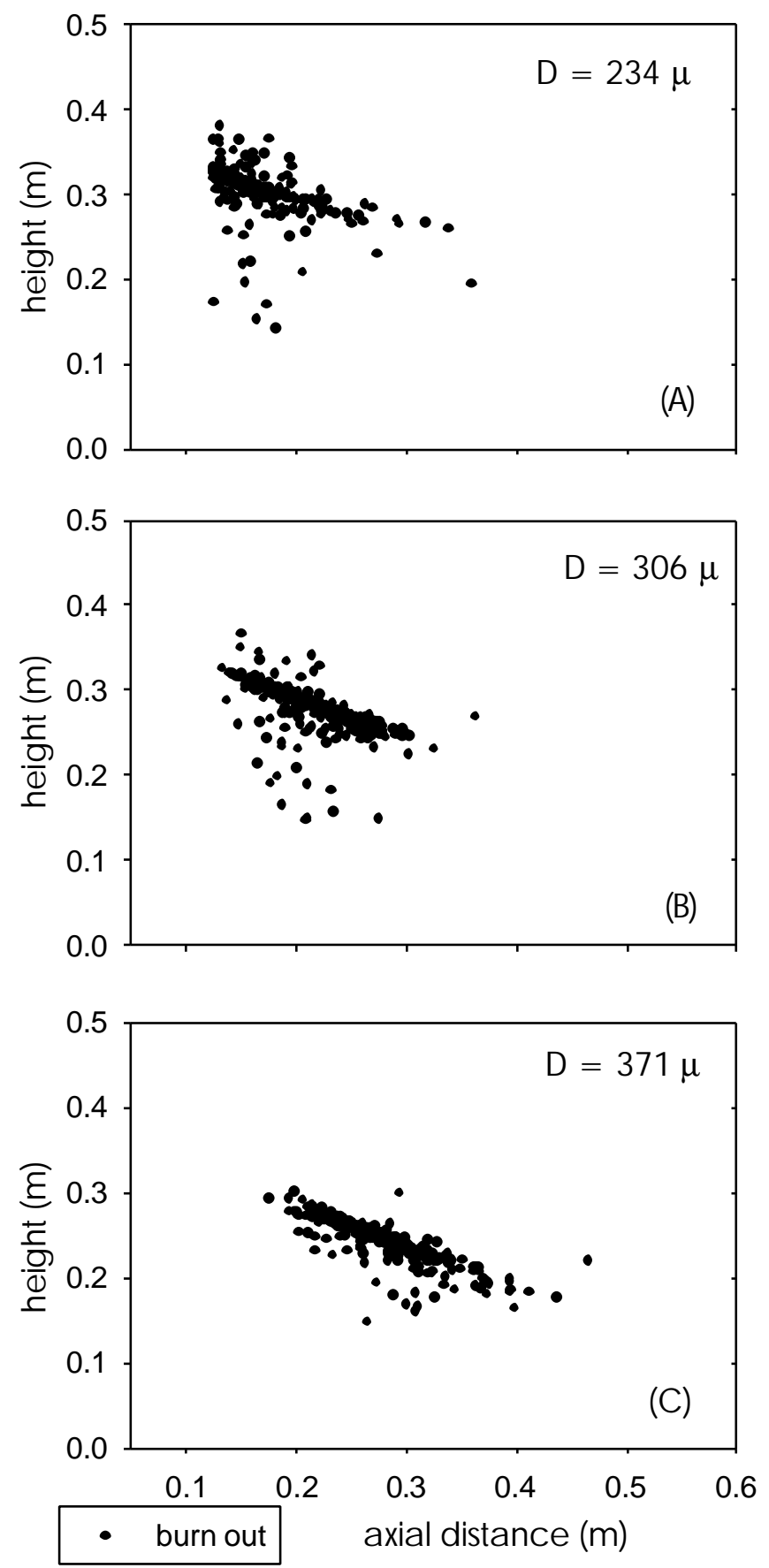

Fig. 11. Burnout points for variation in initial diameter, D. Various scatter plots of the burn out points for different initial diameter are shown in increasing order. An ensemble size of 350 was used to generate these plots. Larger droplets travel further down stream and resist being jostled around as much in the y direction (height). 

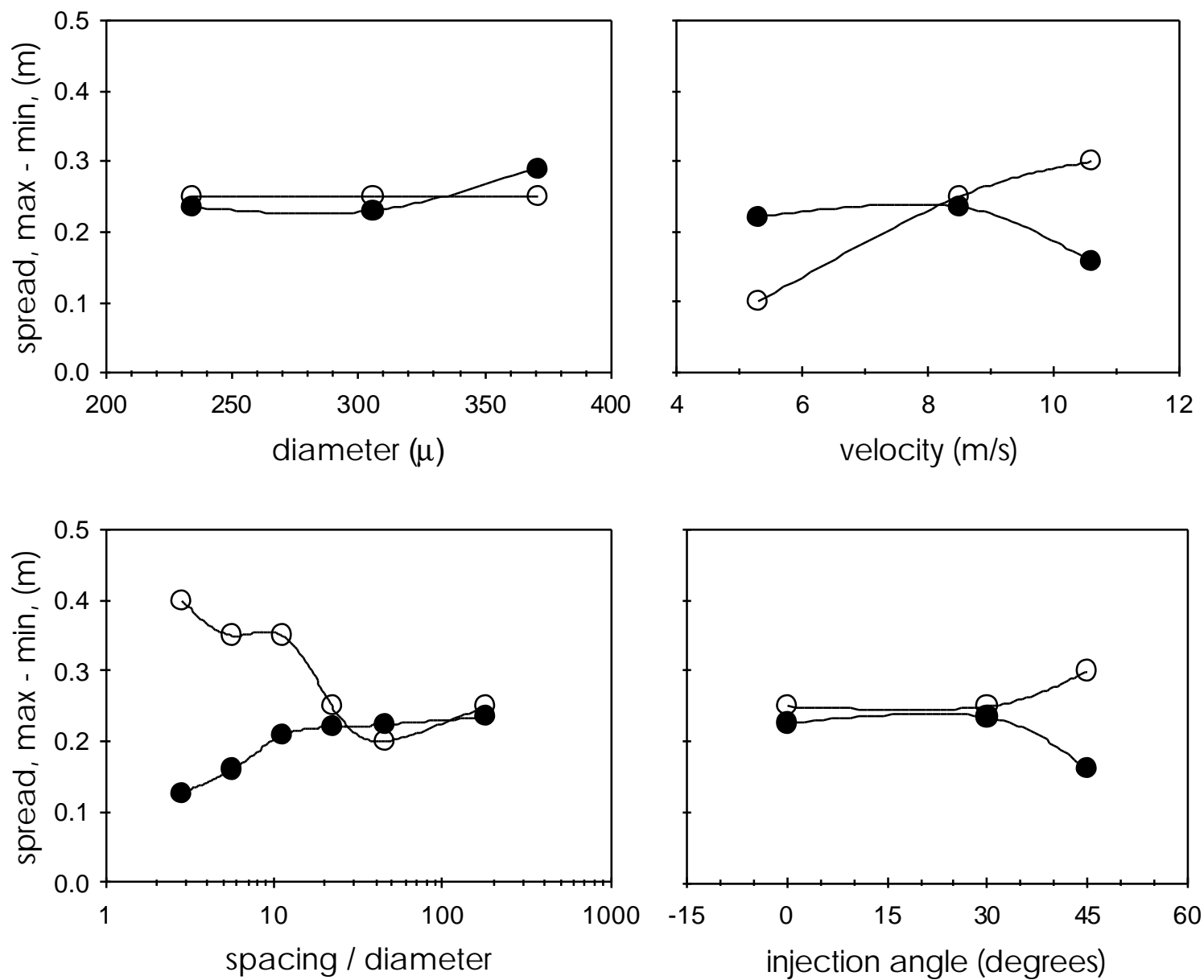

\section{- simulated $\bigcirc$ experimental}

Fig. 12. Comparison between the experimentally measured spread in burnout endpoints and the spread predicted by the simulation. This simulated data comes from an ensemble size of 350 . 